\title{
Philipp Altmann
}

\section{Die Indigenenbewegung \\ in Ecuador \\ Diskurs und Dekolonialität}

[transcript] G । o b a I s t u dies 
Philipp Altmann

Die Indigenenbewegung in Ecuador 
Philipp Altmann hat am Lateinamerika-Institut der Freien Universität Berlin promoviert. Seine Forschungsschwerpunkte sind Lateinamerika, soziale Bewegungen, Indigene und Kultursoziologie. 
Philipp Altmann

\section{Die Indigenenbewegung in Ecuador}

Diskurs und Dekolonialität

[transcript] 


\section{D188}

Unter dem Titel »Die Indigenenbewegung in Ecuador als dekolonisierender Akteur. Eine begiffszentrierte Analyse ihres Diskurses « als Dissertation an der Freien Universität Berlin eingereicht.

\section{(9) $(1 \Theta \Theta$}

Dieses Werk ist lizenziert unter der Creative Commons Attribution-NonCommercial-NoDerivs 4.0 Lizenz (BY-NC-ND). Diese Lizenz erlaubt die private Nutzung, gestattet aber keine Bearbeitung und keine kommerzielle Nutzung. Weitere Informationen finden Sie unter https://creativecommons.org/licenses/by-nc-nd/4.o/deed.de/. Um Genehmigungen für Adaptionen, Übersetzungen, Derivate oder Wiederverwendung zu kommerziellen Zwecken einzuholen, wenden Sie sich bitte an rights@transcript-verlag.de

\section{(C) 2013 transcript Verlag, Bielefeld}

Die Verwertung der Texte und Bilder ist ohne Zustimmung des Verlages urheberrechtswidrig und strafbar. Das gilt auch für Vervielfältigungen, Übersetzungen, Mikroverfilmungen und für die Verarbeitung mit elektronischen Systemen.

\section{Bibliografische Information der Deutschen Nationalbibliothek}

Die Deutsche Nationalbibliothek verzeichnet diese Publikation in der Deutschen Nationalbibliografie; detaillierte bibliografische Daten sind im Internet über http://dnb.d-nb.de abrufbar.

Umschlagkonzept: Kordula Röckenhaus, Bielefeld

Lektorat \& Satz: Philipp Altmann

Druck: Majuskel Medienproduktion $\mathrm{GmbH}$, Wetzlar

Print-ISBN 978-3-8376-2570-7

PDF-ISBN 978-3-8394-2570-1

Gedruckt auf alterungsbeständigem Papier mit chlorfrei gebleichtem Zellstoff.

Besuchen Sie uns im Internet: http://www.transcript-verlag.de

Bitte fordern Sie unser Gesamtverzeichnis und andere Broschüren an unter: info@transcript-verlag.de 


\section{Inhalt}

\section{Einleitung | 7}

1. Theorien und Methoden der Forschung | 15

2. $\mathrm{Zu}$ den Begriffen soziale Bewegung und Organisation der sozialen Bewegung | 21

3. Forschungsdesign $\mid 40$

II. Geschichte der Indigenenbewegung Ecuadors | 55

1. Allgemeines | 55

2. Federación Ecuatoriana de Indios (FEI) $\mid 68$

3. FENOCIN | 86

4. FEINE | 102

5. CONAIE | 108

6. Die Indigenenbewegung Ecuadors aus der Perspektive der Forschung zu sozialen Bewegungen I 177

\section{Begriffe der Indigenenbewegung | 207}

1. Von Land zu Territorialität | 211

2. Von Bauern zu indigenen Völkern und Nationalitäten । 218

3. Plurinationalität $\mid 230$

4. Interkulturalität | 246

5. Zum Sonderfall Sumak Kawsay I 259

6. Zusammenfassung | 267

\section{Plurinationalität/Interkulturalität und kulturelle} Ungleichheit | 287

1. Kolonialität und kulturelle Ungleichheit | 287

2. Plurinationalität und Interkulturalität als dekolonialisierende Begriffe $\mid 296$

V. Schluss | 303

Abkürzungen । 309

Abbildungen und Tabellen | 313

Literatur | 30915 
Sonstige Quellen | 345

Internetseiten | 347 


\section{Einleitung}

In der Verfassungsgebenden Versammlung 2007/2008 kam es zu einem Konflikt, der so nur von wenigen erwartet wurde. Die Frage, wie die multiethnische Zusammensetzung Ecuadors verstanden werden sollte, führte zu zwei Antworten, deren Vertreter sich intensiv bekämpften. Die Konfliktlinien gingen quer durch die Fraktionen der Versammlung und die Zivilgesellschaft. Die Indigenenbewegung, von manchen als homogener Akteur gesehen, war zerstritten. Auf der einen Seite gab es die Verfechter des Begriffs der Plurinationalität. Das war die größte Indigenenorganisation CONAIE ${ }^{1}$, die ihr nahestehende Partei MUPP-NP ${ }^{2}$ und einige Abgeordnete der Mehrheits- und Regierungspartei Alianza PAÍS (AP), vor allem das AP- und CONAIE-Mitglied Mónica Chuji. Auf der anderen Seite befand sich die Sozialistische Partei, die eine Koalition mit der AP formte und weitgehend von einer anderen Indigenenorganisation, der FENOCIN ${ }^{3}$, kontrolliert wurde. Diese Position kritisierte den Vorschlag der Plurinationalität als ethnozentrisch oder Angriff auf das Vaterland und wurde von vielen liberalen und konservativen Abgeordneten unterstützt. Der Gegenvorschlag dieser Gruppe war der Begriff der Interkulturalität. Die Auseinandersetzung wurde dadurch gelöst, dass beide Begriffe ohne genaue Definitionen in die Verfassung übernommen wurden - daher auch nicht so weitgehend, wie das sich beide Gruppen gewünscht hatten (vgl. Simbaña 2008a: 240). So bewegten sich beide Organisationen der Indigenenbewegung in den folgenden Jahren immer weiter von der Regierung weg und begannen, über gemeinsame Aktionen zur Durchsetzung ihrer Forderungen zu verhandeln.

1 Confederación de Nacionalidades Indígenas del Ecuador (Konföderation der indigenen Nationalitäten Ecuadors).

2 Movimiento de Unidad Plurinacional Pachakutik - Nuevo País (Bewegung der Plurinationalen Einheit Pachakutik - Neues Land).

3 Confederación Nacional de Organizaciones Campesinas, Indígenas y Negras (Nationale Föderation der Bauern-, Indigenen- und Schwarzenorganisationen). 
Beide Begriffe, Plurinationalität wie Interkulturalität, waren bereits seit Beginn bzw. Mitte der 1990er Jahre bekannte Forderungen der Indigenenbewegung, die mit ihnen eine stärkere Partizipation der Indigenen und einen Abbau von Diskriminierung und Ungleichheit verbindet. Beide Begriffe spielen eine wichtige Rolle im Kampf gegen das, was die größten Indigenenorganisationen als kulturelle und soziale Ungleichheit beschreiben. Tatsächlich kann man in Ecuador die Verbindung von ethnischen und sozialen Faktoren in einem schon lange bestehenden, weitgehend undurchlässigen sozialen System beobachten - von Aníbal Quijano als Kolonialität der Macht bezeichnet.

Die indigenen Organisationen in Ecuador haben, vor allem seit dem Inti RaymiAufstand 1990, konkrete Forderungen, wie die nach bestimmten Rechten, wirtschaftlicher Unterstützung oder politischer Repräsentation, mit anderen, weniger konkreten oder transzendenten Forderungen, die sich um die Begriffe Interkulturalität und Plurinationalität herum aufbauen, verbunden. Diese beiden Begriffe beinhalten - je nach der Verwendung, die ihnen eine bestimmte Organisation zu einer bestimmten Zeit zukommen lässt - durchaus konkrete Forderungen, integrieren sie aber in ein komplexes, gesamtgesellschaftlich gedachtes Projekt.

Die vorliegende Arbeit widmet sich der Frage, was die Begriffe Plurinationalität und Interkulturalität bedeuten, wie sie sich entwickelt haben und ob sie als dekoloniale oder dekolonialisierende Begriffe im Sinne Quijanos zu verstehen sind. Dazu wird in einem ersten Schritt untersucht, wer diese Begriffe in welchem Kontext entwickelt. Anschließend wird die Entwicklung der Begriffe selbst nachgezeichnet und schließlich werden sie auf ihren dekolonialen Inhalt hin geprüft. Diese drei Schritte sind in drei Teile der vorliegenden Arbeit aufgeteilt, von denen sich jeder einer anderen Methode bedient. Der erste Teil zeichnet die Geschichte der Indigenenbewegung Ecuadors und ihrer Organisationen nach. Er bedient sich vor allem der Texte der Organisationen selbst, die durch Sekundärquellen bzw. wissenschaftliche Arbeiten ergänzt werden. Dieser Versuch einer Geschichte von unten wird von einer weitergehenden Interpretation der ecuadorianischen Indigenenbewegung im Lichte der Theorien sozialer Bewegungen abgeschlossen. Der zweite Teil der Arbeit analysiert die zentralen Begriffe der Indigenenbewegung aus der Perspektive einer begriffszentrierten Diskursanalyse ${ }^{4}$, die sich an Begriffsgeschichte und Diskurstheorie orientiert. Dazu werden zuerst die verschiedenen Begriffe einzeln behandelt, um so ihre Position im Diskurs der Indigenenbewegung und die Wechselbeziehungen zwischen ihnen deutlich machen zu können. Erst im Anschluss werden sie mit diskursanalytischen und begriffsgeschichtlichen Instrumenten als analytische Einheit behandelt und als Bestandteile eines relativ kohärenten Diskurses interpretiert. Dieses Vorgehen wurde gewählt, um die Untersuchung der Begriffe

4 Diese wird im Abschnitt I.3. entwickelt. 
nicht von vorneherein theoretisch und methodisch einzuschränken und die Beachtung theoretisch wenig relevanter Details zu gewährleisten. Der dritte Teil schließlich untersucht in einem ersten Kapitel die Wahrnehmung der Lebenswirklichkeit in Ecuador durch die Organisationen der Indigenenbewegung als kolonial oder kolonialisierend, um anschließend ihre Forderungen und Vorschläge auf gegen diese Kolonialität gewendete Inhalte zu untersuchen. Dabei stützt sich dieser letzte Teil auf die Theorie der Kolonialität der Macht und des Wissens von Aníbal Quijano, Walter Mignolo und anderen. Insgesamt verfolgt die vorliegende Arbeit einen analytischen Ansatz, der sich an den zu untersuchenden Phänomenen orientiert - und nicht in erster Linie an bestimmten Theorien.

Es werden also in drei Teilen drei Fragenkomplexe untersucht, die aufeinander aufbauen und dennoch in sich geschlossen sind. Diese Schließung wird jeweils durch ein theoretisch geprägtes Schlusskapitel deutlich.

1. Wo tauchen die untersuchten Begriffe auf? In welchem Kontext und durch wen werden sie verwendet?

Dieser Fragenkomplex widmet sich den Indigenenorganisationen und ihrer Entwicklung, um so eine Kontextanalyse der untersuchten Begriffe zu erlauben.

2. Wann tauchen die untersuchten Begriffe auf? Wie entwickeln sie sich?

Dieser Fragenkomplex widmet sich der Begriffsgeschichte der einzelnen Begriffe und ihrer Funktion im Diskurs der Indigenenbewegung.

3. Sind die Begriffe und der Diskurs der Indigenenbewegung dekolonial im Sinne der Kolonialität der Macht? Wie ist das Verständnis der Indigenenbewegung von Kolonialität bzw. systematischer Diskriminierung?

Dieser Komplex versucht, die beiden vorangegangenen unter der Perspektive der Kolonialität der Macht zu integrieren.

Die meisten Forschungsarbeiten müssen sich zwischen einem diachronen und einem synchronen Ansatz entscheiden oder zwischen einem Vergleich vieler, gleichzeitig stattfindender Phänomene und der Erforschung der Entwicklung eines Phänomens. Die vorliegende Arbeit hat sich zu einem eher diachronen Ansatz entschlossen. Ein Phänomen - die zentralen Begriffe der ecuadorianischen Indigenenbewegung - soll möglichst vollständig erfasst werden. Das führt dazu, dass synchrone Phänomene - etwa Indigenenbewegungen anderer Länder - tendenziell vernachlässigt werden. Genauso wird die Erforschung des Indigenismus als intellektuelle Bewegung der mestizischen ${ }^{5}$ (nicht-

5 Mestizen (sp. mestizos) verstehen sich in einem kulturellen und biologischen Sinne als Produkt der Mischung von Indigenen und Europäern. 
indigenen) Mittelschicht und der Akademie (siehe etwa Becker 2007) vernachlässigt abgesehen von den wenigen direkten Einflüssen, die nachweisbar sind. Das schließt auch Bedeutungskämpfe über bestimmte Begriffe außerhalb der Indigenenbewegung aus - die Frage, wer in Ecuador warum und wie von Interkulturalität spricht (Walsh 2003: 115) geht weit über den hier entwickelten Forschungsrahmen hinaus. Der erforschte Zeitraum beginnt mit der Gründung der ersten indigenen Gewerkschaften, die 1944 die Ecuadorianische Indio-Föderation (Federación Ecuatoriana de Indios, FEI) gründen sollten, und bezieht sich auf die organisatorischen Aspekte der so beginnenden Indigenenbewegung. Allerdings wird keine Ereignisgeschichte betrieben, wie sie in manchen Schriften zu konkreten Mobilisierungen zu finden ist. Es sollen nicht tagespolitische Ereignisse nachgezeichnet, sondern allgemeine Entwicklungen deutlich gemacht werden.

Auch die vielfältigen Texte, die die Begriffe der Indigenenbewegung philosophisch und ohne den Kontext der Bewegung selbst analysieren - die Begriffe Interkulturalität und Gutes Leben (Sumak Kawsay) sind gute Beispiele dafür - werden in der vorliegenden Arbeit nur am Rande bearbeitet. Die philosophische Interpretation der Begriffe, die die Indigenenbewegung verwendet, und ihr Weiterdenken wird nur dann aufgegriffen, wenn sie unmittelbar dem Verständnis dieser Begriffe und ihrer Entwicklung dient. Insbesondere auf nicht-empirisch orientierte Texte zu Interkulturalität und pluriethnische Zusammensetzung von Staaten wird kein Bezug genommen, um den Schwerpunkt auf den Diskurs der Indigenenbewegung legen zu können und nicht in ein Überprüfen von Theorien übergehen zu müssen.

Viele der bisherigen Arbeiten zur ecuadorianischen Indigenenbewegung sind einem politikwissenschaftlichen Ansatz verpflichtet und konzentrieren sich auf die Analyse staatlicher Institutionen im Sinne der Demokratisierungstheorie. Einflussreich ist dabei Donna Lee Van Cott (2005), die den Begriff der Ethnopolitik von Max Hildebert Boehm, einem Vertreter der völkischen Bewegung und der konservativen Revolution der 1920er, weiterentwickelt und unter anderem auf Ecuador anwendet. Das Politikverständnis dieser Denklinie führt zu einer Überbetonung von Parteien und staatlichen Institutionen - weshalb die Gründung von Pachakutik als erster indigener Partei in Ecuador im Sinne von einem Fortschritt von einer gering institutionalisierten sozialen Bewegung hin zu einer hochgradig institutionalisierten politischen Partei verstanden wird - ein Verständnis, das hier nicht geteilt wird. Auch die Arbeit, die dem hier vorliegenden Ansatz am nächsten kommt, die Dissertation von Robert Andolina (1999), ist wegen dieser Staatsbezogenheit gewissen Beschränkungen unterworfen.

Ein weiterer Ansatz, der hier nicht verfolgt wird, ist die ethnologische Herangehensweise, wie sie etwa Sánchez-Parga anwendet. Es soll der von den Organisationen getragene und weiterentwickelte Diskurs untersucht werden und nicht seine Verankerung in der Basis. Auch wenn es durchaus interessant wäre, das Verständ- 
nis von Plurinationalität in einer indigenen Gemeinde zu untersuchen, oder lokale Indigenenführer zu befragen, was für sie eine indigene Nationalität ausmacht, ist das eine völlig andere Forschung, die völlig andere Methoden nötig macht. Auf den deutschen Kontext übertragen wäre das wie eine Analyse der Parteitagsbeschlüsse der SPD ${ }^{6}$ im Vergleich mit einer Befragung in einem bestimmten Ortsverein der SPD, was denn Sozialdemokratie oder Solidarität bedeutet. Zwei sicherlich interessante Forschungen - die allerdings in ihrer doppelten Dimension als Grundlagenund vergleichende Forschung eine großen institutionellen Rahmen nötig machen.

\section{Was ist der Indigene?}

Eine Arbeit über die ecuadorianische Indigenenbewegung macht ein Verständnis der Kategorie des Indigenen notwendig. Als Indigene versteht die vorliegende Arbeit jene Völker, die in schon vor der europäischen Eroberung in Amerika gelebt haben. Die Erfahrung der Eroberung, der Kolonie und der Republik haben ihre Identitäten im Kontext ihres Landes besonders geformt (Comisión Especial de Asuntos Indígenas 1988: 9f.). Die Kategorie der Indigenen bezeichnet die Kolonisierten, ist also nur im Sinne von Eroberung und Kolonie zu verstehen. Vor diesem Einschnitt gab es keine Indigenen, sondern nur die verschiedenen Völker, etwa Kichwa, Shuar, Maya, usw. - die ebenfalls von Eroberung und Kolonie entscheidend verändert wurden. Also wird der Indigene von den Kolonisierern, den Europäern als Anderer und Unterlegener erschaffen, er ist ein Effekt der Kolonisierung Amerikas (Bonfil Batalla 1981: 19).

„Die Kategorie des Indios ist supraethnisch, das heißt, sie bezieht sich nicht auf die Verschiedenheit der Völker, die unter dem Titel Indios zusammengefasst werden, oder berichtet über sie, denn die Definition selbst (der Begriff des Indios) geht vom Unterschied mit dem NichtIndio aus und diese Unterscheidung ist die einzige, die zählt, das, was dem Indio-Sein Sinn gibt.“ (Bonfil Batalla 1981: 20) ${ }^{7}$

Daher ist die Identität nicht essentiell, sondern prozesshaft; sie wird über die Abgrenzung von Individuen und Gruppen von anderen als grundlegendes Prinzip ihrer Selbsterhaltung erzeugt (Almeida 1997: 175). Die Konstruktion der indigenen und der mestizischen Identität im Kontext der Kolonialität der Macht wird später genauer behandelt.

6 Oder einer beliebigen anderen Partei.

7 Alle Übersetzungen durch den Autor. 


\section{Indigene in Ecuador}

Der Staat Ecuador entstand als ein künstliches Gebilde ohne wirtschaftliche oder soziale Kohärenz oder ein festgelegtes Territorium - und blieb lange Zeit so (Simbaña 2005: 201). Die drei urbanen Zentren Guayaquil, Quito und Cuenca strebten in den ersten Jahrzehnten der Existenz Ecuadors als unabhängiger Staat eine weitgehende Eigenständigkeit an. Gleichzeitig bestimmte die zunehmende Integration des jungen Landes in den Weltmarkt die Entwicklung auch der politischen und sozialen Strukturen Ecuadors (Acosta 2001: 15). So führte eine Gesellschaftsordnung, die in erster Linie auf Großgrundbesitz fußte und daher auf wirtschaftliche Öffnung angewiesen war, zu einem ,abhängigen kulturellen Schema“ (Acosta 2001: 48), dass die Herausbildung einer Gruppe innovativer Unternehmer oder einer nationalen Elite weitgehend verhinderte (Acosta 2001: 135). In diesem Gegensatz zwischen dem Machtstreben lokaler Oligarchien und der marginalisierenden Internationalisierung Ecuadors wurden die Indigenen, bis ca. Mitte des 20. Jahrhunderts die Bevölkerungsmehrheit des Landes, meist ignoriert. Das spiegelt sich am besten in der Sprachenpolitik des Landes wider. So wurde Spanisch als privilegierte Sprache, als Sprache der Oberschicht definiert, wodurch ein „diglossischer Bilingualismus“ (Moya 1987: 391) entstand, in dem die indigenen Sprachen auf „,eingeschränkte und stigmatisierte Kommunikationsfunktionen“ (Moya 1987: 391) beschränkt wurden. Die Versuche der politischen Eliten, ab etwa Mitte des 19. Jahrhunderts eine nationale Identität und entsprechende Institutionen aufzubauen (Selverston 1994: 148), haben diese Marginalisierung der Indigenen auch in anderen Bereichen verstärkt. So blieb etwa der unterschiedliche Zugang zu beruflichen Positionen bestehen, wie die zunehmende Migration von Indigenen in die Städte ab den 1960ern deutlich macht (Ibarra 1999: 78f.).

Die grundlegende Schwäche des ecuadorianischen Staates und seiner politischen Strukturen im Sinne eines „Defizits an Legitimität“ (Burbano 1998: 7) führt zu einer Radikalisierung der verschiedenen Forderungen, die die Strukturen weiter schwächt (Burbano 1998: 7) - während sich die jeweiligen Akteure als Vertreter der wahren Demokratie präsentieren (Burbano 1998: 4). Somit sind die grundlegenden Regeln der Politik in Ecuador ständig umstritten (Burbano 1998: 8) - während der Staat in Gesellschaft und Wirtschaft zentral ist. Das führt dazu, dass der Staat - als Ziel von Eroberung oder Gegner - für die Akteure der Politik in Ecuador im Mittelpunkt steht, während die demokratischen Institutionen relativ schwach geblieben sind (Burbano 1998: 11). Statt den Umweg über Parteien und Parlamente zu nehmen, wird versucht, unmittelbar auf die Regierung Druck auszuüben (Burbano 1998: 13f.). Die Konzentration der Macht im Staat und seine geographische Verortung in der Hauptstadt auch in den Phasen staatlich gelenkter Modernisierung in den 1960ern und 1970ern verstärkte die Bedeutung der urbanen Zentren, vor 
allem Quito und Guayaquil, weiter (Burbano 1998: 13). Diese Fixierung auf den Staat begünstigte die Entwicklung des Populismus als Politikform nach der Rückkehr zur Demokratie 1979, der sein Versprechen einer größeren Teilhabe des Volkes an den Entscheidungsprozessen nicht über eine Veränderung der Strukturen umsetzte (Acosta 2001: 142/143).

Daher lässt sich die politische Rolle der Indigenen gut an der Entwicklung der Verfassungen verfolgen - denen durch die hohe Bedeutung des Staates bei gleichzeitiger Bedeutungslosigkeit der demokratischen Strukturen eine große Zentralität zukommt. Die progressive Verfassung von 1945 erklärte in ihrem 5. Artikel Spanisch zur Amtssprache des Landes und die indigenen Sprachen zu „Bestandteilen der Nationalkultur“ (Trujillo 1992: 168). Dieser Fortschritt wurde schon in der nächsten Verfassung (1946) rückgängig gemacht, in der die Indigenen und ihre Sprachen keine Erwähnung finden. Diese Linie behielt auch die nächste Verfassung (1967) bei, die in ihrem 38. Artikel die indigenen Sprachen ,wo nötig“ für die Erziehung vorsieht, ,,damit der Erzogene in seiner eigenen Sprache die Nationalkultur begreift und dann Spanisch übt." Die Verwendung der indigenen Sprachen in der Erziehung in den von Indigenen besiedelten Gebieten sieht auch die Verfassung von 1979 in ihrem 27. Artikel vor (Trujillo 1992: 168), die zudem die indigenen Sprachen erneut als „Bestandteile der Nationalkultur“ (Art. 1) anerkennt. Im 4. Artikel verurteilt der ecuadorianische Staat ,,alle Formen von Kolonialismus, Neokolonialismus und rassischer Diskriminierung oder Abgrenzung.“ Die Verfassung von 1998 erklärt Ecuador zu einem ,,plurikulturellen und multiethnischen“ (Art. 1) Staat und erkennt die indigenen Sprachen für die „offizielle Verwendung für die indigenen Völker“ (Art. 1) an. Zudem werden in den Artikeln 83, 84 und 85 zum ersten Mal die kollektiven Rechte der indigenen Völker anerkannt - ohne dadurch den Staat zu einem plurinationalen Staat zu erklären (Simbaña 2005: 206). Die Verfassung 2008 schließlich erklärt Ecuador zu einem ,,interkulturellen, plurinationalen [...] Staat“ (Art. 1), und definiert spezielle Rechte für die indigenen Nationalitäten und der Anerkennung indigener Rechts- und Wirtschaftssysteme umsetzt (Larrea 2008: 38). In der vorliegenden Arbeit wird die Integration ethnischer Themen in die politische Kultur und schließlich in die Verfassungen von 1998 und 2008 nicht als „Öffnung des Staates [und] der Zivilgesellschaft“ (Büschges 2009: 46) verstanden, also als eine Art freiwillige Gabe der mestizischen Mehrheitsgesellschaft an die Indigenen, sondern im Sinne der Indigenenbewegung als mühsam und langfristig erkämpfte Rechte. Daher muss die höhere Präsenz dieser Themen keinesfalls eine größere Teilhabe der Indigenen bedeuten - zumindest in einigen Fällen kann davon ausgegangen werden, dass die Betonung des Ethnischen diese Teilhabe verhindern soll. In diesem Sinne beschreibt Aníbal Quijano „die immer weiter anwachsende Familie der Begriffe, die die Frage der Identität umschreiben, um sie von der Frage der Macht fernzuhalten“ (Quijano 2006a: 13). 
Nach den Daten des Zensus von 2010 (INEC 2010) leben 14.483.499 Menschen in Ecuador. Davon verstehen sich 1.018.176 Personen oder 7,0\% der Gesamtbevölkerung als Indigene. Der Zensus von 2001, der zum ersten Mal ethnisches Selbstverständnis abfragte, zählte noch 830.418 Indigene, oder 6,8\% der Bevölkerung (Chisaguano 2008: 100). Die Zahlen machen also deutlich, dass frühere Schätzungen - etwa Lucas (2000: 13), der von 3,5 Millionen Indigenen von 11,5 Millionen Ecuadorianern spricht - unverhältnismäßig hoch ausfallen. In den vorherigen Erhebungen wurden die Muttersprache der Bevölkerung abgefragt, wonach es 1950 347.745 und 1990362.500 indigene Muttersprachler gab (Chisaguano 2008: 97). Die Tatsache, dass im sich Zensus 2001830.418 Personen als Indigene verstehen, aber nur 524.136 Personen eine indigene Muttersprache angeben, zeigt die geringe Vergleichbarkeit der beiden Methoden (Chisaguano 2008: 102). Die meisten der Indigenen $(86,2 \%)$ haben in diesem Zensus Kichwa als Muttersprache angegeben, nur $8,8 \%$ Shuar und jeweils $1 \%$ oder weniger die restlichen indigenen Sprachen (Chisaguano 2008: 103). 49,2\% gaben an, der Nationalität der Kichwa und 6,3\% der Nationalität Shuar anzugehören (Chisaguano 2008: 105).

Den Indigenen stehen etwa gleich viele Mitglieder von drei weiteren Bevölkerungsgruppen gegenüber. So gibt es - zusammengerechnet - eine vergleichbare Anzahl von Afroecuadorianern (615.262), Schwarzen (145.398) und Mulatten (280.899), mit 1.070.728 geringfügig mehr Montubios - eine Volksgruppe von Bauern im Küstengebiet - und mit 882.383 deutlich weniger Weiße. Erwartungsgemäß verstehen sich die meisten Ecuadorianer (10.417.299) als Mestizen, geben also an, sowohl indigene als auch weiße Vorfahren zu haben. Unter den Indigenen ist die größte Gruppe die Hochland-Kichwa mit 328.149, ergänzt von anderen Kichwa-Völkern wie den Puruhá (136.141), den Otavalos (56.675) und anderen, kleineren Kichwa-Völkern. Die Shuar stellen mit 79.709 Mitgliedern die zweitgrößte indigene Nationalität Ecuadors. Die anderen indigenen Völker und Nationalitäten sind kleiner und haben selten mehr als 10.000 Mitglieder (INEC 2010).

Die regionale Verteilung der Indigenen ist relativ klar. Nach den Daten von 2001 leben 71,7\% der Indigenen im Andenhochland, 19,6\% im Amazonasgebiet und 8,5\% an der Küste (Chisaguano 2008: 102). In den Provinzen des Hochlandes leben daher die meisten Indigenen. In Chimborazo leben 18,5\% aller Indigenen, in Pichincha $11,5 \%$, in Imbabura 10,5\%, in Cotopaxi $10,1 \%$ und in Tungurahua $7,9 \%$ (Chisaguano 2008: 101). Alle diese Provinzen befinden sich im Hochland. Während die meisten Indigenen eine Minderheit im stark bevölkerten Andenhochland sind, stellen die wenigen Indigenen, die im Amazonasgebiet leben, einen hohen Anteil an der Bevölkerung dort. Diese Daten können nur als Annäherung genommen werden, da sie politisch hochgradig bedeutsam sind und die verschiedenen Gruppen ein Interesse an besonders hohen oder besonders niedrigen Zahlen haben. 


\section{Theorien Und Methoden der Forschung}

\section{Kolonialismus und Kolonialität}

Die Beschäftigung mit Ungleichheit in Lateinamerika hat in den 1960ern zur Entwicklung des Begriffes des internen Kolonialismus durch Wissenschaftler der neuen Linken (González Casanova 2006: 411) wie Pablo González Casanova und Rodolfo Stavenhagen geführt. Dieser Begriff sollte die Herrschaftsbeziehungen und die sozialen Strukturen innerhalb der unterentwickelten kapitalistischen Nationalstaaten der wirtschaftlichen Peripherie erklären (Quintero 2010: 12). González Casanova führt den Begriff des internen Kolonialismus auf die Analyse Lenins der Lage der unterdrückten Völkern im zaristischen Russland zurück (González Casanova 2006: 412). Darauf aufbauend hat er ihn im Laufe der 1960er zur Analyse der Klassenstruktur und der Staatsmacht in den Staaten Lateinamerikas verwandt. Von besonderem Interesse war dabei die Verbindung ethnischer Faktoren mit dem $\mathrm{Zu}$ gang zu politischer Macht im Staat und die Rolle externer Faktoren im Sinne eines Imperialismus (González Casanova 2006: 415). Interner Kolonialismus findet auf wirtschaftlichem, politischem, sozialem und kulturellem Gebiet statt und entwickelt sich zusammen mit Kapitalismus und Nationalstaat, wodurch diese Kategorie schnell von kritischen Wissenschaftlern und einigen sozialen Bewegungen aufgegriffen wurde (González Casanova 2006: 409). Die unterdrückten Völker werden in Bedingungen gedrängt, die den Kolonialismus charakterisiert haben: sie haben kein Recht auf eine Selbstverwaltung oder eine Beteiligung an Entscheidungen, stattdessen werden sie von Mitgliedern anderer Ethnien oder Völker dominiert (González Casanova 2006: 410). Diese Struktur wird durch den Kolonialismus und seine Konzentration auf die Metropolen bei gleichzeitiger Vernachlässigung der Beziehungen innerhalb der Kolonien und zwischen ihnen (González Casanova 1969: 232f.) gebildet. Damit geht eine enge Einbindung bestimmter Ethnien in politische Entscheidungen einher, die dazu führt, dass „die Herrschafts- und Ausbeutungsbeziehungen Beziehungen zwischen heterogenen, kulturell verschiedenen Gruppen sind" (González Casanova 1969: 236). Tatsächlich spielen Rassismus und Rassentrennung eine entscheidende Rolle im System des Kolonialismus: sie verhindern soziale Mobilität und kulturellen Austausch und garantieren so eine große Anzahl an unqualifizierten Arbeitern (González Casanova 1969: 237). Diese Form des internen Kolonialismus bleibt in den meisten lateinamerikanischen Staaten auch nach dem Ende der Kolonien bestehen und verändert sich auch mit Industrialisierung und Agrarreformen nur wenig (González Casanova 1969: 249). Für González Casanova ist der interne Kolonialismus ,ein weiteres Hindernis für die Integration eines für die industrialisierte Gesellschaft typischen Klassensystems und [kann] die 
Tatsache des Klassenkampfes zugunsten eines Rassenkampfes verdunkeln“ (González Casanova 1969: 249).

Auch innerhalb der Anthropologie wurden seit den 1970er Jahren Überlegungen angestellt über die

„totale Situation, in der die ethnische Grenze die asymmetrische Beziehung zwischen einer Gruppe, die wirtschaftlich, politisch und sozial eine andere beherrscht und ihre herrschende Stellung in Begriffen einer rassischen und/oder kulturellen Überlegenheit begründet, ausdrückt“ (Bonfil Batalla 1978: 211).

So ging auch Bonfil Batalla vom Weiterbestehen einer „kolonialen Beziehung“ (Bonfil Batalla 1978: 211) aus, die die Gesellschaft nicht wahrnehmen will. „Der Staat erkennt den ethnischen Pluralismus an und verweigert ihm Legitimität.“ (Bonfil Batalla 1978: 212) Die Individuen werden nur als solche - und nicht als Mitglieder einer ethnischen Gruppe - vom Staat akzeptiert, während die ethnischen Gruppen über eine spezielle Förderung in die Nationalgesellschaft integriert werden sollen - etwa über die Methoden des Indigenismus von ca. 1940 bis 1970 (Bonfil Batalla 1978: 212). Die damit einhergehende Delegitimierung der ethnischen Gruppen und die beständige Reduktion ihrer - wirtschaftlichen, politischen, sozialen Räume führt dazu, dass sie als unselbstständig und abhängig wahrgenommen werden - und gleichzeitig eine kollektive Erinnerung der Unterdrückung aufbauen können. Dadurch gewinnen sie eine politische Dimension, die nicht unbedingt explizit sein muss (Bonfil Batalla 1978: 217f.), und können ihre Identität und ihr Selbstverständnis als unterdrückte Gruppe mit einer langen Vergangenheit verstärken (Bonfil Batalla 1981: 27). Im Fall der Indigenen Lateinamerikas führt diese geteilte Erfahrung der Kolonisierung zur Ausbildung einer ,panindianischen Identifikation“ (Bonfil Batalla 1981: 20) und zu einem Kampf für das Verschwinden der Kategorie des Indios. „Das Verschwinden des Indios als Kolonisierter wird das Ergebnis der Aufhebung der kolonialen Situation sein, bedeutet aber nicht das Verschwinden der Ethnien.“ (Bonfil Batalla 1981: 20) Damit ist das ethnische Bewusstsein der Indigenen und ihr Kampf für ethnische Forderungen ein dekolonialer Kampf. „Die Dekolonialisierung des Indios übersetzt sich in der Dekolonialisierung der Ethnien.“ (Bonfil Batalla 1981: 20f.)

Die bis zu diesem Zeitpunkt beschriebenen Phänomene beziehen sich vor allem auf die Herrschafts- und Ausbeutungsbeziehungen zwischen verschiedenen Bevölkerungen in verschiedenen Gebieten. Der kulturelle, rassistisch unterfütterte Hintergrund und Produkt des Kolonialismus, den González Casanova und andere mit einem eurozentrischen Verständnis des Nationalstaates als internen Kolonialismus analysieren, wird seit den 1990ern in einem weiteren Kontext als Kolonialität verstanden (Quijano 2000b: 381). Kolonialität umfasst sowohl Kolonialismus als auch 
Imperialismus, geht aber über sie hinaus. Sie ist daher nicht an bestimmte historische Epochen gebunden, sondern dauert durch die Konstitution bestimmter Wissenskategorien und Lesarten der Welt weiter an (Escobar 2004: 219f.). Die Kolonialität entsteht als Resultat des modernen Kolonialismus, bleibt aber nicht auf dessen einfache Struktur als Herrschaftsbeziehung zwischen zwei oder mehr Völkern oder Nationen beschränkt - sie ist Produkt und Kern des Kolonialismus, seine Machtstruktur, die auch ohne ihn weiterbesteht (Quintero 2010: 9). Damit bewegt sich der Begriff der Kolonialität in seiner internationalen Perspektive im Rahmen der ,historisch-strukturellen Abhängigkeit“ (Quijano 2000b: 376) als zentraler Begriff der lateinamerikanischen Dependenztheorie. Kolonialität ist für die Begründer dieser Theorie, die sich in der Gruppe Modernität/Kolonialität austauschen, Grundlage und Gegenseite der Modernität. Beide Phänomene beginnen mit der Eroberung Amerikas ab 1492 und bedingen sich gegenseitig. Ab diesem Zeitpunkt entwickeln sich Kolonialismus und Imperialismus, also die Unterdrückung nichteuropäischer Völker, und ihre grundlegende Struktur der Kolonialität als Bestandteile der Modernität, wodurch diese zu einem weltweiten Phänomen wird. Begleitet wird diese Entwicklung vom Entstehen des „Eurozentrismus als die Wissensform der Modernität/Kolonialität - eine hegemoniale Repräsentation und Wissensmodus, die Universalität für sich behauptet" (Escobar 2004: 217f.) und der Ausbreitung des Kapitalismus als weltweites System der Organisation der Wirtschaft (Quijano 2000b: 342).

Die Modernität ist somit ein europäisches Narrativ, das auf die Kolonialität baut und sie gleichzeitig verdeckt (Mignolo 2009: 39). Sie taucht ab dem 15./16. Jahrhundert, in der Renaissance, als doppelte Kolonisierung der Zeit und des Raumes auf - der Zeit in Form der Konstituierung der Epoche des Mittelalters und des Raumes durch den Prozess der Eroberung Amerikas. Gerade die Kolonisierung des Raumes erzeugt die Kolonialität als ,dunkle Seite“ (Mignolo 2009: 41f.) der Modernität. Mignolo betont die Rolle der Renaissance als Moment der Rationalisierung und Verfestigung der Modernität. Zu diesem Zeitpunkt wurden die europäischen Traditionen über die Abgrenzung von Antike und Mittelalter und die außereuropäischen Traditionen über ihre Eroberung und damit die Trennung zwischen Wilden und Zivilisierten (oder Heiden und Christen, Unterentwickelten und Entwickelten usw.) erfunden bzw. weiter begründet. Dadurch wurde die Modernität zur Neuheit und zu einem Versprechen der Erlösung - zuerst in einem theologischen Sinne, später als Fortschritt. Nach dem Zweiten Weltkrieg wurde sie als Entwicklung und Modernisierung in den Ländern des globalen Südens vertreten (Mignolo 2009: 42f.). Für Walter Mignolo ist die koloniale Differenz eines der zentralen Ergebnisse dieser Entwicklung. Koloniale Differenz meint die Wissenseffekte der Kolonialität, also die kulturellen Unterschiede, die innerhalb der Machtbeziehungen als ,,privilegierter epistemologischer und politischer Raum“ (Escobar 2004: 217f.) 
entstehen. Die Kategorie der kolonialen Differenz führt Mignolo auf den Jurist Francisco de Vitoria zurück, der in seiner Begründung des internationalen Rechtes im 16. Jahrhundert die Indigenen als Menschen klassifizierte, die den Europäern unterlegen und damit auf ihre Führung angewiesen waren. Damit werden Unterschiede in Werte verwandelt und so eine ontologische und epistemische Hierarchie zwischen Menschen aufgebaut (Mignolo 2009: 46). Die koloniale Differenz ist daher Produkt und Grundlage der Kolonialität der Macht, gewissermaßen ihr struktureller Hintergrund.

Deshalb ist die Unabhängigkeit der lateinamerikanischen Länder am Anfang des 19. Jahrhunderts zwar eine Dekolonialisierung - also das Ende des Kolonialismus - aber nicht der Anfang einer Dekolonialität. Die neuen Staaten bilden ein historisches Paradox: ,unabhängige Staaten verbunden mit kolonialen Gesellschaften“ (Quijano 2006a: 18). Die Kolonialität der Macht, die vom Kolonialismus geschaffen wurde, besteht und wirkt weiter. Sie ist ,das zentrale Element der Strukturierung der Gesellschaft in Lateinamerika“ (Quintero 2010: 11). Die Nationalstaaten, die sich in Lateinamerika bilden, kopieren das europäischen Modell des Nationalstaats, ebenso wie seine Grundlagen in klar umgrenzten Nationalidentitäten und der Zentralität der Wirtschaft in den sozialen Beziehungen, insgesamt also das Ideal einer ethnisch homogenen Klassengesellschaft. Daher wurden die Konflikte in den neuen lateinamerikanischen Nationalstaaten als Resultate von Klassengegensätzen verstanden, der grundlegend koloniale Charakter der Staaten aber nicht reflektiert. Tatsächlich „entwickelte sich ein Modell der rassischen Klassifizierung zwischen Weißen und den weiteren Typologien, die als unterlegen angesehen wurden“" (Quintero 2010: 11f.). Die Kolonialität der Macht verhindert eine volle Anerkennung der ethnischen Gruppen in den verschiedenen Ländern und macht somit eine volle Demokratisierung und eine damit einhergehende Stabilität der Nationalstaaten unmöglich (Quintero 2010: 11f.). Diese können nicht auf eindeutige Nationalidentitäten bauen, da sie die Kolonialität und die damit einhergehenden Ungleichheiten nicht reflektieren, und bleiben somit prekär (Quintero 2010: 12). Der lateinamerikanische Nationalstaat war also weder national, in dem Sinne, dass er die Mehrheit seiner Bevölkerung nicht repräsentierte und in einigen Fällen auch nicht kontrollierte, noch war er demokratisch, da die oligarchischen Strukturen eine effektive Vertretung der Mehrheit der Bevölkerung unmöglich machten. „Er war ein enger Ausdruck der Kolonialität der Macht.“ (Quijano 2006a: 19)

Die Konzeption der Kolonialität der Macht, die der peruanische Soziologe Aníbal Quijano mit Bezug auf den peruanischen Kommunisten José Carlos Mariátegui (Jáuregui 2008: 16) entwickelt, präsentiert diese als „ein globales hegemonisches Machtmodell, das seit der Eroberung Amerikas besteht und Rasse und Arbeit, Raum und Menschen nach den Bedürfnissen des Kapitals und zum Vorteil der weißen Europäer artikuliert“ (Escobar 2004: 217f.). Ihre Grundlage ist die „,Rassi- 
fizierung der Beziehungen zwischen Kolonisierern und Kolonisierten“ (Quijano 2006a: 14) und somit ihre Klassifizierung nach ethnischen Kriterien innerhalb eines Machtsystems, das Staat, Wirtschaft und Wissen einschließt (Quijano 2000a: 201). Die Kolonialität der Macht wird im Kampf um die Kontrolle der fünf verschiedenen Bereichen menschlichen Lebens (Arbeit, Sex, Subjektivität, kollektive Autorität und Natur) und ihrer Ergebnisse reproduziert (Quintero 2010: 4). Mignolo reduziert diese Bereiche auf vier (Subjektivitäten, Autorität, Wirtschaft, Wissen), die miteinander verknüpft und traditionell von Europäern kontrolliert werden. Die tiefe Verankerung der Kolonialität in den grundlegenden menschlichen Aktivitäten garantiert ihre Reproduktion und Ausweitung - da das Wissen, das von diesen Aktivitäten ausgehend erzeugt wird, von Beginn an kolonial ist (Mignolo 2009: 48f.). Kolonialität der Macht ist die Verknüpfung dieser Bereiche menschlichen Lebens miteinander durch den kapitalistischen Weltmarkt und die koloniale Differenz (Quintero 2010: 9). Insbesondere die Subjektivität und ihre Elemente - soziales Imaginarium, historisches Gedächtnis und Wissensperspektiven - wird in der Kolonialität durch den Eurozentrismus gesteuert, welcher von den Anforderungen des Kapitalismus und des Kolonialismus als Herrschaftsformen abhängt. Daher sorgt der Eurozentrismus für eine verschobene Selbstwahrnehmung der Beherrschten, die sich „mit den Augen des Beherrschenden sehen“ (Quintero 2010: 10) und dabei ihre eigene Vorgeschichte und Autonomiegrade ignorieren. Er ist also keinesfalls nur die Perspektive der Europäer, sondern aller, die unter der Hegemonie Europas leben und erzogen wurden. Folglich ist der Eurozentrismus mehr als ein reiner Ethnozentrismus.

„Es handelt sich um die kognitive Perspektive, die in der langen Zeit der Gesamtheit der eurozentrierten Welt des kolonialen/modernen Kapitalismus erzeugt wurde und die die Erfahrung der Leute in diesem Machtmuster naturalisiert. Das heißt, sie macht, dass sie als natürliche wahrgenommen werden, somit als gegeben, nicht hinterfragbar.“ (Quijano 2000b: 343)

Die Kolonialität der Macht und der mit ihr verbundene Rassismus sorgt dafür, dass die unterlegene Position der nicht-europäischen Völker nicht als Folge eines Machtkonflikts, sondern einer natürlichen Unterlegenheit dieser Völker verstanden wird. Ihre marginale Einbindung in den entstehenden Kapitalismus als unqualifizierte Arbeiter und - später - Konsumenten ohne Kaufkraft entspricht einem neuen System der weltweiten Arbeitsteilung, das alle Formen der Ausbeutung in dem einzigen System des kapitalistischen Weltmarktes vereint (Quintero 2010: 8). Gesellschaft, Politik, Wissen und Kultur der nicht-europäischen Völker wird durch den Kolonialismus zerstört und ihre Träger zu ungebildeten Bauern reduziert. Wo diese Zerstörung nicht vollständig durchgeführt werden konnte, wurde das nichteuropäische Wissen durch den Eurozentrismus auf eine marginale Position festge- 
schrieben (Quijano 2000b: 378f.). Der Eurozentrismus als Produktionsprinzip von Wissen und Kontrollprinzip der Subjektivität ist also Teil der Kolonialität des Wissens (Walsh 2005: 19). Die Eroberung Amerikas erzeugt so die ,erste moderne und weltweite geokulturelle Identität" (Quijano 2000a: 221), der mit der Konstitution von Schwarzen, Asiaten und Weißen bzw. Ost und West weitere folgen sollten (Quintero 2010: 9). Diese verschiedenen Kategorien sind allerdings nur sinnvoll im Zusammenhang eines globalen Machtsystems, das auf dem Kolonialismus aufbaut und - mit einer Reihe von Veränderungen - bis heute weiterbesteht - der Kolonialität (Quijano 2006a: 14).

Für Quijano bewegen sich die Machtbeziehungen auf globalem Niveau zwischen der Kolonialität der Macht als Prinzip der Strukturierung der Gesellschaft auf rassistischer Basis, dem Kapitalismus als weltweites Ausbeutungssystem, dem Staat als zentrale Kontrollinstanz, insbesondere in seiner Form als Nationalstaat, und dem Eurozentrismus als hegemoniale Art, Subjektivität und Wissensproduktion zu kontrollieren (Quijano 2000: 1; Quijano 2006a: 14-16). Rasse wird also zum zentralen Kriterium für die Einteilung der Bevölkerung in Positionen und Funktionen in der Gesellschaft und somit zum grundlegenden Prinzip der Arbeitsteilung, die wiederum Teil der kapitalistischen Weltwirtschaft ist (Walsh 2005: 19). Daher wird Hautfarbe mit der sozialen Stellung verbunden - und die Idee einer ,Verweißung' der Bevölkerung, etwa im Sinne der mestizaje, als Fortschritt verstanden (Walsh 2009a: 67). Das führt zu „einem Mechanismus der subjektiven De-Indianisierung“ (Quijano 2006a: 24f.) der lateinamerikanischen Gesellschaften und eines Teils der indigenen Bevölkerung bei gleichzeitiger Integration der präkolumbischen indigenen Hochkulturen in die jeweilige Nationalkultur (Quijano 2006a: 24f.).

Verbunden mit einer sich verstärkenden Krise des Machtsystems der Kolonialität in den letzten Jahrzehnten (Quijano 2006a: 31f.) ist das Anwachsen von dekolonialen sozialen Bewegungen, wie der Indigenenbewegung (Quijano 2006a: 25) oder der globalisierungskritischen Bewegungen, die teilweise eine andere Raum-Politik und ein anderes Verständnis von traditionellen oder kulturell bedeutsamen Räumen gegen das globale Machtsystem in Stellung bringen (Escobar 2004: 222f.). Die Verbindung dieser Bewegungen kann eine „Art gegenhegemonische Globalisierung" (Escobar 2004: 223f.) bedeuten, die nicht nur die Globalisierung als kapitalistische und neoliberale in Frage stellt, sondern andere Bedeutungen zur Verfügung stellt, die alternative Verständnisse von Wirtschaft, Natur, Entwicklung enthalten. Daher versuchen die entsprechenden Bewegungen, gleichzeitig Gleichberechtigung und soziale Gerechtigkeit und Differenz umzusetzen (Escobar 2004: 223f.) und so eine andere Welt zu denken (Escobar 2004: 225).

Seit Beginn der Modernität und Kolonialität hat es Widerstand gegen diese gegeben. In der nicht-europäischen Welt „taucht die dekoloniale Art zu denken und zu machen ab dem 16. Jahrhundert als Antwort auf die unterdrückerischen und imperi- 
alistischen Neigungen der modernen europäischen Ideen auf“ (Mignolo 2009: 39). Die Logik der kolonialen Modernität führt zu Unzufriedenheit und Widerstand, der ,sich in Projekte der Dekolonialität übersetzt, die [...] auch der Modernität wesentlich sind“ (Mignolo 2005: 5). Mignolo betont insbesondere das dekoloniale Denken als ein Denken, dass von der Kolonialität verdeckte und verdrängte Möglichkeiten einschließt (Mignolo 2005: 6) und so Alternativen öffnet, die die Modernität und Rationalität in Frage stellen können (Mignolo 2005: 8). Dieses dekoloniale Denken entsteht entlang den Ausbreitungslinien der kolonialen und imperialen Eroberungen - also nicht wie das europäische Denken auf einem relativ begrenzten Raum, von dem es sich dann ausbreiten konnte (Mignolo 2005: 25). Daher kann dieses Denken aus vielen verschiedenen Perspektiven Erinnerungen, Sprachen usw. neu bewerten und so wieder in das allgemeine Denken einführen (Mignolo 2005: 26).

\section{ZU DEN BEGRIFFEN SOZIALE BEWEGUNG UND ORGANISATION DER SOZIALEN BEWEGUNG}

Die Untersuchung der Indigenenbewegung in Ecuador erfordert eine Klärung der verwendeten Begriffe. Auch wenn der Diskurs der Indigenenbewegung im Fokus dieser Arbeit steht, kann sie nicht umhin, die Bewegung selbst und damit die Entstehungsbedingungen ihres Diskurses zu untersuchen. Die Indigenenbewegung in Ecuador unterscheidet sich in einigen Punkten von anderen sozialen Bewegungen in anderen Ländern: 1. in ihrem Zentrum stehen relativ fest strukturierte Organisationen, von denen fast alle Aktionen ausgehen; 2. sie ist - wie die Neuen Sozialen Bewegungen Europas und der USA - von unten nach oben aufgebaut, allerdings und im Gegensatz zu diesen - wiederum in festen Strukturen; 3. sie umfasst an der Basis über eine Strukturierung in Assoziationen, Kommunen oder Zentren im Prinzip alle Bewohner einer bestimmten Region; 4. sie verschmilzt universalistische und partikularistische Forderungen. Deshalb wird hier die Indigenenbewegung als lose Sammlung fester Organisationen gesehen, die wirtschaftliche mit kulturellen Forderungen verbindet und grundlegende Alternativen zum aktuellen Staat und zur aktuellen Gesellschaft entwickelt. Diese vorläufige Theorie der Indigenenbewegung Ecuadors beruft sich auf die Theorie der Ressourcenmobilisierung, ihre poststrukturalistische Überwindung etwa durch Alvarez/Dagnino/Escobar (1998) und die Untersuchung ihres Diskurses als Strategie, wie sie etwa Benford (1997) anbietet.

Da hier eine Theorie sozialer Bewegungen in Ecuador skizziert werden soll, darf nicht vergessen werden, dass die Erforschung sozialer Bewegungen europäisch bzw. US-amerikanisch geprägt ist. Theorien, die an den dort herrschenden Verhältnissen gebildet werden, sind nicht ohne weiteres auf andere Länder zu übertragen. Die seit den frühen 1990er Jahren stark wachsende Beschäftigung mit sozialen 
Bewegungen in Lateinamerika hat sich - trotz einiger Versuche (etwa Alvarez/ Dagnino/Escobar 1998) - nicht von ihren europäischen Ursprüngen emanzipieren können (vgl. Boris 2009: 557). Unterschiede in der Rolle des Staates, der politischen Parteien, der Massenmedien, der Verbände und Gewerkschaften, werden so nicht immer in dem Maße reflektiert, wie es dem lateinamerikanischen Kontext angemessen wäre. Der hier vorliegende Text soll keine Anwendung europäischer Theorien auf einen außereuropäischen Kontext sein, sondern der Versuch, anhand der spezifischen Gegebenheiten Ecuadors eine Theorie mittlerer Reichweite zu entwickeln. Daher werden die verschiedenen Ansätze nicht im Sinne einer Theoriegeschichte getrennt vorgestellt, sondern alle Ansätze gleichermaßen in Bezug auf die Bearbeitung bestimmter Probleme verglichen, um so eine eigene, auf die ecuadorianische Indigenenbewegung anwendbare, Position entwickeln zu können.

\section{a. Struktur sozialer Bewegungen}

Soziale Bewegungen sind rationale Akteure, die Ressourcen - Mitglieder, Unterstützer, Gelder, Räume, etc. - mobilisieren, um bestimmte, relativ allgemeine Ziele zu erreichen (Rodríguez 2008: 158). Damit sind sie „,ein kollektiver Akteur, der in den Prozeß sozialen bzw. politischen Wandels eingreift" (Raschke 1985: 76) und „ein bewußter und kollektiver Versuch einer Anzahl von Leuten, Individuen oder gesellschaftliche Institutionen und Strukturen zu verändern“ (Zald/Ash 1966: 329). Sie engagieren sich in politischen und/oder kulturellen Konflikten, wodurch sie sozialen Wandel auf einer systemischen oder nicht-systemischen Ebene vorantreiben oder verhindern wollen (Diani 1992: 11). Dazu binden sie Individuen ein, ohne aber für diese ein reines Mittel zu sozialem Wandel zu sein. Soziale Bewegungen zeichnen sich durch von ihren Mitgliedern und Unterstützern geteilte Werte und Meinungen aus, die auf einen strukturellen Wandel der Gesellschaft zielen (McCarthy/Zald 1977: 1217f.; Zald/McCarthy 1979: 2). Es findet keine Sicherung der Unterstützung durch Aufbau fester Institutionen statt, daher sind soziale Bewegungen dazu gezwungen, sich permanent um Mobilisierung zu bemühen, immer in Bewegung zu bleiben (Raschke 1985: 77f.). Aus diesen Elementen ergibt sich eine allgemeine Definition, die auch im Falle der Indigenenbewegung Ecuadors zugrunde gelegt werden kann:

„Soziale Bewegung ist ein mobilisierender kollektiver Akteur, der mit einer gewissen Kontinuität auf der Grundlage hoher symbolischer Integration und geringer Rollenspezifikation mittels variabler Organisations- und Aktionsformen das Ziel verfolgt, grundlegenderen sozialen Wandel herbeizuführen, zu verhindern oder rückgängig zu machen." (Raschke 1985: 77) 
Soziale Bewegungen sind also keine einheitlichen und klar umrissenen Akteure, sondern eine Gesamtheit formaler Organisationen, informaler Netzwerke und nicht direkt an die Organisationen gebundener Individuen, die sich mit einer Vielzahl institutioneller und außerinstitutioneller Strategien (Gamson/Meyer 1996: 283) an einem mehr oder weniger kohärenten Kampf für Veränderung beteiligen (Meyer/Whittier 1994: 277). Die verschiedenen Organisationsformen sozialer Bewegungen spielen gerade in dem hier behandelten Fall eine wichtige Rolle. ,Diese Organisationen sind internem und externem Druck unterworfen, der ihre Lebensfähigkeit, ihre interne Struktur und Prozesse und ihren letztendlichen Erfolg im Erreichen von Zielen beeinflusst.“ (Zald/Ash 1966: 327) Organisationen gehören in der Regel zu einer sozialen Bewegung, geben dieser Kontinuität und Koordination, sind aber immer nur ein Teil der Bewegung. „Das Besondere der sozialen Bewegung liegt gerade in der Dialektik zwischen den (Bewegungs-)Organisationen und den fluiden Teilen der Bewegung." (Raschke 1985: 80) Es gibt eine Vielzahl von Arten formaler Organisationen innerhalb sozialer Bewegungen, wobei auch die Bedeutung und Größe formaler Organisationen stark variabel sein kann (Raschke 1985: 78). Nur die Unterordnung der Organisation unter die allgemeinen Ziele der Bewegung ist eine geteilte Eigenschaft - damit man überhaupt von sozialer Bewegung sprechen kann. „Die Organisation definiert nicht die Bewegung, sondern die soziale Bewegung ist immer viel mehr als die Organisationen, die sie umfaßt." (Raschke 1985: 77)

\section{Ressourcen-Mobilisierungs-Ansatz}

Der Ressourcen-Mobilisierungs-Ansatz war der erste Versuch, die Beziehungen zwischen sozialen Bewegungen und den in ihnen aktiven Organisationen zu verdeutlichen. Dazu beschreibt er die Organisationen der sozialen Bewegungen als Social Movement Organizations (SMO), formale Organisationen, die die Ziele der sozialen Bewegung teilen (McCarthy/Zald 1977: 1218; Zald/McCarthy 1979: 2) und ihre Mitglieder in erster Linie über ihre Weltsicht und die zu erreichenden Ziele motivieren (Zald/Ash 1966: 329). SMO repräsentieren und definieren die Ideen und Zielvorstellungen der sozialen Bewegung. Sie sind ,die Quellen von Gegendiskursen und der generativen Ideen, auf denen sie aufbauen“ (Bebbington 2007: 800). SMO koordinieren die Aktivitäten der sozialen Bewegung, verschaffen sich Zugang zu Ressourcen, die für die Bewegung insgesamt wichtig sind und garantieren die Bindung des Führungspersonals in Zeiten geringer Mobilisierung.

In der Terminologie des Ressourcen-Mobilisierungs-Ansatzes bilden alle Organisationen derselben sozialen Bewegung, die die Einstellungen und Ziele dieser Bewegung mit verschiedenen Schwerpunkten teilen (Zald/McCarthy 1979: 2) eine Social Movement Industry (SMI) (McCarthy/Zald 1977: 1219). Diese ist also ist das organisatorische Äquivalent einer sozialen Bewegung, das alle SMO dieser Bewe- 
gung zusammenfasst (Zald 1979: 9). Innerhalb der SMI konkurrieren die verschiedenen Organisationen miteinander um Einfluss, Ressourcen und Mitglieder oder kooperieren, um gemeinsame Ziele zu erreichen (Zald/McCarthy 1979: 1). Die Konkurrenz zielt auf eine ,symbolische Dominanz: welche SMO hat die besten Programme, Taktiken und Führer, um Ziele zu erreichen“ (Zald/McCarthy 1979: 3). Die Übernahme und Anpassung von wichtigen Symbolen ist ein Weg für die SMO, eine Dominanz in ihrer SMI zu erreichen (Zald 1979: 13f.). Bei offenen und inklusiven Organisationen führt die Konkurrenz zu einer „leichten Produktdifferenzierung" (Zald/McCarthy 1979: 7f.) insbesondere der Ziele und der Taktiken. Daher bildet sich eine Vielzahl von SMO, die mit verschiedenen Mitteln - sowohl was Strategien, also auch was Diskurse angeht (Ennis 1987: 523; Bebbington 2007: 812) - ähnliche Ziele erreichen wollen (Zald/McCarthy 1979: 7f.). Alle diese SMO bieten - wie verschiedene Firmen eines Industriezweiges - vergleichbare Produkte an, also vergleichbare Ziele und Strategien. In anderen Worten gibt es in der Regel kein Monopol nur einer Organisation, sondern eine marktähnliche Konkurrenz (Zald 1979: 3). Die Führer der SMO sind Unternehmer, die mehr oder minder rational (Polletta 1997: 438) unbefriedigte Bedürfnisse suchen und im Aufbau der SMO Investitionen tätigen, die sie hoffen, später zurückbekommen zu können. Dazu versuchen sie, den Mitgliedern und Unterstützern der Organisation möglichst viele Ressourcen abzugewinnen (Jasper 2007: 91). Eine SMI wird dabei von der Nachfrage nach ihren Produkten, den Bedingungen ihres Erreichens und dem Einfluss dieser Produkte in den verschiedenen SMO strukturiert (Zald 1979: 12). Die Gesamtheit der SMI einer Gesellschaft werden von der Ressourcen-MobilisierungsTheorie zum Social Movement Sector (SMS) zusammengefasst (Zald/McCarthy 1979: 2), der alle organisierten sozialen Bewegungen enthält. Der SMS gibt Aufschluss über die grundsätzliche Bereitschaft zu Veränderungen in der Gesellschaft (Zald 1979: 3) und die Bandbreite der Positionen bezüglich einer möglichen Veränderung (Zald 1979: 15f.).

Die Grundlagen für Mobilisierung wird von der Ressourcen-MobilisierungsTheorie nur verkürzt behandelt und soll auch in der vorliegenden Studie keine zentrale Rolle spielen. Der Ansatz selbst unterstellt meist eine tiefe und weit verbreitete Unzufriedenheit aufgrund eines Mangels (Jenkins/Perrow 1977: 250), auf die das kollektiven Verhalten eine rationale Antwort ist, die für ihre Entwicklung wiederum ausreichende Ressourcen benötigt (Caniglia/Carmin 2005: 202). Der schwierige Zugang zu den für eine Mobilisierung notwendigen Ressourcen macht das Entstehen von sozialen Bewegungen zu einer Seltenheit, die auf Unterstützung von außerhalb angewiesen ist (Jenkins/Perrow 1977: 251). Erfolg oder Misserfolg sozialer Bewegungen hängt also von Kosten und Nutzen des Handelns und den zur Verfügung stehenden Ressourcen ab (Melucci/Avritzer 2000: 515). Die Organisationen der sozialen Bewegungen sind auf eine Übereinstimmung anderer gesell- 
schaftlicher Gruppen mit ihren Zielen angewiesen, die immer auch von den konkreten Bedingungen in der Gesellschaft abhängt. Diese Übereinstimmung und das Erreichen oder Nichterreichen der Ziele, sowie die Konkurrenz anderer, ähnlich gelagerter Organisationen bestimmen über die Attraktivität der Organisation und ihr Mobilisierungspotential. So kann eine grundlegende Zustimmung oder weitgehende Neutralität der Gesamtgesellschaft die Entwicklung der SMO begünstigen, während eine große Ablehnung der Ziele der SMO ihnen schaden kann (Zald/Ash 1966: 330). ${ }^{8}$ Eine Veränderung der Werte einer Gesellschaft kann die sozialen Bewegungen und ihre Organisationen zu einer Veränderung ihrer Ziele zwingen. Die bestehende Konkurrenz zwischen den verschiedenen Organisationen führt zu einer relativen Offenheit und daher einer gewissen Bereitschaft, die Ziele zu modifizieren ein mangelndes Eingehen auf veränderte Wertvorstellungen kann zum Ende der sozialen Bewegung oder einer ihrer Organisationen führen (Zald/Ash 1966: 332).

Eine erfolgreiche soziale Bewegung, die ihre Ziele in der Öffentlichkeit präsentieren oder die Regierungspolitik beeinflussen kann, kann eine Gegenbewegung provozieren, die - von einer Bevölkerungsgruppe unterstützt, deren Interessen gefährdet sind - versucht, die angestrebten Veränderungen zu verhindern (Zald 1979: 2; Meyer/Staggenborg 1996: 1635). Insbesondere wichtige Ereignisse, wie Massendemonstrationen oder der Erlass von Gesetzen, die der sozialen Bewegung zugute kommen, können diesen Prozess beschleunigen (Meyer/Staggenborg 1996: 1638). Die Gegenbewegung ist ähnlich strukturiert wie die soziale Bewegung selbst - also auch eine soziale Bewegung - , besitzt aber Werte und Meinungen, die denen der bereits vorhandenen sozialen Bewegung entgegenstehen (Zald 1979: 4). Sie versucht oft, bestehende Konflikte zwischen den SMO der angegriffenen Bewegung zu verstärken, um so eine Spaltung herbeizuführen (Bebbington 2007: 812). Das Fortbestehen der Gegenbewegung unterliegt denselben Bedingungen wie das der sozialen Bewegung selbst. Sie muss ihre Ziele ausreichend großen Bevölkerungskreisen nahebringen und diese mobilisieren können (Meyer/Staggenborg 1996: 1639). Auch wenn diese Gegenbewegungen manchmal von der Regierung oder regierungsnahen Organisationen initiiert oder gefördert werden, müssen sie Handlungsoptionen eröffnen, die der Regierung selbst nicht offenstehen - und erfolgversprechend sein (Meyer/Staggenborg 1996: 1630). Somit wird das Entstehen von Gegenbewegungen von einer Schwäche des Staates gegenüber der sozialen Bewegung begünstigt (Meyer/Staggenborg 1996: 1637). Die Gegenbewegung bleibt von

8 Die externen Bedingungen für den Erfolg von sozialen Bewegungen werden von der Political Opportunity Theory behandelt, die nicht immer klar von der RessourcenMobilisierungs-Theorie -auf die sie aufbaut- zu trennen ist. Hier werden beide Ansätze integriert, ohne der Analyse den sozialen Bewegungen externer Faktoren zu viel Platz einzuräumen. 
der Unterstützung durch bestimmte Eliten abhängig, die durch eine Veränderung der Ziele der Gegenbewegung gefährdet werden kann (Meyer/Staggenborg 1996: 1642f.). Konflikte zwischen Bewegung und Gegenbewegung führen dazu, dass keine der Bewegungen ihre Ziele und Taktiken institutionalisiert und daher günstige Rahmenbedingungen nicht genutzt werden können. Stattdessen werden Konflikte über Ziele und Taktiken innerhalb der Bewegungen verstärkt (Meyer/Staggenborg 1996: 1651-1653).

Das Entstehen und Wachstum einer sozialen Bewegung wird meist von der Entwicklung verschiedenen Zielvorstellungen bestimmter SMO oder Gruppen innerhalb der Bewegung begleitet, die in einer Phase hoher Aktivität in einigen wenigen Hauptzielen zusammengefasst werden können. Auf die Formulierung dieser Hauptziele der gesamten sozialen Bewegung haben die einzelnen SMO nur geringen Einfluss (Meyer/Staggenborg 1996: 1641). Ein Nichterreichen dieser Hauptziele kann kurzfristig zu einer verstärkten und langfristig zu einer verringerten Mobilisierung der Mitglieder führen - abhängig von deren Hoffnung, die Ziele noch zu erreichen. Das Offenstehen von zusätzlichen institutionellen Räumen ist besonders für scheiternde Bewegungen interessant, die dort ihre Forderungen neu aufstellen und so weiter bestehen können (Meyer/Staggenborg 1996: 1647f.). SMO bilden somit Strukturen (abeyance structures), in denen Kultur, Werte und ein Teil des Führungspersonals scheiternder sozialer Bewegungen überleben und sich anderen Zielen widmen können (Meyer/Whittier 1994: 279). Das Handeln einer sozialen Bewegung kann spätere Bewegungen, im selben oder einem anderen Bereich, beeinflussen, indem sie die politischen und kulturellen Bedingungen in der Gesellschaft und in der Bewegung selbst verändert (Meyer/Whittier 1994: 282). Somit verändert jede soziale Bewegung die Möglichkeiten für andere soziale Bewegungen (Meyer/Staggenborg 1996: 1635).

Die Ressourcen-Mobilisierungs-Theorie ist trotz ihrer ökonomistischen Tendenz und ihrem Fokus auf Nordamerika (Zald 1979: 3) gut geeignet, die Beziehungen zwischen den verschiedenen Organisationen innerhalb einer sozialen Bewegung zu untersuchen (Zald 1979: 2). Sie untersucht nicht die Gründe, sondern die Bedingungen für das Entstehen sozialer Bewegungen. Psychologische Herangehensweisen oder Vermutungen über kollektives Verhalten werden in den Hintergrund geschoben und den Strukturen und Prozessen der Gesellschaft und des Staates Vorrang eingeräumt (Zald 1979: 1f.). So tritt die individuelle Motivation der Mitglieder sozialer Bewegungen zugunsten der Entwicklung der Organisationen dieser Bewegungen zurück (Polletta 2008: 80). Weiterhin vernachlässigt die Ressourcen-Mobilisierungs-Theorie den Einfluss der politischen Strukturen auf die Entwicklung der sozialen Bewegungen, ihre Bindung an soziale Klassen und ihre kulturellen und symbolischen Hintergründe (Zald 1979: 15/20f.). Auch wenn die mangelnde Integration von Kultur in den Ressourcen-Mobilisierungs-Ansatz oft 
kritisiert wird (Polletta 1997: 431f.), wurde die Rolle der Deutungshoheit und der Übernahme von Symbolen durch die Organisationen schon erläutert. Kultur steht zwar nicht im Mittelpunkt dieser Theorie, wird aber keinesfalls missachtet:

„Obwohl soziale Bewegungen für eine Reihe klar artikulierter Ziele kämpfen, einschließlich Veränderungen in der staatlichen Politik, dem privaten Sektor und kulturellen Normen, beeinflussen sie auch indirekte Ziele. Diese indirekten Ziele umfassen die Praktiken, Perspektiven und Ergebnisse anderer kollektiver Akteure und das Leben der Teilnehmer.“ (Meyer/Whittier 1994: 278)

Diese Beeinflussung führt zu ungewollten Effekten jenseits der formulierten Ziele und kann zu einem Übergreifen (spill over) der Ideen, Taktiken, Stile, Teilnehmer und Organisationen auf andere soziale Bewegungen führen (Meyer/Whittier 1994: 277).

Die Theorie der politischen Gelegenheit oder des politischen Prozesses (Political Opportunity Theory/Political Process Approach), die hier nur ergänzend verwendet wird, beschäftigt sich weniger mit den Strukturen und Prozessen der sozialen Bewegungen und ihrer SMO, als mit Erklärungen für ihren Erfolg oder Misserfolg. Dabei spielen lange Mobilisierungskreisläufe (cycles of mobilization) und externe Faktoren eine besondere Rolle. Sie untersucht die Struktur der politischen Gelegenheiten (political opportunity structure), also die Zugangs- und Einflussmöglichkeiten der sozialen Bewegung auf Öffentlichkeit und Staat. Dazu gehören institutionelle Partizipationsmöglichkeiten, stabile politische Ausrichtungen, Zugang zu und Allianzen mit der Elite, Konflikte innerhalb der Elite und der Grad an Repression. Auch kulturelle Faktoren und soziale Zusammenhänge gehören zu dieser Struktur (Caniglia/Carmin 2005: 203f.). Wichtig sind auch die Massenmedien, die die Ziele und Praktiken sozialer Bewegungen abhängig von ihrer Funktionsweise, ihrer Klientel und ihren Beziehungen zu Staat und Parteien einer breiten Öffentlichkeit präsentieren können und so direkt auf Erfolg oder Misserfolg der Bewegungen Einfluss nehmen können (Gamson/Meyer 1996: 287).

„Normen und Praktiken der Medien und der breiteren politischen Ökonomie, in der sie operieren, beeinflussen die Gelegenheiten und Einschränkungen unter welchen die Bewegungen operieren. Schlüsselorganisationen im Mediensystem [...] gewähren Akteuren Bedeutung.“ (Gamson/Meyer 1996: 287)

Dieser Ansatz definiert soziale Bewegungen als Herausforderung an den Staat, der zu einem bestimmten Verhalten gebracht werden soll. Nach dieser Sichtweise führt die Zentralität des Staates zu Kooperation oder Konkurrenz zwischen verschiedenen sozialen Bewegungen, die versuchen, Zugang zu den begrenzten Einflussmög- 
lichkeiten zu erlangen. Dabei ist ein Austausch über Vor- und Nachteile verschiedener Strategien oder bekannter Reaktionen des Staates zwischen den Bewegungen möglich. Die Aktionen der Bewegungen beeinflussen die Struktur politischer Gelegenheiten und Möglichkeiten für Partizipation für sich selbst und andere Bewegungen. Die Reaktionen des Staates auf soziale Bewegungen verändert also die Handlungsmöglichkeiten für soziale Bewegungen und erhöht oder verringert die Attraktivität bestimmter Strategien (Meyer/Whittier 1994: 279). Perioden ,relativer Offenheit staatlicher Strukturen gegenüber abweichender Meinungen“ (Meyer/ Staggenborg 1996: 1636) eröffnen den sozialen Bewegungen institutionelle Wege, ihre Forderungen zu präsentieren. Das Eingehen vom Staat auf diese Forderungen beeinflusst die Entwicklung der sozialen Bewegungen. Die sozialen Bewegungen hängen also vom Staat und seiner Fähigkeit, Forderungen institutionell zu bearbeiten ab. „Die Kapazität von Bewegungen, politische und Protestfenster zu schließen, ist eine Funktion der staatlichen Kapazität, Konflikte beizulegen." (Meyer/Staggenborg 1996: 1637).

Die Fixierung der Theorie der politischen Gelegenheiten auf den Staat, die Eliten und das Entstehen von Gegenbewegungen ist wenig hilfreich bei einer Anwendung dieser Theorie auf Ländern, in denen der Staat nicht stark genug ist, alleiniger Adressat zu sein. Gerade die Zentralität des Staates und seiner Strukturen (Davis 1999: 586f.) und das daraus folgende Verständnis von sozialen Bewegungen als Agenten der Demokratisierung (Davis 1999: 599) haben die Übernahme dieses Ansatzes in Lateinamerika behindert. Auch andere Theorien sind, wie etwa der Begriff Protestbewegung andeutet, zu sehr auf einen mehr oder minder offenen und pluralistischen, starken Staat ausgerichtet, um ohne weiteres auf die meisten Länder des globalen Südens anwendbar zu sein. Die reduktionistische Sicht auf Kultur als Grundlagen kollektiven Handelns - und nicht etwa Ziel - sozialer Bewegungen (Jasper 2010: 966) ist für die Untersuchung identitärer sozialer Bewegungen wenig hilfreich.

Ein weiteres Problem bei der Anwendung der gängigen Theorien der sozialen Bewegungen ist ihre Konzentration auf Mobilisierung. Der Fokus auf tatsächliche politische Aktionen und die Vernachlässigung der organisatorischen Grundlagen als bloße abayence structures, die nur zur Aufrechterhaltung der Bewegungsnetzwerke dienen, entspricht einer Bevorzugung der Prozesse über die Strukturen. Daher sind diese Theorien nur bedingt geeignet, soziale Bewegungen zu untersuchen, die über lange Zeit hinweg keine größeren Mobilisierungen durchführen. Und gerade das ist der Fall bei der ecuadorianischen Indigenenbewegung.

Weniger formalistisch ist das Verständnis sozialer Bewegungen als Netzwerke, die verschiedene Akteure umfassen die die soziale Bewegung unterstützen und ihre Diskurse und Forderungen weitertragen: Organisationen, Sympathisanten, Parteien, Teile politischer Institutionen und des Staates selbst (Alvarez/Dagnino/Escobar 
1998: 14-16). Dieser Ansatz trägt am ehesten der Tatsache Rechnung, dass es sich bei sozialen Bewegungen um einen schwer abgrenzbaren „Gegenstand mit fließenden Grenzen“ (Raschke 1985: 82) handelt. Eine Variante davon ist das transnationale kollektive Handeln, also koordinierte internationale Kampagnen von Netzwerken von Aktivisten gegen internationale Akteure, andere Staaten oder internationale Institutionen (Della Porta/Tarrow 2005: 2f./7). Die Transnationalisierung sozialer Bewegungen lässt sich an drei Prozessen untersuchen: Streuung (diffusion) meint die Verbreitung von Ideen, Praktiken und Diskursen sozialer Bewegungen von einem Land zum nächsten. Über direkte Kontakte von Aktivisten im Sinne einer Vermittlung (brokerage) oder indirekten Einfluss wandern Bestandteile von sozialen Bewegungen in einem Land zu solchen im nächsten. Domestizierung (domestication) bezieht sich auf das Austragen von externen Konflikten im Heimatland. Mit Externalisierung (externalization) wird die Einbeziehung überstaatlicher Institutionen in nationale Konflikte beschrieben (Della Porta/Tarrow 2005: 2f.). So versuchen Netzwerke von sozialen Bewegungen auf überstaatlicher Ebene mit Unterstützung internationaler Institutionen schwache Bewegungen in bestimmten Ländern durch Allianzen zu unterstützen. Diese Institutionen dienen als Quelle für Ressourcen, die auf nationaler Ebene verwendet werden sollen. Die Vereinten Nationen etwa haben mit der Schaffung internationaler Normen zu den Rechten von Indigenen oder Frauen in einigen Fällen zur Stärkung der jeweiligen Bewegungen beigetragen (Della Porta/Tarrow 2005: 5f.). Diese Erfolge führen wiederum zum ,Aufbau einer Basis von übernationalen Ressourcen und globalen Strategien, die sich deutlich von den in nationalen Bereichen angewandten unterscheiden“ (Della Porta/Tarrow 2005: 10). Dadurch vergrößern sich die Möglichkeiten zu Kooperation und Koordination und zur Formulierung globaler Forderungen und Diskurse (Della Porta/Tarrow 2005: 10).

Ein Sonderfall sozialer Bewegung sind die Neuen Sozialen Bewegungen, ein „schwach strukturierte[r], fluide[r] und offene[r] Typ sozialer Bewegung” (Raschke 1985: 412). Diese Art sozialer Bewegungen hat keine einheitliche Weltsicht, ist thematisch offen und nur schwach organisiert. Neue soziale Bewegungen haben eine Vielzahl von Organisationsformen und bestehen aus vielen autonomen Teilbewegungen, die miteinander vernetzt sind (Raschke 1985: 412). Eines ihrer wichtigsten Charakteristiken ist ihre Autonomie und Unabhängigkeit vom Staat und staatlichen Akteuren (Davis 1999: 586f.). Inwieweit sie sich von ,alten“ sozialen Bewegungen unterscheiden, die genau wie die Neuen Sozialen Bewegungen auch auf kulturellen Wandel ausgerichtet sein können, ist umstritten (Polletta 2008: 82). Aufgrund ihrer zentralen ethnischen Komponente werden die lateinamerikanischen Indigenenbewegungen seit den 1980ern meist als Neue Soziale Bewegungen gesehen (Korovkin 2008: 2) - obwohl sie wichtige Charakteristiken dieser Bewegungen nicht erfüllen. So ist die Indigenenbewegung Ecuadors nach einigen dieser Kriteri- 
en eine Neue Soziale Bewegung, unterscheidet sich aber in wichtigen Punkten von den Neuen Sozialen Bewegungen Europas und der USA. Die ecuadorianische Indigenenbewegung hat durchaus eine relativ einheitliche Weltsicht, zumindest was die Ablehnung von Rassismus und Ausgrenzung angeht. Auch ist sie stark organisiert, in Organisationen, die auch schwere Krisen überdauert haben. Die relativ homogene Sozialstruktur Neuer Sozialer Bewegungen in Europa und den USA (die Akteure sind meist gebildete Mitglieder der Mittelschicht) ist ebenfalls etwas, das auf die Indigenenbewegung Ecuadors nicht zutrifft. In anderen Aspekten ist diese Theorie sehr wohl von Nutzen und auch auf Ecuador übertragbar. Die neuen sozialen Bewegungen ,ergänzen das Prinzip der Repräsentation um das Prinzip der Zugehörigkeit“" (Melucci/Avritzer 2000: 509) und kämpfen so für direkte Teilhabe in repräsentativen Demokratien und Anerkennung von Minderheiten. Die Repräsentation wird als Reduktion der kulturellen Vielfalt zu Zwecken der Politik infrage gestellt, Zugehörigkeit und Identität werden als politische Prinzipien gestärkt (Melucci/Avritzer 2000: 522).

\section{b. Kultur sozialer Bewegungen}

Die Ziele sozialer Bewegungen und die Wege dorthin sind vielfältig, sie kämpfen nicht nur um politische Macht, sondern um kulturellen oder diskursiven Einfluss. Gerade die Indigenenbewegungen Lateinamerikas haben versucht, Einfluss auf die Gesellschaft zu nehmen, indem sie Begriffe wie Staatsbürgerschaft oder politische Repräsentation hinterfragen und neue Deutungen anbieten (Alvarez/Dagnino/Escobar 1998: 2). Kultur ist zu einem Streitfeld um Einfluss geworden. ,[I]n Lateinamerika führen heute alle soziale Bewegungen cultural politics aus. [...] Für alle soziale Bewegungen [...] sind kollektive Identitäten und Strategien unvermeidlich mit Kultur verbunden." (Alvarez/Dagnino/Escobar 1998: 6) Die Konzentration auf cultural politics und die Neuinterpretation von Begriffen, ist ein anderer Weg, Machtverhältnisse in einer Gesellschaft in Frage zu stellen. Neue Bedeutungen alter Begriffe erlauben es, alternative Sichtweisen zu öffnen und so dominante Bedeutungen zu schwächen (Alvarez/Dagnino/Escobar 1998: 7). Cultural politics entstammen den diskursiven Artikulationen bereits bestehender kultureller Praktiken und verändern die politische Kultur eines Landes oder einer Region dadurch, dass sie das Verständnis von Politik selbst verändern. „Cultural politics ist der Prozess, der ausgeführt wird, wenn Reihen von sozialen Akteuren, die von verschiedenen kulturellen Bedeutungen und Praktiken geprägt sind und diese verkörpern, miteinander in Konflikt kommen." (Escobar 1998: 64) Kultur ist also direkt politisch, da sie versucht, soziale Macht neu zu definieren (Escobar 1998: 64). Soziale Bewegungen wollen die politische Kultur ihrer Region öffnen, um sich selbst als ,,soziale Akteure mit politischen Ansprüchen" (Alvarez/Dagnino/Escobar 1998: 8) zu kons- 
tituieren. Diese politischen Kultur in Lateinamerika ist oligarchisch und patriarchalisch verfasst, was sich auch in einer mangelnden Unterscheidbarkeit zwischen Privatem und Öffentlichem ausdrückt. Die Idee eines starken Entwicklungsstaates, der die Gesellschaft erneuern soll, ist kein Bruch, sondern eine modernisierte Weiterentwicklung dieser politischen Kultur (Alvarez/Dagnino/Escobar 1998: 9f.) und wird durch die Neudeutung bestehender Begriffe in Frage gestellt.

Auch wenn dieser Ansatz die institutionellen, historischen und sozialen Bezüge betont (Alvarez/Dagnino/Escobar 1998: 16), ist die Kritik einer „mangelnde[n] Bodenhaftung“ (Boris 2009: 557) eines auf Diskurse orientierten, sich aber gleichzeitig als umfassend verstehenden Ansatzes, nicht von der Hand zu weisen. Die Erforschung der Diskurse der sozialen Bewegungen in Lateinamerika ist wichtig, nur sie erlaubt ein tiefgreifendes Verständnis der Weltsichten, Forderungen und Aktionsformen der einzelnen Organisationen. Jedoch macht eine Diskursanalyse ohne den nötigen historischen Hintergrund wenig Sinn. Daher kann eine umfassende Analyse sozialer Bewegungen weder auf den diskursiven Teil, noch auf soziale, politische, etc. Komponenten verzichten. Die meisten der vorliegenden Studien beschränken sich allerdings auf die klassischen Herangehensweisen, die Institutionalisierung und politische Prozesse in den Vordergrund rücken.

\section{Der Begriff Kultur}

Kultur spielt eine entscheidende Rolle für soziale Bewegungen, sowohl als Grundlage als auch als Ziel ihres Handelns. Kultur meint hier soziale Praktiken und Bedeutungen, die auch individuelle und kollektive Identität umfassen (Escobar 1992: 70f.). Sie ist „flüssig, vielfach verankert und oft uneinheitlich“ (Swindler 1995: 36) und wird im konkreten Handeln der Menschen manifest (Escobar 1992: 76). Sie gibt den Bewegungen „das Vokabular der Bedeutungen“ (Swindler 1995: 27), das den Kontext für einzelne, öffentlich darstellbare, Bedeutungen stellt (Swindler 1995: 28). Kultur besteht aus Symbolen, ,Zeichen, die Bedeutung durch ihre Wechselbeziehungen haben“ (Polletta 1999: 66f.). Sie gestaltet und wird gestaltet, wodurch sie konkret wird (Polletta 1999: 66f.).

„Da die meisten Bewegungen keine politische Macht haben (das ist genau, weshalb sie unkonventionelle politische Taktiken nutzen), können sie die Welt effektiver durch eine Neudefinition ihrer Bedingungen als durch eine Änderung ihrer Sanktionen verändern.“ (Swindler 1995: 34)

Dasselbe trifft natürlich auch auf ihre Gegner zu, die die Bewegungen und ihre Ziele umzudefinieren versuchen. Veränderungen in der Kultur und Weltsicht können langfristiger sein als Veränderungen der Gesetze oder Institutionen. Kultur kann selbst institutionelle Veränderungen erzwingen (Polletta 1997: 443). Sie ist 
strukturell und bestimmt damit das Handeln der Eliten und des Staates (Polletta 1999: 69). Daher ist die kulturelle Sphäre eines der wichtigsten Gebiete für soziale Bewegungen (Diani 1992: 10). Soziale Bewegungen versuchen also nicht nur, bestimmte Forderungen an die staatliche Politik durchzusetzen, sondern das Verständnis von Politik und bestimmten gesellschaftlichen Ideen zu verändern und so ein anderes Wissen zu schaffen (Bebbington 2007: 800). Sie verhandeln die „Praktiken und Rationalitäten der Modernität und Postmodernität in der Vorstellung und Rekonstruktion sozialer Ordnungen“ (Escobar 1992a: 420) neu. Dabei versuchen sie, sich bestimmte, umstrittene Themen anzueignen, in ihrem Sinne umzudeuten und so der Öffentlichkeit zu präsentieren (Melucci/Avritzer 2000: 520). Deshalb kann die Analyse der kulturellen Änderungen, die die sozialen Bewegungen herbeiführen wollen, von größerem Nutzen sein als eine Analyse ihrer konkreten Forderungen (Swindler 1995: 34). Das erfordert allerdings ein hohes Engagement des Forschers vor Ort und eine schrittweise Verfolgung der kulturellen Inhalte und ihrer Entwicklung (Escobar 1992: 76f.).

Auch die Kultur der sozialen Bewegungen selbst verändert sich durch die Situation, in der sie existieren. „Verschiedenen Regimes und verschiedene Repressionsformen erschaffen verschiedene Arten von sozialen Bewegungen mit unterschiedlichen Taktiken und internen Kulturen." (Swindler 1995: 37) So definieren sie bestimmte Begriffe vor, wie etwa Rechte oder ethnische Identität, die von den sozialen Bewegungen aufgegriffen werden, um ihre Forderungen dem Staat und der Öffentlichkeit präsentieren zu können (Swindler 1995: 37). „Soziale Bewegungen [...] bringen soziale Praktiken mit sich, die, teilweise, durch die Schaffung von Räumen für die Produktion von Bedeutungen wirken.“ (Escobar 1992: 73).

Kultur kann, je nach zugrundeliegendem Modell, eine in sich geschlossene Einheit sein, die einfach als eine weitere Variable behandelt werden kann - im Sinne von „kulturellen Möglichkeiten und Einschränkungen“ (Benford/Snow 2000: 629) -, oder etwas, das alle anderen Faktoren durchdringt, und so ein Neudenken dieser Begriffe notwendig macht (Jasper 2007: 60). Kultur als Ansammlung von Ideen darüber, wie Organisation und Mobilisierung funktioniert oder funktionieren sollte, also über das ,wie' sozialer Bewegungen (Polletta 2008: 84), erlaubt es, soziale Bewegungen anders zu verstehen. Alltägliche Praktiken tragen zur Entstehung und Entwicklung sozialer Bewegungen bei und schaffen in ihnen ,institutionelle Schemata" (Polletta 2008: 86f.), die wiederum die interne Kultur der Bewegung und damit ihre Handlungsweisen beeinflussen. Soziale Strukturen hängen also von kulturellen Schemata ab, Kultur ist somit eine mobilisierende Kraft (Polletta 2008: 87-89). 


\section{Identität}

Ein alter und noch immer verwendeter Ansatz zum Verständnis sozialer Bewegungen ist der der kollektiven Identität. Diese ist zu verstehen ,als die kognitive, moralische und emotionale Verbindung eines Individuums mit einer größeren Gemeinschaft, Kategorie, Praktik oder Institution“ (Polletta/Jasper 2001: 285). Somit ist sie die Wahrnehmung einer geteilten Wirklichkeit, die sich kulturell, etwa über Symbole, Narrative oder Praktiken, ausdrückt und positive Gefühle den anderen Gruppenmitgliedern gegenüber erzeugt (Polletta/Jasper 2001: 285). Kollektive Identität beschreibt imaginierte und konkrete Gemeinschaften, weshalb sie Wahrnehmung und Konstruktion und das Neuentdecken von bereits bestehenden Verbindungen umfasst. Sie ist sowohl in traditionellen Praktiken und Wissensformen verankert, als auch ein flüssiges und in wechselnden Beziehungen permanent neu entstehendes Projekt (Escobar 1998: 66). So macht kollektive Identität bestimmte Forderungen und Ideen möglich und andere nicht. Sie schafft Kategorien, durch die Individuen die Welt verstehen (Polletta/Jasper 2001: 298). Soziale Bewegungen mobilisieren auch Identitäten, die sie versuchen, in die Bewegungsidentität zu integrieren (Polletta/Jasper 2001: 289). Dazu bauen sie oft auf bereits bestehende kollektive Identitäten, indem sie ein ,Wir gegen die'-Gefühl erzeugen (Polletta/Jasper 2001: 291). Ein wichtiger Bestandteil des Aufbaus dieser Identität ist die Namensgebung der jeweiligen Gruppe, die nach innen hin Kohärenz gewährleisten und nach außen Druck aufbauen kann (Jenson 1995). Dadurch wirken soziale Bewegungen auf die Identitätskonstruktion der ihnen angehörenden Gruppen, bauen also zugleich die Identität der Bewegung und die der Gruppen, die sie vertritt (Escobar 1992a: 414f.). Die Schwächung der Bewegungsidentität, etwa weil sich die Mitglieder nicht mehr ausreichend repräsentiert fühlen oder sich mehr mit anderen Institutionen oder Bewegungen identifizieren, ist einer der wichtigsten Gründe für die Schwächung sozialer Bewegungen. Daher ist die Verwaltung der kollektiven Identitäten eine zentrale Aktivität sozialer Bewegungen (Polletta/Jasper 2001: 292). Sie werden nach außen hin als kohärenter dargestellt, als sie es in der alltäglichen Praxis sind. Identität ist somit nicht nur ein Mittel der sozialen Bewegungen, sondern auch ein gewolltes oder ungewolltes - Ergebnis; soziale Bewegungen bedienen sich Identitäten, erzeugen sie aber auch (Polletta/Jasper 2001: 296).

Identiät als Haupthandlungsgrund verändert die Eigenschaften von sozialen Bewegungen:

„,identitätsbasierte Bewegungen agieren eher als dass sie reagieren; sie kämpfen, um Freiheit zu erweitern, nicht, um sie zu erreichen; sie mobilisieren eher für Wahlfreiheit als für Emanzipation“ (Cerulo 1997: 393). 
So entsteht „eine selbstbewusste ,kollektive Kraft““ (Cerulo 1997: 393), die das Handeln der sozialen Bewegung steuert. Etwas weniger radikal ausgedrückt: kollektive Identität ist selbstdefiniert und zugeschrieben. Akteure beschreiben sich als Teil einer sozialen Bewegung und werden von dieser Bewegung - aufgrund ähnlicher Ideen - als ihr Teil angesehen. Die Grenzen der sozialen Bewegung werden von der kollektiven Identität ihrer Akteure definiert. Diese kollektive Identität ist die Grundlage für das Handeln sozialer Bewegungen. Daher können auch institutionalisierte Gruppen oder Parteien Mitglied sozialer Bewegungen sein (Diani 1992: $8-15)$.

\section{Framing}

Soziale Bewegungen wollen neben konkret fassbaren Dingen in Gesellschaft und Politik immer auch die Wahrnehmung der Wirklichkeit in der Bevölkerung verändern, „da sie annehmen [...], dass Bedeutung Handlung einleitet.“ (Benford 1997: 410) Sie betreiben „Bedeutungsarbeit - der Kampf um die Produktion von mobilisierenden und gegenmobilisierenden Ideen und Bedeutungen" (Benford/Snow 2000: 613). Dieser Perspektive widmet sich der Framing-Ansatz, der sich ab Mitte der 1980er Jahre entwickelt. Soziale Bewegungen erscheinen so nicht nur als Träger von Ideen, sondern als deren Gestalter und Verbreiter (Benford/Snow 2000: 613). Der Framing-Ansatz baut auf die Arbeit von Goffmann, der mit Frames diejenigen Phänomene meint, die Ereignissen Bedeutung geben und so Erfahrung organisieren und Handlung leiten (Benford/Snow 2000: 614). Es handelt sich also um eine aktive und prozesshafte Tätigkeit der Konstruktion der Wirklichkeit. Framing baut dabei auf kulturelle Grundlagen, wie Bedeutungen, Weltsichten, Praktiken oder Mythen (Benford/Snow 2000: 629). Soziale Bewegungen interpretieren die Wirklichkeit mit diskursiven Handlungen, etwa in Reden oder Schriften. Diese frame articulation meint eine systematische und einheitliche Verknüpfung der Meinungen und Erfahrungen der Bewegung, drückt also ihre Weltsicht aus. Die Verknüpfung von Bewegungswissen lässt sich je nach Erfordernissen erweitern (frame amplification), um so Allianzen mit anderen sozialen Bewegungen aufzubauen oder auf ein bestimmtes Geschehen reagieren zu können (Benford/Snow 2000: 623). Das Ergebnis von framing sind kollektive Handlungsrahmen (collective action frames), innerhalb derer das Verhalten von Gruppen von Menschen stattfindet (Benford/Snow 2000: 614). Sie lassen sich definieren als ,handlungsorientierte Sätze von Glaubensinhalten und Bedeutungen, die Aktivitäten und Kampagnen einer Sozialen Bewegungsorganisation (SMO) inspirieren und legitimieren“ (Benford/Snow 2000: 614). Kollektive Handlungsrahmen sind entscheidend für die Gewinnung neuer Mitglieder und den Aufbau einer kollektiven Identität der sozialen Bewegung, indem sie sowohl die Bewegung, als auch ihre Gegner als kohärente 
Akteure konstruieren. Dazu verwenden sie - wenn möglich - bereits bestehende, unabhängige kollektive Identitäten (Polletta/Jasper 2001: 291).

Da eine soziale Bewegung kein einheitliches Gebilde, sondern ein Feld von Akteuren ist, kann nicht von einem framing die Rede sein - vielmehr ist framing ein interner Prozess sozialer Bewegungen in dem verschiedene Akteure verschiedene Positionen einnehmen (Gamson/Meyer 1996: 283). Kollektive Handlungsrahmen werden innerhalb der sozialen Bewegung und in Bezug auf externe Probleme ausgehandelt. Ein Übereinstimmen über das Problem führt aber keinesfalls zu einem einheitlichen kollektiven Handlungsrahmen. Fragen, welcher Bestandteil des Problems wie angegangen werden soll, führen zu Streitigkeiten zwischen SMO oder innerhalb einer Organisation (Benford/Snow 2000: 615.f). Framing ist also immer von internen und externen Faktoren der sozialen Bewegung abhängig und daher in andauernder Entwicklung (Benford/Snow 2000: 628). Diese Entwicklung kann von strategisch orientierten Anpassungsprozessen, Rahmenausrichtungsprozessen (frame alignment processes) geprägt sein, die bewusst Teile des frames einer sozialen Bewegung verändern (Benford/Snow 2000: 624).

\section{Freiräume}

Soziale Bewegungen müssen, um effektiv agieren zu können, auf langfristige, lokale Strukturen bauen, die als Freiräume (free spaces) Möglichkeiten bieten, das oben beschriebene framing durchzuführen und so die vorhandene Situation und ihre Probleme zu analysieren und Lösungsansätze auszuarbeiten. „Freiräume scheinen eine institutionelle Verankerung für die kulturelle Herausforderung zu bieten, die strukturelle Gefüge sprengt.“ (Polletta 1997: 435/Polletta 1999a: 1) Diese kulturellen und institutionellen Freiräume sind relativ isoliert und dem direkten Einfluss des Staates und seiner Organisationen entzogen (Fantasia/Hirsch 1995: 145f.). Sie werden teilweise von früheren Bewegungen geschaffen, stehen aber nicht unter ihrer Kontrolle - wodurch der Ansatz der Freiräume eine mögliche Verbindung von Ressourcen-Mobilisierungs-Theorie und den Theorien über Neue Soziale Bewegungen sein kann. Diese Freiräume sind meist traditionelle kulturelle Institutionen, wie etwa Kirchengemeinden, Familienbünde (Polletta 1999a: 4f.) oder neuere Institutionen wie Mieterassoziationen, Bars, Gewerkschaftsräume (Polletta 1999a: 3). Sie befinden sich jenseits der direkt mit sozialen Bewegungen assoziierten Strukturen, ,es wird angenommen, dass gegenkulturelle Herausforderung dort entsteht, wo Struktur abwesend ist“ (Polletta 1997: 435). Daher stehen sie außerhalb der direkten Kontrolle durch externe Gruppen und können auf Freiwilligkeit und Informalität bauen.

„In diesen dichten interaktiven Netzwerken gemeinschafts-basierter Institutionen, malen sich die Leute alternative Zukünfte und Handlungsstrategien um sie zu realisieren aus. Freiräume 
stellen den Aktivisten Netzwerke, Fertigkeiten und Solidarität bereit, die den Start einer Bewegung unterstützen. Sie bieten auch den begrifflichen Raum, in dem unterdrückte Gruppen in der Lage sind, den vorherrschenden gesunden Menschenverstand, der die meisten Menschen passiv gegenüber Unrecht hält, zu durchdringen und sind so entscheidend für die Bildung von Identitäten und Interessen, die der Mobilisierung vorangehen.“ (Polletta 1999a: 3)

Dadurch erlauben Freiräume es den Menschen, nicht nur die Gründe ihrer Unterdrückung zu diskutieren, sondern auch, eine kollektive Widerstandserfahrung zu erhalten und auszubauen. Gegenkulturelle Praktiken und oppositionelle Identitäten können sich - auch und gerade bei einer repressiven staatlichen Politik - in diesen Freiräumen entfalten und dabei auf die Erfahrungen und Strukturen vorheriger sozialer Bewegungen bauen (Polletta 1999a: 6-8). In diesem Kontext können kulturelle Einstellungen neu verhandelt und - im Zuge einer Krise oder Rebellion verändert und den neuen Gegebenheiten angepasst werden. Dabei spielt die gewisse Autonomie, die der Staat den in der Regel traditionell ausgerichteten kirchlichen, familiären oder Bildungsgruppen gewährt, eine entscheidende Rolle. Sie sind die organisatorische Grundlage für das Aufrechterhalten und die Verbreitung traditioneller kultureller Werte (Fantasia/Hirsch 1995: 157f.). Durch sozialen Konflikt und starke soziale Bewegungen können diese Freiräume die ihr innewohnende Tradition mit neuen, radikalen Forderungen verbinden. Das kann dazu führen, dass zentrale Institutionen der bestehenden Gesellschaft wie Kirchen, Familien und Gemeindeorganisationen für die sozialen Bewegungen zu „Orten oppositioneller Kultur“ (Fantasia/Hirsch 1995: 157) werden und als solche vom Staat verfolgt werden. Freiräume dienen als ,,soziale Hülle für oppositionelle kulturelle Schöpfung und bieten die räumliche und sozial-organisatorische Grundlage für kulturelle Veränderung“ (Fantasia/Hirsch 1995: 145f.). Dennoch ist die Existenz lokaler und autonomer Strukturen nicht ausreichend, da die engen Kontakte der Mitglieder der Entwicklung globaler und radikaler Ziele eher entgegensteht (Polletta 1999a: 13). Der Ansatz der Freiräume schließt so wieder an die Theorie der politischen Gelegenheiten an, da es externer Ereignisse bedarf, die zu einer überlokalen Mobilisierung führen.

\section{Soziale Bewegungen und Kultur in Lateinamerika}

Der oft kritisierte Formalismus der Ressourcen-Mobilisierungs-Theorie, ihr Fokus auf direkt beobachtbare, materielle Aspekte, ist von Vorteil, wenn man soziale Bewegungen untersucht, die außerhalb des Entstehungskontextes der eher verstehenden oder interpretativen Theorien aktiv sind. Die framing-Theorie oder der Ansatz der Neuen Sozialen Bewegungen bietet tiefer gehende Einblicke in Funktionsweisen sozialer Bewegungen, in die Entwicklung ihrer Bewegungsidentität, ihrer Ziele und Strategien. Jedoch leiden diese Ansätze an der Tatsache, dass sie an 
der Beobachtung US-amerikanischer oder europäischer sozialer Bewegungen entwickelt wurden (Davis 1999). Für sie ist jede soziale Bewegung wie die Studentenund Bürgerrechtsbewegungen der 1960er oder die Friedens- und Umweltbewegungen der 1970er und 1980er. Da diese Bewegungen andere Entstehungsbedingungen haben als die meisten sozialen Bewegungen außerhalb der Länder, in denen sie aktiv waren, haben die an ihnen entwickelten Theorien einen starken eurozentrischen Bias. Sie können mehr erklären als die weitgehend auf Strukturen und Prozesse konzentrierte Ressourcen-Mobilisierungs-Theorie und ihre Weiterentwicklungen in der Politischen-Prozess-Theorie. Sie können aber alles nur so erklären wie die Bewegungen, die sie schon erklärt haben. Aus diesem Grund wird hier der reduktionistische und formalistische Ansatz der Ressourcen-MobilisierungsTheorie vorgezogen, der an bestimmten Stellen um kulturelle oder identitäre Erklärungsmuster ergänzt wird.

Die meisten der Theorien über soziale Bewegungen gehen von den USamerikanischen und europäischen Fällen aus, also von Bewegungen, die unter anderen Bedingungen entstehen und handeln als sie in Lateinamerika vorhanden sind. Diese Theorien bauen auf

„,westliche“ Annahmen über Modernität und geschichtlich spezifische Erfahrungen von Demokratie, Staatsbürgerschaft und Staatenbildung, die für Europa und die Vereinigten Staaten charakteristisch sind und daher nicht in Lateinamerika gelten können“ (Davis 1999: 597).

Gerade die europäischen Ansätze gehen von einer Modernisierung als klare Trennung zwischen Staat und (Zivil-)Gesellschaft aus, eine Trennung, die in Lateinamerika weniger klar ist (Davis 1999: 597). Aus diesem Grund bevorzugen einige lateinamerikanische Forscher die Unterscheidung von Elite und Masse statt die von Zivilgesellschaft und Staat.

Diane Davis arbeitet auf diesen Grundlagen einen staatszentrierten Ansatz aus, der die Besonderheiten der sozialen Bewegungen in Lateinamerika besser erklären soll. Sie geht davon aus, dass der Staat in Lateinamerika zwar im Alltag seiner Bürger präsent ist und zu einer repressiven Politik neigt, die freie Debatten einschränkt. Er hat sich aber ungleich entwickelt und kann diese Wirkung nur in manchen Bevölkerungsgruppen oder Gebieten entfalten. Zum Teil liegt das an der anderen Beziehung zwischen Sozialstruktur und Staat, die sich in Lateinamerika ausgebildet hat. Diese Beziehungen sorgen dafür, dass die in anderen Gegenden klare Trennung zwischen Staat und (Zivil-)Gesellschaft verschwommen ist. So sind einige der wichtigsten sozialen Akteure - wie die Gewerkschaften von Lehrern und öffentlichen Angestellten - selbst an den Staat gebunden (Davis 1999: 598f.). Somit sind die institutionellen Strukturen, auf die die Forderungen der sozialen Bewegun- 
gen gerichtet sind, und die Antworten des Staates andere als in Europa oder den USA. Dazu gehört vor allem ein Mangel an formalen demokratischen Strukturen und Rechtssicherheit - ein Phänomen, das sowohl den Staat als auch die eng mit ihm verbundene Gesellschaft betrifft (Davis 1999: 600f.). Daher ist für Davis ein anderer theoretischer Ansatz vonnöten, der gleichzeitig Staat und Zivilgesellschaft beschreiben kann (Davis 1999: 598f.). Dieser Ansatz ist die „Distanz vom Staat“ (Davis 1999: 601), die geographisch, institutionell, kulturell oder sozial sein kann (Davis 1999: 603).

Geographisch ist die Distanz in dem Sinne, dass in Lateinamerika meist die Hauptstadt das politische und wirtschaftliche Zentrum des Landes ist und ihrer Bevölkerung weiterreichende Möglichkeiten bieten kann als andere Regionen. Soziale Bewegungen, die in der Hauptstadt und den größeren Städten aktiv sind, haben daher besseren Zugang zu den Institutionen und können leichter breite Allianzen bilden (Davis 1999: 603f.). Ländliche soziale Bewegungen haben dagegen Schwierigkeiten, Druck auf den Staat auszuüben, solange sie sich nicht zu größeren Strukturen zusammenschließen, die einen großen Teil des Landes umfassen. Daher sind seit den 1970ern eine Reihe von Einheitsorganisationen und Koordinationen verschiedener kleinerer Organisationen entstanden. Diese können die Distanz vom Staat ausnutzen, um Solidaritätsnetzwerke aufzubauen und so statt zu einzelnen Forderungen an den Staat zu Forderungen nach einer radikalen Umgestaltung von Staat und Gesellschaft kommen (Davis 1999: 604-606).

Die institutionelle Distanz drückt sich im fehlenden Zugang der meisten Bürger zu den Entscheidungsprozesses des Staates aus. Die wichtigsten Institutionen sind national und nicht auf lokale Beteiligung ausgelegt. Die politischen Parteien, ein anderer Zugangsweg, sind meist schwach und zersplittert. Gerade weil nur nationale Akteure mit einiger Aussicht auf Erfolg Druck auf den Staat ausüben können, sind die meisten sozialen Bewegungen in Lateinamerika in erster Linie lokale Bewegungen, die alltägliche Probleme thematisieren und so versuchen, die Distanz zwischen Bürger und Staat zu überbrücken (Davis 1999: 608f.).

Die soziale oder Klassendistanz besteht in einer klassenbasierten bürokratischen und institutionellen Bindung bestimmter Gruppen an den Staat, die die Artikulierung von Interessen außerhalb dieser Strukturen erschwert. Die institutionell integrierten Gruppen sind nur wenig motiviert, die bestehenden Strukturen in Frage zu stellen - solange sie von ihrer Integration Vorteile haben. Marginalisierte Bevölkerungsgruppen haben in diesem korporatistischen System nur geringen Zugang zu den eingebundenen Gruppen (Davis 1999: 614f.).

Diese Strukturen der Distanz und die sie begründenden Diskurse erzeugen Bilder von Staat und Staatsbürgerschaft, gegen die die ausgeschlossenen Bürger mobilisieren, auch, indem sie Alternativen suchen (Davis 1999: 616). Daher stellen die am meisten vom Staat entfernten Bürger seine Strukturen als solche in Frage, wobei 
sie besonders die systematischen Ausschlussmechanismen hervorheben (Davis 1999: 620).

Auch Kultur wirkt für die sozialen Bewegungen in Lateinamerika anders als für die Bewegungen der USA oder Europas. Kultur in Lateinamerika ist hybrid in dem Sinne, dass sie sowohl mit dem Markt und dem transnationalen Kultursystem verbunden ist, als auch mit lokalen und gemeinschaftlichen Systemen der Bedeutungserzeugung. Kulturpolitik im Sinne Escobars beschränkt sich in Lateinamerika nicht auf eine Verwendung oder Aneignung dominant platzierter Produkte oder Texte. Die mangelnde Durchdringung der lateinamerikanischen Gesellschaften durch Markt und Staat führt zu einer anderen Produktion und Zirkulation von Bedeutungen (Escobar 1992: 75f.). Daher ist eine Vermischung verschiedener Mobilisierungsgründe, etwa eine ethnische Bewegung, die gegen wirtschaftliche Missstände protestiert, keine Seltenheit (Bebbington 2007: 796f.). Sie führen gleichermaßen Kämpfe um Bedeutungen und um sozioökonomische Bedingungen - auch wenn dieser letzte Aspekt von Außenstehenden oft eher wahrgenommen wird (Escobar 1992a: 412). Vor allem Privatisierungsprozesse und neue Formen der Ausbeutung von natürlichen Ressourcen können mobilisierend wirken und zu Kooperationen zwischen den verschiedensten sozialen Bewegungen führen. Diese breiten Bewegungen sind Antworten auf etwas, das als Versuch der ,Akkumulation durch Enteignung“ (Bebbington 2007: 802) wahrgenommen wird. Die langjährigen Organisierungsbemühungen verschiedenster sozialer Bewegungen legten die Grundlagen für die Entwicklung vor allem ethnischer Bewegungen als nationale Akteure (Bebbington 2007: 804). Auch die Beteiligung von verschiedenen Wissenschaftlern nicht nur als Erforscher der Bewegungen, sondern auch als ihre Ratgeber hat zu einer breiten Konstituierung ihrer Forderungen beigetragen (Bebbington 2007: 806f.). Die Grundlage für Mobilisierung ist meist nicht absolute Armut, sondern strukturelle Diskriminierung und politische Exklusion (Bebbington 2007: 805). Daher sind die Ärmsten, die unter Mangel an Ressourcen und Zeit leiden, in der Regel nicht an sozialen Bewegungen beteiligt - und ihre Interessen dort nur wenig repräsentiert (Bebbington 2007: 811). Viele der Bewegungen in Lateinamerika kämpfen also eher gegen Ungleichheit als gegen Armut (Bebbington 2007: 813). Dieses Interesse an Partizipation kann durch eine marginale Integration der betreffenden Bewegungen in politische Prozesse - etwa über runde Tische oder Verhandlungen mit der Regierung - zu einer Gefahr für die soziale Bewegung selbst werden. Ein Einfluss von externen Akteuren auf die Bewegung und ihre Ideen lässt ihren gegenhegemonialen Diskurs an Gewicht und sie selbst an Einfluss und Mobilisierungspotential verlieren (Bebbington 2007: 813). 


\section{c. Die Behandlung sozialer Bewegungen im Kontext der vorliegenden Arbeit}

Für die vorliegende Arbeit sind soziale Bewegungen kollektive Bemühungen bestimmter Gruppen, von ihnen als problematisch wahrgenommene Situationen zu verändern. Dazu bilden sich innerhalb der Bewegungen relativ fest strukturierte Organisationen, die im Rahmen der Interessen der Bewegung bestimmte Forderungen in einem kohärenten Diskurs formulieren und bestimmte Mobilisierungsformen entwickeln und durchführen, um diese Forderungen umzusetzen. Forderungen und Mobilisierungen können sich dabei an den Staat oder einen Teil von ihm oder an die Gesellschaft oder bestimmte Gruppen in ihr wenden, haben aber ebenso eine zentrale Funktion innerhalb der Organisation, ihrer Bewegung und der Gruppe, die sie vertritt. Der Erfolg der verschiedenen Organisationen hängt zum einen von ihrer Mobilisierungsfähigkeit innerhalb der Bewegung und ihres Umfeldes ab, die wiederum von der Akzeptanz ihrer Forderungen und Aktionsformen abhängt, zum anderen aber auch von ihrer Fähigkeit, ihre Forderungen umzusetzen oder ihre Umsetzung zumindest als wahrscheinlich erscheinen zu lassen. Daher konkurrieren die verschiedenen Organisationen um begrenzte Ressourcen, die ein Weiterbestehen der Strukturen und die Durchführung von Mobilisierungen zum Erreichen der Forderungen erlauben, und können miteinander kooperieren, wenn die Umsetzung ihrer Forderungen wahrscheinlich erscheint - oder die Bewegung in einer Krise ist. Kultur und Identität sind dabei nicht nur mögliche Ressourcen oder Hindernisse, sondern prägen die grundlegende Struktur der Bewegung und ihres Diskurses. Eine soziale Bewegung mit ihren Mitgliedsorganisationen ist ein Generator für Diskurse, die den bestehenden Staat und die aktuelle Gesellschaftsstruktur kritisieren oder in Frage stellen können.

\section{FORSCHUNGSDESIGN}

Die vorliegende Arbeit versteht sich als begriffszentrierte Diskursanalyse. Es soll der Diskurs der Indigenenbewegung Ecuadors untersucht werden, unter Hervorhebung bestimmter Begriffe, die 1. abstrakt, 2. mit Forderungen verbunden, 3. im Diskurs der Bewegung zentral, 4. innerhalb und außerhalb der Bewegung umstritten sind und 5. eine Geschichte in der Bewegung haben. Diese Begriffe sind Territorium, Nationalität, Plurinationalität und Interkulturalität. Die Analyse dieser Begriffe stützt sich auf ihre Verwendung in den an die allgemeine Öffentlichkeit gerichteten Texten der Organisationen der Indigenenbewegung. Es sollen begriffliche Unklarheiten (etwa Multi- oder Plurikulturalität statt Interkulturalität), Definitionsversuche $\mathrm{zu}$ unterschiedlichen Zeitpunkten und Wechselwirkungen zwischen 
den Begriffen besonders beachtet werden. Dazu wird die Diskurstheorie nach Foucault ${ }^{9}$ mit der Begriffsgeschichte nach Koselleck verbunden.

Für Foucault ist der Diskurs als Möglichkeitsbedingung allen gesellschaftlichen Wissens zutiefst mit der Macht verbunden. Die Machtverhältnisse haben dabei einen ,strikt relationale[n] Charakter“ (Foucault 1983: 96), Macht verläuft parallel zu den sozialen Beziehungen, sie ist ihnen immanent (Foucault 1983: 94). Weder der Diskurs, noch die Macht sind dabei mit Subjekten verbunden, deren Intentionen sie leiten würden (Rusterholz 1998: 2332). Niemand hat also Macht, alles ist Macht. Wissen und Macht vereinen sich im Diskurs, ,dieser [ist] als eine Serie diskontinuierlicher Segmente zu betrachten, deren taktische Funktion weder einheitlich noch stabil ist" (Foucault 1983: 100).

„Die Diskurse sind taktische Elemente oder Blöcke im Feld der Kraftverhältnisse: es kann innerhalb einer Strategie verschiedene und sogar gegensätzliche Diskurse geben; sie können aber auch zwischen entgegengesetzten Strategien zirkulieren, ohne ihre Form zu ändern.“ (Foucault 1983: 101)

Diskurse sind regelhaft (etwa Foucault 1983: 98-100) geordnete Aussagesysteme, die von bisher getätigten Aussagen erzeugt wurden und weitere Aussagen nach den jeweils aktuellen Regeln ermöglichen (Diaz-Bone et al. 2007: 6). Diese Regeln sind nicht als Handlungsanweisungen, sondern als „Regeln der Wissenskonstitution und -strukturierung“ (Busse 1987: 233) zu verstehen. Sie sind produktiv und erzeugen beständig neue Aussagen, Verweise und Bedeutungen. „In diesem Sinn erschaffen Diskurse eine Wahrnehmung und Repräsentation der sozialen Wirklichkeit.“ (DiazBone et al. 2007: 7) Die Untersuchung der Aussagen eines Diskurses ermöglicht es, die Regel des Diskurses zu erfassen - und so das System, das die Aussagen gliedert, analysieren zu können (Diaz-Bone et al. 2007: 6). Ein Diskurs ist also ,eine Menge von Aussagen, die einem gemeinsamen Formationssystem angehören“ (Busse 2003: 23f.). In diesem Formationssystem haben die Aussagen verschiedene Funktionen und werden mit einem bestimmten Sinn verbunden (Busse 1987: 222). „Wesentlich ist nicht die einzelne Aussage [...] sondern die Position, die sie in einem Netz diskursiver Beziehungen einnehmen.“ (Busse 1987: 229f.) Die Aussagen müssen dabei keinesfalls in festen Formen auftreten, sondern können im Sinne von Wissenssegmenten auf verschiedene Weisen artikuliert werden. Als solche steuern sie „die Bedingungen der Möglichkeit der Produktion bestimmter Äußerungen“

9 Es sei darauf hingewiesen, dass Foucault selbst keine Klarheit über sein methodologisches Vorgehen schafft (Busse 1987: 241) und sich daher verschiedene Methoden auf ihn berufen können. 
(Busse 2003: 23f.). Aussagen müssen nicht zwangsläufig sprachlich sein, jede soziale Handlung ist auch eine Aussage im Sinne Foucaults (Busse 1987: 224).

Eine Reihe diskursiver Mechanismen strukturieren die Aussagen eines Diskurses (Busse 2003: 23f.). Dazu gehören „die vier Konzepte Ereignis, Serie, Regelhaftigkeit und Möglichkeitsbedingung“ (Busse 2003: 24), die vom spontanen Auftreten bestimmter Aussagen als Ereignis über ihre Wiederholung als Serie und deren Verdichtung zu einer Regelhaftigkeit, die wiederum die Möglichkeitsbedingung neuer Aussagen ist, die mögliche „Etablierung neuer diskursiver Strukturen“ (Busse 2003: 24) lenken. Diese Strukturierung sorgt dafür, dass bestimmte Inhalte regelmäßig auftreten und sich bestimmten Diskursen zuordnen lassen. Busse spricht von „diskurssemantische[n] Grundfiguren“ (Busse 2003: 28f.), um deutlich zu machen, dass die Aussagen und ihre diskursive Funktion nicht unbedingt den Sprechern, die sie verwenden, bewusst sind. Diese Grundfiguren ordnen - ganz im Sinne von Foucaults Begriffen Regelhaftigkeit und Möglichkeitsbedingung - das Auftreten inhaltlicher Elemente in Diskursen und strukturieren diese. Sie sind nicht an einen bestimmten Diskurs gebunden, sondern können gleichzeitig in verschiedenen Diskursen auftauchen - und so die Diskurse in Beziehung bringen (Busse 2003: 29f.). Weiterhin gehören alle Aussagen zum „Feld der Begleitumstände“ (Busse 1987: 230), das auf jede andere Aussage jedes Bereiches Einfluss nehmen kann. Die diskursiven Grundfiguren müssen keinesfalls Wörtern oder Begriffen entsprechen (Busse 2003: 30), sie sind vielmehr die Regeln, nach denen diese - und andere kommunikative Handlungen - eingesetzt werden.

Die Diskursanalyse versucht nun, anhand der einzelnen Aussagen und ihres Kontextes ihre Regelhaftigkeiten und Möglichkeitsbedingungen und damit die Regeln des Diskurses herauszuarbeiten. Die Analyse der Aussagen in einem Diskurs soll dabei „die Identifizierung von Regelmäßigkeiten“ (Busse 2003: 24) ermöglichen. So kann erklärt werden, warum zu einem bestimmten Zeitpunkt eine bestimmte Art von Aussagen von bestimmten Akteuren verwendet wurde - und nicht unter anderen Umständen (Busse 1987: 233f.). Dazu muss eine Vielzahl von einzelnen Aussagen auf Gemeinsamkeiten untersucht werden, die Aufschluss über die ihnen zugrundeliegenden Regelmäßigkeiten geben können. Von besonderem Interesse ist, wann eine Regel gebrochen wird oder eine Regelmäßigkeit aufhört zu existieren (Busse 1987: 258). Das kann nur über eine Analyse der „,Produktionsbedingungen“ der diskursiven Äußerungen“ (Busse 1987: 259) geschehen, die den Kontext des Entstehens der jeweiligen Aussage klären soll. Insbesondere ist die „soziale Situierung der kommunikativen Akte [...] und der aktuelle Kontext, in dem sie stehen“ (Busse 1987: 260) von Interesse, also die Frage, an welche Aussagen und Diskurse eine neue Aussage anschließt und von welchen sie sich abgrenzt. Diese Situierung entspricht einer diskursiven Periodisierung, die einzelne Serien und Regelhaftigkeiten zeitlich voneinander abgrenzt (Busse 1987: 270). Foucault 
untersucht in seinen Diskursanalysen zuerst den Wissensbereich, der diskursiv erzeugt wird, dann, nach welcher Logik die Begrifflichkeit des Bereiches strukturiert ist, weiterhin, wer oder was den Diskurs kontrolliert und abschließend, welches die strategischen Ziele sind, die im Diskurs verfolgt werden (Diaz-Bone et al. 2007: 5).

Der Diskurs ist für Foucault die Summe alles Sagbaren (Steinmetz 2008: 184). In diesem Sinne sind Begriffe Knotenpunkte des Diskurses, zu denen man sich zwangsweise verhalten muss, wenn man über das jeweilige Thema spricht. Foucault versteht den Diskurs als „,Ort des Auftauchens der Begriffe‘, dessen Formationssystem allererst das Erscheinen und die Kombination von Begriffen ermöglicht“" (Busse 1987: 225). Diese Begriffe oder Aussagen sind einem semantischem Wandel unterworfen, der drei typische Verlaufsformen nehmen kann:

„(1) Wandel durch Plausibilitätsverlust von Wörtern oder Redeweisen infolge überraschender Ereignisse und Umbrüche; (2) Wandel durch $\mathrm{Zu}$ - oder Abnahme des strategischen Gebrauchswerts von Wörtern oder Redeweisen in wiederkehrenden Kommunikationssituationen; (3) Wandel durch Irritation des Wort- und Bedeutungshaushalts einer Sprache durch Wortimporte aus einer anderen Sprache.“ (Steinmetz 2008: 187f.)

Im ersten Fall, dem Plausibilitätsverlust bestimmter Wörter, können diese einen neuen Sachverhalt nicht mehr erfassen und werden daraufhin verändert oder ersetzt. Diese Verlaufsform erklärt die Abschwächung und das Verschwinden, aber nicht das Aufkommen neuer Begriffe (Steinmetz 2008: 188f.). Das lässt sich durch den Wandel des strategischen Gebrauchswerts der Begriffe in den jeweiligen Situationen erklären. ,Wörter gewinnen in der politischen Kommunikation an Gebrauchswert, wenn sie die Fähigkeit besitzen, möglichst viele disparate Einzelerfahrungen zu bündeln." (Steinmetz 2008: 189f.) Wenn sie also sehr anschlussfähig sind und nahezu beliebig mit Inhalt gefüllt werden können. Dazu gehören „Kollektivbezeichnungen, die breite Aktionsbündnisse ermöglichen, also möglichst inkludierend sind" (Steinmetz 2008: 189f.). Eine dritte Erklärung für semantischen Wandel ist die "Irritation infolge von Wortimporten von der einen in die andere Sprache“ (Steinmetz 2008: 190f.). Sprache muss dabei als ein spezifisches Phänomen besonderer sozialer oder kultureller Gruppen gesehen werden - die Wortimporte erfolgen also nicht unbedingt aus einer Fremdsprache, sondern aus einem andern sozialen oder kulturellen Kontext.

An diesem Punkt ist die Diskurstheorie Foucaults mit der Begriffsgeschichte nach Koselleck kombinierbar. Letztere interessiert sich weniger für das erste Erscheinen und eine möglichst genaue Definition der einzelnen Begriffe, sondern für ihre Verbreitung und Bedeutung in der Gesellschaft zu einem gegebenen Zeitpunkt, ,also eine sozialhistorische Konturierung der semantischen Bestimmungen“ (Leon- 
hard 2004: 79). Damit unterscheidet sie sich nicht grundlegend von diskursanalytischen Ansätzen, die ebenfalls mit Aussagen eine Oberkategorie von Begriffen untersuchen.

„Diskurse lassen sich also von begrifflichen Deutungsmustern als unersetzbare Einheiten des politisch-sozialen Vokabulars gar nicht separieren, sondern sind in deren Genese und Transformation notwendig angelegt. Grundbegriffe entstehen und funktionieren nur im Diskurs, der nicht ohne sie zu denken ist. Diskurs und Grundbegriff befinden sich von daher in einem Interdependenzverhältnis, das eine methodische Überordnung der einen über die andere Analyseeinheit ausschließt.“ (Leonhard 2004: 83)

Die Begriffsgeschichte untersucht im Sinne einer historischen Semantik die Bedeutungskonstitution von Begriffen und ihre Bedingungen und ,wählt dazu isolierte, verdichtende Stichwörter, denen eine Schlüsselstellung zugesprochen wird, um sprachförmige Konzeptualisierungen zu erfassen und zu kontextualisieren“ (Kollmeier 2012: 1). Diese Begriffe zeichnen sich dadurch aus, diskursive Entwicklungen nicht nur abzubilden, sondern sie zu gestalten (Koselleck 1996: 61). Es sind „Agenten des historischen Prozesses“ (Busse 2003: 22). Dabei geht es nicht um eine sprachwissenschaftliche Analyse der jeweiligen Begriffe, sondern um die Analyse ihrer Funktionsweisen und Bedeutungen in einem konkreten sozialen Kontext (Kollmeier 2012: 1). Tatsächlich müssen diese Begriffe nicht an einzelne Wörter gebunden sein, sondern können auch durch ähnliche Wortbildungen ausgedrückt werden (Busse 2003: 22) - und so leichte Bedeutungsverschiebungen erfahren. Aus diesem Grund muss gleichzeitig semasiologisch und onomasiologisch vorgegangen werden, also sowohl alle Bedeutungen eines Wortes oder Begriffs, als auch alle Bezeichnungen eines Begriffs untersucht werden (Koselleck 1996: 64). „Begriffsbildungen und der sich wandelnde Wortgebrauch werden als sprachförmig kondensierte Antworten auf spezifische historische Herausforderungen begriffen." (Kollmeier 2012: 4f.) In diesem Sinne analysiert die Begriffsgeschichte die Momente der Begriffsbildung und der Verschiebung der ihm zugeschriebenen Bedeutung (Koselleck 1996: 68; Kollmeier 2012: 5). Dazu werden die Begriffe als 1) politisch, 2) umstritten, 3) mit einer langen Geschichte ausgestattet, 4) Produzenten von oft ungewollten politischen Folgen und 5) auch außerhalb der jeweiligen Elite verwendet gesehen (Richter 2005: 220). Es handelt sich - wie der neuere Ansatz des Netzwerkes Iberconceptos feststellt - um ,soziopolitische und identitäre Grundbegriffe“ (Fernández Sebastián 2007: 167), deren Wirkungen die gesamte Gesellschaft umfassen. Solche Begriffe sind wortgewordene Diskursprozesse, greifbare Einheiten, die auf Entwicklungen verweisen, die nicht ohne weiteres sichtbar gemacht werden können. Sie repräsentieren ,historische Problemfelder in verdichteter Form“ (Kollmeier 2012: 4). 
Die Begriffe im Sinne der Begriffsgeschichte zeichnen sich dadurch aus, nicht auf Erfahrungen aufbauen zu können, also keine Beschreibung oder Interpretation bereits geschehener Ereignisse zu sein. Die mit der Moderne zunehmende Komplexität - eine der Grundvoraussetzungen für Kosellecks Ansatz der Begriffsgeschichte - reduziert die Reichweite der direkten persönlichen Erfahrung und führt zu immer abstrakteren Begriffen, die nicht mehr nur auf die Vergangenheit, sondern vor allem auf die Zukunft verweisen. „Viele Grundbegriffe, vor allem diejenigen, die Bewegungen bezeichnen -ismen-, stimmten darin überein, dass die zukünftige Geschichte grundsätzlich anders sein sollte als die Vergangenheit." (Koselleck 1996: 60f.) Die geringe Bindung an Vergangenes macht diese Begriffe anschlussfähig für Ideologien, die auf eine Veränderung aus sind. Aus diesem Grund kann Begriffsgeschichte sich immer nur mit der spezifischen Sprache spezifischer Sprecher in spezifischen Situationen beschäftigen (Koselleck 1996: 61f.). Begriffe sind beständiger Entwicklung unterworfen, ihre Bedeutung ist nicht fixiert.

Die Arbeiten der Begriffsgeschichte lassen sich nicht ohne weiteres für eine Analyse von Begriffen im heutigen Kontext verwenden. Zum einen hat sie das methodische Problem der Fixierung auf eine Sattelzeit von ungefähr 1750 bis 1850, in der die untersuchten Begriffe geprägt wurden oder ihre Bedeutung erheblich verändert haben (Fernández Sebastián 2007: 167; Kollmeier 2012: 3f.). In diesem Zeitraum fand eine Verdichtung und Abstrahierung von bis dahin im Plural verwendeten Begriffen zu Allgemeinbegriffen statt. Diese begünstigte eine Rezeption solcher Begriffe auch außerhalb der Eliten und damit ihre Verwendung als Mittel politischer Auseinandersetzungen, ihre Verbindung mit bestimmten Erwartungen und schließlich ihre Ausweitung gemäß der gesellschaftlichen Pluralisierung - in der Terminologie der Begriffshistoriker Demokratisierung, Ideologisierbarkeit, Verzeitlichung und Politisierung (Richter 1997: 28-30; Leonhard 2004: 76f.; Kollmeier 2012: 5). Daher ist die Anwendung der Methoden der Begriffsgeschichte auf „die Hochmoderne und das gesamte 20. Jahrhundert weit weniger entwickelt und erprobt“" (Kollmeier 2012: 14).

Ein Problem für die Anwendung der Begriffsgeschichte auf die vorliegende Arbeit ist zudem ihre Orientierung auf Grundbegriffe im Sinne von abstrakten und allgemein gebräuchlichen Begriffen, wie etwa Nation, Volk oder Republik, die von verschiedenen Gruppen unterschiedlich verwendet und interpretiert werden (Koselleck 1996: 65; Fernández Sebastián 2007: 166f.) - und nicht auf Begriffe die als solche oder in ihrer Bedeutung von bestimmten Gruppen neu eingeführt werden und keine Verallgemeinerung in diesem Sinne erreichen. Koselleck etabliert den Sonderbegriff der Grundbegriffe, die sich von normalen Begriffen dadurch unterscheiden, dass sie ,ein unausweichlicher, unersetzlicher Teil des politischen und sozialen Vokabulars“ (Koselleck 1996: 64f.) sind. Diese Grundbegriffe sind zentral im Diskurs des jeweiligen Feldes verankert und repräsentieren verschiedene und 
widersprüchliche Inhalte und Erfahrungen, weshalb sie stets umstritten sind (Koselleck 1996: 65). Ein Grundbegriff verdrängt oder verändert andere, ähnlich gelagerte Begriffe. „Er legt fest und grenzt ab, er erweitert und reduziert das für folgende Generationen verfügbare Vokabular.“ (Koselleck 1996: 66) Damit sind Grundbegriffe gleichzeitig „Indikatoren geschichtlicher Bewegung [und] Faktoren des geschichtlichen Prozesses“ (Busse 1987: 51), gleichzeitig historisches Subjekt und (Forschungs-)objekt. Die Forschungsperspektive hebt dabei die sprachtheoretischen Zweifel Busses (1987: 51) auf: zum Zeitpunkt seiner Untersuchung ist der Grundbegriff für den untersuchten Zeitraum kein Akteur mehr - da dieser Zeitraum schlichtweg vergangen ist.

Schließlich kann die Orientierung auf Begriffe dazu führen, dass Diskurse oder Bedeutungen, die ohne Nennung der jeweiligen Begriffe auskommt, ignoriert wird (Busse 2003: 26f.; Kollmeier 2012: 7). Ebenso werden nicht alle Bedeutungen der jeweiligen Begriffe aufgespürt. Die Orientierung der Begriffsgeschichte auf bestimmte Bedeutungen bestimmter Begriffe führt in diesem Sinne zu einer - teils unnötigen - Verengung der Forschung (Bruners o.J.: 6/9).

Koselleck entwickelt einen Begriff asymmetrischer Gegenbegriffe, die für die vorliegende Arbeit wenig hilfreich ist. Es geht bei der Beschreibung der Begriffe der Indigenenbewegung Ecuadors nicht um asymmetrische Gegenbegriffe, die im Sinne Kosellecks ein Außen markieren (Junge 2011: 10f.). Ein solcher Gegenbegriff ist also der Begriff des Anderen für das Selbst (oder Wir). Die Begriffsgeschichte Kosellecks geht von Begriffen aus, die meist auf einen bestimmten - wenn auch austauschbaren - Gegenbegriff weisen (Junge 2011: 25). Wenn alle - oder fast alle - Begriffe Gegenbegriffe im Sinne Kosellecks haben, ist die Begriffsgeschichte Kosellecks eine zutiefst strukturalistische. Das lässt sich auf politische Begriffe im Sinne dieser Arbeit nicht anwenden. Der hier verwendete Begriffsbegriff bricht mit dieser binären Weltsicht. Politische Begriffe im hier verwendeten Sinne haben nicht die Reichweite der koselleckschen Grundbegriffe. Die vorliegende Arbeit orientiert sich eher an Kosellecks Begriff des Kampfbegriffs, ein Begriff, der dazu verwendet wird, die Vorschläge und Begriffe des Gegners zu diskreditieren (Koselleck 1996: 68) - oder umzudeuten. Der Begriff des Gegenbegriffs meint hier: ein Begriff der sich gegen einen bereits bestehenden Begriff wendet, ihn als Knotenpunkt von Forderungen, Weltsichten und Handlungsweisen kritisiert und sich selbst als Alternative anbietet.

Die Begriffe der Indigenenbewegung werden als politische, das heißt als mit Forderungen an Staat und Gesellschaft verbundene Begriffe verstanden ${ }^{10}$. Damit

10 Wodurch sich die vorliegende Arbeit von Studien unterscheidet, die Indigenität -also ein identitäres Konstrukt, dass als Grundlage von Forderungen dienen kann- ins Zentrum ih- 
unterscheiden sie sich von den Grundbegriffen der Begriffsgeschichte, die nur in einigen Fällen konkrete Forderungen enthalten. Die Begriffe der Indigenenbewegung, die hier untersucht werden sollen, sind also normativ, im Gegensatz zu den deskriptiven Begriffen der Begriffsgeschichte, die zwar die Realität, die sie zu beschreiben vorgeben, erst erschaffen, aber doch immerhin vorgeben, beschreibend zu sein und nur so funktionieren können. Daher wird die deskriptive Begriffsbildung in dieser Arbeit vernachlässigt. Der Begriffsbegriff von Foucault, auf den Busse (1987) aufbaut, ist, ebenso wie die essentially contested concepts (Gallie 1956) vom hier verwendeten Begriff des Begriffs zu unterscheiden. Beide Ansätze verstehen Begriff als Wissenskategorie, im Sinne des Begreifens einer Realität. Die politischen Begriffe, die in dieser Arbeit untersucht werden sollen, sind keine Wissenskategorien in diesem Sinne. Es handelt sich vielmehr um gebündelte Forderungen, die auf eine bestimmte Weltsicht verweisen (Benford/Snow 2000: 623). Politische Begriffe sind somit normativ - und nicht deskriptiv, wie die Begriffe, mit denen Foucault und Gallie arbeiten. Der zentrale Akteur, der hier untersucht wird, ist die Indigenenbewegung, also die in mehr oder weniger formal strukturierten Organisationen tätigen Indigenen und - in geringerem Maße - nicht-Indigenen. Daher wird diese Arbeit nicht näher definieren, was ,Indigen“ ist. Es reicht, die Bewegungsidentität der einzelnen Organisationen als Organisationen der Indigenenbewegung zu betrachten.

Die Begriffe der Indigenenbewegung sind stets politische - also normative Begriffe. Sie beziehen sich auf Veränderungen, die erst durchzusetzen sind. Davon sind wissenschaftliche Begriffe, die deskriptiv funktionieren, zu unterscheiden. Das Verständnis des politischen Begriffs der Plurinationalität als deskriptiver Begriff, der die ethnische Pluralität beschreibt (etwa in Almeida 2008), beschneidet den Begriff also um einen Teil seiner - normativen - Bedeutung. Dasselbe trifft auf den deskriptiven Interkulturalitätsbegriff von Costa/Gurza (2006: 249) zu. In diesem Sinne ist auch der Vergleich zwischen den deskriptiven Begriffen Multi- und Plurikulturalität und dem normativen Begriff Interkulturalität, den (Ramón 2009: 134135) macht, ungenügend. Beide Arten von Begriffen miteinander zu vergleichen, bedeutet auch, die normativen Aspekte des Deskriptiven zu betonen (in dem Sinne, dass Multikulturalität eben nicht nur die Existenz verschiedener Ethnien bedeutet, sondern auch einen bestimmten Umgang zwischen diesen erwartet).

Ein politischer Begriff ist hier ein Wort oder Wortkomplex, das abstrakte - das heißt nicht konkret fassbare -, allgemeine, auf das Zusammenleben der gesamten Gesellschaft gerichtete Forderungen enthält und auf dahinterstehende, komplexe und widersprüchliche Programme oder Weltsichten verweist. Daher ist ein politi-

rer Aufmerksamkeit stellen (etwa Canessa 2007). Fragen der Identität sollen hier nicht untersucht werden. 
scher Begriff immer uneindeutig und verschiedenen Auslegungen oder Interpretationen unterworfen. Er ist eigenständig als Zeichen, dass auf diesen komplexen Forderungskatalog oder diese Reihe an Ideen verweist, umstritten zwischen den Gruppen, die sich zu diesen Forderungen verhalten und ständigem Wandel unterworfen. Die Forderung nach Zugang zu Land für eine bestimmte Gemeinde oder nach günstigen Krediten ist in diesem Sinne nicht direkt an einen politischen Begriff geknüpft, die Forderung nach einer Agrarreform, die unter Teilnahme der betroffenen Gruppen durchgeführt wird, aber sehr wohl - eben den der Agrarreform ${ }^{11}$. Die so definierten politischen Begriffe zeichnen sich dadurch aus, Unterbegriffe zu besitzen, die miteinander in Beziehung stehen. Der politische Begriff "Sozialismus“ verweist so auf die Unterbegriffe ,Klassenkampf', ,Proletariat", usw. Dabei ist die Beziehung zwischen diesen Begriffen nicht zwangsläufig eine hierarchische. Wie am Beispiel der Agrarreform ersichtlich, können sie in unterschiedlichen und teilweise widersprüchlichen Bedeutungszusammenhängen eine Rolle spielen. Die Grundfrage muss dabei immer sein: „Was fordert eine bestimmte Gruppe, wenn sie in einem bestimmten Kontext einen bestimmten Begriff verwendet?" oder als konkretes Beispiel: „Was fordert die CONAIE, wenn sie 1990/1994/2012 einen plurinationalen Staat fordert?“. Der untersuchte Begriff muss dabei eine zentrale Position im Diskurs oder Denken und Sprechen einer bestimmten Gruppe einnehmen. In der vorliegenden Arbeit sind diese zentralen politischen Begriffe (indigene) Nationalität, Territorialität/Territorium, Plurinationalität und Interkulturalität. Der neuere Begriff des Guten Lebens wurde später übernommen, hat aber ebenfalls eine zentrale Position im Diskurs der indigenen Organisationen erlangt.

Die vorliegende Arbeit orientiert sich an der Konzeption von Begriffsgeschichte die das Netzwerk Iberconceptos verwendet. Iberconceptos zeichnet sich insbesondere durch einen Fokus auf das Entstehen und den Wandel von Identitäten aus. Viele der zur Sattelzeit aufkommenden oder ihre Bedeutung verändernden Begriffe in Iberoamerika markieren das Aufkommen neuer kollektiver Akteure mit spezifischer räumlicher, sozialer oder politischer Verortung (Fernández Sebastián 2007: 169). Eine Hypothese der vorliegenden Arbeit ist, dass die Entstehung der neuen, identitär geprägten politischen Begriffe wie indigene Nationalität oder Plurinationalität die Entstehung einer neuen Art von Indigenenbewegung markiert.

Die Konzentration auf bestimmte Begriffe, die dem Diskurs der Indigenenbewegung zentral sind, hat den Vorteil, dass die Untersuchung einiger weniger Texte bereits Ergebnisse bringen kann. So kann die „Konzentration auf die Knotenpunkte im diachronen Bedeutungswandel einzelner Wörter" (Steinmetz 2008: 182) mit einer relativ kleinen Basis an Zitaten auch bei schwierigem Feldzugang oder Daten-

11 Siehe die Auseinandersetzung zwischen den Positionen einer demokratischen und einer integralen Agrarreform in Ecuador, Kapitel III.1. 
lage eine tief gehende Analyse von Diskursen ermöglichen. Gleichzeitig muss jedoch auch das Umfeld der Begriffe und ihres Entstehens untersucht werden, um die vermutete Rolle der jeweiligen Texte und Begriffe absichern zu können. Begriffe können ,immer nur in ihrer Funktion, in ihrer Verwendungsweise in kommunikativen Handlungen verstanden und untersucht werden“ (Busse 1987: 49). So versucht diese Arbeit, eine drohende ,isolierte Betrachtung von Einzelbegriffen“ (Kollmeier 2012: 11) zum einen durch die Betrachtung der Wechselbeziehungen zwischen verschiedenen Begriffen, zum anderen durch eine nähere Untersuchung ihrer Entstehungszusammenhänge in den Indigenenorganisationen selbst zu vermeiden.

Auch wenn sich die vorliegende Arbeit an einzelnen Begriffen orientiert, so gehören diese Begriffe doch einem bestimmten Bereich des Diskurses an, der in der Bearbeitung der Begriffe reflektiert werden soll. „Bezugsebene einer Begriffs- oder Bedeutungsanalyse kann [...] nur der Vollzug des Sprechens über einen Sachbereich sein.“ (Bruners o.J.: 12) Daher muss bei der Untersuchung eines Begriffes auch auf den kommunikativen Zusammenhang dieses Begriffes eingegangen werden. Innerhalb eines Sachbereiches kann der untersuchte Begriff in verschiedenen Situationen verschiedene Bedeutungen annehmen, die also solche zu untersuchen sind (Bruners o.J.: 12-14). So soll das kommunikative Wissen der Beteiligten und die daraus entstehenden Bedeutungszusammenhänge verortet (Busse 1987: 55) und die Analyse der Begriffe erweitert und geschärft werden. Das Sachfeld, dem die zu untersuchenden politischen Begriffe angehören, ist im Fall der vorliegenden Arbeit das der interethnischen Beziehungen, die innerhalb der Indigenenbewegung aber mit gesamtgesellschaftlicher Ausrichtung reflektiert werden. Jeder untersuchte Begriff versucht, diese interethnischen Beziehungen auf eine bestimmte Art zu definieren oder Vorschläge zu machen, wie diese Beziehungen anders und besser gestaltet werden können - und zwar jeweils in einem Kontext, der die gesamte Gesellschaft und den Staat einschließt. Alle Ideen und Überlegungen, die in diesem Sachfeld angestellt werden, werden analysiert und gegebenenfalls einem nahestehenden Begriff zugeordnet.

Die Grundlage für die Erforschung der politischen Begriffe der Indigenenbewegung bildet der Fragebogen, der den Forschungen des Netzwerks Iberconceptos zugrunde liegt. Es sei daran erinnert, dass nicht alle der aufgeführten Punkte für die vorliegende Forschung relevant sind.

\section{„A. Semantik (Diachronie)}

1. Die ersten Erscheinungen des Begriffs datieren (wenn es sich um einen völligen Neologismus handelt). Oder, wenn es sich um einen sinnhaften Neologismus handelt, die ersten Male, in denen das Wort in der neuen Annahme verwandt wird. 
2. Im zweiten Fall, anzeigen bis zu welchem Punkt die alten Bedeutungen, trotz der Resemantisierung, auf die neuen Verwendungen des Begriffs wirken.

3. Die Momente der größten Nutzung des Begriffs feststellen (politische Krisen, parlamentarische Debatten, journalistische Auseinandersetzungen).

4. Ein Entwicklungsschema der nähesten, ähnlichen, angrenzenden und entgegengesetzten Begriffe umreißen.

5. Deutlich machen, ob sich der behandelte Begriff in eine (oder verschiedene) Sprachmodalitäten oder Diskurstraditionen einzufügen scheint.

6. Die Befrachtung mit Vergangenem (Erfahrungsfeld) und die Bestrebung einer zukünftigen Umsetzung (Erwartungshorizont) auswerten, die der Begriff in sich trägt und die Verschiebung des Gewichts beider Bestandteile über den gesamten Zeitraum [...] hinweg.

\section{B. Pragmatik (Synchronie)}

7. So weit wie möglich die Arten von Dokumenten, Situationen oder Reden identifizieren, in denen der Begriff in Frage vorzugsweise auftaucht.

8. Eine Liste von Autoren, Werken oder institutionellen und kulturellen Referenzen machen, die die Autoren zitieren, wenn sie den Begriff behandeln.

9. Die vorherrschende normative Färbung des Begriffs deutlich machen (positiv, negativ oder neutral).

10. Angeben, ob es möglich ist, die übergreifende Bewertung (transvaluación) oder Resemantisierung des Begriffs zu beobachten, deutlich machen, welche individuellen oder kollektiven Agenten bei solchen Versuchen ideologischer Innovation die Hauptrolle spielen und den Grad an Erfolg dieser Bestrebungen bewerten.

11. Die Hauptbruchlinien der Debatten schematisieren, dabei die ihnen zugeschriebenen Hauptbedeutungen und die Achsen der Auseinandersetzung synthetisieren.

12. So weit wie möglich auswerten, ob es sich um einen von den Sprechern der Epoche gewöhnlich verwendeten Begriff handelt oder ob seine Verwendung im Gegenteil nur den politischen und/oder intellektuellen Eliten zukam (und, wenn zutreffend, ob es möglich ist, eine häufigere Verwendung des Begriffs in bestimmten sozialen oder beruflichen Bereichen zu beobachten).

\section{Schluss (Begriffsgeschichte)}

13. Verbindungen und Entsprechungen zwischen bestimmten sozialen, politischen und kulturellen Ereignissen (Revolutionen, Verfassungen oder andere rechtliche Texte) und den Veränderungen in der Bedeutung oder Bewertung des untersuchten Begriffs herstellen.

14. Deutlich machen, wie diese besondere Art die politischen und sozialen Wirklichkeiten zu begreifen auf den Lauf der Ereignisse Einfluss genommen haben kann.“ (Fernández Sebastián 2007: 172f.) 
Die Untersuchung der politischen Begriffe der ecuadorianischen Indigenenbewegung wird sich grob an diesem Fragebogen orientieren, jedoch die bereits herausgearbeiteten Unterschiede der Methode - vor allem was den Begriffsbegriff angeht besonders hervorheben.

\section{Textauswahl}

Schon die Auswahl der zu bearbeitenden Texte ist

„ein interpretativer Akt, der aus der (fiktiven) Menge allen Materials einzelne Texte, und aus diesen einzelne Aussagen, herausgreift, und letztlich zu einem eigenen, neuen Diskurs konstituiert.“(Busse 1987: 65f.)

Dieser neue Diskurs soll dann die untersuchte Gesamtheit repräsentieren. Damit die Repräsentation einigermaßen legitim ist, muss die Gesamtheit des Materials möglichst breit angelegt sein (Busse 1987: 65f.). Nur so kann nicht nur die Auswahl der untersuchten Begriffe, sondern auch ihre jeweilige Verwendung - und damit ihr Wandel - angemessen nachvollzogen werden. Um die relevanten Bedeutungen der Begriffe nicht zu eng zu fassen (Bruners o.J.: 13), werden verschiedene Kategorien in den Blick genommen.

Die Untersuchung sozialer Bewegungen schränkt die Textauswahl auf bestimmte Textarten ein. Meist werden Interviews von zentralen Aktivisten, Berichte in Kommunikationsmedien - vor allem Zeitungen - oder von der Bewegung und ihr nahestehenden Akteuren produzierte Dokumente analysiert (Benford 1997: 421). Im Falle der ecuadorianischen Indigenenbewegung hat der Historiker Hernán Ibarra vier Textkategorien erfasst:

„a) Die offiziellen Dokumente der Organisationen, in denen kulturelle, soziale und politische Forderungen dargelegt und begründet werden; b) Von Indigenen geschriebene Texte über bestimmte ethnische Gruppen; c) Mündliche Wortmeldungen und Interviews, die als geschriebene Texte reproduziert werden und d) Die Produktion von indigenen Intellektuellen, die regelmäßig in der Presse und in akademischen Veröffentlichungen schreiben.“ (Ibarra 1999: 89)

Dieser Textkategorien enthalten „öffentliche Symbole“ (Swindler 1995: 28), die nicht nur das Denken des jeweiligen Autors widerspiegeln, sondern ein „Bedeutungssystem“ (Swindler 1995: 28) repräsentieren, das die Ideen der Bewegung teilweise - öffentlich zugänglich macht. Die vorliegende Arbeit legt das Hauptgewicht auf die offiziellen Dokumente der verschiedenen Organisationen. Diese wurden in allen Fällen von gewählten Repräsentanten der jeweiligen Organisationen zusammengestellt und formuliert, was diesen Texten eine Legitimation gibt, die die 
anderen drei Arten nicht haben. Reden, Interviews und Artikel durch Indigene, genauso wie Texte von Indigenen über ethnische Gruppen werden daher nur ergänzend verwandt, etwa beim Nachzeichnen der ersten Verwendungen bestimmter politischer Begriffe. Die offiziellen Dokumente der verschiedenen Organisationen sind als politische Dokumente von Interesse, also „als Vorschläge, um die Gesamtheit der ganzen Gesellschaft zu verändern, zu denen die Indio-Völker gehören“ (Bonfil Batalla 1981: 11f.). Dahinter steht die Annahme, dass die indigenen Völker politische Einheiten sind, die ein Recht haben, an den Entscheidungen des Staates teilzuhaben. Dokumente, die sich ausschließlich um interne Angelegenheiten der indigenen Gemeinden drehen, werden in diesem Sinne nicht beachtet (Bonfil Batalla 1981: 11f.).

Alle vorgestellten Textarten haben einen wichtigen Nachteil gemeinsam: sie geben vor allem die Sichtweisen des Führungspersonals und der gesellschaftlichen Eliten wider, leiden also unter einer „eingebauten Verzerrung von oben nach unten“ (Benford 1997: 421). Diese Verzerrung kann im Rahmen der vorliegenden Arbeit nicht ausgeglichen werden. Es sei auf ethnographisch inspirierte Studien verwiesen, die sich den Ideen der Basis der Indigenenbewegung nähern, etwa Becker/Tutillo (2009) oder Sánchez-Parga (2007, 2010, 2010a). Ein weiteres Problem ist die Konzentration auf Texte der größten Organisation der Indigenenbewegung. Die meisten der vorliegenden Texte stammen von der CONAIE und ihren Mitgliedsorganisationen - ein Zeichen ihrer diskursiven und organisatorischen Hegemonie. Auch wenn diese Organisationen - wie im Laufe der vorliegenden Arbeit deutlich gemacht wird - die Entwicklung der Indigenenbewegung und ihres Diskurses entscheidend beeinflussen, haben die anderen Organisationen einen gewissen Einfluss, aber eine geringere Textproduktion, die zu ihrer Unterrepräsentation in der Textauswahl führt.

\section{Methodisches Vorgehen}

Die bisher diskutierten Ansätze werden die Bearbeitung der Texte und das Herausarbeiten von Analysekategorien lenken. Jedoch enthält keiner dieser Ansätze eine eindeutige Handlungsanweisung für das methodische Vorgehen. Daher sind Kolonialität der Macht, Theorien sozialer Bewegung und die ad-hoc-Methodologie der begriffszentrierten Diskursanalyse mehr theoretischer Hintergrund der tatsächlichen Forschung als methodologische Instrumente. In allen Fällen wurden die Schwierigkeiten einer direkten Verwendung dieser Ansätze als Methoden herausgearbeitet. Da diese Schwäche offensichtlich ist, wird für die tatsächliche Textanalyse ein theoretisch wenig kompromittierender Ansatz gewählt - ein Ansatz, der die Bearbeitung der verschiedenen Texte für alle drei beschriebenen Ansätze möglich macht. Somit soll neben einem überprüfbaren Minimalinstrumentarium die Methodentriangulation mehr über eine Theorietriangulation oder eine Kombination sich 
ergänzender Kategorien und Interpretationsweisen erreicht werden, als über die Verwendung verschiedener Methoden selbst. Die Auswahl konkreter Methoden ist durch die dieser Arbeit zugrunde liegende Orientierung auf die Texte der verschiedenen Indigenenorganisationen von vorneherein reduziert. Unter den verschiedenen textanalytischen Ansätzen erfüllt die qualitative Inhaltsanalyse nach Mayring die Ansprüche dieses Forschungsdesigns besonders gut.

Dieser Ansatz definiert von der „Einordnung in ein Kommunikationsmodell““ (Mayring 2000: 7) und einer Festlegung des Ziels der Analyse ausgehend feste Regeln, nach denen die Analyseaspekte begründet in Kategorien übersetzt und unter nachvollziehbaren Kriterien auf bestimmte Texte angewendet werden (Mayring 2000: 7). Von besonderer Bedeutung ist der Umgang mit Kategorien, wobei sich ,induktive Kategorienentwicklung und deduktive Kategorienanwendung“ (Mayring 2000: 8) ergänzen. Induktive Kategorienentwicklung meint die Erarbeitung der ,Auswertungsaspekte [...] aus dem Material heraus“ (Mayring 2000: 10) wobei sich die Kategorien aufeinander beziehen und mehrfach überarbeitet werden müssen (Mayring 2000: 11):

„Grundgedanke ist, aus der Fragestellung der Studie abgeleitet und theoretisch begründet ein Definitionskriterium festzulegen, das bestimmt, welche Aspekte im Material berücksichtigt werden sollen, und dann schrittweise das Material danach durchzuarbeiten. Die entwickelten Kategorien werden in einer Rückkopplungsschleife überarbeitet, einer Reliabilitätsprüfung unterzogen, und können später auch zu Überkategorien zusammengefasst [...] werden.“ (Mayring 2000: 12)

Die deduktive Kategorienanwendung bezieht sich auf ,schon vorher festgelegte, theoretisch begründete Auswertungsaspekte“ (Mayring 2000: 13), die methodisch abgesichert auf die Texte angewendet werden.

Beide Ansätze werden nicht nur jeweils für sich überprüft, sondern beziehen sich aufeinander. In der vorliegenden Arbeit wird dasselbe Material (alle zur Verfügung stehenden programmatischen Schriften der Organisationen der Indigenenbewegung) in drei Ansätzen nach unterschiedlichen Kategorien bearbeitet. Dabei werden die beiden Methoden der Kategorienbildung nicht wie von Mayring vorgesehen, nacheinander (Mayring 2000: 11/14), sondern gleichzeitig durchgeführt, nicht zeitlich, sondern thematisch getrennt. Dadurch wird eine Verbindung zur Diskursanalyse und der in ihr enthaltenen ,thematischen Analyse“ (Ruiz 2009: 19) geschaffen, die die Etablierung einer begriffszentrierten Diskursanalyse erlaubt. Dieses Vorgehen ist durch das Fehlen einer überprüfbaren Theorie beim Forschungsdesign begründet - als Grundlagenarbeit kann der vorliegende Text nur in wenigen Punkten an bereits bestehende Theorien anschließen. Diese Theorien müs- 
sen im Prozess der Forschung erst an den ecuadorianischen Fall angepasst werden, sind also selbst noch zu überarbeiten.

Da kein weitreichendes Wissen über die Geschichte der Indigenenbewegung und die ihrer Begriffe vorausgesetzt werden kann, werden diese in den jeweiligen Teilen vor der Interpretation und theoretischen Einordnung aus der Perspektive relativ neutraler Ansätze (im ersten Fall die Geschichte von unten, im zweiten die Begriffsgeschichte) behandelt und erst anschließend weitergehend bearbeitet. So soll eine Kombination von textlicher, kontextueller und interpretativer Ebene erlaubt werden, die sowohl in den einzelnen Teilen, als auch in der gesamten Arbeit im Sinne einer Diskursanalyse greifen soll (Ruiz 2009: 10).

Die dem ersten Teil der vorliegenden Arbeit (II. Geschichte der Indigenenbewegung Ecuadors) zugrundeliegenden Kategorien orientieren sich an Geschichte und Entwicklung der verschiedenen Indigenenorganisationen. Die induktive Kategorienentwicklung hat - aufgrund der relativ häufigen Erwähnung - zu einer Betrachtung zweier Organisationen geführt, die nicht direkt der Indigenenbewegung angehören, aber regelmäßig mit diesen kooperieren. Die Kategorien des zweiten Teils (III. Begriffe der Indigenenbewegung) orientieren sich an dem oben entwickelten Konzept des politischen Begriffs. In diesem Fall hat die Anwendung der Methoden der qualitativen Inhaltsanalyse dazu geführt, dass durch die deduktive Kategorienanwendung mit dem Begriff des Guten Lebens ein Begriff analysiert wurde, der in den Texten der Organisationen selbst kaum eine Rolle spielt - allerdings als politischer Begriff im Sinne dieser Arbeit verstanden werden muss. Der dritte Teil orientiert sich an den Kategorien die im Umfeld der Kolonialität der Macht entwickelt werden, die auf die zuvor erarbeiteten Erkenntnisse angewendet werden. 


\section{Geschichte der Indigenenbewegung Ecuadors}

\section{Allgemeines}

\section{a. Entwicklung der Indigenenbewegung in Ecuador}

Die Entwicklung der Indigenenbewegung in Ecuador lässt sich in verschiedene Etappen einteilen, abhängig vom jeweils gewählten Schwerpunkt der Betrachtung. Die wichtigsten Modelle werden im Anschluss dargestellt. Das sind: das historischidentitäre Modell der CONAIE, das darauf aufbauende zeithistorisch-analytische einiger Wissenschaftler aus dem Umfeld der CONAIE und das sozialwissenschaftlich-historische, das sich auf soziale Bewegungen bezieht.

Für die CONAIE (1989: 275) erstreckt sich die Entstehung der Indigenenbewegung über drei Etappen. Die erste Etappe beginnt mit der spanischen Invasion und geht bis zu den 1920er Jahren. Sie ist durch lokal begrenzte Aufstände und individuellen Widerstand der Indigenen gekennzeichnet; in dieser Zeit wird die Figur des ,indio' als Nicht-Weißer konstruiert. Die zweite Etappe geht von Mitte der 1920er bis zur Agrarreform 1964 und findet vor allem im Hochland statt. Es werden Agrargewerkschaften gegründet und über Vermittler (die sozialistische und die kommunistische Partei, Gewerkschaften) versucht, die Rechte der Indigenen zu verteidigen. Aus dieser Etappe stammen die eher gewerkschaftlich ausgerichteten Organisationen FEI und FENOC. In der dritten Etappe seit 1964 werden die Organisationen gegründet, die später die CONAIE bilden sollen - als erste die Federación Shuar 1961. Dieses Modell unterstreicht die Kontinuität des indigenen Widerstandes seit der spanischen Eroberung und stellt gleichzeitig die CONAIE in das Zentrum der Betrachtung. Andere Organisationen werden als veraltet und von außen gesteuert beschrieben (etwa: CONAIE 1989: 276) und somit delegitimiert. Obwohl die Einseitigkeit dieses Modells offensichtlich ist, wird es auch in der Wissenschaft oft vertreten - die Zentralität der CONAIE also bestätigt.

Simbaña (2009: 154) konkretisiert diese Sichtweise mit einem neutraleren Modell. Er unterteilt die Entwicklung der Indigenenbewegung im 20. und 21. Jahrhun- 
dert in drei Perioden. Die erste Periode, die Periode der Vereinigung, geht von den 1930er Jahren bis zur ersten Agrarreform 1964. Die zweite Periode, die Periode der Transition, erstreckt sich bis 1990. Die dritte Periode schließlich, der Aufbau des plurinationalen Staates, beginnt 1990 und dauert noch an. Auch wenn dieses Modell die Perspektive der CONAIE einnimmt - Simbaña hatte und hat wichtige Positionen im CONAIE-Mitglied ECUARUNARI inne - erlaubt es, die Entwicklung der Indigenenbewegung nicht als unilinear, sondern als Kampf um Deutungshoheit zu verstehen. Seine dritte Periode entspricht der Hegemonie der CONAIE und ihres Diskurses.

Das Modell von Simbaña wird erweitert durch Tibán/García (2006: 19f.), die die erste Etappe in der Periode der Transition von Simbaña sehen, also zwischen 1960 und 1990. Die Indigenenbewegung, durch Vermittler repräsentiert, ist selbst kein anerkannter politischer Akteur, sie bleibt unsichtbar. Ihre Forderungen zielen weitgehend auf Land und Erziehung und werden nur in geringem Maße umgesetzt. In einer zweite Etappe von 1990 bis 2000 wird die Indigenenbewegung in den Staat integriert und als politischer Akteur in staatliche Institutionen - der Erziehung und der Entwicklung - eingebunden. Die Forderungen konzentrieren sich auf eine politische Reform, um einen plurinationalen Staat zu schaffen. Es folgt eine dritte Etappe, die vom Scheitern der bisherigen Strategien und einer Krise der Indigenenbewegung geprägt ist.

Aus dieser Perspektive liest auch Sánchez-Parga (2010) die Entwicklung der Indigenenbewegung in Ecuador. Über die Beziehung zum Staat entwickelt sich aus einer sozialen eine politische Bewegung, die Indigenenbewegung wird immer weiter in den Staat integriert. Diese Entwicklung beginnt mit dem Kommunen-Gesetz von 1937, das über die Schaffung bestimmter sozialer Strukturen die Grundlage für die moderne Indigenenbewegung legt. Eine erste Phase erstreckt sich bis zu den Agrarreformen von 1964 und 1973. Daran anschließend spielt die Politik der Agrarentwicklung (Ende der 1970er bis Ende der 1980er) eine wichtige Rolle in der Institutionalisierung der Indigenenbewegung, die durch ihre Umwandlung in eine politische Partei 1996 beendet wird. Während die Struktur der Kommunen im Niedergang begriffen ist, eröffnen die Aktionsformen einer Partei neue Möglichkeiten (Sánchez-Parga 2010: 16ff.). Der Fokus dieses Modells auf die Integration der Indigenenbewegung in den Staat ist zu reduktionistisch, um einer breiteren Analyse hilfreich zu sein. Die strategische Kooperation gerade der CONAIE mit staatlichen Agenturen, ebenso wie die Konstitution der MUPP-NP als Mittel zum Zweck der Reform des Staates, wird durch die Idee der Transformation eines sozialen in einen politischen Akteur im Rahmen einer unilinearen Entwicklung auf ein formalistisches Verstehen von Politik reduziert.

Ein Modell, das sich oft in der europäisch orientierten Sozialwissenschaft findet, bietet Minkner-Brünjer (2009: 135). Sie sieht eine Abfolge von verschiedenen 
sozialen Bewegungen mit verschiedenen Akteuren. So sind die 1960er und 1970er Jahre die Zeit der Bauernbewegung unter Führung der FENOC. Die 1970er bis Ende der 1980er Jahre sind die Zeit der Gewerkschaftsbewegung, vor allem der Einheitsgewerkschaft FUT. In den 1990ern bis Anfang der 2000er Jahre spricht Minkner-Brünjer von der Indígena-Bewegung, definiert durch die CONAIE. Deren Krise ab 2003 bedeutet ein Führungsvakuum, das bis heute andauert. Diese Perspektive ignoriert Kontinuitäten, wie etwa die Beteiligung der FENOC an der Gewerkschaftsbewegung oder die Präsenz indigener Akteure und Forderungen über den gesamte beschriebenen Zeitraum hinweg. Dazu kommt die Tatsache, dass in den 1960ern und 1970ern die Kategorien ,Indigene“ und ,Bauern' fast deckungsgleich sind und somit die Bauernbewegung auch eine Indigenenbewegung ist selbst wenn ethnische Aspekte in den Hintergrund treten. Auch die jeweiligen Theorien, mit denen Wissenschaft und Gesellschaft die sozialen Bewegungen interpretieren, spielen eine Rolle. Aktivitäten in den 1960ern und 1970ern wurden meist unter dem Aspekt der Klasse verstanden, spätere Aktivitäten unter dem Aspekt der Ethnie. Also werden die ethnischen Organisationen schlicht anders wahrgenommen und anders kategorisiert (Crnic 2009: 46) - ohne deswegen qualitativ anders sein zu müssen.

Die Perspektive von Minkner-Brünjer nimmt auch Sánchez-Parga (2010: 19) ein. Für ihn bildet sich die Indigenenbewegung zwischen Ende der 1970er und den 1990ern in einem Kontext, der von Globalisierung und damit zusammenhängender Ethnisierung bestimmt wird. Man kann also erst ab dem Bedeutungsgewinn der ethnischen Organisationen, die später die CONAIE bilden, von einer Indigenenbewegung im eigentlichen Sinne sprechen. Auf einer Klassenanalyse basierende Organisationen wie die FENOC werden zu Fremdkörpern in der Bewegung, womit Sánchez-Parga die Interpretation der CONAIE teilt. Die Übernahme dieser Sichtweise - die Interpretation des Entstehens der Indigenenbewegung als Folge einer durch Globalisierung und Neoliberalismus erzeugten Re-Ethnisierung - durch die zentralen Denker der Kolonialität der Macht (etwa Quijano 2006a: 31f.) ist ein besonderes Problem, das bislang nicht näher untersucht wurde.

Die anschließende Geschichte der einzelnen Organisationen der Indigenenbewegung wird es erlauben, eine allgemeine Entwicklung nachzuzeichnen, ohne dabei marginale Strömungen zu verschweigen. Hier wird die Indigenenbewegung über ihre Strukturen und formalen Organisationen gelesen. Eine Geschichte der oft als ,spontan ' beschriebenen Aufstände ohne die Aktionen selbst überdauernde Strukturen soll nicht geliefert werden. Das leisten andere, etwa CONAIE (1989). Der Bruch, den das Modell der CONAIE in den 1920er Jahren sieht, ist weniger ein Wandel der Forderungen oder des Mobilisierungspotentials, als vielmehr eine Strukturierung der Bewegung in Organisationen, in Gewerkschaften, Assoziationen und Kommunen/Zentren. Die so geschaffene Kontinuität erlaubt der Indigenenbe- 
wegung die Ausbildung eines vielfältigen Forderungskatalogs bis hin zum Komplex Interkulturalität/Plurinationalität der CONAIE - übernommen, kopiert oder angegriffen durch die anderen Organisationen der Indigenenbewegung.

Zu Recht macht Marc Becker darauf aufmerksam, dass der Übergang von einer lokalen Organisierung der Indigenen mit konkreten Forderungen, die sich an den jeweiligen Großgrundbesitzer oder die Regierung vor Ort richten, hin zu einer Organisierung auf einer Makroebene der - kaum erforschte - Beginn der modernen Indigenenbewegung in Ecuador ist. Dieser Übergang in den 1920er und 1930er Jahren führte zur Entwicklung von neuen Zielen und neuen Fertigkeiten, diese Ziele zu erreichen. Zum ersten Mal verhandelten indigenen Organisationen direkt mit dem Staat (Becker 1997: 211). Drei weitere Einschnitte, die die Entwicklung der Indigenenbewegung maßgeblich beeinflusst haben, sind die Agrarreformen von 1964 und 1973, das Aufkommen explizit ethnischer Tendenzen innerhalb der Bewegung ab Mitte der 1970er (Sánchez-Parga 2007: 11f.) und die eigenständige Beteiligung an der Parteienpolitik ab Mitte der 1990er Jahre. Die Forderung nach einer Landreform hat die Indigenenbewegung seit der Gründung der ersten Gewerkschaften begleitet (Almeida/Prieto 1983: 36). Auch wenn die Reformen nicht die Änderungen brachten, die sich die Organisationen dieser Zeit erhofft hatten, verbesserten sie die Situation der Indigenen, etwa durch die Abschaffung des halbfeudalen Wirtschaftssystems der huasipungaje (Santillana/Herrera 2009: 331). Die huasipungueros, die in enger Abhängigkeit zum jeweiligen Großgrundbesitzer standen, wurden selbst zu Besitzern ihres Landes, das sie - teilweise - in überlieferte Gemeinschaftsformen überführten. Erst ab diesem Moment wird das System der Kommunen bedeutsam - es erlaubt den jetzt freien indigenen Bauern, sich in selbstbestimmten Gruppen zu reorganisieren. Sie entwickeln ein Bewusstsein der kulturellen Differenz und ihrer eigenen ethnischen Identität (Sánchez-Parga 2010a: 62f.). Das ist die Grundlage für die Ausbildung von Institutionen, die später die Organisationen der Indigenenbewegung stützen werden (Simbaña 2009: 157). Die langsame Re-Ethnisierung und das Verschwinden der huasipungueros bedeutet eine Krise für gewerkschaftlich ausgerichtete Organisationen wie die FEI und die FENOC, was auch zum Verlust von Einfluss für die Parteien PSE und PCE führt (Simbaña 2008a: 238). Die Schwäche der gewerkschaftlichen Organisationen ab den 1970ern geht einher mit einer Stärkung eines neuen Organisationstypus: der „Volksorganisation“ (Moya 1987: 389). In diese Kategorie fallen Organisationen in armen Stadtvierteln, Frauen-, Bauern- und Indigenenorganisationen, die sich in erster Linie in lokalen Kontexten engagieren - und nicht wie bis dahin häufig von nationalen Interessen der jeweiligen Muttergewerkschaften vereinnahmen lassen. Die neuen Organisationen schließen sich zu nationalen Bauernföderationen zusammen, um ihren Forderungen Nachdruck verleihen zu können. Dennoch behalten sie einen hohen Grad an Autonomie, der wenige Jahre zuvor noch undenkbar gewesen 
wäre. Der Einfluss der nationalen Organisationen ist geringer, die regionalen Organisationen widmen sich der Repräsentation der Interessen vor Ort, woraus auch neue Forderungen auf nationalem Niveau erwachsen (Almeida/Prieto 1983: 36). Barre (1983: 239f.) interpretiert diesen Prozess als Erneuerung, die ab den 1980ern einen langsamen Eintritt der Indigenenbewegung in die Politik erlaubt.

Tatsächlich ist die Rückkehr zur Demokratie 1979 der Beginn einer politischen Beteiligung der Indigenenbewegung, vor allem in Verbindung mit linken Parteien wie der PSE und der FADI, bis hin zur Wahl einzelner Indigener in die Parlamente (Ayala Mora o. J.: 6). Diese Beteiligung wird erst in den 1990ern systematisch, vorbereitet durch eine starke Präsenz als sozialer Akteur und schließlich vollendet mit der Gründung der MUPP-NP 1996 (Santillana 2006: 217). Die Nutzung von Parteienpolitik zur Erreichung der Ziele der Indigenenbewegung baut auf ein dichtes Netz an Mitgliedsorganisationen (vor allem der CONAIE) und eine allgemeine Krise sowohl der politischen Linken als auch der Parteien insgesamt. Dadurch sind zumindest die vorwiegend indigen besiedelten Gebiete eine gute Grundlage für den Erfolg einer indigenen Partei (García Serrano 2005: 9).

\section{b. Struktur der Indigenenbewegung Ecuadors}

Die Indigenenbewegung Ecuadors zeichnet sich durch eine relativ offene Struktur aus. Die „dreifache Artikulierung“ (Sánchez-Parga 2007: 14) der Indigenenbewegung mit dem Staat, anderen Organisationen und der sozialen Klasse als Bauern und Landarbeiter, sorgte dafür, dass sie sich nie in eine separatistisch-indianistische Bewegung - wie teilweise in Bolivien und Peru - verwandeln konnte, sondern immer eine soziale Bewegung - mit den entsprechenden Bündnissen - blieb ${ }^{1}$. Ob die Beziehung zum Staat für die Bewegung ein Hindernis war und dieser sie so als Bewegung und Partei kooptieren konnte (Sánchez-Parga 2007: 14f.) oder eher förderlich, da die Verbindungen mit dem Staat die Umsetzungen einiger Forderungen erlaubten, kann hier nicht geklärt werden. Festzuhalten bleibt aber, dass gerade auch der Staat an der Entstehung der spezifischen Strukturen der Indigenenbewegung beteiligt war.

Die Organisationen der Indigenenbewegung in Ecuador sind pyramidenförmig angeordnet (Bretón 2003: 219f.). Die untere Stufe bilden Organisationen ersten Grades (OEG) oder Basisorganisationen. Das sind Kommunen, Assoziationen oder lokale Gewerkschaften. Sie sind Teil von Föderationen oder Organisationen zweiten Grades (OZG), die die zweite Schicht der Pyramide bilden. Die OZG lehnen sich meist an die staatliche Struktur der Kantone oder Provinzen an (Maldonado

1 Es wäre zu überprüfen, ob das Argument Sánchez-Pargas in den zeitweise stark korporatistischen Systemen Perus und Boliviens halten lässt. 
2006: 90$)^{2}$. Sie sind eine heterogene und stark verbreitete Organisationsebene in Ecuador. Da sie aus einer Vielzahl teils sehr unterschiedlicher OEG zusammengesetzt sind, sind ihre politische Ausrichtung und ihre kurz- und mittelfristigen Ziele sehr verschieden - auch in Funktion ihrer nationalen und internationalen Einbindung und Finanzierung. So erhalten OZG Unterstützung vom ecuadorianischen Staat, einer Vielzahl von Nichtregierungsorganisationen und internationalen Institutionen wie der Weltbank oder der Interamerikanischen Entwicklungsbank. Daher orientieren sie sich nicht in erster Linie an lokalen Interessen, sondern an den Forderungen ihrer OEG auf der einen, und den Zielsetzungen der nationalen und internationalen Förderer auf der anderen Seite (Martínez Valle 2006: 109f.). Die nächste Stufe der Organisationen, die Organisationen dritten Grades (ODG), sind - im Fall der CONAIE - die regionalen Plattformen ECUARUNARI, CONFENIAE und CONAICE (Maldonado 2006: 90), ansonsten direkt die nationalen Organisationen FEI, FENOCIN (Guerrero/Ospina 2003) und FEINE.

Die CONAIE ist gewissermaßen eine Organisation vierten Grades, während die anderen nationalen Organisationen ODG sind. Dieses Modell ist rein analytisch und hat für die indigenen Organisationen nur wenig Bedeutung - auch wenn sie die Bezeichnungen selbst verwenden. Die einzelnen Organisationen sind autonom und keinesfalls an Weisungen der nächsthöheren Ebene gebunden. Sie können konkrete Forderungen im lokalen Kontext aufstellen und auf die politischen Entscheidungsträger vor Ort wirken, ohne Forderungen und Aktionen mit der nationalen Organisation abzustimmen (Chiriboga 1986: 65). Zumindest die Basisorganisationen können gleichzeitig in verschiedenen $\mathrm{OZG}$ aktiv sein oder von einer zur nächsten wechseln (Botero 1998: 9; Guerrero/Ospina 2003) und diese - in Ausnahmefällen von einer ODG zur nächsten. Die CONAIE und ihre Unterorganisationen sind nach Nationalitäten und nicht Organisationen strukturiert - was teilweise, aber nicht immer, identisch ist. Dazu kommt, dass es in manchen Fällen erheblich mehr organisatorische Ebenen gibt als hier vorgestellt.

Dazu zwei vergleichende Abbildungen:

2 Hier muss dem Modell von Bretón widersprochen werden, der dies erst für die nächste Stufe von Organisation vorsieht - und damit eine Stufe zu viel hat (vgl. FENOCIN 2004: 32, Santillana/Herrara 2009: 332). 


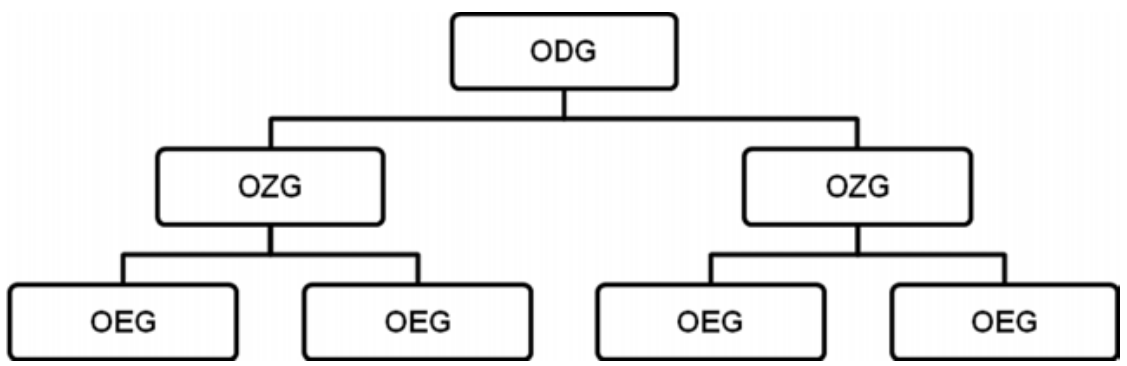

Abbildung 1: Struktur der Indigenenbewegung nach Bretón 2003: 219f., eigene Abbildung

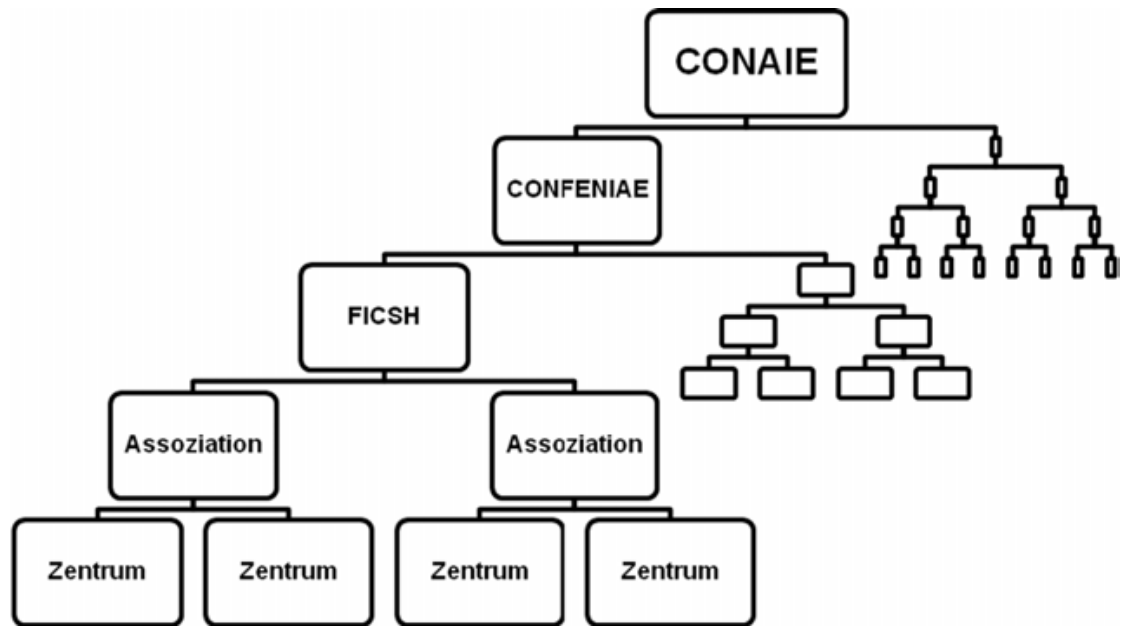

Abbildung 2: Struktur der FICSH/CONAIE, eigene Abbildung

Die meisten der Organisationen der Indigenenbewegung ${ }^{3}$ haben als kleinste Einheit die Gemeinschaft. Es sind meist ganze indigenen Gemeinden, Dörfer, Kommunen oder Viertel, die der Organisation angehören und nicht nur bestimmte Teile von ihnen, wie etwa im Fall der Gewerkschaften. Daher gibt es auch keine formelle Mitgliedschaft für Individuen - man wird in der Organisation aktiv, weil man seine Gemeinde vertreten soll oder weil man sich persönlich mit ihren Zielen identifizieren kann. Zugleich gibt es eine hohe Fluktuation des Führungspersonals. Die meisten Posten werden nur für wenige Jahre besetzt und die Personen, die die jeweilige Funktion ausgeübt haben, ziehen sich nach ihrer Amtszeit nicht selten aus der aktiven Politik zurück. Deshalb ist eine der Funktionen der OEG und OZG die Ausbildung von Führungspersonal (León 1991: 402).

3 Die Organisationen der CONAIE und FEINE, teilweise auch der FENOCIN und FEI. 
Auch wenn es keine neutralen Daten gibt, ist in der Forschung unumstritten, dass die CONAIE die größte Organisation der Indigenenbewegung ist (Tamayo 1996: 66f.). Eine Untersuchung des Erziehungsministeriums von 1989 hat ergeben, dass die CONAIE $61 \%$ der indigenen Gemeinschaften repräsentiert, während die zweitgrößte Organisation, die FEINE, nur 15\% repräsentiert (de la Cruz 1992: 141). Aktuelleren Daten zufolge vertritt die FEINE 30\% und die CONAIE 45\% der organisierten Indigenen (Chisaguano 2008: 99) ${ }^{4}$.

Über Studien des Entwicklungsprojektes der indigenen und schwarzen Völker (Proyecto de Desarrollo de los Pueblos Indígenas y Negros del Ecuador, PRODEPINE) seit 1998 liegen genauere Daten zu den verschiedenen Organisationen ersten und zweiten Grades vor (Martínez Valle 2006: 113). Es wurden insgesamt 7.072 Basisorganisationen (OEG) mit 1.161.870 Einzelmitgliedern oder 193.645 Familien gezählt. Diese haben verschiedene Ausrichtungen, so sind 24\% territoriale Organisationen im Sinne der Kommunen oder Zentren, 21\% sind soziale, kulturelle oder sportliche Vereinigungen, 15\% Wasserkooperativen, usw. (Ramón 2001: 49f.). Die OEG sind nur zu 35\% rein indigen und entsprechen nur zu $24 \%$ den Kommunen ${ }^{5}-$ die große Mehrheit (76\%) sind andersgeartete Organisationen, die meist keine ethnische Exklusivität haben (Martínez Valle 2006: 115). Die OEG sind also weniger an traditionelle Organisationsformen wie Kommunen oder Cabildos gebunden als es oft den Anschein hat - von unten ist die Indigenenbewegung offen und pluralistisch (Martínez Valle 2006: 126). Die OEG bilden 155 OZG (ohne das Amazonasgebiet), von denen $35 \%$ rein indigen und $45,7 \%$ pluriethnisch zusammengesetzt sind (Ramón 2001: 51). Sie entsprechen also nicht dem Selbstverständnis mancher nationaler Organisationen, ausschließlich Indigene zu umfassen (Ramón 2001: 51). Die große Mehrheit der OZG (91\%) konzentriert sich in den Anden und dort vor allem im zentralen Hochland, also den Provinzen Cotopaxi, Tungurahua, Chimborazo und Bolívar - die Provinzen mit dem höchsten indigenen Bevölkerungsanteil Ecuadors. In Chimborazo, Cañar, Cotopaxi, Imbabura und Tungurahua sind 71,6\% aller OZG des Hochlandes angesiedelt. 38,7\% der OZG sind territorial, ihre OEG umfassen das gesamte Territorium und die gesamte Bevölkerung der jeweiligen Provinz. 20,6\% umfassen nur einen Teil der Bevölkerung der jeweiligen Provinz. $11,6 \%$ sind territorial zerstreut, sie umfassen die gesamte repräsentierte Gruppe, diese hat aber keinen zusammenhängenden Siedlungsraum. 4,5\% der OZG bestehen

4 Die Schätzung von Leonidas Iza, der zu seiner Zeit als Präsident der CONAIE davon sprach, dass 75\% der organisierten Indigenen der CONAIE angehören (Iza 2005: 113), sind nur bedingt vertrauenswürdig.

5 Nach Daten des Landwirtschaftsministeriums von 1993 waren 64,2\% der Basisorganisationen in indigenen Gebieten des Hochlandes Kommunen und 16\% Kooperativen (Lucero 2003: 29). 
aus Individuen, die in manchen Fällen für die gesamte Gemeinschaft sprechen und $24,5 \%$ sind gemischte und überlagerte OZG, deren Basisorganisationen gleichzeitig verschiedenen OZG angehören (Ramón 2001: 51f.).

Die Problematik dieser Daten zeigen Guerrero/Ospina (2003) auf. Ein Drittel der OZG, die bei einer Erhebung 1998 registriert wurde, erklärten, keiner ODG oder nationalen Organisation anzugehören, und viele der OEG und OZG sind insbesondere im zentralen Hochland - sowohl in CONAIE als auch in FEINE aktiv - wohingegen die Organisationen der FENOCIN eindeutig unterscheidbar sind (Ramón 2001: 64f.). Dazu kommt eine Bedeutungsverschiebung der Organisationsebenen wegen der verstärkten Förderung durch nationale und internationale Organisationen ab Mitte der 1990er Jahre - die wichtigste wohl das von 1998 bis 2003 aktive PRODEPINE selbst. Dieses förderte nur formell konstituierte OZG und hat so zu einem erheblichen Wachstum der Anzahl dieser Organisationen beigetragen (Martínez Valle 2006: 114ff.). Um bei der Mittelvergabe erfolgreich sein zu können, mussten sich die OZG auf Entwicklungsprojekte etwa der Produktion und Kommerzialisierung bestimmter Waren konzentrieren. Es entstand eine Vielzahl von untereinander konkurrierenden OZG, die sich spezialisierten, um in bestimmte, von außen vorgegebene, Projektbeschreibungen zu passen. Diese neue funktional bestimmte Ausrichtung der OZG führte zu einem politischen Bedeutungsverlust: Projektmanager ersetzten die früheren ethnopolitischen Führer, die Kritik an den Verhältnissen wurde ersetzt durch ein Fachwissen um die Beantragung von Fördergeldern (Bretón 2008: 127f.). Die ODG übernahmen die politische Vermittlerrolle, die die OZG dadurch nicht mehr im selben Maße wahrnehmen konnten (Iturralde 1997: 96) - daher nahm ihre Bedeutung auch in den staatlichen Organen, wie denen der interkulturellen Erziehung oder der Agrarentwicklung, zu (Guerrero/Ospina 2003).

Die PRODEPINE hat ab 1998 eine Reihe von Studien zu den sozialen Bewegungen und vor allem zu den OZG in Ecuador erstellt (Martínez Valle 2006: 113). Diese und andere Daten zeigen, dass sich seit dem Bestehen der PRODEPINE die Anzahl formeller Organisationen auf dem Niveau der OZG erheblich gesteigert hat - eine Folge des erhöhten Angebotes an Projekten und der Bevorzugung dieser Organisationsform durch die PRODEPINE (Martínez Valle 2006: 114). So stieg die Anzahl der OZG von 141 (1998) auf 164 (2002) - die Anzahl der OZG, die tatsächlich an Projekten beteiligt war, reduzierte sich jedoch. 2003 waren nur 107 OZG an Projekten beteiligt, das sind 24\% weniger als 1998 und 34,8\% weniger als 2002. Das Gebiet mit der meisten Förderung durch die PRODEPINE reduzierte sich auf die zentrale Andenregion (mit 52,3\% der geförderten OZG), vor allem die Provinz Chimborazo (30\% der geförderten OZG) und ihren Kanton Colta (mit 38\% der OZG in Chimborazo) (Martínez Valle 2006: 116). Diese Art der Förderung veränderte die Struktur der OZG im Andenhochland. Die beiden Erhebungen von 1998 
und 2002 zeigen, dass 36,5\% der OZG weniger als zehn Basisorganisationen (OEG) hatten, 37\% in den letzten 5 Jahren, also seit 1997, gegründet worden waren und $60,6 \%$ von ihnen multiethnisch strukturiert waren. Von einer organisatorischen Kohärenz mit festen Strukturen und klar definierter Identität kann also keine Rede sein und die Vermutung, dass die Projekte der PRODEPINE ad hoc-Organisationen schufen und andere wiederbelebte (Martínez Valle 2006: 118) scheint wahrscheinlich.

Ein weiteres grundlegendes Problem ist die Auflösung der traditionellen Siedlungsstrukturen. Seit den Landreformen 1964 und 1973, spätestens aber im Zuge der wirtschaftlichen Umgestaltung Ecuadors in den 1980er und 1990er Jahren, mit neuen großflächigen Anpflanzungen und Erdölförderungen, sind die Migrationsbewegungen vom Land in die Stadt stärker geworden. Die Kommunen, die Grundlage der Indigenenbewegung, verlieren ihre Einwohner, die anderswo Arbeit suchen (Korovkin 2008: 7).

\section{c. Ideologien der Indigenenbewegung}

Die Entwicklung der Ideologien der Indigenenbewegung ist vor allem vom Gegensatz von Klassenanalyse und ethnischen Aspekten geprägt. Während die ersten Organisationen eher klassistisch orientiert waren - auch wegen ihrer kommunistischen bzw. sozialistischen Prägung - haben seit den 1970er Jahren die ethnisch orientierten Organisationen stark an Bedeutung gewonnen. Eine klassenbasierte Wahrnehmung der Indigenen als Bauern hat einer ethnischen Wahrnehmung Platz gemacht (Marmon/Kramer 2006: 2). Zu der Analyse einer Ausbeutung der Indigenen als Teil des Proletariats kommt gegen Ende der 1970er ein Selbstverständnis als unterdrückte Nationalität, das die Indigenen dazu bringt, den Aufbau des Staates selbst in Frage zu stellen (Moya 1987: 389). Das bedeutet - wie im Kapitel über die CONAIE näher erläutert wird - nicht, dass Klassenaspekte keine Rolle mehr spielen, sondern vielmehr, dass beide Ansätze unter wechselnden Hegemonien vereinigt werden. Der reine Gegensatz von eher ethnisch orientierten Organisationen des Amazonasgebietes gegen eher klassistisch orientierten Organisationen im Andenhochland (Gnerre/Bottasso 1985: 20) spielt nur im Fall der CONAIE eine Rolle da die anderen Organisationen keine nennenswerte Präsenz im Amazonasgebiet haben $^{6}$ oder, wie die FEINE, andere ideologische Hintergründe. Die Frage, ob dieser Wandel durch eine Veränderung des Bewusstseins der Indigenen stattfindet von innen also - oder - von außen - durch Konstitution bestimmter Organisationen, ist nach wie vor offen.

6 Der Fall der FENOC, der für einige Jahre Organisationen des Amazonasgebietes angehörten, wird im entsprechenden Kapitel näher untersucht. 
Für den Anthropologen Sánchez-Parga steht am Anfang dieses Wandels in den 1970ern und 1980ern das Bewusstwerden der Indigenen, die ihre Besonderheit erkennen und erst dann eine „Ethnie für sich“ (Sánchez-Parga 1988: 108; SánchezParga 2010: 85) bilden. Erst diese Selbsterkenntnis erlaubt die Integration von Klassenaspekten und ethnischer Identität, was schließlich die Grundlage für die Forderungen nach Plurinationalität und Interkulturalität werden sollte (SánchezParga 2010a: 87). Die von ihm als ,nationalistische Kämpfe“ (Sánchez-Parga 2010a: 87f.) bezeichneten Tendenzen erlauben - in Verbindung mit dem Klassenkampf - den Kampf für eine kulturelle und soziale Integration des Landes. Dadurch gehen die ethnisch orientierten Organisationen weiter als die klassistischen, da sie nicht nur für Gleichheit kämpfen, sondern - auch das wird später näher beleuchtet für Einheit in der Vielfalt.

Korovkin erweitert die Idee Sánchez-Pargas. Sie versteht die Verschiebung hin zu einer ethnisch-identitären Sicht der Gesellschaft als Ergebnis der Institutionalisierung der Indigenenbewegung. Indigene Intellektuelle, die von den Strukturen der klassistischen Organisationen - etwa ihren Ausbildungszentren - profitierten, begannen, die klassische linke Sicht wegen ihrer Blindheit für kulturelle Aspekte der sozialen Ungleichheit zu kritisieren. Diese neue Schicht Indigener aus relativ wohlhabenden Familien mit hoher Bildung setzte dem ein ethnisches Verständnis der erlebten Ungleichheit entgegen. Diese neue Sichtweise entsprach der der indigenen Landbevölkerung, die die Machtverhältnisse etwa in den haciendas nicht nur als soziale, sondern vor allem als ethnische Beherrschung begriff. So begann für die klassischen linken Organisationen eine Krise, von der die neuen ethnischen Organisationen profitierten (Korovkin 2008: 2f.).

Bretón analysiert denselben Bruch unter organisatorischen Vorzeichen. Die klassistisch orientierten Organisationen - FEI und FENOC - verlieren Repräsentativität zugunsten neuer, eher ethnisch orientierter Organisationen wie ECUARUNARI, deren Diskurs beide Tendenzen mehr integriert als das vorher der Fall war (Bretón 2003: 228). Ob die Indigenen von einem Klassenbewusstsein zu einem ethnischen Bewusstsein gekommen sind - und wie - wird offengelassen. Stattdessen werden die Organisationen selbst, ihre Forderungen und ihre spezifischen Diskurse näher beleuchtet. Diesem Ansatz wird hier der Vorzug gegeben, auch weil empirische Studien zum Bewusstsein der Indigenen kaum verfügbar sind.

Der Konflikt zwischen einer auf Klasse und einer auf Ethnie basierten Ideologie in der Indigenenbewegung war in den 1970ern akut. Mit der weiteren Entwicklung von ECUARUNARI und spätestens der Gründung der CONAIE wurden beide Ansätze unter dem Schlagwort der ,doppelten Dimension“ (Ibarra 1996: 297f.) in einer gemeinsamen Organisation vereinigt, was vor allem zu einem Bedeutungsverlust der radikal ethnizistischen Strömungen führte. Nachdem die klassistischen Organisationen mit einigem Erfolg für soziale und rechtliche Gleichheit gekämpft 
haben, treten die ethnisch orientierten Organisationen für die Differenz und ihre Anerkennung als Nationalitäten ein (León Trujillo 2010: 14). Die kulturellen Forderungen - etwa die nach zweisprachiger Erziehung - stellten die staatliche Autorität nicht in Frage, erlaubten aber den Organisationen zum einen, einen politischen Raum für identitäre und kulturelle Diskurse zu öffnen, und zum anderen, ihre Strukturen auszubauen (Selverston 1994: 131). Die Neu-Konstitution indigener Identität führte schließlich zu politischen Konzepten und einer organisatorischen Stärke, die für die anderen Akteure unerwartet waren (Selverston 1994: 133) und durchaus Alternativen zum bestehenden Staat darstellen konnten.

Innerhalb der ethnischen Strömungen der Indigenenbewegung unterscheidet Büschges zwischen den Optionen der Integration - vor allem in den gemischt besiedelten Gebieten des Hochlandes - und der politischen Autonomie in eher isolierten Gebieten des Amazonasgebietes (Büschges 2009: 48f.). Die integrativen Gruppen versuchen, sich Räume in den bestehenden politischen Strukturen zu eröffnen, auch in Kooperation mit der mestizischen Bevölkerung - Beispiele wären die Politik des früheren Bürgermeisters von Cotacachi, Auki Tituaña, oder die Gründung der MUPP-NP. Die autonomistischen Gruppen wollen die den jeweiligen Nationalitäten oder Völkern eigene Strukturen (wieder-)aufbauen, ohne sich völlig dem Staat unterzuordnen. So wollen sie etwa eine eigenständige Rechtsprechung, der sich alle im jeweiligen Gebiet lebenden Personen unterwerfen müssen, während die integrativen Gruppen des Hochlandes eine Verbindung von Mehrheitsdemokratie mit „einer formellen Anerkennung eines gewissen Maßes an Autonomie und/oder speziellen Rechten für Minderheitsgruppen“ (Barrera 2002: 101) anstreben.

\section{d. Weitere Organisationen}

Es gibt einige Organisationen im Umfeld der Indigenenbewegung, die sich deutlich von der Geschichte und Entwicklung der bisher vorgestellten Organisationen unterscheiden. Auch wenn häufig Kooperationen und gemeinsame Aktionen stattfinden, nehmen diese Organisationen nicht am Diskurs der Indigenenbewegung teil und sind als reine Gewerkschaften bzw. Interessenvertretungen ohne expliziten ethnischen Anspruch zu verstehen. ${ }^{7}$

Die Nationale Föderation der freien agroindustriellen Arbeiter, Bauern und Indigenen Ecuadors (Federación Nacional de Trabajadores Agroindustriales, Campesinos e Indígenas Libres del Ecuador, FENACLE) wurde 1969 als Filiale der Ecuadorianischen Konföderation der freien gewerkschaftlichen Organisationen (Confederación Ecuatoriana de Organizaciones Sindicales Libres, CEOSL) ge-

7 Daher gibt es kaum Forschung zu diesen Organisationen. Alle Informationen, die nicht anders gekennzeichnet sind, stammen von den jeweiligen Internetseiten. 
gründet. Die CEOSL wurde 1962 als Alternative zur kommunistischen CTE gegründet und war in den ersten Jahren ihrer Existenz betont unpolitisch und auf Verständigung aus. Diese Gründung war auch das Ergebnis einer gezielten Förderung durch die USA seit den 1950ern (León/Ycaza 1989: 187f.). Sie wurde von 135 Arbeiter-, Handwerker- und sonstigen, auch kulturellen, Organisationen gegründet und durch ihre große Mitgliedszahl und starke Präsenz vor allem in der Industrie rasch zu einer der wichtigsten Gewerkschaften des Landes (León/Ycaza 1989: 188). Die CEOSL kämpfte als erste Gewerkschaft in Ecuador für Tarifverträge und sah Streik nur als letztes Mittel an (León/Ycaza 1989: 189). Die Strömung der freien Gewerkschaften wurde auf dem VI Kongress 1975 der Organisation verdrängt, 1984 setzten sich sozialistische Tendenzen durch (León/Ycaza 1989: 192f.). Auf ihrem 16. Kongress 2010 hat sich die CEOSL umstrukturiert und ist in der Gewerkschaftliche Konföderation der Arbeiterinnen und Arbeiter Ecuadors (Confederación Sindical de Trabajadoras y Trabajadores del Ecuador, CSE) aufgegangen.

Die FENACLE ist Mitglied in der Lateinamerikanischen Koordination der Gewerkschaften der Bananenarbeiter (Coordinadora Latinoamericana de Sindicatos Bananeros, COLSIBA), der Lateinamerikanischen Koordination der Landorganisationen (Coordinadora Latinoamericana de Organizaciones del Campo, CLOC) und der Internationalen Union der Lebensmittel-, Landwirtschafts-, Hotel-, Restaurant-, Catering-, Tabak- und anverwandter Arbeitnehmerverbände (IUL). Sie hat ca. 150.000 Mitglieder in 18 Provinz-Föderationen. Auch wenn der regionale Schwerpunkt der FENACLE im Küstengebiet ist, sind einige Indigenenorganisationen auf lokaler und kantonaler Ebene Mitglied (Ayala Mora o.J.: 5f.). Sie unterstützt sowohl die CONAIE und MUPP-NP, als auch Alianza PAÍS und Rafael Correa (Negreiros 2009: 137).

Die 1990 gegründete (Albó 2009: 229) Nationale Einheitskonföderation der Mitglieder der Bauernsozialversicherung-Nationaler Bauernrat (Confederación Única Nacional de Afiliados al Seguro Social Campesino-Consejo Nacional Campesino, CONFEUNASSC-CNC) hat eine Million Mitglieder in 2.300 ländlichen Gemeinden. Sie hat vor allem bei nicht-indigenen Landarbeitern Rückhalt (Albó 2009: 229). Seit ihrer Gründung stand sie der CONAIE nahe, die sie bei vielen Aktionen unterstützte und mit der sie 1996 die Partei MUPP-NP gründete. Die änderte sich mit der Annäherung der CONFEUNASSC-CNC an die Regierung Correa 2006, die bis heute besteht (Wolff 2010: 22). Die CONFEUNASSC-CNC ist ebenfalls Mitglied der CLOC. 


\section{Federación Ecuatoriana de INDIOS (FEI)}

\section{a. Vorgeschichte}

Eine Revolution junger Offiziere im Juli 1925 führt zu einer progressiveren Gesetzgebung, die die politische Organisation der Linken fördert (FENOC o.J.: 10) und die Gründung von Parteien und Gewerkschaften erleichtert. Daher beginnt die Geschichte der modernen Indigenenbewegung in Ecuador mit einer zunehmenden Politisierung auch durch die Gründung der Sozialistischen Partei 1926 und deren Umstrukturierung in die Kommunistischen Partei 1931 (Yumbay 2008: 234). 1926 finden in Cayambe, einem Kanton der Provinz Pichincha mit indigener Bevölkerungsmehrheit, politische Aktionen der indigenen Landarbeiter statt. Die staatlichen haciendas Guachalá und Bonifaz werden von Indigenen besetzt, die ihr Land zurückgewinnen wollen. Die Aktionen scheiterten am entschiedenen Durchgreifen der Großgrundbesitzer und der Armee (Agualsaca o.J.: 1). Aus diesen Aktionen entsteht noch 1926 mit Unterstützung urbaner Linker (Becker/Tutillo 2009: 97) die erste indigene Gewerkschaft (Albornoz 1976: 150), die Gewerkschaft der Landarbeiter von Juan Montalvo (Sindicato de Trabajadores Campesinos de Juan Montalvo) (Becker 1997: 169f.). Ihr Ziel war die Verteidigung des Landes der Bauern, Lohnerhöhungen und Arbeitszeitbegrenzungen. Außerdem bekämpfte sie die semifeudalen Vorrechte der Landbesitzer und der Kirche - etwa das Recht auf unbezahlte Arbeit der Indigenen - und schlechte Behandlungen durch die hacendados. Der erste Generalsekretär dieser Organisation war Jesús Gualavisí, der dieses Amt bis zu seinem Tod 1962 ausübte und an der Gründung anderer Gewerkschaften in der Region beteiligt war (Becker 1997: 169f.; Becker/Tutillo 2009: 96f.). Diese erste Gewerkschaft wurde einige Monate vor dem Entstehen der Sozialistischen Partei im Mai 1926 gegründet, entstammt also nicht den Organisationsversuchen der linken urbanen Parteien, sondern entstand zeitgleich mit ihnen als Reaktion auf ähnliche Bedingungen (Becker 1997: 171; Becker/Tutillo 2009: 97).

1927 werden in Cayambe weitere - damals noch klandestine (Becker/Tutillo 2009: 99) - Gewerkschaften gegründet: die Gewerkschaft ,El Inca“ in Pesillo, ,Pan y Tierra' in La Chimba, ,Tierra Libre“ in Moyurco (GTZ 2004a: 3) ${ }^{8}$. Bei diesen Gründungen spielten die Mitbegründer der Sozialistischen Partei, Jesús Gualavisí (Becker 2006: 134f.; Becker 2009: 1056) und Dolores Cacuango (Rodríguez 2008: 79), eine entscheidende Rolle.

8 Nach Marc Becker wurden El Inca und Tierra Libre vor dem Streik von Dezember 1930 und Januar 1931 gegründet und Pan y Tierra danach. Becker stellt klar: „Jenseits der Namen und ungefähren Gründungsdaten ist nur wenig über diese frühen Organisationen bekannt.“(Becker 1997: 173f.) 
Seit ihrer Gründung hatten die Kommunistischen und Sozialistischen Parteien in Lateinamerika versucht, die Kämpfe der Indigenen und Bauern in ihre Politik zu integrieren, ohne jedoch eine kohärente Linie entwickeln zu können. Als die Kommunistische Internationale (Komintern) ab Mitte der 1920er Jahre in Lateinamerika aktiv wurde, war sie bereits vom Stalinismus und seiner Bestrebungen geprägt, mittels einer Revolution in Etappen den Sozialismus in einem Land aufzubauen. Auf ihrem sechsten Kongress 1928 „entdeckte“ (Becker 2002: 194) sie Lateinamerika. Dort wurde die gestiegene Bedeutung der kommunistischen Parteien in Lateinamerika hervorgehoben und über die Frage diskutiert, ob man die Region als , semikolonial' oder als , abhängig' zu verstehen hatte. Der Vorsitzende der PCE, Ricardo Paredes verteidigte die zweite Position, um die politische Unabhängigkeit von der wirtschaftlichen Integration in den Imperialismus abgrenzen zu können (Becker 2002: 194). Mit Rückgriff auf die kommunistischen Klassiker, allen voran Lenin, forderte die Komintern ,die Selbstbestimmungsrechte der nationalen Minderheiten, einschließlich das Recht, sich von unterdrückerischen staatlichen Strukturen zu trennen“ (Becker 2002: 191f.). So wurde - analog zur Forderung von Schwarzenrepubliken in Südafrika und den USA - der Vorschlag erarbeitet, eine indigene Republik der Quechua und Aymara in den Anden aufzubauen (Becker 2002: 195). Die Hinwendung der Komintern zu Lateinamerika bedeutete auch eine engere Kontrolle der Kommunistischen Parteien dort, wodurch eine ,flexible Anpassung der politischen Strategien an die nationalen Wirklichkeiten“ (Becker 2002: 194f.) durch die lateinamerikanischen Kommunisten schwieriger wurde.

Nach der Rückkehr der lateinamerikanischen Delegierten vom sechsten Kongress der Komintern wurden zwei regionale Kongresse organisiert, auf denen die Beschlüsse der Komintern umgesetzt und die Arbeit der nationalen Kommunistischen Parteien koordiniert werden sollten. Im Mai 1929 fand in Montevideo der Gründungskongress der Lateinamerikanischen Gewerkschaftskonföderation statt, die schon seit Ende 1927 geplant worden war und auf dem Komintern-Kongress viel Unterstützung erfahren hatte. Insbesondere wurde eine Integration der Bauern und Landarbeiter in die Gewerkschaften gefordert (Becker 2002: 198). Die Erste Lateinamerikanische Kommunistische Konferenz in Buenos Aires im Juni 1929 diente der Ausarbeitung eines konkreten, auf die lateinamerikanischen Besonderheiten abgestimmten, Programms. 38 Delegierte aus 15 Ländern arbeiteten an diesem Programm, dessen Grundzüge im August 1929 in der Zeitung der lateinamerikanischen Sektion der Komintern, La Correspondencia Sudamericana, veröffentlicht wurden (Becker 2002: 198). Auf dem Kongress entwickelte der peruanische Kommunist José Carlos Mariátegui die These, dass die Indigenen in erster Linie eine Klasse bilden (Becker 2002: 192) - ohne sich jedoch völlig von den indigenistischen Bestrebungen der Komintern zu distanzieren. Als Landproletariat waren die Indigenen auf Zugang zu Ackerland angewiesen, der ihre Position im Klassen- 
kampf verbessern sollte. Ihre ethnische Verfassung als Nationalität im Sinne der Komintern sollte die Indigenen zu einer Unabhängigkeitsbewegung führen, die in Allianz mit den mestizischen Arbeitern - die ja aus ethnischem Blickpunkt Gegner waren - eine kommunistische Revolution herbeiführen konnte (Becker 2002: 197). Dadurch wurden vorherige, an den USA und Südafrika entwickelte Strategien, ,unabhängige Eingeborenenrepubliken“ (Becker 2002: 191f.) zu gründen, weitgehend verworfen. In den Beschlüssen der Konferenz von 1929 wurde „der Kampf der Indios für den Anspruch ihrer unterdrückten Nationalität" (Correspondencia Sudamericana, August 1929 in: Grupo Internacionalista 2003) verankert - allerdings nur als These zur Diskussion, die auf einem zweiten Kongress konkretisiert werden sollte (Becker 2002: 212). Zu diesem zweiten Kongress kam es jedoch nie (Becker 2002: 198), eine lateinamerikanische Interpretation des Kommunismus konnte sich nicht durchsetzen. In den folgenden Jahren näherten sich die Kommunistischen Parteien der Linie der Komintern an. Mit Hilfe der Bauern und Landarbeiter sollte eine demokratisch-bürgerliche Revolution gegen die feudalen und semifeudalen Verhältnisse durchgeführt werden, die erst die Grundlage für eine kommunistische Revolution schaffen sollte. Ab Mitte der 1930er Jahre wurde in dieser Linie eine Agrarreform ohne politische Revolution eines der wichtigsten Ziele der PCE (Grupo Internacionalista 2003).

Ab Mai 1930 begann die Sozialistische Partei, sich mit den Indigenen in Pesillo zu treffen und sie bei der Organisation und Formulierung ihrer Forderungen zu unterstützen (Becker/Tutillo 2009: 99), wodurch sich die Landarbeiter der Region ihr annäherten (Becker 1997: 174f.). Sie gründete im August 1930 die Arbeiter- und Bauernhilfe (Socorro Obrero y Campesino), die die jungen Gewerkschaften juristisch und logistisch unterstützen sollte. Die ersten Aktionen der Arbeiter- und Bauernhilfe war die Befreiung von gefangengenommenen Gewerkschaftsmitgliedern und Mitgliedern der Kommunistischen Jugend (Juventud Comunista), der Nachwuchsorganisation der PSE (Becker 2008b: 32f. ${ }^{9}$, in Pesillo, die den Organisationen vor Ort beistehen wollten. Der sozialistische Senator Luis Maldonado machte im Nationalkongress auf die Lage der Arbeiter in Cayambe aufmerksam; die PSE sammelte Geld für die gefangengenommenen Arbeiter (Becker 1997: 176; Becker/Tutillo 2009: 100).

Die verstärkte Kooperation zwischen urbanen und ländlichen Aktivisten führte zu einer ideologischen Weiterentwicklung beider Seiten. So berichtet Nela Martínez, dass in den 1920ern und 1930ern die Zeitschrift Amauta, die vom peruanischen Kommunisten José Carlos Mariátegui herausgegeben wurde, von den linken Aktivisten rezipiert wurde (Becker/Tutillo 2009: 114). Die urbanen Aktivisten berieten

9 Nach Gründung der Partido Comunista del Ecuador (PCE) wurde sie ihre Jugendorganisation. 
und unterstützten die Indigenen, diese waren aber die treibende Kraft hinter den Forderungen, die sie weitgehend eigenständig aufstellten, und den Aktionen, die sie durchführten. Obwohl die Indigenen ihren Kampf auch als ethnisch verstanden, wandten sie sich nicht generell gegen alle Weißen, sondern verbündeten sich im Rahmen eines Kampfes für soziale Gerechtigkeit mit den urbanen Marxisten (Becker/Tutillo 2009: 128). Diese gesteigerte Mobilisierung verstärkte den Bruch innerhalb der PSE, der zu ihrer Umbenennung in PCE und der Neugründung einer nicht-kommunistischen PSE führen sollte. Die von der Komintern übernommene Bezeichnung der Indigenen als Nationalitäten wurde so als kommunistische Ideologie wahrgenommen (Becker 2008b: 37f.). Also gab es schon in den 1930ern Versuche, über sowjetische Begriffe Klassenaspekte mit ethnischen Aspekten zu vereinen (Becker 2008b: 45).

1930 und 1931 findet in Pesillo unter der Führung von Cacuango (Becker 2009: 1056) der erste größere Streik der indigenen Organisationen statt, der drei Monate dauern sollte (Yépez 2010). Es wurden Lohnerhöhungen, eine Beschränkung der Arbeitszeit auf 40 Stunden pro Woche, die Rückgabe konfiszierten Landes an die Arbeiter, die Entlohnung der Arbeit der Frauen und ein Ende der unbezahlten Arbeiten für den hacendado und der Erhebung des Zehnten durch die Kirche gefordert. Unter den Forderungen gab es allerdings keine, die sich um ethnische oder gesamtstaatliche Inhalte, wie ein Ende der Diskriminierung, oder um eine Agrarreform drehte (Becker/Tutillo 2009: 102f.). Diese letzte Forderung kam erst später unter dem Einfluss der PCE auf (Becker/Tutillo 2009: 103). Im Anschluss an diesen - erfolglosen - Streik konnte erreicht werden, dass die Regierung Zahlungen für die im Zuge der Aktionen zerstörten Häuser und ausgefallenen Löhne anordnete - eine Anordnung allerdings, die genauso wenig umgesetzt wurde wie die anderen Forderungen der Indigenen, darunter Verbesserung ihrer rechtlichen Situation und eine Erhöhung der Löhne (Albornoz 1983: 44; Agualsaca o.J.: 1).

Im Februar 1931, unmittelbar nach dem Streik, sollte der erste nationale Kongress der Bauernorganisationen (Primer Congreso de Organizaciones Campesinas) mit 2.000 erwarteten Teilnehmer in Juan Montalvo, Cayambe, stattfinden (CONAIE 1989: 30f.). Er war schon vor dem Streik geplant und unter anderem in der Zeitung der PSE angekündigt worden (Becker/Tutillo 2009: 104), gewann durch ihn aber eine größere Bedeutung. Der Kongress wurde von den Gewerkschaften in Cayambe und sechs Kommunen in Otavalo organisiert (Becker 1997: 188). Es sollte dort die Ausweitung des indigenen Kampfes auf ganz Ecuador vorbereitet werden (Albornoz 1976: 150). Er wurde jedoch von der Regierung Isidro Ayora durch Polizei und Militär verhindert (Albornoz 1983: 43f.; Rodríguez 2008: 80) die Regierung befürchtete Unruhen, obwohl Politiker der Region berichtet hatten, dass die Lage ruhig war und es keine direkte Gefahr gab (Becker 1997: 190). Im März 1931 kamen die Streikenden und die Teilnehmer des gescheiterten Kongres- 
ses aus Cayambe zu Fuß in Quito an, um ihre Forderungen direkt der Regierung zu präsentieren. Dabei erhielten sie logistische Unterstützung durch urbane Intellektuelle um die Kommunistin Nela Martínez, die später die zweisprachige indigene Zeitung Nucanchic Allpa herausgeben (Becker/Tutillo 2009: 123) und die indigenen Organisationen mit einer Medienkampagne unterstützen sollten (Yépez 2010). Martínez war seit 1934 Mitglied der PCE und arbeitete mit Indigenen- und Frauenorganisationen zusammen (Becker/Tutillo 2009: 144).

1934 schließlich fand die erste Konferenz der Cabecillas Indígenas in der Casa del Obrero in Quito statt, die die Grundlage für eine nationale Indigenenorganisation werden sollte (Albornoz 1976: 151; Botero 1998: 8). Die Casa del Obrero war ein Freiraum für linke Politik im Zentrum von Quito, an der Plaza del Teatro, die schon zuvor von den Indigenen genutzt worden war, wenn sie ihre Forderungen in Quito vortrugen (Becker/Tutillo 2009: 114). Auf dieser Konferenz wurde der Generalrat der Cabecillas Indios (Consejo General de Cabecillas Indios) gegründet, der als nationale Organisation die indigenen Interessen verteidigen sollte. Er wurde nie offiziell anerkannt (Becker 1997: 240f.). Jesús Gualavisí wurde zum ersten Generalsekretär dieser Organisation gewählt (Becker 2006: 134f.) und versuchte, die vorhandenen indigenen Organisationen in die neue nationale Organisation zu integrieren - ohne größeren Erfolg. Der Generalrat der Cabecillas Indios unterstützte lokale Initiativen und versuchte - allerdings meist ohne Erfolg -, eine Reihe von Streiks zu organisieren (Becker 1997: 208; Becker/Tutillo 2009: 133f.). Dies war der Beginn eines Organisationsprozesses, der 10 Jahre später zur Gründung der FEI führen sollte.

Die erste Ausgabe von Ñcanchic Allpa, ,Nuestra Tierra' erschien direkt im Anschluss und auf Beschluss der Konferenz von 1934 (Becker 2006: 134). Diese Zeitung, deren Titel auf deutsch ,Unser Land“ bedeutet, erschien unregelmäßig zwischen den 1930er und den 1960er Jahren (Albó 2009: 205f.). Ñucanchic Allpa sollte die Aktivitäten der indigenen Organisationen koordinieren und den Kampf gegen die semifeudalen Herrschaftsformen auf dem Land unterstützen (Agualsaca o.J.: 3). Sie war auf spanisch verfasst, enthielt aber meist wenigstens einen Artikel auf Kichwa. Die Ausgaben der 1930er Jahre trugen den Untertitel ,Órgano de los sindicatos, comunidades e indios, en general (Organ der Gewerkschaften, Gemeinschaften und Indios, allgemein), mit der Gründung der Federación Ecuatoriana de Indios (FEI) 1944 wurde sie zu deren offiziellem Organ (Albornoz 1983: 63; Becker 2006: 133). Nucanchic Allpa war ein Instrument der Indigenenbewegung und richtete sich vor allem an Indigene - baute aber von Beginn an auf die Mitarbeit der urbanen Linken. So war das mestizische PCE-Mitglied Nela Martínez in den 1930ern ihre Direktorin (Becker 2006: 135). Nucanchic Allpa verteidigte von einer klassenanalytischen Perspektive aus die Rechte der Indigenen, kritisierte den paternalistischen Indigenismus des Staates und der Mehrheitsgesellschaft und forderte 
einen Ausbau des Bildungssystems für Indigene. So wandte sich ein Leitartikel von Mai 1940 gegen die Idee der Indigenen als , unterlegene Rasse ' und sah die ecuadorianische Gesellschaft als in Proletarier und Kapitalisten gespalten, wobei die Proletarier Indigene, Schwarze und arme Mestizen umfassten. Im Anschluss wurde eine Liste mit 19 Forderungen präsentiert, die eine Abschaffung des huasipungoSystems auf den damaligen haciendas, eine Landreform und die Einhaltung des Arbeitsrechts enthielten. Dazu kam die Forderung nach einer speziellen Bank für indigene Bauern, um so ein angemessenes Angebot an günstigen Krediten zu schaffen - eine Forderung, die die Indigenenbewegung lange begleiten sollte: Vergleichbares forderte die CONAIE auch 1990 (Becker 1997: 208-211).

Das Kommunengesetz (Ley de Comunas) von 1937 war ein Versuch des Staates, über eine Umstrukturierung der indigenen Gemeinschaften die staatliche Kontrolle über sie aufrechtzuerhalten und die Indigenen auf neue Weise in den Staat zu integrieren (CONAIE 1989: 31). Eine Kommune ist eine indigene Gemeinschaft außerhalb der haciendas mit mindestens 50 Mitgliedern. Sie ist eine rechtliche Person und gibt ihren Mitgliedern eine gewisse wirtschaftliche und politische Freiheit, in Form einer eigenen Regierung (cabildo) und gemeinschaftlichem Landbesitz. Die Kommunen sind über die Institution der parroquias in den Staat integriert (Lucero 2003: 29; Van Cott 2005: 103). Da der Landbesitz ein notwendiges Kriterium ist, um eine Kommune bilden zu können, die meisten Indigenen jedoch kein Land hatten, wurde das Kommunengesetz von vielen Indigenenorganisationen als unzureichend kritisiert (Conterón/de Viteri 1984: 20). Das Konstrukt der Kommune ist eine Mischung aus Strukturen der Inka, traditionellen andinen Formen der sozialen Organisation und Strukturen, die die spanischen Eroberer eingeführt hatten. Es war nur als eine Art Übergangslösung geplant. So wurde bereits in der ersten Fassung des Kommunengesetzes festgeschrieben, dass der Staat alles notwendige unternehmen solle, um die Kommunen in Produktionskooperativen umzuwandeln und damit die Modernisierung und Besserstellung der Mitglieder zu befördern (Lucero 2003: 29-31). Dennoch konnten in ihr die traditionellen indigenen Formen der Regierung und Verwaltung fortgeführt und erneuert werden (Becker 1997: 229) - jedoch in einem modernen Gewandt, das den indigenen Traditionen teilweise stark widersprach (Becker 1997: 231). Bis zur Agrarreform 1964 verbreiteten sich die Kommunen nur langsam, erst das Ende der huasipungaje durch diese Reform führte zu einem Anstieg der Anzahl der Kommunen, die von früheren huasipungueros gebildet wurden (Becker 1997: 230). Das Kommunengesetz sollte die Organisation der Indigenen auf überlokaler Ebene unterbinden oder anders kanalisieren, um so den entstehenden Gewerkschaften die Grundlage zu nehmen und die Kontrolle des Staates über die Indigenen zu erhalten (Botero 2000: 44). Der Aufbau neuer und paralleler Strukturen sollte den Einfluss radikalerer Organisationen reduzieren. Das Kommunengesetz war ein Versuch, die landwirtschaftlichen Strukturen in Ecuador 
zu modernisieren und die Entwicklung der Indigenen zu fördern. Das Gesetz war von paternalistischer Natur und sollte die ländlichen indigenen Gemeinschaften schützen und leiten, der Staat behielt dabei die Autorität über die organisatorischen Strukturen, die er verändern konnte, wenn nötig (Becker 1997: 231).

Ein weiteres wichtiges Gesetz, das Arbeitsgesetzbuch (Código del Trabajo), wurde am 5. August 1938 erlassen. Es war der erste Versuch, die Beziehungen zwischen Arbeitern und Arbeitgebern zu regeln. So wurde ein Mindestlohn und der 8-Stunden-Arbeitstag festgelegt und das Recht der Arbeiter, sich zu organisieren und zu streiken, formalisiert (Becker 1997: 233). Durch diese Grundlage des gemeinsamen Kampfes näherten sich die ländlichen und die urbanen Organisationen weiter an und bereiteten über ihre verstärkten Kooperationen den Boden für die Gründung nationaler Organisationen (Becker/Tutillo 2009: 135).

Schon vor der Gründung der FEI begannen die Indigenen mit dem Aufbau eines Systems zweisprachiger Schulen für Indigene (Becker 2006: 141) - ein Thema, das die gesamte Indigenenbewegung prägen sollte. Auch die Auseinandersetzung mit dem Indigenismus, vor allem dem Ecuadorianischen Indigenistischen Institut (Instituto Indigenista Ecuatoriano, IIE) spielte in den frühen 1940ern eine Rolle (Becker 2006: 141). Der Indigenismus wurde als paternalistisches Projekt der mestizischen Mittelschicht abgelehnt, das die Indigenen langfristig in eine einheitliche Nationalkultur integrieren sollte. 1940 fand auf Betreiben des mexikanischen Präsidenten Lázaro Cárdenas in Pátzcuaro, Michoacán, der erste Interamerikanische Indigenistische Kongress (I Congreso Indigenista Interamericano) statt, der die Grundlinien dieses Indigenismus vorgeben sollte. Eine Assimilation der Indigenen in die mestizische Mehrheitsgesellschaft wurde in Teilen auch von der PCE unterstützt (Grupo Internacionalista 2003) - eine Quelle für Konflikte zwischen der PCE und der Indigenenbewegung.

Die Revolution vom 28. Mai 1944, die die Regierung von Carlos Alberto Arroyo del Río stürzte, führte zu einer Öffnung der Zivilgesellschaft (Albornoz 1976: 151). Vom 4. bis zum 9. Juli 1944 fand unter Teilnahme von 1.036 Teilnehmern der Gründungskongress der Konföderation der Arbeiter Ecuadors (Confederación de Trabajadores del Ecuador, CTE) statt (CTE o.J.: 4f.). Pedro Saad, Mitbegründer der kommunistischen Partei, wurde zu ihrem Generalsekretär gewählt (Yépez 2010). Auf diesem Kongress kündigte die CTE an, Landarbeitergewerkschaften und Bauernorganisationen, sowie indigene Kommunen in sich integrieren $\mathrm{zu}$ wollen und zu diesem Zweck eine Nationale Bauern- und Indioföderation (Federación Nacional Campesina e India) innerhalb der CTE gründen zu wollen (Becker 1997: 240f.) - die einige Monate später als Ecuadorianische Indioföderation (Federación Ecuatoriana de Indios, FEI) gegründet wurde (Tamayo 1996: 89). 


\section{b. Gründung}

Vom 6. bis zum 8. August 1944 fand der erste Ecuadorianische Indigenen-Kongress (Primer Congreso Ecuatoriano de Indígenas) in der Casa del Obrero in Quito statt. Die Delegierten kamen vor allem aus den Provinzen des nördlichen und zentralen Andenhochlandes, Imbabura, Pichincha, Cotopaxi und Chimborazo, wobei der Kanton Cayambe (Pichincha) von besonderer Bedeutung war. So entstammten fast alle wichtigen Figuren des Kongresses diesem Kanton (Becker 1997: 242). Kommunisten und Linke aus Quito unterstützten den Kongress und die daran teilnehmenden Organisationen und halfen mit ihren spezifischen Erfahrungen (Becker 1997: 242). Nela Martínez etwa nahm an der Gründung der FEI teil und war persönliche Sekretärin von Dolores Cacuango, die sie auf den zweiten Kongress der Arbeiterkonföderation Lateinamerikas (Confederación de Trabajadores de América Latina, CTAL) in Cali, Kolumbien 1944 begleitete (Becker/Tutillo 2009: 144). Der Kongress war offen konzipiert, Presse und sonstige Interessierte wurden über Anzeigen in Tageszeitungen ebenso eingeladen wie eine Reihe von Ministern und Parlamentariern (Becker/Tutillo 2009: 142). Auf diesem Kongress wurde die Ecuadorianische Indioföderation (Federación Ecuatoriana de Indios, FEI) als ländliche Filiale der kommunistischen, der PCE nahestehenden, Gewerkschaft CTE gegründet (Büschges 2009: 54). Während die CTE national agierte, war die FEI auf die Andenregion beschränkt, was vor allem an ihrer Orientierung auf huasipungueros und Landarbeiter auf den haciendas lag (CONAIE 1989: 31f.; Guamán 2003: 82). In ihr schlossen sich die seit den 1920ern aktiven, von PSE und PCE geförderten Landarbeitergewerkschaften zusammen, wobei sie sich explizit auch anderen indigenen Organisationen öffnete (Albornoz 1983: 63). Die CTE, Mitglied im kommunistischen Weltgewerkschaftsbund ${ }^{10}$ (Lechón 1976: 86), kämpfte für

„die Verbesserung der sozialen Sicherheit, der Arbeitsbedingungen, aber genauso Demokratisierung, Antiimperialismus und die Überwindung ,prä-kapitalistischer' Ausbeutungsverhältnisse auf den Haciendas durch eine Agrarreform“ (Kaltmeier 2009: 344; siehe auch: CTE o.J.: 5f.).

Dieser letzte Punkt war die Hauptforderung der FEI, in Verbindung mit anderen Forderungen bezüglich des Arbeitsrechts und der Lebensbedingungen auf den - oft staatlichen - haciendas (Becker 2007: 138f.). Dazu kam in den Statuten, die auf dem Gründungskongress beschlossen wurden, die ,wirtschaftliche Emanzipation der ecuadorianischen Indios“ (Albornoz 1983: 63). Auch enthielten die Statuten Elemente einer pan-indigenen Ideologie, die Organisation verpflichtete sich zur

10 Und nicht im Weltverband der Arbeitnehmer, wie León (1994: 55) behauptet. 
Kooperation mit den anderen indigenen Organisationen des Landes und des Kontinents (Becker/Tutillo 2009: 140). Die FEI hatte ihre Schwerpunkte im Norden und Zentrum der Andenregion, in Cayambe, Cotopaxi und Chimborazo (Kaltmeier 2009: 344). Sie war die erste Organisation, die sich explizit als indigen verstand und verwendete die Bezeichnung indio im Sinne des Mottos ,mit dem Wort indio wurden wir erobert und mit dem Wort indio werden wir uns befreien“ (Yumbay 2008: 234 $)^{11}$. Das Weiterbestehen der kolonialen Strukturen sollte nicht durch das neutralere , indígena 'überdeckt werden, sondern durch die Umdeutung des Begriffs ,indio' gegen den Kolonialisierer gewendet werden (Barre 1983: 19; Quijano 2006a: 33). Dadurch, dass sich die FEI nicht allein als Bauern- oder Landarbeiterorganisation bezeichnete, distanzierte sie sich sowohl vom liberalen Diskurs der Integration in die Gesellschaft, als auch vom marxistischen Verständnis der Indigenen als Landproletariat. So bereitete sie das Entstehen eines ethnischen Bewusstseins der Indigenen in Ecuador vor (Becker/Tutillo 2009: 138).

Am 29. Januar 1945 wurde die FEI staatlich anerkannt (FEI o.J.), das Sozialministerium akzeptierte die Statuten der FEI mit zwei Änderungen. Kommunen konnten nicht, wie ursprünglich geplant, Mitglied der FEI werden, wodurch die Basis der neuen Organisation erheblich beschränkt wurde. Diese Maßnahme der Regierung, die Ausbreitung der FEI zu behindern, war erfolgreich, konnte sie sich doch nie in den Regionen durchsetzen, in denen die Kommunen als Organisationsform vorherrschten. Die zweite Änderung betraf die Forderung der FEI, den Vertreter der Indigenen Rasse im Nationalkongress selbst zu bestimmen. Das Ministerium erlaubte der FEI, diesen Vertreter zu nominieren, aber nicht, seine Funktion völlig zu kontrollieren (Becker 1997: 252; Becker/Tutillo 2009: 140f.). Die erste Generalsekretärin der FEI wurde das indigene PCE-Mitglied Dolores Cacuango (Albornoz 1983: 63; Tamayo 1996: 89), ihr erster Präsident der indigene PCE-Mitbegründer Jesús Gualavisí (Becker 1997: 247). Somit entstammten die wichtigsten Funktionäre der neuen Organisation dem Kanton Cayambe, die dort die zentralen Einrichtungen des Exekutivkomitees der FEI aufbauten - obwohl die Statuten der FEI vorsahen, dass sie in Quito sein müssen (Becker/Tutillo 2009: 137). Die FEI wurde in der Verfassungsgebenden Versammlung 1944/1945 vom Gründer der PCE, Ricardo Paredes, als Funktioneller Repräsentant für die indigene Rasse vertreten (Becker 2006: 143; Becker/Tutillo 2009: 141). Die dort ausgearbeitete Verfassung, die nur im Jahr 1945 in Kraft war, sah einen Abgeordneten der indigenen Organisationen im Parlament vor, der auch an den Aktivitäten dieser Organisationen beteiligt sein musste - also nicht wie zuvor ein Außenstehender. Diese Funktion wurde schon in

11 Das Motto ist mit einigen Veränderungen seit den 1970ern wieder zu finden, etwa 1980 auf dem ersten Kongress der Indio-Bewegungen Südamerikas (I Congreso de Movimientos Indios de Sudamérica) in Ollantaytambo, Peru (AAVV 1985: 163). 
der Verfassung von 1946 gestrichen (Becker/Tutillo 2009: 141). Paredes vertrat die FEI auch später auf internationalem Niveau, etwa bei den jeweiligen Gewerkschaftsbünden ${ }^{12}$ (Becker/Tutillo 2009: 142).

FEI und PCE teilten sich in den 1940ern und 1950ern einen großen Teil ihres Führungsapparates und ihrer Funktionäre (Santana 1983: 159). Diese strukturellen Verbindungen haben zu einer heftigen Diskussion in der Forschung darüber geführt, ob die FEI eine reine Massenorganisation der PCE war - also von außen bestimmt war, ethnische Probleme nicht anerkannte und die Indigenen nur als Bauern oder Landproletariat wahrnahm. Diese Position ist die ältere, auch von der CONAIE (1989: 276) selbst vertretene. Die Blindheit den ethnischen Bestandteilen der Lage der Indigenen gegenüber verhinderte in diesem Sinne eine weiteres Wachstum der Organisation unter der indigenen Bevölkerung. Auch wenn einige Indigene in Führungspositionen der FEI aufsteigen konnte, war die Organisation selbst immer unter Kontrolle externer Akteure, vor allem der PCE und der CTE (CONAIE 1989: 31f.; GTZ 2004a: 3). Die FEI war, wie auch die CTE, eine Massenorganisation der PCE (Santana 1983: 159) ohne wirkliche Eigenständigkeit. Ihre Aktionsformen und Forderungen wurden von der PCE geprägt (Rodríguez 2008: 130). Sie beachtete in ihrem Programm nicht Probleme wie ethnische Diskriminierung, kulturelle Gewalt oder Erziehungsfragen (Yumbay 2008: 234); die sozialistischen Gruppen versuchten, die politische Mobilisierung zu „de-ethnisieren“ (Büschges 2009: 45) und betrachteten die Indigenen als Teil der Bauernschaft (Rodríguez 2008: 131). Die indigenen Führer, die den Klassenkampf mit der Verteidigung indigener Rechte verbinden wollten (Büschges 2009: 45), waren stets in der Minderheit und konnten die rein marxistische Sichtweise der PCE nicht in Frage stellen. Die fehlende Teilhabe Indigener an den Führungspositionen der PCE und der FEI führte, zusammen mit einer fehlenden Integration ethnischer und kultureller Forderungen (Santana 1983: 162), zu einer quasi-patriarchalischen Situation, in der das mestizische Führungspersonal für die Indigenen sprach, ohne dass diese wirklich an den Entscheidungen beteiligt gewesen wären (García Serrano 2005: 1f.).

Die Kritik an dieser Sichtweise ist relativ neu und mit der Person des Historikers Marc Becker verbunden. Für Becker basiert das Verständnis der FEI als eine von urbanen Mestizen gesteuerte, quasi-indigenistische Organisation auf der fehlenden Kenntnis der Geschichte der indigenen Organisationen in Ecuador (Becker 2008b: 2). Es stimmt nicht, dass erst in den 1970ern oder 1980ern eine neue Generation indigener Intellektueller die Führung der Indigenenbewegung übernommen hat. Stattdessen liegt dem eine lange Entwicklung der Bewegung zugrunde, in der

12 Die Teilnahme von Paredes am Zentralrat der amerikanischen gewerkschaftlichen Konföderation (Consejo Central de la Confederación Sindicalista Americana) in Bukarest in den 1950ern (Becker/Tutillo 2009: 142) ist nicht gesichert. 
die FEI eine wichtige Rolle spielt (Becker 2007: 136). Nach Becker wurde die FEI nicht von der PCE gesteuert, vielmehr teilten sich beide Organisationen - und die CTE - Strukturen und Personal. Die PCE war nicht ausschließlich urban, sondern hatte schon früh einige indigene Mitglieder in wichtigen Positionen (Becker 2007: 139) - wie Dolores Cacuango und Jesús Gualavisí. Durch den starken Einfluss der PCE auf die Gewerkschaften der 1920er und 1930er Jahre, die in der FEI aufgehen sollten, kamen die meisten Führer der FEI aus den Reihen der PCE. „[D]ie Kommunistische Partei bildete nicht die Indigenenbewegung, sondern beide wurden im selben Kampf geboren.“ (Becker 2007: 139). Sowohl Indigene als auch nichtIndigene waren an der Bildung der FEI beteiligt, wobei sie sich - trotz vielfältiger Konflikte - ergänzen konnten. So verankerten die indigenen Führer die Organisation in den Gemeinschaften vor Ort (Botero 1998: 8), während die urbanen Kommunisten Fachwissen über politische Prozesse und rechtliche Möglichkeiten beitrugen (Becker 2007: 140). Die FEI kämpfte nicht nur für eine Landreform, sondern auch für die Rechte der Indigenen und gegen ihre Diskriminierung (Minkner-Brünjer 2009: 138; FEI o.J.a). Es ist nicht nachweisbar, dass die Kommunisten ethnische Forderungen explizit abgelehnt hätten, wie es in Peru der Fall war (Becker 2008b: 45). Vielmehr enthielt schon das Gründungsprogramm der FEI ethnische (die Kultur der Indigenen bewahren und verbessern) neben ökonomischen (wirtschaftliche Emanzipation der Indigenen) Aspekten, was im Sinne eines Klassenkampfes mit ethnischer Zielrichtung zu verstehen ist (Becker 2007: 141). Die starke Präsenz ethnischer Forderungen widerspricht der Wahrnehmung einer Entwicklung von klassistischen Organisationen vor den 1960ern hin zu ethnischen in den 1970ern und schließlich zu den ethnischen Nationalitäten der 1980er und 1990er.

„Ideologien von Klasse, Ethnizität und Nationalität waren in verschiedenen Formen und Ebenen bei der Gründung der FEI präsent, mit Aktivisten, die verschiedenen Aspekten den Vorzug gaben, um die aktuellen Notwendigkeiten und Forderungen zu treffen.“ (Becker 2007: 141)

Diese verschiedenen Aspekte waren auch in CTE und PCE präsent. Der sowjetische Anthropologe Yuri Zubritski beschreibt die Bedeutung der nationalen Eigenheiten der indigenen Völker für die PCE und ihre Forderungen nach einem Ende der Diskriminierung, nach Unterricht in der Muttersprache, Förderung der indigenen Kultur und politischer Autonomie für die Indigenen (Zubritski 1979: 151f.).

\section{c. Entwicklung}

Die FEI war immer mit dem Kampf der huasipungueros und Landarbeiter für Arbeitsrechte und eine Landreform verbunden. Diese sah sie als die Grundlage für die 
Entstehung eines Landproletariats (Korovkin 1999: 10). Schon im Gründungsmanifest forderte sie die Übergabe des Landes an die, die es bearbeiten, seine Parzellierung, die Regulierung der Arbeitszeiten und der Löhne, das Verbot der huasipunga$j e$, den Aufbau von Gesundheits-, Erziehungs- und Wohnungsinfrastruktur, die Technisierung der Landwirtschaft, die Verteidigung der indigenen Sprachen und Kulturen und die Teilnahme der Indigenen am politischen Leben des Landes (Rodríguez 2008: 82). Dazu kam ein spezifisches Recht auf Wasser (GTZ 2004a: 3). Die FEI kämpfte gegen das System der haciendas (Rodríguez 2008: 82) und die damit verbundenen Landenteignungen der indigenen Gemeinden und verteidigte die einzelnen Gewerkschaften der huasipungueros und Landarbeiter (Tamayo 1996: 64).

Die Basis der FEI waren huasipungueros und Landarbeiter auf den haciendas, dennoch wäre es falsch, sie als reine Landarbeitergewerkschaft zu verstehen. Der Aufbau von - zuerst klandestinen (Martínez Novo 2009: 3f.) - zweisprachigen Schulen auf dem Land, verbunden mit der Person Tránsito Amaguañas und Dolores Cacuangos (Becker 1997: 205f.; Lucas 2000: 10), war seit ihrer Gründung ein wichtiges Anliegen der FEI. Das Zentrum dieses parallelen Bildungssystems, dessen Ursprünge älter sind als die FEI, war Cayambe (Becker 1997: 293). Dort, in der Umgebung von Pesillo, entstand im Oktober 1945 unter der Führung von Dolores Cacuango die erste von später drei indigenen Schulen in der Region (Montaluisa 2003: 86). Diese Bemühungen wurden von einigen urbanen Gruppen aus dem Umfeld der PCE, wie der Ecuadorianischen Frauenallianz (Alianza Femenina Ecuatoriana, AFE) aus Quito, unterstützt (Becker 1997: 296; Becker/Tutillo 2009: 164). Die Grundidee war, das indigene Lehrer die Kinder in ihrer Muttersprache, also Kichwa, unterrichten sollten (Becker 1997: 296). Zu den ersten Lehrern gehörten viele indigene Aktivisten aus der FEI selbst, wie etwa Dolores Cacuango, Alberto Tarabata, José Amaguaña, Neptalí Ulcuango und Luis Catucuamba (Lazos/Lenz 2004: 6). Das Ziel des Aufbaus eines Erziehungssystems war eine Stärkung der Indigenen sowohl in ihrer ethnischen als auch in ihrer Klassenlage. Über Bildung und Alphabetisierung sollten Benachteiligungen abgebaut und ein ethnisches und Klassenbewusstsein aufgebaut werden (Moya 1987: 391f.). Gut ausgebildete Arbeiter, die in der Lage waren, Papiere und Abrechnungen ihrer Arbeitgeber zu überprüfen würden Betrug und Missbrauch durch die Großgrundbesitzer schwieriger machen (Becker 1997: 296). Dieser erste Versuch einer selbstverwalteten Erziehung wirkt bis heute nach und hat sich zu einer der wichtigsten Forderungen und Projekte der Indigenenbewegung entwickelt. Ñucanchic Allpa berichtet, dass dieses Projekt nach einiger Zeit vom Staat und der Journalistengewerkschaft Unión Nacional de Periodistas begrüßt wurde. In einem Artikel wurde die Ausweitung auf alle staatlichen haciendas gefordert und eine genuin indigene Ausbildung gegen Einflussnahme des Staates - in Bezug auf die Unterrichtssprache und -inhalte - verteidigt 
(Becker 2006: 148). 1963 begann die Regierung, die indigenen Schulen zu regulieren und sie in die Institutionen des Bildungsministeriums $\mathrm{zu}$ integrieren (Lazos/Lenz 2004: 6).

Die Statuten der FEI sahen einen jährlichen Kongress vor, was sich wegen fehlender Ressourcen nicht durchhalten ließ. Der zweite Kongress der FEI orientierte sich noch an diesem Vorhaben und fand im Februar 1946 in Quito statt. Dolores Cacuango wurde die Nachfolgerin von Gualavisí als Präsidentin der Organisation. Auf dem Kongress wurde über die Aktivitäten der Organisation, Gesetze, die die Indigenen betrafen, wie das Kommunengesetz und das Arbeitsgesetz, und den Bericht des funktionellen Abgeordneten der Indigenen in der Nationalversammlung diskutiert. Es wurden die Durchführung von Weiterbildungskursen für die Mitglieder der FEI und weitere Aktionen beschlossen (Becker/Tutillo 2009: 148).

1949 findet der erste gemeinsame Streik von FEI und CTE statt, auf dem eine Agrarreform und eine Abschaffung des Großgrundbesitzes gefordert wird. Ein Gesetz, das die Indigenen zu unbezahlter Arbeit beim Bau von Straßen in den haciendas zwang, wird danach abgeschafft (Agualsaca o.J.: 3).

Die aktivste Zeit der FEI waren die 1950er und 1960er Jahre, in denen bereits die Krise der Organisation begann. Sie war fast ausschließlich in der Andenregion tätig, während die Föderation der Landarbeiter des Küstengebietes (Federación de Trabajadores Agrícolas del Litoral, FTAL) - eine weitere Organisation der CTE, 1954 gegründet - in der anderen großen Region Ecuadors Bedeutung hatte (Albornoz 1983: 70; CONAIE 1989: 32). Die FTAL wurde von Aktivisten der FEI wie Dolores Cacuango und Tránsito Amaguaña unterstützt, konnte aber wegen mangelnder Ressourcen keinen großen Einfluss erlangen (Becker/Tutillo 2009: 155). Dennoch spielte sie ab 1967 eine Rolle im Kampf für die Umsetzung der Agrarreform und ermöglichte so den Aufbau einer sozialen Bewegung auf dem Land (Guerrero 2010: 4).

Die FEI blieb weitgehend auf Cayambe beschränkt, den Einfluss, den sie in anderen Teilen Pichinchas und Chimborazos gewinnen konnte, verlor sie nach der ersten Agrarreform an FENOC und ECUARUNARI. Andere Provinzen wie Imbabura konnten - trotz einer vergleichbaren soziokulturellen Struktur wie Cayambe nie von der FEI erschlossen werden. Dies lag zum einen an der je nach Provinz unterschiedlichen Verbreitung der huasipungaje, zum anderen am Weiterbestehen einer starken ethnischen Identität um Otavalo. Das ethnische Selbstverständnis stand der Organisation in klassistischen Gewerkschaften also entgegen (Korovkin 1999: 10).

Die Beschränkung der FEI auf eine bestimmte Bevölkerungsgruppe in einer bestimmten Gegend erlaubte es ihr nicht, die Mehrheit der Indigenen zu organisieren. Dennoch hat sie eine gewisse Bedeutung entfalten können, wie ihr VI. Kongress 1952 mit über 400 Teilnehmern aus 6 Provinzen des Andenraumes beweist (Albor- 
noz 1976: 151). Ende 1961 organisiert die FEI einen Marsch von 12.000 (Guerrero 1983: 110) huasipungueros nach Quito, um für eine Agrarreform und eine Verbesserung ihrer Lebensbedingungen zu protestieren (Becker 1997: 298). Direkt im Anschluss an diesen Marsch fand der dritte Kongress der FEI statt, auf dem Miguel Lechón, ein Indigener aus Cayambe und Mitglied der PCE, zu ihrem Präsident gewählt wurde. Am Kongress nahmen auch der Präsident der CTE und der ecuadorianische Präsident, Carlos Julio Arosemena Monroy, teil (Becker 1997: 258f.). Arosemena, der nur einen Monat zuvor die Präsidentschaft von Velasco Ibarra übernommen hatte, führte mit einigen seiner Minister den Marsch in Quito an und versprach den Demonstranten eine Agrarreform und die Abschaffung der huasipungaje (Becker/Tutillo 2009: 205f.). Das Ziel des Kongresses war es, Leitlinien für eine Agrarreform zu formulieren, was durch eine Reihe von Märschen unterstützt wurde (Becker/Tutillo 2009: 203). Die Konflikte zwischen Ende der 1950er und Anfang der 1960er zeigten die programmatischen Beschränkungen der FEI. Kommunale Landkonflikte passten nicht in die Konzeption der FEI und konnten nicht angemessen aufgenommen werden (Botero 1998: 8). Der problematische Umgang mit einem Aufstand von 2.000 Indigenen in einer hacienda in Colta/Chimborazo 1961 (Yépez 2010) ist ein Beispiel dafür. Auch die Protestformen waren zu dieser Zeit eher konservativ, etwa gab es kaum Besetzungen umstrittenen Landes, wie es in anderen Ländern der Region üblich war (Albó 2009: 206). Diese fehlerhafte Strategie im Hinblick auf gewerkschaftliches Handeln sollte später zum Scheitern der FEI beitragen (Moreno/Figueroa 1992: 33f.).

$\mathrm{Zu}$ dieser Zeit beginnt die Krise der FEI (Albornoz 1983: 63). Bereits 1960 beschwerte sich der Generalsekretär der PCE, Pedro Saad, dass die FEI keine funktionierende Organisation mehr sei (Santana 1983: 159; Santana 1992: 86f.) und man sie umbilden und mit der FTAL vereinigen solle (Becker 2006: 149). Die neue Organisation sollte den Namen Nationale Bauern- und Indioföderation (Federación Nacional de Campesinos e Indios) tragen. Im Oktober 1960 organisierte die CTE als Muttergewerkschaft von FEI und FTAL die Erste Nationale Bauernkonferenz (Primera Conferencia Nacional Campesina) in Quito, an der 350 Abgeordnete von 130 Organisationen, in denen insgesamt 200.000 Bauern des Küsten- und Andengebietes vereinigt waren, teilnahmen. Es sollte die Gründung der neuen Organisation vorbereitet werden, wofür sich Anfang 1961 sowohl FEI als auch FTAL in eigenen Kongressen aussprechen sollten. Im Anschluss hätte der Gründungskongress der neuen Organisation stattgefunden. Dazu kam es jedoch nicht. Statt sich an die montubios des Küstengebietes anzunähern, begannen die Indigenen, ein stärkeres Bewusstsein ihrer ethnischen Eigenständigkeit zu entwickeln. Das bestimmte den Verlauf der indigenen Kämpfe in den 1960er Jahren, die sich zunehmend vom nationalen Gewerkschaftskampf weg und zu lokalen, ethnischen Kämpfen hin entwickelten (Becker/Tutillo 2009: 156f.). 
Tatsächlich bedeutete erst die Agrarreform von 1964, die das System der huasipungaje beendete, für die FEI den Verlust eines Großteils ihrer bisherigen Interessengruppen. Sie hatte seit ihrem Bestehen für die Landreform gekämpft und war an der Reform selbst beteiligt (Guamán 2006: 37), konnte jedoch ihre Forderungen kaum durchsetzen, so dass die Reform für die Indigenen ein Scheitern war, die Krise der FEI verschärfte und den Aufstieg anderer Organisationen begünstigte (Simbaña 2008a: 238). Es fand eine Verschiebung von klassenorientierten Organisationen $\mathrm{zu}$ ethnischen Organisationen statt, was sich in der Gründung wichtiger Organisationen ab Anfang der 1970er Jahre ausdrückte, die sich meist mehr an ethnischer Identität als an der Klassenlage ihrer Mitglieder orientierten - etwas, das sich auch in einer neuen Generation von Führern ausdrückte. Dennoch bauten die neuen Organisationen auf die Strukturen und Forderungen, die seit den 1920ern entstanden waren, auf - brachen also nicht vollständig mit ihren Vorläufern (Becker 1997: 310). Da der Kampf um Land ab 1964 über das Agrarreformgesetz und das Institut der Agrarreform IERAC lief, verlor die FEI ihren Handlungsspielraum und damit ihre Einflussmöglichkeiten (CONAIE 1989: 33). Mit der Abschaffung der huasipungaje wurde eine ihrer zentralen Forderungen erfüllt - und ihr Mobilisierungspotential entsprechend reduziert (Becker/Tutillo 2009: 225). Die FEI konzentrierte sich nun vor allem auf eine Analyse und Kritik des Gesetzes.

Schon seit den 1930ern war die Agrarreform ein permanentes Projekt des ecuadorianischen Staates. 1936 wurde das Brachland- und Kolonisierungsgesetz (Ley de tierras baldías y colonización) erlassen, um die Ungleichheit im Landbesitz über die Verteilung unproduktiven Landes zu lösen, ohne dadurch die bestehende haciendaStruktur in Frage zu stellen. Dieses Gesetz hatte nur geringe Folgen und konnte die Erwartungen nicht erfüllen. 1954 führte der ecuadorianische Staat einen Agrarzensus durch, der die Ungleichheit im Besitz und Zugang zu Land deutlich machte und den Staat unter Zugzwang stellte. 1957 schuf das Landwirtschaftsministerium das Nationale Kolonisierungsinstitut (Instituto Nacional de Colonización), das vor allem das unproduktive Land in den östlichen Anden verteilte. Zur gleichen Zeit begannen einige der hacendados, wohl wissend, dass eine Agrarreform kurz bevor stand, einen Teil ihrer Ländereien an die auf ihnen lebenden Indigenen zu verteilen (Becker/Tutillo 2009: 210f.). Mit der kubanischen Revolution von 1959 und der daran anschließenden Agrarreform und Welle von Verstaatlichungen wurde der Druck auf die lateinamerikanischen Staaten weiter erhöht. Das von John F. Kennedy angestoßene Programm Allianz für den Fortschritt wurde 1961 auf einem Treffen der Organisation amerikanischer Staaten in Punta del Este, Uruguay, vorgestellt. Weitere Revolutionen in der Region sollten über technische und wirtschaftliche Zusammenarbeit und landwirtschaftliche Entwicklungsprojekte verhindert werden. Dazu gehörten Agrarreformen, ein Mittel, das zwischen 1960 und 1964 von elf Ländern der Region angewendet wurde (Becker/Tutillo 2009: 212). 
Die Agrarreform sollte einer Neuordnung der landwirtschaftlichen Produktion und einer Integration der Bauern in die Wirtschaft dienen. Am 11. Juli 1964 erließ die regierende Militärjunta das Agrarreform- und Kolonisierungsgesetz (Ley de Reforma Agraria y Colonización), das das Ecuadorianische Institut für Agrarreform und Kolonisierung (Instituto Ecuatoriano de Reforma Agraria y Colonización, IERAC) schuf. Mit diesem Schritt wollte die Regierung die prekäre Situation auf dem Land durch den Aufbau einer Schicht von Besitzern mittelgroßer Ländereien als Puffer zwischen den Großgrundbesitzern und Kleinbauern entschärfen. Dazu wurde den bisherigen huasipungueros Land gegeben, dass in der Praxis allerdings meist nur das Land war, das sie ohnehin bewirtschafteten. So wurden die Interessen der Großgrundbesitzer bedient und nur die Zahl der Kleinbauern erhöht und damit die Migration in die Städte verschärft. Diese ,agrotechnische kapitalistische Reform“ (CONAIE 1994: 31) war auf die Interessen der Investoren und nicht auf die der Indigenen und Bauern ausgerichtet. Die zweite Phase der Agrarreform, mit einem neuen Agrarreformgesetz, wurde ebenfalls von einer Militärjunta gesteuert. Über eine weitere Verteilung des Landes nach neuen Richtlinien sollte der Einfluss des Staates auf die landwirtschaftliche Produktion erhöht werden. Dazu wurden vor allem die als unproduktiv betrachteten Teile der haciendas an Kleinbauern, indigene Gemeinden und neue Siedler verteilt. Die von den haciendas gebundenen Arbeitskräfte sollten so dezentraler verteilt werden (Botero 2000: 42).

Nach dem Ende der Militärdiktatur, die von 1963 bis 1966 herrschte, bemühten sich die indigenen Aktivisten um eine Reorganisation der FEI. So wurde im Oktober 1966 der vierte Kongress der FEI in Quito mit 350 Abgeordneten durchgeführt. Wie schon seit der Gründung der FEI spielten die Abgeordneten aus Cayambe eine besondere Rolle. Von ihnen ging der Impuls aus, für eine Agrarreform zu kämpfen, die auf die Bedürfnisse der Landbevölkerung ausgerichtet ist (Becker/Tutillo 2009: 226). Miguel Lechón wurde als Präsident der Organisation bestätigt (Becker/Tutillo 2009: 225). Ein wichtiger Bestandteil des Versuchs des Neuaufbaus der FEI war die Wiederbelebung von Ñucanchic Allpa ab April 1968 (Becker/Tutillo 2009: 227). Diese beschäftigte sich in ihren letzten Ausgaben vor allem mit der Durchführung der Agrarreform und der Kritik daran (Becker 2006: 149).

Die Landreform, ihr Scheitern und der Kampf für eine weitere Reform bestimmten die Politik der FEI bis 1973, dem Jahr, in dem - auch wegen den Forderungen von FEI und FENOC - die zweite Landreform stattfand (Guamán 2003: 31). Der Kampf für eine „demokratische Agrarreform“ (Lechón 1976: 91f.) unter Beteiligung der Landarbeiter war mit dieser Reform gescheitert und die FEI verlor einen Großteil ihres Mobilisierungspotentials (Santana 1992: 86, Rodríguez 2008: 94). Seit der zweiten Agrarreform akzentuierte sich in der FEI das Verständnis der Indigenen als „Nation mit ihrer eigenen Sprache, Kultur, Gebräuchen, Traditionen, Territorium, Wirtschaft usw.“ (Lechón 1976: 85f.), die von fremden Kulturen im 
Rahmen von Kolonialismus, Kapitalismus und Imperialismus unterdrückt und ausgebeutet werden. Daher verstand sie sich als nationale Befreiungsbewegung einer diskriminierten und ausgebeuteten nationalen Minderheit, die zusammen mit der internationalen Arbeiterbewegung und den sozialistischen Staaten für eine bessere Menschheit kämpft (Lechón 1976: 87f.). Sie verlor an Einfluss bei den Landarbeitern und Indigenen und stürzte in eine Krise, verbunden mit einem Verlust ihrer Stellung innerhalb der PCE (Rodríguez 2008: 87). Die FEI konnte durch ihre Orientierung auf ländliche Entwicklung (Botero 1998: 8) nur unter den wenigen Begünstigten der Agrarreform einige Mitglieder halten und verlor ansonsten fast jede Repräsentativität zugunsten erst der FENOC und später ECUARUNARI (Santana 1983: 159; Kaltmeier 2009: 344). Ein Ergebnis dieser Orientierung ist die Übertragung von haciendas auf Kooperativen, die der FEI angehören. Das ist der Fall in La Chimba, Pesillo, Mollurco, Pisambillo, Chaupimuyuco, Santo Domingo (Escobar 1983: 48).

Die Aktionen der Muttergewerkschaft CTE treten in den Vordergrund. Ab Oktober 1969 bemüht sie sich um die Gründung einer einheitlichen Gewerkschaftsfront der größten Gewerkschaften woraus die Ecuadorianische Arbeiterkraft (Fuerza Laboral Ecuatoriana, FLE) entsteht, die im Juni 1971 zum Kern der Einheitsfront der Arbeiter (Frente Unitario de los Trabajadores, FUT) wird (CTE o.J.: 7). Diese umfasste ab 1973 die drei größten Gewerkschaften, neben der CTE selbst, die CEDOC und die CEOSL (Paz y Miño 2009: 34). Die FUT bestimmte die Arbeitskämpfe zwischen Mitte der 1970er und Mitte der 1980er Jahre (Becker 2009a: 1058). Insbesondere zu Beginn der 1980er organisierte die FUT eine Reihe nationaler Streiks, um für wirtschaftliche und soziale Rechte und die nationale Eigenständigkeit zu kämpfen. Der Regierungsantritt von Febres Cordero 1984 bedeutete wegen seines harten Durchgreifens einen erheblichen Bedeutungsverlust aller Gewerkschaften (Paz y Miño 2009: 37).

Die Strategie der FEI, sowohl für die Rechte der Indigenen als auch für deren Politisierung und Mobilisierung zu kämpfen, war zumindest in dem Sinne erfolgreich, dass sie über ihre Bildungsarbeit und Schaffung eines gemeinsamen Bewusstseins den Boden für andere Organisationen auf lokaler Ebene geebnet haben (Albornoz 1976: 151; Botero 1998: 8f.) - eine Leistung, die auch ECUARUNARI und CONAIE anerkennen (ECUARUNARI/CONAIE 1989: 40). Im Kampf für eine Agrarreform spielte die FEI eine wichtige Rolle, auch wenn ihre Bemühungen letztendlich gescheitert sind (CONAIE 1989: 276). Dennoch konnten andere Organisationen an die Arbeit der FEI anknüpfen.

$\mathrm{Zu}$ Beginn der 1980er Jahre konnte die FEI kaum Unterstützung außerhalb der CTE und der PCE mobilisieren (Santana 1983: 158). Seit dieser Zeit war die FEI nur noch zu Aktionen in Kooperation mit anderen Organisationen fähig, was zu einem Strategiewechsel und einer Modernisierung in der FEI führte (Agualsaca o.J.: 
3; Becker/Tutillo 2009: 228). Ihre Rolle als Vermittler zwischen dem Ecuadorianischen Institut für Agrarreform (IERAC) und den Gemeinden verhalf ihr zu einer gewissen Stabilisierung - etwa 1980-1981 in den Gemeinden um die Kanäle Guanguilquí und Porotog. Sie konnte das allerdings nicht ausbauen, auch wegen ihrem radikalen und konflikthaften Politikstil, der oft zu direkten Auseinandersetzungen innerhalb der Gemeinden führte. Diese von manchen Gemeinden als nicht zielführend empfundene Stil (Perreault/Bebbington/Carroll 1998: 8) begünstigte andere indigene Organisationen, die eher auf Verhandlung setzten.

Die FEI nahm an den indigenen Erhebungen 1990, 1992 und 1994 teil und anschließend zusammen mit FENOCIN, CONAIE und FENACLE an der Gründung der Nationalen Agrar-Koordination (Coordinadora Nacional Agraria, CAN). Die CAN sollte den Widerstand gegen das Landentwicklungsgesetz der Regierung Durán Ballén organisieren, das eine Integration des gemeinschaftlichen Besitzes der indigenen Gemeinschaften in den Markt zum Ziel hatte (Agualsaca o.J.: 3; Yépez 2010).

Anschließend hat die FEI fast jede Aktivität eingestellt. Dennoch besteht sie noch und verfügt weiterhin über einige Strukturen in wenigen Provinzen (Botero 1998: 9). Programmatisch ist der Einfluss der CONAIE kaum zu übersehen. So gehört zu den Zielen der Organisation die Orientierung der Indigenenbewegung hin auf eine Suche nach sozialer Gleichheit bei Respekt der kulturellen Unterschiede. Identitären Forderungen wird große Bedeutung zugemessen (FEI o.J.). Im Jahr 2002 hat die FEI die Bezeichnung indios in ihrem Namen durch indígenas ersetzt (Sánchez-Parga 2009: 97). Sie arbeitet für eine ,breite Klasseneinheit aus interkultureller Perspektive“ (Agualsaca o.J.: 5), wobei der „revolutionäre Klassenkampf“ (Agualsaca o.J.: 3) in einer Kooperation zwischen Bauern und Arbeitern gestärkt werden soll. Die Selbstbeschreibung auf den Internetseiten der CTE ist wahrscheinlich übertrieben: „Im Jahr 2006 ist die FEI eine Organisation mit organischer Präsenz in allen Provinzen Ecuadors; sie bietet politische Ideen, die das nationale Tagesgeschäft beeinflussen.“ (FEI o.J.) Nach einer Schätzung des CODENPE von 2007 verfügt die FEI über zwei OZG in den Provinzen Chimborazo und Esmeraldas und insgesamt 5.655 Mitglieder (IEE o.J.) ${ }^{13}$.

Die FEI ist Mitglied in der CLOC, der lateinamerikanischen Filiale der Vía Campesina. Ende 2010 tauchte eine neue Webseite der FEI auf (http://www.confe deracionfei.com/), die nur ein Jahr lang online war. Auf dieser Seite bezeichnet sich die FEI als Konföderation der indigenen bäuerlichen Völker und Organisationen Ecuadors (Confederación de Pueblos y Organizaciones Indígenas Campesinas del

13 Nach einer persönlichen Kommunikation von Pablo Ospina. Die Schätzungen beziehen sich auf die OZG. 
Ecuador ${ }^{14}$ und bekundet ihre Unterstützung der Regierung Correas. Sie steht weiterhin der PCE nahe und hat ihren Hauptsitz im Gebäude des Landwirtschaftsministeriums - was Zweifel an ihrer Eigenständigkeit erlaubt.

\begin{tabular}{|c|c|c|c|c|}
\hline Jahr & Nummer des Kongresses & Teilnehmer & Ort & $\begin{array}{l}\text { Gewählter } \\
\text { Präsident }\end{array}$ \\
\hline 1944 & $\begin{array}{l}\text { Primer Congreso } \\
\text { Ecuatoriano de Indígenas }\end{array}$ & & Quito & Jesús Gualavisí \\
\hline 1946 & $\begin{array}{l}\text { Segundo Congreso } \\
\text { de Indios Ecuatorianos }\end{array}$ & & Quito & Dolores Cacuango \\
\hline 1950 & $\begin{array}{l}\text { Außerordentlicher } \\
\text { Kongress }\end{array}$ & & & \\
\hline 1952 & VI. Kongress & 400 & & \\
\hline 1961 & 3. Kongress & & Quito & Miguel Lechón \\
\hline 1966 & 4. Kongress & 350 & Quito & Wiederwahl \\
\hline 1971 & 5. Kongress & & Quito & Manuel Escobar \\
\hline 1989 & VII. Congreso FEI & & Quito & \\
\hline 1995 & VIII. Congreso Ordinario & & Riobamba & \\
\hline 2010 & $\begin{array}{l}\text { XI. Congreso nacional ordi- } \\
\text { nario ,Tránsito Amaguaña“ }\end{array}$ & & Quito & José Agualsaca \\
\hline
\end{tabular}

Tabelle 1: Kongresse der FEI, eigene Abbildung

\section{FENOCIN}

\section{a. Gründung}

Im September 1938 wurde auf Betreiben der katholischen Kirche und der konservativen Partei der erste katholische Arbeiterkongress durchgeführt (Becker 1997: 224f.), um den Einfluss der linken Parteien auf die Arbeiter zu begrenzen (CONAIE 1989: 32). Dort wurde die Ecuadorianische Konföderation Katholischer Arbeiter

14 Der Präsident der FEI, Agualsaca (o.J.: 1), nennt die FEI in einem Text aus den frühen 2000er Jahren Konföderation der bäuerlichen indigenen Völker Ecuadors (Confederación de Pueblos Indígenas Campesinos del Ecuador). 
(Confederación Ecuatoriana de Obreros Católicos, CEDOC) ${ }^{15}$ gegründet, die erste gesamtecuadorianische Gewerkschaft. Ihre Basis waren - fast ausschließlich Handwerker und städtische Arbeiter (Albó 2009: 207f.), ihre Politik mehr auf individuelle Moral als auf die Analyse struktureller Faktoren gerichtet - es ging um gegenseitige Hilfe und individuelle Organisation statt um den Klassenkampf (Becker 2009a: 1057). Obwohl die CEDOC als konservative, pro-katholische und antisozialistische Organisation konzipiert worden war $^{16}$, waren in ihrem Denken auch progressive Elemente zu finden. Sie forderte etwa einen 8-Stunden-Arbeitstag, eineinhalb arbeitsfreie Tage pro Woche, einen Mindestlohn und eine Unfallversicherung - Forderungen, die auch die kommunistischen und sozialistischen Gruppen aufstellten. Daher konnte sie schnell an Bedeutung gewinnen und zur Entwicklung von nichtstaatlichen Organisationen in Ecuador beitragen (Becker 1997: 224f.).

Auf ihrem fünften Kongress 1955 beschließt die CEDOC, sich mehr auf Industrie- und Landarbeiter zu konzentrieren (Becker 1997: 224f.). Im Anschluss beginnt eine stärker werdende christdemokratische Strömung die klerikal-konservative Strömung, die die CEDOC zu Beginn prägte, zu verdrängen. Die CEDOC öffnete sich den Ideen eines christlichen Gewerkschaftswesen, die sich auf die päpstlichen Enzykliken stützten (FENOCIN 1999: 23). Diese Modernisierung ist verbunden mit dem steigenden Einfluss der Katholischen Jugend und einer bedeutenden Unterstützung durch die Konrad-Adenauer-Stiftung (León/Ycaza 1989: 190) vor allem zwischen 1965 und 1975 (CEDOC 1976: 19). Die CEDOC näherte sich im Land den christdemokratischen Parteien und auf kontinentaler Ebene der CLAT an (CEDOC 1976: 25). Auch durch diesen programmatischen Wandel konnte die CEDOC in den 1960ern ihre Aktivitäten auf dem Land verstärken und dort einen großen Mitgliederzuwachs verzeichnen (León/Ycaza 1989: 191). Die Organisation verstand sich zu dieser Zeit weniger als soziale Bewegung denn als großer Dienstleister und wollte die Landarbeiter durch das Bereitstellen von Rechtsberatung, Erziehung, technischer Unterstützung und Krediten in die Volkswirtschaft integrieren, um so die Entwicklung des Landes zu beschleunigen (CEDOC 1976: 17f.). In dieser Zeit

15 Die CEDOC änderte mehrfach ihren Namen. So hieß sie ab 1957 Ecuadorianische Konföderation der katholischen Arbeiter, Angestellten und Handwerker (Confederación Ecuatoriana de Obreros, Empleados y Artesanos Católicos), ab 1965 Ecuadorianische Konföderation christlicher Gewerkschafts-organisationen (Confederación Ecuatoriana de Organizaciones Sindicales Cristianas) und ab 1972 Ecuadorianische Zentrale klassistischer Organisationen (Central Ecuatoriana de Organizaciones Clasistas) (León/Ycaza 1989: 186).

16 Für einige Zeit war die CEDOC unter der Kontrolle der offen faschistischen Nationalrevolutionären Ecuadorianischen Aktion (Acción Revolucionaria Nacional Ecuatoriana, ARNE) (León/Ycaza 1989: 186). 
gründete die CEDOC das Ecuadorianische Institut für die soziale Entwicklung (Instituto Ecuatoriano para el Desarrollo Social, INEDES), das seinerseits 1968 die Ecuadorianische Zentrale für Landdienstleistungen (Central Ecuatoriana de Servicios Agrícolas, CESA) gründen sollte (CEDOC 1976: 19f.), die in der Entwicklung der indigenen Organisationen eine wichtige Rolle spielen wird.

1965 wurde die Föderation der Landwirtschaftlichen Arbeiter (Federación de Trabajadores Agropecuarios, FETAP) gegründet (Santana 1983: 159) - auch, um den Einfluss der kommunistisch dominierten Organisationen auf dem Land einzudämmen (Becker 1997: 224f.). Dies war ebenso eine Reaktion auf das erste Agrarreformgesetz von 1964 und geschah aus dem Kampf gegen die huasipungaje und das System der haciendas heraus. Auf dem Gründungskongress der FETAP im März 1965 waren Vertreter von 26 Basisorganisationen anwesend (FENOCIN 2004: 7). Die FETAP war als eine Alternative zur kommunistischen FEI gedacht und sollte der Vermittlung der Forderungen der Landbevölkerung an die Regierung dienen. Sie forderte eine Revision des Agrarreformgesetzes, eine Verteilung des Landes der haciendas, und eine Regulierung des Landverleihs für den Reisanbau (Rodríguez 2008: 87f. ${ }^{17}$. Dabei vertrat sie eine konservative Politik und hielt sich streng an die Regeln des Landreforminstituts IERAC (FENOC o.J.: 19). Eine Reihe von OEG, die zuvor der FEI angehörten, traten der FETAP und später der FENOC bei, von der sie - stärker als von der FEI - Unterstützung bei den Verhandlungen mit den Großgrundbesitzern bekamen, bis hin zu anwaltlicher Betreuung. So konnten sie über das Landgesetz (Ley de Tierras) ungenutztes Land zu verbilligten Preisen erwerben (Perreault/Bebbington/Carroll 1998: 8). Daher erlebte die FETAP ein rasches Wachstum. Bereits Ende 1965 waren in ihr 10 Landgewerkschaften und 73 Assoziationen, die noch nicht rechtlich anerkannt waren, organisiert (FENOC o.J.: 17).

Auf ihrem zweiten Kongress im April und Mai 1966 waren bereits Delegierte von 38 Basisorganisationen anwesend. $\mathrm{Zu}$ Beginn dieses Kongresses, am 29.4.1966, wurde die FETAP rechtlich anerkannt (FENOC o.J.: 17). Im selben Jahr gründeten CEDOC, CTE und einige kleinere Gewerkschaften die Front der klassistischen Einheit (Frente de Unidad Clasista, FUC) als gemeinschaftliche Plattform (FENOCIN 2004: 13), die eine wichtige Rolle beim Kampf gegen die damals herrschende Militärregierung und dem anschließenden Wiederaufbau der Gewerkschaftsbewegung spielte (León/Ycaza 1989: 191; Ycaza 1991). Sie war auch für den Gründungsprozess der FETAP bedeutend.

17 Die Behauptung von Rodríguez, die FETAP sei schon von Beginn an an die PSE gebunden gewesen, ist nicht haltbar. Diese Verbindung entsteht erst in den 1970ern, wie später erläutert wird. 
1967 konnte sich die FETAP auf das Küstengebiet ausweiten, vor allem auf die Provinzen Los Ríos mit 44 Organisationen und Guayas mit 7 Organisationen (FENOC o.J.: 18).

Auf dem dritten Kongress der FETAP in Quito im November 1968, an dem 200 Vertreter der Basisorganisationen aus 8 Provinzen (Rodríguez 2008: 87f.; FENOC o.J.: 18) teilnahmen, wurde - neben inhaltlichen Punkten - die Umbenennung in Nationale Föderation der Bauernorganisationen (Federación Nacional de Organizaciones Campesinas, FENOC) beschlossen (FENOCIN 2004: 8; FENOCIN o.J.: 2).

\section{b. Entwicklung}

Die FENOC wurde zur Repräsentantin der Landbevölkerung im Kampf für die Agrarreform (Minkner-Brünjer 2009: 138). Dadurch erlebte sie ein schnelles Wachstum und eine Erweiterung ihrer Strukturen und Mitgliedsorganisationen im ganzen Land (Tamayo 1996: 89f.). Gerade im Küstengebiet konnte sie Mitglieder gewinnen (Santana 1992: 86) - etwas, das der FEI nie gelungen war. Sie unterschied sich von der FEI auch durch ihre Konzentration auf Landarbeiter und Bauern, ohne dabei ethnische Zugehörigkeit zu einem Kriterium zu machen - wodurch sie schon zu ihren katholisch-konservativen Zeiten eine klassistische Organisation war (Albó 2009: 208).

Die FENOC kämpfte gegen Velasco Ibarra, der sich im Juni 1970 zum Diktator erklärt und 1972 vom Militär gestürzt wird. In diesem Kontext findet der zweite Kongress der FENOC in Riobamba im Oktober 1971 statt (FENOCIN 2004: 9). Auch die Gewerkschaftsbewegung konsolidierte sich. Um die Arbeitskämpfe zu koordinieren, gründeten CTE, CEDOC und einige kleinere Gewerkschaften 1971 die Einheitsfront der Arbeiter (Frente Unitario de Trabajadores, FUT), der später auch die CEOSL beitrat (León/Ycaza 1989: 191, Ycaza 1991). Ein Jahr darauf, im Juni 1972, fand in Quito die Erste Lateinamerikanische Bauernkonferenz über die Agrarreform (Primera Conferencia Latinoamericana Campesina sobre la Reforma Agraria) statt (FENOCIN 2004: 9), die den Beginn der Suche der FENOC nach internationalen Bündnissen markierte. Sie wurde von der Lateinamerikanischen Bauernföderation (Federación Campesina Latinoamericana, FCL) und ihrer Oberorganisation Lateinamerikanische Arbeiterzentrale (Central Latinoamericana de Trabajadores, CLAT) durchgeführt, Organisationen, denen FENOC und CEDOC angehörten (FENOC o.J.: 21). Das starke Wachstum der FENOC führte zu einem Konflikt zwischen einer konservativen und einer christdemokratischen Gruppe in den Jahren 1972 und 1973, bei dem sich die christdemokratische Tendenz durchsetzen konnte. Als direkte Folge verließen einige Organisationen die FENOC zuguns- 
ten der neu entstandenen ECUARUNARI (FENOCIN 2004: 10), die zu diesem Zeitpunkt kirchlich geprägt war (ECUARUNARI o. J.: 2).

Der dritte Kongress der FENOC im Juni $1973^{18}$ - die FENOC war bereits unter Kontrolle der Christdemokraten (FENOCIN 1999: 27) - bringt programmatische Neuerungen. Aus einem Studium der ländlichen Probleme wird die Unterstützung der kleinen Grundbesitzer, der minifundistas, der Landarbeiter und der Bauern in ihrem Kampf für bessere Löhne und leichteren Zugang zu Land, Wasser und günstigen Krediten abgeleitet. Außerdem wurde eine direkte Teilhabe der Bauernorganisationen an einer neuen Agrarreform gefordert (FENOCIN 2004: 10). Die kämpferischen Gruppen, vor allem aus dem Küstengebiet, konsolidieren ihre Position und beginnen, Druck auf die CEDOC auszuüben (CEDOC 1976: 24). Die FENOC verstärkte ihre Aktionen - etwa Landbesetzungen (Guamán 2003: 31) - gegen Großgrundbesitzer und forderte mit Protesten eine neue Agrarreform. Am 27. Juni 1973 finden Demonstrationen von 15.000 Bauern in Cañar, 50.000 in Guayaquil und 5.000 in Quito statt (FENOC o.J.: 23). In diesem Zusammenhang wurde, zusammen mit anderen Bauern- und Indigenenorganisationen wie der FEI und ECUARUNARI, die Einheitsfront der Agrarreform (Frente Unido de Reforma Agraria, FURA) gegründet (Chiriboga 1986: 86) und das erste Nationale Bauerntreffen für die Agrarreform (Encuentro Nacional Campesino por la Reforma Agraria) in Quito im August 1973 organisiert (FENOC o.J.: 23; FENOCIN 2004: 10f.; Albó 2009: 209). Die dort aufgestellten Forderungen wurden auf dem zweiten Nationalen Bauerntreffen in Balzar, Guayas, im Oktober 1973 weiter konkretisiert (FENOCIN 2004: 11). Vor allem FENOC und ECUARUNARI organisierten große Demonstrationen und übten Druck auf den Staat aus (Chiriboga 1986: 68). Gefordert wurde eine weitreichende Agrarreform unter Mitwirkung der verschiedenen Organisationen. Die beiden FENOC-Mitgliedsorganisationen aus dem Amazonasgebiet, FOIN und Federación Shuar, kritisieren die Siedlungspolitik in ihren Regionen (FENOCIN 1999: 32). Wenige Tage später, noch im Oktober 1973 wurde ein neues Agrarreformgesetz verabschiedet, dass die Interessen der Großgrundbesitzer bewahrt. Als Reaktion darauf wurde auf dem dritten Nationalen Bauerntreffen in Sucúa, Morona Santiago, im November 1973 eine neue Siedlungspolitik auf dem Land vorgeschlagen (FENOCIN 2004: 11).

Der Kampf für eine Agrarreform unter Mitwirkung der Bauernorganisationen führt zu einem politischen Wandel in der FENOC. Sie versteht sich jetzt als revolutionäre Führerin der Bauernbewegung und konkretisiert ihre Klassenanalyse weiter (FENOCIN 2004: 11). Ihre Aktionen erlauben der FENOC, ihre Struktur auf fast

18 Auf diesem Kongress wird zum ersten Mal ein Vertreter der Föderation Indigener Organisationen Napos (Federación de Organizaciones Indígenas del Napo, FOIN) in die Nationaldirektion der FENOC gewählt (FENOCIN 1999: 29). 
das ganze Land auszuweiten. Es werden neue lokale und regionale Organisationen gegründet, die innerhalb der FENOC an Bedeutung gewinnen (FENOCIN 2004: 12). Vor allem Kommunen und Kooperativen werden wichtiger als die klassischen Gewerkschaften (Simbaña I-2) ${ }^{19}$. Sie wurde zur Vermittlerin zwischen Bauernbewegung und Staat (Büschges 2009: 54). Diese organisatorische und politische Entwicklung führte zu weiteren Auseinandersetzungen um die Richtung der Organisation.

Ein Treffen des Nationalrates der FENOC in Quito im Oktober 1974 wurde im Nachhinein zum IV. Kongress der Organisation erklärt (FENOC o.J.: 24f.). Dieser Kongress führt zu einer Spaltung der FENOC und etwas später zum Aufbau einer christdemokratisch geprägten FENOC-CLAT (FENOCIN 1999: 34), die nur wenig Einfluss erlangen konnte und nicht lange bestand.

Zwischen 1974 und 1975 wurden in der CEDOC (León 1994: 72f.) die christdemokratischen Gruppen von sozialistischen Gruppen verdrängt, die der PSE nahestanden (Ospina 2008: 71; Barrera o.J.: 151). Das führte zur Spaltung der CEDOC ${ }^{20}$ auf ihrem XI. Kongress in Portoviejo 1975 in eine sozialistische und eine christdemokratische Gruppe, die später den Namen CEDOC-CLAT annahm (CEDOC 1976: 25; León/Ycaza 1989: 189). Die Präsidentschaft von Jorge Cuisana ${ }^{21}$ in der CEDOC von April 1975 bis Mai 1976 war von den internen Konflikten der Organisation und einem letzten Umsturzversuch durch die christdemokratischen Gruppen geprägt, dessen Scheitern zum Aufbau der CEDOC-CLAT beitrug (CEDOC 1976: 27). Die rebellierenden Gruppen wurden aus der CEDOC ausgeschlossen und die sozialistische Führung konnte sich konsolidieren. Das führte auch zu einer Stärkung dieser Tendenzen innerhalb der FENOC (FENOCIN 1999: 38). Die ausgeschlossenen Gruppen erhielten weiterhin Unterstützung von der CLAT und der KonradAdenauer-Stiftung (CEDOC 1976: 33). Dieser Prozess war 1977 beendet (de la Torre 2005: 33). Der Kampf für eine Verteilung des Landes verstärkte sich (Botero 1998: 8), die Agrarreform blieb aber ab 1975 stecken (Minkner-Brünjer 2009: 139) - was zu einer Schwächung der FURA führte (Chiriboga 1986: 86).

Seit 1974 war es vermehrt zu Kooperationen zwischen den großen Gewerkschaftsverbänden gekommen, die sich zunehmend politisch annäherten und begannen, gemeinsam Streiks durchzuführen. Die bislang größte gemeinsame Aktion fand am 1. Mai 1975 statt und führte wenige Monate später zum Aufbau einer ge-

19 Die mit Simbaña I-1 und Simbaña I-2 bezeichneten Quellen sind zwei längere Interviews. Die genaueren Daten sind unter „Sonstige Quellen“ verzeichnet.

20 Die CEDOC heißt nach einer Abspaltung und Wiedervereinigung jetzt CEDOCUT. Die FENOCIN gehört ihr weiterhin an (Tamayo 1996: 89f.).

21 Der auf dem Kongress 1975 gewählten Exekutivkommission der CEDOC gehörte mit Ernesto Tseremp ein Vertreter der Federación Shuar an (CEDOC 1976: 26). 
meinsamen Plattform. Am 20. August 1975 (Dávila 1995: 60f.) gründeten CTE, CEDOC und CEOSL die Klassistische Einheitsfront (Frente de Unidad Clasista, FUC) (FENOC o.J.: 27), ein Ausdruck der politischen Verschiebung innerhalb der Gewerkschaftsbewegung. Von diesem Moment an, nahm die Gewerkschaftsbewegung eine Avantgardeposition unter den sozialen Bewegungen Ecuadors ein, die sie bis Mitte der 1980er Jahre behalten sollte (Dávila 1995: 56).

Im Mai 1977 wurde der FENOC wegen ihrer Opposition gegen die Militärdiktatur ihr rechtlicher Status aberkannt. Dennoch fand im Oktober desselben Jahres in Quito der fünfte Kongress der FENOC statt, an dem mehr als 400 Vertreter der Basisorganisationen teilnahmen (FENOCIN 2004: 14). Auf diesem Kongress setzt sich die sozialistische Strömung innerhalb der FENOC endgültig durch (FENOCIN 1999: 39). Das bedeutete nicht eine Annäherung an die PSE, sondern bezieht sich vielmehr auf die Selbstbeschreibung einiger Führungspersonen der FENOC. Tatsächlich stand die Organisation der heute verschwundenen Revolutionären Bewegung der Arbeiter (Movimiento Revolucionario de los Trabajadores, MRT) nahe, die keine klare politische Linie definieren konnte (FENOCIN 1999: 37). Die neue Führung widmete sich vor allem der Konsolidierung der verschiedenen regionalen Mitgliedsorganisationen, wodurch die FENOC schließlich 21 OZG mit 1.000 OEG umfasste. Gleichzeitig konzentrierte sie sich weitgehend auf die Landfrage und vernachlässigte andere Forderungen (FENOCIN 1999: 40). Die FENOC umfasste $\mathrm{zu}$ dieser Zeit Organisationen von kleinen Grundbesitzern und Landarbeitern des Küstengebietes und der Andenregion (FENOCIN 2004: 15). Allerdings führte die politische Verschiebung zum Verlust einiger Mitgliedsorganisationen, darunter die Föderation indigener Organisationen Napos (Federación de Organizaciones Indígenas del Napo, FOIN), die 1980 die FENOC verließ (Tamayo 1996: 64; CONAIE 1989: 50) und die Interprovinzielle Föderation der Shuar- und Achuar-Zentren (Federación Interprovincial de Centros Shuar y Achuar, FICSHA) (CEDOC 1976: 26). Beide Organisationen nahmen 1980 an der Gründung der politisch anders ausgerichteten Konföderation der indigenen Nationalitäten des ecuadorianischen Amazonasgebietes (Confederación de Nacionalidades Indígenas de la Amazonía Ecuatoriana, CONFENIAE) teil (CONAIE 1989: 50).

Ab 1978 bemühte sich die FENOC um Bündnisse im Kampf für eine Umsetzung der Agrarreform. Sie gründete zusammen mit der FEI und ECUARUNARI die Einheitsfront des Bauernkampfes (Frente Único de Lucha Campesina, FULC) (FENOCIN 2004: 15; Albó 2009: 209), die später einige Organisationen des Amazonasgebietes einschloss und sich Einheitsfront des Bauern- und Indigenenkampfes (Frente Único de Lucha Campesina e Indígena, FULCI) nannte (FENOCIN 2004: 17). Diese Organisation sollte nicht lange bestehen und konnte kaum Aktivitäten entfalten (Chiriboga 1986: 86). Dennoch waren die späten 1970er Jahre von gemeinsamen Aktionen der FENOC mit der ECUARUNARI geprägt, die auch zu 
einem gemeinsamen Programm jenseits des Kampfes für eine Agrarreform führte. So verständigten sich die beiden Organisationen auf Forderungen nach kollektiven Rechten, Bildung und Demokratie. Die FENOC nahm zu Beginn auch an den Koordinationsbemühungen der Indigenenorganisationen teil, die schließlich in der Gründung der CONAIE münden sollte (Ayala Mora 2011: 8).

1978 begann die FENOC, regelmäßig das Mitteilungsblatt ,Die FENOC informiert' (,La FENOC informa') zu publizieren, aus der später die Zeitung ,Bauernkampf' (,Lucha Campesina`) werden sollte (FENOC o.J.: 30). Seit November 2011 heißt diese Zeitung, Vom Land`(,Del Campo ').

Im März 1979 erließ die Militärregierung auf Druck der Großgrundbesitzer das Gesetz der landwirtschaftlichen Förderung und Entwicklung (Ley de Fomento y Desarrollo Agropecuario), das die wirtschaftliche Unterstützung auf die bereits bestehenden Betriebe konzentrierte und die Bauernbewegung mit Strafandrohungen zu befrieden suchte (FENOC o.J.: 8). Das Scheitern der Agrarreform führte die FENOC in eine Krise (Santana 1992: 86), auf die sie mit einer verstärkten Suche nach Bündnissen reagierte. Trotz einer Unterstützung durch verschiedene Organisationen, wie etwa die Ecuadorianische Zentrale der Land-Dienstleistungen (Central Ecuatoriana de Servicios Agrícolas, CESA) oder die katholische Kirche, konnte sie sich nur mit Mühe behaupten und kaum neue Ideen entwickeln (Santana 1983: 159). Die FENOC verlor im Hochland an Einfluss, was auf das mangelnde Mobilisierungspotential der sozialistischen Führung der Organisation in den indigenen Gemeinden und eine allgemeinen Krise der politischen Linken durch den Druck der Militärregierung in den 1970ern zurückgeführt werden kann (Black 1999: 27). Auch die kritische Teilnahme an der Alphabetisierungskampagne der Regierung Roldós (1979-1981), wo sie zusammen mit ECUARUNARI didaktisches Material entwickelte (Moya 1987: 400), bedeutete mehr eine Bindung des engagierten Personals als eine Öffnung für andere Inhalte. In diesem Kontext tritt die FENOC 1978 der Sammlungsbewegung Breite Linksfront (Frente Amplio de Izquierda, FADI) bei, einem Wahlbündnis, das bis 1986 besteht, als die FENOC ein Bündnis mit der PSE eingeht (Van Cott 2005: 104f.). In diesem Zusammenhang stellte sie in verschiedenen Provinzen eigene Kandidaten auf (FENOCIN 2004: 15). Die FADI war eine Plattform von verschiedenen linken Parteien, die größten unter ihnen die prosowjetische Kommunistische Partei (PCE) und die pro-kubanische Revolutionäre Sozialistische Partei (PSRE), die sich 1962 von der PSE abgespaltet hatte (Hanratty 1989; Maugé 2008: 117; Huarcaya 2011: 255). Ihre Strategie war die Einbindung aller zivilgesellschaftlichen Gruppierungen, also auch der Gewerkschaften der Einheitsfront der Arbeiter (Frente Unido de los Trabajadores, FUT) (Maugé 2008: 118) und linker christlicher Gruppen (Huarcaya 2011: 255). Auch wenn einige der OZG der FENOC eigene Kandidaten auf den Listen der FADI aufstellte, traten sie nicht zwangsläufig der Partei bei, um so lokal Einfluss gewinnen zu können, poli- 
tisch aber weiterhin unabhängig zu sein. Der Fall der UNORCAC, die mehrere Kandidaten in Cotacachi auf der Liste der FADI aufstellte und in einigen Fällen auch Erfolg hatte, ist ein Beispiel für diese Strategie (Chiriboga 1987: 108).

Das mangelnde Entgegenkommen der Regierung Roldós/Hurtado (1979-1983) brachte die FENOC in offene Opposition. So wurde im Oktober 1980 der Nationale Bauern- und Indigenenmarsch (Marcha Nacional Campesina e Indígena) organisiert, um von der Regierung die Einlösung der gegebenen Versprechen zu fordern (FENOCIN 2004: 15). Auch die Teilnahme an den 5 nationalen Streiks von Mai 1981 bis März 1983 ist so zu verstehen. Im April 1982 findet das erste Bauern- und Indigenentreffen (Encuentro Campesino e Indígena) statt, auf dem alle Bauern- und Indigenenorganisationen eine Agrarreform unter Kontrolle der Bauern fordern und eine Nationalen Bauern- und Indigenenkoordination (Coordinadora Nacional Campesina e Indígena) gründen, um gemeinsame Aktionen durchzuführen (FENOCIN 2004: 17). Die Aktionen in Kooperation mit der Gewerkschaftsbewegung wurden in den indigenen Gemeinden nicht gut aufgenommen und führten zu einer weiteren Schwächung der FENOC (Rodríguez 2008: 94). Die FENOC versteht sich zu dieser Zeit als eine klassistische Organisation, was sie von den anderen Indigenenorganisationen wie ECUARUNARI oder CONFENIAE unterscheidet (Andrango 1983: 50). Dennoch sieht sie die Bedeutung des Landes für die Bewahrung der Identität der Indigenen (Andrango 1983: 50).

Der Wandel von einer Bauernbewegung der 1960er und 1970er zu ethnischen Bewegungen ab den 1980er Jahren geht einher mit einer Krise der gewerkschaftlichen Organisationen in den 1980ern. So sind die Landarbeiterorganisationen bis zu diesem Zeitpunkt nicht nur Gewerkschaften, sondern auch Genossenschaften. Es besteht ein „Modell der Bauernorganisation, das dadurch charakterisiert ist, dass es seine eigenen Unternehmen entwickelt" (Guerrero 2010: 5f.). Diese sind Produktionskooperativen, Kreditinstitutionen und Vermarktungs- und Handelsassoziationen für landwirtschaftliche Produkte. Sie sind als private Firmen in der Hand der Organisationen der Landarbeiterbewegung verfasst und bilden einen Schnittpunkt zwischen den Landarbeitern und der landwirtschaftlich orientierten Mittelschicht, etwa Ingenieure oder Anwälte. Dafür erhalten sie Förderung staatlicher und internationaler Organisationen (Guerrero 2010: 18). Dieses genossenschaftliche Modell - verbreitet vor allem in den Organisationen der CEDOC und FENOC - sollte die Nachfrage der Bauern befriedigen und zum Aufbau einer Schicht kleiner landwirtschaftlicher Produzenten beitragen - während die Organisationen der CTE, etwa FEI oder FTAL, dieselben Maßnahmen vom Staat forderten, sich also nicht in gleichem Maße genossenschaftlich engagierten (Guerrero 2010: 6). Ab Ende der 1970er Jahre befand sich das Modell von lokalen Genossenschaften neben dem eigentlichen Markt in der Krise, sie wurden zunehmend durch lokale Händler und Geldverleiher ersetzt (Guerrero 2010: 15). Daher verschwanden die meisten dieser Organi- 
sationen ab 1985 oder konzentrierten sich auf nicht-genossenschaftliche Politik (Guerrero 2010: 17).

Die Regierung von Febres Cordero (1984-1988) ist von einer repressiven Politik und einer Verfolgung der Gewerkschaften geprägt. Im Oktober 1986 findet in Picalquí, Pedro Moncayo der sechste Kongress der FENOC statt, an dem 3000 Bauern und Landarbeiter teilnehmen. Es wird eine neue Untersuchung der Situation auf dem Land unternommen und ein Programm entwickelt, das bis in die Jahre nach 2000 Bestand hat (FENOCIN 2004: 18). Dennoch markierte dieser Kongress den Beginn einer langen Krise der FENOC. Zwischen 1986 und 1995 ist sie nicht zu größeren Aktionen oder programmatischen Neuerungen fähig und verliert an Einfluss in ihren lokalen Organisationen (FENOCIN 2004: 18f.). Sie kann nur unter den Gewinnern der Agrarreform Mitglieder halten, was sie zu lokal spezifischen Forderungen und Programmen führt - etwa für genossenschaftliche Dienstleitungen (Zamosc 1994: 48) -, die eine einheitliche Politik nicht erlauben (Verdesoto 1986: 42f.). Während die nationale Führung der FENOC weiterhin die Agrarreform als ihr Ziel angibt, unternimmt sie keine größeren Anstrengungen, um sie auch zu erreichen. Gleichzeitig ist sie nicht in der Lage, die Organisation an die sich verändernden Bedingungen anzupassen und die Bedürfnisse ihrer Mitgliedsorganisationen in einem nationalen Projekt zu vereinen. Mitglieder und Beobachter geben für diese Schwäche zwei Gründe an: die Verfestigung und Inflexibilität der Führungsbürokratie in der FENOC und die anhaltende Schwäche der ecuadorianischen Linken (Zamosc 1994: 48). Eine Reihe der Mitgliedsorganisationen trat aus der FENOC aus, andere lösten sich auf. Die FENOC, die 1986 noch 22 Mitgliedsorganisationen (OZG) hatte, bestand 1995 nur noch aus 10 mäßig funktionierenden Unionen und stand kurz vor ihrem Ende (FENOCIN 1999: 13, 83). Das nun offensichtliche Scheitern des Kampfes um eine Agrarreform (Ospina 2008: 71) und die mit der Präsidentschaft Febres Corderos beginnende Schwäche der Gewerkschaften führt dazu, dass die FENOC ihre Position korrigiert. 1988 wird der Name der Organisation in Nationale Föderation der Bauern- und Indigenenorganisationen (Federación Nacional de Organizaciones Campesinas-Indígenas, FENOC-I) geändert und so der Präsenz der Indigenen in der Organisation (FENOCIN 2004: 19) und der stärker werdenden ethnischen Organisationen in der Bauern- und Indigenenbewegung allgemein (Wolff 2008: 116) Rechnung getragen. $\mathrm{Zu}$ diesem Zeitpunkt stellten die Indigenen 40\% der Mitgliedschaft der FENOC-I (Tamayo 1996: 89f.). Dieser Wandel brachte auch gewisse programmatische Neuerungen mit sich, so nahm die FENOC-I am Aufstand 1994 und an der Gründung der Nationalen Agrar-Koordination (Coordinadora Agraria Nacional, CAN) zusammen mit FEI, CONAIE und FENACLE teil, um gegen das Landentwicklungsgesetz (Ley de Desarrollo Agrario) der Regierung Durán Ballén (1992-1996) zu protestieren, das die Agrarreform formell beenden sollte (FENOCIN 2004: 19). 
Ende Februar 1994 fand in Lima ein Kongress von Indigenen- und Bauernorganisationen Lateinamerikas statt, an dem 238 Delegierte von 84 Organisationen aus 18 Ländern Lateinamerikas und 3 Ländern Europas teilnahmen. Aus Ecuador nahmen FENOC-I, CEDOCUT und FENACLE teil (FENOCIN 1999: 168). Auf dem Kongress wurde die Lateinamerikanischen Koordination der Landorganisationen (Coordinadora Latinoaméricana de Organizaciones del Campo, CLOC) gegründet, eine Plattform von Organisationen von Landarbeitern und Bauern, die 1996 der weltweiten Organisation Vía Campesina auf ihrem zweiten Kongress in Tlaxcala, Mexiko, beitreten sollte (FENOCIN 2004: 25). Diese wurde 1993 auf einer internationalen Konferenz von Bauernorganisationen in Belgien gegründet und lehnte sich in ihrer Struktur der sich bereits im Entstehen befindenden CLOC an. Bereits der Name der Vía Campesina verrät den starken Einfluss lateinamerikanischer Organisationen und wurde auch nach einer stärkeren Beteiligung asiatischer und afrikanischer Organisationen beibehalten (Martínez-Torres/Rosset 2010: 157). Seit ihrer zweiten Konferenz hat Vía Campesina den Begriff der Ernährungssouveränität entwickelt, mit dem sie und ihre Mitglieder für eine stärkere Unterstützung von Kleinbauern kämpft und eine Umstrukturierung der Wirtschaft fordert. Die Nahrungsmittel sollen nicht als reine (Export-)Waren gesehen werden, sondern als Mittel, Hunger und Armut vor Ort zu bekämpfen und die Umwelt zu schützen (Martínez-Torres/Rosset 2010: 159f.). Vía Campesina hatte 2010148 Mitgliedsorganisationen aus 69 Ländern, die insgesamt bis zu 500 Millionen Familien weltweit repräsentieren (Martínez-Torres/Rosset 2010: 164f.).

Im November 1997 fand der zweite Kongress der CLOC in Brasilia statt, an dem 338 Delegierte von 49 Organisationen aus 23 Ländern und acht Organisationen aus Asien, Europa und Nordamerika teilnahmen. Ecuador wurde von der FENOC-I und der CUT $^{22}$ vertreten (FENOCIN 1999: 171).

Der siebten Kongress der FENOC-I im Oktober 1995 in Ibarra bedeutete eine grundlegenden Wandel der Organisation von einer ,strikt bäuerlichen und klassistischen Organisation zu einer Organisation, die sich als pluriethnisch [...] definiert [...] und eine nachhaltige Entwicklung mit Identität sucht" (FENOCIN 1999: 103). Gestützt auf eine Selbstanalyse mithilfe der OEG wurden Entwicklung, Interkulturalität und interne Demokratie zu zentralen Prinzipien der FENOC-I erklärt und die Führung im Sinne einer Repräsentation der Regionen, Ethnien, Geschlechter und Generationen gewählt (FENOCIN 1999: 13). Die FENOC-I beginnt einen Wandel hin zu einer pluriethnischen, interkulturellen, demokratischen Föderation, die für eine nachhaltige und gleichberechtigte Entwicklung kämpft (FENOCIN 1999: 53). Sie öffnet sich verstärkt ethnischen Aspekten (Tamayo 1996: 66f.; Ayala Mora o.J.: 5f.) und beschreibt sich selbst als ,pluriethnisch, interkulturell“ (FENOCIN 2004:

22 Es ist keine Organisation diesen Namens nachweisbar. 
20), um Anschluss an die von der CONAIE geführten Indigenenbewegung zu halten. So nimmt sie auch an der Gründung des Comité del Decenio teil, einer Plattform der Indigenen-, Schwarzen- und Bauernorganisationen, die ein gemeinsames, interkulturelles Programm erarbeitet (FENOCIN 2004: 20). In diesem Kontext unterstützte sie auch den Aufbau der MUPP-NP und den Kampf für die Schaffung von Institutionen wie CONPLADEIN und PRODEPINE (FENOCIN 1999: 152).

Die neu gewählte Führung um Pedro de la Cruz bemühte sich um eine Wiederbelebung der FENOC-I. Zu den 10 damals aktiven OZG stießen 8 weitere, die weitgehend inaktiv waren. 15 dieser insgesamt 18 OZG nahmen 1996 und 1997 an dem Prozess der Selbstanalyse und Bestandsaufnahme teil, in dessen Rahmen 521 Personen in verschiedenen Arbeitskreisen die Situation der FENOC-I untersuchten (FENOCIN 1999: 94). Dieses weitgehende Programm interner Demokratisierung und partizipativer Planung stützte sich auf Selbstanalysen aller Mitgliedsorganisationen und den Aufbau von Frauen-, Jugend- und Schwarzenorganisationen innerhalb der FENOC-I (FENOCIN 1999: 57). Die Entwicklung partizipativer Methoden orientierte sich an den Ideen der Befreiungstheologie und des action research, wie ihn einige Nichtregierungsorganisationen in den Organisationen betrieben (FENOCIN 1999: 90). Institutionell wurde die FENOC-I von der Stiftung Paulo Freire (Fundación Paulo Freire), heute AGRITERRA, unterstützt (FENOCIN 1999: 94). Der Prozess verfolgte vier Ziele: die Teilnahme der Basis an der Demokratisierung der FENOC-I, Erneuerung der Gesellschaftsanalyse der FENOC-I unter Einbeziehung der Erfahrungen der Mitgliedsorganisationen, der Aufbau eines neuen Arbeitsprogramms der FENOC-I und die Institutionalisierung partizipativer Methoden in der Organisation (FENOCIN 1999: 94).

Die Ergebnisse dieses Prozesses werden auf dem außerordentlichen Kongress in Guayaquil im Januar 1997 vorgestellt (FENOCIN 1999: 96). Der Name der FENOC-I wird auf Nationale Föderation der Bauern-, Indigenen- und Schwarzenorganisationen (Federación Nacional de Organizaciones Campesinas, Indígenas y Negras, FENOCIN) erweitert und die Teilhabe von Indigenen, Schwarzen und Bauern in der Führung der Organisation institutionalisiert (FENOCIN 1999: 102). Sie versteht sich als ,die einzige nationale Organisation die zur gleichen Zeit den Unterschied der Identitäten und die Interkulturalität anerkennt“ (FENOCIN 2004: 20).

Die Schaffung des Entwicklungsrates der Völker und Nationalitäten Ecuadors (Consejo de Desarrollo de las Nacionalidades y Pueblos del Ecuador, CODENPE) unter Kontrolle der CONAIE, die schon die Vorgängerorganisation Nationaler Planungs- und Entwicklungsrat der indigenen und schwarzen Völker (Consejo Nacional de Planificación y Desarrollo de los Pueblos Indígenas y Negros, CONPLADEIN) und das Entwicklungsprojekt der indigenen und schwarzen Völker (Proyecto de Desarrollo de los Pueblos Indígenas y Negros del Ecuador, PRODE- 
PINE) zumindest teilweise kontrollierte, führte zu Aktionen wie der Besetzung der Büros der CODENPE, um die Position der FENOCIN zu erhalten (FENOCIN 2004: 21).

1999 ändert die FENOCIN auf ihrem achten Kongress in Quito ein letztes Mal ihren Namen in Nationale Konföderation der Bauern-, Indigenen- und Schwarzenorganisationen (Confederación Nacional de Organizaciones Campesinas, Indígenas y Negras, FENOCIN) (FENOCIN 2004: 33). Zu diesem Zeitpunkt umfasst sie 27 OZG und ungefähr 1000 Basisorganisationen (FENOCIN 1999: 13).

\begin{tabular}{|c|c|c|c|c|c|}
\hline Jahr & $\begin{array}{l}\text { Nummer des } \\
\text { Kongresses }\end{array}$ & Teilnehmer & Ort & $\begin{array}{l}\text { Besondere } \\
\text { Neuerungen }\end{array}$ & $\begin{array}{l}\text { Gewählter } \\
\text { Präsident/ } \\
\text { Generalsekretär }\end{array}$ \\
\hline 1965 & $\begin{array}{l}\text { Gründungskon- } \\
\text { gress FETAP }\end{array}$ & $26 \mathrm{OEG}$ & Quito & & \\
\hline 1966 & $\begin{array}{l}\text { 2. Kongress } \\
\text { FETAP }\end{array}$ & 38 OEG & & & \\
\hline 1968 & $\begin{array}{l}\text { 3. Kongress FE- } \\
\text { TAP/ Grün- } \\
\text { dungskongress } \\
\text { FENOC }\end{array}$ & $\begin{array}{l}200 \text { aus } 8 \\
\text { Provinzen }\end{array}$ & Quito & & Francisco Checa \\
\hline 1971 & 2. Kongress & & Riobamba & & Wiederwahl \\
\hline 1973 & 3. Kongress & & & $\begin{array}{l}\text { Christdemokraten } \\
\text { setzen sich durch }\end{array}$ & $\begin{array}{l}\text { Adolfo } \\
\text { Tutivén }\end{array}$ \\
\hline 1974 & 4. Kongress & & Quito & $\begin{array}{l}\text { Treffen des Consejo } \\
\text { Nacional, erst später } \\
\text { zum Kongress erklärt }\end{array}$ & Emilio Velasco \\
\hline 1977 & 5. Kongress & 400 & Quito & $\begin{array}{l}\text { Sozialisten setzen } \\
\text { sich durch }\end{array}$ & $\begin{array}{l}\text { Mesías } \\
\text { Tatamuez }\end{array}$ \\
\hline 1986 & 6. Kongress & 3000 & $\begin{array}{l}\text { Picalquí, } \\
\text { Pedro } \\
\text { Moncayo }\end{array}$ & & Wiederwahl \\
\hline 1988 & $\begin{array}{l}\text { Außerordentli- } \\
\text { cher Kongress }\end{array}$ & & & $\begin{array}{l}\text { Namensänderung in } \\
\text { FENOC-I }\end{array}$ & - \\
\hline 1995 & 7. Kongress & & Ibarra & $\begin{array}{l}\text { Aufnahme der Inter- } \\
\text { kulturalität in Pro- } \\
\text { gramm }\end{array}$ & Pedro de la Cruz \\
\hline
\end{tabular}




\begin{tabular}{|c|c|c|c|c|c|}
\hline 1997 & $\begin{array}{l}\text { Außerordentli- } \\
\text { cher Kongress }\end{array}$ & & Guayaquil & $\begin{array}{l}\text { Namensänderung in } \\
\text { FENOCIN }\end{array}$ & - \\
\hline 1999 & 8. Kongress & & Quito & $\begin{array}{l}\text { Namensänderung in } \\
\text { „Konföderation” }\end{array}$ & \\
\hline 2004 & 9. Kongress & 953 & Ambato & & \\
\hline 2008 & $\begin{array}{l}\text { X. Nationaler } \\
\text { Kongress }\end{array}$ & 1200 & Portoviejo & & Luis Andrango \\
\hline 2013 & $\begin{array}{l}\text { XI. Nationaler } \\
\text { Kongress }\end{array}$ & & $\begin{array}{l}\text { Santa Lu- } \\
\text { cia, Gua- } \\
\text { yas }\end{array}$ & & Santos Villamar \\
\hline
\end{tabular}

Tabelle 2: Kongresse der FENOCIN, eigene Abbildung

Die FENOCIN ist in 18 Provinzen Ecuadors aktiv, in denen sie 200461 regionale und provinziale Organisationen (OZG) und 1.200 Basisorganisationen (FENOCIN 2004: 26/32) hatte. Diese sind, im Gegensatz zur rein gewerkschaftlichen FEI, thematisch organisiert, etwa in Händlerassoziationen (Simbaña I-2). Neueren Zahlen zufolge, wie sie auch auf den Internetseiten der FENOCIN zu finden sind, besteht die FENOCIN aus 52 regionalen und provinzialen Organisationen, in denen etwa 1.300 Basisorganisationen und etwa 200.000 Familien zusammengeschlossen sind (Minkner-Brünjer 2009: 135) ${ }^{23}$. Diese Zahlen werden auch von den ersten Ergebnissen einer von der FENOCIN 2011 selbst durchgeführte Studie gedeckt, die von 52 OZG in 16 Provinzen spricht (FENOCIN 2011: 7). Einer - wohl älteren Selbstdarstellung nach hat die FENOCIN 2011 über 60 OZG und 1.200 OEG in 20 Provinzen (Ayala Mora 2011: 2). Nach einer Schätzung des CODENPE von 2007 hat die FENOCIN nur 17 OZG in 7 Provinzen (Cañar, Carchi, Esmeraldas, Imbabura Loja, Pichincha und Azuay) und 109.278 Mitglieder (IEE o.J.). Das größte Gewicht hat die FENOCIN im Küstengebiet und dem Andenhochland, während sie im Amazonasgebiet kaum präsent ist (Barrera o.J.: 151; Guerrero/Ospina 2003). Die wichtigste und aktivste OZG der FENOCIN ist die 1977 gegründete Union der indigenen Bauernorganisationen von Cotacachi (Unión de Organizaciones Campesinas Indígenas de Cotacachi, UNORCAC) (León 1991: 406f.), die 41 Gemeinschaften und einige andere Basisorganisationen in diesem Kanton der Provinz Imbabura repräsentiert. Auch wenn sie im Umfeld des politischen Kampfes der FENOC entstanden ist, finanziert sie sich jetzt über kleine Entwicklungsprojekte (Martínez Valle 2006: 111).

23 Die Behauptung von Minkner-Brünjer (2009: 135), die FENOCIN sei eine Mitgliedsorganisation der CONAIE, ist falsch. 
Obwohl die FENOCIN ihre Basis ausbauen konnte und an vielen Aktionen der CONAIE und der Gewerkschaften teilnimmt (FENOCIN 2004: 22), konnte sie im letzten Jahrzehnt keine Aktionen auf nationaler Ebene selbstständig durchführen (Barrera o.J.: 151; Minkner-Brünjer 2009: 145f.). Die Krise der CONAIE nach ihrer Regierungsbeteiligung 2003 strahlte auch auf die FENOCIN aus, die nur einige kleine Aktionen gegen den von der Regierung Gutiérrez geplanten Freihandelsvertrag realisieren konnte (Simbaña 2007: 23). Diese Situation hat sich erst mit der Verbindung der FENOCIN mit der Regierung Correa verändert. Über ihre Beteiligung an der PS-FA war die FENOCIN von 2007 bis 2010 in der Regierung und der Regierungspartei Alianza PAÍS repräsentiert, deren politisches Projekt sie unterstützt (FENOCIN 2008). Anders gesagt: „die völlige Anpassung der FENOCIN an die Regierung Correa hat ihr im Moment größeren Handlungsspielraum und Anerkennung verschafft" (León Trujillo 2010: 16). Der ehemalige Präsident der FENOCIN, Pedro de la Cruz, übernimmt auch weiterhin Funktionen in Partei und Regierung (Wolff 2010: 22). Er war bereits Ersatzabgeordneter ${ }^{24}$ für die PS-FA von 1998 bis 2003 und ist seit den Wahlen zur Verfassungsgebenden Versammlung Abgeordneter für Alianza PAÍS (Becker 2011: 50). Auch der Bruch der PS-FA mit Alianza PAÍS 2010 hat daran nichts geändert.

\section{c. Programm}

Die FENOCIN versteht sich als unabhängige Repräsentantin der Einheit von Bauern, Indigenen und Schwarzen in Ecuador (FENOCIN 2004: 32f.). Dieses Verständnis beginnt mit dem siebten Kongress von 1995 und dem außerordentlichen Kongress 1997, auf dem sie ihr Programm ändert hin zu einem „Aufbau der Autonomie und Plurikulturalität der indigenen, schwarzen und mestizischen Gesellschaften Ecuadors, die verschiedenen Identitäten und interkulturellen Räume stärkend“ (FENOCIN 2004: 54). Der achte Kongress 1999 bestätigte diesen Beschluss als drittes in einer Strategie mit vier einzelnen Programmen: „1. Programm der organisatorischen Stärkung, 2. Programm der nachhaltigen und gerechten Entwicklung mit Identität, 3. [...], 4. Programm der Teilnahme an der Globalisierung mit Identität und in Opposition zum Neoliberalismus." (FENOCIN 2004: 25) Mit diesen Programmen wollte sich die FENOCIN als interkulturelle Organisation festigen und ein interkulturelles Denken entwickeln, das die gesamte Mitgliedschaft durchdringt (FENOCIN 1999: 127). Für besonders wichtig wird dabei der Einfluss auf die Öffentlichkeit erachtet. Auf dem Kongress 1999 wird kritisiert, dass sich die FENO-

24 In Ecuador wird gleichzeitig mit dem jeweiligen Abgeordneten ein Ersatzabgeordneter gewählt, der ihn im Fall von Krankheit oder Tod vertritt, ansonsten aber als sein Assistent fungiert. 
CIN mit ihrem Vorschlag der Interkulturalität bislang nicht als von den anderen Indigenen-, Schwarzen- und Bauernorganisationen verschieden präsentieren konnte (FENOCIN 1999: 108). Weiterhin soll die finanzielle Abhängigkeit der FENOCIN von externen Akteuren durch einen allgemeinen Mitgliedsbetrag, den Aufbau eigener Unternehmen, den Export von fair gehandelten Produkten durch strategische Allianzen mit den entsprechenden Agenturen und den Aufbau von Vermarktungsnetzwerken mit anderen lateinamerikanischen Organisationen reduziert werden (FENOCIN 1999: 126).

Zur Landentwicklung wird der Aufbau selbstverwalteter Territorien der Indigenen, Schwarzen und Bauern verlangt, die vom Staat geschützt und ausreichend finanziert werden sollen (FENOCIN 1999: 143-146). Zudem soll ein nationales Programm der technischen Unterstützung durch die nationalen Bauernorganisationen und mit Finanzierung des Staates aufgebaut werden, das einer Methode ,von Bauer zu Bauer“ (FENOCIN 1999: 145) folgt. Die bereits bestehenden Kreditprogramme wie PRODEPINE und CODENPE sollen gestärkt und in einem ländlichen Finanzsystem integriert werden, das parallel zum nationalen Finanzsystem bestehen soll, seine Ressourcen aber völlig auf die kleine und mittlere Agrarwirtschaft ausrichtet (FENOCIN 1999: 146).

In diesem Zusammenhang interpretiert die FENOCIN die politische Lage in Ecuador als ein Kampf zwischen zwei Projekten. „Das eine ist das der Macht, der Exklusion, des Erstarrens und der Unterwerfung unter die internationalen Gläubiger, das das soziale Kapital, die ethischen Werte, die Identität und das Vertrauen zwischen den Ecuadorianer zerstört.“ (FENOCIN 2004: 27). Es reduziert die indigenen und schwarzen Bauern auf ,ethnische Minderheiten“ (FENOCIN 2004: 29). Dem steht ein anderes Projekt gegenüber, ,das der Ecuadorianer des Volkes, der Bewegung, der Teilhabe, der Inklusion und der Hoffnung“ (FENOCIN 2004: 29). Es will ,in unserem Land einen interkulturellen, flexiblen und demokratischen sozialen Zusammenhang schmieden, der die Identitäten und die Rechte aller Bürger besser unterstützt" (FENOCIN 2004: 29).

Auf dem 10. Kongress der FENOCIN 2008 wurden die strategischen Leitpunkte organisatorische Stärkung, Interkulturalität, Agrarrevolution, Ernährungssouveränität und Aufbau des Sozialismus beschlossen. Die FENOCIN will nicht staatliche Macht erlangen, um ihre Ziele zu erreichen, sondern am Staat teilhaben, um die Zivilgesellschaft und die sozialen Bewegungen zu unterstützen (FENOCIN 2008). Sie versteht sich als eine interkulturelle Organisation, die die besonderen Identitäten ihrer Mitglieder respektiert (FENOCIN 2004: 24). Sie hat einen ,offenen, pluralen, einbeziehenden, einheitlichen Charakter, ohne einseitigen Ethnizismus und [ist] nicht divisionistisch“ (FENOCIN 2004: 32). Die Interkulturalität soll die Teilhabe der Bürger, das Zusammenleben und die Entwicklung der Fähigkeiten jedes Einzelnen erlauben, um die nationalen Probleme zu lösen (FENOCIN 2004: 39). Die 
FENOCIN versteht Interkulturalität als Kampf gegen ,internen und externen Kolonialismus [...] [und] alle Formen des Rassismus und Paternalismus“ (FENOCIN o.J.: 11). Interkulturalität ist ein Prozess, der die Forderungen aller Bürger aufgreift. „Dies bedeutet persönliche Veränderungen und Veränderungen des Verhaltens, sowie strukturelle Veränderungen der gesamten Gesellschaft.“ (FENOCIN o.J.: 11) Dem stellt die FENOCIN den Begriff der Plurinationalität gegenüber, der als eine bereits jetzt bestehende - Zersplitterung des Landes in ethnische Gruppen verstanden wird. Diese Situation soll durch die Interkulturalität überwunden werden (Simbaña I-2) ${ }^{25}$.

Das Ziel der FENOCIN ist die Verteidigung der Rechte ihrer Mitglieder und der Aufbau einer gerechten Gesellschaft. Dieses andere Modell des Landes soll über die Entwicklung der Interkulturalität möglich gemacht werden und sich durch die Verteidigung der Rechte der Pachamama, die Ernährungssouveränität und das Gute Leben auszeichnen (Ayala Mora 2011: 12). Die FENOCIN

„,bemüht sich, ein plurikulturelles Land aufzubauen, in dem die Unterschiede respektiert werden, aber zur gleichen Zeit plurikulturelle Organismen und ein interkulturelles Denken geschaffen werden, die das ermöglichen sollen“ (FENOCIN 1999: 103).

\section{FEINE}

Schon in den 1950ern begann - zusammen mit einer allgemeinen Verstärkung der christlichen Missionsbemühungen - der Prozess der Organisation der evangelischen Indigenen, die sich von sozialistisch und/oder katholisch geprägten Organisationen wie FEI, FENOC und ECUARUNARI nicht repräsentiert fühlten (FEINE o.J.; Becker 2009: 1056). Es bildeten sich - mit Unterstützung und weitgehend unter Kontrolle US-amerikanischer Kirchen (Tamayo 1996: 67; Guamán 2006: 91; Korovkin 2008: 6) - einige Basisorganisationen, die sich $1980 \mathrm{zu}$ einer nationalen Organisation, der Ecuadorianischen Föderation der Evangelischen Indigenen (Federación Ecuatoriana de Indígenas Evangélicos, FEINE), zusammenschlossen. Diese wiederum brauchte bis Ende der 1980er Jahre, um ihre Strukturen zu festigen. Diese beiden ersten Etappen ihrer Entwicklung sind von einer nach innen gewandten und betont unpolitischen Ausrichtung der Organisation geprägt, während sie in den 1990ern verstärkt soziale Themen aufgreift (Guamán 2006: 91). 1989 bis 1994 traten die evangelischen Indigenen in die Öffentlichkeit - schon Mitte der 1990er hatte die FEINE 14 Provinz-Organisationen (Tamayo 1996: 67) - und er-

25 Im Teil III.4 wird der Begriff der Interkulturalität und die Diskussion zwischen FENOCIN und CONAIE näher beleuchtet. 
weiterten ihre Beziehungen zu anderen Organisationen von 1995 bis 1998 (Guamán 2003: 81). Erst in dieser Etappe entwickelt die FEINE Charakteristiken einer sozialen Bewegung, wie eigenständige Strukturen und definierte soziale und kulturelle Forderungen (Guamán 2006: 91). 1998 gründete die FEINE eine politische Partei in Konkurrenz zur Partei der CONAIE, MUPP-NP, Amauta Jatari, jetzt Amauta Yuyay (Andrade 2005: 53f.), die ab 2000 auf lokalem und provinzialem Niveau des Andengebietes einige Wahlerfolge hatte (Freston 2004: 144). Das war das Ergebnis einer Diskussion, die schon 1996 begonnen hatte. 1997 wurde innerhalb der FEINE eine politische Kommission gebildet, die Partei wurde aber erst zu den Wahlen im Mai 2000 offiziell beim Provinz-Wahltribunal (Tribunal Electoral Provincial) in Chimborazo angemeldet (Guamán 2006: 86). Amauta Yuyay ist eine rein indigene Partei, deren Stärken in den ländlichen Gebieten des zentralen Andenhochlandes liegen, vor allem im Kanton Colta der Provinz Chimborazo. Ab 2002 tritt sie damals noch unter dem Namen Unabhängige Bewegung Amauta Jatari (Movimiento Independiente Amauta Jatari) - auch zu nationalen Wahlen an und konnte so an Bedeutung gewinnen (Guerrero/Ospina 2003; Guamán 2006: 48/86).

Die FEINE und ihre Mitgliedsorganisationen entstammen den Missionsbemühungen verschiedener fundamentalistischer evangelischer Organisationen, die sich besonders um die Missionierung der Indigenen bemühen (Rohr 2006: 11f.). Die wichtigste unter diesen Organisationen ist die Evangelische Missions-Union (Unión Misionera Evangélica, UME) (Guamán 2006: 32). Dazu kommen die Christliche und Missionarische Allianz (Alianza Cristiana y Misionera, ACM) - heute unter dem Namen The Alliance bekannt -, das seit den 1980er Jahren aus Ecuador vertriebene Linguistische Sommer-Institut (Instituto Lingüístico de Verano, ILV) und eine Reihe kleinerer Organisationen (Guamán 2006: 32). Die Missionstätigkeit wurde in den 1970ern schrittweise von Indigenen übernommen, die auch die Gründung evangelischer Organisationen vorantrieben. So entstanden eine Reihe von OZG, die später die FEINE gründen sollten. Auffallend ist der hohe Anteil evangelikaler, vor allem pfingstkirchlicher Gruppen, die rund $80 \%$ der protestantischen Religionsgemeinschaften in Lateinamerika ausmachen. In Ecuador sind $70 \%$ der Anhänger der Pfingstkirchen Indigene (Rohr 2006: 2f.), insgesamt sind zwischen 1,5 und 2 Millionen Ecuadorianer, also ca. 12\% der Bevölkerung Mitglied einer protestantisch-fundamentalistischen Kirche (Rohr 2006: 11f.).

Eine der wichtigsten OZG ist die Konföderation der indigenen evangelischen Völker, Organisationen, Gemeinschaften und Kirchen Chimborazos (Confederación de Pueblos, Organizaciones, Comunidades e Iglesias Indígenas Evangélicas de Chimborazo, CONPOCIECH); sie war die erste OZG evangelischer Indigener und bestimmte die Entwicklung anderer evangelischer OZG und der FEINE selbst entscheidend mit (Guamán 2006: 52f.). Sie wurde 1967 als Assoziation evangelischer Indigener der Provinz Chimborazo (Asociación de Indígenas Evangélicos de la 
Provincia de Chimborazo) gegründet, 1983 in Indigene Evangelische Assoziation Chimborazos (Asociación Indígena Evangélica de Chimborazo, AIECH) umbenannt, 1994 in Assoziation indigener evangelischer Kirchen Chimborazos (Asociación de Iglesias Indígenas Evangélicas de Chimborazo, AIIECH) und bekam 2001 schließlich ihre aktuelle Bezeichnung (Guamán 2006: 52).

1972 wurde die Assoziation Evangelischer Indigener Bolívars (Asociación de Indígenas Evangélicos de Bolívar, AIEB), 1974 die Assoziation Evangelischer Indigener Cotopaxis (Asociación de Indígenas Evangélicos de Cotopaxi, AIEC) und 1976 die Assoziation Evangelischer Indigener Napos (Asociación de Indígenas Evangélicos de Napo, AIEN) gegründet (Guamán 2006: 65). Diese vier OZG begannen ab 1975, eine nationale Organisation aufzubauen. 1977 fand in Quito ein Kongress der bereits staatlich anerkannten OZG statt, an dem auch noch in der Gründung befindliche Organisationen aus Imbabura, Pichincha, Tungurahua und Cañar teilnahmen (Guamán 2006: 65). Auf dem Kongress wurde die Nationale Föderation evangelischer Assoziationen (Federación Nacional de Asociaciones Evangélicas, FENAE) gegründet, die sich auf ihrer Generalversammlung 1979 in Ecuadorianische Föderation evangelischer Indigener (Federación Ecuatoriana de Indígenas Evangélicos, FEINE) umbenannte und als solche im November 1980 staatlich anerkannt wurde (Guamán 2006: 65). Dies beförderte das Wachstum evangelischer Indigenenorganisationen. So wurde 1981 die Assoziation Evangelischer Indigener Shuar Ecuadors (Asociación de Indígenas Evangélicos Shuar del Ecuador, AIESE), 1984 die Assoziation Evangelischer Indigener Sucumbíos (Asociación de Indígenas Evangélicos de Sucumbíos, AIES) und die Assoziation Christlicher Evangelischer Indigener Azuays (Asociación de Indígenas Evangélicos Cristianos del Azuay, AINECA) und 1985 die Assoziation Evangelischer Kirchen ,Chimborazo“ der Provinz Napo (Asociación de Iglesias Evangélicas ,Chimborazo“ de la provincia de Napo) und die Assoziation Evangelischer Indigener Pastazas, Amazonas (Asociación de Indígenas Evangélicos de Pastaza Región Amazónica, AIEPRA) gegründet (Guamán 2006: 66).

Die FEINE hat 17 Provinz-Organisationen, denen ca. 600 evangelische Kirchengemeinden und $17 \%$ der indigenen Bevölkerung angehören (Wolff 2008: $116)^{26}$. Andere Angaben (Chisaguano 2008: 99) sprechen von 30\% Repräsentativität unter den Indigenen. Dazu kommen über 300 evangelische Pfarrer (Andrade 2005: 54). Nach Angaben der FEINE zu ihrem 30-jährigen Jubiläum 2010 hat sie 18 OZG in 21 Provinzen, die 2.800 Gemeinden und Kirchen ${ }^{27}$ und 1.200 Pastoren

26 Die Zahlen von Lucero (2006: 40), der von über 5.000 Kirchengemeinden und ca. 2,5 Millionen Mitgliedern spricht, sind -vorsichtig gesagt- unwahrscheinlich.

27 Andere Angaben aus demselben Jahr sprechen von 2.323 Kirchengemeinden (FEINE 2010: 15). 
umfassen (FEINE o.J.; FEINE 2010: 5) ${ }^{28}$. Die Basisorganisationen sind in der Regel Kirchengemeinden der ländlichen Gemeinschaften oder städtischen Viertel, die sich auf zweiter Ebene - in Chimborazo - in Unionen auf Kantonalniveau und auf dritter in Provinz-Assoziationen zusammenschließen. Die OEG widmen sich in erster Linie kirchlichen (oder „sozio-kirchlichen“ (Guamán 2006: 90f.)), die Organisationen der höheren Ebenen politischen Aufgaben (Guamán 2006: 90f.; FEINE 2010: 2). Schwerpunkt der FEINE ist die zentrale Andenregion (Lucero 2006: 40). Die wichtigsten Mitgliedsorganisationen sind die OZG in Imbabura mit 8 und die OZG in Chimborazo mit 6 Gemeinschaften (GTZ 2004a: 35f.). Nach einer Schätzung des CODENPE von 2007 hat die FEINE 5 OZG in 4 Provinzen (Chimborazo, Imbabura, Pastaza und Cañar) und 26.384 Mitglieder (IEE o.J.). Trotz ihrer zahlenmäßigen Stärke und weitverzweigten Strukturen hat sie nicht die organisatorische Bindung und ideologische Stärke der CONAIE (Lucero 2006: 39f.). Dennoch konnte sie in den letzten Jahren weiter an Bedeutung gewinnen (Barrera o.J.: 151). Nach eigenen Angaben hat die FEINE in den letzten 10 Jahren ein jährliches Wachstum von 65\% erlebt (FEINE 2010: 15). Die FEINE hat - im Gegensatz zu CONAIE und FENOCIN - eine starke Repräsentativität unter den Indigenen in den Städten (Rohr 2006: 11f.).

Die FEINE identifiziert sich klar mit ihrem evangelisch-religiösen Ursprung, aus dem sie eine Kritik der Verhältnisse ableitet. So will sie die Bibel „holistisch“ (Freston 2004: 143) verstehen und - im Gegensatz zu den US-amerikanischen Missionaren, die viele der evangelischen Kirchen der Region aufgebaut haben - die politische Dimension des Evangeliums stärken (Freston 2004: 143). Das Programm der FEINE ist mehr als der „Indianized Protestant Evangelicalism“ (Lucero 2006: 33f.), den Lucero diagnostiziert. Die Verteidigung der indigenen Kulturen - etwa über Gesundheits- und Erziehungsprojekte (Tamayo 1996: 67) - und die Verbesserung der Lebensbedingungen der Indigenen über eine Reform der öffentlichen Verwaltung und Schaffung wirtschaftlicher Instrumente (Rodríguez 2008: 97), sind schon lange Forderungen der FEINE, die in den letzten Jahren weiter an Bedeutung gewonnen haben (Ayala Mora o.J.: 5f.).

Die FEINE engagiert sich vor allem in ihren Gemeinden selbst, wobei Bildungsprogramme und Projekte der Gemeindeentwicklung eine besondere Rolle spielen (FEINE o.J.). Dazu kommen spezifisch kulturelle oder ethnische Aspekte, die durch die CONAIE an Bedeutung innerhalb der Indigenenbewegung gewonnen haben (Lucero 2006: 41). So hat die FEINE im Zuge eines Politikwechsels ab 1998 Teile der Sprache und Politik der CONAIE übernommen (Lucero 2006: 52). Sie will nun von der evangelischen indigenen Identität aus für Menschenrechte, Ge-

28 Dem entsprechen die Zahlen von Rohr (2006: 11f.), die von 2.500 Kirchengemeinden in 18 Mitgliedsorganisationen spricht. 
rechtigkeit und Interkulturalität kämpfen, wobei sie sich dem Kampf für kollektive Rechte der Indigenen verbunden fühlt, aber nicht dem Projekt der Plurinationalität (Guamán 2006: 80f.). Bestandteil dieser Erneuerung war die Reform der Statuten im Jahr 2000, durch die sich die FEINE - unter Beibehaltung ihres Akronyms - von Ecuadorianische Föderation der Evangelischen Indigenen (Federación Ecuatoriana de Indígenas Evangélicos) in Rat der indigenen evangelischen Völker und Organisationen Ecuadors (Consejo de Pueblos y Organizaciones Indígenas Evangélicas del Ecuador) umbenannte (FEINE o.J.; Guamán 2006: 81; Lucero 2006: 41). Ebenso wurden die Strukturen der Organisation verändert, so dass sie jetzt einen Nationalkongress hat, in dem alle vier Jahre Vertreter der OEG und OZG zusammentreffen, eine Nationalversammlung, in der sich die Vertreter der OZG sammeln und einen Regierungsrat, der auf dem Nationalkongress für jeweils vier Jahre gewählt wird und die laufenden Geschäfte der Organisation in verschiedenen Bereichen kontrolliert (Guamán 2006: 81).

Auch die Hegemonie der CONAIE innerhalb der Indigenenbewegung hat der FEINE neue Räume eröffnet, die sie für ein weiteres Wachstum nutzen konnte (Lucero 2006: 43). Dennoch ist die FEINE wegen ihrer ,politische[n] Enthaltsamkeit" (Rohr 2006: 11f.) und teilweiser Zusammenarbeit mit der Regierung innerhalb der Indigenenbewegung eher isoliert (Barrera o.J.: 151). In den ersten Jahren der 1990er versuchten die Führer der FEINE, sich von den anderen Organisationen der Indigenenbewegung, vor allem der als rebellisch verstandenen CONAIE, zu unterscheiden, indem sie sich als rechtschaffen und staatstreu präsentierten (Lucero 2006: 36). Weder die nationale Führung der FEINE noch ihre OZG waren in der Lage, angemessen auf die nach 1990 stärker in der Öffentlichkeit präsenten Forderungen der Indigenenbewegung zu reagieren. Vor allem nationale Projekte wie die Plurinationalität, die Forderung nach einer neuen Verfassung und nach kollektiven Rechten für die indigenen Völker wurden von der FEINE kaum aufgegriffen - was ihre Distanz von der Indigenenbewegung vergrößerte (Guamán 2006: 75). Große Unterschiede in der politischen Ausrichtung der Führung der FEINE erschwerten eine klare Positionierung innerhalb der Indigenenbewegung Ecuadors. Ein offener und auf Koalitionen und die Bekämpfung sozialer Probleme ausgerichteter Stil (1989-1990) und ein geschlossener und nur an religiösen Themen interessierter Stil (1991-1994) wechselten sich ab, ihre jeweiligen Projekte - im ersten Fall Erziehungs- und Sozialprojekte auf Gemeindeniveau und im zweiten Fall die Konstitution der FEINE als völlig andere im Hinblick auf die Indigenenbewegung und so die Stärkung ihrer ethnoreligiösen Identität - führten zu einer unklaren Entwicklung mit vielen Brüchen (Guamán 2006: 73). Dennoch beteiligte sich die FEINE an der Erhebung des Inti Raymi 1990 (FEINE o.J.) und den Aktionen der Jahre 1992 und 1994, wobei die Forderungen der anderen Organisationen für die FEINE von wenig Interesse waren - sie sah sich ihrem Begriff von Interkulturalität verbunden und 
nicht den Forderungen nach Plurinationalität, Verteidigung der indigenen Völker oder Landverteilung (Guamán 2006: 70f.). Auch wenn er keine weitere Definition erfährt, verwendet die FEINE den Begriff der indigenen Nationalitäten (etwa FEINE 2010: 1). Nach dem Interkulturalitätsbegriff der FEINE

,treten die indigenen Völker, ausgehend von freier Entscheidung, Stärkung der Identität und Anerkennung der Diversität, in Verbindung, Dialog und Interaktion mit den anderen Völker, um eine solidarische, gerechte und demokratische Gesellschaft und Staat aufzubauen“ (Guamán 2006: 71).

Dazu sollen Mechanismen der Teilhabe der evangelischen indigenen Völker und Nationalitäten an den staatlichen Entscheidungen geschaffen werden und eine interkulturelle Gesellschaft aufgebaut werden, die sich ,auf die Gleichberechtigung, den Respekt vor den Unterschieden, die Gerechtigkeit, die Toleranz und die Harmonie mit der Natur stützt“" (FEINE 2010: 1). Die Interkulturalität wird so - ähnlich dem Diskurs der FENOCIN - als Alternative zum bestehenden System der sozialen, politischen und kulturellen Trennung und Diskriminierung gesehen, die neue Identitäten schafft. Als Prozess des Dialogs und des harmonischen Zusammenlebens zwischen den verschiedenen Völkern erlaubt sie eine Transformation der Strukturen des Staates und die Suche nach einer neuen Art der Organisation der Gesellschaft. Dazu müssen allerdings Ethnozentrismus und Diskriminierung beendet werden (FEINE 2010: 19). In den letzten Jahren wurde auch der Begriff des Guten Lebens von der FEINE übernommen, die jetzt ,die organisatorische kirchliche Identität durch den Aufbau einer indigenen Theologie stärken will, um zum Guten Leben beizutragen“ (FEINE 2010: 7). Die FEINE versteht sich als kirchliche Leitung der Entwicklung der evangelischen indigenen Völker Ecuadors, wobei sie diese repräsentiert und eine ganzheitliche Entwicklung mit Identität und auf Basis der christlichen Werte anstrebt (FEINE 2010: 3).

Der Politikwechsel 1998 mit der Wahl von Marco Murillo zum Präsidenten der FEINE markierte eine endgültige Wendung hin zur Politisierung der Organisation. Die im Vergleich zu den anderen Indigenenorganisationen autoritäre Ausrichtung und große Treue zur jeweiligen Führung der Organisation (Rohr 2006: 11f.) erlaubt ein relativ geschlossenes Auftreten. So beteiligte sie sich entscheidend an den Aktionen der Indigenenbewegung in den Jahren 2000 und 2001. 2000 organisierte sie Märsche in Riobamba und Quito und besetzte das Büro der CODENPE. Die Erhebung von Januar und Februar 2001 fand unter anderem auf ihre Initiative hin statt (Guamán 2006: 76) - die FEINE konstituierte sich unter einer neuen Führung und unter dem Druck ihrer Basisorganisationen als Teil der sozialen Bewegungen und der Indigenenbewegung (Guamán 2006: 76f.). 
In den frühen 2000ern zogen FEINE und Amauta Yuyay Aufmerksamkeit und Feindseligkeiten der anderen indigenen Organisationen auf sich, als sie den ehemaligen Präsidenten der CONAIE, Antonio Vargas, als Präsidentschaftskandidat 2002 unterstützten (Freston 2004: 144) - während CONAIE und MUPP-NP Lucío Gutiérrez unterstützten. Spätestens ab diesem Zeitpunkt arbeitete die FEINE mit den Regierungen - vor allem der von Gutiérrez nach seinem Bruch mit der CONAIE zusammen, wodurch sie wirtschaftliche Förderung (Kaltmeier 2009: 355) ${ }^{29}$ und politische Posten bekam (Andrade 2005: 60). Die FEINE kritisiert die Regierung Correa, bleibt aber der offenen Opposition der CONAIE fern (Wolff 2010: 22).

Auf internationalen Niveau hat die FEINE Beziehungen zu kirchlichen und religiösen Verbunden aufgebaut. Die wichtigsten sind der Weltkirchenrat oder Ökumenische Rat der Kirchen und seine Regionalorganisation, der Lateinamerikanische Kirchenrat (Consejo Latinoamericano de Iglesias, CLAI), der Lateinamerikanische Rat indigener evangelischer Völker (Consejo Latinoamericano de Pueblos Indígenas Evangélicos) und der US-amerikanische Church World Service (Guamán 2006: 84; FEINE 2010: 13). Diese Institutionen verstehen sich nicht als Teil sozialer Bewegungen, sondern als Kooperationsinstanzen vor allem protestantischer Kirchen. Daher sind sie zwar auf Sozialarbeit und Entwicklungsprojekte ausgerichtet, aber nicht auf Interessenvertretung jenseits des rein Religiösen.

\section{CONAIE}

\section{a. Federación Shuar und CONFENIAE}

Seit den 1950er Jahren nahmen die Missionsversuche der katholischen Kirche im Amazonasgebiet zu. Vor allem der religiöse Orden der Salesianer, der unter Luis Bolla und Juan Shutka ab 1952 in der Region aktiv wurde (Diario Hoy 2000; Bottasso 2003: 24), spielte eine wichtige Rolle bei der Missionierung der Shuar, der größten indigenen Gruppe der Region. Ihre Strategie bestand in einer Reorganisation der Shuar in Zentren, einem Äquivalent zu den Kommunen des Andenhochlandes, die mehrere Familien, die an einem Ort leben, zusammenfassen (CONAIE 1989: 91). $\mathrm{Zu}$ einem Zentrum gehören über eine formale Mitgliedschaft in der Regel 25 bis 30 Familien, die meist miteinander verwandt sind. Es muss als solches durch das Arbeits- und Sozialministerium anerkannt werden, wodurch es Recht auf

29 Bis hierher sollte klar geworden sein, dass keinesfalls von einer „Einheitsorganisation“ (Kaltmeier 2009: 355) der Indigenen gesprochen werden kann. Außerdem muss darauf hingewiesen werden, dass die Gründung von Amauta Jatari einige Jahre vor der Regierung Gutiérrez (2002-2005) stattfand, also kein gezielter Spaltungsversuch sein kann. 
ein eigenes Territorium erwirbt, dessen Größe von der Größe der Mitgliedsfamilien abhängt. Ein Shuar-Zentrum ist kein Dorf, da seine Bevölkerung nicht direkt benachbart sein muss. Dennoch verfügt es über einige zentrale Strukturen, wie einen Platz, der von wichtigen Verwaltungsgebäuden umgeben ist (Salazar 1981: 65). Damit ist ein Shuar-Zentrum:

„Eine politische und verwaltungstechnische Einheit mit umgrenztem Territorium, zusammengesetzt aus einer Bevölkerung, deren Besonderheit formal definiert ist, und errichtet unter der Autorität eines Rates, der gewählt wurde, um für die sozioökonomische Entwicklung seiner Mitglieder zu sorgen.“ (Salazar 1981: 65)

Eine gewisse Anzahl von Shuar-Zentren - Salazar (1981: 66) spricht von 2 bis 15, mit einem Durchschnitt von 8 - bilden eine Assoziation, alle Shuar-Assoziationen bilden zusammen die Föderation der Shuar-Zentren (Federación de Centros Shuar). 1961 organisierten die Salesianer den ersten Kurs für Shuar-Dirigenten, auf dem die Gründung der ersten Assoziation vorbereitet wurde (Zallez/Gortaire 1978: 59). Diese wurde 1961 von 263 Shuar-Zentren (Porras 2005: 98) als „Lokale Assoziation der Jíbaro-Zentren“30 (Asociación Local de Centros Jíbaros) gegründet und 1962 als „Shuar-Assoziation Sucuas“ (Asociación de Centros Shuar de Sucúa) rechtlich anerkannt. Daraufhin wurden in den Gegenden Sevilla, Méndez und Limón Kongresse und Treffen veranstaltet, um in der ganzen Provinz Morona Santiago ähnliche Assoziationen zu schaffen (Federación de Centros Shuar 1976: 115). Diese führten im Januar 1964 die Erste Provinzielle Tagung der Shuar-Führer (Primera Convención Provincial de Dirigentes Shuar) mit 52 Delegierten durch, auf der die Gründung einer selbstbestimmten und selbstverwalteten Föderation beschlossen wurde (Zallez/Gortaire 1978: 60). Noch im selben Jahr wurde die Interprovinzielle Föderation der Shuar- und Achuar-Zentren (Federación Interprovincial de Centros Shuar y Achuar, FICSHA) ${ }^{31}$ gebildet (León 1991: 389). Ihre Statuten wurden im Oktober 1964 vom zuständigen Ministerium angenommen (Zallez/Gortaire 1978: 60). Die Föderation soll die Arbeit aller Assoziationen koordinieren und wird von einer Generalversammlung (Asamblea General) gelenkt, in der drei Delegierte für jede Assoziation und ein Delegierter jedes Zentrums sitzt. Sie tritt einmal im Jahr in Sucúa zusammen, wo sich ihr Hauptgebäude befindet, das mit einer Radiostation, einer kleinen Bibliothek und Versammlungsräumen ausgestattet ist (Salazar 1981: 66). Dort arbeiten eine Reihe von Personen hauptberuflich für die Föderation, Ende der 1970er waren das 52 Personen (Zallez/Gortaire 1978: 49). Ihr Organisationsziel ist die soziale, wirtschaftliche und moralische Verbesse-

30 Jíbaro ist die damals übliche Fremdbezeichnung für die Shuar.

31 Seit dem Aufbau einer eigenen Achuar-Organisation FICSH. 
rung ihrer Mitglieder und die Koordinierung von Entwicklungs- und Kolonisierungsprojekten (Salazar 1981: 65).

In den ersten Jahren war der Einfluss der Geistlichen in der Organisation erheblich. Ihre Unterstützung der neuen Organisationen ergab sich auch aus ihrem Versuch, den Einfluss von sozialistischen und kommunistischen Gruppen unter den Indigenen - wie etwa der FEI - zu begrenzen (Becker 2009: 1056). Jede Assoziation hatte einen religiösen Berater, der regelmäßig die verschiedenen Zentren besuchte und bei der Ausarbeitung und Umsetzung der Statuten der Organisation mitarbeitete (Federación de Centros Shuar 1976: 115). Die Geistlichen trugen auch zur zunehmenden Strukturierung und Organisation der Shuar-Zentren bei, ohne dabei ausschließlich die Mission im Auge zu haben (Barre 1983: 179). Der erste geistliche Berater und Mitbegründer der FICSHA war der Salesianer Juan Shutka, der auch bei der Assoziation in Sucúa diesen Posten innehatte (Zallez/Gortaire 1978: 60). Ihm wurde ein Büro im Zentralgebäude der FICSHA in Sucúa zur Verfügung gestellt, wo er sich ganztägig dieser Aufgabe widmete (Salazar 1981: 67).

Auch bei der Erforschung der Sprache der Shuar spielten die Salesianer - neben protestantischen Missionaren - eine wichtige Rolle. Diese Arbeit, die in den 1950er und 1960ern unter Siro Pellizzaro und P.A. Germani als Unterstützung der Mission begann, führte zur Ausarbeitung eines vereinheitlichten Alphabets, dass 1970 von den Salesianern, der FICSHA und dem Linguistischen Sommerinstitut akzeptiert wurde (Bottasso 2003: 23).

Nach einigen Jahren ging der Einfluss der Missionare und religiösen Berater zurück und die Shuar-Zentren konnte ihre Autonomie ausbauen (Albó 2009: 209). Diese politische Eigenständigkeit sollte die Grundlage für das Verständnis als soziale Bewegung der Indigenen sein (García Serrano 2005: 2). Die Anwesenheit von meist europäischen Freiwilligen des Friedenscorps ab 1964 und vor allem ihre Arbeit mit der FICSHA selbst ab 1968 trug zur Säkularisierung der Organisation und der Öffnung ihrer ideologischen Position bei (Gnerre/Bottasso 1985: 19). So wurden die Shuar-Zentren der Ausgangspunkt für eine regionale ethnische Bewegung (Ruiz 1991: 472). Die anfängliche Zielsetzung, die Shuar vor dem Einfluss mestizischer Siedler zu schützen, das Territorium der Shuar zu verteidigen (CONAIE 1989: 91; Kaltmeier 2009: 353) und den Einfluss der katholischen Kirche und des salesianischen Ordens zu erhalten, verschob sich hin zu einer Verteidigung der kulturellen Identität (Chiriboga 1986: 83). Diese Veränderungen führten in den 1970ern zu einer Annäherung der Organisation an andere soziale Bewegungen im Land, darunter CEDOC und FENOC, denen sie von spätestens 1973 (CEDOC 1976: 26; FENOCIN 1999: 32) bis Ende der 1970er angehörte (Gnerre/Bottasso 
1985: 19) ${ }^{32}$. Diese Annäherung drückte sich im dritten Nationalen Bauerntreffen (Encuentro Nacional Campesino) in Sucúa im November 1973 aus, an dem Vertreter der meisten Bauernorganisationen Ecuadors teilnahmen und über die Situation der Landarbeiter und die Agrargesetzgebung diskutierten (Zallez/Gortaire 1978: 79; FENOCIN 2004: 11). Die FICSHA war schon früh erfolgreich in der Suche nach Unterstützung bei staatlichen und internationalen Organisationen. So konnte sie einige Entwicklungs- und Bildungsprojekte aufbauen und das Führungspersonal technisch fortbilden, das später diese Projekte kontrollieren sollte (Porras 2005: 99).

Man kann durchaus von einer „Ethnisierung des Politischen“ (Büschges 2009: 45f.) sprechen. Diese frühe Entwicklung hat auch mit der geographischen Lage der Shuar zu tun:

„Obwohl der Kampf der Shuar zunächst ökonomischen und sozialen Überlegungen folgte (Erhaltung des Landes ihrer Vorfahren, Zugang zu produktiven Ressourcen), kann er keinesfalls als reiner ,Klassenkampf' bezeichnet werden, sondern er unterscheidet sich von Landverteilungskämpfen, die gleichzeitig zwischen Bauern und Großgrundbesitzern in den Andenhochländern stattfanden. Indem die Shuar und andere indigene Tieflandvölker nicht eindeutig in eine agrarische Klassenstruktur eingebunden waren, trug ihre Organisation eher kommunale und ethnische Züge als die eher klassenorientierten Bewegungen indigener Bauern in anderen Teilen Lateinamerikas.“ (Stavenhagen 1997: 18)

Eine der wichtigsten Errungenschaften der Shuar-Zentren ist der Aufbau von Radiostationen und -programmen. Dazu gehört eine Station, die seit 1966 in Riobamba als Teil der Radiophonen Volksschulen (Escuelas Radiofónicas Populares) und seit 1968 als Radio Föderation (Radio Federación) mit einem eigenen kleinen Sender (Zallez/Gortaire 1978: 65) ein simultan zweisprachiges Programm sendet, seit 1970 nur noch auf Shuar. Die Generalversammlung der FICSHA wollte so Sprache und Kultur der Shuar vor allem unter der jungen Generation verbreiten (Rodríguez 1982: 200f.). Ab 1971 wird ein Sender des Radio Föderation im Hauptgebäude der FICSHA in Betrieb genommen, der schon in den 1980er 16 Stunden täglich auf mehreren Kanälen sendet und vollständig von Shuar-Personal betrieben wird. Aus diesen Radiostationen entstehen schließlich die zweisprachigen Radio-Schulen, die seit 1972/73 in Betrieb sind (Federación de Centros Shuar 1976: 127). Dieses SERBISH (Shuar-System der bikulturellen radiophonen Erziehung/Sistema de Educación Radiofónica Bicultural Shuar) genannte System begann als zweisprachige Alphabetisierungskampagne und wurde schrittweise ausgebaut (Lazos/Lenz

32 Nach Angaben von Zallez/Gortaire gehörte die FICSHA weder FENOC oder CEDOC noch sonstigen politischen Organisationen an, unterhielt mit der CEDOC jedoch eine „brüderliche Beziehung“(Zallez/Gortaire 1978: 79). 
2004: 7f.). Mit Unterstützung der katholischen Kirche wurde ein komplettes Erziehungssystem - mit dem Fokus auf Bildung auf Grundschulniveau - über Radio aufgebaut (Moya 1987: 394). Diese Infrastruktur erlaubt nicht nur eine grundlegende Schulbildung für die oft isoliert lebenden Shuar, sondern trägt auch zur Aus- und Weiterbildung der Mitglieder und politischen Führer der Assoziationen bei. Es soll eine Bildung ermöglicht werden, die ein Bewusstsein der spezifischen Probleme der Shuar erlaubt und eine Wertschätzung der eigenen Kultur und eine Teilhabe an der Gesellschaft als Träger dieser Kultur fördert (CONAIE 1989: 92). Dabei wird das Kursprogramm von den mit mindestens Abitur qualifizierten Fachkräften im Sender zusammengestellt und aufgezeichnet und vor Ort in den Zentren von einer Hilfskraft der Schulklasse präsentiert (Salazar 1981: 69). 1972 erreichte das Programm 31 Shuar-Zentren und hatte 4 Lehrer und 40 Hilfskräfte, die Unterrichtsmaterial für zwei Kurse zusammenstellten und die Unterstützung des Bildungsministeriums gewinnen konnten. 1975 umfasste das SERBISH 13 Radio-Schulen mit 10 Lehrer, 230 Hilfskräften und 3.109 Schülern - das sind zwei Drittel der Shuar im Grundschulalter - die bis zum vierten Schuljahr unterrichtet werden konnten ${ }^{33}$. 1976 wurden 180 Schulräume verwendet, die in Zusammenarbeit mit dem Bildungsministerium gebaut worden waren, 140 Mittelschüler und 15 Universitätsstudenten erhielten Stipendien. 1978 wurde eine Radio-Sekundarschule eingerichtet (Rodríguez 1982: 200f.). Ende der 1970er Jahre wurden 138 Radioschulen mit etwa 3.500 Schülern betrieben (Salazar 1981: 69). Die Radioschulen der FICSHA wurden 1979 vom Staat anerkannt und 1988 in die DINEIB integriert, ohne dadurch ihre Autonomie zu verlieren (Lazos/Lenz 2004: 7f.). Kurz vor der Integration umfasste das Schulsystem der FICSHA 163 Schulen mit 3.876 Schülern und ungefähr 100 Lehrer und Hilfskräfte (Chiriboga 1987: 97).

Eine wichtige Rolle bei der Verteidigung der Identität der Shuar spielte die Politik der FICSHA, eigene Verlage zu gründen. Seit 1972 wird ihre zweisprachige Verbandszeitung CHICHAM (deutsch: Nachricht) veröffentlicht, die über die Aktivitäten der Shuar-Organisationen und weitere Themen in Bereich indigene Völker und indigene Bewegungen in Ecuador und Südamerika berichtet, sowie eine Textreihe in Shuar über verschiedene Themen (Salazar 1981: 70; Rodríguez 1982: 200f.). 1975 wurde von der Föderation und den Salesianern unter Juan Bottasso (Bottasso 2003: 25; Abya-Yala o.J.) der Verlag Mundo Shuar gegründet, der eine Reihe von Büchern über die Shuar und andere Völker des Amazonasgebietes veröffentlichte. Dadurch sollte das kulturelle Erbe der Shuar bewahrt und bekanntgemacht werden (Becker 1997: 330) - die Verlage spielten aber auch eine wichtige Rolle bei der Verbreitung von Informationen über die indigenen Organisa-

33 Nach anderen Daten 99 Schulen mit 133 Hilfskräften und 2023 Schülern, die 47\% der schulpflichtigen Shuar entsprechen (Zallez/Gortaire 1978: 71). 
tionen und deren Forderungen. Bis 1983 veröffentlicht Mundo Shuar 140 Bücher in 6 Serien, darunter anthropologische Studien, Übersetzungen und eine kleine Enzyklopädie über die Shuar. 1980 wird, begünstigt vom Wachstum der Indigenenorganisationen des Hochlandes, der Verlag Mundo Andino gegründet, der sich den anderen indigenen Völkern des Landes widmete. Bis 1983 hatte der neue Verlag bereits 60 Bücher herausgegeben (Abya-Yala o.J.). Im gleichen Jahr (Dietz 1996: 1; Diario Hoy 2000; Bottasso 2003: 25) werden die beiden Verlage unter dem Namen AbyaYala $^{34}$ zusammengeschlossen. Der neue Verlag entwickelte sich schnell. 1995 veröffentlichte er unter dem Geschäftsleiter José Juncosa mit mehr als 100 Titeln schon 20\% aller in Ecuador veröffentlichten Publikationen (Dietz 1996: 1f.). AbyaYala ist heute einer der größten sozialwissenschaftlichen Verlage Lateinamerikas und verantwortlich für 70\% der Publikationen Ecuadors. Juan Bottasso ist weiterhin sein Präsident (Abya-Yala o.J.) und Abya-Yala gehört weiterhin den Salesianern (Dietz 1996: 1).

Ein wichtiges Projekt der 1970er und 1980er Jahre war die Korrektur der Eintragungen der Shuar im Standesamt (Registro Civil) und der ausgegebenen Personalausweise, die sehr viele Fehler enthielten. Der FICSHA wurde ein eigenes Büro zur Ausstellung von Ausweisen gewährt, das innerhalb weniger Jahre die meisten Fehler des Standesamtes korrigieren konnte (Zallez/Gortaire 1978: 69; Salazar 1981: 75).

Die FICSHA erlebte ein schnelles Wachstum. Mitte der 1970er waren 154 Zentren in 13 regionalen Assoziationen und damit 20.040 Einzelpersonen (Zallez/Gortaire 1978: 48-50, 61) in ihr Mitglied, Ende der 1970er 138 Zentren in 14 Assoziationen und 26.800 Einzelpersonen (Salazar 1981: 66). Anfang der 1980er umfasste sie 240 Zentren mit insgesamt 30.000 Personen (Conterón/de Viteri 1984: 31). Mitte der 1980er Jahre organisierte sie schon 35.000 der insgesamt 40.000 Shuar (Chiriboga 1986: 84) und Anfang der 1990er waren 263 Zentren in ihr organisiert (León 1991: 389). Den Daten der Organisation selbst zufolge ${ }^{35}$ umfasst sie $^{2}$ heute 500 Zentren in 50 Assoziationen und insgesamt 120.000 Mitglieder.

Die FICSHA kann als Ausgangspunkt für die Entwicklung der modernen Indigenenbewegung in Ecuador gesehen werden. Sie war die erste Organisation, die offen eine wirtschaftliche und politische Autonomie forderte und die Aufwertung der kulturellen Identität der in ihr vereinigten Indigenen betrieb (Zallez/Gortaire 1978: 3; Salazar 1981: 76). Die jungen Shuar, die in diesem Umfeld aufwuchsen, verbreiteten ihre politischen Ideen auch im Andenhochland, in dem einige von ihnen über staatliche Stipendien studierten. Das wiederum hatte starken Einfluss auf die bereits bestehenden, meist sozialistisch orientierten Organisationen dort (Sel-

34 Zur Genealogie des Begriffes Abya Yala siehe Kapitel III.6.

35 Nach ihrer Webseite. 
verston 1992: 13). Selverston stellt diesen ,amazonischen Faktor“ (Selverston 1992: 13) dem „Otavalo-Faktor“ (ebd.) gegenüber, der über wirtschaftliche Erfolg und nicht konkrete Bedrängung etwa durch erdölfördernde Firmen - zu Ideen kultureller Unabhängigkeit führt.

In den 1960er und 1970er Jahren wurden im Amazonasgebiet weitere Organisationen mit ähnlicher Stoßrichtung (Ramón 1992: 84) gegründet. Im Gegensatz zum Andengebiet war hier die ethnische Identität der Faktor, der eine Mobilisierung jenseits lokaler Strukturen erlaubte (Stavenhagen 1997: 18). Während dort die ungleiche Verteilung des Landes der wichtigste Konflikt war, wurde das Amazonasgebiet durch Besiedlung, Erdölförderung und industrielle Plantagen bedroht; der Kampf der Organisationen dort war also nicht für eine Agrarreform, sondern für eine Verteidigung des - ethnisch definierten - Territoriums (Viteri 1983: 44; Ruiz 1991: 472; Rodríguez 2008: 95). Dazu gehörte auch die Abwehr der Missionierungsversuche religiöser Sekten, vor allem des Linguistischen Sommerinstituts (Chiriboga 1986: 84).

Als Reaktion der Gründung der FICSHA durch die Salesianer wurde 1963 von der Evangelistischen Missionsunion (Unión Misionera Evangelista) die Entwicklungsassoziation Jíbaros des Ostens (Asociación de Desarrollo Jíbaros del Oriente, ADJOE) gegründet, die den katholischen Einfluss auf die Shuar begrenzen sollte. 1976 löste sich die ADJOE von ihren Gründern und veränderte ihren Namen in Unabhängige Assoziation des Ecuadorianischen Shuar-Volkes (Asociación Independiente del Pueblo Shuar Ecuatoriano, AIPSE). Von diesem Zeitpunkt an fand eine Annäherung der beiden Shuar-Organisationen statt, die 1986 in einem Abkommen mündete (Fontaine 2007: 232).

In den 1960ern gründeten die Kichwa der Provinz Napo eine Organisation, die seit 1973 Föderation der indigenen Organisationen Napos (Federación de Organizaciones Indígenas del Napo, FOIN) heißt. Diese war bis 1980 ein aktives Mitglied der FENOC (CONAIE 1989: 50; Tamayo 1996: 64). Im Laufe der 1970er Jahre erweiterte sich ihr Programm von einer reinen Verteidigung des Landes der Indigenen hin zu einer Verteidigung ihrer Kultur und somit einem Widerstand gegen das Eindringen der Nationalkultur und ihrer Ideologie der Entwicklung und mestizaje in Napo (Black 1999: 22). 1978 wurden von weiteren Kichwa des Amazonasgebietes die Föderation der Kommunen Union der Eingeborenen des Ecuadorianischen Amazonasgebietes (Federación de Comunas Unión de Nativos de la Amazonía Ecuatoriana, FCUNAE) ${ }^{36}$ und die Föderation der indigenen Organisationen Su-

36 Nach den Angaben auf der Internetseite der FCUNAE wurde sie 1975 gegründet und 1978 staatlich anerkannt. 
cumbíos (Federación de Organizaciones Indígenas de Sucumbíos, FOISE) ${ }^{37}$ gegründet, 1979 die Organisation indigener Völker Pastazas (Organización de Pueblos Indígenas de Pastaza, OPIP) (Ruiz 1991: 473; Albó 2009: 210). Die OPIP widmet sich der Verteidigung der Landrechte der Indigenen und fordert einen verbesserten Schutz der Umwelt und der Naturschätze der Provinz. Darüber hinaus kämpft sie für eine gerechte Gesellschaft ohne Rassismus, an der die Indigenen als Gleiche mit eigenen Rechten teilhaben können (Viteri 1983: 45/47). Sie war seit ihrer Gründung sehr aktiv mit Forderungen nach einer Autonomie der Territorien der Indigenen, so forderte sie im August 1990 von der ecuadorianischen Regierung die Übergabe von $90 \%$ des Landes im Osten Pastazas an die Indigenen. Das hätte nicht nur eine innere politische Autonomie bedeutet, sondern auch eine Ende der Erdölförderung in der Region (Becker 1997: 331).

Auf den Ersten Kongress der indigenen Nationalitäten des Amazonasgebietes in Puyo/Pastaza im August 1980 (Black 1999: 23) gründeten diese die Konföderation der indigenen Nationalitäten des ecuadorianischen Amazonasgebietes (Confederación de Nacionalidades Indígenas de la Amazonía Ecuatoriana, CONFENIAE) ${ }^{38}$, die noch im selben Jahr zusammen mit ECUARUNARI den Koordinationsrat der indigenen Nationalitäten Ecuadors (Consejo de Coordinación de las Nacionalidades Indígenas del Ecuador, CONACNIE) aufbaute (CONAIE 1989: 50). Die CONFENIAE sollte neben den konkreten Forderungen ihrer Mitgliedsorganisationen abstraktere ,politische Aspekte kanalisieren“ (Viteri 1983: 44). Auf ihrem zweiten Kongress 1982 begann die CONFENIAE, ein Programm zu entwickeln, dass über den Verweis auf die Selbstbestimmung über Kultur und Ressourcen eine Verbindung von lokalen materiellen Forderungen und Kämpfen um Bedeutung und Identität vorbereitete. So forderte die CONFENIAE die rechtliche Anerkennung der indigenen Territorien durch die Regierung, einen Ausbau der zweisprachigen Erziehung als Mittel zum Schutz der indigenen Sprachen und Kulturen, einen Schutz der indigenen Medizin und eine Stärkung und Einigung der autonomen indigenen Organisationen (Black 1999: 24). Auf beiden Kongressen wurde Alfredo Viteri zum Präsidenten der CONFENIAE gewählt (Viteri 1983: 44). Die CONFENIAE gründete 1984 die regionale Koordination der indigenen Organisationen des Amazonasbeckens (Coordinadora de las Organizaciones Indígenas de la Cuenca Amazónica, COICA) mit (GTZ 2004: 33), die Organisationen aus Peru, Bolivien, Brasilien, Kolumbien, Venezuela und den Guayanas umfasst. Die COICA beschäftigt

37 Nicht etwa Föderation der indigenen Nationalitäten Sucumbios (Federación de Nacionalidades Indígenas de Sucumbíos), wie Albó (2009: 210) meint.

38 Seit dem 14. Kongress der amazonischen Nationalitäten im September 2013 heißt diese Organisation Regierung der Ureinwohnernationen des ecuadorianischen Amazonasgebietes (Gobierno de las Naciones Originarias de la Amazonía Ecuatoriana, GONOAE). 
sich unter zwischen den Organisationen wechselnder Führung mit den gemeinsamen Problemen (Albó 2009: 323) und war eine der Plattformen für das Wiederaufkommen von Forderungen nach territorialer Autonomie im Sinne der indigenen Gemeinschaften in Südamerika (Quijano 2006a: 33).

Die FICSH und die anderen Organisationen des Amazonasgebietes kämpfen vor allem für ihre Selbstbestimmung und Autonomie, Forderungen, die sie in einer „neuen Konzeption eines pluralistischen ecuadorianischen Staates“ (Federación de Centros Shuar 1976: 129) verwirklichen wollen. Der Staat soll die indigenen Völker und ihre - ethnisch begründeten - spezifischen Interessen anerkennen und sich dem Kulturell-Ethnischen im Sinne eines "pluriethnischen und multinationalen Staates“ (Chiriboga 1986: 85) öffnen. Diese Stärkung der Ethnizität und der wirtschaftlichen Eigenständigkeit soll keinesfalls ausschließlich über eine Modernisierung erfolgen, wie Verdesoto (1986: 42) behauptet, sondern ist - in einem ersten Schritt - eine Kritik der staatlich gesteuerten, kapitalistisch geprägten Modernisierung. Die ideologische Entwicklung der amazonischen Organisationen sollte der Indigenenbewegung auf nationalem Niveau zugutekommen. Die Begriffe des Territoriums und der Nationalität in ihren aktuellen Ausprägungen wurden in den 1970er Jahren auch von den Organisationen, die später die CONFENIAE bilden sollten, begründet (Ruiz 1991: 476). Schon die - im Vergleich zur ECUARUNARI - enorme ethnische Vielfalt der CONFENIAE machte eine Begrifflichkeit der Differenz, als Grundlage etwa der Interkulturalität und der Plurinationalität, nötig (Rodríguez 2008: 95; Santillana/Herrera 2009: 332).

\section{b. ECUARUNARI}

Der Aufbau von ECUARUNARI beginnt bereits in den 1960er Jahren. Sie speist sich aus drei verschiedenen Quellen: Organisationen der linken Parteien, Organisationen der progressiven katholischen Kirche und aus autonomen Prozessen der indigenen Gemeinschaften (Simbaña 2009: 159). Schon zu Beginn der 1960er Jahre - noch vor der ersten Agrarreform von 1964 - verändert sich die Politik der katholischen Kirche hin zu einem sozialen Ansatz, was sich durch eine ,private“ (ECUARUNARI o. J.: 1) Agrarreform in den Ländereien der Kirche unter Mitwirkung der Ecuadorianischen Zentrale der landwirtschaftlichen Dienstleistungen (Central Ecuatoriana de Servicios Agrícolas, CESA) ausdrückte. Dieser Wandel steht im Zusammenhang mit der kubanischen Revolution und der wahrgenommenen Stärke der linken Parteien ebenso wie mit der Befreiungstheologie und den Ansätzen des zweiten vatikanischen Konzils (1962-1965) und der II. Generalversammlung des Lateinamerikanischen Episkopats von Medellín (1968), die mit der ersten und zweiten Konvention der Presbyterianer (Convención de Presbíteros) 1970 und 1971 von den progressiven Kräften der katholischen Kirche in Ecuador übernommen werden. 
Die Bischöfe beschließen, die Forderungen der Indigenen und Bauern zu unterstützen. Sie fördern Alphabetisierungskampagnen, Gesundheitsprogramme, lokale Entwicklung und politische Bildung (Rodríguez 2008: 88). Die Internationale Bewegung der katholischen Landjugend (Movimiento Internacional de Juventudes Agrarias Católicas, MIJARC), eine Organisation der Option für die Armen (Botero 1998: 10), arbeitet seit Mitte der 1960er Jahre mit indigenen Gemeinschaften unter anderem in Riobamba, Cañar, Tungurahua (León 1994: 72f.), ursprünglich, um eine eher ethnisch ausgerichtete Parallelorganisation zur FEI und zur sich nach links bewegenden FENOC aufzubauen und so deren Einfluss zu beschränken (MinknerBrünjer 2009: 139f.; Becker 2009: 1056). Die MIJARC organisierte zusammen mit der Organisation Kirche und Gesellschaft in Lateinamerika (Iglesia y Sociedad en América Latina, ISAL) und der Unterstützung der Kirche von Chimborazo unter Bischof Leónidas Proaño ${ }^{39}$ (Albó 2009: 208) die erste nationale Konferenz der Indigenen und Bauern in Quito (ECUARUNARI o. J.: 1).

Die Politik der Militärregierung, die Indigenen in die vermeintliche Mehrheitsgesellschaft zu integrieren, führte zu einer Stärkung der regionalen und lokalen Indigenenorganisationen, die - im Gegensatz zu den nationalen Organisationen der Zeit, FEI und FENOC - ethnische Forderungen entwickelten (Minkner-Brünjer 2009: 139f.). Eine Reihe von gewerkschaftlichen Organisationen, die sich von den nationalen Gewerkschaftsverbänden nicht vertreten fühlten, näherten sich diesem Prozess an (Simbaña I-2). Dazu kommt die Unzufriedenheit mit der Agrarreform von 1964 und die Forderung nach einer weiteren, wirklichen Agrarreform (Simbaña 2009: 158). Der gestiegene staatliche Einfluss durch die Agrarreform förderte die Prozesse der Selbstorganisation der Indigenen. Diese griffen die diskursiven und organisatorischen Anreize der kommunistischen und klerikalen Mobilisierungsversuche auf, um so ihre eigenen Strukturen zu stärken (Ramírez Gallegos 2009: 68). ECUARUNARI baut mehr als die anderen Organisationen der Indigenenbewegung auf das System der Kommunen, das in den 1930er Jahren eingerichtet wurde und den indigenen Gemeinden eine gewisse Autonomie erlaubt (Simbaña I-2).

39 Leonidas Proaño war von 1954 bis 1985 Bischof von Riobamba und von 1985 bis zu seinem Tod 1988 Präsident der Abteilung für Indigene der Ecuadorianischen Bischofskonferenz. Von Beginn an arbeitete er mit den Indigenen und organisierte die Übergabe des kirchlichen Landbesitzes in Riobamba an die indigenen Gemeinden bis 1960 und den Aufbau der Volks-Radioschulen (Escuelas Radiofónicas Populares, ERPE) ab 1964, die der Alphabetisierung der Indigenen in deren Muttersprachen dienten (Krainer 1996: 43). 1988 gründete er die Stiftung Indio-Volk (Fundación Pueblo Indio), die neben der Weiterbildung von indigenen Geistlichen und Laien auch Forschung zu den indigenen Völkern betreibt (Espinosa 1992: 185f.). 
Der Gründungskongress von ECUARUNARI (Ecuador Runacunapac Riccharimui, Das Erwachen der ecuadorianischen Indigenen) fand im Juni 1972 in der Kommune Tepeyac der Provinz Chimborazo unter Teilnahme von mehr als 200 Repräsentanten verschiedener indigener Organisationen (León 1991: 392; León 1994: 72f.) aus Imbabura, Pichincha, Cotopaxi, Bolívar, Chimborazo und Cañar statt (CONAIE 1989: 215; ECUARUNARI o. J.: 1). Es gab dort Auseinandersetzungen zwischen einer Position, die eine integrative Bewegung von Indigenen und Nicht-Indigenen befürwortete, und einer anderen, von den Geistlichen gestützten, Position, dass ECUARUNARI eine rein indigene Organisation sein sollte. Die zweite Strömung konnte sich durchsetzen (Moreno/Figueroa 1992: 46f.), ECUARUNARI definierte sich ,als eine INDIGENE Bewegung, deren Hauptziel es sein wird, in der indigenen Bevölkerung eine Bewusstwerdung zu ermöglichen, um eine soziale, wirtschaftliche und politische Erholung zu erreichen" (ECUARUNARI o. J.: 1$)^{40}$. Die Organisation sollte ein eigenständiger Ausdruck der Indigenen sein, mit Führern aus den indigenen Gemeinden. „Ihr Hauptziel war es, die indigene Kultur aufzuwerten, sie zu verteidigen und von dort den Kampf für Land zu beginnen.“ (Chancoso 1983: 41) ECUARUNARI gewinnt ein ethnisches Profil (Rodríguez 2008: 89) ${ }^{41}$, das von den klassischen Gewerkschaftlern und linken Parteien heftig kritisiert wurde (Santana 1983: 166). So bekam der Kampf für eine Agrarreform für ECUARUNARI eine kulturelle Bedeutung. Die Forderung nach Land war nicht mehr nur eine wirtschaftliche, das Land war jetzt zentral für die Reproduktion der indigenen Gemeinschaften (Chancoso 1983: 41). Auch der Kampf für die Anerkennung der indigenen Sprachen in den jeweiligen Verbreitungsgebieten war von Beginn an eine wichtige Forderung (Chancoso 1983: 42). Die ethnischen Inhalte, die die Kirche förderte, standen im Gegensatz zu einem Klassenbewusstsein als Bauern und Landarbeiter (León 1991: 392), wodurch sich die Indigenenbewegung weiter von den linken Parteien entfernte. Die Provinzen, die die Klassenanalyse unterstützen, zeichnen sich meist durch eine kleinere indigene Bevölkerung aus, die relativ stark in die mestizische Gesellschaft integriert ist, während die in Kommunensystem strukturierten Bevölkerungen eher eine ethnische Politik befürworten. Dieser Konflikt, und der Versuch, beide Positionen zu integrieren, prägt ECUARUNARI bis heute und führt immer wieder zu Auseinandersetzungen (León 1991: 393). Auch um die Frage der Beziehungen der Organisation zur Kirche gab es Konflikte, in denen die Strömung, die sich „,völlig mit der Kirche identifiziert“ (ECUARUNARI o. J.: 1) durchsetzen konnte. Es wurde beschlossen, dass ECUARUNARI

40 Dennoch waren von Beginn an auch nicht-indigene Bauern Mitglied in ECUARUNARI (Chancoso 1983: 41; Conterón/de Viteri 1984: 16).

41 Damit ist Ospina (2008: 71) widerlegt, der schreibt, dass die ursprüngliche Zielrichtung umstritten ist und ECUARUNARI sich erst zu einer ethnischen Organisation entwickelt. 
eine klerikale Organisation mit geistlichen Beratern in jeder OZG sein sollte (ECUARUNARI o. J.: 2), ähnlich der FICSHA (Rodríguez 2008: 89). Seit ihrer Gründung bildete ECUARUNARI in allen Provinzen des Andenhochlandes Filialen, die huahua riccharimui (Das Erwachen der Kinder) genannt wurden (León 1991: 406f.; Albó 2009: 208). Die erste Generalsekretärin war Blanca Chancoso (Albó 2009: 208).

Schon früh versuchte ECUARUNARI, ihre ethnische Sichtweise mit den Ansätzen der anderen Organisationen der Bauern und Landarbeiter zu verbinden (Tamayo 1996: 65). So forderte sie die Verteidigung von und den Respekt für Identität, Sprachen und Traditionen der indigenen Völker und zugleich eine neue Agrarreform (Tamayo 1996: 91). Dabei betrachtete die ECUARUNARI ihre Konkurrenzorganisationen als ,von ,fremden Ideologien“ manipuliert oder den ,Bürokratien“ der Gewerkschaftszentralen untergeordnet“ (Moreno/Figueroa 1992: 46f.). Sie war die erste Organisation, die eine wirkliche Kombination von ethnischen und klassistischen Aspekten in ihrem Programm anstrebte (Botero 2000: 68) - und brachte damit die klassischen Organisationen in Schwierigkeiten (Botero 1998: 10). ECUARUNARI verstand sich als eine Organisation der Kichwa, die mit einem bestimmten Territorium verbunden sind, kombinierte also einen klassistischen Diskurs mit ethnischen Begriffen (Pacari 2007: 21). Später bewegte sich ECUARUNARI weiter in Richtung ethnischer Forderungen und brach - teilweise - mit ihrem klassistischen Diskurs, um so besser auf die Lage der Indigenen eingehen zu können (León 1991: 401). Gerade die Öffnung hin zu ethnischen und identitären Inhalten erweiterte auch das Spektrum der klassischen Linken. Die Begriffe der kulturellen Differenz und der ethnischen Diversität - im später entwickelten Konzept der Plurinationalität artikuliert- und ihre Verbindung zu Forderungen nach Umverteilung und sozialer Gerechtigkeit brachte die Indigenenbewegung in eine Avantgarde-Position im Vergleich mit den anderen sozialen Bewegungen (Ramírez Gallegos 2009: 65). Über ihr Verständnis der Beziehung zwischen Indigenen und Mutter Erde kam ECUARUNARI zur Forderung, eine Territorialität für die indigenen Nationalitäten zu erobern, um so das Weiterbestehen der Indigenen zu garantieren (Santana 1992: 194).

Auf ihrem ersten Kongress beschloss ECUARUNARI, dass die Aufgabe der Organisation die Schaffung eines Bewusstseins der ecuadorianischen Indigenen sei, damit sie sich ,als eine einzige Masse erheben“ (ECUARUNARI o. J.: 2). Die Organisation bekennt sich zu friedlichen Aktionen, nicht ohne sich die Möglichkeit einer Revision dieses Punktes in besonderen Situationen offen zu halten. Sie versteht sich von Beginn an als eine basisdemokratische Organisation: ,Es soll die Basis sein, die die Bewegung trägt.“ (ECUARUNARI o. J.: 2) ECUARUNARI versteht sich als Repräsentation der Indigenen, womit sie die Vertretung der Indigenen durch Nicht-Indigene - etwa in der CTE oder PCE - kritisierte (Guamán 2003: 
83). Sie kämpft für Land, gegen Diskriminierung, für Selbstrespekt, für die offizielle Anerkennung der Kichwa als Volk und ihr Recht, eigene Autoritäten zu ernennen. Sie versucht weiterhin, die indigene Identität zu definieren und die Geschichte und Kultur ihres Volkes wiederzuentdecken (León 1994: 72f.). Somit war ECUARUNARI die erste Organisation, die nicht nur dafür kämpft, dass Indigene , volle“ Staatsbürger sein können - also für Gleichheit -, sondern auch dafür, dass ihre Unterschiede anerkannt werden - also für Differenz (ECUARUNARI/CONAIE 1989: 40).

Trotz dieser eigenständigen Politik sucht ECUARUNARI nach Allianzen innerhalb der Indigenen- und Landarbeiterbewegung. Sie versteht ihren Kampf als einen für die Unterdrückten, der nur mit vereinten Kräften zu gewinnen ist (ECUARUNARI/CONAIE 1989: 41). Daher kooperierte sie bei allgemeinen sozialen Forderungen - vor allem bei Aktionen für eine neue Landreform (ECUARUNARI o. J.: 2) - mit der FENOC (Barre 1983: 150). Beide Organisationen führten große Demonstrationen auf nationalem Niveau durch und forderten vom Staat eine Umverteilung des Reichtums (Chiriboga 1986: 68). Als 1973 das neue Agrarreformgesetz verkündet wurde, widmete sich ECUARUNARI dem Studium der neuen rechtlichen Lage, um so die Landforderungen ihrer Gemeinden vor Gericht und über Petitionen durchsetzen zu können. Zur gleichen Zeit hält sie den Druck auf den Staat mit Landbesetzungen aufrecht. Dieses Engagement in sozialen Fragen bedeutete aber keinesfalls eine Abschwächung der ethnischen Ausrichtung der Organisation, die von 1972 bis 1975 nicht nur den Kampf für eine effektive Landverteilung verschärfte, sondern auch großangelegte Aktionen für politische Rechte und eine größere Teilhabe der Indigenen an Entscheidungsprozessen durchführte. Bei diesen Mobilisierungen stützte sich die ECUARUNARI auf die traditionellen indigenen Strukturen - vor allem die Kommunen -, die ihre Basis ausmachen, und schloss sie in ihren Forderungskatalog ein - etwas, das die Gewerkschaften vernachlässigt hatten (Botero 2000: 61f.). Auch wegen dieser Linie traten einige Basisorganisationen der in einer Krise befindlichen FENOC zur ECUARUNARI über (Ibarra Illanez 1987: 95).

Zwischen dem ersten und dem zweiten Kongress 1975 verschärften sich die Spannungen, die schon bei der Gründung der ECUARUNARI bestanden und führten zu einer tiefen Krise. Der zweite Kongress der ECUARUNARI findet im Juli 1975 in Ambato unter Teilnahme von ungefähr 120 Abgeordneten aus den acht Provinzen Cañar, Loja, Chimborazo, Tungurahua, Pichincha, Imbabura, Azuay und Bolívar statt (ECUARUNARI o. J.: 2-3). Es wurde die institutionelle Trennung von der katholischen Kirche beschlossen (Ibarra Illanez 1987: 95). Um die Krise zu beheben wurden, statt einer nationalen Führung, drei Führer für die drei geographischen Zonen Nord, Mitte, Süd, mit jeweils unterschiedlichen Aufgabenbereichen (Nord: Bildung, Mitte: Finanzen, Süd: Organisation) bestimmt (ECUARUNARI o. 
J.: 3). Diese Strategie war allerdings nicht hilfreich, denn bereits ein Jahr darauf, im Februar 1976, rief ein Teil von ECUARUNARI zu einem dritten nationalen Treffen auf, das in Pichincha stattfand ${ }^{42}$. Da einige Provinzorganisationen, wie die aus Tungurahua und Loja, und die Mehrheit der Mitglieder der ECUARUNARI den Kongress nicht anerkannten, wurde auch das neu gewählte nationale Direktorium nicht akzeptiert. Um diese Probleme zu überwinden, fand kurz darauf ein nationales Treffen des Führungspersonals in der Provinz Bolívar statt, auf dem die Durchführung eines vierten Kongresses beschlossen wurde. Dieser fand im Juli 1977 in Chibuleo, Tungurahua statt. Es nahmen ungefähr 180 Bauern teil, von denen 100 offizielle Delegierte der Provinzen Carchi, Imbabura, Pichincha, Tungurahua, Chimborazo, Cañar, Azuay und Bolívar waren (ECUARUNARI o. J.: 3). Auf diesem Kongress bewegte sich ECUARUNARI zu einer eher klassistischen Politik, wodurch sie sich weiter der Gewerkschaftsbewegung annäherte (ECUARUNARI o. J.: 4; Becker 1997: 336). Eine „marxisierende [sic] christliche Linke“ (Santana 1983: 166) mit Basis in Pichincha und Azuay setzte sich gegen die ethnisch orientierten Gruppen durch (Santana 1983: 166).

Die neue, klassistisch orientierte Linie führte zu verstärkten Kooperationen mit anderen, nicht-ethnisch ausgerichteten Organisationen. Dazu gehörte eine Annäherung an die Gewerkschaften der FUT, der ECUARUNARI beitreten wollte, um so größere Unterstützung für ihre Forderungen zu gewinnen. Die Gewerkschaften, allen voran die CTE, lehnten einen Beitritt ECUARUNARIs allerdings mit der Begründung ab, es gäbe mit ihrer Landarbeitergewerkschaft FEI bereits eine vergleichbare Organisation, die über ihre Mitgliedschaft in der CTE Mitglied der FUT ist. In diesem Sinne wurde der ECUARUNARI geraten, sich einer der Gewerkschaften der FUT anzuschließen. Später, als die Stärke der Indigenenbewegung offensichtlich wurde, kam es zu Kooperationen zwischen diesen Organisationen, die dazu führten, dass die FUT auch ethnische Forderungen der Indigenenbewegung übernahm (Dávila 1995: 168f.).

Im April 1978 gab es in Columbe, Chimborazo, ein Treffen des Führungspersonals von ECUARUNARI, FENOC und FEI, um die Einheitsfront des Bauernkampfes (Frente Único de Lucha Campesina, FULC) zu gründen, die später Einheitsfront des Bauern- und Indigenenkampfes (Frente Único de Lucha Campesina e Indígena, FULCI) heißen sollte. Diese Plattform forderte die Umsetzung des Agrarreformgesetzes von 1973 und die Abschaffung von einigen Gesetzen, wie dem Landwirtschaftsförderungsgesetz und dem nationalen Sicherheitsgesetz (ECUA-

42 Es ist unklar, ob das der Kongress ist, auf dem, laut Van Cott (2005: 105), nicht-Indigene ausgestoßen wurden. Van Cott spricht von einem Kongress in Riobamba 1976 - einen solchen gab es nicht. Auch ist die Teilnahme von nicht-Indigenen in ECUARUNARI sowohl vor als auch nach diesem Jahr nachweisbar. 
RUNARI o. J.: 4; CONAIE 1989: 220). In diesem Zusammenhang fand im September 1979 der fünfte Kongress von ECUARUNARI in Cuatro de Octubre, Pintag, Pichincha, mit 350 Delegierten aus 10 Provinzen und Vertretern von FEI, FENOC und CTE statt (ECUARUNARI o. J.: 5). Die Organisation wurde durch die Wahl eines nationalen Direktoriums, in dem alle Provinzorganisationen repräsentiert waren, gestärkt (ECUARUNARI o. J.: 6). Dem kam der zuvor erfolgte Aufbau von besseren Kommunikationsstrukturen innerhalb der Organisation zugute - eine Entwicklung, die das weitere Wachstum der ECUARUNARI begünstigte (Rodríguez 2008: 91). Auf diesem Kongress wurde die Stärkung der FULC und weitere Zusammenarbeit mit FENOC und FEI beschlossen, ebenso wie eine Kooperation mit den Organisationen, die Monate später die CONFENIAE bilden sollten und mit Indigenen- und Bauernorganisationen der Region, wie dem Regionalen Indigenen Rat des Cauca (Consejo Regional Indígena del Cauca, CRIC), der Assoziation der ländlichen Nutzer (Asociación de Usuarios Campesinos, ANUC), der Bauernkonföderation Perus (Confederación Campesina del Perú, CCP), und der Konföderation Tupac Katari aus Bolivien (Confederación Túpac Katari) (ECUARUNARI o. J.: 5f.; CONAIE 1989: 222). Die linke, klassistisch geprägte Strömung setzte sich endgültig gegen die ethnisch geprägte Strömung durch, was zu einer kurzzeitigen Schwächung der Organisation führte. ECUARUNARI identifiziert sich mit dem „Klassenkampf [...] zwischen AUSGEBEUTETEN und AUSBEUTERN“ (ECUARUNARI 1989: 88), jenseits von ethnischer Zugehörigkeit. Die ethnischen Organisationen distanzieren sich und 1980 gehen kurzzeitig alle Provinzen - mit Ausnahme Pichinchas - verloren. Dadurch verschwand die ethnische Strömung nicht, sie blieb in den lokalen und überlokalen Organisationen des Andenhochlandes bestehen, ohne aber nationale Bedeutung erlangen zu können (Santana 1983: 166). Dennoch zeichnet sich der fünfte Kongress dadurch aus, dass sowohl indigene, als auch klassistische Forderungen in das Programm aufgenommen wurden, um die „doppelte Dimension des indigenen Problems“ (ECUARUNARI o. J.: 6; CONAIE 1989: 223) erfassen zu können. Themen wie die zweisprachige Erziehung und die Verteidigung der indigenen Kulturen wurden in das Programm der ECUARUNARI integriert (Black 1999: 27). Die organisatorische Krise der ECUARUNARI - zusammen mit dem Aufbau einer nationalen Indigenenorganisation ab 1980 - führte zu einer erneuten Integration der ethnischen Tendenzen (Santana 1992: 98f.), wodurch das Motto der doppelten Dimension mit Leben gefüllt wurde. Die FULCI, als erste gemeinschaftliche Plattform aller Organisationen der Indigenenbewegung (Rodríguez 2008: 91) und der CTE, diente bei der Rückkehr zur Demokratie und in der Phase der Transition von 1978 bis 1984 dazu, die Forderungen der Arbeiter, Bauern und Indigenen sichtbar zu machen (Rodríguez 2008: 91). In diesem Zusammenhang hat ECUARUNARI - zusammen mit der FENOC, der 
PCE und anderen - bis 1984 an der Sammlungsbewegung Breite Linksfront (Frente Amplio de Izquierda, FADI) teilgenommen (Van Cott 2005: 104f.).

Im Rahmen der Alphabetisierungskampagne der Regierung Roldós (1979-1981) verstärkte ECUARUNARI - teilweise in Kooperation mit der FENOC (Moya 1987: 400) - ihre Bemühungen, eine zweisprachige Erziehung für die Indigenen aufzubauen. Im Dezember 1979 fand in Colta, Chimborazo, ein Treffen der Indigenenorganisationen zu diesem Thema statt, auf dem gefordert wurde, dass die Alphabetisierung von den Organisationen selbst durchgeführt werden sollte. Diese sollten Vergangenheit und aktuelle Situation der jeweiligen Völker in die Bildung integrieren. Auch wurde die Betonung der weiteren Forderungen der Indigenenbewegung, wie nach Land und nach Maßnahmen gegen Diskriminierung und Armut, angeregt (ECUARUNARI 1984: 47).

In den 1980er Jahren beginnt ein Prozess der Distanzierung von der Arbeiterbewegung, die bis Ende des Jahrzehnts einen klaren Protagonismus innerhalb der ecuadorianischen Zivilgesellschaft hatte. Daher nimmt ECUARUNARI an den meisten Aktionen der FUT nicht teil, sondern unterstützt sie nur in Kommuniqués (Rodríguez 2008: 94). Eine Ausnahme war der Nationale Indigenen- und Bauernmarsch (Marcha Nacional Indígena y Campesina), Mártires de Aztra ${ }^{43}$ am 16. Oktober 1980, den ECUARUNARI zusammen mit der FENOC gegen das nationale Sicherheitsgesetz und das Landwirtschaftsförderungsgesetz und für eine demokratische Agrarreform unter Kontrolle der Bauern und die Verstaatlichung der Ölreserven durchführte. An dieser Demonstration nahmen über 10.000 Bauern und Indigene teil (ECUARUNARI o. J.: 7; ECUARUNARI 1984: 47f.). Eine der dort aufgestellten Forderungen bezog sich auf die Territorien und Kultur der indigenen Nationalitäten (ECUARUNARI 1998: 99). Im Anschluss wurde der Nationale Koordinierungsrat der indigenen Nationalitäten Ecuadors (Consejo Nacional de Coordinación de las Nacionalidades Indígenas del Ecuador, CONACNIE) gegründet, um zwischen alle indigenen Organisationen des Landes zu vermitteln. 1981 übernahm ECUARUNARI leitende Funktionen in dieser Instanz (ECUARUNARI o. J.: 6; CONAIE 1989: 223). Auch durch die Präsenz von ECUARUNARI und anderen, teilweise ethnisch orientierten Organisationen, beginnt eine ,Ethnisierung des Politischen“ (Büschges 2009: 45f.), den offiziellen Diskurs des Staates zu beeinflussen - eine Entwicklung, die vor allem in der Politik des Präsidenten Jaime Roldós (1979-1981) ihren Ausdruck findet.

Durch diese Entwicklungen erweiterte sich das politische Spektrum der ECUARUNARI. Sie übernahm den Begriff der indigenen Nationalitäten (Chancoso

43 Der Name nimmt Bezug auf einen Streik der Zuckerarbeiter auf der staatlichen hacienda Aztra in der Provinz Cañar am 18.10.1977, der von der Armee blutig niedergeschlagen wurde. Es gab ca. 100 Todesopfer (Huarcaya 2011: 261). 
1983: 41), für deren Befreiung in ,einer neuen Gesellschaft ohne Ausbeutung, ohne Diskriminierung“ (Conterón/de Viteri 1984: 21) sie nun kämpfte. In diesem Sinne forderte sie die Verteidigung der Naturschätze und die Verstaatlichung der gesamten Erdölförderung. Die Gewinne aus den natürlichen Ressourcen sollten an die Indigenen- und Bauernorganisationen gehen. Diese sollten auch an den politischen Entscheidungen auf dem Land teilhaben können, ohne dadurch ihre Autonomie und Unabhängigkeit zu verlieren (Conterón/de Viteri 1984: 24). Auch wenn ECUARUNARI sich weiterhin als ,,antiimperialistische Organisation, die für die endgültige Befreiung unseres Vaterlandes, für eine Gesellschaft ohne Ausbeutung kämpft" (ECUARUNARI 1984: 45) versteht und ihren Gegner im „Imperialistischen Bürgerlichen Staat“ (ECUARUNARI 1984: 46) sieht, der die Indigenen und Bauern in das kapitalistische System integrieren und ausbeuten will (ECUARUNARI 1984: 48), so betont sie nun die Kondition der Indigenen als Nationalität und Klasse und verstärkt ihren Kampf gegen Diskriminierung und für die Verteidigung der indigenen Nationalitäten (ECUARUNARI 1984: 46f.). Sie versteht das Land als „Grundlage für die Entwicklung unserer Nationalität, unserer Kultur, unserer Sprache“ (ECUARUNARI 1984: 44f.). Daraus leitet ECUARUNARI Forderungen nach einer Anerkennung der Territorien der indigenen Nationalitäten in den Küstenregionen und im Amazonasgebiet ab (ECUARUNARI 1984: 48).

Im November 1985 findet in Quito der achte nationale Kongress der ECUARUNARI statt, an dem 510 Delegierte aus 8 Provinzen teilnehmen (ECUARUNARI o. J.: 10) - der letzte Kongress über den detaillierte Informationen vorliegen. Bis zu diesem Zeitpunkt hatte sich die ECUARUNARI nicht vollständig im Andenraum durchsetzen können ${ }^{44}$. Einige Organisationen zogen eine radikalere ethnische Linie vor, die ECUARUNARI zu dieser Zeit nicht bieten konnte. Daher behielten Organisationen der Provinzen Chimborazo, Cotopaxi und Tungurahua ihre Unabhängigkeit, eine Situation, die sich erst mit der Gründung der CONAIE 1986 und einer stärkeren ethnischen Ausrichtung der ECUARUNARI änderte (León 1991: 394). 1988 beantragt ECUARUNARI die Eintragung in das Vereinsregister und damit die Anerkennung als juristische Person, die 1989 gewährt wird (ECUARUNARI 1998: 191).

1996 gründet ECUARUNARI die Escuela Nacional de Formación de Mujeres Líderes ,Dolores Cacuango“, die die indigenen Frauen in Führungspositionen der Organisationen von ECUARUNARI weiterbildet. Ab 1998 wird diese Initiative von der norwegischen Entwicklungshilfeorganisation Norsk Folkehjelp unterstützt

44 Anfang der 1980er Jahre hatte ECUARUNARI auch einige Basisorganisationen im Küstengebiet (Conterón/de Viteri 1984: 16), die mittlerweile anderen Organisationen angehören. 
(Palacios/Chuma 2001; Flores 2005: 104), seit 1999 auch von der dänischen Entwicklungshilfeorganisation IBIS (Palacios/Chuma 2001).

Von besonderer Bedeutung sollte der erste Kongress der Kichwa-Völker Ecuadors von 2003 sein, auf dem die in den 1990ern dominierende ethnische Strömung mit der Wahl Humberto Cholangos zum Präsidenten ECUARUNARIs abgelöst wurde. Der zweite Kongress der Kichwa-Völker Ecuadors von 2006, der die Teilnahme an den Protesten gegen das Freihandelsabkommen das Lucio Gutiérrez (2003-2005) beschloss, markierte das Ende der Krise, die der Beteiligung an der Regierung Gutiérrez 2003 folgte. Ab diesem Zeitpunkt öffnete sich ECUARUNARI der Kooperation mit anderen sozialen Bewegungen, etwa der Schwulen- und der Rocker-Bewegung (Simbaña I-1). Im Juli 2006 wurde von ECUARUNARI, der peruanischen CONACAMI und der bolivianischen CONAMAQ in Cusco die Andine Koordination der indigenen Organisationen (Coordinadora Andina de Organizaciones Indígenas, CAOI) gegründet, der später Organisationen aus Kolumbien, Chile und Argentinien beitraten (Simbaña 2007: 23). Ihr Präsident ist der Repräsentant der CONACAMI, Miguel Palacín. Die CAOI ist im Beratenden Rat der Indigenen Völker der Andinen Gemeinschaft CAN vertreten (Albó 2009: 324).

ECUARUNARI unterstützte - mehr als die anderen CONAIE-Filialen - in den ersten Jahren die Correa-Regierung kritisch. Im Juli 2008 entschied sie, die neue Verfassung im Referendum über ihre Annahme zu unterstützen, wozu sie mit anderen Organisationen die Front für das Ja und den Wandel (Frente por el Sí y el Cambio) bildete. Die Unterstützung der neuen Verfassung bedeutete aber, nach den Aussagen ECUARUNARIs, keinesfalls die kritiklose Unterstützung Correas oder seiner Partei Alianza PAÍS. Die Verfassung wurde als das Ergebnis des langen Kampfes der verschiedenen sozialen Bewegungen verstanden und als solche unterstützt (Becker 2011: 59).

\begin{tabular}{|l|l|l|l|l|l|}
\hline Jahr & $\begin{array}{l}\text { Nummer des } \\
\text { Kongresses }\end{array}$ & $\begin{array}{l}\text { Teilneh- } \\
\text { mer }\end{array}$ & Ort & $\begin{array}{l}\text { Gewählter } \\
\text { Präsident/ } \\
\text { Generalse- } \\
\text { kretär }\end{array}$ & $\begin{array}{l}\text { Besondere } \\
\text { Neuerungen }\end{array}$ \\
\hline 1972 & $\begin{array}{l}\text { Gründungs- } \\
\text { kongress }\end{array}$ & $\begin{array}{l}200 \text { aus 6 } \\
\text { Provinzen }\end{array}$ & $\begin{array}{l}\text { Tepeyac, } \\
\text { Chimborazo }\end{array}$ & $\begin{array}{l}\text { Blanca } \\
\text { Chancoso }\end{array}$ & $\begin{array}{l}\text { Trennung von der } \\
\text { katholischen } \\
\text { Kirche; schwere } \\
\text { Krise }\end{array}$ \\
\hline 1975 & 2. Kongress & $\begin{array}{l}120 \text { aus 8 } \\
\text { Provinzen }\end{array}$ & Ambato & & Nicht anerkannt \\
\hline 1976 & 3. Kongress & & Pichincha & & \\
\hline
\end{tabular}




\begin{tabular}{|c|c|c|c|c|c|}
\hline 1977 & 4. Kongress & $\begin{array}{l}180, \\
\text { davon } 100 \\
\text { Delegierte } \\
\text { aus } 8 \\
\text { Provinzen }\end{array}$ & $\begin{array}{l}\text { Chibuleo, } \\
\text { Tungurahua }\end{array}$ & $\begin{array}{l}\text { José } \\
\text { Lligalo }\end{array}$ & \\
\hline 1979 & 5. Kongress & $\begin{array}{l}350 \text { aus } \\
10 \text { Pro- } \\
\text { vinzen }\end{array}$ & $\begin{array}{l}\text { Pintag, } \\
\text { Pichincha }\end{array}$ & $\begin{array}{l}\text { Blanca } \\
\text { Chancoso }\end{array}$ & \\
\hline 1981 & 6. Kongress & & Chimborazo & $\begin{array}{l}\text { Wieder- } \\
\text { wahl }\end{array}$ & \\
\hline 1983 & 7. Kongress & & $\begin{array}{l}\text { Pijal, } \\
\text { Imbabura }\end{array}$ & $\begin{array}{l}\text { Wieder- } \\
\text { wahl }\end{array}$ & \\
\hline 1985 & 8. Kongress & $\begin{array}{l}510 \text { aus } 8 \\
\text { Provinzen }\end{array}$ & Quito & & \\
\hline 1992 & 11. Kongress & $\begin{array}{l}1300 \text { aus } \\
10 \text { Pro- } \\
\text { vinzen }\end{array}$ & Quito & & $\begin{array}{l}\text { Teilnahme an den } \\
\text { nationalen Wahlen } \\
\text { mit ungültigen } \\
\text { Stimmen }\end{array}$ \\
\hline 1996 & 13. Kongress & & Guamote & $\begin{array}{l}\text { Ricardo } \\
\text { Ulcuango }\end{array}$ & \\
\hline 1998 & 14. Kongress & & & & \\
\hline 2000 & 15. Kongress & $\begin{array}{l}372 \text { aus } \\
13 \text { Pro- } \\
\text { vinzen }\end{array}$ & Guanujo & $\begin{array}{l}\text { Salvador } \\
\text { Quishpe }\end{array}$ & \\
\hline 2003 & $\begin{array}{l}\text { 16. Kon- } \\
\text { gress/ } 1 . \\
\text { Kongress der } \\
\text { Kichwa- } \\
\text { Völker } \\
\text { Ecuadors }\end{array}$ & 600 & $\begin{array}{l}\text { Cacha, } \\
\text { Chimborazo }\end{array}$ & $\begin{array}{l}\text { Humberto } \\
\text { Cholango }\end{array}$ & $\begin{array}{l}\text { Kampf gegen die } \\
\text { Regierung Gutiérrez }\end{array}$ \\
\hline 2006 & $\begin{array}{l}\text { 2. Kongress } \\
\text { der Kichwa- } \\
\text { Völker } \\
\text { Ecuadors }\end{array}$ & 1460 & Cañar & $\begin{array}{l}\text { Wieder- } \\
\text { wahl }\end{array}$ & $\begin{array}{l}\text { Kampf gegen den } \\
\text { TLC und OXY }\end{array}$ \\
\hline
\end{tabular}




\begin{tabular}{|l|l|l|l|l|l|}
\hline 2009 & $\begin{array}{l}\text { 3. Kongress } \\
\text { der Kichwa- } \\
\text { Völker } \\
\text { Ecuadors }\end{array}$ & $\begin{array}{l}\text { Cochapamba, } \\
\text { Cangahua, } \\
\text { Pichincha }\end{array}$ & $\begin{array}{l}\text { Delfín } \\
\text { Tenesaca }\end{array}$ & $\begin{array}{l}\text { Opposition zu } \\
\text { Correa wird } \\
\text { beschlossen }\end{array}$ \\
\hline 2013 & $\begin{array}{l}\text { 4. Kongress } \\
\text { der Kichwa- } \\
\text { Völker } \\
\text { Ecuadors }\end{array}$ & $\begin{array}{l}\text { Saquisilí, } \\
\text { Cotopaxi }\end{array}$ & $\begin{array}{l}\text { Carlos } \\
\text { Pérez } \\
\text { Guartambel }\end{array}$ & \\
\hline
\end{tabular}

Tabelle 3: Kongresse ECUARUNARI, eigene Abbildung

Neben den klassischen politischen Strategien wie Streiks oder Besetzungen, spielt die Bildungspolitik der ECUARUNARI eine wichtige Rolle. Die „Interkulturelle Zweisprachige Erziehung“ (ECUARUNARI 1989: 89) dient nicht nur der Ausbildung und Alphabetisierung der indigenen Bevölkerung, sondern auch - ähnlich wie die vergleichbaren Projekte im Amazonasgebiet - der Ausbildung eines Bewusstseins, um so die Bevölkerung mobilisieren zu können (ECUARUNARI 1989: 87). Das Radiobildungssystem im andinen Raum war auf Alphabetisierung und Weiterbildung ausgerichtet und fand, trotz seiner Orientierung auf Indigene, weitgehend in Spanisch und nur in wenigen Fällen in Kichwa statt (Moya 1987: 394). Auch führten die Projekte der interkulturellen zweisprachigen Erziehung zu neuen Kontakten, etwa zu einem Abkommen zwischen der CONAIE, Ecuador und Deutschland, insbesondere der GTZ, wodurch nicht nur neue Ressourcen, sondern auch neue Erfahrungen gewonnen werden konnten (ECUARUNARI 1989: 89; Yumbay 2008: 245).

\section{c. CONAIE}

\section{Der CONACNIE und die Gründung der CONAIE}

Seit Ende der 1970er Jahre gab es Pläne, eine nationale Indigenenorganisation zu gründen. 1978 fand in Sucúa das Erste Indigenen-Treffen Ecuadors (Primer Encuentro de Indígenas del Ecuador) statt, auf dem die Konföderation der indigenen Völker Ecuadors (Confederación de Pueblos Indígenas del Ecuador), eine Vorläuferorganisation von CONACNIE und CONAIE, gegründet wurde (CONAIE 1989: 50).

Am 16. Oktober 1980 fand auf Betreiben der CONFENIAE und der ECUARUNARI (Fontaine 2001: 58f.) der Nationale Bauern- und Indigenenmarsch (Marcha Nacional Campesina Indígena) statt (Ibarra Illanez 1987: 141), an den sich das erste Treffen der indigenen Nationalitäten Ecuadors (Primer Encuentro de Nacionalidades Indígenas del Ecuador) in Sucúa anschloss, auf dem der Koordinationsrat 
der indigenen Nationalitäten Ecuadors (Consejo de Coordinación de las Nacionalidades Indígenas del Ecuador, CONACNIE) gegründet wurde (CONAIE 1989: 261). Auf beiden Veranstaltungen wurde die Rücknahme der Gesetze der Nationalen Sicherheit und der Agrarentwicklung, die Bestrafung der Schuldigen am Massaker von Aztra 1977 und die Ausweisung des Linguistischen Sommerinstituts gefordert und der Nationale Alphabetisierungsplan kritisiert (Ibarra Illanez 1987: 141). Auf dem Treffen von Sucúa wurde zudem von der Regierung die Respektierung der indigenen Nationalitäten und ihrer Territorien, die Anerkennung der indigenen Sprachen als Amtssprachen und die Einführung einer zweisprachigen und bikulturellen Erziehung gefordert (Ibarra Illanez 1987: 142).

Der CONACNIE sollte als Plattform zur Stärkung der Indigenenbewegung und für eine größere Koordination zwischen den regionalen Organisationen dienen (León 1994: 72f.; Rodríguez 2008: 96), um so - langfristig - eine nationale Organisation aufzubauen (CONAIE 1989: 261). Weiterhin sollte sie die politische Linie und die Forderungen der verschiedenen Indigenenorganisationen vereinheitlichen (Chancoso 1983: 43) und die Ausbildung von Führungskadern unterstützen (Conterón/de Viteri 1984: 47). Die nationale Koordination sollte eine einheitliche Kommunikation der Indigenen mit dem Staat ermöglichen und dafür die Ressourcen der einzelnen Organisationen integrieren (Rodríguez 2008: 95). Zu Beginn nahm auch die FENOC an diesen Bemühungen teil - nicht hingegen die FEI und die gerade gegründete FEINE (Almeida/Prieto 1983: 39). Die erste Exekutivkoordinatorin des CONACNIE war Blanca Chancoso, zur selben Zeit Generalsekretärin der ECUARUNARI (Chancoso 1983: 40).

Von Beginn an hatte der CONACNIE den Plan, eine Organisation der Indigenen des Küstengebietes aufzubauen (CONAIE 1989: 261), wo die anderen Indigenenorganisationen kaum Repräsentativität hatten (León 1991: 406f.) ${ }^{45} .1978$ identifizierten sich weniger als 5000 Personen im Küstenraum als Indigene und erst 1980 wurde eine Föderation des Volkes der Awa gegründet. Erst 1986 - kurz vor der Gründung der CONAIE - konnte der CONACNIE eine Konföderation der Indigenen der ecuadorianischen Küste (Confederación de Indígenas de la Costa Ecuatoriana, COICE) aufbauen46 (Rodríguez 2008: 95).

Die Gründung des CONACNIE war auch eine Reaktion auf den Druck von auBen, den die indigenen Völker zu dieser Zeit erlebten. Die - vom Staat geförderte -

45 Also irrt Tamayo (1996: 65), der von einem Zusammenfließen von Organisationen aller drei Gebiete im CONACNIE spricht.

46 Aus der 1999 die Konföderation der indigenen Nationalitäten der ecuadorianischen Küste (Confederación de Nacionalidades Indígenas de la Costa Ecuatoriana, CONAICE) hervorging (CONAIE o.J.: 6). Diese ist nach wie vor die schwächste Filiale der CONAIE was Mitgliederzahlen und organisatorische Strukturen angeht. 
Kolonisierung des Amazonasgebietes und die Ausbeutung der Rohstoffe dort nahm bedrohliche Züge an. Eine nationalistische Politik des Staates, der über Alphabetisierungskampagnen und den Aufbau eines Instituts der eingeborenen Kulturen und gemeinschaftlicher Aktion (Instituto de Culturas Aborígenes y Acción Comunitaria) die Indigenen in die Mehrheitsbevölkerung assimilieren wollte, setzte die lokalen Organisationen unter Druck. Dazu kamen die Aktivitäten des Linguistischen Sommer-Instituts (Instituto Lingüístico del Verano, ILV), einer evangelikalen Gruppe, die die Missionierung der Indigenen betrieb und so ihre Kultur bedrohte (Moreno/Figueroa 1992: 35f.). Das ILV wurde 1934 in den USA mit dem Ziel gegründet, die Bibel in alle Sprachen der Welt zu übersetzen, wofür es mit vielen Regierungen von Ländern der dritten Welt Abkommen schloss, die ihm Forschungs- und Bildungsprojekte in den jeweiligen Gebieten erlaubten (Krainer 1996: 41). Seit den 1950er Jahren arbeitete es mit der ecuadorianischen Regierung zusammen, die ihm ab 1952 erlaubte, im Amazonasgebiet tätig zu werden. So begann das ILV, die indigenen Sprachen - vor allem Kichwa - zu studieren und in ihnen Missionierung zu betreiben (Krainer 1996: 41f.) und Programme der zweisprachigen Bildung durchzuführen (Moya 1987: 390). Es gründete eine eigene indigene Organisation, den Rat der indigenen Völker (Consejo de Pueblos Indígenas), um seinen Einfluss auszuweiten. Sowohl das ILV als auch seine indigene Organisation wurden von den anderen indigenen Organisationen bekämpft (FENOCIN 2004: 17). Obwohl das ILV formal schon 1981 auf Druck der sozialen Bewegungen von der Regierung Roldós des Landes verwiesen wurde (Moya 1987: 396f.; Krainer 1996: 41f.), hatte es sich unter anderen Namen reorganisiert und konnte so seine Arbeit weiterführen. Dazu waren ihm auch seine internationalen Verbindungen und die daraus resultierenden wirtschaftlichen Möglichkeiten von Nutzen. Das Linguistische SommerInstitut wurde von den indigenen Organisationen beschuldigt, die Indigenen passiv machen und von einer Organisation abhalten zu wollen, um sie so besser missionieren zu können (ECUARUNARI/FENOC 1989: 27). Diese Konflikte führten zu einer Phase der Schwäche des CONACNIE, die erst 1986 überwunden werden konnte (Fontaine 2001: 58f.).

Der CONACNIE vereinte die aus unterschiedlichen Traditionen stammenden Organisationen des Andenhochlandes und des Amazonasgebietes unter einer Perspektive, die die ,doppelte Dimension unseres Kampfes, die der Klasse und die ethnische" (CONAIE 1989: 261) verbinden sollte. Für den CONACNIE hatten die verschiedenen indigenen Völker und Nationalitäten dieselben Probleme, vor allem Land, Gesundheitsversorgung, Erziehung und soziale Gleichheit. Nur die nationale Zusammenarbeit aller Indigenenorganisationen erlaubt es, diese Probleme im Rahmen einer neuen, anderen Gesellschaft lösen zu können (Conterón/de Viteri 1984: 2). Die Auseinandersetzung der ethnischen und klassistischen Tendenzen, mit ihren Schwerpunkten auf einer autonomistischen Politik oder der Bildung einer Allianz 
mit anderen sozialen Bewegungen, beförderte die Entwicklung des CONACNIE und der CONAIE - und gefährdete sie zugleich (Barrera o.J.: 153). Tendenzen, die aus dem Kampf gegen latifundistas und für Arbeiterrechte hervorgegangen waren, standen Tendenzen gegenüber, die die indigenen Kultur wiedergewinnen und verteidigen wollten (Petras/Veltmeyer 2005: 119). Aus den Forderungen nach ethnischer Autonomie und nach einer Landverteilung wurde so die Forderung nach einem Neuaufbau des Staates im Rahmen des Begriffs der Plurinationalität (Rodríguez 2008: 96). Diese Orientierung war allerdings bedeutender für die Koalition der indigenen Organisationen der beiden Regionen als für eine Ausweitung der Beziehungen zu anderen indigenen Organisationen oder sozialen Bewegungen (Becker 2008: 168), die sich nicht für ethnische Aspekte interessierten oder - wie FENOC und FEI - eine Hegemonie ihrer eigenen Organisation in der Indigenenbewegung anstrebten. So liefen die Bemühungen des CONACNIE, enge Beziehungen mit anderen Organisationen, wie der FENOC und der FUT aufzubauen (CONAIE 1989: 263), oft ins Leere. Die Gefahr, dadurch an Einfluss zu verlieren oder in verschiedene Fraktionen aufgespalten zu werden, war der Organisation durchaus bewusst (ECUARUNARI/CONAIE 1989: 42), weshalb die Diskussion um eine weitere Öffnung hin zu anderen sozialen Bewegungen die gesamte Entwicklung des CONACNIE und der CONAIE markieren sollte.

Die unterschiedlichen Interessen von CONACNIE und Gewerkschaftsbewegung wurden im landesweiten Streik von Oktober 1982 offensichtlich, in dem die FUT - als Alliierter der Indigenenbewegung - die Forderungen der Indigenen nicht zu ihren Hauptforderungen machte. Statt auf die indigenen Forderungen nach Land, Wasser und günstigen Krediten einzugehen, fokussierte sich die FUT auf den Kampf um Lohnerhöhungen und bessere Dienstleistungen für die Arbeiter - etwas, das den Indigenen, die meist keine Arbeiter waren, nicht nützte. Als der Präsident Osvaldo Hurtado einige der Forderungen der FUT erfüllte, stiegen mit den Löhnen der Arbeiter auch die Preise, ohne dass die Indigenen ein höheres Einkommen hätten. Erst im Rahmen des Inti Raymi 1990 strebte die FUT nach ihrer schweren Krise ab Mitte der 1980er erneut nach einer Allianz mit der Indigenenbewegung, wobei sie darauf aufmerksam machte, dass der neue Staat, den die CONAIE wollte, keine soziale Gruppe, also auch nicht die Arbeiter, außen vor lassen durfte (Botero 2000: 67).

Das Nationale Alphabetisierungsprogramm der Regierung Roldós/Hurtado (1979-1983) nahm in sein Exekutiv-Kommitée und ins Nationalen Alphabetisierungsbüro Vertreter der Indigenen- und Bauernbewegung auf. Das Unterprogramm der Alphabetisierung in Kichwa wurde so teilweise von den Organisationen des CONACNIE kontrolliert, die sich auch maßgeblich in den Projekten engagierten (Moya 1987: 395). Es unterstand dem Forschungszentrum für die indigene Erziehung (Centro de Investigaciones para la Educación Indígena, CIEI) der Päpstlichen 
Katholischen Universität Ecuadors (Pontificia Universidad Católica del Ecuador, PUCE), das schon 1978 erste Pilotstudien erstellt hatte (Moya 1987: 396), in den Jahren 1980 bis 1984 im ganzen Land aktiv werden sollte (Krainer 1996: 45) und 1986 aufgelöst wurde (Flores 2005: 30). Das CIEI war ein Ergebnis der Bemühungen von indigenen Akademikern, darunter Luis Macas, Luis Montaluisa, Cristóbal Quishpe und Rosa Bacasela, die ab Mitte der 1970er in der PUCE den Aufbau von Kichwa-Sprachkursen betrieben (Lazo/Lenz 2004: 10), die 1977 zu einem licenciatura-Studium in Kichwa am Sprachwissenschaftlichen Institut derselben Universität wurden (Sánchez-Parga 1988: 125) und sich an Modellstudien des CIEI zu zweisprachiger Bildung in Cotopaxi beteiligten (Lazo/Lenz 2004: 10). Das CIEI hatte mit 724 Erwachsenenschulen und 300 Schulen für Kinder die größte Abdeckung aller Bildungsprojekte für Indigene zu dieser Zeit (Montaluisa 2003: 88). Es baute auf die Erfahrungen und teilweise auch auf die Strukturen indigener und kirchlicher Erziehungsprojekte, allen voran die in den 1960ern auf Betreiben vom Bischof von Riobamba, Leonidas Proaño, aufgebauten Radio-Volksschulen Ecuadors (Escuelas Radiofónicas Populares del Ecuador, ERPE) in Cayambe und anderen Provinzen des Hochlandes und der anderen Regionen Ecuadors (Black 1999: 35; Lazos/Lenz 2004: 7), das Shuar-System der bikulturellen radiophonen Erziehung (Sistema de Educación Radiofónica Bicultural Shuar, SERBISH) im Amazonasgebiet und das System indigener Schulen Cotopaxis (Sistema de Escuelas Indígenas de Cotopaxi, SEIC), die schon lange eine wichtige Rolle nicht nur in der allgemeinen Ausbildung der Indigenen, sondern auch in der Weiterbildung des Führungspersonals der indigenen Organisationen gespielt hatten (Lazos/Lenz 2004: 6). In einem Nationalen Seminar indigener Delegierter Ecuadors (Seminario Nacional de Delegados Indígenas del Ecuador) im April 1980 in Quito wurde in Zusammenarbeit des CIEI und der indigenen Organisationen ein einheitliches Alphabet für Kichwa entwickelt, das eine wichtige Rolle für die Identität der Indigenen im Rahmen ihres Selbstverständnisses als Nationalitäten spielt (Moya 1987: 398). In diesem Rahmen wurde auch die kulturalistische Herangehensweise des CIEI kritisiert. In den Materialien, die es ausgearbeitet hatte, wurden soziale Unterschiede ignoriert, kulturelle hingegen überbetont. So wurde auf den Unterschied zwischen Indigenen und Mestizen hingewiesen, keinesfalls aber auf die Klassenstruktur der ecuadorianischen Gesellschaft. Diese Kritiken spielten eine wichtige Rolle für die Entwicklung des Diskurses der doppelten Diskriminierung der Indigenen als Indigene und als Landarbeiter (Moya 1987: 399). Trotz dieser Kritiken beteiligten sich die indigenen und gewerkschaftlichen Organisationen federführend an der Alphabetisierungskampagne (Lazo/Lenz 2004: 10). Im Januar 1982 wurde die zweisprachige Erziehung in indigenen Gemeinschaften von Präsident Hurtado offiziell anerkannt und im Mai desselben Jahres im Amt für ländliche Erziehung (Departamento de Educación Rural, DER) koordiniert, das später in der DINEIB aufgehen sollte (Black 1999: 35). 
Die indigenen Organisationen forderten eine ausschließliche Kontrolle über die Erziehungsprogramme, die nicht nur der Alphabetisierung, sondern auch der politischen Bildung dienen sollten. Dadurch wurde die Orientierung auf die Bereiche Kultur und Bildung innerhalb der Indigenenbewegung verstärkt und ein Ausbau der Kompetenzen in diesem Bereich ermöglicht - eine Grundlage für die spätere Ausrichtung der CONAIE (Moya 1987: 396f.). Auch lernten die Organisationen, ihre kulturellen Forderungen, in ihrem Zentrum die zweisprachige Erziehung, gegen den Staat durchzusetzen (Moya 1987: 394). Die Teilnahme der Indigenenorganisationen an den Projekten der Alphabetisierungskampagne hatten den Effekt, dass die Identität der Indigenen und die Repräsentativität ihrer Organisationen gestärkt wurden, da sie ihre eigenen Antworten entwickeln und beständig um die Kontrolle der staatlichen Programme kämpfen mussten (Chiriboga 1983: 124). Im Rahmen der durchgeführten Projekte konnte die Indigenenbewegung und vor allem der CONACNIE und seine Filialen Erziehungs- und Weiterbildungsprogramme aufbauen, die auch auf ihr eigenes Personal ausgerichtet waren, etwa technologische oder administrative Fortbildungen und die Ausbildung von Lehrern und Forschern (Moya 1987: 390f.). So bildete sich im Umfeld der Alphabetisierungskampagne und der daraus entstehenden Institutionen und Programme eine Gruppe indigener Intellektueller, die sich in der Führungsebene der Indigenenorganisationen etablieren konnten (Sánchez-Parga 1988: 126).

Die Regierung Roldós-Hurtado versuchte, die Beziehung zwischen Staat und Indigenen neu zu fassen. Dazu wurde seit 1980 an einem Gesetz über Garantien an die Ureinwohner-Gruppen gearbeitet, das die Indigenen als Teil des kulturellen Erbes Ecuadors definierte und ihnen die Grundlagen für die Entwicklung ihrer Identität, Sprache und Traditionen, sowie die Möglichkeit, sich in die nationale Gemeinschaft zu integrieren, versicherte. Zudem wurden die Richtlinien für die Gründung ethnischer Organisationen (mindestens 20 Mitglieder einer Ethnie) und eine Rücksichtnahme der Entwicklungs- und Siedlungsprogramme für die Indigenen festgelegt. Auf dieser Grundlage wurde 1981 die Beratende Kommission für Ureinwohner-Angelegenheiten (Comisión consultiva de Asuntos Aborigenes) als Abteilung des Sozialministeriums gegründet (Sánchez-Parga 1988: 89). Aus dieser Kommission wurde 1983 das Büro für Indigene Angelegenheiten (Oficina de Asuntos Indígenas), ebenfalls im Sozialministerium, das die Leitlinien des staatlichen Indigenismus aufgriff. Das Büro für Indigene Angelegenheiten sollte die indigenen Kulturen beschützen und entwickeln, ihre Autonomie respektieren und die Indigenen- und Bauernorganisationen fördern. Weiterhin sollte es die Politik des Staates hinsichtlich der Indigenen definieren und Mechanismen der Partizipation für die Indigenen schaffen (Sánchez-Parga 1988: 88f.). Somit sind auch die Verwendung des Kichwa in der Eröffnungsrede von Roldós vor dem Kongress und die Beschriftung der Präsidentschaftsbinde von Hurtado auf Shuar mehr als nur populistische 
Gesten. Sie sind Teil der symbolischen Umdeutung der Indigenen (Sánchez-Parga 1988: 106), die auch dem gestiegenen Einfluss der Indigenenorganisationen geschuldet sind.

Im April 1984 fand der zweite Kongress des CONACNIE in Quito statt, an dem Vertreter von neun Nationalitäten teilnahmen. Es wurde die Unabhängigkeit von allen politischen Parteien erklärt (Rodríguez 2008: 96; Albó 2009: 210), eine Position, die die CONAIE beibehalten wird (CONAIE o.J.: 4f.).

1986 gründete der CONACNIE 1986 das Wissenschaftliche Institut der Indigenen Kulturen (Instituto Científico de Culturas Indígenas, ICCI), um die praktische Arbeit von CONACNIE und später CONAIE theoretisch zu begleiten und zwischen Indigenenbewegung und Wissenschaft zu vermitteln (ICCI o.J.). Das im September 1986 staatlich anerkannte Institut sollte einen Raum für die Reflexion über die Begriffe der Indigenenbewegung und ihre politischen Projekte bieten (ICCI o.J.). Von Beginn an gab es eine hohe personelle Kontinuität zwischen ICCI und CONAIE, was sich auch in der wichtigen Rolle des ehemaligen Präsidenten der CONAIE, Luis Macas, im ICCI widerspiegelt.

Im November 1986 findet in Quito der dritte Kongress des CONACNIE statt, gleichzeitig der erste Kongress der indigenen Nationalitäten Ecuadors (Primer Congreso de Nacionalidades Indígenas del Ecuador), auf dem die Konföderation der indigenen Nationalitäten Ecuadors (Confederación de Nacionalidades Indígenas del Ecuador, CONAIE) gegründet wird (CONAIE 1989: 50; CONAIE o.J.: 4f.). Ihr erster Präsident ist der Gründer der FICSHA, Miguel Tankamash (Pajuelo 2004: 60). Damit kommt der Prozess der Gründung einer dezentralen Vielfalt lokaler indigener Organisationen, der in den 1960ern begonnen hat, zu einem vorläufigen Höhepunkt, der Sammlung in einer nationalen Organisation (CONAIE 1989: 277; Santillana/Herrara 2009: 332). Die Perspektiven der Organisationen des zentralen Andenraums, beeinflusst von der katholischen Kirche und linken Parteien, des Amazonasgebietes, die um den Aufbau eines plurinationalen Staates und die Verteidigung des Territoriums kreisen, und des nördlichen Andenraums um Cayambe und Otavalo, in denen, auch wegen ihrer Sozialstruktur, ein ethnischer Ansatz vorherrscht, wurden in einer nationalen Organisation vereinigt (Cholango in Tamayo 2007: 7; Santillana/Herrara 2009: 332 ${ }^{47}$. $\mathrm{Zu}$ diesem ideologischen Kompromiss gehört auch, dass der Begriff der Nationalitäten, wie die Namen CONACNIE und CONAIE verdeutlichen, Wiedereinzug in die Diskurse der Organisationen des Andenhochlandes halten. Der Begriff wurde - in seiner neuen, nicht-kommunistischen Bedeutung - zuerst im Amazonasgebiet verwendet und wanderte seit Gründung der CONFENIAE auch in andere Regionen (Ospina 2008: 71). Die CONAIE

47 Also täuscht sich Le Quang (und andere), der davon spricht, dass die CONAIE ,typisch indigene Forderungen“ (Le Quang 2010: 112) anderen Perspektiven vorzieht. 
war so eine der ersten Organisationen in Lateinamerika, die ein dezidiert ethnisches Programm entwickelten und auch Forderungen nach Autonomie und kollektiven Rechten aufstellten (Ojeda Segovia 2004: 54).

Die Gründung der CONAIE fand zu einem Zeitpunkt relativer Schwäche der politischen Linken - auch wegen der Regierung Febres Cordero (1984-1988) statt, wodurch die Übernahme explizit ethnischer Forderungen, die nicht in ein klassistisches Programm integriert wurden, als Infragestellung der sozialistischen Perspektive verstanden wurde (Simbaña 2009: 163). Die CONAIE sollte dennoch gerade für eine allgemeine Belebung der sozialen Bewegungen und über ihre Kooperation auch der geschwächten Arbeiterbewegung sorgen, woraus einige Jahre später der breite Widerstand gegen den Neoliberalismus erst möglich wurde (Ayala Mora o.J.: 5f.).

Die CONAIE hatte von Beginn an den Anspruch, alle indigenen Organisationen in sich zu vereinigen und sich als allumfassende indigene Repräsentation zu konstituieren, die die Indigenen in allen erdenklichen Bereichen vertritt (Moreno/Figueroa 1992: 43). Die Forderungen, die sie auf ihrem Gründungskongress ausarbeitete, umfassten die Rückgabe der indigenen Länder in gemeinschaftlichen Besitz, die Verteidigung der indigenen Kulturen über Programme der zweisprachigen Erziehung unter der Kontrolle der indigenen Gemeinden (Montaluisa 2003: 88) und das Bewusstmachen über den Wert der traditionellen Medizin. Sie beschloss, Weiterbildungskurse für die Indigenen zu organisieren und für die Projekte der indigenen Nationalitäten Finanzierungsquellen zu erschließen, etwa über den Aufbau einer indigenen Bank (Moreno/Figueroa 1992: 43f.). Es wurde die Forderung nach einem autonomen und staatlich geförderten System der zweisprachigen interkulturellen Erziehung erhoben und der Aufbau einer Universität der indigenen Nationalitäten angekündigt (Montaluisa 2008: 102).

Erst im August 1989 wurde die CONAIE - unter ihrem damaligen Präsidenten Cristóbal Tapuy - vom ecuadorianischen Staat anerkannt (CONAIE 2005).

\section{Entwicklung der CONAIE}

Die Gründung der CONAIE bedeutete vorerst einen Kompromiss zwischen den ethnischen und klassistischen Tendenzen der Mitgliedsorganisationen, wofür das Ende des klaren Klassismus der ECUARUNARI 1982 steht (Santana 1992: 98f.). Die Stärkung des Begriffes der Territorialität seit dem Kongress von 1984 erlaubte es, die gewerkschaftlichen Forderungen nach einer Landverteilung mit ethnischen Forderungen zu kombinieren und machte so CONACNIE/CONAIE zu einer politischen Option für alle Indigenen in Ecuador (Santana 1992: 194). In der Indigenen Koordination des Amazonasgebietes (Coordinadora Indígena de la Cuenca Amazónica, COICA), die 1984 von der CONFENIAE mitgegründet wurde (Albó 2009: 
323), wurde zur gleichen Zeit über territoriale Autonomie innerhalb eines Konzeptes der indigenen Gemeinschaften debattiert (Quijano 2006a: 33).

Die CONAIE blieb dem Engagement des CONACNIE für den Aufbau eines Bildungssystems für die Indigenen treu, was durch Abkommen mit der deutschen GTZ und der ecuadorianischen Regierung 1985 und 1986 gestärkt wurde (ECUARUNARI 1989: 89; Krainer 1996: 46; Yumbay 2008: 245). Das daraus hervorgegangene Projekt der interkulturellen zweisprachigen Erziehung (Proyecto de Educación Bilingüe Intercultural) kooperierte mit der CONAIE, um in 74 Grundschulen in 8 Provinzen des Hochlandes diese Art der Erziehung zu erproben und anschließend auf das ganze Land auszuweiten. Im November 1988 gründete das Erziehungsministerium auf Druck der Indigenenbewegung hin (CONAIE 1994: 44; Montaluisa 2003: 88) die Nationale Direktion der interkulturellen zweisprachigen Erziehung (Dirección Nacional de Educación Intercultural Bilingüe, DINEIB) als unabhängige Abteilung innerhalb des Ministeriums (Lazo/Lenz 2004: 11), um die verschiedenen regionalen Projekte zu vereinheitlichen (Martínez Novo 2009a: 179). Sie wurde zusammen vom Erziehungsministerium und der CONAIE als Vertreterin der Indigenenbewegung verwaltet (Moreno/Figueroa 1992: 44; Krainer 2010: 39), ohne dass der Staat den Indigenen völlige Autonomie gewährt hätte (CONAIE 1994: 44). Die Kontrolle, die die CONAIE über die DINEIB ausübte, wurde von den anderen indigenen Organisationen kritisiert - bis auch sie eingebunden wurden (Martínez Novo 2009a: 179f.). 1992 wurde an der Staatlichen Universität von Bolívar in Guaranda, unter der Leitung der CONAIE die Schule für andine Erziehung und Kultur (Escuela de Educación y Cultura Andina) eingerichtet. An dieser Institution wurden nicht nur Lehrer für das interkulturelle, zweisprachige Schulsystem ausgebildet, sondern ausdrücklich auch Führungskader der Indigenenbewegung (Botero 2000: 65). Auch wenn der Staat das Budget der DINEIB kontrollierte, verfügten die indigenen Organisationen über Entscheidungsgewalt über den Lehrplan, die Lehrmaterialien und das Personal des Systems der interkulturellen zweisprachigen Erziehung. Diese Autonomie wurde am 20. Februar 2009 beendet, der Staat übernahm die alleinige Kontrolle über die DINEIB (Martínez Novo 2009a: 174). Die DINEIB hat immer unter einer starken Unterfinanzierung gelitten (CONAIE 1994: 44), so dass Investitionen in die Infrastruktur fast unmöglich und die Lehrkräfte unterbezahlt waren (Martínez Novo 2009a: 180f.). Etwa die Hälfte der Lehrkräfte spricht - obwohl das eine formale Voraussetzung für diese Arbeit ist keine indigene Sprache (Montaluisa 2003: 90). So wurde eine geringe und weiter sinkende Akzeptanz der interkulturellen zweisprachigen Erziehung festgestellt selbst bei den Führern der indigenen Organisationen (Martínez Novo 2009a: 181f.). Tatsächlich ist der Unterricht gerade in Englisch und Informatik sehr schlecht, die Schülerzahlen haben sich etwa in Imbabura von 11.5001989 auf 10.7952006 reduziert (Martínez Novo 2009a: 183). 
Die Konzentration der CONAIE auf Fragen der Erziehung war auch mit einer darüber hinausgehenden Mobilisierung verbunden. So fand am 12. Oktober 1989 ein nationaler Marsch der an der interkulturellen Erziehung Beteiligten nach Quito statt, auf dem zum ersten Mal die Wiphala als Banner der Indigenen und der CONAIE gezeigt wurde. Neben einer Verbesserung der zweisprachigen interkulturellen Erziehung forderten die Indigenen Respekt vor ihren Territorien. Dies war eine der ersten Demonstrationen mit genuin indigenen Themen, ohne gewerkschaftliche oder parteipolitische Einbindung, über die die nationale Presse berichtete (Montaluisa 2008: 102f.).

Von November 1987 bis August 1988 erarbeitete eine Sonderkommission für indigene Angelegenheiten einen Entwurf für ein Gesetz der indigenen Nationalitäten, der anschließend dem ecuadorianischen Parlament unterbreitet wurde (Comisión Especial de Asuntos Indígenas 1988: 4). Die Kommission unter Führung der Sozialistischen Partei arbeitete mit CONAIE, ECUARUNARI, FENOC, CONFENIAE, der Shuar-Föderation und anderen Indigenenorganisationen des Landes zusammen (Comisión Especial de Asuntos Indígenas 1988: 2). In diesem Text wurde Ecuador zum plurinationalen Staat erklärt und gefordert, dass die Regierung die indigenen Nationalitäten, Territorien, Organisationsformen, Kulturen, Medizin und rechtlichen Systeme anerkennt und garantiert (Comisión Especial de Asuntos Indígenas 1988: 17; Becker 2008: 172). Kichwa sollte neben Spanisch zur Amtssprache und die anderen indigenen Sprachen zu Amtssprachen in den Gebieten ihrer Verbreitung gemacht werden (Comisión Especial de Asuntos Indígenas 1988: 19; Bernal 2000: 42f.). Die zweisprachige interkulturelle Erziehung wird von den Indigenen mitbestimmt und in den indigenen Gebieten verpflichtend (Comisión Especial de Asuntos Indígenas 1988: 15). Bei der Ausarbeitung des Gesetzesvorschlags spielten der ,Bischof der Indios ‘ Leonidas Proaño und Enrique Ayala Mora, damals Abgeordneter der PSE, eine besondere Rolle (Comisión Especial de Asuntos Indígenas 1988: 2). Auch wenn alle indigenen Organisationen beteiligt waren, wird im Vorschlag selbst der CONAIE eine besondere Stellung eingeräumt und ihre Beteiligung an verschiedenen Institutionen bestimmt (Guerrero/Ospina 2003). Die CONAIE wird als Repräsentativorgan der indigenen Völker definiert, das dafür als eigener Posten im Staatshaushalt geführt wird und so seine Aktivitäten finanzieren kann (Comisión Especial de Asuntos Indígenas 1988: 28). Für alle Verträge, die die Indigenen betreffen ist die Zustimmung der jeweiligen Indigenenorganisationen notwendig (Comisión Especial de Asuntos Indígenas 1988: 20). Der Gesetzesvorschlag wurde vertagt und nie im Parlament diskutiert. Dennoch ist dieser Text von großer Bedeutung, da in ihm zum ersten Mal die Ideen des plurinationalen Staates der Öffentlichkeit präsentiert wird (CONAIE 1999: 51). Die CONAIE sollte sich später immer wieder auf diesen Gesetzesvorschlag beziehen (Bernal 2000: 42f.). 
Der Amtsantritt des Sozialdemokraten Rodrigo Borja 1988 war ein Hoffnungsschimmer für die CONAIE, da er schon in seiner Antrittsrede Ecuador als ein plurinationales und multikulturelles Land bezeichnete und die indigenen Nationalitäten als solche anerkannte (Black 1999: 31). Er baute das Anfang der 1980er geschaffene Büro der indigenen Angelegenheiten (Oficina de Asuntos Indígenas) aus, das für die Beziehungen zwischen Regierung und Indigenen zuständig war (Rodríguez/Martínez 2000: 73). Ebenso organisierte er wöchentliche Treffen von drei Repräsentanten seiner Regierung mit den Repräsentanten der Indigenenorganisationen - die allerdings nur spärlich besucht wurden. Acht Monate später wurden Mitglieder des IERAC in Sarayacu für mehrere Tage festgehalten und so die Unterzeichnung der Sarayacu-Verträge erzwungen, die weitgehende Konzessionen in territorialer Autonomie, Kontrolle über die Ressourcen, zweisprachige Erziehung und Entwicklungsprogramme enthielt - die Themen über die bei den wöchentlichen Treffen gesprochen werden sollte (Macdonald 1998: 89). Darauf hin veränderte sich Diskurs der Regierung und Borja verzichtete auf weitere Referenzen auf den plurinationalen Charakter Ecuadors.

In den ersten Jahren nach ihrer Gründung war die CONAIE relativ schwach und konnte nur einen geringen Teil der indigenen Bevölkerung mobilisieren (Santana 1992: 216) - eine Schwäche, die erst 1990 überwunden wurde (Guerrero/Ospina 2003). Diese langsame Stärkung wurde begleitet von einer Reihe gemeinsamer Aktionen der CONAIE mit der schwächer werdenden Gewerkschaftsbewegung. Im April 1987 organisierten die Einheitsgewerkschaft FUT, die den maoistischen Parteien PCMLE (Partido Comunista Marxista Leninista del Ecuador, Kommunistische Marxistische Leninistische Partei Ecuadors) und MPD (Movimiento Popular Democrático, Demokratische Volksbewegung) nahestehende Frente Popular (Volksfront) aus der Lehrergewerkschaft UNE (Unión Nacional de Educadores, Nationaler Erzieherverband) und den Schüler- und Studentenorganisationen FESE (Federación de Estudiantes Secundarios del Ecuador, Föderation der Sekundärschüler Ecuadors) und FEUE (Federación de Estudiantes Universitarios del Ecuador, Föderation der Universitätsstudenten Ecuadors), die der christdemokratischen Partei nahestehende CNT, die auch öffentliche Angestellte vertritt, und die CONAIE die erste Convención Unitaria (Einheitskonvent), die am 1. Mai 1987 den ersten gemeinsamen Marsch aller vier Organisationen organisierte (Dávila 1995: 169). Die zweite Convención Unitaria fand am 12.11 .1988 statt, und führte einen Streik gegen die Regierung Borja am 24.11. durch (Dávila 1995: 120). Die dritte Convención Unitaria fand am 20.5.1989 (Dávila 1995: 121), die vierte, an der die CONAIE nicht teilnahm, am 1.7.1989 (Dávila 1995: 128) und die fünfte und letzte am 16.2.1991 (Dávila 1995: 135) statt. Auf diesem letzten Einheitskonvent wurde der Generalstreik beschlossen (Dávila 1995: 135). Die FUT begann in dieser Zeit, 
öffentlich die Forderungen der CONAIE zu unterstützen, etwa zu Aktionen am 20.9.1988 (Dávila 1995: 243) und am 26.1.1991 (Dávila 1995: 245).

Zum traditionellen indigenen Sonnenwendfest Inti Raymi im Juni 1990 fand die erste Erhebung der Indigenen in Ecuador statt. Eine Gruppe indigener Bauern hielt ab dem 28. Mai die Kirche Santo Domingo in Quito besetzt, daraufhin beschlossen andere Organisationen der Indigenenbewegung, sie zu unterstützen. Weitere Gruppen blockierten ab dem 4. Juni die wichtigsten Zufahrtsstraßen zur Hauptstadt, besetzten öffentliche Gebäude und führten andere Aktionen durch (Lucas 2000: 10; de la Torre 2005: 40). Die CONAIE als wichtigster Akteur hatte bereits im April 1990 eine noch nicht näher definierte Erhebung beschlossen, um die Regierung unter Druck zu setzen und breitere Bündnisse unter den sozialen Bewegungen zu erzwingen (Barrera 2001: 116). Sie kooperierte mit anderen Organisationen wie der FENOC-I, der FEI (León 1994: 72f.) und der Koordination der Landkonflikte (Coordinadora de Conflictos Agrarios) (Guerrero/Ospina 2003). Letztere wurde 1988 und 1989 als Organisation innerhalb der CONAIE (Maldonado 2006: 93) gegründet, um die Landkonflikte in Chimborazo, Imbabura, Tungurahua und Cotopaxi zu lösen. 1989 hatte sie bereits das Gebäude des IERAC besetzt, wodurch sie auf die Lösung von 75 Landkonflikten drängen wollte (Barrera 2001: 115f.). Die Koordination der Landkonflikte war die treibende Kraft hinter der Besetzung der Kirche Santo Domingo und Vorreiterin der Erhebung (Maldonado 2006: 93).

Die Beteiligten veröffentlichten am 6. Juni 1990 (de la Torre 2005: 41) ihre Forderungen in einem Mandat für die Verteidigung des Lebens und der Rechte der indigenen Nationalitäten (Mandato por la defensa de la vida y los derechos de las nacionalidades indígenas), das 16 Punkte enthielt. Diese lassen sich in drei Bereiche einteilen: Forderungen nach ethnischer Anerkennung, nach politischer und wirtschaftlicher Teilhabe und nach finanzieller Unterstützung wegen der benachteiligten Position der Indigenen, also in die Bereiche Ethnie, Staatsbürgerschaft und Klasse. Dieses Manifest spiegelt die Politik der CONAIE wider: die Verbindung von Forderungen nach Gleichheit, im Sinne gleicher Rechte, und nach Differenz, im Sinne der Anerkennung der ethnischen Identität (León 1994: 61). Die erste der 16 Forderungen war die Reform des ersten Artikels der ecuadorianischen Verfassung, wodurch das Land zu einem plurinationalen Staat erklärt werden sollte (León 1994: 19). Das wurde durch Forderungen nach territorialer Autonomie untermauert, wodurch der Bruch $\mathrm{zu}$ den traditionellen Forderungen nach Land deutlich wird (Rodríguez 2008: 100). Ein anderes Dokument dieser Erhebung ist der Brief vom 28. Mai 1990, den die Besetzer der Kirche Santo Domingo an den Präsidenten sandten. Die Absender nennen sich Volkskoordination (Coordinadora Popular), definieren sich als Erben des Widerstands des Volkes gegen Kolonialismus und Neokolonialismus und unterstützen die Forderung nach einer Anerkennung Ecuadors als plurinationales Land - und kritisieren zugleich die Weigerung der Regie- 
rung, auf diese Forderung einzugehen (Coordinadora Popular, in: León 1994: 90). Die Volkskoordination setzte sich zu einem großen Teil aus Mitgliedern der Koordination der Landkonflikte zusammen, die - ohne Abstimmung mit der CONAIE - diese durch ihre Besetzung zu Handlungen zwang (Guerrero/Ospina 2003).

Die Reaktionen der Mehrheitsgesellschaft auf die Forderungen der Indigenen sind vielsagend. Insbesondere die Forderung nach Plurinationalität wurde als Versuch der Spaltung des Landes abgelehnt (Pajuelo 2004: 47). Jorge León (1994) hat Artikel des El Comercio, einer der meistverkauften Zeitungen Ecuadors, zusammengestellt. Fabián Corral beschreibt dort am 11. Juni 1990 die Forderung nach Plurinationalität als zentraler Punkt der 16 Forderungen. Diese bedeuten einen „,neue[n] Partikularismus“ (Corral, in: León 1994: 34), der die Integrität des Staates, und seine Basis, die Ideologie der mestizaje, in Frage stellt. In einem späteren Text vom 3. September 1990 wirft er den Indigenen vor, in der Provinz Pastaza einen autonomen indigenen Staat, der nur lose mit Ecuador verbunden ist, bilden zu wollen (Corral, in: León 1994: 35). Ein Artikel von Diego Romero vom 24. Juni 1990 beschreibt, wie die Indigenen wahrgenommen werden: ,zur Mehrheit ist [die indigene Bevölkerung] analphabetisch, unproduktiv und eine Last für die ecuadorianische Gesellschaft“ (Romero, in: León 1994: 37). So können sie ohne Schwierigkeiten von irrationalen Organisationen mobilisiert werden, um an internationale Gelder zu kommen.

Ein Ergebnis der Inti Raymi 1990 war die der Vorlage des „,Vertrags über das territoriale Recht der Kichwa, Shiwar und Achuar-Völker der Provinz Pastaza zur Unterschrift mit dem ecuadorianischen Staat" (Acuerdo sobre el derecho territorial de los pueblos Quichua, Shiwiar y Achuar de la provincia de Pastaza a suscribirse con el Estado ecuatoriano), kurz „Vertrag von Sarayacu“ (Acuerdo de Sarayaku), zur Unterzeichnung im August desselben Jahres. In diesem Vertrag wird definiert, was die Indigenen unter Territorium verstehen, welches die Territorien der drei genannten Völker sind und wie sie über Gesetze und Normen geschützt werden sollen. Darin ist eine weitgehende Autonomie und Kontrolle der Indigenen über ihre Territorien und den dort befindlichen Rohstoffen im Sinne des traditionellen indigenen Rechts vorgesehen (Ojeda Segovia 2004: 33f.; Ortiz 2010: 476f.).

Auch wenn die Erhebung des Inti Raymi ihre Forderungen nicht durchsetzen konnte - die Verhandlungen mit der Regierung zogen sich hin und wurden schließlich ergebnislos abgebrochen - etablierte sich die Indigenenbewegung, und in ihrem Zentrum die CONAIE, als politischer Akteur der ecuadorianischen Gesellschaft (Guamán 2003: 102). Sie war nun die wichtigste soziale Bewegung des Landes mit dem größten Mobilisierungspotential (Lucas 2000: 11). Eine Reihe kleinerer, unabhängiger Organisationen banden sich stärker an die CONAIE oder traten ihr bei und verliehen ihr so eine weitergehende Repräsentativität. Guerrero und Ospina gehen vorsichtig - soweit, die CONAIE mehr als Produkt, denn als Produzentin des Auf- 
stands von 1990 zu bezeichnen (Guerrero/Ospina 2003). Allerdings sollte die Präsenz gerade der CONAIE im öffentlichen Raum und den Medien nicht darüber hinweg täuschen, dass ihre tatsächliche Verankerung in der indigenen Bevölkerung schwer abzuschätzen war und ist (Santana 1992: 217). Ein weiterer Effekt der Erhebung von 1990 ist die Öffnung der Streitkräfte, etwa über Projekte der Sozialarbeit, hin zur indigenen Bevölkerung (Lucas 2000: 10) - eine Entwicklung, die ein Jahrzehnt später zu einer offenen Kooperation führen sollte.

Leon Zamosc (1994: 37) sieht in der Namensgebung der Aktion als levantamiento, also Aufstand oder Erhebung, einen Versuch, an die indigenen Aufstände des 16. bis 19. Jahrhunderts anzuknüpfen. Auch weil die konkreten Aktionsformen nur wenig mit einem Aufstand gemein haben (Zamosc 1994: 37), gibt es Gründe, ihm zuzustimmen. Tatsächlich nahm die CONAIE (1989) schon in den Jahren vor 1990 auf die Aufstände zur Zeit der Kolonie Bezug. Dazu gehört auch die ständige Erwähnung und Abbildung der Führer dieser Aufstände, wie etwa Rumiñahui.

Auf dem III. Nationalen Kongress der CONAIE, der im selben Jahr stattfand, wurde Luis Macas, ein Vertreter der ,harten', konfliktiven Linie in der CONAIE (Barrera 2001: 119), zum Präsidenten der Organisation gewählt. Macas stand - und steht bis heute - für eine Verbindung ethnischer bzw. kultureller und sozialer bzw. wirtschaftlicher Inhalte in einem einheitlichen Programm (Guerrero/Ospina 2003), wodurch sich die CONAIE Koalitionen mit anderen Organisationen und Bewegungen weiter öffnen konnte. Es wurde beschlossen, 1992 eine weitere nationale Erhebung durchzuführen, wenn bis dahin nicht die 16 Forderungen des Inti Raymi erfüllt worden waren. Außerdem wurde beschlossen, nicht an den Wahlen 1992 teilzunehmen und keine Partei zu unterstützen (Barrera 2001: 119).

Die Kampagne der CONAIE, bis 1992 alle haciendas abzuschaffen, fügte sich in ihre Forderungen nach einer rechtlichen Anerkennung der indigenen Territorien und nach einer neuen Landreform ein (Moreno/Figueroa 1992: 44). Da die Regierung weder auf diese Forderungen noch die des Inti Raymi einging, kam es im April 1992 (Martínez Novo 2009a: 176) zu einem Protestmarsch der Indigenen, dem ,Marsch für das Leben“ (Marcha por la Vida) (Pacari 2007: 36), der von der OPIP unter ihrem Präsidenten Antonio Vargas und der CONFENIAE ausging. Von ihrem Regionalsitz marschierten die Indigenen über das Zentrum und den Norden des Landes nach Quito, um so die rechtliche Anerkennung ihrer Territorien als ersten Schritt des Aufbaus der indigenen Nationalitäten (García Serrano 2001: 71) und eines plurinationalen Staates zu fordern (Barrera 2001: 124). Dazu wurde die Einberufung einer Verfassungsgebenden Versammlung gefordert (Viteri 1997: 54). Die Regierung Rodrigo Borja (1988-1992) erkannte daraufhin die Rechte der Indigenen über mehr als eine Million Hektar Land an (Pajuelo 2004: 47; Guatemal 2006: 9), die Hälfte der geforderten Fläche (Ojeda Segovia 2004: 35) - lehnte aber die Begriffe Territorialität und Plurinationalität als Gefährdung der nationalen Ein- 
heit ab (Barrera 2001: 124; Maldonado 2006: 93). 1992 war auch das Jahr der amerikaweiten Kampagne für die 500 Jahre indigenen Widerstands seit der Eroberung Amerikas. Die vielfältigen Aktionen, die im Rahmen dieser Kampagne stattfanden, erlaubten den indigenen Organisationen, ihre internationalen Kontakte zu intensivieren (Pajuelo 2004: 47).

Zu Beginn der 1990er wurde die Nationale Agrar-Koordination (Coordinadora Agraria Nacional) als Plattform der CONAIE, der FENOCIN, der CONFEUNASCC, der FEI und der FEINE gegründet, um gegen die Pläne zu kämpfen, die Agrarreform formell zu beenden (Buendía 2000: 60; Agualsaca o.J.: 3). 1993 trat der Koordination auch die FENACLE bei, die sich ab diesem Zeitpunkt der Politik der CONAIE annäherte (Negreiros 2009: 136).

Der konservative Präsident Sixto Durán-Ballén schuf noch 1992 das Sekretariat der indigenen Angelegenheiten und ethnischen Minderheiten (Secretaria de Asuntos Indígenas y Minorías Étnicas, SENAIN), das sein geringes Budget für kleinere Infrastrukturmaßnahmen in den dieser Institution politisch und geographisch nahestehenden indigenen Gemeinden verwendete (Rodríguez/Martínez 2000: 73).

Der IV. Nationale Kongress der CONAIE im Dezember 1993 war vom Eindruck der Erfolge der letzten Jahre geprägt. Die offensichtliche Stärke der CONAIE und der Indigenenbewegung führte zu Überlegungen, wie sich diese Stärke in die staatlichen Institutionen übertragen lässt, wie man also die Entscheidungen des Staates im Sinne der Indigenenbewegung beeinflussen kann. Der Schritt zu einer Überlegung, wie direkte politische Teilhabe möglich ist, war also nicht groß (Pacari 2007: 36). Auch wegen der schlechten Erfahrungen, die die Indigenenbewegung mit den politischen Parteien hatte, wurde eine direkte Unterstützung der CONAIE für Parteien und indigenen Kandidaten in Parteien ausgeschlossen (CONAIE 1994: 26)48. Es hatte bei den Wahlen 1988 eine teilweise Unterstützung Borjas und der Izquierda Democrática (ID) gegeben - die die Erwartungen der Indigenen enttäuschten. Die Teilnahme an Wahlen ,unter besonderen politischen Umständen“ (CONAIE 1994: 26) und unter direktem Bezug auf das auf demselben Kongress ausgearbeitete Politische Projekt wurde von der Entscheidung eines Kongresses der CONAIE abhängig gemacht. Dazu wurde eine Verfassungsreform angestrebt, um die Kandidatur außerhalb der großen Parteien zu erlauben - ein Vorschlag, der 1994 dem Nationalkongress präsentiert wurde (Pacari 2007: 41). Gleichzeitig wurde eine Taktik des Aufbaus indigener und Volksparlamente eingeschlagen, die als Alternative zum staatlichen Parlament gedacht waren (Guerrero/Ospina 2003). Der bis dahin betriebene Aufbau einer eigenen Institutionalität der Indigenenbewegung - unter Führung der CONAIE - wurde zugunsten der Forderungen nach einer

48 Der entsprechende Absatz wurde in der zweiten (CONAIE 1997: 25f.) und dritten (CONAIE 2001: 17) Version des Politischen Projektes entfernt. 
grundlegenden Reform des Staates im Sinne der Plurinationalität zurückgestellt (García Serrano 2005: 4).

1993 erstellte eine Arbeitsgruppe der CONAIE ein ,Politisches Projekt" (Proyecto Político) als Manifest der Forderungen und Pläne der Organisation, das 1994 veröffentlicht (Barrera 2001: 189) und 1997 und 2001 verändert wurde und bis heute die Grundlage des Handelns der CONAIE ist. Die Festlegung politischer Forderungen und Prinzipien in einem abgeschlossenen Text ist auch eine Konsequenz der Reaktionen, die die Forderungen des Inti Raymi 1990 provoziert hatten (Pajuelo 2004: 47). Im Zentrum der ersten Ausarbeitung steht der Aufbau eines plurinationalen Staates über eine Reform und Ausweitung der Demokratie (Van Cott 2005: 110; Tibán/García 2006: 4; Larrea 2008: 36). Die Thesen des Politischen Projektes der CONAIE sind das Ergebnis von Diskussionen, die schon seit der Gründung des CONACNIE stattfanden. Der plurinationale Staat ist die Grundlage für den Kampf für eine Anerkennung der verschiedenen indigenen Völker und Nationalitäten und ihrer Autonomie gegenüber dem Staat. Er ist dem uninationalen, exkludierenden Staat entgegengesetzt, als den die CONAIE den derzeitigen ecuadorianischen Staat beschreibt. Es soll eine gemischte Wirtschaftsordnung aufgebaut werden, die einen gleichen und gerechten Zugang zu den Ressourcen in Harmonie mit der Natur ermöglicht. Diese Verbindung ethnischer und klassistischer Forderungen erlaubte der CONAIE, sich dezidiert an Aktionen gegen wirtschaftsliberale Reformen zu beteiligen (Santillana/Herrera 2009: 334f.)49.

Zwischen dem 15. und 26. Juni 1994 fand unter dem Namen Mobilisierung für das Leben und das Agrargesetz (Movilización por la Vida y la Ley Agraria) die zweite nationale Erhebung der Indigenen statt, die sich gegen das Gesetz der Agrarentwicklung (Ley de Desarrollo Agropecuario) richtete (Pajuelo 2004: 48). Dieses Gesetz der Regierung Sixto Durán (1992-1996) bedeutete das Ende der Agrarreform. Es sollte das als unproduktiv betrachtete Gemeindeland der indigenen Gemeinschaften in den Markt integrieren und marktwirtschaftliche Mechanismen auf dem Land fördern (Maldonado 2006: 93f.). Nach langen Verhandlungen, die für die Indigenen von Nina Pacari geführt wurden, wurden die entsprechenden Abschnitte im Gesetz verändert (García Serrano 2001: 71) - ohne das Gesetz allerdings völlig zu entkräften (Ospina 2006: 104; Martínez Valle 2006: 108). Die traditionellen indigenen Territorien wurden als Besitz der jeweiligen Völker anerkannt und mit Eigentumstiteln garantiert (Pajuelo 2004: 48). Das IERAC wurde durch das Nationale Institut für Landentwicklung (Instituto Nacional de Desarrollo Agrario, INDA) ersetzt, das wirtschaftsnähere Aufgaben wahrnehmen sollte (Becker 1997: 325; Becker/Tutillo 2009: 219). Das Ende der Agrarreform bedeutete für die Indi-

49 Im zweiten Teil dieser Arbeit wird näher auf die Inhalte des Politischen Projektes eingegangen. 
genenbewegung und besonders für die CONAIE eine Verschiebung ihres Programms weg von den klassischen Forderungen nach Umverteilung hin zu einer Behauptung und Verteidigung der Ethnizität. Während sich die Indigenenbewegung ideologisch weiterbewegt hat, hat sich die Situation auf dem Land kaum verändert. Seit 1974, kurz nach der zweiten Agrarreform, hat sich die Landverteilung nicht verändert und ist nach wie vor mit einem Gini-Index von 0,80 sehr hoch konzentriert. Die Indigenen sind in einer Position von Klein- und Kleinstbesitzern stecken geblieben, ohne die Chance zu haben, ihre Situation gemäß den Regeln des Marktes zu verbessern (Martínez Valle 2006: 108).

Die Regierung Durán Ballén organisierte im August 1994 eine Volksbefragung über eine Reform der Verfassung und die Möglichkeit, ohne Bindung zu den registrierten Parteien zu Wahlen anzutreten (Ibarra 2010: 39f.). Diese Vorschläge wurden mehrheitlich unterstützt und daraufhin das Wahlrecht reformiert (Guerrero/Ospina 2003). Im Oktober 1994 präsentierte die CONAIE ihr Projekt für eine Verfassungsreform. Der Staat sollte seine Vielfältigkeit anerkennen und sich zum plurinationalen Staat erklären (Barrera 2001: 172). Die indigenen Sprachen sollten als Amtssprachen anerkannt werden und die lokalen Regierungen in indigen besiedelten Gebieten größere Autonomie erhalten. Weiterhin sollten von 30\% der Sitze des nationalen und der regionalen Parlamente der indigenen Repräsentation vorbehalten sein (Ibarra 2010: 41)50. Diese sollten gemäß den internen Wahlformen der jeweiligen Völker bestimmt werden (Barrera 2001: 173; Pacari 2007: 41). Der Staat sollte die Plurinationalität und die interkulturelle, zweisprachige Erziehung fördern (Rodríguez 2008: 105). Auch sollte eine Novelle zur Einberufung einer Verfassungsgebenden Versammlung durch die Zivilgesellschaft (Bernal 2000: 44) in die Verfassung integriert werden - eine Strategie, um über eine neue Verfassung die Plurinationalität formal verankern zu können. Der Kongress verweigerte wieder eine Diskussion über die Vorschläge (Pajuelo 2004: 47). Durch die Verfassungsreform wurde Ecuador in der im Januar 1996 verabschiedeten Verfassung zum ,plurikulturellen und multiethnischen Einheitsstaat“ (Art. 1) erklärt (Bernal 2000: 44; Pajuelo 2004: 49) und die indigenen Sprachen in ihren jeweiligen Verbreitungsgebieten und die zweisprachige Erziehung anerkannt - wodurch die Forderungen der indigenen Organisationen teilweise übernommen wurden. Diese Reform ist durchaus als Erfolg der Indigenenbewegung und vor allem der CONAIE zu werten - auch wenn noch, wie auch in der Verfassung 1998, die Begriffe der CONAIE vermieden wurden (Maldonado 2006: 139).

Bis Mitte der 1990er konzentrierte sich die CONAIE darauf, die Landkonflikte, die oft schon seit den 1960ern bestanden, über verschiedene Wege - politisch,

50 Ein ähnlicher Vorschlag für die Verfassungsgebende Versammlung 1997/1998 wurde ebenfalls abgelehnt (Guerrero/Ospina 2003). 
sozial und rechtlich - zu lösen, womit sie bei den meisten dieser Konflikte Erfolg hatte (Simbaña 2007: 21).

Im November 1995 versuchte Präsident Sixto Durán über ein Referendum mit elf Fragen, eine Reihe von Gesetzen wirtschaftsliberalen Inhalts durchzusetzen. Die Reformvorschläge erstreckten sich von einer Reform der Sozialversicherung über eine Deregulierung der Arbeit im öffentlichen Dienst und einer Dezentralisierung des Staates hin zu neuen Möglichkeiten, das Parlament aufzulösen und einer neuen politischen Instanz, den Distrikt-Abgeordneten im Parlament (Ibarra 2010: 42). Die CONAIE beteiligte sich aktiv am Kampf für das „Nein“ in dieser Abstimmung (Santillana/Herrera 2009: 335) und konnte sich über ihre Kooperation mit nichtindigenen Organisationen als wichtigster sozialer und politischer Akteur des Landes behaupten (Rodríguez 2008: 181f.). 58\% der abgegebenen Stimmen waren gegen die Vorschläge (Ibarra 2010: 42). Diese Erfahrung führte zur Entscheidung, eine Partei der sozialen Bewegungen zu gründen. Die Plurinationale Bewegung Pachakutik-Neues Land (Movimiento Plurinacional Pachakutik-Nuevo País, MUPP-NP) nahm an den nationalen und regionalen Wahlen 1996 teil und konnte 53 Indigene in Wahlämter bringen (García Serrano 2001: 71).

Der bei den Wahlen 1996 gewählte Präsident Abdalá Bucaram (1996-97) setzte sein schon 1988 aufgestelltes (Frank/Patiño/Rodriguez 1992: 42) Versprechen um, ein Ministerium für indigene Angelegenheiten zu schaffen. Dieses Ministerium ersetzte das unter Sixto Durán geschaffene Sekretariat der indigenen Angelegenheiten und ethnischen Minderheiten (Barrera 2001: 221). Das und andere Versuche, Teile der Indigenenbewegung - vor allem im Amazonasgebiet - für seine Regierung zu gewinnen, interpretierte die CONAIE als Versuch, sie zu spalten. Die Unterstützung, die Bucaram von einigen Organisationen des Amazonasgebietes bekam, beantwortete er mit der Unterstützung von Antonio Vargas, dem Kandidat dieser Organisationen, innerhalb der CONAIE - wohl auch um die eher klassenkämpferisch eingestellte Führungsriege um Luis Macas zu schwächen (Barrera o.J.: 154). Insbesondere konnte Bucaram die Unterstützung vom Shuar und CONFENIAE-Mitglied Rafael Pandam, dem Vizepräsidenten der CONAIE, gewinnen, den er zum Minister für indigene Angelegenheiten ernannte (Pacari 2007: 64) - woraufhin Pandam seine Posten in der CONAIE verlor und aus ihr ausgeschlossen wurde.

Tatsächlich verschärften sich interne Konflikte auf dem V. Kongress 1996/97 soweit, dass die Organisation kurz vor dem Zerbrechen stand. Nachdem die Abstimmungen auf dem Kongress im Dezember 1996 unter Abwesenheit der ECUARUNARI stattfanden, musste der Kongress im Januar 1997 weitergeführt werden (Pacari 2007: 65f.). Der Präsident der OPIP, Antonio Vargas, setzte sich in den Wahlen zum Präsidenten der CONAIE gegen den amtierenden Luis Macas durch (Guerrero/Ospina 2003). Bis 2001 war mit ihm ein Vertreter der ethnischen Tendenzen der Indigenenbewegung Präsident der CONAIE. Dadurch identifizierte sich 
die CONAIE mit ethnologisch inspirierten Konzepten wie Ethnie - im Gegensatz zu politischeren Begriffen wie Nationalität oder Volk (Simbaña I-1). Während die CONAIE durch Vargas als radikal ethnozentrische Organisation wahrgenommen wurde, wurden die spezifischen Forderungen der Indigenen zugunsten nationalen Projekten zurückgestellt. Statt für indigene Territorien oder den Erhalt der Biodiversität zu kämpfen, konzentrierte sich Vargas auf ,Themen von nationaler Transzendenz" (Pacari 2007: 73f.) - und schwächte so die Position der indigenen Organisationen. Die CONAIE reagierte auf diese Krise mit dem Aufbau der Patriotischen Front (Frente Patriótico), die neben der CONAIE die Volksfront (Frente Popular), die Koordination der sozialen Bewegungen (Coordinadora de Movimientos Sociales, CMS) und die FUT umfasste (Pajuelo 2004: 50). Die CMS war im Juni 1995 als Koordinationsinstanz derjenigen Organisationen gebildet, die der CONAIE nahestanden. In ihr waren die CONFEUNASSC, die Gewerkschaften der Öl- und Energiearbeiter und einige kleinere Organisationen vertreten (Barrera 2001: 177). CONAIE und MUPP-NP waren Teil der Opposition gegen Präsident Bucaram und konnte ihn - über gemeinsame Aktionen aller sozialen Bewegungen und parlamentarische Mittel - im Februar 1997 absetzen (García Serrano 2001: 71).

$\mathrm{Zu}$ den Kompromissen, die die Indigenenbewegung dem Nachfolger Bucarams, Fabián Alarcón, noch im März 1997 abringen konnte, gehörte die Ersetzung des ethnischen Ministeriums Bucarams durch einen Rat für Entwicklungsplanung der Indigenen und Schwarzen Völker Ecuadors (Consejo de Planificación del Desarrollo de los Pueblos Indígenas y Negros, CONPLADEIN). Der CONPLADEIN war dem Präsidenten direkt zugeordnet, sein Oberster Rat setzte sich aus Vertretern aller sieben nationalen Indigenen- und Bauernorganisationen zusammen. Die erste Exekutivsekretärin war Nina Pacari (Barrera 2001: 235). Auch wenn der CONPLADEIN einen hohen Grad an Autonomie hatte, war seine tatsächliche Effektivität wegen permanenter Unterfinanzierung gering. So wurde vom ersten Budgetentwurf nur 1,7\% vom Staat bewilligt (Rodríguez/Martínez 2000: 73; Pajuelo 2004: 50f.). Ein Ergebnis der ersten Monate der CONPLADEIN war der Aufbau des von der Weltbank geförderten Entwicklungsprojektes der Indigenen und Schwarzen Völker Ecuadors (Proyecto de Desarrollo de los Pueblos Indígenas y Negros del Ecuador, PRODEPINE) (Barrera 2001: 236) in Kooperation mit der CONAIE 1998. Auch wenn das PRODEPINE beachtliche finanzielle Mittel für die indigenen Organisationen bereitstellte, nahm nach einiger Zeit die Kritik der indigenen Organisationen zu. PRODEPINE

,erlaubte den Eintritt für NRO und versuchte, die politische und ideologische Position der Indigenenbewegung zu verzerren, indem es sie in eine Entwicklungsbewegung auf Projektbasis verwandeln wollte, so dass man von der Regierung aus versuchte, die Struktur der Organisation zu zerstören“ (Cholango in Tamayo 2007: 8f.). 
Unter der Regierung Mahuad (1998-2000) wurde 1999 (Büschges 2009: 45f.) der CONPLADEIN durch den Entwicklungsrat der Nationalitäten und Völker Ecuadors (Consejo de Desarrollo de las Nacionalidades y Pueblos, CODENPE) ersetzt, dessen Kontrollgremien nicht mehr von den Organisationen, sondern nach Zugehörigkeit zu den Nationalitäten und Völkern bestimmt werden (Rodríguez/Martínez 2000: 74). So sollte die staatliche Verpflichtung, die Indigenen in Planung und Entscheidungsfindung einzubinden, umgesetzt werden (Maldonado 2006: 133). Diese Institution soll Entwicklungsprojekte für Indigene auf dem Land koordinieren (FEI o.J.a), Forschungsprojekte anstoßen und indigene Repräsentanten in die staatlichen Institutionen entsenden (Maldonado 2006: 133).

Die durch die Absetzung Bucarams hervorgerufene Krise des Staates sollte durch eine neue Verfassung behoben werden. 1997 fanden Wahlen zu einer Verfassungsgebenden Versammlung statt, die die konservativen Parteien mit 37 von 70 Sitzen gewannen (Ibarra 2010: 42f.). Die parallel zum Kongress tätige Verfassungsgebende Versammlung trat im Dezember 1997 zusammen. Als Reaktion auf die eher geringe Repräsentation der sozialen Bewegungen beriefen CONAIE und CMS am 12. Oktober 1997 eine Verfassungsgebende Versammlung des Volkes (Asamblea Constituyente del Pueblo) ein, die weitgehend von den Organisationen des Andenhochlandes dominiert wurde. Gefordert wurde neben der Plurinationalität die Verteidigung der bäuerlichen Sozialversicherung und der staatlichen Unternehmen, so wie das Nichtbezahlen der Auslandsschulden. Diese Versammlung unterstützte das Verfassungsprojekt der CONAIE (Ibarra 2010: 42f.), das als ,Verfassung des Volkes“ (Constitución del Pueblo) am 19. Dezember 1997 verabschiedet wurde. Die offizielle Verfassungsgebende Versammlung weigerte sich, die vorgeschlagenen Begriffe ,Nationalitäten“ und ,Plurinationalität‘ zu verwenden und sprach stattdessen von ,Völkern“ und ,Ethnien“ (Pajuelo 2004: 51). Im Juni 1998 trat die neue Verfassung in Kraft, die die kollektiven Rechte der indigenen Völker anerkannte, das Abkommen 169 der OIT ratifizierte und Ecuador zu einem pluriethnischen und multikulturellen Staat erklärte (Lucas 2000: 13; Pajuelo 2004: 51 ). Die CONAIE konnte sich mit ihrer Forderung nach Plurinationalität, die über die vier Achsen Autonomie, Territorium, Identität und Entwicklung (Noboa 2005: 102) verankert werden sollte, nicht durchsetzen (CONAIE 2007a: 3).

Schon 1999 kämpfte die CONAIE gegen den nächsten Präsidenten, Mahuad, mit der Indigenen Erhebung für das Leben und gegen den Hunger (Levantamiento Indígena por la Vida y Contra el Hambre). Diese hatte zwei Teile, eine Erhebung im März 1999, eine weitere im Juli 1999 - beide waren Reaktionen auf bestimmte Beschlüsse der Regierung. Im März protestierten die Indigenen gegen die Sperrung der privaten Konten im Rahmen des feriado bancario, wobei über eine Million Indigener in zehn Provinzen des Hochlandes und sechs des Amazonasgebietes vier Tage lang die wichtigsten Straßen sperrten. Auch andere soziale Bewegungen 
schlossen sich dem Protest an. Im Juli beschloss die Regierung eine Steigerung des Benzinpreises, wodurch ähnliche Aktionen provoziert wurden (Pajuelo 2004: 52). Diesmal fand auch ein Marsch der indigenen Organisationen der Provinzen Cotopaxi, Tungurahua, Pichincha und Imbabura nach Quito statt, über den 12.000 Indigene direkte Verhandlungen mit dem Präsidenten erzwingen wollten. Nach zwei Tagen Protest in Quito unterzeichnete die Regierung eine Vereinbarung mit den Organisationen, die sie allerdings nicht einhielt. Daher beschloss die CONAIE auf ihrem Kongress im November 1999, die Verhandlungen abzubrechen und mit weiteren Aktionen zu beginnen. Der VI. Nationale Kongress der CONAIE wurde mit der Unterstützung der Armee, die Antonio Vargas erbeten hatte, durchgeführt. Auf ihm konnte sich der Präsident der ECUARUNARI, Ricardo Ulcuango, nicht gegen Vargas durchsetzen, der als Präsident der CONAIE bestätigt wurde (Pacari 2007: 73).

$\mathrm{Zu}$ den Zugeständnissen, zu denen die CONAIE den Staat bringen konnte, gehörte die Schaffung der Nationalen Gesundheitsdirektion der indigenen Völker (Dirección Nacional de Salud de los Pueblos Indígenas, DNSPI) als Bestandteil des Gesundheitsministeriums. Über eine solche Institution, die die traditionelle Medizin der Indigenen erforschen und in die öffentliche Gesundheitsversorgung integrieren sollte, war bereits seit 1993 verhandelt worden (Maldonado 2006: 133).

Nachdem die Regierung den Umstieg auf den US-Dollar als Leitwährung ab Januar 2000 beschlossen hatte und sich die wirtschaftliche Lage weiter verschlechterte, marschierten die Indigenen erneut nach Quito und setzten zusammen mit anderen sozialen Bewegungen und Teilen der Armee die Regierung ab, um am 21. Januar 2000 eine neue, demokratische Staatsform zu etablieren (García Serrano 2001: 72). Damit hatte die Armee die Rolle des Vermittlers zwischen sozialen Bewegungen und dem Rest des Landes und der Regierung übernommen, was sich auch in der Absetzung Mahuads und der Einsetzung seines Vizepräsidenten Noboas durch die Armee ausdrückte - ein offensichtlicher Verstoß gegen die Verfassung (García Serrano 2001: 73).

Die weiteren Aktionen der CONAIE im Jahr 2000 zeigten ihre Abhängigkeit von ihren nicht-indigenen Verbündeten. Im August versuchte sie, eine Volksabstimmung über eine Verfassungsänderung durchzusetzen, im September, eine weitere nationale Erhebung zu organisieren. Beide Aktionen scheiterten (García Serrano 2001: 79), im Fall der Volksabstimmung, die weitgehend von Vargas selbst ausging, an gefälschten Unterschriften (Pacari 2007: 73f.). Diese Niederlagen bedeuteten vor allem für Antonio Vargas, den Präsidenten der CONAIE, einen Vertrauensverlust, da er sich vehement für diese Aktionen ausgesprochen hatte, aber nicht genug Unterstützung mobilisieren konnte (Pajuelo 2004: 53; Mayer 2008: 45). Als Reaktion wurde die Mesa Agraria als Plattform der Organisationen, die Landarbeiter vertreten, ins Leben gerufen. Sie umfasste neben der CONAIE die CON- 
FEUNASSC-CNC und die FENOCIN und organisierte gemeinsame Aktionen für die Verteidigung der Landbevölkerung (Negreiros 2009: 137).

Im Juni 2000 wurde der Entwicklungsfond der indigenen Völker (Fondo de Desarrollo de los Pueblos Indígenas, FODEPI) als autonome, staatliche Institution gegründet, die von Vertretern der indigenen Völker verwaltet wird. Daher forderten die indigenen Organisationen von Beginn an einen größeren Einfluss und die Möglichkeit, direkt an Planung und Durchführung der Entwicklungsprojekte beteiligt zu sein (Maldonado 2006: 134).

Im Februar 2001 rief die CONAIE erneut zu einer nationalen Erhebung, diesmal mit deutlich mehr Erfolg (Guerrero/Ospina 2003). Es wurde gegen eine Preiserhöhung für Gas und Benzin und für eine Verbesserung des öffentlichen Nahverkehrs protestiert. Ab März 2001 kam es so zu neuen Verhandlungen zwischen der Regierung und der CONAIE, die zu teilweisen Zugeständnissen führten (García Serrano 2001: 73). In einem neuen Projekt für ein Nationalitätengesetz im selben Jahr präsentierte die CONAIE erneut ihre politischen Forderungen, wieder ohne Erfolg (CONAIE 2007a: 3). Im Oktober 2001 fand ein - vorgezogener - Kongress der CONAIE statt, in dem sie sich, entsprechend den Beschlüssen auf dem Kongress von 1999 (Guerrero/Ospina 2003; Pacari 2007: 74), nach Nationalitäten und Völkern - anstatt nach Organisationen - neu organisierte. So lässt sich auch der neue Name erklären: ,Erster Kongress der Völker und Nationalitäten Ecuadors ‘ (Albó 2009: 231). Auf diesem Kongress konnten die im selben Jahr offensichtlich gewordenen Konflikte innerhalb der CONAIE über den Austausch des Führungspersonals gelöst werden (Pajuelo 2004: 55). Der amtierende Präsident Antonio Vargas konnte mit Hilfe seiner Unterstützer aus dem Amazonasgebiet Leonidas Iza, einen anderen Vertreter der ethnischen-indigenistischen Gruppe (Mayer 2008: 74), bei den Wahlen zum Präsidenten der CONAIE durchsetzen (Mayer 2008: 45).

Vargas selbst verstand das als eine Unterstützung seiner geplanten Präsidentschaftskandidatur bei den nationalen Wahlen 2002, die er im Dezember 2001 verkündete (Mayer 2008: 45). MUPP-NP und CONAIE entschlossen sich allerdings, Auki Tituaña statt Vargas aufzustellen, woraufhin dieser sich der FEINE anschloss und über ihre Partei Amauta Jatari kandidierte. Vargas wurde daraufhin aus der CONAIE ausgeschlossen, genoss aber weiterhin die Unterstützung bestimmter Gruppen, gerade in der CONFENIAE (Guerrero/Ospina 2003; Mayer 2008: 45). Um die direkte Konkurrenz zweier indigener Kandidaten zu verhindern, entschlossen sich MUPP-NP und CONAIE dazu, keinen eigenen Kandidaten aufzustellen, sondern die Kandidatur eines der Protagonisten des 21. Januar 2000, Lucio Gutiérrez, zu unterstützen. Die Kandidatur von Vargas scheiterte mit nur 0,8\% der Stimmen, woraufhin die CONFENIAE wieder die MUPP-NP und Gutiérrez unterstützte (Mayer 2008: 46f.). 
Die Koalition der MUPP-NP mit Gutiérrez 2003 (Crnic 2009: 46) hielt nur wenige Monate bis die Indigenen in Opposition zur liberalen Wirtschaftspolitik der Regierung gingen. Das Jahr 2004 war geprägt vom Widerstand der CONAIE gegen die Regierung - bei gleichzeitiger struktureller Schwäche und weitgehender Unfähigkeit, ihre Mitglieder zu mobilisieren (Martínez Novo 2009a: 177). Ein Aufruf zur nationalen Erhebung im Juni 2004 wurde von den Basisorganisationen nicht befolgt (Guatemal 2006: 11). Die meist genannten Gründe dieser Schwäche sind der Legitimitätsverlust durch die Regierungsteilnahme, die Schwäche und fehlende Klarheit des Führungspersonals und als ethnozentrisch wahrgenommene Bestandteile der Forderung nach Plurinationalität (Simbaña 2007: 21). Dazu kam die staatliche Förderung der kleineren indigenen Organisationen vor allem seit der Regierung Gutiérrez, mit dem Ziel, die Indigenenbewegung zu spalten und so zu schwächen (Martínez Novo 2009a: 176). Im März 2004 etwa ernannte Gutiérrez Antonio Vargas zum Sozialminister (Mayer 2008: 68), um so dessen Unterstützer in der CONFENIAE wieder für sich gewinnen zu können. Erst auf ihrem Kongress 2004 konnte die CONAIE mit der Wahl Luis Macas zu ihrem Präsidenten eine Phase der Erneuerung einleiten, die im Januar 2005 zu ihren ersten Protesten seit 2001 führte, als 2.000 Personen gegen das geplante Freihandelsabkommen demonstrierten (Albó 2009). Die Teilnahme an den Aktionen gegen das Freihandelsabkommen erlaubten der CONAIE, sich innerhalb der sozialen Bewegungen als politischer Akteur neu zu positionieren (Simbaña 2007: 23). Das Freihandelsabkommen scheiterte schließlich 2006 wegen der Aufhebung der Verträge mit dem US-amerikanischen Erdölunternehmen OXY durch den ecuadorianischen Staat - ein Vorgang, den die USA als Enteignung interpretierten (Ibarra 2010: 48). Die Pläne der Regierung Gutiérrez, den Sitz der CONAIE in Quito zu besetzen und eine neue, ,offizielle' CONAIE um Antonio Vargas, Nelson Chimbo, José Quenama und andere aufzubauen, wurden nur von seinem Sturz im März 2005 vereitelt (Guatemal 2006: 11). Auch die wichtige Rolle, die die CONAIE und ihre Filialen in verschiedenen transnationalen Indigenenorganisationen spielten (etwa die COICA und CAOI) (Becker 2008a: 167), halfen ihr, die Krise zu überwinden und in verschiedenen Kontexten wieder aktiv zu werden.

Die beiden Abgeordneten des Nationalkongresses, Luis Macas und Leonidas Iza, bildeten im Jahr 1996 eine Arbeitsgruppe, die einen Vorschlag für den Aufbau einer indigenen Universität ausarbeiten sollte (Sarango 2009: 196). Nach neun Jahren der Vorbereitung wurde die von der CONAIE und dem ICCI gegründete Interkulturelle Universität der indigenen Nationalitäten und Völker ,Amawtay Wasi“ (Haus der Weisheit) (Universidad Intercultural de las Nacionalidades y Pueblos Indígenas ,Amawtay Wasi', UINPI) 2004 per Gesetz geschaffen und 2005 vom Nationalen Rat der höheren Erziehung (CONESUP) anerkannt (Sarango 2009: 199; Mato 2010: 112). Sie ist eine private und gemeinschaftliche (universidad 
comunitaria) Universität im Sinne der ecuadorianischen Verfassung von 2008 mit Autonomie in Lehre, Forschung und Verwaltung (Sarango 2009: 193f.). Die UINPI finanziert sich zu einem Großteil durch Spenden von internationalen und nationalen Organisationen, darunter der schweizer Entwicklungshilfeagentur Swissaid, CARE International und der CODENPE (Sarango 2009: 194). Sie hat zusammen mit der Autonomen Indigenen Interkulturellen Universität (Universidad Autónoma Indígena Intercultural, UAIIN) Kolumbiens und der Universität der Autonomen Regionen der Nicaraguanischen Karibikküste (Universidad de las Regiones Autónomas de la Costa Caribe Nicaragüense, URACCAN) das Netzwerk der Indigenen Universitäten Abya Yalas (Red de Universidades Indígenas de Abya Yala, RUIA) gegründet, das die Qualitätsstandards seiner Mitgliedsuniversitäten festlegen und überprüfen soll (Sarango 2009: 195). Der RUIA sind mittlerweile weitere Universitäten aus Mexiko und Bolivien beigetreten. Die UINPI ist auf Studiengänge in Menschenrechte und indigenen Völker, Agrarökologie, Erziehungswissenschaften und Architektur spezialisiert (Mato 2010: 112) und untersteht der CONESUP, dem ICCI und der CONAIE (Sarango 2009: 191; Mato 2010: 113). Sie bietet sowohl formelle Studiengänge an, bis hin zum Grad des Masters, als auch informelle Bildung in Lerngemeinschaften, die sich vor allem an indigene Gemeinden richten (Sarango 2009: 192). Dennoch ist die UINPI eine sehr kleine Universität. 2009 waren 156 Studenten und 35 Lehrkräfte an den grundständigen Studiengängen beteiligt und weitere 32 Studenten und 24 Lehrkräfte an den Masterprogrammen, die in Kooperation mit der Zentraluniversität Ecuadors (Universidad Central del Ecuador) durchgeführt werden. Auch an den Lerngemeinschaften der informellen Bildung haben 2009 nur 70 Studenten und 8 Lehrkräfte teilgenommen. Seit der Gründung der UINPI 2004 bis zum Jahr 2009 hatten sich nur insgesamt 90 Studenten in den Lerngemeinschaften immatrikuliert. Insgesamt sind 70\% der Studenten und 60\% der Lehrkräfte Indigene (Sarango 2009: 193-195).

2006 erarbeitete die CONAIE ihren Entwurf für ein Gesetz der indigenen Institutionen (CONAIE 2007a: 3), der eine größere Autonomie der indigenen Gemeinschaften vorsah. Im selben Jahr lehnten die CONAIE und die MUPP-NP eine Wahlallianz mit Rafael Correa und seiner Alianza PAÍS ab (Minkner-Brünjer 2009: 141), da dieser nicht als Vizepräsidentschaftskandidat neben Luis Macas antreten wollte. Die Wahlergebnisse 2006 und 2007 bei den Wahlen zur Verfassungsgebenden Versammlung waren für die MUPP-NP enttäuschend (Martínez Novo 2009a: 177). Dennoch nahm die CONAIE und ihre Filialen großen Einfluss auf die Verfassungsgebende Versammlung, es gab Verfassungsentwürfe bzw. -vorschläge der ECUARUNARI, der Shuar-Organisationen, der CONAIE selbst und der MUPP-NP (CONAIE 2007a: 3), die zumindest in den Diskussionen eine wichtige Rolle spielten. 
In der Verfassungsgebenden Versammlung 2007 und 2008 kam es zu Auseinandersetzungen zwischen den Forderungen der Plurinationalität, die MUPP-NP und CONAIE erhoben und denen nach Interkulturalität, die PS-FA, FENOCIN und FEINE erhoben (Walsh 2009b: 235). Wieder wurde der Plurinationalität vorgeworfen, die indigenen Völker von der Gesellschaft isolieren zu wollen, während die Interkulturalität als Möglichkeit der Verständigung der verschiedenen Völker präsentiert wurde (Larrea 2008: 36). Die Plurinationalität und die dazugehörigen Begriffe der Territorialität, der politischen Autonomie und der Kontrolle über die natürlichen Ressourcen wurden als separatistisches Projekt begriffen (Ibarra 2010: 56). Auch wenn die Erklärung Ecuadors zu einem plurinationalen und interkulturellen Staat im ersten Artikel der neuen Verfassung für die CONAIE ein wichtiger Fortschritt ist, wurden in diesen Auseinandersetzungen beide Vorschläge erheblich abgeschwächt und auf bloße Erklärungen reduziert oder ,gezähmt“ (Simbaña 2008a: 240). Der Umbau des Staates und seiner Strukturen, den sich die CONAIE von der Plurinationalität erhoffte, fand nicht statt, neue Mechanismen der Beteiligung wurden nur unzureichend aufgebaut (Resina de la Fuente 2011: 109f.). So blieben staatliche Eingriffsrechte in die territoriale Autonomie der Indigenen bestehen, die Ressourcen in den Territorien gehören dem ecuadorianischen Staat. Die Indigenen werden weiterhin nur über die traditionellen demokratischen Mechanismen vertreten (Martínez Novo 2010: 12). Die Forderung der Indigenenbewegung nach einer Formalisierung der bindenden vorherigen Befragung der betroffenen Gemeinden über den Abbau von Rohstoffen dort verdeutlicht die Unterschiede zwischen Indigenenbewegung und Regierung. Für die CONAIE ist diese bindende Befragung ein Teil der Autonomierechte der Nationalitäten und somit die Grundlage für die Plurinationalität, die Regierung folgt aber einer auf Entwicklung ausgerichteten Wirtschaftspolitik, die auf die Einnahmen aus dem Export von Rohstoffen angewiesen ist (Resina de la Fuente 2011: 109f.). Schließlich setzte sich die Regierung durch und die vorherige Befragung wurde als nicht bindend in die Verfassung integriert. Dieser Konflikt spiegelt sich auch in der Stärkung des Nationalen Planungs- und Entwicklungssekretariats (Secretaría Nacional de Planificación y Desarrollo, SENPLADES) ab 2007 wider. Dieses verfügt nur über beschränkte Partizipationsmöglichkeiten und integriert die indigenen Organisationen nur punktuell, was sich in seinem Nationalem Plan für das Gute Leben (Plan Nacional para el Buen Vivir) 2009-2013 ausdrückt, der weitgehend einer klassischen staatlichen Planung entspricht (Resina de la Fuente 2011: 111). Im Gegensatz zur tiefgreifenden Umgestaltung des Staates und der Gesellschaft, wie die CONAIE sie anstrebt, steht das SENPLADES für einen schrittweisen Umbau, in dem nicht die Indigenen und ihre Organisationen die Initiative haben, sondern der Staat selbst, der die indigenen Zielsetzungen nurmehr integriert (Resina de la Fuente 2011: 113). 
Dennoch zeigt die Integration der Plurinationalität in die Verfassung auch den Einfluss der den Indigenen gegenüber freundlich gesonnenen sozialen Bewegungen innerhalb der Regierungspartei, die sich auch gegen die ablehnende Haltung des Präsidenten durchsetzen konnten (Ramírez 2010: 96). Die Präsenz der Begriffe der Indigenenbewegung in der Verfassung gibt dieser Bewegung eine bessere Position, da sie jetzt die Prinzipien des Staates selbst einfordern kann - und nicht mehr potenziell separatistische Ideen einer ethnischen Minderheit.

„Auch wenn es nur Schmuck ist, aber in der Verfassung ist die Pachamama, auch wenn nur als Fassade, die Plurinationalität ist drin. Aber diese Fassade, dieser Titel, dieser Schmuck sind da, uns bringt das sehr viel, denn wir werden die Plurinationalität einfordern und die Ausübung der Plurinationalität.“ (Tenesaca 2010: 108f.)

Die Beziehung der CONAIE zur Regierung Correa entwickelte sich von einer kritischen Unterstützung 2006 bis 2008 zur offenen Opposition ab 2009. Noch 2007 bezeichnet der damalige Präsident der ECUARUNARI, Humberto Cholango, die Regierung Correa als progressiv und sagt, dass sich dadurch die Indigenenbewegung reorganisieren und von innen heraus stärken könnte (Cholango in Tamayo 2007: 15). Tatsächlich verkörpert Correa den Wunsch der sozialen Bewegungen nach einem Wandel, wobei er einige der direkten Forderungen übernimmt, ihren radikalen Kern aber verwirft. Dadurch erhält er den Gedanken des Wandels, macht ihn aber kontrollier- und realpolitisch verwendbar (Saltos/Vázquez 2007: 354f.). Kurz nach der Annahme der neuen Verfassung kippt die Stimmung im Konflikt über ein Bergbaugesetz.

„Eine Klage der CONAIE auf Verfassungswidrigkeit eines im Jahr 2009 verabschiedeten, umstrittenen Minengesetzes - u.a. wegen Verletzung des Buen Vivir - wurde allerdings abgelehnt. Auch der Versuch, ein neues Wassergesetz zu verabschieden, wurde von der Regierung erst nach monatelangen Protesten durch indigene Organisationen fallen gelassen.“ (Schilling-Vacaflor 2010: 4)

Die Erteilung neuer Konzessionen für die Erdölförderung im Amazonasgebiet, der systematische Ausschluss indigener Expertise aus den Bereichen Wassermanagement und zweisprachige Erziehung und das geringe Interesse des Präsidenten, einen partizipativen Politikstil zu entwickeln, trugen zu einer weiteren Distanzierung der Indigenenbewegung von der Regierung bei (CONAIE 2011: 25).

Die CONAIE versteht sich als Regierung der Indigenen und wird als solche von der ecuadorianischen Regierung nicht akzeptiert. Die Autonomie der bestehenden staatlichen Institutionen unter Kontrolle der CONAIE und der anderen indigenen Organisationen, vor allem CODENPE und DINEIB (CONAIE 2011: 17), wurde 
unter Correa erheblich eingeschränkt und diese - teilweise - von einem Sekretariat der Völker, Bewegungen und Teilhabe der Bürger (Secretaría de Pueblos, Movimientos y Participación Ciudadana) ersetzt, das mit den lokalen anstatt der nationalen Organisationen kooperiert (Wolff 2010: 21). Die Regierung Correa betrachtet diese Institutionen als eine unzureichende Antwort auf die indigenen Forderungen aus der Zeit neoliberaler Politik, zudem hätten sich DINEIB und CODENPE in klientelistische und korrupte Räume verwandelt (Resina de la Fuente 2011: 111). Vor allem die DINEIB, die im Februar 2009 per Dekret dem Bildungsministerium untergeordnet wurde, ist von hoher symbolischer und praktischer Bedeutung für die CONAIE, da viele ihrer Führungspersönlichkeiten in ihr aktiv sind (Martínez Novo 2010: 15f.). Obwohl die CONAIE diese beiden Institutionen stets als unzureichend und fehlerhaft betrachtet hat, waren es autonome Behörden unter der Kontrolle der Indigenen. Ihre Schwächung bedeutet für die indigenen Organisationen einen direkten Angriff auf sie selbst und eine erneute Zentralisierung der indigenen Angelegenheiten in staatlichen Institutionen, ohne angemessene Partizipation der Indigenen (Resina de la Fuente 2011: 111). Der geringe Widerstand gegen diese Maßnahmen wird oft als Indiz für die Krise der CONAIE gewertet (Martínez Novo 2009: 2f.; Martínez Novo 2009a: 177). Der Aufbau einer Reihe von Sozial- und Entwicklungsprogrammen für Basisorganisationen, wie socio páramo und socio bosque, die Umweltschutzmaßnahmen auf lokalem Niveau unterstützen, wird von der CONAIE als Vereinnahmung ihrer Mitgliedsorganisationen gesehen (Martínez Novo 2010: 7). Im Februar 2010 beendete die CONAIE ihre Verhandlungen mit der Regierung, die sie als Verteidiger ,der Interessen des transnationalen Kapitals und der Gruppen der wirtschaftlichen Macht“ (CONAIE 2010: 1) betrachtet. Rafael Correa wird als Verräter bezeichnet, der den „Kolonialismus des 21. Jahrhunderts“ (CONAIE 2010: 2) vertritt. Gleichzeitig wird die Suche nach Allianzen im Kampf gegen die Politik der Regierung beschlossen und dass Regierungsvertreter nur noch mit Erlaubnis der jeweiligen Indigenenorganisationen indigene Territorien betreten dürfen (Martínez Novo 2010: 1).

Die Konflikte mit der Regierung Correa haben zu einer Stärkung der CONAIE geführt, die gemeinsam mit anderen, von der Regierung vernachlässigten Gruppen, wie den Umweltschützern, die Durchsetzung der Verfassung und ihrer rechtlichen Neuerungen verlangt (Martínez Novo 2010: 7/22).

\begin{tabular}{|l|l|l|l|l|l|}
\hline Jahr & $\begin{array}{l}\text { Nummer des } \\
\text { Kongresses }\end{array}$ & $\begin{array}{l}\text { Teilneh- } \\
\text { mer }\end{array}$ & Ort & $\begin{array}{l}\text { Besondere } \\
\text { Neuerungen }\end{array}$ & $\begin{array}{l}\text { Gewählter } \\
\text { Präsident }\end{array}$ \\
\hline 1980 & $\begin{array}{l}\text { Erstes Treffen der } \\
\text { indigenen } \\
\text { Nationalitäten } \\
\text { Ecuadors }\end{array}$ & Sucúa & $\begin{array}{l}\text { Gründung des } \\
\text { CONACNIE }\end{array}$ & $\begin{array}{l}\text { Blanca } \\
\text { Chancoso }\end{array}$ \\
\hline
\end{tabular}




\begin{tabular}{|c|c|c|c|c|c|}
\hline 1984 & $\begin{array}{l}\text { II. Kongress des } \\
\text { CONACNIE }\end{array}$ & & Quito & $\begin{array}{l}\text { Unabhängigkeit } \\
\text { von allen Parteien } \\
\text { wird erklärt }\end{array}$ & \\
\hline 1986 & $\begin{array}{l}\text { III. Kongress des } \\
\text { CONACNIE, I. } \\
\text { Nationaler } \\
\text { Kongress der } \\
\text { CONAIE }\end{array}$ & & Quito & $\begin{array}{l}\text { Gründung der } \\
\text { CONAIE }\end{array}$ & $\begin{array}{l}\text { Miguel } \\
\text { Tan- } \\
\text { kamash }\end{array}$ \\
\hline 1988 & $\begin{array}{l}\text { II. Nationaler } \\
\text { Kongress der } \\
\text { CONAIE }\end{array}$ & & Cañar & $\begin{array}{l}\text { Abkommen über } \\
\text { Interkulturelle } \\
\text { Zweisprachige } \\
\text { Erziehung } \\
\text { (DINEIB) }\end{array}$ & $\begin{array}{l}\text { Cristóbal } \\
\text { Tapuy }\end{array}$ \\
\hline 1990 & $\begin{array}{l}\text { III. Nationaler } \\
\text { Kongress der } \\
\text { CONAIE }\end{array}$ & & $\begin{array}{l}\text { Pujilí, } \\
\text { Cotopaxi }\end{array}$ & $\begin{array}{l}\text { Beschluss der } \\
\text { Erhebung im Juni } \\
1990\end{array}$ & $\begin{array}{l}\text { Luis } \\
\text { Macas }\end{array}$ \\
\hline 1993 & $\begin{array}{l}\text { IV. Nationaler } \\
\text { Kongress der } \\
\text { CONAIE }\end{array}$ & & & $\begin{array}{l}\text { Beschluss der } \\
\text { Teilnahme an den } \\
\text { nächsten Wahlen }\end{array}$ & $\begin{array}{l}\text { Wieder- } \\
\text { wahl }\end{array}$ \\
\hline $\begin{array}{l}1996 \\
17\end{array}$ & $\begin{array}{l}\text { V. Nationaler } \\
\text { Kongress der } \\
\text { CONAIE }\end{array}$ & & $\begin{array}{l}\text { Shindar, } \\
\text { Saraguro, } \\
\text { Loja }\end{array}$ & $\begin{array}{l}\text { Starker Druck der } \\
\text { Regierung, } \\
\text { Kongress muss } \\
\text { wiederholt werden }\end{array}$ & $\begin{array}{l}\text { Antonio } \\
\text { Vargas }\end{array}$ \\
\hline 1999 & $\begin{array}{l}\text { VI. Nationaler } \\
\text { Kongress der } \\
\text { CONAIE }\end{array}$ & & $\begin{array}{l}\text { Santo } \\
\text { Domingo } \\
\text { de los } \\
\text { Colorados }\end{array}$ & $\begin{array}{l}\text { Unter Anwesenheit } \\
\text { und Hilfe des } \\
\text { Militärs }\end{array}$ & $\begin{array}{l}\text { Wieder- } \\
\text { wahl }\end{array}$ \\
\hline 2001 & $\begin{array}{l}\text { I. Kongress der } \\
\text { indigenen Völker } \\
\text { und } \\
\text { Nationalitäten } \\
\text { Ecuadors }\end{array}$ & $\begin{array}{l}1100, \text { da- } \\
\text { von } 745 \\
\text { Vertreter } \\
\text { der } 35 \mathrm{Na}- \\
\text { tionalitäten } \\
\text { und Völker }\end{array}$ & Quito & $\begin{array}{l}\text { Vorgezogener } \\
\text { Kongress }\end{array}$ & $\begin{array}{l}\text { Leonidas } \\
\text { Iza }\end{array}$ \\
\hline
\end{tabular}




\begin{tabular}{|l|l|l|l|l|l|}
\hline 2004 & $\begin{array}{l}\text { II. Kongress der } \\
\text { indigenen Völker } \\
\text { und Nationalitä- } \\
\text { ten Ecuadors }\end{array}$ & 1070 & Otavalo & & $\begin{array}{l}\text { Luis } \\
\text { Macas }\end{array}$ \\
\hline 2008 & $\begin{array}{l}\text { III. Kongress der } \\
\text { indigenen Völker } \\
\text { und } \\
\text { Nationalitäten } \\
\text { Ecuadors }\end{array}$ & $\begin{array}{l}1300 \text { aus } \\
24 \text { Provin- } \\
\text { zen }\end{array}$ & $\begin{array}{l}\text { Santo } \\
\text { Domingo } \\
\text { de los } \\
\text { Colorados }\end{array}$ & $\begin{array}{l}\text { Marlón } \\
\text { Santi }\end{array}$ \\
\hline 2011 & $\begin{array}{l}\text { IV. Kongress der } \\
\text { indigenen Völker } \\
\text { und } \\
\text { Nationalitäten } \\
\text { Ecuadors }\end{array}$ & 3500 & $\begin{array}{l}\text { Puyo, } \\
\text { Pastaza }\end{array}$ & & $\begin{array}{l}\text { Humberto } \\
\text { Cholango }\end{array}$ \\
\hline
\end{tabular}

Tabelle 4: Kongresse von CONACNIE und CONAIE, eigene Abbildung

Die CONAIE ist die größte und einflussreichste Organisation der Indigenenbewegung, dennoch ist sie nicht umfassend im Land vertreten. Die OZGs der CONAIE sind in $60 \%$ der Kantone und $40 \%$ der parroquias Ecuadors vertreten (Rodríguez 2008: 159). Insbesondere FEINE und FENOCIN (Petras/Veltmeyer 2005: 153) sind eine direkte Konkurrenz für sie.

Die CONAIE setzt sich aus den drei regionalen Filialen ECUARUNARI, CONFENIAE und CONAICE zusammen, die Föderationen der Provinzen, Kantone und Parroquias bzw. Assoziationen, Zentren, Gemeinschaften usw. in den jeweiligen Regionen umfassen (CONAIE o.J.: 6). Von besonderer Bedeutung sind die OEG, im Andenhochland vor allem die Kommunen, im Amazonasgebiet vor allem die Zentren. Sie spielen eine wichtige Rolle im Entscheidungsprozess und bei den konkreten Aktionen der CONAIE und liefern eine „eigene zivilisatorische Vision“ (Saltos 2000: 7f.). Durch die zentrale Stellung der Basis in der Gesamtorganisation verfügen die CONAIE und ihre Filialen über einen hohen Organisationsgrad. Die jeweiligen Mitgliedsorganisationen sind autonom und verhalten sich weitgehend eigenständig zu Problemen in ihrer jeweiligen Region (Minkner-Brünjer 2009: 149). Das ist die Grundlage für den Aufbau der CONAIE von unten nach oben sowohl in der Organisationsstruktur als auch bei der Mobilisierung (MinknerBrünjer 2009: 158). Eine ständige Präsenz der Führungsebene der CONAIE in den lokalen Organisationen und Regierungen ermöglicht erst die Bildung von Allianzen und eine Mobilisierung der Basis (Resina de la Fuente 2011: 112). Tatsächlich werden alle größeren Aktionen der CONAIE mit den verschiedenen Mitgliedsorganisationen abgesprochen - genauso wie das Führungspersonal nur durch die Zu- 
stimmung der Basisorganisationen legitimiert ist. Diese erwarten, dass die gewählte Person nach Ende ihres Mandats in die jeweilige Ortschaft zurückkehrt und sich erneut in der OEG engagiert (Collins 2006: 226-228). Viele Forscher betonen diesen konsensuellen Aspekt in der internen Entscheidungsfindung der CONAIE, der zu einer hohen Interaktion zwischen den verschiedenen Mitgliedsorganisationen der CONAIE führt und Konflikte reduziert. Dennoch wird der Konsens mit den Mechanismen repräsentativer Demokratie, insbesondere bei der Wahl von Repräsentanten, kombiniert, so dass auch schnelle Entscheidungen möglich sind. Manchmal wird diese Erweiterung auch als eine Ersetzung des gemeinschaftlichen Konsenses gesehen (Viteri 1997: 55f.). Zwar wird bei den Wahlen darauf geachtet, dass es allgemeine Übereinstimmung über die jeweilige Entscheidung gibt (Porras 2005: 97), oft reduziert sich dieses Verständnis von Konsens jedoch auf eine Parität zwischen den größten Organisationen und Nationalitäten. Das hat zu einer „Hegemonie der ,großen“ Nationalitäten in der Führung der regionalen Organisationen“ (Viteri 1997: 56) geführt. Die schiere Größe der Nationalitäten der Kichwa und Shuar macht somit eine Beachtung der internen Diversität schwierig.

Die CONAIE selbst hat drei exekutive Instanzen: der alle drei Jahre stattfindende Kongress ist die höchste Instanz, auf der alle weiteren gewählt werden. Die zweithöchste Instanz ist die Versammlung, die dritthöchste der Regierungsrat (CONAIE o.J.: 5). Nach einer Schätzung des CODENPE von 2007 sind 804.801 Personen Mitglied in einer der 141 OZG der CONAIE in 16 Provinzen (Azuay, Bolívar, Cañar, Chimborazo, Cotopaxi, Esmeraldas, Imbabura, Loja, Morona Santiago, Napo, Orellana, Pastaza, Pichincha, Sucumbíos, Tungurahua, Zamora Chinchipe) (Ospina 2009: 124; IEE o.J.) ${ }^{51}$. Gerade das Mobilisierungspotential der CONAIE ist ein Alleinstellungsmerkmal auf internationalem Niveau. Zumindest bis 2002 gab es in Lateinamerika keine indigene Organisation mit vergleichbaren Strukturen und Möglichkeiten (Zamosc 2005: 197). Dazu kommt der innovative Diskurs und Forderungskatalog, der die Verbindung von ethnischen und sozialen Aspekten ermöglicht hat (Barrera o.J.: 152).

\section{Politik der CONAIE}

Einige der politischen Ideen und Strategien der CONAIE und ihrer Tochterorganisationen stammen aus den internationalen und kontinentalen Organisationsversuchen indigener Bewegungen um den Begriff des Indianismus ab Mitte der 1970er Jahre. Von besonderer Bedeutung ist der I. Kongress der Indio-Bewegungen Südamerikas (I Congreso de Movimientos Indios de Sudamérica) in Ollantaytambo,

51 Diese Ziffer war nicht überprüfbar. Auf Nachfrage hat die CODENPE mitgeteilt, keinerlei Daten über die Organisationen der Indigenenbewegung zu erheben - sondern nur über die indigenen Völker und Nationalitäten. 
Peru, 1980, an dem Vertreter von Organisationen Perus, Ecuadors, Kolumbiens, Venezuelas, Brasiliens, Boliviens, Paraguays, Argentiniens, Chiles und einige Beobachter anderer Länder teilnahmen. Er baute auf dem I. Indioparlament des Cono Sur (I Parlamento Indio Americano del Cono Sur) auf, das 1974 in Asunción, Paraguay, stattgefunden hat und zur Gründung des Weltrates der Indio-Völker (Consejo Mundial de Pueblos Indios, CMPI) 1975 in Port Alberni, Kanada, geführt hat. Dieser, wie der auf dem I. Kongress der Indio-Bewegungen Südamerikas gegründete Indio-Rat Südamerikas (Consejo Indio de Sudamérica, CISA) mit Sitz in La Paz, Bolivien, vertritt die indigenen Organisationen international bis in die 1990er und wirkt bis heute nach (AAVV 1985: 162).

Einige der auf dem Kongress 1980 beschlossenen Positionen spiegeln sich in denen der CONAIE wider. So widmet sie sich - ohne das als Indianismus zu bezeichnen - dem ,Wiederfinden und der Identifikation mit unserer historischen Vergangenheit" (AAVV 1985: 163), also dem traditionellen Kommunitarismus der indigenen Völker (AAVV 1985: 163) als Alternative zum bestehenden Wirtschaftssystem und den traditionellen moralischen Prinzipien des ,ama swa, ama llulla y ama qhe 1la“ (AAVV 1985: 164), nicht lügen, nicht stehlen, nicht faul sein. Auch die damals vorgeschlagenen Strategien wurden von der CONAIE und ihren Mitgliedsorganisationen aufgegriffen:

„a) Wenn das Indio-Volk die Mehrheit stellt, wird sein direktes Ziel die Machtergreifung sein. b) Wenn das Indio-Volk in der Minderheit ist, sollte es eine Autonomie erreichen, ohne auf das Recht zu verzichten, mit anderen Gruppen des Volkes Aktionen zu planen, ohne aber seine Unabhängigkeit und kulturelle Identität zu gefährden.“ (AAVV 1985: 164)

Die CONAIE und die FENOCIN waren bis 1987 Mitglieder der CISA, sie schieden wegen der geringer werdenden externen Finanzierung dieser aus. Ihre letzte Teilnahme in diesen Institutionen war auf der IV. Generalversammlung des CMPI 1992 in Tromsoe, Norwegen. Der CMPI existiert heute nicht mehr ${ }^{52}$.

Da die beschriebenen Institutionen vor allem für Peru und Bolivien von Bedeutung sind, während die ecuadorianischen Organisationen andere Strukturen aufzubauen versuchten, überrascht die Übernahme der Positionen von CISA und CMPI durch die CONAIE. So kombinierte sie von Beginn an in ihrer Strategie den sozialen Protest in breiten Bündnissen mit der Schaffung eigener Räume innerhalb des Staates. Über konstante Verhandlungen mit staatlichen Organen erlangte sie Kontrolle über einige der Institutionen, die sich mit indigenen Angelegenheiten beschäftigten, zuerst die der zweisprachigen Erziehung. Auch die Erhebungen von 1990, 1992 und 1994 führten - trotz der weitgehenden Forderungen - zu wenig radikalen

52 Mit Dank an Tomás Condori für die wertvollen Informationen. 
Verhandlungen der CONAIE mit dem Staat, die ihre Position stärkten ohne dabei durch überzogene Forderungen zum Scheitern verurteilt zu sein (Ospina 2006: 104). Die CONAIE ist also gespalten zwischen sozialer Bewegung und politischem Akteur. Auf der einen Seite dient ihr anti-systemisches, kritisches Programm als Mobilisierungsstrategie und Garant für ihren Erfolg, auf der anderen Seite wird dieser Erfolg durch die realpolitischen Verhandlungen mit dem Staat gefährdet (Ojeda Segovia 2004: 23) - die aber gleichzeitig Gewinne und Kompromisse erlauben, die das Weiterbestehen der Organisation sichern.

Vielleicht ist diese doppelte Strategie der Verhandlung mit dem Staat (innen) bei gleichzeitigem Druck auf dem Staat (von außen) mit der ,doppelten Dimension" (Becker 2008b: 45) der Unterdrückung der Indigenen in Verbindung zu bringen, also ihrer Konstituiertheit als Klasse und als Ethnie. „Die Forderungen haben sich [...] um zwei Achsen gedreht, die eine verbunden mit dem Wohlergehen der gesamten armen Bevölkerung des Landes und die andere mit Aspekten des spezifischen Wohlergehens der indigenen Völker." (García Serrano 2001: 76) Eine Verbindung beider Strategien, der der Teilhabe und der der Autonomie, kann man im Diskurs der CONAIE finden. Begriffe wie Plurinationalität, kollektive Rechte, Territorialität oder Autonomie „bildeten den Rahmen für ihre Aktionen während der gesamten Dekade [der 1990er, P.A.]“ (Minkner-Brünjer 2009: 157) und der daran anschließenden Zeit. Die CONAIE versteht sich als „Regierung der indigenen Völker und Nationalitäten Ecuadors“ (CONAIE o.J.: 6), die „den Prozess des Aufbaus des Plurinationalen Staates und der Ausübung der kollektiven Rechte anführt“ (CONAIE o.J.: 6). Diese Forderung - zusammen mit den anderen Prinzipien der CONAIE, Plurikulturalität, Interkulturalität, Einheit in der Verschiedenheit, Solidarität (CONAIE o.J.: 7) - hat sich seit 1990 zum Kristallisationspunkt des Kampfes der CONAIE und der Zivilgesellschaft als Ganzes entwickelt (García Serrano 2001: 74f.). Die Indigenenbewegung und die CONAIE schufen „begriffliche Instrumente“ (CONAIE 1989: 279), wie indigene Nationalität, Territorien, kulturelle Differenz, selbstbestimmte Entwicklung. Auch dadurch unterscheiden sich die Aktionen der CONAIE von denen der anderen großen indigenen Organisationen - die Einbindung lokaler kultureller Praktiken und Traditionen in die nationale Politik über Begriffe wie Plurinationalität ist etwas völlig neues in Ecuador (Korovkin 1999: 10).

„Diese Symbiose des Ethnisch-Kulturellen mit dem Klassenbegriff und seiner Dimension in Bezug auf das Staats- und Entwicklungsmodell [...] bedeutet, dass die indigenen Völker und Nationalitäten einen qualitativen Fortschritt in der doktrinären Entwicklung eines politischen Denkens, das auf die Wirklichkeiten seiner Völker und der Ecuadors heute antworten kann, gemacht haben.“ (Pacari 2007: 24) 
Die CONAIE will sich mit dem Kampf um Identität als „Referenzpunkt für den Rest der ecuadorianischen Gesellschaft“ (CONAIE 1992a) behaupten. Ihr Einstehen für Differenz und Andersheit konnten sich mit den Begriffen der traditionellen Linken im Kampf gegen die wirtschaftliche Umgestaltung der letzten 20 Jahre verbinden und so konnte sich ,die ecuadorianische Indigenenbewegung in der Avantgarde des gesellschaftlichen Widerstands gegen die neoliberale Politik platzieren“ (Ramírez Gallegos 2009: 65). Dadurch konnte sie sich der Gesamtgesellschaft im Rahmen einer ,Strategie der Sichtbarkeit“ (Resina de la Fuente 2011: 114) als attraktiver zivilgesellschaftlicher Akteur mit moralischem Führungsanspruch zu präsentieren. Die Suche nach Allianzen drückt sich auch im Spruch „,nichts für die Indios, alles für alle““ (Resina de la Fuente 2011: 112) aus, der eine kommunitäre Neugründung des Staates für die gesamte Gesellschaft ausdrückt. Die CONAIE sieht den Kampf gegen Kolonialismus und Neokolonialismus, sowohl von Seiten des Staates und der Gesellschaft als auch von Seiten transnationaler Unternehmen (CONAIE 2005), als grundlegendes Ziel ihrer Arbeit (CONAIE o.J.: 7f.). So konstituiert sich die CONAIE als ,post-marxistisch“ (Black 1999: 4), in dem Sinne, dass sie die klassischen marxistischen Begriffe ablehnt und statt der Machtergreifung einer Klasse eine radikale und inklusive Demokratie anstrebt, in der alle Gruppen gleichermaßen repräsentiert sind. Die Mischung von identitären und klassistischen Sichtweisen erlaubte die Entwicklung ,alternativer Pfade zu einem modernen Verständnis der Nationalidentität“" (Black 1999: 4).

Die CONAIE ist eine pluralistische Organisation, die kollektive Forderungen nach sozialen Wandel mit unkonventionellen Taktiken und alternativen politischen Aktionen in einem Kontext sozialer Konfliktivität umsetzen will (Sánchez/Freidenberg 1998: 71). Dem neoliberalen Staat soll eine inklusive Alternative entgegengestellt werden, der anti-neoliberale und globalisierungskritische mit ethnokulturellen Forderungen verbindet: der plurinationale Staat (García Serrano 2005: 4). Als eine solche Alternative wurde er schon früh definiert - etwa auf dem zweiten Kongress der CONAIE 1988 (CONAIE 1988 in Simbaña 2006: 66f.). Die CONAIE sucht - mit unterschiedlichen Schwerpunkten im Laufe ihrer Entwicklung Allianzen und Koalitionen mit anderen sozialen Bewegungen und Sektoren wie den Gewerkschaften, politischen Organisationen, Studentenorganisationen etc., die im Rahmen gegenseitigen Respekts und gleichartiger Ziele für die politische und wirtschaftliche Befreiung kämpfen - und in letzter Instanz für den Aufbau des „Neuen Plurinationalen Staates und der Neuen Interkulturellen Gesellschaft“ (CONAIE 2001: 17). Dieser soll sich durch eine partizipative Demokratie und eine Dezentralisierung der Macht und der wirtschaftlichen Ressourcen auszeichnen (CONAIE 2005). Da die CONAIE - im Gegensatz zu den Gewerkschaften und FEI und FENOCIN - nicht unter dem Einfluss einer politischen Partei entstanden ist, konnte sie ihren Diskurs freier entwickeln und sozialistische Elemente mit ethnisch- 
kulturellen Elementen verbinden (Salamea 2006: 54f.). Die CONAIE ist also eine kollektive und identitäre Organisation, die der Gesamtgesellschaft die Idee eines plurinationalen Staates anbieten kann (Pacari 2007: 26f.) - und damit auch einen Schwachpunkt hat. Spätestens seit die CONAIE 1990 ins Zentrum der sozialen Bewegungen getreten ist, ist ihre wichtigste Forderung die nach dem Aufbau eines plurinationalen Staates. Diese Beschränkung ist zusammen mit ihrer eingeschränkten Basis - Indigene - und ihrer - zumindest von 1996 bis 2005 - fehlgeleiteten Koalitionspolitik ein Grund für die schwere Krise, die die CONAIE seit 2003 durchlitten hat (Hidalgo 2005: 344).

Die Integration verschiedenster Gruppen und politischer Einstellungen innerhalb der CONAIE ist nicht ohne Konflikte abgelaufen, die immer noch andauern und zu bestimmten Zeiten wieder aufflammen. In den ersten Jahren hat das an den Mitgliedsorganisationen - und nicht den Völkern und Nationalitäten - ausgerichtete Organisationsmodell interne Kritik hervorgerufen, da es die interne Pluralität nur bedingt widerspiegelt (Viteri 1997: 56). Ein Weg, diese Probleme zu reduzieren, war die Umgestaltung der CONAIE von einer Konföderation von Organisationen zu einer Konföderation von Völkern und Nationalitäten, die ab etwa 1996 angestrebt (Viteri 1997: 57), allerdings nur bedingt erreicht wurde. Ein Zeichen dieses versuchten Wandels ist die Namensänderung der Kongresse der CONAIE und ihrer Mitgliedsorganisationen ab 2000, die auch deren Zählweise verändert hat.

\section{Strömungen in der CONAIE}

In der Indigenenbewegung Ecuadors gibt es zwei politische Richtungen - der traditionellen Klassenanalyse, wie sie vor allem FEI und FENOCIN betreiben, steht eine eher ethnisch orientierte Position gegenüber, die die sozialen Probleme als Konsequenz der Unterdrückung der Indigenen sieht. Dazu kommt die originäre Position der CONAIE, die beide Perspektiven zu integrieren versucht (CONAIE 1989: 281). Bis in die 1970er Jahre waren die Kategorien ,Indigene “ und ,Bauern “ im Sinne der traditionellen Linken weitgehend identisch - sie wurden als Teile eines entstehenden Landproletariats verstanden, also als Klasse. Auch das sich daran anschließende Verständnis der Indigenen als Ethnie sah sie als Landarbeiter und somit als Teil einer Klasse. Die Strategie, die sich aus diesem Verständnis ableitete, war ein breites Bündnis aller Arbeiter und Bauern als einziger Weg zu einer gerechteren Gesellschaft (Ruiz 1991: 477). Die Krise der politischen Linken in den 1980er und 1990ern führte sowohl innerhalb als auch außerhalb der Indigenenbewegung zu einer Abkehr von sozialistischen Positionen, wodurch die ethnischen Tendenzen an Bedeutung gewinnen konnten (Simbaña 2009: 163). Der Aufstieg von ECUARUNARI und CONFENIAE etablierte ein Verständnis der Indigenen als qualitativ von den Bauern verschieden - eben durch ihre Zugehörigkeit zu einer bestimmten Ethnie und die damit verbundene Identität und Geschichte. Daraus leitete sich - in 
einigen Fällen - eine Strategie der Autonomie und ethnischen Eigenständigkeit ab, in dem Sinne, dass die Probleme der Indigenen von den Indigenen selbst gelöst werden müssen. Statt gemeinsam mit anderen Unterdrückten Gleichheit zu fordern, wurde die Verteidigung des Eigenen, des in der Gesellschaft Anderen, angestrebt. Diese teilweise offen rassistische Position wurde nur von einer Minderheit vertreten - die aber internationale Unterstützung bekam, etwa von NRO wie ,Cultural Survival' (Ruiz 1991: 477). Beide Tendenzen treffen vor allem in der CONAIE, die Organisationen aus beiden Traditionen umfasst, aufeinander (Minkner-Brünjer 2009: 140).

So ist die Entwicklung der CONAIE auch eine Geschichte der Konflikte zwischen diesen beiden Strömungen und dem Versuch, sie in einer Organisation zu integrieren. Eine Strömung, die - über den Aufbau eines plurinationalen Staates eine größere Teilhabe der Indigenen an staatlichen Entscheidungen erreichen will, steht einer anderen Strömung gegenüber, die sich mehr an sozialer Gerechtigkeit orientiert - und so auch andere soziale Bewegungen leichter einbinden kann (Barrera 2002: 100). Innerhalb dieser Strömungen gibt es jeweils radikale Gruppen, die die jeweiligen Forderungen weiter zuspitzen. Eine Gruppe stellt rein ethnische Forderungen in das Zentrum ihres Handelns und ist teilweise rassistisch in dem Sinne, dass sie die Indigenen den Mestizen gegenüberstellt. Einige Personen dieser Gruppe wollen eine völlige Vertreibung der ,Invasoren “ und eine Rückkehr zu präkolumbischen Verhältnissen ${ }^{53}$. Eine weitere Gruppe ordnet den ethnischen Aspekt dem Klassenaspekt unter und versteht die Indigenen in erster Linie als Bauern. Die dritte Strömung will die beiden vorigen vereinigen und geht davon aus, dass „der indigene Kampf eine doppelte Dimension hat: Klasse und Ethnie“ (CONAIE 1989: 281). Sie will im Rahmen eines Kampfes um Respekt alle Formen von Unterdrückung und Diskriminierung abschaffen (Maldonado 2006: 90). Diese Position hat die CONAIE von Beginn an eingenommen und von dort aus nach Allianzen für eine Veränderung der Gesellschaft gesucht. Dennoch bestehen die beiden anderen Strömungen auch innerhalb der CONAIE weiter. Die Bezeichnungen für die Strömungen sind nicht einheitlich. Die ethnische Strömung wird von Beteiligten und Forschern „,indigenistisch“ (Saltos/Vázquez 2005: 372), „ethnisch oder indianistisch“ (Ruiz 1991: 477), ,indianistisch-rassistisch“ (Barrera o.J.: 160f.), „ethnizistisch“ (Simbaña 2007: 22), ,kulturalistisch“ (Simbaña 2008a: 237), ,,anthropologisch“ (Simbaña 2009: 163), ,ethnozentrisch“ (Simbaña 2009: 163), ,ethnopopulistisch“ (Rodríguez/Martínez 2000: 76) genannt, die klassenanalytische Strömung ,alternativ“ (Saltos/Vázquez 2005: 372), „klassistisch“ (Barrera o.J.: 160f.;

53 Eine solche Gruppe ist in Ecuador nicht nachweisbar - ein politischer Gebrauch dieser Bezeichnung, um bestimmte Strömungen innerhalb der CONAIE auszugrenzen, ist wahrscheinlich. 
Simbaña 2008a: 237), „historisch“ (Simbaña 2007: 22), „links“ (Simbaña 2007: 23).

Die CONAIE hatte schon früh den Anspruch, beide Strömungen zu integrieren. In diesem Sinne spricht sie von einem ,doppelten Charakter unserer Problematik: als Mitglieder einer Klasse und als Teil verschiedener indigener Nationalitäten“ (CONAIE 1989: 261f.). Diese Vorstellung hat ihren Ursprung zu Beginn der 1980er Jahre. Nina Pacari, die den Aufbau von ECUARUNARI und CONAIE eng begleitet hat, formulierte schon 1984:

„Wir sind ein Teil der Ausgebeuteten dieses Landes, aber wir werden auch politisch und kulturell beherrscht, denn wir sind unterdrückte Nationalitäten. Unser Problem ist nicht nur eines des Klassenkampfes, sondern es ist auch ein Volkskampf, als Volk. Wir wollen, dass unsere Existenz anerkannt wird. Das bedeutet nicht, dass wir uns isolieren, im Gegenteil, wir sind in den Kämpfen und mit den Kämpfen.“ (Pacari 1984: 118f.)

Die Unfähigkeit der früheren indigenen Organisationen wie der FEI und der FENOCIN, diesen Komplex angemessen zu erfassen, trug dazu bei, dass die Probleme der Indigenen nicht gelöst werden konnten (CONAIE 1989: 261f.). Also versucht die CONAIE, eine einseitige Sicht zu vermeiden. „Klasse und Ethnizität erschienen zur gleichen Zeit konflikthaft und sich gegenseitig verstärkend.“ (Becker 2008a: 167) Dennoch war meist ein Übergewicht der klassistischen Strömung zu erkennen, was einige Forscher zum Schluss kommen ließ, dass die „CONAIE ethnische Identitäten benutzte, um eine wirtschaftliche Agenda zu befördern“ (Becker 2008: 170). Sie sah sozioökonomische Ungleichheiten - und nicht die Anwesenheit unterschiedlicher ethnischer Gruppen - als Grund für die nationalen Probleme (Becker 2008: 173).

Die Strömungen haben - tendenziell - verschiedene geographische Zentren, abhängig von der Struktur und Geschichte der Indigenen in den jeweiligen Regionen. So fand die wirtschaftliche Integration der Indigenen im Andenhochland viel früher und intensiver statt als in anderen Gebieten Ecuadors. Die ersten Mobilisierungsversuche und Gründungen von Gewerkschaften in den 1920ern, ebenso wie die Bemühungen der befreiungstheologischen Strömungen der katholischen Kirche ab den 1960ern legten die Grundlage für eine eher sozialistische Ausrichtung der heutigen Organisationen dieser Region. Daher war das Ziel dieser ,,indigenen Bauernströmung“ (Barrera 2001: 142) lange Zeit die Selbstbestimmung der Indigenen, im Sinne einer Stärkung der traditionellen Strukturen in den indigenen Siedlungen (Ramírez Gallegos 2009: 70), bei gleichzeitiger Suche nach Allianzen mit anderen klassistischen Organisationen (Ibarra Illanez 1987: 142). So verbindet sich der Kampf um Land als klassistische Politik mit dem Bezug auf die indigenen Kommunen als ethnischer Hintergrund (Barrera 2001: 143). Typische Aktionsformen dieser 
Strömung entstammen der gewerkschaftlichen und linken Tradition: direkte Aktion, Streik, Märsche, Besetzungen. Viele der Führer dieser Strömung, wie etwa Ricardo Ulcuango, José María Cabascango, Estuardo Remache, Miguel Lluco, Leonidas $\mathrm{Iza}^{54}$ und Luis Macas haben akademische Bildung und entstammen den OZG des Hochlandes (Barrera 2001: 142).

Ein Sonderfall im Andenhochland ist die ,intellektuelle, ethnisch-kulturelle Strömung“ (Barrera 2001: 143) in und um Otavalo, die sich in den 1970er Jahren entwickelt. Otavalo ist nicht von Bauern, sondern von Händlern und Handwerkern geprägt, die wenig Interesse an einer Landreform haben, sondern eher ethnische Forderungen aufstellen und mit neuen Organisationsformen erreichen wollen (Ibarra Illanez 1987: 144). Sie legen großen Wert auf die Bildung ihrer Kinder, die sie dank ihrer finanziellen Ressourcen fördern können (Flores 2005: 55f.). Kulturelle Forderungen wie der Kampf gegen Diskriminierung und die Verteidigung historisch bedeutsamer Orte werden in dieser Zeit für die Organisationen Otavalos zentral (Barrera 2001: 143). In Otavalo entstanden in den 1970ern eine Reihe kultureller Organisationen wie Conjunto Rumiñahui, Indo América, Ñanda Mañachi und Obraje, die die Traditionen der Kichwa erforschten und versuchten, erneut zu beleben, darunter das Fest des Inti Raymi, der die frühere Bezeichnung San Juan ersetzte (Kowii o.J.: 1). Er wurde 1976 zum ersten Mal wieder gefeiert (Kowii o.J.: 1f.). $\mathrm{Zu}$ diesen Organisationen gehörte auch ein Studentenklub in Peguche, der 1976 zur einflussreichen Kulturwerkstatt Taller Cultural Causanacunchic ${ }^{55}$ wurde. In dieser Werkstatt versuchten ungefähr 50 Schüler und Studenten der urbanen Mittelschicht Otavalos und Cotacachis (Hoy 2001; Ortiz Crespo 2009: 16) die Identität der Kichwa zu stärken und zu verteidigen. Dazu organisierten sie kulturelle Veranstaltungen, Diskussionsrunden und Unterstützungskomitees für indigene Schüler und Studenten. Weiterhin baute die Kulturwerkstatt 1978 die sozialwissenschaftliche Bibliothek Amauta (Kowii o.J.: 1f.) mit Schwerpunkt auf indigene Themen auf und eine Studiengruppe, die die Beziehungen zwischen Indigenen und Nichtindigenen untersuchte (Huarcaya 2011: 208f.). So begannen die daran teilnehmenden Studenten, ihre indigene Identität über ihre Kleidung, wie Hüte oder Ponchos auszudrücken (Flores 2005: 39) und kamen zum Vorschlag, dass Indigene in der Öffentlichkeit nicht mehr ihre Hüte abnehmen sollen - wie es seit der Kolonie üblich war, wenn ein Indigener einem Weißen begegnete (Huarcaya 2011: 210). Von 1976 bis 1980 leitete Ariruma Kowii (UASB o.J.), von 1980 bis 1985 Nina Pacari die Kulturwerkstatt. Auch Luis Maldonado war von 1976 bis 1982 Mitglied (Cultura Andi-

54 Der sich später als Vertreter der eher ethnisch orientierten Strömung herausstellen sollte (Mayer 2008: 74).

55 Causanacunchic bedeutet: wir haben gelebt, wir leben und wir werden leben (Pacari 1984: 118). 
na o.J.), ebenso Auki Tituaña, Blanca Chancoso (Huarcaya 2010: 312f.) und Mario Conejo (Hoy 2001). Gemeinsam las man Klassiker der lateinamerikanischen Literatur aber auch sozialistische und wissenschaftliche Texte. In diesem Zusammenhang beschlossen einige der Teilnehmer, ihre mestizischen Namen abzulegen. So wurde aus Segundo Antonio mit Bezug auf einen Sohn des Inkas und einen peruanischen Berg Auki Tituaña, aus María Esther Vega Nina Pacari (was Licht des Sonnenaufgangs bedeutet) und aus Jacinto Conejo Ariruma Kowii (nachdenklicher Mann) (Abad o.J.). Im Taller Cultural Causanacunchic wurde eine Kritik der sozialistischen und klassistischen Strömungen artikuliert, die die Ethnie nur als einen Nebenwiderspruch oder gar als falsches Bewusstsein verstanden (Huarcaya 2010: 311f.). Diese wirkte mit dem zunehmenden Engagement seiner Mitglieder in den Organisationen der Indigenenbewegung auch auf die OZG der Region, die FICI. Die Mitglieder der Kulturwerkstatt konnten die FICI beim Ausbau ihrer Programme in den Bereichen Land, Bildung, Gesundheit und Kommunikation unterstützen und so die Organisation stärken und ihre eigenen Ideen einbringen (Ortiz Crespo 2009: 18). Wegen der bis zu diesem Zeitpunkt eindeutig klassistischen Ausrichtung der FICI fand diese Integration allerdings nicht ohne Probleme statt (Flores 2005: 39).

Eine vergleichbare wirtschaftliche Integration erlebte das Amazonasgebiet erst in den 1960er Jahren, verbunden vor allem mit der wachsenden Erdölförderung. Die Indigenen des Andenhochlandes waren zu diesem Zeitpunkt schon lange als Bauern in die Nationalgesellschaft integriert - daher auch ihre Forderung nach einer Landverteilung. Im Amazonasgebiet kam es erst in den letzten 50 Jahren zu einer vergleichbaren Integration, die mit einer Verteidigung der ethnischen Identität beantwortet wurde - und nicht mit den klassischen Forderungen der Bauernbewegung (Fontaine 2001: 61). Die ethnische Zugehörigkeit war und ist stärker ausgeprägt als im Andenhochland - weshalb sie die Forderung nach - kulturell definierter - Territorialität der andinen Forderung nach Land zur Bewirtschaftung entgegenstellten (Korovkin 2008: 3). Im Amazonasgebiet spielte die „Wiedergewinnung und Verteidigung der indigenen Territorien“ (Ramírez Gallegos 2009: 70) eine wichtige Rolle - etwas, das die Organisationen des Andenhochlandes kaum anstrebten. Daher wurden hier die Begriffe von Territorialität, Nationalität und Autonomie entwickelt, die heute zentral im Diskurs der CONAIE sind (Barrera 2001: 144). Insbesondere die Kichwa des Amazonasgebietes, vor allem die aus Pastaza und der OPIP, haben eine Gruppe von Führungspersönlichkeiten entwickelt, die aus einflussreichen Familien stammen und die Entwicklung der CONAIE lange bestimmen sollten. Dazu gehören Alfredo Viteri, Leonardo Viteri, César Cerda und Antonio Vargas (Barrera 2001: 144).

Auch wenn die geographischen Unterschiede eine wichtige Rolle für das Entstehen der verschiedenen Begriffe und ideologischen Positionen spielen, sollte ihre Bedeutung nicht überschätzt werden. Tatsächlich ist zu betonen, dass die verschie- 
denen Strömungen - trotz gewisser geographischer Schwerpunkte - in allen Organisationen der CONAIE und teilweise auch den anderen Organisationen der Indigenenbewegung vorhanden sind (Ruiz 1991: 477). So kommt Floresmilo Simbaña zu folgender Einschätzung:

„Mir scheint, es ist nicht richtig zu sagen, dass es eine klassistisch gefärbte und eine andere, ethnisch oder kulturalistisch gefärbte Organisation gibt. Ich habe den Eindruck, dass diese Tendenzen nicht nach Organisationen auftreten, sondern als Tendenzen innerhalb jeder Organisation.“ (Simbaña 2008a: 237)

Weiterhin sind diese Tendenzen meist eher Neigungen als radikale, nicht verhandelbare Positionen. Es liegen keine Texte vor, die sich eindeutig den radikal klassistischen oder ethnizistischen Strömungen innerhalb der Indigenenbewegung zuordnen lassen. Daher ist zu vermuten, dass diese Bezeichnungen in erster Linie Kampfbegriffe innerhalb (etwa in FENOCIN 1999) oder außerhalb (in einigen Reden von Rafael Correa) der Bewegung sind. Die radikalen Strömungen konnten sich spätestens seit den 1980ern nicht behaupten - ganz im Gegensatz etwa zu Bolivien und dem radikal ethnizistischen Diskurs der Kataristas und von Felipe Quispe. Auch wenn sich einzelne Personen den radikalen Strömungen zuordnen lassen, dient die Beschuldigung, rein klassistisch oder rein ethnizistisch zu argumentieren, vor allem zur Ausgrenzung der gemäßigt klassistischen oder ethnizistischen Strömungen.

Die Wahl Antonio Vargas zum Präsidenten der CONAIE 1996 hat die Konflikte zwischen den beiden Strömungen offensichtlich gemacht. Er und seine Unterstützer konnten die eher klassenanalytisch orientierten Tendenzen aus den meisten Führungsinstanzen verdrängen (Simbaña 2007: 22). Somit gab es ein „Wiederauferstehen einer ethnizistischen und ethnopopulistischen Tendenz, die man als aus der Indigenenbewegung verdrängt betrachtet hatte“ (Rodríguez/Martínez 2000: 76) Diese Strömung sollte bis zur Krise der CONAIE 2003/2004 die Politik der Indigenenbewegung bestimmen.

„[D]ie soziopolitischen Konflikte der zweiten Hälfte der 1990er und der ersten Jahre nach 2000 werden als klare politisch-kulturelle Probleme gelesen [...]. Es ist kein Zufall, dass in dieser Periode die Mehrzahl der Berater des Führungsstabes indigene und nicht-indigene Anthropologen waren.“(Simbaña 2009: 164)

Die Forderung der Plurinationalität wurde nun als Konzept der ethnischen Identität und sozialen Differenz verstanden - und nicht mehr als gesamtgesellschaftlichen Vorschlag, eine auf Ausgrenzung und Ungleichheit basierende Gesellschaft zu verändern, wie sie bis dahin gemeint war (Simbaña 2009: 164). Daher geht das 
Interesse der CONAIE an Allianzen und Kooperationen mit anderen sozialen Bewegungen in dieser Zeit zurück - eine Entwicklung, die von einigen Regierungen gefördert wird (Rodríguez/Martínez 2000: 78). „[D]er politisch-wirtschaftliche Konflikt wird [...] als soziokulturelles Problem gesehen“ (Simbaña 2007: 22), durch den Einfluss des neuen Führungspersonals um Vargas wird ,der kulturelle Konflikt auf ein ethnisches Problem reduziert" (Simbaña 2007: 22). In der zweiten Hälfte der 1990er stellte die CONAIE die kulturellen Konflikte in das Zentrum ihrer Aufmerksamkeit (Simbaña 2008a: 237). Daher wird das eher wirtschaftlich und politisch ausgerichtete, ,antikapitalistische“ (Simbaña 2007: 22) Politische Projekt von 19941997 und 2001 reformiert, um es weniger radikal zu gestalten und die ethnische Sichtweise - anstelle der sozio-kulturellen - zum Ausgangspunkt für Gesellschaftsanalyse und politische Aktionen zu machen (Simbaña 2007: 22).

Die CONAIE als ,,identitäre Organisation mit bis jetzt größter Mobilisierungsund Forderungskapazität Lateinamerikas“ (Bretón 2003: 219), bediente sich zunehmend der „Ethnizität als Strategie“ (Bretón 2003: 219). Die Neu-Konstruktion der indigenen Identität ,,als Quelle der Einheit und Stärke in ihrem Kampf“" (Crnic 2009: 45) hatte den Aufstieg der CONAIE erst erlaubt. Durch den Bezug auf die indigene Identität konnte sie internationale Ressourcen und Projekte in die indigenen Gemeinden bringen - wodurch die Organisationen allerdings ihrer Charakter als Plattformen für Forderungen und soziale Kämpfe verloren und zu Entwicklungsagenturen wurden. Das Bemühen um Entwicklungsprojekte schwächte die politische Ausrichtung der Organisationen ab, sie akzeptierten den ideologischen Hintergrund der Entwicklungshilfe, um sich mit deren Projekten in ihrem Stammland behaupten zu können (Bretón 2008: 129f.). Die Führer der Epoche der Landreform wurden durch neue Führer ersetzt, die die Sprache der Entwicklungshilfe beherrschten und wussten, wie man Projekte anlocken kann. Ihre Rhetorik wurde ethnizistischer und weniger kompatibel mit den anderen armen Sektoren der Gesellschaft, sie bereiteten den Boden für die „scharfe Repräsentativitätskrise“ (Bretón 2008: 130) der CONAIE. Die Organisationen der Indigenenbewegung wurden durch ihre Integration in staatliche und nicht-staatliche Entwicklungsprojekte zu einer „Logik der Ergebnisse auf Kosten der Logik der Beratung“ (Fontaine 2001: 65) gezwungen, wodurch sie die Einbindung in ihre Basis und damit ihre Legitimität verloren.

Nichtsdestotrotz bleiben die Forderungen nach Land und Territorium für die CONAIE zentral und entfalteten vor allem zwischen 2000 und 2005 ein hohes Mobilisierungspotential - das zur Überwindung der Krise der CONAIE nach 2003 beitrug (Simbaña 2008a: 239). Ein anderer Faktor beim Wiedererstarken der CONAIE ab 2005 war der Kampf gegen das von der Regierung Gutiérrez geplante Freihandelsabkommen mit den USA. Er schwächte die ethnische Tendenz, die keinerlei Antworten auf die sozialen Probleme geben konnte, und eröffnete der 
linken Strömung neue Räume. Die indigenen Gemeinden waren von der mangelnden Effektivität der ethnischen Strömung enttäuscht, die weder die Machtstrukturen noch die Einkommensverteilung im Land verändern konnte (Büschges 2009: 58). Der Widerstand gegen die als neoliberal empfundene Politik spielte ab den 1990ern die Rolle, die in den 1970ern der Kampf um Land gespielt hatte (Korovkin 2008: 4). In diesem Prozess wurden die beiden Repräsentanten der soziopolitischen, eher klassistischen Strömung Humberto Cholango und Luis Macas zu den Präsidenten der ECUARUNARI und der CONAIE gewählt. Die Umstrukturierung der CONAIE erlaubte ihr, sich national und international weiter zu vernetzen (Simbaña 2007: 23; Simbaña 2009: 166) und zur traditionellen Politik der Bündnissuche in einer ,,breiteren Volksbewegung“ (CONAIE 1989: 261f.) zurückzukehren. In diesem Kontext konnte sich die traditionelle Verbindung beider Strömungen erneut behaupten, erkennbar etwa in einer neuen Zentralität des Begriffs der Plurinationalität, als ein Begriff, der den sozialen Kampf gegen den Neoliberalismus und den kulturellen Kampf für Selbstbestimmung verbindet (Larrea 2008: 36).

\section{d. MUPP-NP/Pachakutik}

\section{Gründung}

Seit den 1980er Jahren gab es Initiativen in der Indigenenbewegung, eine indigene Partei zu gründen, um in der Parteipolitik von der traditionellen Bindung an die linken Parteien (Marmon/Kramer 2006: 2) unabhängig zu werden. Für die damaligen Parteien spielten die Themenbereiche Ethnie und Kultur - zentrale Interessenfelder der neuen Indigenenorganisationen der CONAIE - nur selten eine Rolle, weshalb sich die Indigenen von diesen Parteien nicht vertreten fühlten und andere Organisationsformen bevorzugten (Barre 1982: 70). Das kurzzeitige Engagement von CONAIE und FENOC in der linken Sammelbewegung FADI in den 1980ern wurde aus diesem Grund beendet (Huarcaya 2011: 255). Innerhalb der CONAIE wurde im August 1987 in Pujilí der Aufbau einer ,Indigenen Politischen Front" diskutiert, die allerdings nie umgesetzt wurde (Santana 1992: 200). 1988 unterstützte die CONAIE die Kandidatur des Präsidentschaftskandidaten Rodrigo Borja der sozialdemokratischen Izquierda Democrática (ID) im zweiten Wahlgang (Rodríguez 2008: 157). Die Politik Borjas nach der gewonnenen Wahl entsprach nicht seinen Versprechen oder den Vorstellungen der indigenen Bewegung. Daraufhin rief die CONAIE seit 1990 stets zu einem Wahlboykott auf und erkannte die Wahlen nicht für sich an (Massal 2010: 23f.). Das änderte sich schrittweise ab dem dritten Kongress der CONAIE 1993, auf dem die Teilnahme an der Parteipolitik gründlich diskutiert wurde (Rodríguez 2008: 157). Es wurde die Teilnahme an lokalen Wahlen beschlossen, auch, weil in den mehrheitlich indigen besiedelten Gebieten Wahl- 
erfolge erwartet wurden (Sánchez/Freidenberg 1998: 71)56. Seit einer Gesetzesänderung 1994 war es auch unabhängigen Bewegungen möglich, bei Wahlen anzutreten, ohne formal einer Partei angehören zu müssen (Sánchez/Freidenberg 1998: 72; Rodríguez 2008: 167f.).

Die Aktionen gegen die Wirtschaftsreformen der Regierung Durán Ballén, vor allem der Sieg des ,Nein“ bei der Volksabstimmung 1995, erlaubten eine politische Neupositionierung der sozialen Bewegungen (Ramírez Gallegos 2009: 73). Die Indigenenbewegung konnte einen ersten Erfolg feiern bei ihrer neuen Strategie, Druck von außerhalb mit Teilhabe innerhalb der Politik zu verbinden (Santillana/Herrera 2009: 335). Viele der an den Protesten beteiligten Organisationen unterstützten die Gründung einer eigenen Partei - die zu Beginn auch breite Unterstützung von den sozialen Bewegungen erfuhr (Simbaña 2009: 162). Ende 1995 verhandelten die wichtigsten Organisationen, die CONAIE, die CONFEUNASSC, die Gewerkschaft der Ölarbeiter, die Koordination der sozialen Bewegungen (Coordinadora de Movimientos Sociales, CMS) ${ }^{57}$, die Bürgerbewegung für ein Neues Land (Movimiento de Ciudadanos por un Nuevo País) und einige Gruppen der politischen Linken über die Gestalt der zu gründenden Partei (Le Quang 2010: 112f.). Jede Strömung trug bestimmte Inhalte bei, der gemeinsame Nenner war der Aufbau einer Macht von unten in einem Kampf innerhalb und außerhalb der Institutionen (Becker 2008a: 168). Die Machterlangung auf lokaler und regionaler Ebene als langfristiges Ziel war ein zentraler Bestandteil dieser Strategie (García Serrano 2001: 71; Jijón 2006: 48; Büschges 2009: 49). Man kann gerade die Teilhabe indigener Politiker als Strategie der symbolischen Eröffnung einer Möglichkeit einer anderen Art von Macht verstehen, die ohne Indigene nicht mehr auskommt (Santillana/Herrera 2009: 340).

Auf einem Außerordentlichen Kongress der CONAIE im Januar 1996 wurde ihre Teilnahme an Wahlen auf allen Ebenen endgültig beschlossen. Es wurde darüber diskutiert, ob eine rein indigene Partei oder eine Frontbewegung verschiedener sozialer Bewegungen gebildet werden sollte, wobei die Form ,Bewegung ' auf begrifflicher Ebene eine Integration beider Bestrebungen erlaubte (Rodríguez 2008:

56 Dass diese Strategie nicht das Ende alternativer Mobilisierungsstrategien oder gar eine Institutionalisierung der Indigenenbewegung bedeutete, wie Sánchez/Freidenberg (1998: 71) behaupten, dürfte in der Geschichte der CONAIE klar geworden sein.

57 Die CMS wurde im Juni 1995 als Allianz verschiedener sozialer Bewegungen (der Afroecuadorianer, Frauen, Studenten und Bürgervereinigungen) unter der Führung von Marcelo Román gegründet. Ab der Volksabstimmung 1995 kooperierte sie mit der CONAIE (Alcántara/Marenghi o.J.: 9). 
167f.)58. Die amazonischen Organisationen konnten ihre Forderung nach einer rein indigenen Partei nicht durchsetzen (Guerrero/Ospina 2003; Ramírez Gallegos 2009: 74) - eine Quelle für Spannungen bis heute (Sánchez/Freidenberg 1998: 72).

Nur einen Monat später wurde die Bewegung der Plurinationalen Einheit $\mathrm{Pa}-$ chakutik-Neues Land (Movimiento de Unidad Plurinacional Pachakutik-Nuevo País, MUPP-NP) formal gegründet (Sánchez/Freidenberg 1998: 72). Der Name der Bewegung spiegelt die verschiedenen Interessen wider, die in der MUPP-NP zusammenfließen. Der Bestandteil ,Plurinationale Einheit' stammt von den andinen Organisationen der CONAIE (Santillana/Herrera 2009: 335), ,Pachakutik‘ von den indigenen Organisationen des Amazonasgebietes und ,Neues Land" von einer urbanen, politisch linken Gruppe aus Cuenca (Albó 2009: 217), die sich für eine ethische Politik einsetzte und ein Wirken der indigenen Forderungen auf nationaler Ebene ermöglichte (Sánchez/Freidenberg 1998: 72; Barrera 2001: 207f.). Zur gleichen Zeit konnte der Präsidentschaftskandidat dieser Gruppe, der Fernsehjournalist Freddy Ehlers, so Rückhalt in den ländlichen Gebieten gewinnen (Sánchez/Freidenberg 1998: 72). Die MUPP-NP stellte sich als neue Partei innerhalb der politischen Linken auf. Die traditionellen linken Parteien sind seit der Rückkehr zur Demokratie 1979 relativ schwach geblieben, wodurch die MUPP-NP mit ihrer inklusiven Ausrichtung und ihrer Kooperation mit urbanen Gruppen und Intellektuellen im Rahmen ihrer Begriffe von Interkulturalität und Plurinationalität großen Einfluss erlangen konnte (García Serrano 2005: 6). Gleichzeitig sollte die MUPP-NP autonom sein und ihre Unabhängigkeit auch von den Organisationen, aus denen sie entstammte, bewahren (Ramírez Gallegos 2009: 73). Sie war-anders als die bisherigen politischen Parteien - mit flachen Strukturen ausgestattet (Lalander/ Gustafsson o.J.: 3) und baute auf eine kollegiale Teilhabe sowohl indigener als auch nicht-indigener Mitglieder, ohne charismatische Führer (Ramírez Gallegos 2009: 74). Daher sind die internen Strukturen der MUPP-NP denen einer sozialen Bewegung näher als denen der traditionellen Parteien in Ecuador - ohne dass sie dadurch etwas anderes als eine Partei im weiteren Sinne wäre (Lalander/Gustafsson o.J.: 3).

Die Teilnahme an den Wahlen 1996 war ein Erfolg für die MUPP-NP. Bei den Parlamentswahlen konnte die MUPP-NP 8 Abgeordnete, also 10\% aller Abgeordneten, in den Kongress bringen, wodurch sie die viertstärkste Fraktion stellte (Alcántara/Marenghi o.J.: 19f.). Auch wenn der Präsidentschaftskandidat Freddy Ehlers (Sánchez/Freidenberg 1998: 72) mit einem beachtlichen Ergebnis von 20,6\% als Drittplatzierter (Alcántara/Marenghi o.J.: 19) scheiterte, konnte die Bewegung eine Reihe ihrer Politiker auf den verschiedenen Ebenen in Ämter bringen, darunter

58 Der begrifflichen Klarheit zuliebe, und um eine Reduktion auf Begriffe wie soziale oder politische Bewegung zu vermeiden, wird die MUPP-NP hier als Partei bezeichnet. 
53 Indigene - ein absolutes Novum im Land (García Serrano 2001: 71). Besonders hervorzuheben ist Auki Tituaña, der - mit der Unterstützung der FENOCIN-Filiale UNORCAC und der Sozialistischen Partei - zum Bürgermeister von Cotacachi gewählt wurde (Lalander/Gustafsson o.J.: 2). Dieser Fall ist auch insoweit besonders, als dass die CONAIE keine starke Präsenz vor Ort hat, sich die UNORCAC hingegen mit der MUPP-NP, also einer von der CONAIE dominierten Organisation, identifiziert. Tatsächlich bot die UNORCAC selbst Tituaña das Wahlbündnis an (Lalander/Gustafsson o.J.: 5f.). Die MUPP-NP übernahm also in Cotacachi die Rolle des Vermittlers, der ein erfolgreiches Wahlbündnis mit der Einbindung lokaler Akteure erst möglich machte (Lalander/Gustafsson o.J.: 6). Tituaña übte dieses Amt von 1996 bis 2009 aus.

Auch wenn die MUPP-NP eine klare politische Grundrichtung hat - den Kampf gegen den Neoliberalismus und für eine andere Art der Entwicklung, in deren Mittelpunkt der Mensch steht (Santillana 2006: 218) - konnte sie sich nicht auf eine einheitliche Strategie einigen. Die Idee eines politischen Instrumentes, um die Souveränität des Volkes und der Völker wiederherzustellen und die Macht von unten nach oben aufzubauen, wurde nicht mit einer Perspektive der Machtübernahme verbunden. Das lag auch an den sehr breiten Allianzen, die der MUPP-NP zugrunde lagen und nicht in erster Linie politische oder ideologische Wurzeln hatten (Cholango in Tamayo 2007: 8). Dadurch wurde das Herausbilden einer politischen Linie jenseits eines grundsätzlichen Anti-Neoliberalismus erschwert (Simbaña 2009: 162).

Pachakutik war von Beginn an besonders im Hochland stark, wo sie die sporadische Kooperation indigener Organisationen mit den linken Parteien Izquierda Democrática und PS-FA weiterführte. Die zunehmende Schwäche dieser Parteien ab Mitte der 1990er Jahre erlaubte der MUPP-NP, deren Basis mit dem Versprechen einer Erneuerung der politischen Linken für sich zu gewinnen (Alcántara/Marenghi o.J.: 20). Die relative Hegemonie in den weniger bevölkerten Hochlandprovinzen führt dazu, dass die nationalen Wahlergebnisse der MUPP-NP meist hinter den Ergebnissen bei den lokalen Wahlen in diesen Provinzen zurückbleibt (Porras 2005: 103). In den ersten Jahren wurde die MUPP-NP auch von den anderen großen Indigenenorganisationen unterstützt, vor allem der FEINE (Porras 2005: 103) und der FENOC-I (FENOCIN 1999: 152). Diese Unterstützung endete mit dem steigenden Einfluss der CONAIE und der Hinwendung dieser Organisationen zu anderen Parteien.

\section{Entwicklung}

Nach den Wahlen 1996 verliert Ehlers seine Stellung in der MUPP-NP und wird von CONAIE und CMS verdrängt (Sánchez/Freidenberg 1998: 72) - ein erster Führungsstreit, der sich in den nächsten Jahren wiederholen sollte. Im November 
1997 trennte sich die Bürgerbewegung für ein Neues Land wieder von der MUPPNP (Massal 2010: 12). Sie war der einzige Bestandteil der MUPP-NP, der keine soziale Bewegung war, sondern eine regionale Partei, die zwar einen kritischen Diskurs pflegte, jedoch deutlich hierarchischer ausgerichtet war. Diese Besonderheit verhinderte ihre Integration in die MUPP-NP (Collins 2006: 222f.).

Bei den Wahlen zur Verfassungsgebenden Versammlung Ende 1997 konnte die MUPP-NP 10\% der Sitze erringen (Zamosc 2005: 201). In diesem neuen Kontext spielte die neue politische Sprache der MUPP-NP um Begriffe wie Differenz, Autonomie, Plurinationalität oder Biodiversität eine wichtige Rolle. Neue Räume wurden eröffnet und altbekannte Vorstellungen, wie die der Nation, veränderten ihre Bedeutung (Sánchez/Freidenberg 1998: 76). Tatsächlich konnte die MUPP-NP eine Reihe ihrer Forderungen in der neuen Verfassung unterbringen, etwa die Erklärung Ecuadors zum plurikulturellen und pluriethnischen Staat im ersten Artikel und eine Reihe von sozialen und kulturellen Rechten. Die Integration der Territorialität in die Verfassung stellte eine rechtliche Grundlage für eine gewisse Autonomie in den indigenen Gebieten dar (Zamosc 2005: 201).

Die erneute Präsidentschaftskandidatur von Ehlers bei den Wahlen 1998 brachte ihn mit 14,74\% der Stimmen auf den vierten Platz. Auch wenn er Kandidat der MUPP-NP war, trat er offiziell für seine eigene Partei Bürgerbewegung für ein Neues Land (Ciudadanos por un Nuevo País) an. Bei den Parlamentswahlen 1998 konnte die MUPP-NP auch in Verbindung mit anderen Parteien 9 Abgeordnete stellen, was 6\% der Gesamtzahl an Abgeordneten entsprach (Alcántara/Marenghi o.J.: 19f.).

Im August 1999 fand in Quito der erste Kongress der MUPP-NP statt, auf dem zum ersten Mal Statuten der Partei beschlossen wurden. Dies sollte nicht nur die Funktionen innerhalb der Partei klären, sondern vor allem die Unterscheidung zwischen politischer und sozialer Bewegung erleichtern. Der Aufbau einer vergleichsweise traditionellen Struktur mit einem politischen Komitee und einem elfköpfigen Nationalen Exekutivrat sollte die Autonomie der Partei von ihren Gründungsorganisationen garantieren (Guerrero/Ospina 2003).

2000 wurde Mario Conejo für die MUPP-NP zum Bürgermeister von Otavalo gewählt, gründete jedoch 2006 eine eigene Partei, die Minga Intercultural (Lalander/Gustafsson o.J.: 2). Heute ist er Mitglied von Alianza PAÍS und weiterhin Bürgermeister Otavalos.

Die Wahlen 2002, bei denen die MUPP-NP eine Koalition mit der Partei des Ex-Militärs und Protagonisten des 21. Januar 2000, Lucio Gutiérrez, Partei Patriotische Gesellschaft (Partido Sociedad Patriótica 21 de Enero, PSP) anstrebte, brachten ihr Gewinne. Sie konnte zum ersten Mal in Guayas und anderen Küstenprovinzen Kandidaten aufstellen (Collins 2006: 180f.). In der Koalition mit PSP und der postmaoistischen Demokratische Volksbewegung (Movimiento Popular Democrá- 
tico, MPD) konnte sie 11 von 100 Sitzen im Parlament erobern (Alcántara/Marenghi o.J.: 16). Die MUPP-NP stellte sechs Minister, darunter den Minister für Landwirtschaft (Luis Macas), den Vizestaatsminister (Virgilio Hérnandez), die Minister für Erziehung, Tourismus und Sozialwesen sowie mit Nina Pacari die Außenministerin. Der spätere Bürgermeister von Quito für Alianza PAÍS, Augusto Barrera, war für die MUPP-NP Sonderbeauftragter für Dezentralisierung (Massal 2010: 19f.). Die Koalition zerbrach allerdings noch im August 2003 wegen der unterschiedlichen Vorstellungen der Parteien über Wirtschaftspolitik. Die MUPPNP warf Gutiérrez vor, seine Wahlversprechen gebrochen zu haben und der Politik des Internationalen Währungsfonds zu folgen (Alcántara/Marenghi o.J.: 19f.). Dies markierte den Beginn einer tiefen Krise sowohl der MUPP-NP als auch der CONAIE.

Eine Folge der Krise nach der Regierungsbeteiligung unter Lucio Gutiérrez 2003 war eine „Indigenisierung“ (Wolff 2008: 126) der MUPP-NP, also eine Distanzierung von der früheren offenen Orientierung. Sie lehnte sich wieder näher an die CONAIE und deren Diskurs an (Alcántara/Marenghi o.J.: 28) und entwickelte sich in Richtung einer rein indigenen Partei (Wolff 2008: 126), die als ,,politischer Arm der CONAIE“ (FENOCIN 2004: 24; Wolff 2010: 19) gesehen wird und sich als alleinige Vertretung der Indigenen versteht. Dennoch ist es übertrieben, die MUPP-NP als „Wahlapparat“ (Petras/Veltmeyer 2005: 124) der CONAIE zu bezeichnen, da sie nach wie vor autonom agiert und nicht direkt von der CONAIE abhängig ist. Auch sind weiterhin nicht-indigene Mitglieder in der MUPP-NP aktiv und treten für ihre jeweiligen Interessen ein, etwas, das auf die CONAIE nicht zutrifft, die ausschließlich aus Indigenen besteht ${ }^{59}$ und nicht den Anspruch erhebt, für andere Bevölkerungsgruppen zu sprechen (Tibán/García 2006: 11). Das Programm der MUPP-NP von 2006 beinhaltet soziale und wirtschaftliche Themen, jedoch nicht die genuin indigenen Themen wie Interkulturalität und Plurinationalität (MUPP-NP 2006). Diese Umorientierung - und damit der Bruch mit den anderen sozialen Bewegungen - soll eine Stärkung der indigenen Basis erlauben, die sich gerade in der Zeit der Regierungsbeteiligung nicht von der MUPP-NP vertreten fühlten (Wolff 2010: 19). Als Konsequenz verließen viele der urbanen und mestizischen Gruppen die MUPP-NP. Einige dieser Mitglieder der MUPP-NP aus dem Umfeld der Bürgerbewegung für ein Neues Land (NP) waren Mitbegründer der aktuellen Regierungspartei Alianza PAÍS (AP). So sind heute Fernando Cordero, von 1996 bis 2004 Bürgermeister von Cuenca für die NP, und Augusto Barrera und Virgilio Hernández mit ihrem Foro Urbano Mitglieder der AP und haben wich-

59 Das wurde bereits widerlegt (siehe Kapitel II.1.b) - entspricht allerdings dem Selbstverständnis der CONAIE. 
tige politische Positionen für sie inne (Wolff 2010: 19). Freddy Ehlers ist seit 2010 ist Tourismusminister in der Regierung Correa.

Seit der Gründung der AP im Vorfeld der Wahlen 2006 entstand eine direkte Konkurrenz zwischen AP und MUPP-NP, die sich auch auf die indigenen Stimmen bezog. Die Verhandlungen über eine Koalition der beiden Parteien und eine gemeinsame Präsidentschaftskandidatur von Correa und Macas 2006 scheiterten aufgrund der Ablehnung der CONAIE und MUPP-NP, die sich nicht dem weitgehend unbekanntem Correa als Präsidentschaftskandidat unterordnen wollten - während Macas Vizepräsidentschaftskandidat geworden wäre (Minkner-Brünjer 2009: 141). Die AP stützte sich deshalb auf die anderen indigenen Organisationen, vor allem die FENOCIN und in geringerem Maße die FEINE, wodurch diese an Einfluss gewinnen und CONAIE und MUPP-NP ihren Alleinvertretungsanspruch streitig machen konnten. Einige der Mitglieder dieser Organisationen schlossen sich auf verschiedenen Ebenen der Regierung und der Regierungspartei an (León Trujillo 2010: 17).

Die Wahlergebnisse für die MUPP-NP bei den Wahlen 2006 und den Wahlen zur Verfassungsgebenden Versammlung 2007 waren enttäuschend (Martínez Novo 2009: 2f.; Martínez Novo 2009a: 177). Bei der Verfassungsgebenden Versammlung konnte die MUPP-NP nur 4 Sitze für sich behaupten und wurde daher zusammen mit den traditionellen Parteien als marginalisiert und irrelevant betrachtet (Becker 2011: 50). Auch wenn sich die MUPP-NP bei den folgenden Wahlen stabilisieren konnte, ist ihre Position zur Regierung problematisch. Ähnlich der CONAIE entwickelte sie sich von einer kritischen Unterstützung hin zu einer klareren Opposition, wobei sie manche Vorhaben der Regierung unterstützt, andere wiederum kritisiert. 2009 näherte sie sich wieder der post-maoistischen MPD an, mit der sie eine Kooperation begann (León Trujillo 2010: 18).

\begin{tabular}{|l|l|l|}
\hline Art der Wahl/Jahr & Prozent & Sitze (Gesamtsitze) \\
\hline 1996 Kongresswahlen & & $8(80)$ \\
\hline 1996 Präsidentschaftswahlen (Ehlers) & $20,61 \%$ & \\
\hline 1998 Kongresswahlen & & $9(100)$ \\
\hline 1998 Präsidentschaftswahlen (Ehlers) & $14,74 \%$ & \\
\hline 2002 Kongresswahlen & & $10(100)$ \\
\hline 2006 Kongresswahlen & & $6(100)$ \\
\hline 2006 Präsidentschaftswahlen (Macas) & $2,19 \%$ & \\
\hline 2007 Verfassungsgebende Versammlung & & $4(130)$ \\
\hline
\end{tabular}




\begin{tabular}{|l|l|l|}
\hline 2009 Nationalversammlung & & $4(124)$ \\
\hline $\begin{array}{l}2013 \text { Nationalversammlung (im Parteienbündnis } \\
\text { Unidad Plurinacional de las Izquierdas) }\end{array}$ & $5(137)$ \\
\hline 2013 Präsidentschaftswahlen (Acosta) & $3,26 \%$ & \\
\hline
\end{tabular}

Tabelle 5: Wahlergebnisse MUPP-NP, nach Meléndez (2007), eigene Abbildung

$\mathrm{Zu}$ Beginn ihrer Existenz waren die formalen Strukturen der MUPP-NP kaum ausgeprägt und ihre Basis nicht von der der Organisationen, die sie unterstützten, zu trennen. Auch wenn einige Führungsfunktionäre bestimmt wurden, waren ,die Diskussions- und Entscheidungsräume zwischen CONAIE, CMS und MUPP-NP unter den Bedingungen der Logik der sozialen Bewegung mit großen Versammlungen völlig ununterscheidbar“ (Barrera 2001: 220). Das änderte sich erst mit den Kongressen der MUPP-NP ab 1999, die zu einer Strukturierung der Partei führten. Bis zum zweiten Kongress der MUPP-NP 2001 gab es - wie bei den Indigenenorganisationen - keine Mitgliedschaft von Individuen, sondern nur von Organisationen (Collins 2006: 214).

Die MUPP-NP wird von einem Nationalen Exekutiv-Komitee (Comité Ejecutivo Nacional) geführt, vergleichbar mit dem Regierungsrat (Concejo de Gobierno) der CONAIE, das sich aus einem Koordinator, zwei Sub-Koordinatoren und jeweils 4 Sprechern aus den drei geographischen Regionen Ecuadors besteht. Früher bestand das Nationale Exekutiv-Komitee aus einem Koordinator, einem SubKoordinator, Abgeordneten der Mitgliedsorganisationen (CONAIE und Unterorganisationen, CONFEUNASSC und CMS). Dazu kamen 5 thematische Sprecher, ein Repräsentant der Bürgermeister der MUPP-NP und ein Repräsentant der Fraktion der Partei in der Nationalversammlung (Rodríguez 2008: 117). Es fand also ein Wandel von einer Struktur nach Mitgliedsorganisationen zu einer Struktur nach Regionen statt. Diese Führungsstruktur soll auch die Unabhängigkeit von den Gründungsorganisationen, allen voran der CONAIE, garantieren. Verschiedene Autoren machen darauf aufmerksam, dass dieses Ziel nicht erreicht wurde und das Nationale Exekutiv-Komitee mehrheitlich von Vertretern der CONAIE kontrolliert wird (Alcántara/Marenghi o.J.: 8).

\begin{tabular}{|l|l|l|l|l|l|}
\hline Jahr & $\begin{array}{l}\text { Nummer des } \\
\text { Kongresses }\end{array}$ & $\begin{array}{l}\text { Teil- } \\
\text { neh- } \\
\text { mer }\end{array}$ & Ort & Besondere Neuerungen & $\begin{array}{l}\text { Gewählter } \\
\text { Ko- } \\
\text { ordinator }\end{array}$ \\
\hline 1999 & I. Kongress & & Quito & $\begin{array}{l}\text { Strukturierung der } \\
\text { MUPP-NP }\end{array}$ & $\begin{array}{l}\text { Miguel } \\
\text { Lluco }\end{array}$ \\
\hline
\end{tabular}




\begin{tabular}{|c|c|c|c|c|c|}
\hline 2001 & II. Kongress & 500 & & & $\begin{array}{l}\text { Wieder- } \\
\text { wahl }\end{array}$ \\
\hline 2003 & $\begin{array}{l}\text { III. } \\
\text { Kongress }\end{array}$ & 650 & Riobamba & $\begin{array}{l}\text { Aufarbeitung der Regie- } \\
\text { rungsbeteiligung, } \\
\text { Opposition zu Gutiérrez }\end{array}$ & $\begin{array}{l}\text { Gilberto } \\
\text { Talahua }\end{array}$ \\
\hline 2005 & $\begin{array}{l}\text { IV. } \\
\text { Kongress }\end{array}$ & & Ambato & & $\begin{array}{l}\text { Wieder- } \\
\text { wahl }\end{array}$ \\
\hline 2007 & V. Kongress & 342 & Quito & & $\begin{array}{l}\text { Jorge } \\
\text { Guamán }\end{array}$ \\
\hline 2010 & $\begin{array}{l}\text { VI. } \\
\text { Kongress }\end{array}$ & 1200 & Sucúa & & $\begin{array}{l}\text { Rafael } \\
\text { Antuní }\end{array}$ \\
\hline 2013 & $\begin{array}{l}\text { VII. } \\
\text { Kongress }\end{array}$ & & $\begin{array}{l}\text { Santo } \\
\text { Domingo de } \\
\text { los } \\
\text { Tsáchilas }\end{array}$ & & $\begin{array}{l}\text { Fanny } \\
\text { Campos }\end{array}$ \\
\hline
\end{tabular}

Tabelle 6: Kongresse der MUPP-NP, eigene Abbildung

\section{Politik}

Die politischen Ziele der MUPP-NP spiegeln den Ursprung der Partei wider. Sie steht für eine grundsätzliche Infragestellung des intransparenten politischen Systems und der wenig demokratischen traditionellen Parteien, fordert also sowohl durch ihre offene Gestalt, als auch durch ihr politisches Programm eine Demokratisierung des Staates (Sánchez/Freidenberg 1998: 76; Santillana 2006: 218). Die jeweiligen Regierungen werden kritisch gesehen als „Ent-Regierungen im Dienst der Oligarchie und der transnationalen Unternehmen“ (MUPP-NP 2006: 1), die die von außen vorgegebenen Strukturanpassungsprogramme nur noch umsetzen. Dazu kommt die schon im Namen vertretene Plurinationalität, im Sinne einer Umgestaltung des Staates, damit die verschiedenen Kulturen anerkannt und durch kollektive Rechte geschützt werden (Santillana 2006: 218). Plurinationalität und Interkulturalität bedeuten für die MUPP-NP auch gelebte politische Wirklichkeit durch das Zusammenwirken verschiedener Kulturen in einem inklusiven Kontext (Tibán/García 2006: 11). Dies geht einher mit einer Verteidigung der Umwelt und der Forderung, die Wirtschaft so umzugestalten, dass sie gerechter wird und dem gesellschaftlichen Wohl dient. Dazu werden Umverteilungsmechanismen angestrebt (Le Quang 2010: 112f.). Die MUPP-NP will also eine „Umverteilung des nationalen Reichtums und des Einkommens bis zum Erreichen der sozialen Gerechtigkeit“ (MUPP-NP 2006: 5). Diese Forderungen stimmen zu einem großen Teil mit denen 
der CONAIE, insbesondere ihres politischen Projekts von 1994, überein, die nur über Reformen innerhalb des politischen Systems umsetzbar sind (Ramírez Gallegos 2009: 73).

Die MUPP-NP folgt einer Strategie des Aufbaus der politischen Macht von unten, vor allem in den größtenteils indigen besiedelten Gebieten. So sollen die Beziehungen zwischen Staat und Gesellschaft neu definiert (Sánchez/Freidenberg 1998: 73) und gleichzeitig Methoden des Wandels erprobt werden. Diese Strategie hängt auch mit der Nähe zu den lokalen indigenen Organisationen zusammen, die die Basis der Partei bilden (Santillana 2006: 257). Die indigenen Basisorganisationen sind um die Gemeinschaft herum aufgebaut (Santillana 2006: 222) und daher an lokaler Politik besonders interessiert.

Die MUPP-NP verfolgt eine „,kontra-hegemoniale“ und ,anti-systemische“ Machtstrategie“ (Santillana 2006: 241f.), die Teilnahme an den Institutionen mit Aktionen außerhalb der Institutionen verbinden will. Über eine Übergangsregierung will sie eine neue Gesellschaft schaffen; das „,beinhaltet eine Strategie der Konstruktion einer kontra-hegemonialen Macht, die sich nicht auf die Aktionen im Bereich der öffentlichen Institutionen beschränkt" (MUPP-NP 2006: 1). Auch in diesem Kontext spielt die lokale Machteroberung eine wichtige Rolle; die Regierungen der niederen Ebenen sind nötig, um den plurinationalen Staat aufbauen zu können. Ähnlich der CONAIE sieht die MUPP-NP die wichtigsten Probleme der ecuadorianischen Gesellschaft sowohl im Konflikt zwischen Arm und Reich, als auch im Konflikt zwischen dem „,Uninationalen bürgerlichen Staat““ (Santillana 2006: 242) und den indigenen Nationalitäten und Völkern.

Die gemischte ethnische Zusammensetzung ist ein charakteristisches Merkmal der MUPP-NP. Sie baut nicht auf einer traditionellen indigenen Identität auf, sondern auf der Mischung ihrer verschiedenen Komponenten. Sowohl ihre politischen Forderungen, als auch ihre interne Organisation und ihre Strategien spiegeln die Einflüsse der indigenen Traditionen, der Kirche, der Gewerkschaften und linken Parteien und des Staates selbst wider (Wolff 2008: 113). Interkulturalität und Plurinationalität werden also schon in der Partei gelebt - was mit Lernprozessen und der Überwindung vieler Vorurteile verbunden war und ist (Jijón 2006: 49). Dennoch ist die indigene Identität und die Erfahrungen der indigenen Bewegung und der CONAIE der zentrale Bestandteil des Zusammengehörigkeitsgefühls der MUPP-NP, das schon mehrfach das Auseinanderbrechen der Partei verhindert hat (Santillana/Herrera 2009: 344). Das ist die andere Funktion der „neuen Sprache“ (Sánchez/Freidenberg 1998: 76), die MUPP-NP und CONAIE in der ecuadorianischen Politik etablieren konnten. Begriffe wie Interkulturalität oder Plurinationalität bieten - auch wegen ihrer offenen Definition - ein hohes Identifikationspotenzial.

Die MUPP-NP konnte seit ihrer Gründung über ihre Strategie einer lokalen, partizipativen Demokratie und eines Aufbaus des Staates und der Partei von unten 
nach oben eine Reihe von Erfolgen feiern und beweisen, dass sich der abstrakte Diskurs um die Begriffe Plurinationalität und Interkulturalität praktisch umsetzen lässt (Santillana/Herrera 2009: 341). Dennoch stieß das Modell der MUPP-NP spätestens mit der Regierungsbeteiligung 2003 an seine Grenzen. Eine effektive Verbindung der Strategie der doppelten Teilhabe außerhalb und innerhalb der Institutionen funktionierte nur in wenigen Fällen. Stattdessen wurde die MUPP-NP seit Mitte der 2000er Jahre mehr und mehr zu einer reinen parlamentarischen Vertretung der sozialen Bewegungen und vor allem der Indigenenbewegung, ohne über deren Perspektive hinausgehen zu können (Ramírez Gallegos 2009: 73f.). Dass die Etablierung der MUPP-NP „Teil des Schrittes vom politischen Subjekt zum politischen Akteur“ (Santillana 2006: 219) der Indigenenbewegung ist, also einen qualitativen Wandel darstellt, wie in der Politikwissenschaft oft behauptet wird, sollte spätestens mit der Krise der MUPP-NP im Anschluss an ihre Regierungsbeteiligung 2003 widerlegt sein. Ende 2005 zog sich die linke, nicht-ethnische Strömung in der MUPP-NP weitgehend zurück (Maldonado 2006: 132). Die schlechten Wahlergebnisse für die MUPP-NP in den Wahlen 2006 und 2007 verstärkte diese Tendenz einer „Indigenisierung“ (Wolff 2008: 126) in dem Sinne, dass die Partei sich jetzt klar als eine indigene Partei begreift und präsentiert - wodurch weitere der urbanen Gruppen die MUPP-NP verließen (Wolff 2008: 126). Die Bevorzugung der sozialen vor der politischen Bewegung führt auch dazu, dass die soziale Bewegung, in Gestalt der CONAIE, von den möglichen Wählern deutlicher als Vertretung ihrer Interessen wahrgenommen wird und schlechthin effektiver ist (Marmon/Kramer 2006: 4).

\section{Die IndigenenbeWegung Ecuadors aUS Der Perspektive der Forschung ZU SOZIALEN BEWEGUNGEN}

Eine kurze Einführung in die hier angewandten Theorien wurde im Kapitel I.1 gegeben. Zum Verständnis der folgenden Analyse ist es dennoch notwendig, einige Voraussetzungen für das Entstehen sozialer Bewegungen zu wiederholen. Soziale Bewegungen sind kollektive Versuche einer Anzahl von Menschen gesellschaftliche Institutionen und Strukturen zu verändern. Die Motivation liegt dabei in erster Linie in den $\mathrm{zu}$ erreichenden Zielen, die von den Mitgliedern geteilt und weitergetragen werden (Zald/Ash 1966: 329). Das bedeutet keinesfalls, dass soziale Bewegungen über diese Ziele definierte einheitliche Akteure sind. Vielmehr sind es „Ansammlungen formeller Organisationen, informeller Netzwerke und selbstständiger Individuen, die sich einem mehr oder weniger kohärenten Kampf für einen Wandel widmen“ (Meyer/Whittier 1994: 277). Als Mittel, Veränderung zu errei- 
chen, sind sie eine vergleichsweise kosten- und zeitintensive Methode. Daher entstehen soziale Bewegungen nur, wenn sie als potentiell effizient wahrgenommen werden. Das bedeutet, dass es auf der einen Seite eine „,,relative Offenheit“ staatlicher Strukturen abweichenden Meinungen gegenüber" (Meyer/Staggenborg 1996: 1636) geben muss, sie also toleriert und eventuell aufgegriffen werden. Weiterhin müssen die für eine Mobilisierung notwendigen Ressourcen zur Verfügung stehen oder von außerhalb zur Verfügung gestellt werden (Jenkins/Perrow 1977: 251). Auf der anderen Seite müssen andere Einflusswege versperrt sein. Eine grundsätzliche Bereitschaft der Regierung, die geforderten Änderungen ohne Druck und von sich aus durchzusetzen (Meyer/Staggenborg 1996: 1630), würde das Entstehen von sozialen Bewegungen bremsen. Verstärkt wird das Entstehen dieser Bewegungen hingegen, wenn die wichtigen politischen Parteien nicht auf die Forderungen reagieren oder die Interessen der jeweiligen Gruppen nicht repräsentieren (Zald 1979: 17).

Diese Faktoren haben auch das Entstehen der ecuadorianischen Indigenenbewegung begünstigt. Der ecuadorianische Staat hat seit der Abschaffung der kolonialen Indio-Gesetze Mitte des 19. Jahrhunderts keine klare Indigenenpolitik gehabt. Daher schwankte seine Politik den Indigenen und ihren Organisationen gegenüber zwischen Repression (etwa beim Indigenenkongress 1931, auf dem eine nationale Organisation gegründet werden sollte, oder zu Zeiten autoritärer Politik wie der Militärdiktatur 1972-1979, die einige Organisationen verfolgte) und Entgegenkommen (der Erlass des Kommunengesetzes von 1937, die Agrarreformen von 1964 und 1973, die Schaffung staatlicher Organe für die Indigenen ab Anfang der 1980er). Das Entgegenkommen des Staates konnte von der Indigenenbewegung als Kompromiss und - wenn auch unzureichende - Erfüllung ihrer Forderungen interpretiert werden, ihre Mobilisierung war also ein erfolgreiches Mittel, den Staat zu einer bestimmten Politik zu zwingen. Die politischen Parteien waren nie ein erfolgversprechender Weg, um die indigenen Forderungen umzusetzen. Auch wenn einige linke und liberale Parteien (vor allem die Sozialistische und Kommunistische Partei, die FADI, in geringerem Maße auch Democracia Popular (DP) und ID als Parteien der Mitte) eine gewisse Nähe zu den Indigenen suchten und zeitweise den Anspruch hatte, sie zu vertreten, so blieb diese Repräsentationspolitik immer nur an einige allgemeine Forderungen, wie die nach einer Landreform, oder bestimmte Personen, wie Ricardo Paredes von der PCE, gebunden. Vor dem Entstehen der Indigenenparteien MUPP-NP und Amauta Jatari gab es keine Partei, die als allgemeine Interessenvertretung der Indigenen anerkannt wurde.

Eine kaum untersuchte Frage ist die, inwieweit die Organisationen der Indigenenbewegung die Interessen der wirtschaftlich Armen vertreten, denen es an den nötigen Ressourcen mangelt, sich aktiv zu beteiligen (Bebbington 2007: 811) - wie also die Sozialstruktur der Indigenenbewegung beschaffen ist. Auf eine mangelnde 
Repräsentation der Armen in der Indigenenbewegung deutet der Fall der Ortschaften - vor allem Otavalo und Cotacachi - hin, in denen die Indigenenbewegung über lange Zeit die Politik bestimmen konnte - ohne jedoch die Armut signifikant zu verringern (Bebbington 2007: 805).

Auch gibt es einen grundsätzlichen Unterschied zwischen den sozialen Bewegungen Lateinamerikas und denen Europas und der USA. Alison Spedding bezieht sich auf David Llanos, einen bolivianischen Soziologen, der die Theorie aufstellt, dass die Beschäftigungsstruktur mit der Bereitschaft zu Protest zusammenhängt. Seine Untersuchungen über die hohe Mobilisierungsfähigkeit in El Alto bei La Paz haben diese weniger auf eine Hegemonie der jeweiligen Organisationen zurückgeführt, sondern auf die Tatsache, dass die meisten Anwohner einer informellen Arbeit nachgehen. Daher haben sie keine festen Arbeitszeiten und kein festes Einkommen, also keine großen Verdienstausfälle durch einige Tage politische Mobilisierung. Das unterscheidet sie von Angestellten oder Arbeitern, bei denen Verspätungen oder ein Fernbleiben vom Arbeitsplatz mit scharfen wirtschaftlichen Sanktionen einhergeht. Da nur wenige der Anwohner El Altos feste Arbeit in diesem Sinne haben, ist die Bevölkerung insgesamt eher zu Protesten und Mobilisierungen bereit (Spedding 2010: 11). Ähnlich könnte es sich bei der Indigenenbewegung Ecuadors verhalten, die sich in erster Linie aus Bauern und Landarbeitern rekrutiert. Diese können abseits der Saat- und Erntezeiten ohne größere Schwierigkeiten einige Tage in Protestmärsche und ähnliches investieren. Es wäre zu überprüfen, ob deshalb viele der Aktionen in den Monaten März bis Juli und nur in besonders kritischen Situationen - etwa der Sturz Bucarams Anfang 1996 oder Mahuads Ende 1999/Anfang 2000 - größere Mobilisierungen außerhalb dieses Zeitraums stattfinden.

Bei den folgenden Ausführungen sollte zudem bedacht werden, dass die ecuadorianische Indigenenbewegung nicht allein eine soziale Bewegung als ,,andauernde Herausforderung staatlicher Politik“ (Meyer/Whittier 1994: 279) ist, sondern auch gewerkschaftliche und genossenschaftliche Bestandteile hat. Diese haben insbesondere seit den Landreformen an Bedeutung gewonnen, was sich durch die Gründung von Kooperativen und Genossenschaften mit den dazugehörenden kleinen Betrieben ausdrückt. Diese Tendenz ging in den 1980er bis hin zu einer Umwandlung der Basisorganisationen in gemeinschaftliche Betriebe (,,empresarialización comunitaria“" (Almeida/Prieto 1983: 36)). Nach einer Krise dieser Bestrebungen gegen Mitte der 1980er Jahre (Guerrero 2010: 17), ist ab Mitte der 1990er eine Tendenz hin zu einer engen Einbindung der Indigenenorganisationen in Entwicklungsprojekte festzustellen, begünstigt vor allem durch die Kooperation mit PRODEPINE 1998 bis 2003 (Martínez Valle 2006, Bretón 2008). Die Entwicklungsagenturen haben Einfluss auf die Entwicklung der Organisationen gewinnen können, die zur Erhaltung ihrer Strukturen dazu übergegangen sind, selbst kleine 
Entwicklungsprojekte durchzuführen und so Ressourcen zu gewinnen - auf Kosten ihrer politischen Positionen, die von den jeweiligen nationalen Föderationen verteidigt werden (Iturralde 1997: 96).

\section{Struktur und Entwicklung der ecuadorianischen Indigenenbewegung}

Im Modell des Ressourcen-Mobilisierungs-Ansatzes manifestieren sich soziale Bewegungen in formalen Organisationen, den Social Movement Organizations (SMO) (Zald/Ash 1966: 327), in Ecuador etwa die CONAIE oder die CTE. Da soziale Bewegungen zum Funktionieren auf die Mobilisierung von Ressourcen angewiesen sind, hängen sie von diesen relativ festen Strukturen ab, die Aktivitäten koordinieren, Zugang zu Ressourcen sichern, Führungspersonal bereitstellen und die Bewegung nach außen repräsentieren können (Bebbington 2007: 800). SMO mit relativ ähnlichen Zielen bilden eine Social Movement Industry (SMI), etwa die Indigenen- oder die Gewerkschaftsbewegung. SMI ist also die organisatorische Entsprechung der sozialen Bewegung, die ihr angehörenden SMO identifizieren sich mit den Zielen der Bewegung (Zald 1979: 9). Alle SMI einer Gesellschaft bilden den Social Movement Sector (SMS) (Zald/McCarthy 1979: 2), also die Gesamtheit aller sozialer Bewegungen. Dieser spiegelt die allgemeine Mobilisierungsbereitschaft in einer Gesellschaft wider (Zald 1979: 3).

Diese Kategorien sind nicht ohne Probleme auf die Indigenenbewegung Ecuadors zu übertragen. Die oben beschriebenen SMO sind zum einen als Mitglieder der SMI ,Indigenenbewegung ' von anderen, ihnen nahestehenden SMO abzugrenzen. Die CONAIE, die FENOCIN, die FEINE und die FEI sind SMO der SMI ,Indigenenbewegung'. Die FENACLE, die CONFEUNASSC-CNC und andere kooperieren häufig mit diesen Organisationen oder teilen sich gar Strukturen, sind aber SMO einer anderen SMI, der ,Bauern- und Landarbeiterbewegung‘. Diese zeichnet sich durch andere Ziele und - meist - eine andere Mitgliedschaft aus. Auf einer zweiten Ebene sind die SMI ,Indigenenbewegung' und die SMI ,Bauern- und Landarbeiterbewegung' voneinander abzugrenzen. Bestimmte SMO, wie die FENOCIN und die FEI, sind Mitglied in beiden SMI, wie ihre Zugehörigkeit zu den nicht-ethnisch ausgerichteten Plattformen CLOC und Vía Campesina zeigt, die sie etwa mit der ebenfalls nicht-ethnischen CONFEUNASSC-CNC gemein haben. Sie vertreten nicht nur Indigene im Kampf um ethnische Rechte, sondern auch Bauern und Landarbeiter im Kampf um soziale und wirtschaftliche Rechte. Auf einer dritten Ebene sind Partnerorganisationen der SMO der Indigenenbewegung, die anderen SMI angehören, von diesen analytisch zu trennen. Die FEI der Indigenenbewegung ist eine Filiale der CTE der Gewerkschaftsbewegung, die wiederum der politischen Partei PCE nahesteht. Die FENOCIN ist eine Filiale der Gewerkschaft 
CEDOCUT. FEINE und CONAIE gehören keinen Gewerkschaften an, haben aber Parteien gegründet, die sie zumindest teilweise kontrollieren. Diese Verbindungen erschweren eine klare Trennung der SMI, da es wechselseitige Einflüsse und Abhängigkeiten gibt, die sich oft verschieben und nur schwer nachzuzeichnen sind. Diese Verknüpfungen werden im nachfolgenden Schaubild skizziert.

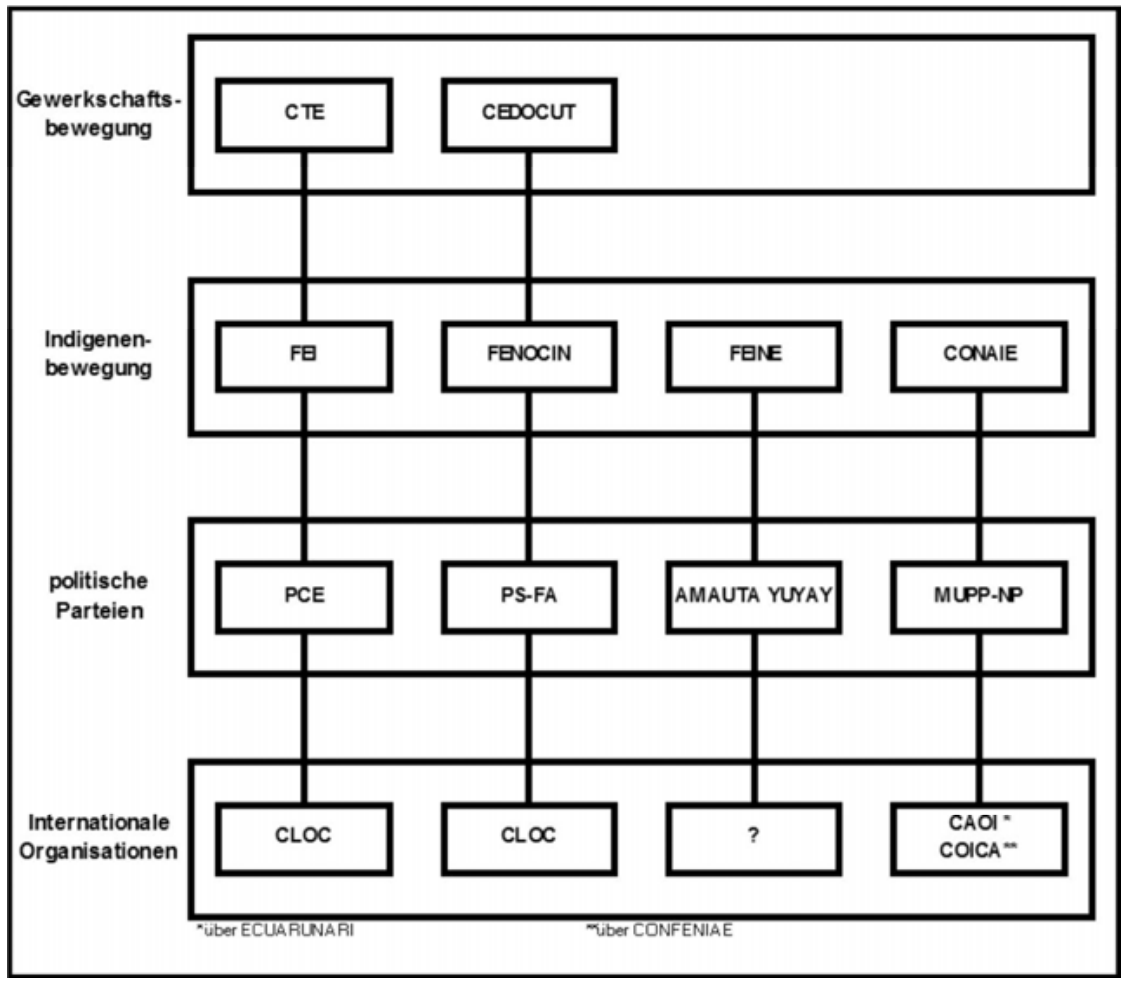

Abbildung 3: Struktur und Verbindungen der sozialen Bewegungen in Ecuador, eigene Abbildung

Eine SMI wird von der Nachfrage nach ihren Produkten, also ihren Forderungen oder Aktionen, den Voraussetzungen zur Bereitstellung dieser Produkte und der ideologischen und organisatorischen Hegemonie ihrer Ziele in der Gesellschaft strukturiert. Sie braucht kleine und lokale Einheiten, die vor Ort die Mitglieder und Unterstützer der Bewegung einbinden können (Zald 1979: 12). Dabei ist eine SMI nur selten einheitlich, sie besteht aus einer Reihe von Organisationen, die mit verschiedenen Teilen der Mitgliedschaft und der Unterstützer verbunden sind und untereinander um Ressourcen und Einfluss innerhalb der Bewegung konkurrieren, kooperieren und sich ergänzen und/oder bekämpfen (Zald/McCarthy 1979: 1). Eine 
SMI ist also eine Gruppe von Organisationen die derselben Gruppe von Interessenten ähnliche Produkte anbieten und dabei miteinander in Konkurrenz stehen - was gelegentliche Kooperation nicht ausschließt (Zald 1979: 3).

In diesem Sinne sind CONAIE, FENOCIN, FEINE, FEI und ihre verschiedenen Unter- und Mitgliedsorganisationen SMO der SMI Indigenenbewegung. Sie alle kämpfen für eine Verbesserung der Lage der Indigenen, setzen dabei aber unterschiedliche Schwerpunkte und sprechen andere Gruppen innerhalb der Indigenenbewegung an. Diese unterschiedlichen Schwerpunkte führen zum Verschwimmen der Grenzen der sozialen Bewegung. Alle der erwähnten SMO kämpfen auch für eine Verbesserung der Lage der Bauern, was sowohl an einer geteilten Basis zwischen Indigenen- und Bauernbewegung - besonders wichtig im Falle von CONAIE und FENOCIN - als auch an der Tatsache liegt, dass viele der organisierten Indigenen Bauern sind. Die FEINE mit ihrer Konzentration auf evangelische, in Kirchengemeinden organisierte, Indigene besitzt die am klarsten definierte Unterstützergruppe. Im Fall der CONAIE wurde in Kapitel II.1.b beschrieben, dass sich ihr Selbstverständnis als ethnische Organisation nicht völlig mit der Zusammensetzung ihrer Basisorganisationen deckt. Die FENOCIN vertritt - wie schon ihr Name sagt - nicht nur Indigene, sondern auch Schwarze und Bauern. Die FEI war lange Zeit hindurch eine SMO, die sowohl der Indigenenbewegung, als auch der Bauernbewegung angehörte. In ihrem aktuellen Namen und ihren ersten Texten vertritt sie explizit sowohl Bauern, als auch Indigene - und konnte in ihrem Einflussbereich die meisten Interessenten mit beiden Kategorien mobilisieren. Alle diese Organisationen zeichnen sich durch ein Netz von Basisorganisationen aus, die die Interessenten in den jeweiligen Verbreitungsgebieten organisieren und ihre konkreten Forderungen vor Ort artikulieren.

Auch die Wechselwirkungen zwischen ganzen SMI ist wichtig, um deren Entwicklung angemessen verstehen zu können. Die allgemeine Krise der SMI ,Gewerkschaftsbewegung' ab 1982 nach einer Phase hoher Aktivität gegen Mitte der 1970er (Dávila 1995: 56) war ein unterstützender Faktor für die Stärkung der Indigenenbewegung gegen Ende der 1980er, die wiederum zu einer Stärkung urbaner Bewegungen führte (Acosta 2001: 180).

Im Gegensatz zu vielen theorieprägenden sozialen Bewegungen hat die Indigenenbewegung in Ecuador nie eine Gegenbewegung im Sinne von Meyer/Staggenborg (1996) provoziert. Es gab und gibt zwar Widerstände und Kritik an bestimmten Forderungen oder Aktionen - im Umfeld des Inti Raymi 1990 wurde die Indigenenbewegung etwa als Infragestellung der mestizaje verstanden (León 1994: 34-37) -, diese wurden jedoch nie zu einer sozialen Bewegung. Es trifft zu, dass die Außenwahrnehmung der Indigenenbewegung oft von kleinen radikalen Gruppen bestimmt wird, die als ihre Sprecher - oder Träger der , wahren Ziele ‘ der Bewegung - gesehen werden, obwohl sie die Bewegung selbst nicht repräsentieren 
können und nicht von den einzelnen SMO kontrolliert werden (Meyer/Staggenborg 1996: 1641). Beispiele hierfür sind die zuerst eigenmächtigen Aktionen in Saraya$\mathrm{ku}$, die zur Unterzeichnung des Vertrags von Sarayaku führten, oder der OPIP mit ihrem Marsch 1992. Die Ablehnung dieser radikalen Ziele führte jedoch nie zum Aufbau einer eigenen sozialen Bewegung. Tatsächlich finden die schärfsten Auseinandersetzungen über Forderungen und Taktiken zwischen Organisationen innerhalb der Indigenenbewegung statt. So gab es statt verschiedenen Arten von Gegenbewegungen (Meyer/Staggenborg 1996: 1639), nur Versuche innerhalb der Indigenenbewegung Gegenorganisationen zu schaffen bzw. zu unterstützen. Diese wurden von anderen sozialen Bewegungen, Parteien und der Regierung betrieben, um so die Bewegung zu spalten und zu schwächen. Viele dieser Gegenorganisationen wurden zwar von den jeweiligen Gruppen und teilweise den Medien (Meyer/Staggenborg 1996: 1642) unterstützt, konnten jedoch nie eine wirkliche Basis entwickeln. Erfolgreiche SMO, die zumindest teilweise als Gegenorganisationen geschaffen wurden, sind die FENOC als konservative und christliche Alternative zur kommunistischen FEI in den 1960ern, die befreiungstheologisch beeinflusste ECUARUNARI als ethnische Alternative zu den gewerkschaftlichen FEI und FENOC Anfang der 1970er und schließlich die protestantische FEINE als Alternative zu den katholisch geprägten Organisationen der CONAIE Ende der 1970er. Andere Versuche, Gegenorganisationen aufzubauen, blieben erfolglos. Gegenorganisationen verhalten sich in mancher Hinsicht wie Gegenbewegungen, wenn man davon absieht, dass sie nicht monothematisch sind. So erfuhr jede der erwähnten erfolgreichen Gegenorganisationen Unterstützung der Elite (Meyer/Staggenborg 1996: 1642), das waren in allen Fällen Teile der Kirchen und im Falle der FENOC und in geringerem Maße - der ECUARUNARI bestimmte Parteien. Daher führten Richtungsänderungen dieser Organisationen zu einem Ende der jeweiligen Unterstützung, verbunden mit einer Krise der SMO (Meyer/Staggenborg 1996: 1643). Während etwa der Übergang der FENOC von einer konservativen zu einer christdemokratischen Linie 1973 ohne größere Folgen blieb, da die neue Linie auch international unterstützt wurde, führte der Übergang zum Sozialismus als Leitlinie $1977 \mathrm{zu}$ einer Krise und organisatorischen Umorientierung. Auch die Distanzierung der ECUARUNARI von den kirchlichen Kreisen, die an ihrer Gründung mitgewirkt hatten, führte Mitte der 1970er zu einer Krise, die mit einem erheblichen Verlust an Mitgliedern und Einfluss verbunden war. Die Vermutung von Meyer und Staggenborg, dass sich Bewegung und Gegenbewegung in denselben politischen Bereichen betätigen, die eine Bewegung die andere zu einer Aktivität im selben Bereich geradezu zwingt (Meyer/Staggenborg 1996: 1649), trifft auf die ecuadorianische Indigenenbewegung und die Gegenorganisationen in ihr zu. So ist es kein Zufall, dass in den 1970er Jahren alle bedeutenden Organisationen sozialistisch geprägt waren, in den 1980ern ethnische Forderungen aufstellten und sich in den 1990ern gesamt- 
staatliche Vorschläge zu eigen machten, die auf ein Zusammenleben verschiedener Gruppen ohne Diskriminierung oder erzwungene Integration ausgerichtet sind. Die Gründung von Amauta Jatari als Partei der FEINE Ende der 1990er ist eine auch Reaktion auf den Erfolg der MUPP-NP als der CONAIE nahestehende Partei, ebenso wie das verstärkte Engagement der FENOC-I/FENOCIN in der Sozialistischen Partei. In einem weiteren Punkt funktionieren die Gegenorganisationen innerhalb der Indigenenbewegung wie Gegenbewegungen: Erfolge einzelner Organisationen - etwa die Aktionen 1990, 1992 und 1994, die weitgehend von der CONAIE gesteuert wurden - können zur Schwächung des Mobilisierungspotentials führen, weil bestimmte Forderungen - etwa die Anerkennung kollektiver Rechte in der Verfassungsänderung 1996 und der Verfassung 1998 - erfüllt werden. Das kann gleichzeitig zu einer Schwächung der Gegenorganisationen führen, auf die sie mit höherer Aktivität reagieren (Meyer/Staggenborg 1996: 1652). So lässt sich die Restrukturierung und anschließende Stärkung der FENOC-I/FENOCIN ab 1995 erklären - in einer Zeit, in der die CONAIE die bei weitem wichtigste SMO der Indigenenbewegung war. Auch trifft zu, dass die internen Konflikte über bestimmte Weltsichten oder Forderungen von den Konflikten mit Gegenorganisationen verstärkt wird (Meyer/Staggenborg 1996: 1653). Die Auseinandersetzungen zwischen ethnizistischen und klassistischen Akteuren in der CONAIE wurden von den Konflikten mit der eindeutig klassistischen FENOCIN verstärkt. Andere Besonderheiten der Beziehung Bewegung-Gegenbewegung treffen auf die ecuadorianischen Gegenorganisationen allerdings nicht zu. Es kann keine Rede davon sein, dass SMO die Vorteile nach errungenen Siegen nicht nutzen konnten (Meyer/Staggenborg 1996: 1652). Die CONAIE konnte ihre Position und ihren Einfluss in den 1990ern - der Zeit ihrer großen Erfolge - beständig erweitern.

Soziale Bewegungen verändern immer die Bedingungen für ihre Nachfolger und Gegner, politisch handeln und Ressourcen mobilisieren zu können (Meyer/Whittier 1994: 282; Meyer/Staggenborg 1996: 1635). Neben direkten, eindeutig artikulierten Zielen versuchen sie auch, weniger eindeutige Strukturen, wie die Kultur oder Weltsicht der Gesamtgesellschaft, zu ändern. Das führt zu einem spill over-Effekt von Einstellungen, Praktiken und Ideen auf andere Organisationen und soziale Bewegungen (Meyer/Whittier 1994: 277f.). Die Theorie politischer Prozesse sieht den Aufbau von abeyance structures als eine andere Möglichkeit für soziale Bewegungen, ihre Umgebung auch zu Zeiten geringer Mobilisierung zu beeinflussen. Das sind formale Organisationen sozialer Bewegungen, in denen die Kultur und Praxis der sozialen Bewegung, sowie ein Teil ihres Führungspersonals bis zur nächsten Mobilisierung aufgehoben sind (Meyer/Whittier 1994: 279). In ihnen werden Erfahrungen über die Erfolge und Misserfolge bisheriger Strategien und über die Kosten und Nutzen von Mobilisierungen weitergegeben, was die Entwicklung neuer Mobilisierungsmechanismen ermöglicht. So erlauben sie den Erhalt der 
Identität der sozialen Bewegung bei der gleichzeitigen Möglichkeit, ihre Ziele zu verändern (Meyer/Whittier 1994: 280).

Da die ecuadorianische Indigenenbewegung in einem hohe Maße auf langfristig bestehende, in den jeweiligen Gemeinden verankerte Organisationen baut und spontane Mobilisierung eher außerhalb der Indigenenbewegung stattfindet, ist der Begriff der abeyance structures von wenig Nutzen. Wichtiger kann der spill overEffekt sein, der die Übernahme bestimmter Mobilisierungsformen der Indigenenbewegung - etwa Märsche oder Besetzungen - durch andere soziale Bewegungen erklären kann. Das langjährige Eintreten der Indigenenbewegung für ein neues Modell der ethnischen Beziehungen in Staat und Gesellschaft, etwa unter den Begriffen Interkulturalität und Plurinationalität, hat spürbar auf Staat und Zivilgesellschaft ausgestrahlt, wie die Verwendung dieser Begriffe durch Ministerien und Parteien, die sich nur wenig mit den Zielen der Indigenenbewegung identifizieren, deutlich macht.

\section{Struktur und Entwicklung der SMO der Indigenenbewegung}

Die Theorien des Ressourcen-Mobilisierungs-Ansatzes zur Entwicklung der Organisationen einer sozialen Bewegung erwarten in Anlehnung an das Weber-MichelsModell, dass das Erlangen einer wirtschaftlichen und sozialen Basis in der Gesellschaft zu einer Ersetzung der ursprünglichen charismatischen durch eine bürokratische Führung und einer relativen Anpassung der sozialen Bewegung an die Gesellschaft führt. Die Teilnehmer an der Organisationsbürokratie haben ein Interesse an der Erhaltung der Organisation, unabhängig von ihrer Möglichkeit, ihre Ziele zu erreichen. Dazu ist eine relativ konfliktfreie Beziehung zur Gesellschaft von Vorteil, was zu Veränderungen der SMO führen kann (Zald/Ash 1966: 332). Zald und Ash sprechen von drei möglichen Veränderungen innerhalb der SMO: eine Veränderung der Ziele, in dem Sinne, dass bereits erreichte oder nicht mehr erreichbare Ziele durch neue Ziele ersetzt werden, die Konzentration auf Erhaltung der Organisation, ohne weiter an der ursprünglich angestrebten Veränderung $\mathrm{zu}$ arbeiten (Zald/Ash 1966: 334), und die Ausbildung einer Oligarchie innerhalb der Organisation (Zald/Ash 1966: 327). Da die Mobilisierungsfähigkeit einer SMO über ihr Weiterbestehen entscheidet, ist sie von externen Faktoren abhängig, die das allgemeine Interesse der Teilnehmer an einer Mobilisierung oder an bestimmten Zielen der SMO beeinflussen, und der Entwicklung anderer SMO, die zu Konkurrenz oder Allianzen führen kann (Zald/Ash 1966: 330). Auch die Art und Weise, wie die Gesamtgesellschaft die soziale Bewegung und ihre Ziele sieht, ob sie sie unterstützt oder ablehnt, beeinflusst über die daraus folgende Veränderung der Anzahl der Interessenten entscheidend die Entwicklung einer SMO (Zald/Ash 1966: 330). Eine exklusive Mitgliedschaft mit hohen Eintrittskriterien und eine damit einhergehende 
Isolation der SMO führt zu einer geringeren Anfälligkeit der Organisation für Druck hin zu einer Verschiebung der Ziele oder zum bloßen Aufrechterhalten der SMO (Zald/Ash 1966: 332).

Die offenen Strukturen der Organisationen der Indigenenbewegung - oft werden alle Anwohner einer Gemeinde als passive Mitglieder gesehen, die je nach individuellem Interesse aktiv werden können (León 1991: 402) - führen zu einer breiten und relativ stabilen Basis. Die Tatsache, dass die meisten SMO fast ausschließlich auf dem Land über eine organisierte Basis verfügen, könnte jedoch ein Indiz für eine relativ kohärente Interessenlage sein, die den Effekten einer exklusiven Mitgliedschaft entspricht. Zumindest seit den 1980er Jahren zeichnen sich die SMO der Indigenenbewegung durch eine hohe Fluktuation ihres Führungspersonals aus, das zudem von der jeweiligen Basisorganisation legitimiert sein muss (Collins 2006: 226). Das verhindert zumindest teilweise die Ausbildung einer Oligarchie. Die zum Teil konsensuellen Wahlmechanismen innerhalb der CONAIE führen zudem zu einem beständigen Wechsel zwischen Führungspersonen aus den beiden wichtigsten Regionen Ecuadors, bei gleichzeitiger bedeutender Repräsentation der anderen Regionen. Daher sind die Effekte der Organisationsbürokratie andere. Auch in den anderen SMO ist und war der Einfluss der Basis entscheidend, so dass sich in ihnen nur in Zeiten der Krise - und der damit verbundenen Schwäche der Basis - eine oligarchische Führung herausbilden konnte, die außerdem zu anderen Reaktionen führte. Eine Konzentration auf den Erhalt der Organisation passiert nur selten. In den meisten Fällen - etwa die Krise der FEI in den 1960ern oder die der FENOC in den 1980ern - wechseln die Mitgliedsorganisationen ihre Mutterorganisation oder lösen sich auf. Häufiger ist eine Zielverschiebung zu beobachten, die von externen Faktoren - in den 1960er und 1970er Jahren der Kampf für eine Agrarreform und ihr Scheitern - und internen Faktoren - Stärke oder Schwäche bestimmter Strömungen in der Muttergewerkschaft im Falle der FENOC und CEDOC, die Nähe zu bestimmten Organisationen oder Parteien außerhalb der Indigenenbewegung - beeinflusst wird. So kann die Ablösung einer konservativen durch eine christdemokratische und dieser durch eine sozialistische Führung in der FENOC der 1970er Jahre, die jeweils auch von externen Akteuren unterstützt wurden, als Zielverschiebung und Austausch des Führungspersonals gewertet werden. Dem folgen zwar jeweils Aktionen der organisatorischen Stärkung, aus denen aber immer eine neue Politik mit neuen Forderungen erwächst.

Die Zielverschiebung der jeweiligen SMO hängt auch von den Interessen und Sympathien der Basis für bestimmte Ziele oder Taktiken zusammen. Daher spielt der Wettbewerb der verschiedenen SMO innerhalb einer sozialen Bewegung bei der Veränderung der Ziele und Taktiken eine entscheidende Rolle (Zald/Ash 1966: 332f.). Ein allgemeines Interesse an einer Landreform führt dazu, dass sich die SMO der Indigenenbewegung Ecuadors in den 1960ern und 1970ern auf dieses Ziel 
einigen können, in ihren Taktiken (etwa Landbesetzungen oder legale Strategien) aber voneinander abweichen. Ähnliches trifft auf das allgemeine Interesse der Indigenenbewegung am Aufbau eines offenen und pluralistischen Landes in den 1990ern zu, das von CONAIE und FENOCIN mit verschiedenen Vorschlägen verarbeitet wird. Die Tatsache, dass die Organisationen der Indigenenbewegung stets spezifische Ziele - etwa die Lösung bestimmter Landkonflikte oder die Veränderung bestimmter Gesetze - mit allgemeineren Zielen - der Landreform oder dem Aufbau eines plurinationalen und interkulturellen Staates - kombinierten, ist der Grund, dass sie nicht nach einem Erfolg aufhörten zu existieren (Zald/Ash 1966: 334) - es gab, zumindest mit Bezug auf die allgemeinen Ziele, aus der Sicht der Indigenenbewegung keinen Erfolg.

Organisationen sozialer Bewegungen, die einige institutionelle Macht erlangen und einige ihrer Ziele umsetzen konnten, aber nicht mehr weiter wachsen und vom Erreichen ihrer Ziele weit entfernt sind, neigen dazu, sich in eine „beruhigte Bewegung" (becalmed movement) (Zald/Ash 1966: 334) zu verwandeln. Eine solche SMO wird von der Basis und der Gesellschaft als relevant angesehen und kann so weiterbestehen. Sie folgt mit hoher Wahrscheinlichkeit dem Weber-Michels-Modell und bildet eine an der Erhaltung der SMO interessierte Oligarchie heraus (Zald/Ash 1966: 334). Beispiele für diese Entwicklung sind die FEI nach der ersten Agrarreform 1964, die nur einen Teil ihrer Basis weiter mobilisieren konnte und sich nun eher auf die Verteidigung der errungenen Erfolge konzentrierte (Becker/Tutillo 2009: 225) oder die FENOC in den 1980ern, die nach dem Scheitern ihrer Strategie der Kooperation mit den großen Gewerkschaften erheblich an Mobilisierungspotential einbüßte, die Krise aber überleben konnte. Beide Fälle sind durch lange Präsidentschaften einer bestimmten Person und unregelmäßige Kongresse - und daher mangelnde demokratische Kontrolle - gekennzeichnet. Die FENOC etwa führte im Zeitraum von 1977 bis 1995 unter ihrem Präsidenten Mesías Tatamuez nur drei Kongresse durch.

Für die Entwicklung der Indigenenorganisationen sind auch Freiräume (free spaces), die der direkten Kontrolle und dem ideologischen Einfluss (Fantasia/Hirsch 1995: 156f.) der herrschenden Gruppen entzogen sind, von entscheidender Bedeutung. Diese Freiräume sind relativ kleine, lang bestehende Institutionen in den Gemeinschaften, die über freiwillige Beteiligung die Erzeugung kultureller Herausforderungen erlauben, die politische Mobilisierungen begleiten. In Lateinamerika können sie die ungleiche Entwicklung des Staates ausnutzen, der den Bürgern in vielen Belangen fern ist (Davis 1999: 598/601). Insbesondere die geographische und die institutionelle Entfernung (Davis 1999: 603) vom Staat trägt zur Entwicklung von Freiräumen bei - die nicht selten Aufgaben des Staates übernehmen. In ihnen werden die hegemonialen Glaubensinhalte in Frage gestellt, Alternativen entwickelt, interne Differenzen behoben, traditionelle kulturelle Bedeu- 
tungen verändert und neue kulturelle Manifestationen geschaffen (Fantasia/Hirsch 1995: 145f.). „Freiräume scheinen den institutionellen Anker für die kulturelle Herausforderung zu bieten, die die strukturellen Gegebenheiten sprengt." (Polletta 1999a: 1) Außerdem wird in ihnen die soziale Wirklichkeit analysiert und Strategien entwickelt, um sie zu verändern. Freiräume versorgen die Aktivisten bei der Mobilisierung einer sozialen Bewegung mit Verbindungen, Fortbildung und Solidarität. In ihnen kann sich ohne Einfluss von außen eine gemeinsame Wahrnehmung von Problemen und Unterdrückung und damit eine kollektive Identität ausbilden (Fantasia/Hirsch 1995: 145f.). Vor allem bieten sie Möglichkeiten zur Analyse der gesellschaftlichen Diskurse und ihrer Begriffe und sind so ,entscheidend in der Ausbildung von Identitäten und Interessen, die Mobilisierung vorbereiten“ (Polletta 1999a: 3). In den Freiräumen vor Ort kann ebenso die Erfahrung vorheriger Mobilisierungen weiterbestehen. In ihnen können die kulturellen Praktiken der unterdrückten Gruppen als politisch verstanden werden (Polletta 1999a: 4f.), so erlauben sie den Aufbau ,einer kollektiven Aufzeichnung des Widerstandes“ (Polletta 1999a: 6) auf die Aktivisten zurückgreifen können. Sie sind also die Verknüpfung der lokalen Wirklichkeit mit den Erfahrungen früherer sozialer Bewegungen und erlauben damit den Aufbau neuer Identitäten und liefern zur gleichen Zeit die Motivation zu einer aktiven Beteiligung (Polletta 1999a: 7f.). Bei ethnischen Gruppen erlauben Freiräume den Aufbau von ,unberührbarem Kapital“ (Bonfil Batalla 1981: 27):

„,traditionelles Wissen, Kampf- und Widerstandsstrategien, Erfahrungen, ausprobierte Haltungen; ein ganzes Arsenal, das nur schwer enteignet werden kann, eine wachsende Grundlage von unterscheidenden Elementen die die Identität erlauben und untermauern“ (Bonfil Batalla 1981: 27).

$\mathrm{Zu}$ diesen Freiräumen gehören Klubs, Mieter- und Anwohnerorganisationen, Bars, Gewerkschaftsräume, Familien und Kirchen (Polletta 1999a: 3). Diese Institutionen bauen auf ,institutionalisierte normative Prinzipien“ (Polletta 1999a: 13), die ihnen eine gewisse Autonomie erlauben. Dieselben Prinzipien können einer Mobilisierung allerdings auch entgegenstehen. Daher ist die reine Existenz von Freiräumen noch keine Erklärung für ihre Funktion in besonderen Fällen (Polletta 1999a: 13). Sie sind eher stabile Elemente, die zu Zeiten hoher sozialer Konfliktivität eine innovative Rolle annehmen können (Fantasia/Hirsch 1995: 158) - die Wirkung von Freiräumen hängt also von ihrer Funktion innerhalb sozialer Bewegungen ab.

Für die ecuadorianische Indigenenbewegung sind Kirchengemeinden, (Groß)Familien und Produktionsgenossenschaften wichtige Freiräume. Ihre Tradition und relativ unpolitische Ausrichtung erlaubt ihnen einen gewissen Grad an Autonomie, der die Möglichkeit zur Reflexion und zum Bewahren von früheren Erfahrungen 
eröffnet. So ist es keine Überraschung, dass viele der Organisationen wie FENOCIN und ECUARUNARI mit Unterstützung und Anfangs unter Kontrolle der katholischen Kirchen gegründet wurden. Diese konnte durch ihre langjähriges Engagement nicht nur die nötigen Freiräume für die Entwicklung eigener Forderungen und schließlich Organisationen bereitstellen, sondern diese auch teilweise beeinflussen - wodurch sich die kirchlich geförderten Organisationen zumindest anfangs stark von den klassenbasierten Konkurrenzorganisationen unterschieden (Bebbington 2007: 804). Die Indigenenbewegung selbst hat spätestens in den 1980er Jahren damit begonnen, sich staatlichen Institutionen vor allem der Entwicklungs- und Bildungspolitik zu öffnen und konnte so neue Freiräume aufbauen. Die Institutionen der zweisprachigen interkulturellen Erziehung dienen so neben der allgemeinen Schulbildung indigener Kinder auch der Weiterbildung und der wirtschaftlichen Absicherung eines Teils des Führungspersonals der Indigenenorganisationen. In diesem Sinne handelt es sich um Freiräume (free spaces) im Sinne von Poletta und Fantasia/Hirsch.

Zumindest in manchen Gegenden ist die Rolle von Großfamilien als Freiräume der Indigenenbewegung zentral. So ist es kein Zufall, dass einer relativ kleinen Anzahl von Familien eine Reihe der wichtigen Führungspersonen der verschiedenen Organisationen entstammt. Dazu gehören die Familien Conejo Maldonado und Tituaña um Quinchuquí, Imbabura, im Norden Ecuadors, und die Viteri Gualinga und Villamil um Sarayacu, Pastaza, im Amazonasgebiet. Diese Ortschaften sind wirtschaftliche und handwerkliche Zentren, wodurch den jeweiligen Familien die nötigen Ressourcen zur Verfügung standen, ihren Kindern eine gute Ausbildung zukommen zu lassen - von denen einige die zentralen Figuren der Indigenenbewegung der letzten 30 Jahre werden sollten (Hoy 2001).

\section{Wettbewerb und Konflikte der SMO}

Die Tatsache, dass SMO auf begrenzte Ressourcen - wirtschaftliche Mittel, Mitglieder bzw. Unterstützer und Infrastruktur - angewiesen sind, führt dazu, dass verschiedene SMO innerhalb einer SMI und verschiedene SMI miteinander um den Zugang zu diesen Ressourcen konkurrieren. Die Konkurrenz dreht sich um die „symbolische Dominanz“ (Zald/McCarthy 1979: 3), also die Fähigkeit der SMO, über Programme, Taktiken und charismatische Führungspersonen Sympathisanten zu gewinnen. Eine Verringerung der vorhandenen Ressourcen lässt eine stärkere Konkurrenz erwarten (Zald/McCarthy 1979: 6), genauso wie exklusivere Organisationen mit einer stark engagierten Mitgliedschaft zu einer stärkeren Konkurrenz neigen als inklusivere Organisationen mit einer weniger stark engagierten Mitgliedschaft (Zald/McCarthy 1979: 7). Letztere neigen stattdessen dazu, ihrer Ziele und Taktiken leicht zu verschieben (Zald/McCarthy 1979: 7f.). Da die inklusiveren 
SMO sich über eine heterogene Mitgliedschaft definieren, entwickeln sie meist auch eine größere Bandbreite an zur Verfügung stehenden Zielen und Taktiken. Eine heterogene Basis erlaubt eine Vielzahl von Definitionen einer bestimmten Situation (Zald/McCarthy 1979: 8). Gerade für diese Art der SMO ist Produktdifferenzierung bzw. das Ausbilden origineller und eindeutig mit der Organisation identifizierbarer Ziele und Taktiken entscheidend (Zald/McCarthy 1979: 9).

Die Organisationen der Indigenenbewegung Ecuadors konkurrieren um Mitglieder und Unterstützer vor allem in den indigenen Gemeinden und um wirtschaftliche Förderung durch staatliche und nicht-staatliche Akteure auf regionaler, nationaler und internationaler Ebene. Die Mobilisierung der Mitglieder und Unterstützer hängt auch von der Vertretung ihrer Interessen durch die SMO ab - weswegen die Organisationen ersten und zweiten Grades die Regional- oder Nationalorganisation vor allem dann wechseln, wenn sie sich nicht ausreichend repräsentiert fühlen. Beispiele hierfür sind die politischen Richtungswechsel in der FENOC Mitte der 1970er (FENOCIN 1999: 39) und in der ECUARUNARI Anfang der 1980er (Santana 1983: 166), bei denen sich sozialistische Strömungen durchsetzen konnten und die oppositionellen Mitgliedsorganisationen ersten und zweiten Grades mit einem Wechsel hin zur anderen nationalen Organisation reagierten. Auch die Erwartung, dass die jeweilige SMO langfristig ihre Ziele erreichen kann, führte in der Geschichte der Indigenenbewegung Ecuadors zum Wechsel von Organisationen ersten und zweiten Grades von einer nationalen Organisation zu einer anderen. Dieser Wechsel findet weniger spektakulär statt und manifestiert sich meist als eine allmähliche Entwicklung. Das Sichabschwächen bestimmter nationaler Organisationen und die Stärkung anderer sind die Folge. Beispiele für diese Entwicklung ist der langsame Verlust von Mobilisierungsfähigkeit der FEI, deren Mitgliedsorganisationen in den 1960ern und 1970ern zur FENOC und vor allem zur ECUARUNARI die in den Verbreitungsgebieten der FEI stärker war - übertraten (Perreault/Bebbington/Carroll 1998: 8). Auch die Krise der FENOC in den 1970ern führte zum Übertritt einiger Organisationen zur ECUARUNARI (Ibarra Illanez 1987: 95) und zum Austritt zwei wichtiger Organisationen zweiten Grades - FOIN und FICSHA - , die kurz darauf die CONFENIAE gründen sollten, die am Aufbau der CONAIE großen Anteil hatte. Die Unterscheidung zwischen exklusiver und inklusiver Organisation trifft auf die Organisationen der Indigenenbewegung in Ecuador nicht zu, da sie in der Regel keine individuelle Mitgliedschaft kennen. Es ist meist die gesamte Gemeinde Mitglied, wobei sich Einzelpersonen bei Interesse stärker engagieren und aktiv werden können. Zur gleichen Zeit ist der Kreis des Führungspersonals relativ geschlossen und wird durch Lehrgänge und Fortbildungen gezielt auf weitere Aufgaben innerhalb der Organisationen vorbereitet (León 1991: 402). Daher ist die Konkurrenz zwischen den nationalen Organisationen - vor allem CONAIE, FENOCIN und FEINE - in der Regel größer als die Konkurrenz der Organisationen 
ersten und zweiten Grades vor Ort. Während auf nationaler Ebene das Herausstellen der inhaltlichen und personellen Unterschiede wichtig für die Mobilisierungskraft der Organisationen ist, ist auf lokaler Ebene eine pragmatische und zielgerichtete Politik, die auf breite Allianzen baut, von größerem Interesse. Die Krise der FEI in den 1960ern (Albornoz 1983: 63), der FENOC in den 1980ern (FENOCIN 2004: 18f.) und der CONAIE nach 2003 (Martínez Novo 2009a: 177) ist auch der Vernachlässigung dieser lokalen Ebene geschuldet.

Alle nationalen Organisationen versuchen, über originelle, tief mit der Organisation selbst verwurzelte Ziele und Inhalte symbolische Dominanz innerhalb der Indigenenbewegung zu erlangen. Ein wichtiger Teil dieses Versuches sind die politischen Begriffe, denen sich die vorliegende Arbeit widmet. So ist die Forderung nach einem plurinationalen Staat mit selbstverwalteten indigenen Territorien eindeutig mit der CONAIE, die Forderung nach Interkulturalität und Mechanismen der Verständigung zwischen den Kulturen eindeutig mit der FENOCIN verbunden. Das Verständnis der indigenen Völker als Nationalitäten - ebenfalls eindeutig mit der CONAIE verbunden - konnte von bestimmten indigenen Eliten des Hochlandes und des Amazonasgebietes ausgehend in der gesamten ecuadorianischen Gesellschaft verankert werden (Lucero 2008: 151). Diese Begriffe und ihre Definitionen sind „Schlüsselsymbole“ (Zald 1979: 13f.), die zur Artikulation der Ideen der jeweiligen SMO beitragen und so Unterstützung mobilisieren können. Die Forderung der CONAIE nach Plurinationalität ab den 1980er Jahren hat in diesem Sinne die Antwort der FENOCIN mit dem Begriff der Interkulturalität ab 1995 (FENOCIN 1999: 53) geradezu herausgefordert. Die FENOCIN musste die symbolische Dominanz und die Schlüsselsymbole der CONAIE in Frage stellen, um ihre Position innerhalb der Indigenenbewegung halten zu können (Zald 1979: 13f.). Auch das Aufkommen des Begriffs des Guten Lebens ab 2003 durch die Basisorganisation in Sarayacu (Sarayaku 2003) ist ein Versuch, über die Etablierung eines neuen Schlüsselsymbols die Kräfteverhältnisse innerhalb der CONAIE zu verändern.

Diese Auseinandersetzungen innerhalb der Bewegung werden nicht selten von externen Akteuren befördert, die ein Interesse daran haben, dass ein bestimmter Diskurs dominant wird oder schlichtweg die Bewegung als Ganzes schwächen wollen. Das können Wirtschaftsunternehmen, Entwicklungsagenturen oder der Staat selbst sein. Wenn es keine einheitliche, starke Führung gibt, kann es schwer sein, die so zerstrittene Bewegung zusammenzuhalten - wodurch sie an Bedeutung verliert (Bebbington 2007: 812). Der Einfluss staatlicher und internationaler Organisationen auf die Entwicklung und Verbreitung der beiden Begriffe Interkulturalität und Gutes Leben wird in den folgenden Kapiteln näher untersucht.

Gerade im Zusammenhang mit den Begriffen der Indigenenbewegung spielt die Mobilisierung wirtschaftlicher Ressourcen eine wichtige Rolle. Die SMO stellen die Legitimität der jeweiligen Konkurrenzorganisation in Frage, indem sie ihr vor- 
werfen, die Indigenen nicht oder nur wenig zu repräsentieren. Dadurch, dass die CONAIE der FEI vorwirft, von außen gesteuert zu sein (CONAIE 1989: 276), und die FENOCIN der CONAIE, unter der Kontrolle radikaler Ethnizisten zu stehen (FENOCIN 1999: 153), erhoffen sich beide Organisationen nicht nur die Mobilisierung von Unterstützern, sondern gerade auch wirtschaftliche Förderung durch die verschiedenen Entwicklungsorganisationen. So lässt sich die Politik der FENOCIN als Reaktion auf die weitgehende Vereinnahmung staatlicher und internationaler Mittel durch die CONAIE erklären (Zald/McCarthy 1979: 11) - was zumindest teilweise stimmt, wenn man die Rolle der zweisprachigen interkulturellen Erziehung bei der Aus- und Fortbildung des Führungspersonals gerade der CONAIE bedenkt (Maldonado 2006: 91).

Neben den Auseinandersetzungen über die Begriffe und den Diskurs, spielen die Auseinandersetzungen zwischen den verschiedenen Indigenenorganisationen über die anzuwendenden Strategien eine wichtige Rolle. Die Frage, wer mit welchen Mitteln bekämpft oder beeinflusst werden soll, kann die Konflikte in einer sozialen Bewegung verschärfen (Bebbington 2007: 812). Dabei werden Strategien und Taktiken von externen Faktoren, wie etwa dem Vorhandensein von Ressourcen oder politischen Gelegenheiten und der erwarteten Reaktion des Staates, und von internen Faktoren, wie etwa der Ideologie der jeweiligen Organisation und dem zu erwartenden Gewinn der Aktion, beeinflusst (Ennis 1987: 521). Politische Aktionen haben nicht nur direkte politische, sondern auch indirekte symbolische Folgen, die Wahl einer bestimmten Taktik oder Strategie beeinflusst nicht nur die Erfolgschancen, sondern auch die Bedeutung einer Aktion. Daher ist die Wahl einer bestimmten Taktik auch eine symbolische (Ennis 1987: 523). Soziale Bewegungen können mit bestimmten Aktionen die Aufmerksamkeit der Öffentlichkeit auf ihre Probleme lenken (Meyer/Staggenborg 1996: 1638) - und so Druck auf den Staat ausüben.

Ein Beispiel für diese Art strategischer Konflikte sind die Auseinandersetzungen zwischen FEI und FENOC in den Jahren nach der ersten Agrarreform 1964. Während die FEI zu eher militanten Aktionen neigte, die nur wenig Erfolg brachten, konnte die FENOC mit rechtlicher Unterstützung und direkten Verhandlungen mit den betroffenen Behörden größere Erfolge verbuchen - die zu ihrem schnellen Wachstum beitrugen (Perreault/Bebbington/Carroll 1998: 8). Auf nationalem Niveau spielt die Strategie der CONAIE, mit nationalen Erhebungen und groß angelegten Märschen und Demonstrationen ihre Ziele durchzusetzen, seit dem Inti Raymi 1990 eine besondere Rolle. Diese Mobilisierungsformen werden als erfolgversprechendster Weg gesehen, Forderungen umzusetzen und eine direkte Repräsentation innerhalb des Staates zu erlangen - somit „eine eigene Macht aufzubauen, die zur Gegenmacht der etablierten Macht wird“ (Maldonado 2006: 91). Die zahlenmäßige Überlegenheit der CONAIE hat seit diesem Zeitpunkt bis zu ihrer Regierungsbeteiligung 2003 strategische Konflikte auf die Frage reduziert, ob sich die 
anderen Organisationen den von der CONAIE und ihren Mitgliedsorganisationen geplanten und durchgeführten Aktionen anschließen oder nicht. Diese großen nationalen Aktionen integrieren lokale, regionale und nationale Forderungen der verschiedenen Organisationen, die sich gegenseitig verstärken und in einem konflikthaften Prozess integriert werden müssen (Barrera 2001: 153).

Die Entwicklung der verschiedenen Organisationen innerhalb der ecuadorianischen Indigenenbewegung war seit ihren Anfängen in den 1930ern bis zur Regierungsbeteiligung der CONAIE 2003 von der weitgehenden Hegemonie jeweils einer Organisation geprägt, die eine symbolische Dominanz in der Bewegung hatte und ihre Forderungen und Aktionsformen zu einem großen Teil bestimmten konnte.

\begin{tabular}{|l|l|l|l|l|}
\hline & $1930-1965$ & $1965-1980$ & $1980-2003$ & 2003-heute \\
\hline FEI & $\mathrm{X}$ & - & - & - \\
\hline FENOCIN & & $\mathrm{X}$ & - & $\mathrm{X}$ \\
\hline FEINE & & - & - & $\mathrm{X}$ \\
\hline CONAIE & & - & $\mathrm{X}$ & $\mathrm{X}$ \\
\hline
\end{tabular}

Tabelle 7: Organisatorische Hegemonie und symbolische Dominanz in der Indigenenbewegung $^{60}$, eigene Abbildung

Seit Anfang des neuen Jahrtausends und bedingt durch die zunehmende Stärke von FEINE und FENOCIN gibt es zum ersten Mal seit dem Bestehen einer formal strukturierten Indigenenbewegung in Ecuador eine Situation, in der verschiedene Organisationen großen Einfluss erlangen konnten. Das ist nicht unbedingt gleichbedeutend mit einer Schwächung der CONAIE, sondern als Entwicklung weg von einer weitgehenden Hegemonie und hin zu einer polyzentrischen Struktur zu verstehen. Die Folge ist, dass die Indigenenbewegung nicht mehr als einheitlicher Akteur wahrgenommen wird, der von einer Organisation repräsentiert wird und mit einer Stimme spricht - wie es bis vor wenigen Jahren auch viele Wissenschaftler taten (etwa Santillana 2006). Verschiedene Diskurse und Begriffe konkurrieren miteinander, gleichzeitig gewinnt die traditionelle Politik der Indigenenorganisationen, nach breiten Bündnisse zu suchen, weiter an Bedeutung. Vor allem im Zuge der Konflikte der Indigenenbewegung mit der Regierung Correa ist eine Annäherung der verschiedenen Organisationen zu beobachten, deren Folgen sich erst in einigen Jahren zeigen werden.

60 ,X`- weitgehende Hegemonie/Dominanz, ,-` - gewisser Einfluss, , ‘- kein Einfluss/nicht existent; es werden auch die jeweiligen Mitglieds- und Vorläuferorganisationen miteinbezogen. 


\section{Koalitionen}

Die Politik der Organisationen sozialer Bewegungen ist nicht nur von Konkurrenz um Ressourcen, sondern auch von Interaktion geprägt. Diese tritt in drei Idealtypen auf: eine punktuelle Kooperation in besonderen Fällen, in denen spezielle Fähigkeiten nötig sind, über die nur eine der SMO verfügt; die Zusammenlegung von Ressourcen und Infrastruktur - unter Beibehaltung der Organisationsidentitäten - im Rahmen einer Koalition, insbesondere, wenn diese Strategie die Erreichung eines Ziels erleichtern kann; und die Verschmelzung der SMO (Zald/Ash 1966: 335).

Kooperation erfolgt meist über Gebietsvereinbarungen (domain agreements), in denen SMO mit verschiedenen aber nicht widersprüchlichen Stärken und Strategien kooperieren. Sie kann durch eine gestiegene Repression des Staates erzwungen werden, um die Existenz der sozialen Bewegung und ihrer Organisationen zu verteidigen. Im Kontext eines repressiven Umfeldes kooperieren SMO auch über die Grenzen ihrer SMI hinweg (Zald/McCarthy 1979: 16). Kooperation kann ebenfalls durch eine strukturelle oder personelle Verzahnung zwischen den jeweiligen SMO befördert werden (Zald/McCarthy 1979: 18).

Eine intensivere Kooperation etwa in Allianzen lässt sich meist beobachten, wenn die Erreichung der Ziele aus der Perspektive der SMO unmittelbar bevor steht. Zielerreichung wird dann - anstelle von Organisationserhaltung - zur Priorität, Ressourcen lassen sich leicht und schnell mobilisieren und in einer einheitlichen Organisation besser verwalten, so dass die Aufrechterhaltung der verschiedenen Einzelorganisationen kein unmittelbares Problem ist (Zald/McCarthy 1979: 23). Engere Allianzen von SMO treten in besonderen Umständen auf, etwa zu bestimmten Ereignissen wie Massendemonstrationen oder Märschen, die von verschiedenen Organisationen gemeinsam organisiert werden. Die größte Organisation der Bewegung trägt dann in der Regel mehr als ihren proportionalen Anteil an Ressourcen bei (Zald/McCarthy 1979: 22). Dieses Phänomen ist im Fall der ecuadorianischen Indigenenbewegung bei den meisten größeren Aktionen (die Erhebungen von 1990 und 1994, der Marsch von 1992, die Aktionen gegen die Regierungen Bucaram und Mahuad, die Proteste gegen das Freihandelsabkommen 2005) zu beobachten, die neben der CONAIE als dominierende SMO - die dementsprechend höhere Investitionen in die jeweilige Aktion tätigte und die erste Nutznießerin der Erfolge war FENOCIN, FEINE und FEI ebenso wie einige Organisationen anderer sozialer Bewegungen, wie die CONFEUNASSC-CNC der Bauernbewegung, vereinigen konnten. Jedoch waren diese gemeinsamen Bemühungen in allen Fällen nur kurzfristig. Für einige Wochen oder Monate koordiniert eine gemeinsam gebildete formelle oder informelle Instanz die Aktionen und löst sich nach Ende der jeweiligen Mobilisierung wieder auf oder wird bedeutungslos. Das ist der Fall bei der Koordination der Landkonflikte (Coordinadora de Conflictos Agrarios) (Guerrero/Ospina 
2003) und der Volkskoordination (Coordinadora Popular), die beim Aufstand 1990 eine wichtige Rolle spielten, sowie bei der Nationalen Agrar-Koordination (Coordinadora Agraria Nacional), die an der Organisation des Aufstands 1994 beteiligt war (Buendía 2000: 60; Agualsaca o.J.: 3). Der Umweg über Koordinationsinstanzen, die außerhalb der eigentlichen SMO stehen, erlaubt es allen SMO, sich an den Aktionen zu beteiligen, ohne ihre Eigenständigkeit aufgeben zu müssen. Die Tatsache, dass diese Koordinationsinstanzen tatsächlich von der CONAIE kontrolliert wurden (Koordination der Landkonflikte: Maldonado 2006: 93; Nationale AgrarKoordination: Negreiros 2009: 136), führt aber dazu, dass die Aktion von der Öffentlichkeit als eine Aktion der CONAIE gesehen wird, diese also einen überproportionalen Gewinn hat.

Auch auf niedrigerem Niveau neigen die lokalen Organisationen der Indigenenbewegung nicht zu einer Verschmelzung mit anderen SMO. So sind alle großen Organisationen Föderationen bzw. Konföderationen. Die Einzelorganisationen „,behalten ihre Identität aber geben gewisse Entscheidungsrechte an die neue Organisation ab" (Zald/McCarthy 1979: 21). Daraus resultiert ein hoher Grad an Autonomie, den die lokalen und Provinzorganisationen, wie etwa die OPIP, innerhalb der nationalen Organisationen, wie etwa der CONAIE, haben.

\section{Transnationalisierung der ecuadorianischen Indigenenbewegung}

Um die Entwicklung der ecuadorianischen Indigenenbewegung verstehen zu können, ist es wichtig, auch ihre inter- und transnationalen Aspekte ins Auge zu fassen. Trotz seiner Beschränkung auf internationale Kampagnen von Aktivistennetzwerken gegen Staaten und internationale Akteure oder Institutionen, kann der Ansatz der transnationalen kollektiven Handlung (transnational collective action) (Della Porta/Tarrow 2005: 2f./7) in einigen Punkten zum Verständnis der ecuadorianischen Indigenenbewegung beitragen. Nach diesem Ansatz gibt es drei wichtige Transnationalisierungsprozesse: die Verbreitung (diffusion) von Taktiken, Praktiken und Ideen sozialer Bewegungen von einem Land zum anderen, die Austragung von Konflikten externen Ursprungs im eigenen Land im Sinne einer Domestizierung (domestication) und die Externalisierung (externalization) nationaler oder lokaler Konflikte auf supranationale Institutionen (Della Porta/Tarrow 2005: 2). Die Verbreitung von Ideen und Praktiken von einem Land in ein anderes ist ein bekanntes und häufiges Phänomen. Dazu sind nicht unbedingt Verbindungen zwischen den sozialen Bewegungen nötig, sondern nur eine Annahme und Anpassung der Ideen oder Praktiken einer sozialen Bewegung eines anderen Landes. In einigen Fällen findet diese Vermittlung über eine Person statt (brokerage), die mit beiden Bewegungen in Kontakt steht (Della Porta/Tarrow 2005: 3). Eine genauere Untersuchung dieser Transnationalisierungsprozesse im Fall der ecuadorianischen Indigenenbe- 
wegung steht noch aus. Bestimmte Einflüsse - die Beschäftigung mit der Indigenenbewegung Kolumbiens in der Kulturwerkstatt Causanacunchic (Flores 2005: 37), eine teilweise Übernahme von Empfehlungen kontinentaler Organisationen wie des CISA - wurden bereits herausgestellt, ihr genauer Charakter sollte noch näher untersucht werden.

Als Variante der Externalisierungsthese stufen Della Porta und Tarrow den Aufbau transnationaler Koalitionen internationaler Nichtregierungsorganisationen ein, die soziale Bewegungen des globalen Südens in internationalen Institutionen unterstützen (Della Porta/Tarrow 2005: 5). Dabei hilft die Kooperation hoch spezialisierter NRO mit Institutionen wie der UNO (Della Porta/Tarrow 2005: 6) bei einer Präsentation spezifischer Forderungen auf internationaler Ebene. Gerade die UNO selbst wurde in diesem Kontext zu einer Bühne der Artikulierung von Forderungen, die sie durch die Festsetzung internationaler Normen stärkt und legitimiert (Della Porta/Tarrow 2005: 5f.). Diese Art der Transnationalisierung ist auch bei der ecuadorianischen Indigenenbewegung zu beobachten. Internationale Verbünde sozialer Bewegungen wie Vía Campesina (FENOCIN 2004: 25), die Andine Koordination der indigenen Organisationen (Coordinadora Andina de Organizaciones Indígenas, CAOI) (Simbaña 2007: 23) und die Koordination der indigenen Organisationen des Amazonasbeckens (Coordinadora de las Organizaciones Indígenas de la Cuenca Amazónica, COICA) (GTZ 2004: 33) dienen nicht nur als Basis für die Mobilisierung übernationaler Ressourcen und Strategien oder Möglichkeiten zum internationalen Austausch und Zusammenarbeit (Della Porta/Tarrow 2005: 10), sie sind auch institutionell in die Apparate supranationaler Organisationen integriert, etwa in der Andengemeinschaft CAN oder der UNO. Diese transnationalen Organisationen sind auch als Antwort auf transnationale Probleme zu begreifen. Viele der Indigenenorganisationen Lateinamerikas sind von Vertreibung und Enteignung im Rahmen von Freihandelsabkommen und Rohstoffförderung betroffen und suchen deshalb verstärkt nach Allianzen auf lokalem, nationalem und internationalem Niveau (Bebbington 2007: 802).

\section{Kultur und soziale Bewegungen}

Eine für die Indigenenbewegung Ecuadors besonders wichtige Ressource ist die Kultur im Sinne von Ethnie und Identität auf der einen und von Diskurs oder gesellschaftlich konstituiertem Sinn auf der anderen Seite. Diese Bedeutung ergibt sich aus den identitären Aspekten der Bewegung und den daraus folgenden Fragen nach ihrer Kulturpolitik im Sinne von cultural politics. Cultural politics meint dabei die von verschiedenen kulturellen Bedeutungen und Praktiken geprägten Konflikte zwischen verschiedenen sozialen Akteuren. Kultur im Sinne von Bedeutungen und Praktiken kann also die Quelle für politische Prozesse sein. Aus diesen Bedeutun- 
gen und Praktiken entstehen Diskurse, die sich durch ihre Unterschiede zu den dominanten Kulturen auszeichnen (Escobar 1998: 64).

„Kultur ist politisch, denn Bedeutungen sind grundlegend für Prozesse, die, implizit oder explizit, anstreben, soziale Macht neu zu definieren. Wenn Bewegungen alternative Vorstellungen von Frau, Natur, Entwicklung, Wirtschaft, Demokratie oder Bürgerschaft aufstellen, die dominante kulturelle Bedeutungen erschüttern, üben sie Kulturpolitik aus.“ (Escobar 1998: 64)

Durch ihre zentrale Rolle prägen die SMO den Diskurs, die Politik und das Weltbild der sozialen Bewegung, die sie über Vorträge, Publikationen und andere Aktionen der Öffentlichkeit präsentieren. Sie ,neigen dazu, die Quellen von Gegendiskursen und der generativen Ideen, auf denen diese erbaut wurden, zu sein“ (Bebbington 2007: 800). Daher ist die Kultur, im Sinne von Bedeutungen und Praktiken, einer der zentralen Bereiche, in denen soziale Bewegungen aktiv sind und den sie verändern wollen. Kultur ist nicht nur eine Ansammlung möglicher Ziele sozialer Bewegungen, sondern ,das Vokabular von Bedeutungen, die ausdrucksvollen Symbole und das emotionale Repertoire, mit dem sie überhaupt etwas anstreben können“ (Swindler 1995: 27). Somit ist ein Großteil der Erfahrungen sozialer Bewegungen im Bereich der Kultur zu finden. Es geht ihnen nicht nur um politische oder wirtschaftliche Ungleichheit, ,gesellschaftlich geteilte Bedeutungen“ (Diani 1992: 10), die auf verschiedenen Ebenen die Interpretation der Wirklichkeit steuern, sind ebenfalls ein zentrales Ziel sozialer Bewegungen (Escobar 1992a: 412). Sie beantworten Initiativen des Staates oder anderer Akteure nicht nur mit Gegenvorschlägen, sondern sie kritisieren die Bedeutung, die hinter den Debatten liegt und machen Alternativen deutlich. Somit sind ,,soziale Bewegungen am besten zu verstehen als Vektoren besonderer Diskurse und Arten, die Welt zu hinterfragen“ (Bebbington 2007: 800). Diese gegenhegemonialen Ideen sind allerdings von der Entwicklung ihrer sozialen Bewegung abhängig - wenn diese in eine Krise tritt und an Unterstützung oder Legitimität verliert, verliert auch der Gegendiskurs an Einfluss (Bebbington 2007: 813).

Kultur im Sinne von Bedeutungen ist durch den Kontext, in den ,ein Text oder Ritual eingebettet ist“ (Swindler 1995: 28) bestimmt; erst so wird dieser Text im kulturwissenschaftlichen Sinne für alle Beteiligte sinnvoll. Die Einbettung führt dazu, dass die Kultur sozialer Bewegungen über eine Analyse ihrer öffentlichen Äußerungen und Aktionen greifbar ist (Swindler 1995: 28) - und nicht in erster Linie der persönlichen Überzeugungen ihrer Akteure (Swindler 1995: 36). Der schwierige Zugang zu formellen Entscheidungsprozesses macht die Veränderung von Kultur und Bedeutungen zu einer erfolgversprechenderen Strategie - über eine Veränderung bestimmter Bedeutungen kann eher die gewünschte Veränderung der 
Gesellschaft erreicht werden, als über eine Teilnahme an politischen Strukturen. Daher ist der „Kampf um symbolische Kodierung“ (Swindler 1995: 34) und damit nicht nur die mobilisierende Wirkung, sondern auch die Sichtbarkeit der Aktionen sozialer Bewegungen von nicht zu unterschätzender Wichtigkeit (Swindler 1995: 34). Diese Fokussierung auf eine Veränderung von Bedeutungen führt dazu, dass gesellschaftliche Institutionen als verfestigte Bedeutungskomplexe zu einem zentralen Ziel sozialer Bewegungen werden. Sie sind die Träger der Regeln, die das Verhalten der Individuen lenken und erzeugen damit Einschränkungen und Möglichkeiten für Individuen und soziale Bewegungen (Swindler 1995: 36). Somit beeinflussen die Institutionen einer Gesellschaft entscheidend Kultur und Strategie sozialer Bewegungen (Swindler 1995: 37); eine Dominanz bestimmter Diskurse innerhalb der Institutionen kann dazu führen, dass soziale Bewegungen ihre Forderungen in den Termini dieser Diskurse ausdrücken. Wenn Institutionen etwa auf Recht oder ethnische Identität reagieren, werden soziale Bewegungen mit höherer Wahrscheinlichkeit ihre Forderungen in diesen Begriffen formulieren (Swindler 1995: 37).

„Bedeutungsarbeit“ (Benford/Snow 2000: 613) als Kampf um Ideen und Bedeutungen ist also ein zentraler Bestandteil sozialer Bewegungen. Sie sind Agenten einer Bedeutungskonstitution, die in Konflikten und Kooperationen mit anderen Akteuren, wie dem Staat, den Medien oder der Zivilgesellschaft geschieht (Benford/Snow 2000: 613). Diese diskursive Sinngebung wird von manchen Forschern sozialer Bewegungen als framing umschrieben. Damit soll die aktive Rolle, die die Bewegungen in diesem Prozess spielen, hervorgehoben werden. Im Prozess des framing werden aus vorhandenem „kulturellem Material“ (Benford/Snow 2000: 629), wie Ideologien, Glaubensinhalten, Praktiken oder Mythen, neue kulturelle Elemente geschaffen und Interpretationsweisen angeboten (Benford/Snow 2000: 629). Das Ergebnis des framing sind kollektive Handlungsrahmen (collective action frames), die die Aktionen einer bestimmten Organisation oder Bewegung inspirieren und legitimieren und ihren Diskursivierung steuern. Diese Handlungsrahmen erlauben den Mitgliedern der Bewegung, ein geteiltes Verständnis einer Situation zu entwickeln (Benford/Snow 2000: 614f.). framing ist dabei ein andauernder Prozess, der von verschiedenen Faktoren beeinflusst werden kann und beständig neue Handlungsrahmen erzeugt (Benford/Snow 2000: 628).

Die Aufgaben des framing sind die Definition und Diagnose einer problematischen Situation, wer für sie verantwortlich ist und wie sie verändert werden kann, die Prognose der möglichen Aktionen, um diese Situation zu verändern, und die Motivation der Mitglieder, damit sie die entwickelten Aktionen unterstützen (Benford/Snow 2000: 616f.). Die ersten beiden Aufgaben können zu Konflikten zwischen verschiedenen SMO oder sozialen Bewegungen führen. So kann es etwa zu Auseinandersetzungen über die Frage kommen, wer oder was für die problematische Situation verantwortlich ist (Benford/Snow 2000: 616) - im Falle der Indige- 
nenbewegung Ecuadors ist eine der zentralen Auseinandersetzungen die Frage, ob die Indigenen in erster Linie als Ethnie unterdrückt oder als Klasse ausgebeutet werden. Das hat zu Konflikten zwischen den gewerkschaftlich ausgerichteten Organisationen wie der FENOCIN und den eher ethnischen Organisationen der CONAIE geführt (CONAIE 1989: 281) und gleichzeitig zu Konflikten zwischen den beiden Positionen innerhalb der CONAIE (Simbaña 2007). Die Frage, wie die einmal als problematisch definierte Situation behoben werden kann, führt meist dazu, dass bestimmte SMO explizit die Strategien ihrer Konkurrenten ablehnen (Benford/Snow 2000: 616f.) - wie im Fall der Konflikte zwischen CONAIE und FENOCIN und innerhalb der CONAIE selbst. So wurde der CONAIE vorgeworfen, sich mit einem korporativen Modell der Integration zufrieden zu geben, statt eine völlige Veränderung des Staates im Sinne der Plurinationalität anzustreben (Viteri 1997: 55; FENOCIN 1999).

Die kollektiven Handlungsrahmen werden mit zwei interaktiven, diskursiven Prozessen verändert: frame articulation, die Integration verschiedener Ereignisse und Ideen in den bestehenden Rahmen, der damit neue Blickwinkel erlaubt, und frame amplification, die Hervorhebung bestimmter Themen oder Ideen, die den gesamten Rahmen repräsentieren sollen (Benford/Snow 2000: 623). Dazu wird versucht, diese Themen oder Ideen mit bereits bestehenden Werten oder Glaubensinhalten zu verbinden. Dieser Prozess ist besonders relevant, wenn die betreffende Bewegung für Personen kämpft, die nicht in ihr vertreten sind oder die Werte der Bewegung den Hauptwerten der vorherrschenden Kultur widersprechen (Benford/Snow 2000: 624). Zu diesen hervorgehobenen Ideen gehören im Fall der ecuadorianischen Indigenenbewegung mit Sicherheit die hier behandelten Begriffe, die auf komplexe Programme verweisen und Teile älterer Programme einbinden - wie im anschließenden Teil dieser Arbeit näher untersucht wird.

Weiterhin finden Rahmenanpassungsprozesse (frame alignment processes) statt, mit denen die kollektiven Handlungsrahmen der SMO an die Interessen und Erwartungen ihrer Mitglieder und Sympathisanten sowie der möglichen Ressourcengeber angepasst werden sollen. $\mathrm{Zu}$ diesen Anpassungsprozessen gehört neben dem frame amplification das frame bridging, also die Verbindung verschiedener, aber ideologisch übereinstimmender frames in Bezug auf ein bestimmtes Problem, das frame extension, das die frames und Interessen einer SMO erweitert, um so mögliche Unterstützer ansprechen zu können, und das frame transformation, das alte Bedeutungen verändern und neue erzeugen will (Benford/Snow 2000: 624f.). frame bridging wird bei gemeinsamen Aktionen der verschiedenen SMO der Indigenenbewegung in Ecuador deutlich. Bei den Erhebungen 1990 und 1994 haben sich mit FENOCIN, FEINE und FEI Organisationen beteiligt, die mit dem Diskurs der CONAIE nicht übereinstimmten - aber bestimmte Ziele, wie die Verbesserung der Situation der Landbevölkerungen, sehr wohl unterstützen. Eine ähnliche Situation 
kann bei den Protesten gegen die Regierung Correa in den letzten Jahren beobachtet werden. Als frame extension kann die Betonung umweltpolitischer Aspekte im Diskurs vor allem der CONAIE seit den 1990ern gesehen werden, mit der neue Verbündete und - gerade auf internationalem Niveau - Geldgeber mobilisiert werden können. In diesem Zusammenhang ist das Auftauchen des Begriffs Sumak Kawsay oder Gutes Leben interessant, der das bereits seit den 1980ern (etwa Pacari 1984) anzutreffende Selbstverständnis der Indigenen und Indigenenbewegung als ökologisch und auf Harmonie mit der Umwelt bedacht weiter konkretisiert. Ein Fall von frame transformation ist die Umwidmung der FENOCIN in eine interkulturelle Organisation ab 1995 (FENOCIN 1999: 53) - und damit eine teilweise Abkehr von der vorherigen klassistischen Politik.

Bestehende kollektive Handlungsrahmen können auf mindestens drei verschiedene Arten herausgefordert werden: durch Gegner der sozialen Bewegung, die counterframing, also Umdeutung der framing-Inhalte der Bewegung, betreiben, durch Auseinandersetzungen über die frames innerhalb der sozialen Bewegungen, und durch die Beziehung zwischen frames und Ereignissen (Benford/Snow 2000: 625). Im Fall der ecuadorianischen Indigenenbewegung kann der Vorwurf, der um die Begriffe indigene Nationalitäten und Plurinationalität gebaute Diskurs der CONAIE wolle den ecuadorianischen Staat teilen und Indigenenrepubliken schaffen (Walsh 2009b: 233) - meist von Seiten der Medien (siehe León 1994: 35ff.) oder der Armee vorgebracht - als counterframing gesehen werden. Der Angriff auf dieselben Begriffe vor allem durch die FENOCIN ab 1995 mit dem Begriff Interkulturalität, wobei diese der CONAIE und ihrem Diskurs vorwirft, radikal indigen ausgerichtet zu sein (FENOCIN 1999; Ayala Mora 2011: 53), ist eine der internen Auseinandersetzungen über frames in der Indigenenbewegung. Die eindeutige Definition der Begriffe Nationalität und Plurinationalität im Politischen Projekt der CONAIE 1994 ist auch den Schwierigkeiten bei der Vermittlung dieser Begriffe in der Gesamtgesellschaft bei den Massenaktionen 1990 und 1992 geschuldet, die der CONAIE die Anklage einbrachte, nur die indigenen Interessen zu vertreten (Walsh 2008: 17) - also eine Wirkung von externen Ereignissen.

Diese Rahmenanpassungsprozesse werden durch intentionale Prozesse ergänzt. Das sind strategic selection, die Auswahl und Integration bestimmter Elemente anderer sozialer Bewegungen in die Bewegung vor Ort durch bestimmte Personen, und strategic fitting, die aktive Propagierung bestimmter Elemente durch andere soziale Bewegungen, einschließlich ihrer Anpassung an die jeweilige Kultur (Benford/Snow 2000: 627). Der folgende Teil wird zeigen, dass im Fall der ecuadorianischen Indigenenbewegung eine klare Zuordnung nicht möglich ist. Zu den wenigen Ausnahmen gehören die eindeutigen Strategieempfehlungen des Indio-Rates Südamerikas CISA (AAVV 1985: 162). 
Diese hier nur kurz angerissene Analyse des Diskurses der Indigenenbewegung in Ecuador wird im folgenden Teil unter methodisch anderen Gesichtspunkten systematisiert. Auch wenn der Ansatz des framing die Analyse von Diskursen oder Diskursbereichen in sozialen Bewegungen erleichtert, so bleibt sie den Inhalten des Diskurses doch fern. Sie erlaubt es, seine strategische Verwendung innerhalb und außerhalb der Bewegung zu erfassen, geht jedoch nicht über eine Umfeldanalyse hinaus und greift so nicht auf die tatsächlichen Inhalte des jeweiligen Diskurses zu. Man kann mit framing feststellen, welche Aufgaben ein Diskurs in einer sozialen Bewegung zu erfüllen hat und - teilweise - warum er zu bestimmten Phänomenen neigt - im Sinne des framing wäre es jedoch unnütz, bestimmte Inhalte eines Diskurses näher zu untersuchen. Diese Inhalte erfüllen ihre Funktion - oder eben nicht. Woher sie kommen und welche Bedeutung ihnen zugrunde liegt, ist uninteressant.

\section{Identität und soziale Bewegungen}

Die Untersuchung der Indigenenbewegung in Ecuador zwingt zu einer grundlegenden Unterscheidung, die auch methodologische Auswirkungen hat: die Unterscheidung zwischen Bewegungsidentität (Polletta/Jasper 2001: 289) und ethnischer Identität. Dieser Unterscheidung wird in der Forschung zu sozialen Bewegungen nicht immer Rechnung getragen. Der Fokus auf kollektive Identität sozialer Bewegungen ist dabei keine rein kulturelle Herangehensweise. Es sollen im gleichen Maße auch „strukturelle“ (Polletta/Jasper 2001: 285) Prozesse untersucht werden, die die Beziehungen zwischen Identität und Strategie, Politik oder den geteilten Interessen prägen.

So sieht eine einflussreiche Schule innerhalb der Theorien zu sozialen Bewegungen eine geteilte kollektive Identität als einer der entscheidenden Faktoren sozialer Bewegungen (Diani 1992: 13). Diese kollektive Identität ist sowohl eine Selbst- als auch eine Fremdzuschreibung als Mitglied der Bewegung bzw. Gruppe (Polletta/Jasper 2001: 285). Mitglieder der Indigenenbewegung etwa müssen sich selbst also nicht nur als solche verstehen, sondern auch von Außenstehenden als solche gesehen werden. Das Selbstverständnis als Mitglied einer sozialen Bewegung meint vor allem, dass die grundlegenden Ideen und Ziele dieser Bewegung geteilt werden und es ein Gefühl der Zugehörigkeit gibt. Durch die Interaktionen der verschiedenen Individuen oder SMO, die sich einer bestimmten Bewegung zugehörig fühlen, wird die Grenze dieser Bewegung definiert (Diani 1992: 8f.). In diesem Sinne können auch Mitglieder der öffentlichen Verwaltung oder politische Parteien einer sozialen Bewegung angehören (Diani 1992: 14f.) - somit lässt sich die Position der MUPP-NP als Mitglied der Indigenenbewegung eindeutig klären, ebenso die Zugehörigkeit einiger nicht-indigener Intellektueller als Sympathisanten. 
Soziale Bewegungen versuchen, bereits bestehende und fest institutionalisierte kollektive Identitäten in ihre Bewegungsidentität zu integrieren und über ihre Diskurse neue kollektive Identitäten zu erzeugen, um so Mitglieder und Unterstützer zu gewinnen. Dabei ist vor allem die Abgrenzung der eigenen Gruppe von einer zu konstituierenden Gruppe der Gegner wichtig, die das Verständnis der eigenen Situation als , ungerecht" erlaubt. Diese Bewegungsidentität muss die verschiedenen kollektiven Identitäten ihre Mitglieder aufgreifen und in ihrer Verschiedenheit respektieren. Zudem muss die Identifikation der Mitglieder mit der sozialen Bewegung durch gewisse Rituale oder andere Bestätigungen der Bewegungsidentität bestärkt werden. Wenn die Bewegungsidentität geschwächt wird, etwa weil die soziale Bewegung nicht mehr als Repräsentant der eigenen Interessen wahrgenommen wird oder andere Institutionen diese Funktion besser erfüllen, gerät die soziale Bewegung in eine Krise (Polletta/Jasper 2001: 289-292). Die Aktivisten der sozialen Bewegungen können dabei ihre Identitäten je nach strategischer Situation umdefinieren, um so die Einheit der Bewegung zu verstärken (Polletta/Jasper 2001: 294). Die Integration der Indigenen in die Indigenenbewegung Ecuadors hat je nach allgemeiner politischer Situation nach anderen Regeln funktioniert. So wurde auf die kollektive Identität als Landarbeiter und Kleinbauern verwiesen, als die Indigenenbewegung in erster Linie gewerkschaftlich geprägt war und auf die kollektive Identität als Indigene oder Indios, als die Gewerkschaften und PCE und PSE in eine Schwächephase eintraten und statt dessen ethnisch geprägte Organisationen an Bedeutung gewannen.

Die Besonderheiten ethnischer kollektiver Identität (Escobar 1998: 66) - im Gegensatz zu etwa lokaler oder sozialer bzw. Klassenidentität - ist die Hervorhebung systematischer Diskriminierung, die kulturell oder politisch sein kann (Bebbington 2007: 796f.). Auch der historisch bedingte Einfluss kirchlicher und Erziehungsorganisationen hat zu einem Fokus auf Anerkennung, Partizipation und ethnische Rechte geführt (Bebbington 2007: 804), der den gewerkschaftlichen Bewegungen meist fremd blieb. In Ecuador konnte sich ethnische Identität als wichtiger Grund für politische Mobilisierung etablieren (Selverston 1992: 1). Ethnische Identität wird damit zu einer politischen Ressource, die von sozialen Bewegungen mobilisiert werden kann (Selverston 1992: 2). So lässt sich das Aufkommen des Begriffes der indigenen Nationalitäten auch daraus erklären, dass die CONAIE und ihre Mitgliedsorganisationen „fähig waren, mit diesem Diskurs zu mobilisieren“ (Becker 2008: 173).

Eine wichtige Rolle bei der Mobilisierung von Identitäten spielt die Namensgebung der verschiedenen Völker. Dabei geht es nicht nur darum, einen Namen zu finden, der zur Suche nach Anerkennung beiträgt, sondern auch um die Beziehung, die das jeweilige Volk zum Staat, in dem es lebt, haben soll (Jenson 1995: 107). Die Namensgebung der jeweiligen Gemeinschaft bezieht sich dabei notwendigerweise 
auf ihre Vergangenheit, die zu diesem Zweck neu imaginiert werden muss. Diese Vergangenheit ist eine der wichtigsten Begründungen für aktuelle Forderungen an den Staat und die Gesellschaft (Jenson 1995: 107f.). Der Aufbau einer kollektiven Identität über die Konstruktion einer Vergangenheit und eine daraus abgeleitete Namensgebung ist ein politischer Prozess, mit dem in bestimmten Situationen Druck ausgeübt werden kann. Die Repräsentation ,,einer Gemeinschaft durch einen Namen hat reale, materielle Konsequenzen; es ist nicht nur ein Kampf um Wörter“ (Jenson 1995: 108). Die Namenspolitik ist Bestandteil eines diskursiven Wandels, der - etwa im Fall Kanadas, den Jenson untersucht - zur Verdrängung von Forderungen nach einer poli-ethnischen Politik durch nationalistische Forderungen nach neuen Rechten und Einflussmöglichkeiten für bestimmte Gruppen geführt hat (Jenson 1995: 108). In diesem Sinne haben die Indigenen Kanadas mit einem nationalistischen Diskurs und einem antikolonialen Selbstverständnis die Unterstützung verschiedener Völker in verschiedenen Gegenden Kanadas mobilisieren können (Jenson 1995: 112). Dazu bedienen sie sich einem Diskursstil, der die indigenen Völker als gleichberechtigte Nationen begreift, die mit der Nation Kanada verhandeln können - ein Stil, den es bereits im 19. Jahrhundert gegeben hatte (Jenson 1995: 113). Dieses neue, antikolonial geprägte (Jenson 1995: 112), Selbstverständnis führte zu Forderungen nach Autonomie, die sich auf das internationale Recht beriefen, und eine Neuordnung des Staates und eigenständige Institutionen der Indigenen beinhielten (Jenson 1995: 113).

Die Namenswahl für eine bestimmte Gruppe hat vier strategische Aspekte:

„Erstens, ein Name erzeugt strategische Ressourcen. Grenzen um eine Gemeinschaft zu ziehen macht die Ressourcen dieser Gemeinschaft für die Bewegung verfügbar und schafft die notwendige Solidarität für erfolgreiches Handeln. Zweitens, einen Namen über einen anderen auszuwählen setzt diskursive Grenzen, so dass manche Forderungen sinnvoll werden und andere weniger relevant sind. Drittens, jede Definition der eigenen Gemeinschaft verortet sie in Bezug zu anderen. Daher ergibt sie Möglichkeiten für Allianzen und für die Identifizierung von Gegnern. Wahrscheinliche Konflikte und Kooperationsmuster folgen den Grenzen, die die Gemeinschaft abgrenzt. Viertens, jeder Name hat Konsequenzen für das Führen von Forderungen durch staatliche Institutionen. Wege zur Repräsentation werden in Übereinstimmung mit dem ausgewählten Namen verfügbar.“(Jenson 1995: 116)

Namenspolitik hat die Indigenenorganisationen Ecuadors seit ihrem Entstehen begleitet. So ist sowohl die Namensgebung der Konferenz der Cabecillas Indígenas 1934, als auch die der 1944 daraus entstehenden FEI (Ecuadorianischen IndioFöderation) als Versuch der Erzeugung oder Verstärkung einer kollektiven Identität als Indigene bzw. Indios - also nicht nur als Bauern oder Landarbeiter - zu verstehen. Gleichzeitig ist mit der Anspielung auf den kolonialen Marker ,Indio` hier 
schon das antikoloniale Element zu finden, das Jenson (1995: 112) bei den kanadischen Indigenen in den 1970ern zu entdecken glaubt. Es sei an das Motto erinnert, das in den 1970ern in Ecuador wieder geläufig wurde: ,mit dem Wort indio wurden wir erobert und mit dem Wort indio werden wir uns befreien" (Yumbay 2008: 234).

Ein zweites Moment der strategischen Namenspolitik ist die systematische Bekämpfung von oft diskriminierenden Fremdbezeichnungen. Ein gutes Beispiel dafür sind die Organisationen der Shuar, die 1961 noch unter dem Namen ,Lokale Assoziation der Jíbaro-Zentren" (Asociación Local de Centros Jíbaros), also unter der Fremdbezeichnung ,Jíbaro' gegründet, schon 1962 aber als als ,Shuar-Assoziation Sucuas" (Asociación de Centros Shuar de Sucúa), also unter der Selbstbezeichnung der Shuar, rechtlich anerkannt wurden (Federación de Centros Shuar 1976: 115). Auch spätere Organisationsgründungen behielten die Selbstbezeichnung bei, etwa die auch heute noch größte Organisation Indigene Föderation der Shuar-Zentren (Federación Interprovincial de Centros Shuar, FICSH) (León 1991: 389).

Ein drittes Moment der Namensgebung ist das Wiederaufkommen der Bezeichnung ,indigene Nationalitäten ' in den Namen der Organisationen ab 1980. Dieser Begriff ist ein „Organisationswerkzeug“ (Becker 2008: 173), das von den 1930er bis zu den 1950ern verwendet wurde, dann aber bis in die 1970er an Bedeutung verlor. Die ersten Organisationen, die sich schon in ihrem Namen auf indigene Nationalitäten beriefen wurden im selben Jahr gegründet: die Konföderation der indigenen Nationalitäten des ecuadorianischen Amazonasgebietes (Confederación de Nacionalidades Indígenas de la Amazonía Ecuatoriana, CONFENIAE) und der Koordinationsrat der indigenen Nationalitäten Ecuadors (Consejo de Coordinación de las Nacionalidades Indígenas del Ecuador, CONACNIE) (CONAIE 1989: 50).

\section{Die Indigenenbewegung Ecuadors als soziale Bewegung}

Die Indigenenbewegung in Ecuador besteht aus einer Reihe von fest strukturierten Organisationen, deren Konkurrenz und Kooperation die Entwicklung der gesamten Bewegung und ihre Beziehungen zu anderen sozialen Bewegungen beeinflussen. Die zentralen Faktoren in der Beziehung zwischen den verschiedenen Organisationen der Bewegung sind ihre Aktionsformen und ihre Diskurse, wobei bis in die 1970er die Aktionsformen als Unterscheidungsmerkmal wichtiger zu sein scheinen und seit den 1980ern die Diskurse. Die zunehmende Distanzierung der Organisationen von externen Akteuren, wie Parteien oder Gewerkschaften, und die wachsende strukturelle Bedeutung ihrer lokaler Organisationen können Gründe für diesen Wandel sein. So wird ein konsensorientierter Politikstil befördert, der die Umsetzung lokaler Forderungen über Verhandlungen und Kompromisse beinhaltet.

Neben dem konsequenten Aufbau von unten nach oben im Rahmen fester, föderaler Strukturen, unterscheiden sich die Organisationen der ecuadorianischen Indi- 
genenbewegung von den Organisationen und Bewegungen, die die Theorien sozialer Bewegungen inspiriert haben, vor allem durch ihre Mehrdimensionalität. Tatsächlich sind sie nicht nur soziale Bewegungen, sondern übernehmen auch gewerkschaftliche und genossenschaftliche Funktionen, agieren als parteipolitische Akteure und sind in staatlichen und quasi-staatlichen Institutionen der Bildung, der Erziehung und der Entwicklung tätig. Diese Mehrdimensionalität führt zu einer auch institutionellen Verankerung der Organisationen vor Ort, die wiederum zur Entwicklung eines eigenständigen und für die lokale Bevölkerung relevanten Diskurses beiträgt und gleichzeitig Grundlage für Mobilisierung ist.

Alle Indigenenorganisationen haben die ethnische Identität der Indigenen in ihr Denken und Handeln integriert, aber in ihren spezifischen Ideologien als Landproletariat, Bauern oder Völker und Nationalitäten je anders definiert. Daher ist es wenig verwunderlich, dass erst die von Gewerkschaften weitgehend unabhängigen Organisationen des Amazonasgebietes und ECUARUNARI im Andenhochland einen eigenständigen Diskurs entwickeln konnten, in dem das Identitäre nicht eine Funktion des Klassenkampfes oder anderer, externer Faktoren ist, sondern ein Mobilisierungsgrund, der für sich spricht. Erst in diesem Zusammenhang konnten verschiedene Begriffe entwickelt und etabliert werden, die den Schwerpunkt auf verschiedene Bereiche der ethnischen Identität legen und so als Instrumente der Mobilisierung und Unterscheidungsmerkmale zwischen den Organisationen dienen. Damit prägte sich ein eindeutiger und distinktiver Diskurs der Indigenenbewegung heraus, der so nur von der Bewegung selbst vertreten wird, ihr also als Alleinstellungsmerkmal dient. 



\section{Begriffe der Indigenenbewegung}

Die Indigenenbewegung in Ecuador hat neben ihrem Kampf um konkrete Rechte auch immer einen Kampf um das gesellschaftliche Verständnis ihrer Forderungen geführt. „Die indigene Bewegung schuf begriffliche Instrumente und wir haben die Kohärenz unserer theoretischen Forderungen mit der alltäglichen Praxis diskutiert.“ (CONAIE 1989: 279) Damit bezieht sich die CONAIE auf eine „klare Ideologie, die auf unserem eigenen historisch-kulturellen Handeln gründet“" (CONAIE 1994: 5; CONAIE 1997: 7) ${ }^{1}$. So dienen die Begriffe der Indigenenbewegung einem politischen und einem epistemologischen Kampf (Macas 2005: 40). In den Worten von ECUARUNARI und CONAIE:

,wir kämpfen nicht mehr für etwas Punktuelles, sondern wir handeln, um eine andere Gesellschaft aufzubauen, eine andere Welt, in der wir Respekt vor uns selbst, vor unserer Kultur haben [...]. Das heißt, mit den Organisationen sind wir uns bewusst was wir wollen, warum wir so handeln, warum wir ein Ideal umsetzen müssen.“ (ECUARUNARI/CONAIE 1989: 39)

Die Begriffe des Territoriums, der Nationalität und der Plurinationalität wurden spätestens 1988 und 1989 von der CONAIE als konkrete Forderungen ausformuliert (CONAIE 1989: 279). Auch die Interkulturalität wurde - noch als weniger weitreichende Plurikulturalität - in diesen Forderungskatalog integriert, nicht hingegen das sumak kawsay oder Gute Leben. Von Beginn an waren die Forderungen der CONAIE nicht als rein indigene Forderungen formuliert, sondern als Möglichkeiten zur Veränderung des Staates für alle. 1992 schrieb die CONAIE:

1 Im Politischen Projekt von 2001 ist die Rede von einer ,klaren Weltanschauung und Philosophie der Nationalitäten, die auf unserem eigenen historisch-kulturellen Handeln gründet“" (CONAIE 2001: 1). 
„Die Indigenenbewegung hat die folgenden Begriffe in die Diskussion gebracht: Territorialität, Plurinationalität, Nationalität, Identität. Diese Themen müssen auf nationalem Niveau diskutiert werden, da sie jeden betreffen können. All das bedeutet Modernisierung.“ (CONAIE 1992a)

Über diese Begriffe will die CONAIE den „,bürgerlichen uninationalen Staat“ (CONAIE 1994: 6; CONAIE 1997: 8) ${ }^{2}$ und seine Konzeption der Indigenen als ,,pädagogisches, kirchliches oder Verwaltungsproblem“ (CONAIE 1994: 5; CONAIE 1997: 7; CONAIE 2001: 2) in Frage stellen und unter Einschluss aller Schichten eine demokratische und inklusive Alternative aufbauen (CONAIE 1994: 6). Dazu strebt die CONAIE keine Machtübernahme an, sondern ,die Transformation der Natur der aktuellen Macht des uninationalen hegemonialen Staates“ (CONAIE 1994: 7; CONAIE 1997: 9) über die die politischen und wirtschaftlichen Rechte wiederhergestellt werden sollen (CONAIE 1994: 7). Dass dieser Kampf als dekolonial oder antiimperialistisch verstanden werden kann (CONAIE 1994: 1), wird im nächsten Teil dieser Arbeit diskutiert.

Die Alternative, die die CONAIE anbietet, ist der Aufbau eines plurinationalen Staates und einer interkulturellen Gesellschaft, die einen Wandel in den Machtbeziehungen, eine Umverteilung des Vermögens und Einkommens und eine Bekämpfung der Armut beinhalten (Tibán/García 2006: 21). Diese Alternative baut auf eine Integration der Ausgeschlossenen, soll also über breite Koalitionen der Indigenen mit Arbeitern, Bauern und anderen Marginalisierten realisiert werden. So erklärt sich auch die Strategie des Aufbaus eines plurinationalen Staates von unten, die, von den lokalen Strukturen ausgehend, eine schrittweise Ausweitung des Einflusses der sozialen Bewegungen und der Indigenenbewegung vorsieht (Rodríguez/Martínez 2000: 74). Die Integration des Anderen in ein alternatives Modell zum unikulturellen Staat soll so nicht nur die Politik, sondern auch die Kultur Ecuadors öffnen und erneuern (Almeida 1997: 174). Abgesehen vom Ideal eines neuen Staates dienen die unter Rückgriff auf eine indigene Vergangenheit gebildeten Konzepte vor allem dem Kampf gegen wirtschaftliche und ethnische Ungleichheit und Diskriminierung,

„solche Bezüge bilden wichtige Reservoirs von Wissen oder ,historische Ansammlungen“ die in die aktuellen Strategien als Bezüge für die Reorganisation der aktuellen Gesellschaft passen“ (Almeida 1997: 177) ${ }^{3}$.

22001 außerdem als ,weiß-mestizisch“ (CONAIE 2001: 2) beschrieben.

3 Damit baut Almeida auf den Begriff der „ethnischen Ansammlungen“ (Sánchez-Parga 1988: 107) auf, mit dem Sánchez-Parga die beständige Neulektüre und Reinterpretation der indigenen Traditionen und Geschichte durch diese selbst beschreibt. 
Die Verfassungsänderung von 1996 und die Verfassung von 1998 erklären Ecuador zu einem plurikulturellen und multiethnischen Staat und erkennen die kollektiven Rechte der Indigenen teilweise an. Die Verfassung von 2008 erklärt Ecuador zu einem interkulturellen und plurinationalen Staat, wobei sie - im Gegensatz zu ihrer Vorgängerin - diese Begriffe auch in speziellen Rechten für die indigenen Nationalitäten und der Anerkennung indigener Rechts- und Wirtschaftssysteme umsetzt (Larrea 2008: 38). Dafür hatte die CONAIE seit spätestens 1990 gekämpft (CONAIE 1994: 17). Auch wenn dieser Fortschritt das Ergebnis des Kampfes der indigenen Organisationen ist, fand er nicht allein unter ihrer Führung statt. Die CONAIE, als wichtigste und größte Organisation der Indigenenbewegung, war seit ihrer Beteiligung an der Regierung von Lucio Gutiérrez 2003 in einer tiefen Krise, die andere Indigenenorganisationen und soziale Bewegungen für sich ausnutzten. Als 2006 die heutige Regierungspartei Alianza PAÍS gegründet wurde, konnte sie die Indigenenbewegung als anti-neoliberale Kraft teilweise verdrängen. Unter der Regierung Rafael Correas (seit 2007) wurden eine Reihe der indigenen Forderungen umgesetzt, allerdings aus der Position des Staates heraus und nicht der der jeweiligen Organisationen. Plurinationalität und Interkulturalität wurden nicht nur in die Verfassung, sondern auch in die konkrete Regierungspolitik integriert - aber nicht als Freiräume für die indigenen Organisationen, sondern als offizielle Politik. Daher sollen sie nicht die Grundlage einer indigenen Emanzipation sein, sondern die einer Neugründung der ,,nationalen Identität nach der , bürgerlichen Revolution “" (Kaltmeier 2010: 5f.). Correa steht für eine staatliche Aneignung der institutionellen Räume, die vorher den indigenen Organisationen gehörten. Dazu kommt:

„Es ist sehr zweifelhaft, dass Teile der mestizisch-weißen Elite, so wie sie in der Regierung Correa sind, für eine Interkulturalität kämpfen, die ihre eigenen Positionen der materiellen und symbolischen Macht in Frage stellt.“ (Kaltmeier 2010: 6)

Diese Situation lässt sich am besten an der Einschränkung der Souveränität der Nationalen Direktion der Zweisprachigen Interkulturellen Erziehung (Dirección Nacional de Educación Intercultural Bilingüe, DINEIB) verdeutlichen, die unter der fast alleinigen Kontrolle der indigenen Organisationen stand, von Correa aber rückverstaatlicht wurde (Kaltmeier 2010: 6).

Die Geschichte der Begriffe der Indigenenbewegung fällt zusammen mit dem Entstehen und der Entwicklung der CONAIE als erster nationaler Indigenenorganisation, die amazonische und andine Organisationen verbinden konnte. Insbesondere die Organisationen des Amazonasgebietes, die CONFENIAE und ihre Mitglieder, vor allem die FICSH, waren in der Lage, durch ihre marginale Position in der ecuadorianischen Gesellschaft, ein ethnisches - und kein soziales, wie die meisten andinen Organisationen - Selbstverständnis zu entwickeln. Daher entsprach ihr Denken 
eher einem Versuch, die Selbstständigkeit der indigenen Völker zu erhalten oder wiederzuerlangen, als gesamtgesellschaftlichen Forderungen nach Gleichheit und Gerechtigkeit (Korovkin 2008: 3). In diesem Kontext eignete die Indigenenbewegung sich die heute verwendeten Begriffe an, deren Grundlage die Ideen von indigenen Nationalitäten und dazugehörigen Territorien ist (Ruiz 1991: 476). Der enge Kontakt zu den Organisationen des Hochlandes erlaubte eine Migration der Begriffe und ihre Weiterentwicklung innerhalb der CONAIE (Guerrero/Ospina 2003). Dieser komplexe Zusammenhang soll bei der Untersuchung der Geschichte und Bedeutung der einzelnen Begriffe genauer analysiert werden.

Das Entstehen der CONAIE wurde von einem wachsenden Bewusstsein der Indigenen begleitet, die sich durch die Bestärkung und Aufwertung ihrer Besonderheiten und Unterschiede als relativ homogene Gruppe neu konstituieren konnten. Gerade der Bezug auf eine idealisierte Vergangenheit erlaubte es ihnen, die Idee einer neuen, kollektiven Gesellschaft als Alternative zur Bestehenden zu präsentieren und als Referenz für die verschiedenen Indigenenorganisationen und die Mehrheitsgesellschaft als Ganzes zu positionieren (León Trujillo 1983: 8). Die Indigenen als ethnische Minderheit begreifen sich neu als Nationalitäten, die sich durch ein „historisches Projekt“ (León Trujillo 1983: 8) auszeichnen und auf dieser Basis einen anderen Umgang mit Staat und Gesellschaft einfordern. Als Nationalitäten verlangen die Indigenen nun mehr als Rechte für ,einfache Bürger, ohne Sprache, ohne Bräuche, ohne Territorium“ (ECUARUNARI 1984: 46).

„Der in einen politischen Akteur verwandelte Indio verlangt keine ,Integration“. Zusammen mit Land und wirtschaftlichem Wohlstand fordert er seine Identität als Indio und eine Bürgerschaft mit Recht auf die Differenz. Der Indigene will nicht nur das Ende der Ausbeutung, sondern auch das Ende der Unterdrückung.“ (León Trujillo 1983: 10)

Der Beginn einer nationalen Organisation und eines landesweiten Bewusstseins der Indigenen führte $\mathrm{ab}$ den 1970ern $\mathrm{zu}$ einer großen Produktion programmatischer Texte oder Diskurse, die auf dem Indigenismus der vorherigen Jahre und den damals aktuellen sozialistischen Ideen aufbaute, sie aber zugleich überwinden wollte. Dabei spielte insbesondere die Beziehung zwischen Staat oder Gesellschaft und den Indigenen und ihrer Bewegung eine wichtige Rolle. Das Entstehen nationaler Indigenenorganisationen, das ethnische Bewusstwerden der Indigenen und ihr Selbstverständnis als Völker und Nationalitäten in einem eigenständigen Diskurs trugen so dazu bei, die „Indigenenbewegung zu nationalisieren“ (Sánchez-Parga 1993: 177), sie also von einer Reihe lokaler und regionaler Bewegungen zu einer nationalen Bewegung zu machen. Die eher kulturalistischen Ideen der 1970er, die eine Anerkennung der indigenen Sprachen und Traditionen forderten, entwickelten sich vor allem in den breit angelegten Alphabetisierungskampagnen und dem all- 
gemeinen Bildungsausbau der frühen 1980er Jahre weiter (Sánchez-Parga 1993: 178f.). Dadurch, dass viele dieser Programme in Kooperation mit den Indigenenorganisationen durchgeführt wurden, stärkten sie die Identität der Indigenen und die Repräsentativität der Indigenenorganisationen, die in diesem Kontext versuchten, alternative Lösungen $\mathrm{zu}$ finden und um die Kontrolle der staatlichen Programme kämpften (Chiriboga 1983: 124).

Die Auseinandersetzung der Indigenenorganisationen mit ethnischer bzw. kultureller Vielfalt konnte an die nationalistische und integrationistische Ideologie der Militärdiktatur der 1970er anknüpfen und so zu einer Beschreibung Ecuadors als plurikulturelles und multiethnisches Land kommen. Über das Verständnis der Indigenen als Nationalitäten (Sánchez-Parga 1993: 179), kamen die Organisationen in den 1980ern zu der Forderung nach Selbstbestimmung innerhalb eines neuen pluralistischen ecuadorianischen Staates, die sich auf die politische Einheit der Indigenen und ihre wirtschaftliche Selbstverwaltung stützen sollte (Chiriboga 1987: 101). Der Kampf der Indigenen für eine gerechte Gesellschaft ohne Rassismus und Ausgrenzung (Viteri 1983: 45) führte in den 1980ern auch zu einem größeren Bewusstsein innerhalb der Gesamtgesellschaft für die indigenen Forderungen (Comisión Especial de Asuntos Indígenas 1988: 3).

Es sei darauf hingewiesen, dass sich die Begriffe der Indigenenbewegung in ständiger Veränderung befinden und immer auch politische oder wirtschaftliche Einflüsse von außerhalb aufgreifen und widerspiegeln. Die neuere Geschichte der Indigenenbewegung und ihrer Begriffe fällt zusammen mit der Politik der wirtschaftlichen Anpassung, die ab Anfang der 1980er in Lateinamerika herrschte und zur Verarmung großer Teile der Bevölkerung führte. In diesem Kontext ließen sich die Forderungen nach Gerechtigkeit für alle leicht mit den Forderungen für Gerechtigkeit für die Indigenen kombinieren (García Serrano 2001: 76).

\section{VON LAND ZU TERRITORIALITÄT}

Die Indigenenbewegung in Ecuador war immer auch eine Bauernbewegung. Daher war die Forderung nach einer gerechten Verteilung des Ackerlandes von Beginn an ein zentraler Bestandteil der Programme ihrer Organisationen, etwa der ersten Indigenenorganisation Conferencia de Cabecillas Indígenas (1935). Die gewerkschaftlichen Indigenenorganisationen FEI und FENOC teilten die Politik ihrer Muttergewerkschaften, für eine Landreform zu kämpfen, eine Politik, die sich erst mit der Krise dieser beiden Organisationen und der gesamten Gewerkschaftsbewegung in den 1980ern abschwächte.

Das Aufkommen ethnisch orientierter Organisationen in den 1970ern und ihre weitgehende Dominanz ab den 1980ern führte zu einer Bedeutungsverschiebung in 
diesem Kampf. Die Bedeutung des Landes für die Indigenen in der „Bewahrung ihrer ethnisch-kulturellen Identität“ (Andrango 1983: 50) wurde prominenter. Das Land gewinnt einen doppelten Sinn: als Mittel zum Überleben der Bauern und als kultureller Raum, in dem sich die verschiedenen Völker entwickelt haben. Die 1970er Jahre sind von Auseinandersetzungen zwischen den gewerkschaftlichen und den ethnischen Indigenenorganisationen geprägt, deren gemeinsame Forderung nach einer Landreform auf zwei widersprüchliche Weisen interpretiert wird. FEI, FENOC und zu Beginn ECUARUNARI (Ibarra Illanez 1987: 159/162) verstehen den monopolistischen Landbesitz und die kapitalistischen Produktionsbeziehungen als größtes Problem der Indigenen und fordern daher eine ,demokratische Agrarreform“ (Ibarra Illanez 1987: 117), also die Verteilung des Landes an die Armen. Die ethnisch ausgerichteten Organisationen vor allem des Amazonasgebietes verstehen das Land auch als kulturell definiertes Territorium und fordern daher eine ,integrale Agrarreform“ (Ibarra Illanez 1987: 119), die die Schaffung und Verteidigung von selbstverwalteten indigenen Territorien beinhaltet (Fontaine 2001: 61f.).

So sehen die ethnischen Organisationen das Problem der Indigenen nicht länger nur als Ergebnis kapitalistischer Ausbeutung - wie es die klassistischen Organisationen weiterhin tun -, sondern als Folge der Koexistenz sich entgegenstehender Völker und damit als ethnische Unterdrückung. Daraus leitet eine Strömung die wirtschaftliche Umformung der gesamten Gesellschaft, eine andere die Integration der kulturellen und wirtschaftlichen Andersheit der Indigenen in diese Gesellschaft ab (Ibarra Illanez 1987: 119) - die nicht unbedingt auf die gesamte Gesellschaft ausstrahlen muss (Ibarra Illanez 1987: 159/162). Diese Suche nach einer Gleichberechtigung der Indigenen im Rahmen eines pluralen Staates (Ibarra Illanez 1987: 123f.) auch unter Vernachlässigung der anderen Marginalisierten, hat zu einer Reihe von Anklagen linker Intellektueller geführt. Die ethnisch ausgerichteten Indigenenorganisationen verstehen den Staat als eine neutrale Instanz, die die Indigenen in ein System regionaler Autonomien integrieren kann - ohne dass das etwas mit einem Klassenkonflikt zu tun hätte (Ibarra Illanez 1987: 129). Damit werden Allianzen mit nicht-indigenen Armen und Ausgeschlossenen erschwert und so die Mobilisierungsfähigkeit dieser Bewegungen reduziert.

Dieser Wandel hin zu einer ethnisch fundierten Forderung nach Territorien ist in den 1970er Jahren bei den meisten Indigenenbewegungen Lateinamerikas zu beobachten. Ein wichtiger Faktor ist die Forderung nach einer staatlichen Anerkennung der traditionellen Territorien als gemeinschaftlicher Besitz der jeweiligen Gemeinden im Sinne eines Kommunitarismus, der nicht nur wirtschaftlich, sondern auch kulturell, politisch, rechtlich usw. ist. So sollen die Territorien von den jeweiligen Gemeinden nach ihren eigenen Regeln selbst verwaltet werden - wodurch der gemeinschaftliche Besitz an Land zu einer Alternative zum individuellen kapitalistischen Besitz wird (Barre 1982: 63/74). 
Schon auf dem Nationalen Indigenen- und Bauernmarsch, den ECUARUNARI und FENOC im Oktober 1980 durchführten, wurde der Respekt des Staates vor den Territorien und der Kultur der indigenen Nationalitäten gefordert (ECUARUNARI 1998: 99). So wird die Landfrage zu einem zentralen Problem der indigenen Kämpfe, sowohl für die ethnischen, als auch für die klassistischen Strömungen (Ramón 1983: 101). Das Land wird als „materielle Grundlage“ (Conterón/de Viteri 1984: 17) der indigenen Völker und ihrer Kultur verstanden. ECUARUNARI erklärt in einem Text Anfang der 1980er ,das Land [zur] Grundlage für die Entwicklung unserer Nationalität, unserer Kultur, unserer Sprache“ (ECUARUNARI 1984: 44f.) Auf dem ersten Bauern- und Indigenentreffen (Encuentro Campesino e Indígena) im April 1982 wird das Recht auf Land als Grundlage für die Entwicklung der Nationalität, Kultur und Sprache der Indigenen definiert und als zentrales Element des Kampfes gegen den Imperialismus und für eine wirkliche Demokratie (Lucha Campesina 1982, in: Becker 2011a: 199). Die FENOC schreibt 1984:

„,der Kampf der indigenen Bauernbewegung hat sich im grundsätzlichen Prinzip des Rechts auf das Land als Hauptgrundlage für die Entwicklung der Nationalität, unserer Kultur, unserer Sprache, des Rechts auf die Organisation ausgedrückt“ (FENOC [1984], in: Ibarra Illanez 1987: 143).

Daher steht das Land lange Zeit im Zentrum der Konflikte zwischen dem Staat und der Indigenenbewegung (Chiriboga 1983: 124) - nicht nur als Forderung, sondern in Form von Vertreibungen oder Verkauf - auch als Mittel der Repression gegen die Indigenen (Barre 1982: 62). Vor allem die Organisationen des Amazonasgebietes entwickelten wegen ihrer besonderen Geschichte (Korovkin 2008: 3) Konzepte, die statt auf sozialen Wandel - wie bei vielen Organisationen des Hochlandes - auf Autonomie ausgerichtet waren (Ruiz 1991: 476). Schon auf ihrem ersten Kongress stellt die CONFENIAE eine Reihe von Forderungen auf, die sie bis heute beibehalten sollte (Ibarra Illanez 1987: 150). Für sie war die Forderung nach Land mit der Forderung nach kommunalen oder gemeinschaftlichen Besitzformen verbunden (Viteri 1983: 46). Ihr Kampf für Land wurde so seit den 1970ern zum Kampf für die „Eroberung einer ethnischen Territorialität“ (Sánchez-Parga 1988: 111), der direkt mit dem Aufbau eines neuen, explizit ethnisch ausgerichteten Projektes dieser Organisationen verbunden ist (Sánchez-Parga 1988: 111). Die Föderation der Kommunen Union der Eingeborenen des Ecuadorianischen Amazonasgebietes (FCUNAE), die weitgehend aus Kichwa besteht, entwickelte in einem Text von 1986 eine Definition von Territorialität als Grundlage für die Indigenen und die Indigenenbewegung. Die Territorialität schafft - neben Sprache, Geschichte, Gebräuchen - die Einheit und damit die Stärke des indigenen Volkes (UNAE o.J.: 18). Daher sind die Kämpfe der Indigenen und ihrer Bewegung ,,Verteidigungskämpfe 
des Territoriums, des Überlebens der Gruppe“ (UNAE o.J.: 22). Das Land gewinnt so eine weitere Bedeutung:

„Das Land bedeutet für den Eingeborenen nicht das Gebiet, das das Gesetz ihm zuschreiben kann oder das Familienerbe. [...] Das Land meint einen bestimmten Ort, wo er lebt und wo er den Sinn seiner Existenz findet. Sein Begriff von Land ist der eines Territoriums, eines umfassenden Vaterlandes, in dem er sich frei in Beziehung mit den anderen Mitgliedern der Gruppe bewegen kann.“ (UNAE o.J.: 34)

Die Organisationen des Amazonasgebietes verlangten die rechtliche Anerkennung der Territorien, die schon ihren Vorvätern gehörten, während die HochlandOrganisationen mehr an der Lösung ihrer Konflikte um Landbesitz - meist zwischen Großgrundbesitzern und indigenen Gemeinden - interessiert waren (Pacari 2007: 24). So setzte sich ECUARUNARI mehrfach für die Anerkennung der Territorien der Nationalitäten im Küsten- und Amazonasgebiet ein (ECUARUNARI 1984: 48) - aber nicht für ähnliche Forderungen in ihrem Stammland. Die FOIN der Kichwa des Amazonasgebietes versuchte ab 1987, Fragen des Landbesitzes mit denen der nachhaltigen Landnutzung zu verbinden (Macdonald 1998: 94). Auch wenn weiterhin eine gerechte Landverteilung verlangt wird, wie die Manifeste des Aufstands 1990 oder die aktuelle Forderung nach einer ,Agrarreform durch eine Agrarrevolution“ (Cholango 2011: 62) zeigen, ging es jetzt um mehr. „Der Zugang $\mathrm{zu}$ Land ist die Grundlage für die Schaffung einer multiethnischen, plurikulturellen Gesellschaft“ (Moreno/Figueroa 1992: 23f.). Der Kampf für Land und eine Agrarreform wurde um einen Kampf für die Anerkennung indigener Territorien ergänzt (Rodríguez 2008: 100). Damit verbunden ist das Recht der indigenen Nationalitäten, innerhalb ihrer Territorien über ihr Schicksal selbstbestimmt zu entscheiden (ECUARUNARI/CONAIE 1989: 42). Im Begriff des Territoriums wird das Land als gleichzeitig wirtschaftliches und kulturelles Problem gesehen und somit als Grundlage für die „kulturelle Reproduktion“ (Fontaine 2001: 61f.) der indigenen Völker. Die Territorialität der indigenen Nationalitäten ergänzte also die Forderung nach einer gerechten Landverteilung (ECUARUNARI/CONAIE 1989: 39). Diese Forderung ist allerdings nach wie vor zentral für das politische Handeln der Indigenenorganisationen: „Wir glauben, dass es keine Lösung des Indigenenproblems geben wird, wenn man nicht das Landproblem löst, das Grundlegende für uns ist das Wiedererlangen des Landes.“ (Macas 1991: 10)

Der Wandel von einer wirtschaftlichen Forderung nach Land hin zu einer auch kulturellen und sozialen Forderung nach selbstverwalteten Territorien hat gegen Mitte der 1980er Jahre die Indigenenorganisationen ganz Ecuadors erfasst und wurde zum Grundstein für die CONAIE und ihre Mitgliedsorganisationen (CONAIE 1989: 279; Santana 1992: 194). Zu dieser Zeit hat innerhalb der Indigenen- 
bewegung der Wandel von den Forderungen nach Land im Sinne einer den indigenen Interessen angepassten Landreform hin zu der Forderung nach der Anerkennung der Territorien der verschiedenen indigenen Völker und Nationalitäten stattgefunden. Ein Text des CONACNIE von 1984 fordert Land für die Indigenen als Grundlage für das Fortbestehen und die Entwicklung der Kultur - auch wenn die kulturellen Aspekte bereits eine wichtige Rolle spielen, wird der Begriff Territorium noch nicht verwandt (Conterón/de Viteri 1984: 5). Die bedeutende Indigenenführerin Nina Pacari fordert in einem Text aus demselben Jahr, dass jeder indigenen Nationalität der Besitz ihres Territoriums anerkannt und garantiert werden soll. Das Territorium soll kollektiv, unveräußerlich und ausreichend groß sein, um das Wachstum und die Entwicklung der Nationalität abzusichern (Pacari 1984: 122). Im Entwurf für ein Gesetz der Nationalitäten von 1988 wird das Land der Indigenen als ,unteilbar, unpfändbar und unverletzbar, unter Bewahrung ihrer kommunitären Logik“ (Comisión Especial de Asuntos Indígenas 1988: 12) definiert. Den indigenen Nationalitäten wird das Recht auf ihr Territorium eingeräumt, das nicht nur ihren Fortbestand, sondern auch die Weiterentwicklung ihrer Kultur und den Schutz der Naturschätze des Landes garantieren soll (Comisión Especial de Asuntos Indígenas 1988: 21). Zur genaueren Klärung der Situation sollte dem Staat die Schaffung eines Territorialitätsstatuts aufgetragen werden, in dem die jeweiligen Grenzen für die ausschließliche Nutzung des Landes und der Naturschätze festgelegt werden sollten (Comisión Especial de Asuntos Indígenas 1988: 12) ${ }^{4}$. Da der Gesetzesvorschlag nicht behandelt wurde, kam es auch nie zu diesem Statut. Eine wichtige Rolle in der Verbreitung der Forderung nach territorialer Autonomie - verbunden mit einer Rückbesinnung auf ein Leben in traditionellen Gemeinschaften - spielte die 1984 gegründete Koordination der indigenen Organisationen des Amazonasgebietes (Coordinadora de Organizaciones Indígenas de la Cuenca Amazónica, COICA), die die amazonischen Organisationen von Kolumbien, Venezuela, Ecuador, Peru, Brasilien und Bolivien umfasst. Auf den Kongressen der COICA wurden kommunitäre Alternativen zu den bestehenden Staaten, die den Indigenen kaum Interesse entgegenbrachten, diskutiert. In den Texten der Kommunistischen Internationale in den 1920er und 1930er Jahren fanden die Indigenen den Vorschlag, die Region über autonome, selbstverwaltete Territorien neu zu gliedern (Quijano 2006a: 33) - der andere Begriff aus diesem Kontext, indigene Nationalitäten, wird im nächsten Kapitel behandelt.

Die Indigenenorganisationen des Amazonasgebietes, allen voran die OPIP, entwickelten so eine weitgehende Definition von Territorialität, die sie der nationalen Öffentlichkeit präsentieren konnten. Dies geschah zum ersten Mal mit dem Vertrag

4 Damit ist der Vorwurf von Santana widerlegt, der Begriff des Territoriums würde bis in die 1990er nicht genauer definiert (Santana 1992: 194). 
von Sarayacu von Juli 1990, in dem die Indigenen Pastazas eine rechtliche Anerkennung der Territorien der Kichwa, Achuar und Swiviar dieser Provinz erreichen wollten. Die Territorien sollten nicht nur das Land, sondern auch den Luftraum und die Bodenschätze des jeweiligen Gebietes umfassen, und unter weitgehender Kontrolle der Indigenen stehen. Die Autonomierechte der Indigenen sollten eine alleinige Wirksamkeit des traditionellen indigenen Rechtes im jeweiligen Territorium und ein Verbot der Ausbeutung der Rohstoffe und der Manöver der ecuadorianischen Armee dort umfassen (Ojeda Segovia 2004: 33f.). Die ersten Zugeständnisse erreichte die OPIP mit ihrem Marsch von April 1992, nach dem die ecuadorianische Regierung den jeweiligen Völkern eine eingeschränkte Kontrolle über einen Teil ihrer Territorien zusprach (Ojeda Segovia 2004: 35).

Nach der Definition der CONAIE ist das Territorium ein „,bestimmter physischgeographischer Raum, wo ein Volk lebt und sich entwickelt“ (CONAIE 1994: 20) und als solcher die Grundlage für das Überleben der indigene Nationalitäten. Ein Territorium in diesem Sinne ist ein autonomer Raum, der von selbstbestimmten Autoritäten verwaltet wird (CONAIE 1994: 20). Die Kontrolle der indigenen Territorien durch die indigenen Nationalitäten ,,hat einen harmonischen und ausgeglichenen Umgang mit den natürlichen Ressourcen erlaubt“" (CONAIE 1994: 20) und so „die integrale Entwicklung aller Leben garantiert“ (CONAIE 1994: 20). Beim Vergleich des Politischen Projektes der CONAIE in den Versionen von 1994 und 1997 fallen einige Unterschiede auf. Die Definition von 1994 versteht das Territorium als „Habitat, das die indigenen Völker und Nationalitäten besetzen und auf unterschiedliche Art und Weise nutzen“ (CONAIE 1994: 52). Drei Jahre später ist von einer Nutzung nicht mehr die Rede, stattdessen wird darauf verwiesen, dass das Territorium „,die Oberfläche der Erde und ihren Untergrund umfasst“ (CONAIE 1997: 50), ein Hinweis auf die gestiegene Bedeutung der amazonischen Indigenen innerhalb der CONAIE und ihren Kampf gegen die Ausbeutung der Bodenschätze in ihrer Region.

Der Aufbau von autonomen Territorien soll die Grundlage für die Etablierung von Strukturen der Selbstverwaltung im Bereich etwa der Erziehung, der Gesundheitsversorgung und der Verwaltung der Ressourcen sein (Cholango in Tamayo 2007: 10) - hat aber wenig mit dem manchmal unterstellten Versuch der Auflösung des Staates zu tun. Manche der großen Massenkommunikationsmedien konstruieren von der Forderung nach autonomen Territorien ausgehend das Bild der ,,separatistischen Indios“ (Rodríguez/Martínez 2000: 81), die das Vaterland auflösen wollen. Bereits in der Verfassung von 1998 war die Möglichkeit verankert, „territoriale indigene und afroecuadorianische Eingrenzungen“ (Art. 224) im Sinne staatlicher Strukturen, wie etwa der Regionalregierungen, zu schaffen. Diese Möglichkeit wurde in der Verfassung von 2008 (Art. 257) erweitert - ohne dass es bis 2013 tatsächlich anerkannte indigene Territorien gäbe. De facto gibt seit den 1990ern in 
einigen Teilen des Amazonasgebietes eine gewisse Selbstverwaltung außerhalb der staatlichen Strukturen (Büschges 2009: 49f.). Diese Entwicklung zeigt auch die Schwierigkeiten des Aufbaus von Gebieten mit territorialer Autonomie auf. Während es im Amazonasgebiet Gegenden gibt, die fast ausschließlich von den Angehörigen einer indigenen Nationalität besiedelt sind - sich also als indigenen Territorien definieren ließen -, ist die Situation im Andenhochland anders. Dort lebt der Großteil der indigenen Bevölkerung des Landes, allerdings meist in gemischt besiedelten Gebieten ohne klare Mehrheitsverhältnisse, die eine indigene oder mestizische Selbstverwaltung rechtfertigen würden (Rhon 2003: 134). Die meisten Gebiete sind von „einem starken indigen-bäuerlichen Kontinuum“ (FENOCIN 1999: 153f.) geprägt, das sich der Vorstellung von von nur einem Volk besiedelten Territorien nur schwer anpassen lässt. Auch die indigenen Völker und Nationalitäten, die über kein zusammenhängendes Territorium verfügen oder in andere Gegenden migriert sind, können sich im Begriff des Territoriums nicht wiederfinden (FENOCIN 1999: 153). Die Schlussfolgerung von Alejandra Santillana: „Die Debatten über Territorialität wurden nur für die indigene Bevölkerung formuliert“ (Santillana 2006: 250) greift also zu kurz. Diese Debatten wurden nur für einen kleinen Teil der indigenen Bevölkerung formuliert.

Eine andere Kritik am Begriff der indigenen Territorien ist seine mangelnde Klarheit und sein fehlender Inhalt (Chuji 2008). Tatsächlich hat selbst die CONAIE Schwierigkeiten, die Territorien über das bisher Erläuterte hinaus zu konkretisieren. So fordert das politische Projekt der CONAIE eine territoriale Neuordnung über ,eine Studie der historischen Entwicklung der Landnutzung und -ordnung in der Periode 1400-1900“ (CONAIE 1994: 21) und die Sammlung von „,notwendiger grundlegender Information, um eine Karte anzufertigen, die die indigenen territorialen Einheiten bestimmt und festlegt" (CONAIE 1994: 21). Auch die FENOCIN fordert ein Programm der Legalisierung der traditionellen indigenen Territorien ${ }^{5}$, das neben der Schaffung klarer Mechanismen und der Ausbildung indigener Experten die Erstellung von Landkarten der Territorien umfasst (FENOCIN 1999: 143f.). Der Staat soll die indigenen Territorien also nicht nur anerkennen, sondern sie zuerst bestimmen und anschließend institutionell integrieren (CONAIE 1994: 21f.; Chuji 2008).

5 Sie spricht nicht von Territorien, sondern von tierras ancestrales (,,von den Vorfahren überliefertes Land“) (FENOCIN 1999: 143f.). 


\section{VON BAUERN ZU INDIGENEN VÖLKERN UND NATIONALITÄTEN}

Im Laufe der 1970er Jahre fand eine Veränderung des Selbstverständnisses der organisierten Indigenen statt. Hatten sie sich bis dahin in erster Linie als Bauern definiert, verstanden sie sich nun als indigene Völker und Nationalitäten. Diese Veränderung beginnt um 1975 (Ramón 1992a: 9) und erreicht mit der Gründung von CONACNIE und CONFENIAE, die beide die Bezeichnung, indigenen Nationalitäten“ im Namen tragen, im Jahr 1980 ihren Höhepunkt (Ospina 2008: 71). Von da an konnte sich die neue Begrifflichkeit ausbreiten und an Kraft gewinnen und die bisherigen Konzepte des Klassenkampfes und des ethnischen Konfliktes überwinden (Ramón 1992a: 9). Bereits Anfang der 1980er Jahre war der Begriff der indigenen Nationalitäten als Bezeichnung für alle indigenen Gruppen von der Regierung und den meisten Organisationen angenommen worden (Viteri 1983: 46).

Der Begriff der Nationalität ist jedoch wesentlich älter ${ }^{6}$. Die wahrscheinlich erste Abgrenzung von Nation und Nationalitäten unternahm der österreichische Marxist Otto Bauer (1907) in Bezug auf den Vielvölkerstaat Österreich-Ungarn. Ein anderer möglicher Ursprung ist der russische sozialistische Zionist Ber Borochov (Sánchez-Parga 1992: 70), der in einem Text von 1905 Nationalitäten und Klassenkampf untersucht (Borochov 1905). Von da an wurde der Begriff zunehmend in den kommunistischen Diskurs integriert und stetig verändert (Almeida 2008). Lenin übernahm den Begriff der Nationalitäten (Becker 2002: 191f.) und nach ihm Stalin. So wurden in der Sowjetunion seit den 1920er und 1930er Jahren ethnische Minderheiten als Nationalitäten bezeichnet (Ayala Mora o.J.: 10f.). Über die Kommunistische Internationale kam der Begriff nach Lateinamerika, wo er schon auf der ersten Konferenz der Kommunistischen Parteien Lateinamerikas in Buenos Aires 1929 in Bezug auf die Indigenen als ,unterdrückte Nationalität“ (Correspondencia Sudamericana, August 1929, in: Grupo Internacionalista 2003) in einem Report des peruanischen Kommunisten José Carlos Mariátegui verwendet wurde. Danach wurde der Begriff der Nationalität in den 1930ern von der Kommunistischen Partei Perus (Partido Comunista del Perú, PCP) aufgegriffen, die von Quechua- und Aymara-Nationalitäten in Peru sprach (Ibarra 2010: 44). Während die peruanischen Kommunisten dem weitergehenden Vorschlag der Komintern, für die Gründung einer indigenen Republik in den Anden zu kämpfen, nicht folgen wollten, wurden diese Ideen von den ecuadorianischen Kommunisten begrüßt und die Begrifflich-

6 Der vorliegende Text widmet sich der begrifflichen Entwicklung und nicht der Interpretation indigener Aufstände bis zum 19. Jahrhundert, die von einigen Wissenschaftlern als nationalistisch in dem Sinne gesehen werden, dass sie „Indio-Nationen und unabhängige indigene Königreiche oder Staaten“ (Barrientos o.J.: 4) schaffen wollten. 
keit der Nationalitäten auch für die ecuadorianischen Indigenen übernommen. So bezeichneten Ricardo Paredes, der Vorsitzende der PCE, und der zu diesem Zweck gegründete Arbeiter-Bauern-Block, schon im Präsidentschaftswahlkampf 1933 Indigene und Schwarze nicht nur als ausgebeutete Klassen, sondern auch als unterdrückte Nationalitäten (Becker 2011a: 192). Auf Druck der Komintern wurde der Begriff der indigenen Nationalitäten weiter in Diskurs und Strategie der PCE verankert. „Indigene Nationalitäten waren grundlegend für die Art, wie die Komintern ihren Kampf in den Anden verstand." (Becker 2011a: 195) Dadurch näherten sich die urbanen Kommunisten Ecuadors an die indigene Landbevölkerung an und legten das Fundament für das heutige indigene Selbstverständnis (Becker 2008b: 2). Bereits auf der Conferencia de Cabecillas Indígenas Ende 1935 wurden die Indigenen als „indigene Nationalitäten“ (Conferencia de Cabecillas Indígenas 1935) bezeichnet und über die Nationalitätenpolitik und ,[d]as was die Sowjetunion den Minderheitsnationalitäten gegeben hat" (Conferencia de Cabecillas Indígenas 1935) diskutiert. In einem Text, der einige Monate später in der Zeitung Ñucanchic Allpa erschien, beschrieben die Organisatoren der Konferenz ihr Verständnis der Unterschiede zwischen Indigenen und den anderen Ethnien Ecuadors:

„die Indios haben Sprachen, die nur sie sprechen (Kichwa, Cayapa, Cofan, usw.), sie haben ihre eigene Kleidung und Gebräuche, sie gehören zu ihren eigenen Rassen und Nationalitäten oder Völkern, die über vierhundert Jahre lang frei gelebt haben, ohne wie heute Weißen und Mestizen unterworfen zu sein. Das ist der Grund weshalb die Indios über vier Jahrhunderte lang einer großen Unterdrückung ihres Volkes oder Nationalität unterworfen worden waren, zurückgewiesen wurden, als ob sie eine unterlegene Rasse wären.“ (Conferencia de Cabecillas Indios, 1936, in: Becker 2011a: 196)

Leider sind weitere Details dieses Diskurstransfers nicht belegt (Becker 2008b: 2), weshalb die Begriffsgeschichte der Nationalitäten bis in die 1970er nur bruchstückhaft nachverfolgt werden kann. Nachprüfbar ist, dass sich die Indigenen bis in die 1950er und 1960er Jahre immer wieder als Nationalitäten bezeichneten, etwa in einem Leitartikel in Ñucanchic Allpa aus den 1940ern: „die indigenen Völker sind unterdrückte Nationalitäten, gestern durch die Kolonie und heute durch die Republik. Ihre wahre Lösung ist das Recht zu Selbstbestimmung ihrer Schicksale.“ (Becker 2006: 143f.) Noch in den Vorbereitungen zum sechsten Kongress der PCE 1957 konnten sich Indigene innerhalb der Partei gegen eine geplante Minimierung ihrer Forderungen in den Beschlüssen des Kongresses durchsetzen. Der verabschiedete Text sprach schließlich davon, dass die „Indio-Masse unbestreitbar eine Reihe von nationalen Elementen hat“ (PCE 1957, in: Becker 2011a: 197). Dieselbe Wendung wurde auch in den Beschlüssen des achten Kongresses der PCE 1968 wiederholt (PCE 1968, in: Becker 2011a: 197). 
Bis in die 1970er Jahre hinein konzentrierten sich die ecuadorianischen Sozialwissenschaften auf Begriffe wie Rasse oder soziale Klasse im Sinne einer „Fusion der Rassen“ (Moreno Yánez 1997). Die Masterarbeit ,Interethnische Beziehungen in Otavalo-Ecuador. Die Ausbildung einer Indio-Nationalität?' der unter anderem in Mexiko ausgebildeten ecuadorianischen Anthropologin Gladys Villavicencio von 1973 ist die erste wissenschaftliche Arbeit, die das Gefühl der ethnischen Kohärenz und die Überbewertung des Eigenen als Schritt zur Ausbildung einer eigenständigen aber sekundären Nationalität deutet. In diesem Sinne wollen die Indigenen Otavalos als klar unterschiedenen Ethnie mit allgemein anerkannten Werten in die nationale Gesellschaft integriert werden und in ihr ihre eigene Zukunft selbstständig bestimmen können (Moreno Yánez 1997). Villavicencio definiert Nationalität als

„menschliche Gruppe, die durch besondere Verbindungen kultureller Homogenität (ethnische Gruppe) geeint ist, Besitzer eines solchen wirtschaftlichen und politischen Systems, das sie zu einem größeren Einfluss in der allgemeinen Gesellschaft führt. [...] Das bedeutet, eine ethnische Gruppe könnte sich in dem Moment, in dem ihr politisch-wirtschaftlicher und sozialer Einfluss ihre Grenzen als lokale Gruppe überschreitet, in eine Nationalität verwandeln, um Bedeutung in der nationalen Sphäre einzufordern. Dafür ist es wichtig, dass die ethnische Gruppe eine breite Bevölkerung umfasst, nicht nur auf eine Ortschaft beschränkt, sondern eher auf eine Region, und dass zwischen dieser Bevölkerung eine solche Kommunikation besteht die die Konstitution einer wichtigen kulturellen, sozialen und wirtschaftlichen Ähnlichkeit erlaubt.“(Villavicencio 1973: 6)

Auch international wendet sich die Anthropologie in den 1970ern zunehmend anderen Begriffen zu. Dazu gehört auch die Wiederverwendung von Begriffen wie Nationalität und Nation zur Beschreibung ethnischer Gruppen. Diese Begriffe für ethnische Gruppen zu verwenden, bedeutet in diesem Zusammenhang, ihnen ,ppolitische Sichtbarkeit“ (Bonfil Batalla 1977: 97) zu bescheinigen. Eine Nationalität ist in diesem Sinne eine Ethnie, die sich selbst annimmt und daraus politische Forderungen ableitet. Oder ,eine Ethnie ist eine Nationalität an sich, die sich aber politisch für sich annehmen muss, wenn sie sich als Nationalität konstituieren will“ (Barabas/Bartolomé 1979: 7). Damit ist gemeint, dass eine Nationalität eine Ethnie ist, die sich als solche - und nicht etwa als Kooperative oder Gewerkschaft - organisiert und spezifische Forderungen erhebt (Bartolomé 1979: 14) ${ }^{7}$. Schon in dieser frühen Definition liegt der Schwerpunkt auf der geschichtlich bestimmten Identität der jeweiligen ethnischen Gruppe und ihrer Fähigkeit, sich autonom selbst zu verwalten - eine Fähigkeit, die vom jeweiligen Staat im Sinne eines ethnischen Plura-

7 Die Metapher der Ethnie für oder an sich wurde auch in Ecuador aufgenommen (etwa in Sánchez-Parga 1988: 108). 
lismus anerkannt werden soll (Bonfil Batalla 1977: 98). Nationalitäten zeichnen sich durch eine Reihe von Charakteristiken aus, die sie von ihrer Umgebung unterscheiden, darunter linguistische, soziale, kulturelle, organisatorische oder religiöse Besonderheiten (Bartolomé 1979: 13f.). Bartolomé macht auch schon darauf aufmerksam, dass es sich beim Entstehen einer Nationalität um den Versuch eines im Zuge der Integration der Indigenen in die kapitalistische Wirtschaft „entstehenden indigenen Bürgertums“ (Bartolomé 1979: 19) handeln kann, zu politischer Macht zu gelangen.

Das spätere Wiederaufleben des Begriffes der Nationalitäten in Ecuador ist eng mit dem sowjetischen Anthropologen Yuri Zubritski verbunden (Moreno Yánez 2010), der in seiner Forschung über die Indigenen in Ecuador diesen Begriff verwendet: „Die große indigene Masse lebt weiterhin nicht nur unter dem Joch einer harten Ausbeutung, sondern auch in Zuständen einer unterdrückten Nationalität.“ (Zubritski, in: Almeida 1979: 14) Ende der 1970er hat er am Anthropologieinstitut Otavalos (Instituto Otavaleño de Antropología) (Moreno Yánez 1997) mit jungen ecuadorianischen Wissenschaftlern zusammengearbeitet - darunter Nina Pacari und Auki Tituaña - und als Programmverantwortlicher von Radio Moskau einigen von ihnen - etwa Ileana Almeida - Stipendien in der Sowjetunion besorgt. Diesen jungen Ecuadorianern sollten als Aktivisten und Wissenschaftler das Schicksal der Indigenenbewegung ab den 1980ern bestimmen (Albó 2009: 211) - insbesondere im Umfeld der Zentraluniversität Ecuadors (Universidad Central del Ecuador), die viele der späteren Führungspersonen der CONAIE besuchten (Lucero 2003: 33f.). In einem Artikel vom Juli 1977 führte Zubritzki den Begriff Nationalität in die ecuadorianische Wissenschaft ein ${ }^{8}$. Noch in einem Seminar des der CTE nahestehenden Ecuadorianischen Institutes für Sozioökonomische Untersuchungen (Instituto de Investigaciones Socioeconómicas del Ecuador, INIESEC) in Quito 1985 verwendet Zubritzki die Begriffe Rasse, Ethnie und Nationalität ohne sie voneinander abzugrenzen (Sánchez-Parga 1992: 70). Dieses Entstehungsumfeld trug dazu bei, dass der Begriff der indigenen Nationalitäten zuerst vor allem von der nichtindigenen Linken verwendet wurde. So hat Marc Becker eine Reihe von Texten zusammengetragen, die seit 1980 von ,der Existenz unterdrückter indigener Nationalitäten innerhalb des Staates“ (Movimiento 1980, in: Becker 2011a: 198) sprechen. 1982 sendet die CEDOC ,revolutionäre Grüße an die indigenen Nationalitäten Ecuadors“ (CEDIS/CEDOC 1982, in: Becker 2011a: 198), 1983 spricht der Vizepräsident der FADI, Rafael Quintero, von den Forderungen der Indigenenbewegung die sich nicht länger nur als Bauern, sondern auch als Völker und Nationalitäten verstehen (in: Becker 2011a: 198).

8 Leider gibt Sánchez-Parga keine genauere Quelle an. 
Die sowjetische Theorie, die auf Lenin und Stalin zurückgeht, von Zubritski (Almeida 1979: 16) ausformuliert und von Ileana Almeida auf Otavalo angewandt (Ibarra 1999: 83) wurde, sieht Nationalitäten als einen Entwicklungsschritt hin zum Nationalstaat, in der Reihenfolge: Stämme, Nationalitäten, Nationen, Staaten (Albó 2009: 211). Der Begriff entstammt also einer Evolutionstheorie der Staaten (Albó 2009: 304). Der Begriff der Nationalität verortet die indigenen Völker in einem „historischen Prozess“ (Almeida 1979: 14), wobei die Nationalitäten selbst auch historisch definiert sind, sich also verändern können. Er bezieht sich ,auf eine stabile menschliche Gruppe, die ein Territorium, eine wirtschaftliche Lebensweise, eine Kultur und eine Sprache teilt“ (Almeida 1979: 15). Die Nationalität steht vor dem Aufbau der Nation, wobei die Sprache der Nationalität eine besondere Rolle spielt. Im Vergleich zu den Dialekten der Stämme, bildet sich in der Nationalität eine einheitliche Sprache aus, wodurch die kulturelle und soziale Einheit der Nationalität verstärkt wird (Almeida 1979: 15). Die Nation kann erst im Kapitalismus entstehen und wird durch eine höhere Normativität der Sprache, eine wirtschaftliche Zentralisierung, ein Zusammenwachsen des Territoriums und eine weitere Ausprägung der kulturellen Eigenheiten bestimmt. Dadurch wird ein Nationalbewusstsein aufgebaut (Almeida 1979: 15). Eine Kontinuität von den ethnischen Gemeinschaften über die Nationalitäten hin zur Nation erleichtert deren Entstehen, ist aber keine unabdingbare Voraussetzung. Tatsächlich sind einige Nationen aus verschiedenen Nationalitäten und verschiedenen Stämmen entstanden (Almeida 1979: 16). Diese Vorstellung von Entwicklung war schon in den 1970er Jahren fragwürdig und wurde von den Indigenenorganisationen in der Regel nicht übernommen.

Die ethnisch orientierten Organisationen der Indigenenbewegung, die ab den 1960ern gegründet wurden, besannen sich auf den Begriff der Nationalitäten jedoch ohne den sowjetischen Hintergrund zu übernehmen (Albó 2009: 304). Vor allem die Organisationen des Amazonasgebietes - in dem sich die FEI und ihr Diskurs nie verankern konnten - und eine Reihe von urbanen Intellektuellen übernahmen diesen Begriff als ethnische Alternative zu den klassenzentrierten Begriffen (Lucero 2008: 142). Jorge León Trujillo etwa beschreibt schon 1983 die Indigenen als Nationalitäten, wobei seine Definition eine gemeinsame Geschichte und Sprache und geteilte Traditionen und Kultur vorsieht, von denen ausgehend die jeweilige Nationalität Forderungen nach Selbstverwaltung und territorialer Autonomie aufstellen kann (León Trujillo 1983: 8). Diese frühe nicht-stalinistische Definition enthält die Grundlagen, innerhalb derer sich spätere Formulierungen von Nationalität bewegen sollten. Auch auf internationalem Niveau wurde der Begriff der indigenen Nationalitäten übernommen. Besonders der 1980 gegründete Indiorat Südamerikas (Consejo Indio de América del Sur, CISA) verbreitete die neue Begrifflichkeit in ihren Publikationen (etwa AAVV 1985). So wurde sie in den 1980er von 
den indigenen Organisationen Lateinamerikas zunehmend verwendet und auch von Presse und Zivilgesellschaft diskutiert (Ibarra 1999: 83).

Der Begriff der indigenen Nationalitäten wurde in Ecuador bis Mitte der 1980er oft als ein kultureller Begriff und Synonym für Ethnie verstanden und mit den kulturellen Forderungen der Indigenen assoziiert (Ibarra 1999: 85) - vor allem die offizielle Anerkennung ihrer Sprachen und die Förderung der zweisprachigen Bildung. Dadurch konnte er schon früh auf Gesellschaft und Staat ausstrahlen. Jaime Roldós, ecuadorianischer Präsident von 1979 bis 1981, verwendete bereits den Begriff der Nationalitäten, wenn er über die indigenen Völker sprach. Roldós strengte auch ein Garantiegesetz für die indigenen Nationalitäten an, das er durch seinen frühen Tod nicht mehr realisieren konnte (Iturralde 2000: 168).

Der wiederentdeckte Begriff bewegte sich im Zuge der Versuche, eine eigenständige nationale Indigenenorganisation zu gründen, in das Andenhochland. Die erste regionale Organisation des Amazonasgebietes, die CONFENIAE, war die treibende Kraft hinter dieser Bewegung. ECUARUNARI als eher sozialistische Organisation des Andenhochlandes hatte bis dahin andere Begriffe bevorzugt, begann nun aber auch von indigenen Nationalitäten zu sprechen - ohne dadurch die Forderungen nach territorialer Autonomie der Organisationen des Amazonasgebietes zu übernehmen (Guerrero/Ospina 2003). Diese gängige Interpretation deckt sich nicht mit den vorliegenden Daten, die zwar einen Diskurstransfer ethnischer und identitärer Inhalte vom Amazonasgebiet und dort insbesondere von der Federación Shuar aus ins Hochland bestätigen, aber gerade für den Begriff der indigenen Nationalitäten etwas anderes vermuten lassen. Tatsächlich ist die Wiederentwicklung des Begriffs der Nationalitäten in Otavalo - also im Hochland - gegen Ende der 1970er nachweisbar, die Zentralität des dortigen Anthropologischen Instituts deutet auf eine umgekehrte Ausbreitung hin. Die Namensgebung der CONFENIAE allein ist kein ausreichender Beweis für eine Vorreiterrolle des Amazonasgebietes; nur wenige Monate später wurde unter entscheidender Mitwirkung von ECUARUNARI die CONACNIE gegründet, die ebenfalls die indigenen Nationalitäten im Namen trägt. Spätestens seit dem Nationalen Indigenen- und Bauernmarsch im Oktober 1980 sind Territorien und Kultur der indigenen Nationalitäten (ECUARUNARI 1998: 99) ein Teil des Diskurses von ECUARUNARI. Schon Anfang der 1980er beschreibt sich die Organisation als ,eine Bewegung, die das Banner des Kampfes gegen die Diskriminierung und für die Verteidigung der indigenen Nationalitäten erhebt“ (ECUARUNARI 1984: 46). Sie beginnt, ethnische und soziale Faktoren zu integrieren und versteht die Indigenen als ,ausgebeutete Nationalität und Klasse“ (ECUARUNARI 1984: 47), denen der Nationalstaat als Produkt des Kapitalismus gegenübersteht (ECUARUNARI 1984: 48). In diesem Rahmen verlangt sie nicht nur „das grundlegende Prinzip des Rechts auf das Land“ (ECUARUNARI 1984: 44f.), sondern eine weitgehende Autonomie, ,das Recht, eigene Autoritäten zu 
haben, um so die Diskriminierung zu verhindern, die gegen die Bauern und insbesondere gegen die Indigenen ausgeübt wird“"(ECUARUNARI 1984: 44f.).

Das ECUARUNARI-Mitglied Nina Pacari entwickelt in einem Text von 1984 den Begriff der Nationalität mit Bezug auf die Kichwa. Dabei folgt sie in einigen Grundsätzen der Theorie von Almeida. So wurden für Pacari die Festigung der Kichwa-Nationalität und der Aufbau eines Kichwa-Staates von der spanischen Invasion unterbrochen ${ }^{9}$ (Pacari 1984: 116). Dennoch zeichnet sich die KichwaNationalität durch „das Auftauchen staatlicher Elemente“ (Pacari 1984: 115f.) aus. Dazu zählt für Pacari die Struktur Ayllu-Llacta Ayllu-Mama Ayllu oder Familie, erweiterte Gemeinschaft und Volk. Das Volk der Kichwa entspricht in diesem System der Mama Ayllu, die sich durch gemeinsame Sprache, Kultur, Territorium und wirtschaftliche Verbindungen auszeichnet (Pacari 1984: 115). Dieser Sozialstruktur entspricht auch eine moralische und rechtliche Struktur, die von Ayllu Camachic über Llacta Camachic zu Mama Ayllu Camachic geht, also von familiären Normen über sozialen Normen einer Gemeinschaft zu den rechtlichen Normen des ganzen Volkes. Diese Struktur der Normen orientiert sich an der harmonischen Beziehung zwischen Pachamama-Allpamama-Runa, Universum-Erde-Mensch, und drückt sich in Moralvorstellungen wie Ama Quilla, Ama Llulla, Ama Shua (nicht faul sein, nicht lügen, nicht stehlen) aus (Pacari 1984: 115). Mit diesen vorstaatlichen Strukturen begründet Pacari die Forderung nach einer rechtlichen Anerkennung (Pacari 1984: 122) und Selbstbestimmung (Pacari 1984: 115) der indigenen Nationalitäten. Jeder der indigenen Nationalitäten soll der kollektive und unveräußerliche Besitz ihres ausreichend großen Territoriums garantiert werden (Pacari 1984: 122).

Auch der CONACNIE, die Vorgängerorganisation der CONAIE, gründete auf einem Verständnis der Indigenen als Nationalitäten (Conterón/de Viteri 1984: 6). In einem Text von 1984 definiert diese Organisation Nationalitäten als Völker mit einer eigenen Kultur und Geschichte, eigener Sprache und Literatur, eigenen Formen sozialer, wirtschaftlicher und politischer Organisation, eigenen Legenden und Traditionen und in Teilen eigener Religion und eigenen Territorien (Conterón/de Viteri 1984: 7). Diese Eigenständigkeit begründet für den CONACNIE die Andersheit und die besonderen Forderungen der indigenen Nationalitäten.

Die FCUNAE formuliert aus der Perspektive der Kichwa des Amazonasgebietes in einem Text von 1986 ihr Verständnis der Indigenen als Volk und somit - aus ihrer Perspektive - als etwas Umfassenderes als eine Klasse. Der Kampf der Indigenen ,[b]richt mit den Formen der klassischen Organisation der Gesellschaft in soziale Blöcke. Er ist eher ein Problem der Kultur und Nationalität, denen der Klas-

9 Das gleiche nimmt Bartolomé (1979: 14) von den mittelamerikanischen Nationalitäten an. 
senkampf nicht nur eine stereotypische Lösung der sozialen Revolution geben kann.“ (UNAE o.J.: 22) Daher ist das Selbstverständnis der Indigenen als Völker und Nationalitäten innerhalb eines Staates für die FCUNAE der Beginn ihrer Befreiung innerhalb eigener Modelle (UNAE o.J.: 22f.). Die indigenen Nationalitäten definieren sich durch ihr Territorium, in dem die Produktionsweisen und kulturellen Ausdrücke des jeweiligen Volkes entwickelt haben (UNAE o.J.: 47). Sie sind daher unterdrückte Nationalitäten, die für ,,ihre ethnisch-kulturelle Befreiung“ (UNAE o.J.: 46) kämpfen - ohne deswegen einen eigenen Staat anzustreben.

„Der wirkliche Begriff der Nationalität ruht auf einem Autonomiestatus oder Charta der Nationalität durch die Territorien, Regierungssysteme und autonome kulturelle Prozesse anerkannt werden, innerhalb eines multiethnischen Staates, der sie umfasst.“ (UNAE o.J.: 43)

So war der Begriff der Nationalitäten zuerst ,eine weitere Form des Kampfes um Respekt" (Guerrero/Ospina 2003), der die Forderungen nach territorialer Autonomie klären sollte. Nationalität sollte die Eigenständigkeit der indigenen Völker betonen und ihre Forderungen nach kollektiven Rechten und Autonomie unterstützen (Ayala Mora o.J.: 10f.) - und nebenbei den bestehenden Konflikt zwischen ethnischen und klassistischen Tendenzen in den Organisationen überwinden (Ramón 1992a: 9). Der Begriff der indigenen Nationalitäten half, die Frage zu beantworten, ob die Indigenen als Bauern eine unterdrückte Klasse oder als Kichwa, Shuar, usw., ein unterdrücktes Volk sind. Er konnte zugleich eine relative Einheit der verschiedenen Kichwa-Völker des Hochlandes, einen Wiedergewinn der Würde der amazonischen Völker - die bis dahin oft Wilde genannt wurden - und eine Einheit aller indigener Völker Ecuadors fördern - so spielte er eine wichtige Rolle beim Aufbau einer nationalen Indigenenorganisation (Iturralde 2000: 168). Der Begriff der indigenen Nationalitäten wird nicht zufällig meist von Referenzen an den Kampf um Land und an die Anerkennung der indigenen Kulturen und Werte begleitet (Sánchez-Parga 1988: 110). Die Selbstverwaltung der Nationalitäten als Bevölkerungsgruppen soll die verlorenen Rechte dieser Gruppen wiederherstellen. „Wir sind nicht nur ,Individuen“, Bürger, wir sind Völker und Nationalitäten. Deshalb fordern wir kollektive Rechte zusätzlich zur Individualität.“ (ECUARUNARI/CONAIE 1989: 42) Daraus entstand die Überzeugung, Ecuador sei ein plurikulturelles, plurinationales und mehrsprachiges Land, die mit der Gründung der CONAIE 1986 (CONAIE 1989: 279) eine organisatorische und mit der Forderung nach einem plurinationalen Staat, der die Charakteristiken des Landes widerspiegelt und anerkennt, eine diskursive Heimat fand (Ramón 1992a: 9f.).

Der Begriff der Nationalitäten ermöglichte den noch jungen Indigenenorganisationen auch, sich vom staatlichen Diskurs und den verschiedenen indigenistischen Institutionen zu distanzieren, deren Forderungen sich nicht sehr von ihren eigenen 
unterschieden - abgesehen eben vom hochgradig politischen Selbstverständnis als indigene Nationalitäten (Sánchez-Parga 1988: 110). Dieses Selbstverständnis konnte der Staat und seine Institutionen nicht in vollem Umfang übernehmen ohne den Indigenen weitgehende Autonomierechte einzugestehen. Der traditionelle Staat kann durch seine starre und vertikale Struktur nicht angemessen auf die Existenz von Nationalitäten als „aktive soziale Subjekte“ (Viteri 1997: 52) reagieren und Verhandlungen mit ihnen nur als Konzessionen oder Unterwerfung verstehen. Daher muss dieser Staat die Indigenen als Gefahr für seine Einheit sehen (Viteri 1997: 52).

Auch die gewerkschaftlich ausgerichteten Organisationen übernahmen in den 1980ern den Begriff der indigenen Nationalitäten. 1987 forderte die FENOC eine Verteidigung der Rechte als Völker und Nationalitäten (FENOC 1987, in: Becker 2011a: 199). Seit 1987 arbeiteten FENOC, FEI und CONAIE im Rahmen einer Parlamentskommission an einem Projekt für ein Gesetz der indigenen Nationalitäten, das 1988 dem Nationalkongress vorgelegt wurde. Die indigenen Organisationen und das Gesetz wurden von Bischof Proaño und dem Vorsitzenden der Sozialistischen Partei, Enrique Ayala unterstützt (Albó 2009: 211f.). Dennoch wurde es im Kongress nicht diskutiert. Das Projekt enthielt eine Anerkennung der indigenen Territorien und Sprachen, der zweisprachigen interkulturellen Erziehung und der indigenen Medizin, sowie eine erste Definition autonomer indigener Nationalitäten im Rahmen eines plurinationalen Staates (Albó 2009: 212). Es sollte zu einer Anerkennung und Verteidigung der indigenen Nationalitäten durch den Staat führen (Comisión Especial de Asuntos Indígenas 1988: 6). Dabei macht der Text deutlich, dass die Anerkennung der indigenen Nationalitäten keine Rückkehr zur Situation vor der spanischen Eroberung bedeuten kann (Comisión Especial de Asuntos Indígenas 1988: 7f.) und dass der Begriff der indigenen Nationalitäten nicht abschlieBend definiert ist. Das sollte über die Herausbildung eigener und autonomer Entwicklungsformen der jeweiligen Nationalitäten erfolgen (Comisión Especial de Asuntos Indígenas 1988: 9). Auch die genauen Formen der Repräsentation und Selbstverwaltung der Nationalitäten durch formale Instanzen konnte noch nicht geklärt werden (Comisión Especial de Asuntos Indígenas 1988: 13).

Bis in die 1990er Jahre hatte sich der Begriff der Nationalitäten in den Organisationen der CONAIE weitgehend durchgesetzt, ohne jedoch genau definiert zu werden (Ramón 1992a: 9f.). Die Tatsache, dass die indigenen Organisationen und in zunehmendem Maße die Wissenschaft von indigenen Nationalitäten spricht, bedeutet nicht, dass dieser Begriff genauer erklärt wurde, sondern dass ,,mit der Zeit seine Vieldeutigkeit und Verwirrung zunahmen“" (Ayala Mora o.J.: 11). Die Diskussion über den Begriff der Nationalitäten ist dynamisch und kontrovers und baut kaum auf feste Definitionen. So sind die geographischen und historischen Grenzen der Nationalitäten meist nicht klar - die CONAIE selbst fordert Studien dazu, um 
so ihre Politik schärfen zu können (Macas 1991: 11; CONAIE 1994: 21). Von größerer Bedeutung sind die Beziehungen zwischen den Nationalitäten und ihr Ziel, als gleichberechtigte Partner in einen pluralen oder plurinationalen Staat integriert zu werden (Macdonald 2002: 184).

Die CONAIE hat sich mit der Arbeit an ihrem Politischen Projekt ab 1993 um eine Definition ihrer Begriffe bemüht und konnte so diese Vieldeutigkeiten einschränken. Das Politische Projekt definiert schon seit 1994 Nationalitäten als indigene Völker mit ,derselben Herkunft, einer gemeinsamen Geschichte, eigenen Sprachen“ (CONAIE 1994: 49; CONAIE 1997: 47; CONAIE 2001a). Dazu kommen eigenen Gesetze, Gebräuche, Glaubensarten und eigene Formen der sozialen, wirtschaftlichen und politischen Organisation in den Territorien der jeweiligen Nationalitäten. Die Version des Politischen Projekts von 2001 ergänzt, dass die Nationalitäten schon vor der Gründung des ecuadorianischen Staates bestanden und dass sie ,eine Reihe von eigenen und besonderen kulturellen Charakteristiken hat, die sie vom Rest der Gesellschaft unterscheiden“ (CONAIE 2001a). Die wichtigste dieser Eigenheiten, in dieser Hervorhebung ebenfalls eine Neuerung des Politischen Projekts von 2001, ist eine eigene Sprache. So bewegte sich der Begriff der Nationalität von einer Infragestellung des uninationalen Staates und seiner Strukturen im Zuge der Integration des Begriffes in die Institutionen dieses Staates und der Indigenenbewegung selbst langsam zu einer wenig radikalen Idee mit der schrittweise Reformen gefordert werden konnten (Guerrero/Ospina 2003). Nach der Definition der CONAIE gibt es in Ecuador folgende Nationalitäten: Kichwa, Shuar-Achuar, Chachi, Tsáchila, Siona-Secoya, Huaroani, Cofán, Awá, Epera (CONAIE 2001a). Nationalitäten

,sind historische und politische Einheiten, aus denen der ecuadorianische Staat besteht, die eine Identität, Geschichte, Sprache, eigene Kultur gemein haben, die in einem bestimmten Territorium mit ihren eigenen Institutionen und traditionellen Formen der sozialen, wirtschaftlichen, rechtlichen, politischen Organisation und Ausübung eigener Autorität leben“ (Saltos/Vázquez 2007: 383).

Innerhalb der Nationalitäten gibt es verschiedene Völker, die sich vor allem über ihre Identität (UIAW 2004: 151f.), ihr lokales Zugehörigkeitsgefühl, ihre Sprache und ihre gemeinsame Geschichte und Kultur als besondere Teile der jeweiligen Nationalität definieren (CONAIE 1994: 49; Maldonado 2006: 99f.). Sie haben eigenständige und unabhängige Institutionen und Organisationsformen, die sie von den anderen Völkern derselben Nationalität und der Gesamtgesellschaft unterscheiden (CONAIE 2001a). Die indigenen Völker mitsamt ihrer Institutionen sind integriert ,,in die Institutionalität einer anderen herrschenden Gesellschaft, die ihre ursprüngliche Umgebung besetzt“ (CONAIE 1994: 49). Daher wird ein indigenes 
Volk in Relation zu einer nicht-indigenen Gesellschaft definiert und über sein Bewusstsein dieser Situation (CONAIE 1994: 49). Wichtig bei der Definition von Nationalitäten und Völkern in diesem Sinne ist die Selbstbezeichnung (autodefinición), die schon in der Verfassung von 1998 (Art. 83) die Nationalitäten ausmacht und auch von der Indigenenbewegung vorgesehen ist (CONAIE 2004: 116f.). Die Verfassung von 1998 war die erste, die die indigenen Nationalitäten offiziell anerkannte (Ibarra 1999: 88; Radcliffe 2002: 159f.; Grijalva 2008: 55).

Die Definition von Nationalitäten, die die CONAIE entwickelt, bleibt der europäischen Definition von Nation verhaftet, wobei die Nationalitäten die Grundlage der Nation sind (Rhon 2003: 134) - und im Sinne der CONAIE auch mehrere Nationalitäten, indigene wie nicht-indigene, eine Nation bilden können (Ibarra 2010: 44). Die indigenen Nationalitäten und die ethnische und kulturelle Diversität sind also nicht ein marginaler Teil der Nation, sondern ihr Kern und der Ausgangspunkt einer neuen, plurinationalen Identität (Macdonald 2002: 182). Dieses neue Selbstverständnis hat auch direkte und praktische Auswirkungen. Da die Nationalitäten eigene soziale Strukturen haben und sich selbst verwalten können, ist ihr entwicklungspolitischer Anspruch die Hilfe zur Selbsthilfe - und nicht der traditionelle Paternalismus des Staates (Macdonald 2002: 183).

Die Nationalitäten haben das grundlegende Recht auf Selbstbestimmung in ihrem eigenen Territorium, zu dem auch die freie Wahl und Ausübung ihres eigenen politischen und wirtschaftlichen Systems gehört (CONAIE 2001: 6). Darauf aufbauend konnten die Strukturen des traditionellen Staates im Namen der Nationalitäten in Frage gestellt und Alternativen entwickelt werden. Das erlaubte einen Umbau von staatlichen Strukturen und der Strukturen der Indigenenbewegung selbst CONAIE und ECUARUNARI wurden nach 2000 so reformiert, dass sie sich als Vertretung der indigenen Völker und Nationalitäten verstehen und nicht nur die ihrer Mitgliedsorganisationen ${ }^{10}$ (Guerrero/Ospina 2003). Die moderne Entwicklung des Begriffs der Nationalitäten ist untrennbar verbunden mit der Entwicklung der CONAIE. Nationalität als Begriff, der ethnische, politische und wirtschaftliche Forderungen kombiniert, ist das Ergebnis einer Koordination der Organisationen des Hochlandes und des Amazonasgebietes. Seine gesellschaftliche Verbreitung und weitgehende Akzeptanz auch in nicht-indigenen Kreisen ab den 1990er Jahren ist Beweis der Hegemonie der CONAIE und ihrer Fähigkeit, gesamtgesellschaftliche Bedeutungen zu beeinflussen (Lucero 2008: 151).

Die starke Verbindung des Begriffs der Nationalität mit der CONAIE führte zum Widerstand der anderen Indigenenorganisationen. Vor allem die FENOCIN verstand die Idee, die Repräsentation der Indigenen nach Nationalitäten und Völ-

10 Gut nachvollziehbar an der Namensänderung ihrer Kongresse, vergleiche Abbildungen 5 und 6 . 
kern zu gliedern - statt nach Organisationen - als ,ethnisch fundamentalistischen Vorschlag" (FENOCIN 1999: 152) und direkten Angriff auf die verschiedenen Organisationen ersten und zweiten Grades der Indigenenbewegung ${ }^{11}$. Sie kämpfte gegen die Umsetzung dieses Vorschlages in den staatlichen Organisationen, wo sie zwischen 1999 und 2002 weitgehend durchgesetzt wurde, meist - wie im Fall der CODENPE - zu ihrem Nachteil (FENOCIN 1999: 152). So interpretiert die FENOCIN die Tatsache, dass nur die Nationalität der Kichwa verschiedene Völker umfasst, als tagespolitische Entscheidung der CONAIE, die mehr mit den internen Spannungen der Organisationen zu tun hat als mit der Realität der jeweiligen Gruppen (FENOCIN 1999: 153). Es ist wohl kein Zufall, dass nur die Völker der Nationalität der Kichwa verschiedene, territorial und ethnisch voneinander getrennte Organisationen, besitzen - und somit die notwendige Infrastruktur haben, um ihre Verschiedenheit als Völker zu begründen ${ }^{12}$. Auch die Grenzen der indigenen Nationalitäten, die die CONAIE umschreibt, werden von der FENOCIN in Frage gestellt. Für den Begriff der indigenen Nationalität wird die indigene Identität durch die Zugehörigkeit eine Individuums zu einer spezifischen ethnischen Gruppe definiert. Dieser relativ strikten Zugehörigkeit stellt die FENOCIN numerisch bedeutende Sonderfälle entgegen. So lässt sich durch dieses Modell der einfachen Zugehörigkeit nur schwer die Identität von Indigenen klären, die in die Städte migriert sind. Auch Gruppen, die einige Aspekte ihrer indigenen Kultur aufgegeben haben, sich aber dennoch als Indigene verstehen, sind so nur schwer einzuordnen. Dasselbe Problem besteht bei indigenen Gruppen, die sich vor Jahrhunderten in die Kichwa integriert und ihre vorherige Identität weitgehend verloren haben (FENOCIN 1999: 155f.). Daher fordert die FENOCIN einen flexibleren Umgang mit indigenen Identitäten, der auch weniger eindeutige Zuschreibungen erlaubt und so die Wirklichkeit besser widerspiegelt (FENOCIN 1999: 156).

Der Begriff der indigenen Nationalität ist eine Möglichkeit, die indigenen Kulturen zu bewahren und eine völlige Assimilation in die Mehrheitsgesellschaft aufzuhalten (Andolina 1998: 65). Er dient als „Synthetisierer der Differenzrechte als Völker“ (Viteri 1997: 55f.). Die damit einhergehende Essenzialisierung dient der Verstärkung der wiederentdeckten Identitäten und ist somit eine Absicherung gegen die verstärkten Integrationstendenzen im Zuge der Globalisierung (Andolina 1998: 65). Gerade deshalb ist die Kritik der FENOCIN gerechtfertigt. Tatsächlich überdeckt ,,der Begriff der Nationalität Differenzen innerhalb der Nationalität“ (Andolina 1998: 70), vereinheitlicht also die indigenen Gruppen.

11 Damit bezieht sich die FENOCIN nicht auf offizielle Positionen der CONAIE und ihrer Organisationen, sondern auf Diskussionen und Vorschläge, die im Umfeld der CONAIE stattfanden (etwa Viteri 1998: 97) und keine Mehrheiten gewinnen konnten.

12 Vgl. die Kritik von Viteri (1997: 56). 


\section{Plurinationalität}

\section{a. Eine kurze Begriffsgeschichte}

Der Begriff der Nationalitäten und die Forderung nach ihrer staatlichen Anerkennung richtet sich bereits auf eine plurinationale Umgestaltung des ecuadorianischen Staates. ,[I]n einer Nation kann eine Vielfalt von Nationalitäten existieren, ohne die Souveränität und die Existenz der ecuadorianischen Nation als Einheit zu gefährden.“ (Saltos/Vázquez 2005: 145) Ohne den Begriff der Plurinationalität zu verwenden, wurden vergleichbare Zielsetzungen schon in den 1970ern von der FICSHA entwickelt: ,das Ziel, nach dem [die FICSHA, P.A.] strebt, ist eindeutig eines: die Selbstbestimmung der Shuar-Gruppe in einer neuen Konzeption eines pluralistischen ecuadorianischen Staates“ (Federación de Centros Shuar 1976: 129).

Der Begriff der Plurinationalität entstand in Ecuador in den 1980er Jahren, wobei der genaue Zeitpunkt seiner ersten Verwendung umstritten ist. Nach Almeida und Arrobo wurden die ersten Überlegungen über den plurinationalen Staat schon in den 1970ern am Anthropologieinstitut Otavalos unter der Leitung Yuri Zubritskis angestellt (Almeida/Arrobo o.J.: 6). Moreno Yánez sieht die Forderung nach einem plurinationalen Land als eine konsequente Weiterentwicklung des Begriffes der Nationalität außerhalb der Wissenschaft. Er beschreibt ein Treffen indigener Organisationen in Puyo, auf dem Anfang der 1980er Jahre diese Forderung zum ersten Mal aufgestellt wird (Moreno Yánez 1997). Galo Ramón spricht einem Zeitraum zwischen 1983 und 1988, in dem Ecuador zuerst als plurinationales Land verstanden wurde (Ramón 1992a: 9f.). Die erste nachweisbare Erwähnung eines multinationalen Staates findet sich in einer Sonderausgabe der Zeitschrift Cuadernos de Nueva von 1983. Dort knüpft Jorge León an seine Erfahrungen in Kanada an und skizziert als Konsequenz des Selbstverständnis der Indigenen als Nationalitäten verschiedene Staatsformen ${ }^{13}$.

\footnotetext{
„Was auch immer die politische Form eines neuen Staates sein mag: multinational (die Nationalitäten werden nicht nur anerkannt, sondern nehmen an den Entscheidungszentren teil) oder multiethnisch (die verschiedenen Ethnien haben ein Recht auf die Anerkennung ihrer Besonderheiten: Sprache, Erziehung, Gewohnheiten, agrarische und Gemeinschaftsorganisation, usw.), föderal oder anders, das ethnische Problem verlangt die Anerkennung des Verschiedenen, das Zusammenleben mit dem Anderen und auch Konfliktiven." (León Trujillo 1983: 8)
}

13 Mit Dank an Jorge León für seine hilfreichen Kommentare. 
In derselben Zeitschrift spricht Manuel Chiriboga bereits vom Aufbau eines pluriethnischen und plurinationalen Landes, das durch eine neue Beziehung zwischen Indigenen und Staat und eine territoriale Autonomie unter Verwaltung und Repräsentation der jeweiligen indigenen Organisationen geprägt ist (Chiriboga 1983: 123). Er macht auf den Einfluss der Intellektuellen aufmerksam, die ,den ethnischnationalen Widerspruch“ (Chiriboga 1983: 124), also die Unterdrückung bestimmter Ethnien durch andere Ethnien, als eines der wichtigsten Probleme des Landes definieren. Dieser Widerspruch kann für ihn nur durch ,einen plurinationalen Staat und Gesellschaft" (Chiriboga 1983: 124) gelöst werden.

Kurze Zeit später wurden diese neuen wissenschaftlichen Begriffe von zentralen Personen der Indigenenbewegung aufgegriffen. Nina Pacari beschreibt die Existenz einer Vielzahl von Nationalitäten in Ecuador, von denen jede ihre eigene Kultur bewahrt hat. Somit stellt sie die Multinationalität und Plurikulturalität Ecuadors den Integrations- und Assimilationsversuchen des ecuadorianischen Staates entgegen, der die Indigenen politisch und kulturell unterdrückt (Pacari 1984: 118f.). Darauf aufbauend fordert Pacari ,die Schaffung eines wahrhaft multinationalen und plurikulturellen Staates, in dem jede Nationalität das Recht auf Selbstbestimmung und die freie Wahl sozialer, politischer und kultureller Alternativen hat" (Pacari 1984: 119). Durch eine Veränderung der sozialen Strukturen sollen die indigenen Nationalitäten als gleichberechtigte aber andere Völker gesehen werden (Pacari 1984: 121). Das Ziel dieses Staates ist das Ende der „Herrschaft eines Volkes über ein anderes Volk“ (Pacari 1984: 121). Im Anschluss stellt sie Forderungen auf, die bis Mitte der 1990er Jahre mit dem Begriff der Plurinationalität verbunden sein sollten. So werden Funktionale Indigene Senatoren im Nationalkongress gefordert, die die Interessen der indigenen Völker vertreten sollen. Das Erziehungssystem in den indigenen Gebieten soll den jeweiligen indigenen Organisationen unterstellt sein. Ein Autonomer Indiorat soll Vertreter aller Nationalitäten versammeln und in allen Institutionen des Staates vertreten sein, ,für die korrekte multinationale und plurikulturelle Koexistenz" (Pacari 1984: 122).

Diese Diskussion knüpft an die Entwicklungen der internationalen Anthropologie an - ohne sie allerdings zu zitieren. Mit Rückgriff auf die Theorie des internen Kolonialismus beschreibt Edelberto Torres zu Beginn der 1970er „eine Unabhängigkeit der indigenen Nation oder ihre Integration in ein multinationales Guatemala“ (Torres 1972: 121) als unwahrscheinlich. Einige Jahre später entwickelt Bonfil Batalla eine weitergehende Definition dieser Begriffe. Im Anschluss an das zweite Treffen von Barbados 1977 spricht er von ethnischen Gruppen, die als Nationalitäten Autonomie einfordern. Die jeweiligen Staaten bezeichnete er als ,multiethnische oder multinationale Staaten“ (Bonfil Batalla 1977: 98), die - nach den Forderungen der Indigenenbewegungen - die indigenen Nationalitäten als politische Einheit und Teil der staatlichen Organisation und des Staates selbst anerkennen 
sollen. Dadurch ,wird dieser indigen oder erkennt zumindest seinen plurinationalen Charakter an“ (Bonfil Batalla 1977: 99). Selbst das Verständnis dieser Forderungen als Gefahr für die nationale Einheit hat Bonfil Batalla vorausgesagt (Bonfil Batalla 1977: 99). Er nennt Finnland, die Schweiz und Spanien als Beispiele für multi- oder plurinationale Staaten mit weitgehenden Autonomierechten für ethnische Gruppen - um so zu zeigen, dass die Forderung nach einem plurinationalen Staat durchaus realisierbar ist (Bonfil Batalla 1977: 100). Die Anregungen Bonfil Batallas wurden in Mexiko schnell aufgegriffen, etwa durch die Zeitschrift Revista Mexicana de Ciencias Sociales y Políticas der Universidad Nacional Autónoma de México (UNAM), die 1979 das Sonderheft ,Die indigenen Nationalitäten in Mexiko ' herausgibt. Dort betonen die Anthropologen Alicia Barabas und Miguel Bartolomé die politischen Aspekte des ,indigenen Problems', das sich nicht durch eine Vermehrung von Hilfsprogrammen, sondern nur durch Selbstbestimmung und Autonomie lösen lässt (Barabas/Bartolomé 1979: 6). Dabei heben sie hervor, dass „ein pluriethnischer und plurinationaler Staat der Zukunft“" (Barabas/Bartolomé 1979: 7) politisch durchsetzbar ist. Bartolomé spricht von der „entstehenden Plurinationalität in Mexiko“ (Bartolomé 1979: 15) und bezieht sich damit auf „seine Bestimmung als multinationalen Staat, in den Ebenen der Politik und Verwaltung" (Bartolomé 1979: 15).

Diese Ideen und Begriffe wurden im Umfeld des Indianismus, also der explizit ethnisch ausgerichteten Strömungen der lateinamerikanischen Indigenenbewegungen, ab Ende der 1970er Jahre aufgegriffen. Die auf dem ersten Kongress der IndioBewegungen Südamerikas 1980 festgelegten Ziele der politischen Selbstbestimmung der indigenen Völker als regionale Autonomien im Fall einer indigenen Minderheit im jeweiligen Land und als Umgestaltung des Staates im Falle einer indigenen Mehrheit führten zu Überlegungen, wie diese Umgestaltung passieren könnte. „Mit dem Ziel, dass man die verschiedenen Indio- und nicht-Indio-,Nationalitäten“ respektiert, taucht auch die Idee eines ,plurinationalen' Staates auf.“" (Barre 1982: $74)^{14}$

Die kataristische Bewegung in Bolivien und die Bolivianische Einheitsgewerkschaftskonföderation der Landarbeiter (Confederación Sindical Única de Trabajadores Campesinos de Bolivia, CSUTCB) haben schon seit den 1970er Jahren auf Grundlage eines Diskurses der indigenen Nationen ähnliche Forderungen entwickelt und - nach Catherine Walsh - die Forderung nach einem plurinationalen Staat schon vor der indigenen Bewegung in Ecuador aufgestellt (Walsh 2008: 16). Xavier Albó erwähnt einen Nationalen Kongress der CSUTCB 1983, auf dem sich das

14 Leider bietet Barre keinerlei weiterführenden Verweise, welche Organisationen welcher Länder wann diese Begriffe verwendet haben; sie beschreibt lediglich einen einheitlichen indianistischen Diskurs. 
beginnende Selbstverständnis einiger Indigener als Nationen innerhalb des bolivianischen Nationalstaates in der Forderung nach einem plurinationalen Staat widerspiegelte (Albó 2007: 342) ${ }^{15}$. Bolivien war der zweite Staat nach Ecuador, der sich mit der Annahme der neuen Verfassung im Januar 2009 als plurinational erklärt. Die dort unter dem Begriff der Plurinationalität subsumierten Begriffe Dekolonisierung, Pluralismus, Pluralität der Nationen im Nationalstaat und der Aufbau einer interkulturellen Gesellschaft (Walsh 2009a: 73) entsprechen grob den in Ecuador verwendeten.

In den ersten Jahren war die Forderung nach einem plurinationalen Staat weitgehend auf die Indigenenbewegung beschränkt und wurde kaum von der Mehrheitsgesellschaft aufgegriffen. Pablo Ospina bezieht sich auf den Historiker Hernán Ibarra, der feststellt,

„dass das Konzept der Plurinationalität bis in die Mitte der 1980er Jahre vor allem mit kulturellen Forderungen in Verbindung gebracht wurde, wobei es einen breiten Konsens verschiedener politischer und ideologischer Positionen gab“ (Ospina 2006a: 458).

Daher verwendete Rodrigo Borja, von 1988 bis 1992 ecuadorianischer Präsident, den Begriff häufig und sprach schon in seiner Antrittsrede im August 1988 von der Plurinationalität des Landes (Black 1999: 31). Diese positive Aufnahme der Plurinationalität in Teilen der Politik und Zivilgesellschaft veränderte sich mit der stärkeren Integration der Begriffe Territorialität und Autonomie. „Die nationale Forderung gewann an revolutionärer Tiefe, verlor aber an öffentlicher Zustimmung.“ (Ospina 2006a: 458) Die 1986 gegründete CONAIE setzte sich das Ziel, Ecuador zu einem plurinationalen Staat mit besonderen Rechten für die Indigenen zu machen (Ramón 1992a: 9f.) So wurde der Begriff der Plurinationalität ab Ende der 1980er (García/Tuasa 2007: 17) und in den 1990er Jahren innerhalb der CONAIE viel diskutiert, von der mestizischen Mehrheitsgesellschaft aber meist als ,Sonderangelegenheit der Ethnien“ (Santillana 2006: 240f.) missverstanden und abgelehnt (Walsh 2008: 17).

Ein erster Höhepunkt in der Formulierung dieser neuen Forderung war der Vorschlag für ein Gesetz der Nationalitäten von 1988, das die Anerkennung der indigenen Nationalitäten und den Aufbau eines plurinationalen Staates enthielt (García/Tuasa 2007: 36) - allerdings nie vom Kongress diskutiert wurde und daher nur wenig Bekanntheit erlangte. Der erste Artikel des Vorschlags definiert die Beziehung zwischen Staat und Indigenen neu:

15 Auch an anderen Stellen wird behauptet, dass bereits in den 1970ern von verschiedenen Indigenenorganisationen des Kontinents der Aufbau eines plurinationalen Staates verlangt wurde (etwa Huanacuni 2010: 10) - leider ohne Belege. 
„Die Republik Ecuador ist ein plurinationaler Staat in dem die indigenen Völker mit ihrer Kultur, Organisation und Rechten, insbesondere dem der Selbstbestimmung den dieses Gesetz garantiert und normiert, teilnehmen." (Comisión Especial de Asuntos Indígenas 1988: 17)

Die indigenen Völker und Nationalitäten sollen innerhalb dieses ,plurinationalen und multiethnischen Staates“ (Comisión Especial de Asuntos Indígenas 1988: 8) das Fortbestehen und die Weiterentwicklung ihrer Territorialität, Kultur, Sprache und wirtschaftlichen Organisation suchen (Comisión Especial de Asuntos Indígenas 1988: 8f.). Es werden also Forderungen nach Territorialrechten für die indigenen Völker und Nationalitäten mit dem Begriff der Plurinationalität verbunden (Ospina 2010: 203f.). Schon in dem Gesetzesvorschlag wird deutlich gemacht, dass die Schaffung von autonomen Territorien für die indigenen Nationalitäten nicht „einen Staat innerhalb eines anderen Staates“ (Comisión Especial de Asuntos Indígenas 1988: 13f.) bedeutet, ein Vorwurf, der bis heute gegen die Plurinationalität verwendet wird. Die CONAIE selbst sieht diesen Zeitpunkt als Geburt der Forderungen nach einem plurinationalen Staat als Alternative zum bestehenden Staat (CONAIE 1999: 51). Bestimmte Bestandteile, die damals zum ersten Mal formuliert wurden, blieben mit dem Begriff der Plurinationalität verbunden. So wurde eine Anerkennung des Kichwa als nationale und der anderen indigenen Sprachen als lokale Amtssprachen neben dem Spanischen gefordert, eine Anerkennung der indigenen Territorien und ihrer sozialen, politischen und wirtschaftlichen Strukturen, sowie der indigenen Medizin. Der plurinationale Staat wurde definiert als

„Aufbau einer neuen dezentralisierten, kulturell heterogenen politischen und Verwaltungsstruktur, offen für die eigene und teilnehmende Repräsentation aller indigener Nationalitäten und gesellschaftlicher Sektoren [...] eine institutionelle Erweiterung, die die soziokulturelle Verschiedenheit Ecuadors in einem neuen Begriff von Staat, Entwicklung und Staatsbürgerschaft aufgreift" (Bernal 2000: 42f.).

Auf ihrem zweiten Kongress 1988 bestätigte die CONAIE diese Interpretation und betont die Bedeutung der regionalen und sozialen Unterschiede. Insbesondere der Klassengegensatz soll in einem plurinationalen Staat beachtet werden; dieser soll also nicht nur die Rechte der indigenen Nationalitäten garantieren, sondern alle Formen von Unterdrückung und Ausbeutung, ob individuell oder kollektiv, reflektieren und zu lösen versuchen (CONAIE 1988, in Simbaña 2006: 66f.).

Auch die zu diesem Zeitpunkt bereits weitgehend bedeutungslose FEI fordert auf ihrem siebten Kongress 1989 die Anerkennung Ecuadors als multinationales und multikulturelles Land, wobei sie auf die Russische Revolution verweist, die als 
erste das Problem der Nationalitäten lösen konnte (FEI 1989, in: Becker 2011a: 199).

In den 1990ern wurde der Begriff der Plurinationalität von vielen der indigenen Organisationen der Amerikas übernommen und den jeweiligen lokalen und nationalen Gegebenheiten angepasst. Auf dem II. Kontinentalen Gipfeltreffen der indigenen Völker Amerikas (II Cumbre Continental de los Pueblos Indígenas de América) im Juni 2004 in Quito wurde die Plurinationalität als kontinentales Projekt angenommen (Simbaña 2008: 106f.).

Unumstritten ist, dass der Begriff der Plurinationalität im Sinne einer Neugründung der ecuadorianischen Nation zum ersten Mal im Rahmen der indigenen Erhebung des Inti Raymi 1990 eindeutig formuliert und der Gesellschaft präsentiert wurde (Dávalos 2003: 44; Pacari 2007: 24). Zusammen mit den in den 16 Punkten aufgestellten Forderungen nach einer Lösung der bestehenden Landkonflikte, wurde gefordert, dass Ecuador im ersten Artikel seiner Verfassung zu einem plurinationalen Staat erklärt wird. Diese Erklärung sollte eine Anerkennung der indigenen Nationalitäten als der mestizischen Nationalität gleichberechtigte Gruppen sein (Walsh 2008: 17f.). Eingebettet in diese Forderung - aber zu diesem Zeitpunkt noch nicht ausformuliert - war die Wiedergewinnung und Verteidigung der indigenen Territorien und der Aufbau von Strukturen der Selbstverwaltung für die indigenen Nationalitäten (Pacari 2007: 24). Schon 1990 kamen unter den Indigenen Anklagen gegen einen ,kolonialen und rassistischen Staat und eine ausgrenzende Gesellschaft" (Cholango, in Tamayo 2007: 7f.) auf, von denen die Forderung nach einer Verfassungsgebenden Versammlung abgeleitet wurde, die den Aufbau eines plurinationalen Staates, territorialer Autonomie und einer neuen lateinamerikanischen Ordnung des gegenseitigen Respekts betreiben sollte (Cholango in Tamayo 2007: 7f.).

Seit der ersten öffentlichen Formulierung der Forderung nach Plurinationalität hat die CONAIE zu weiteren Gelegenheiten diese Forderung wiederholt - nicht in immer genauerer (Walsh 2009: 174), sondern in stets leicht veränderter Ausformulierung. Walsh sieht diese Momente nur im Bereich der Parteienpolitik, für sie sind die weiteren wichtigen Präsentationen der Plurinationalität die Verfassungsgebende Versammlung von 1997/98, die Regierungsbeteiligung unter Gutiérrez 2003 und die Verfassungsgebende Versammlung von 2007/08 (Walsh 2008: 17-19). Auch wenn diese Gelegenheiten unzweifelhaft eine große öffentliche Wirkung entfalten konnten, sind die internen Prozesse der CONAIE und ihre Reaktionen auf gesellschaftliche Entwicklungen hilfreicher, um den Begriff der Plurinationalität zu verstehen. So wurde der Begriff der Plurinationalität zum ersten Mal im Politischen Projekt der CONAIE (1994) ausgearbeitet, das auf ihrem IV. Nationalen Kongress 1993 verabschiedet und im folgenden Jahr veröffentlicht wurde. Daher ist die Forderung nach einer Integration der Plurinationalität in die Verfassung von 1998 
durch die MUPP-NP (García/Tuasa 2007: 36) von großer politischer Bedeutung war aber keinesfalls eine Öffnung des Begriffs durch den Einfluss der anderen sozialen Bewegungen innerhalb MUPP-NP (Walsh 2008: 18). Die zweite genaue Ausarbeitung der Plurinationalität findet in der CONAIE zwischen 1996 und 1997 statt, die dritte - und bislang letzte - zwischen 1999 und 2001. 1997 wird eine neue Version des Politischen Projekts (CONAIE 1997) veröffentlicht, im dem die Plurinationalität um den Begriff der Interkulturalität ergänzt wird. Das wird im Politischen Projekt von 2001 weiter ausgearbeitet. Dieser Text erlaubt es auch, die Politik für den Nationalen Regierungsplan (Políticas para el Plan de Gobierno Nacional) von MUPP-NP und CONAIE von 2003 zu verstehen. Begriffe wie Selbstbestimmung, plurinationale Demokratie und Interkulturalität als vom Staat garantierter, auf gegenseitigem Respekt und Anerkennung basierender Prozess (Walsh 2008: 19), stammen direkt aus dem Politischen Projekt. Aus dieser Perspektive lassen sich die Konflikte in der Verfassungsgebenden Versammlung von 2007/08 verstehen und die Schwierigkeiten der CONAIE, die Entgegensetzung der Begriffe Plurinationalität und Interkulturalität nachzuvollziehen.

Obwohl die CONAIE dies nie impliziert hat, wurde ihre Forderung nach Plurinationalität oft als Versuch missverstanden, die Nation zu spalten und indigene Staaten auf ecuadorianischen Grund zu errichten (Walsh 2009b: 233). Der CONAIE wird vorgeworfen, unter dem Namen der Plurinationalität das Land teilen zu wollen, anstatt für die Rechte der Indigenen zu kämpfen (Ayala Mora 2011: 53). Die Plurinationalität wird als eine radikal indigene Forderung verstanden, die sich nicht auf die Gesamtgesellschaft richtet, sondern die nationale Einheit in Frage stellt (Walsh 2009: 174). Sie wird als ethnozentrisch oder rassistisch verstanden und den Vertretern dieses Vorschlags wird vorgeworfen, in die Vergangenheit zurückkehren und eine Gesellschaft unter Vorherrschaft der Indigenen aufbauen zu wollen (Ayala Mora 2011: 51). Die CONAIE selbst hat diesem Vorwurf immer widersprochen und erklärt, dass der Vorschlag der Plurinationalität den Staat nicht teilen soll, sondern eine neue, dezentrale, heterogene und offene politische und Verwaltungsstruktur aufbauen will (CONAIE 1999: 51f.). Auch einige mestizische Wissenschaftler haben früh auf die gesamtgesellschaftliche Bedeutung der Plurinationalität hingewiesen: „Der Vorschlag war an alle Gesellschaften gerichtet, die Ecuador bilden, und geht durch alle Regionen und alle Klassen hindurch.“ (Ramón 1992a: 20$)^{16}$

16 Ramón hat seine Auffassung später geändert und glaubt jetzt dasselbe von der Interkulturalität -vergleichbar mit Walsh- während er behauptet, die Plurinationalität würde die Differenz anerkennen, aber nicht die Einheit in der Vielfalt und „den Staat dazu bringen, die indigenen Völker als nationale Minderheiten zu behandeln und nicht auf aktive Weise alle rassistischen, ausschließenden, ungerechten und herrschenden monokulturellen 
Eine andere Kritik am Begriff der Plurinationalität ist der Vorwurf mangelnder Klarheit. So sei es sich statt ,einer Parole, die ein klares und in sich abgeschlossenes politisches Projekt enthält, nur [...] eine vage Hoffnung, die auf sehr unterschiedliche Weise interpretiert werden konnte" (Ramón 1992a: 9f.). Forderungen nach Territorien, Selbstverwaltung, Autonomie, politischer Partizipation und Wandel in Staat und Gesellschaft vermischen sich in einem ,komplexen, ungenügend behandelten und verstandenen Problem“ (Ramón 1992a: 10), dem der Plurinationalität. Diesem Begriff wird vorgeworfen, dass man seinen Inhalt nie definiert hat und die vielfältige Produktion von Texten über die Plurinationalität nur zur Verwirrung beigetragen hat (Ayala Mora 2011: 45). „Die Begriffe erweisen sich als mehrdeutig und mit der Zeit haben sich ihre Vieldeutigkeit und Verwirrung verstärkt.“ (Ayala Mora o.J.: 11) Ein repräsentatives Beispiel für diese Verwirrung ist die Gegenüberstellung von zwei Arten von Plurinationalität, die Sánchez-Parga (1997: 118) unternimmt. Für ihn ist die Plurinationalität zum einen die einfache Forderung ,aller verschiedenen Kulturen und ethnischer Identitäten“ (Sánchez-Parga 1997: 118) nach Anerkennung und Teilhabe an der nationalen Identität. Davon unterscheidet er die Forderung, die ethnischen Gruppen ,als ,Nationen“ [anzuerkennen, P.A.], was nicht anderes bedeutet als eine besondere Annahme des Begriffs ,Ethnie", dass sich aber vom Begriff des Nationalen das dem Nationalstaat eigen ist, unterscheidet" (Sánchez-Parga 1997: 118). Sánchez-Parga ist also nicht in der Lage, die vermeintliche Unterscheidung, die er konstruiert - und die die indigenen Organisationen schon mit dem Begriff der Nationalitäten deutlich machen -, als Ausgangspunkt für Forderungen $\mathrm{zu}$ sehen, die das Ethnische und nur die Indigenen Betreffende überschreitet. Ein weiteres Beispiel ist Cabrera (2002: 96), die versucht, Plurinationalität als deskriptives Konzept dem Multikulturalismus unterzuordnen. Nach ihr unterschieden sich innerhalb der Kategorie der multikulturellen Staaten plurinationale Staaten mit territorial voneinander abgegrenzten und mit mehr oder weniger festen Institutionen versehenen Ethnien von pluriethnischen Staaten, in denen das Ethnische rein privater Natur ist, also keine eigenen Institutionen hat (Cabrera 2002: 96). Darin folgt sie den Definitionen von Kymlicka (etwa: 2003: 154f.). Es ist offensichtlich, dass diese Unterscheidung nicht zur Klärung der Begriffe der Indigenenbewegung in Ecuador beiträgt, die den Begriff der Ethnie in der Regel nicht verwendet und Plurinationalität nicht nur deskriptiv versteht. Neben diesen nicht-indigenen Wissenschaftlern gibt es auch innerhalb der Indigenenbewegung selbst Stimmen, die von einer mangelnden Klarheit des Projektes der Plurinationalität sprechen und die Bewegung aufrufen ,,in ihren Aktionslinien und pro-

Strukturen ändern wollen.“ (Ramón 2009: 125f.) Dieses Kapitel beweist, dass er mit seinem späteren Urteil Unrecht hat. 
grammatischen Konkretisierungen des Projektes der Plurinationalität“ (Simbaña 2006: 88) zu klareren Definitionen zu kommen.

In den drei Jahrzehnten ihres Bestehens hat sich die Plurinationalität weiterentwickelt, ist jedoch „ein Vorschlag im Aufbau“ (Ramón 1998) geblieben, der sich nicht weiter konkretisieren ließ und so an Mobilisierungsfähigkeit einbüßte (Ramón 1998). So ist die Plurinationalität weniger ein fester Begriff als eine „Einstellung für den andauernden Aufbau der Nationalidentität, die die Besonderheiten der Nationalgesellschaft ausdrücken muss" (UIAW 2004: 152). So erklärt sich auch das unklare Verhältnis der Plurinationalität zur Interkulturalität (Ospina 2009: 142), die ähnlich offen ist. Als offener und pluraler Begriff umfasst die Plurinationalität eine Reihe von verschiedenen Vorschlägen, die von verschiedenen Organisationen getragen werden (Ramón 1992: 20) - also auch konfliktiv sein können. Einige Organisationen vor allem des Amazonasgebietes verstehen die Plurinationalität radikal im Sinne einer weitgehenden Verbannung des Zentralstaates aus ihren Territorien, die sie selbstständig verwalten wollen. Andere Organisationen, etwa ECUARUNARI, versprechen sich mehr von einer stärkeren Rolle des Staates in der Kontrolle von strategischen Ressourcen. Also auch in der Definition des Begriffes der Plurinationalität sind die Differenzen zwischen ethnischen und klassistischen Positionen innerhalb der CONAIE erkennbar (Larrea 2008: 36).

Die Behauptung, die CONAIE hätte sich 1993 für den Begriff des Multinationalismus ausgesprochen (Macdonald 2002: 183) ist falsch. Dem liegt ein Übersetzungsfehler zugrunde, der in der englischen Übersetzung (CONAIE 1993) der Beschlüsse des IV. Nationalen Kongresses der CONAIE vorkommt, die später als Politisches Projekt (CONAIE 1994) veröffentlicht wurden. Es gab keine Verschiebung des ,Lexikon der Ethnizität von ,multinational“ zu ,plurinational““ (Macdonald 2002: 183) in der Mitte der 1990er Jahre, der Begriff der Plurinationalität wurde seit spätestens 1988 (Comisión Especial de Asuntos Indígenas 1988: 8) nicht mehr verändert - ganz im Gegensatz zu anderen Begriffen der Indigenenbewegung. Ob der Begriff der Multinationalität tatsächlich wegen seiner Ähnlichkeit zur Bezeichnung ,multinationale Unternehmen' verworfen wurde (Trujillo 2009: 64) kann nicht bestätigt werden. Die Verwendung des Begriffs multinational gerade zu Beginn der 1990er dürfte weniger durch eine mangelnde Klarheit der indigenen Organisationen, sondern eher durch ein mangelndes Verständnis der Intellektuellen, die beide Begriffe bedeutungsgleich verwenden (etwa: Moreno/Figueroa 1992: 57) zu erklären sein.

Der Begriff der Plurinationalität wurde in erster Linie von der CONAIE entwickelt und verwendet, einer Organisation, die den Aufbau eines plurinationalen Staates als ihr oberstes Ziel betrachtet (CONAIE 2001: 3, 8). Jedoch hat sie dabei punktuelle Unterstützung durch andere Organisationen erfahren. Vor allem die FENOCIN war an der Entwicklung des Begriffes im Rahmen des Gesetzes der 
Nationalitäten von 1988 beteiligt und unterstützte die CONAIE in der Verfassungsgebenden Versammlung von 1998 bei ihrem Kampf, die Plurinationalität in die neue Verfassung aufzunehmen (Vogel 2008: 15). Die Verbindung der beiden Organisationen schwächte sich aber 2001 und 2002 im Streit um die Leitung der CODENPE ab (Vogel 2008: 15). Tatsächlich ist die CONAIE die einzige nationale Organisation, die die Indigenen konsequent als Nationalitäten begreift - mit allen Inhalten, die in den vorherigen Kapiteln beschrieben wurden. Die anderen Indigenenorganisationen und die Organisationen der Afroecuadorianer können sich mit diesem Begriff nicht identifizieren (García/Tuasa 2007: 31) und sehen daher auch keinen Gewinn im Begriff der Plurinationalität. Obwohl sie kollektive Rechte für die Indigenen und Afroecuadorianer befürworten, lehnen sie die Plurinationalität ab. Stattdessen fordern sie eine Interkulturalität (Ayala Mora o.J.: 11). FENOCIN, FEINE und FEI verstehen die Plurinationalität als einen Versuch der CONAIE, sich als alleinige Repräsentation aller Indigenen zu definieren und als solche die entsprechenden staatlichen Institutionen zu kontrollieren. Daher ist für sie die Plurinationalität ein Versuch, die Indigenenbewegung zu spalten (Ayala Mora 2011: 47f.).

Die Verfassungsgebende Versammlung 2007/2008 war auch von Auseinandersetzungen über diese Positionen geprägt. FENOCIN und FEINE sahen die Plurinationalität als Angriff auf die Einheit der Nation und stellten dem einen interkulturellen und inklusiven Staat mit einem egalitären Staatsbürgerschaftsbegriff gegenüber (Grijalva 2008: 55). Die Dynamik der Kulturen sollte über Zusammenarbeit, Verständnis und den Abbau von Diskriminierung im Rahmen der interkulturellen Verfassung gewährleistet werden (Vogel 2008: 16f.). Die Plurinationalität wurde von den anderen indigenen Organisationen als ethnozentrisch und auf Autonomie und Differenz ausgerichtet wahrgenommen, wohingegen die Interkulturalität auf die Beziehungen und Begegnungen zwischen Verschiedenen abzielt und so inklusiv wirkt (Grijalva 2008: 55; Ayala Mora o.J.: 16). Der ideologische Konflikt wird also überlagert von einem organisatorischen Konflikt um die CONAIE, die sich als Regierung der Indigenen begreift und dementsprechend auch über die anderen indigenen Organisationen bestimmen will (Ospina 2009: 139).

\section{b. Definition der Plurinationalität}

Der Begriff der Plurinationalität setzt die Existenz von nicht nur kulturell, sondern auch politisch, wirtschaftlich und historisch unterschiedlichen Völkern und Nationalitäten in Ecuador voraus (CONAIE 2001: 5). Diese unterschiedlichen Völker und Nationalitäten sollen nicht nur staatlich anerkannt, sondern als autonome und selbstverwaltete Einheiten in den Staat integriert werden (Ojeda Segovia 2004: 49). Ausgehend von einer kulturellen Anerkennung der indigenen Völker und Nationalitäten soll die Plurinationalität den Staat und die politischen, wirtschaftlichen und 
soziokulturellen Organisationsformen neu ordnen (Simbaña 2008: 108). Die Plurinationalität ,garantiert die volle Ausübung der Rechte aller Nationalitäten, die es im Land gibt“" (CONAIE 2001a). Dieses politische Prinzip (CONAIE 1999: 72) macht eine Neuordnung und Öffnung der politischen, rechtlichen, wirtschaftlichen und Verwaltungsstrukturen nötig (CONAIE 1994: 12; Walsh 2009b: 234). Der Aufbau der Plurinationalität wird verstanden

„als ein Modell der politischen, wirtschaftlichen und soziokulturellen Organisation und ein Regierungssystem, das die Gerechtigkeit, die Freiheit und die gleichberechtigte Entwicklung der Gesamtheit der ecuadorianischen Gesellschaft und aller ihrer Regionen und Kulturen sucht" (CONAIE 2010: 2).

Daher unterscheidet die CONAIE in ihrem Politischen Projekt die Nation als „menschliche Gruppe, vereint durch spezielle Verbindungen der kulturellen, geschichtlichen, politischen, wirtschaftlichen und sprachlichen Homogenität" (CONAIE 2001a) von der plurinationalen Nation, in der die Nationalitäten, die auf einem zusammenhängenden Territorium und unter einer Regierung leben (CONAIE 2001a), durch eine kulturelle Identität, eine gemeinsame Geschichte und den Willen, der Nation anzugehören (Acosta 2009: 19), vereint werden. Die plurinationale Regierung ist dezentralisiert und autonom; die Nationalitäten haben im Rahmen der plurinationalen Demokratie (CONAIE 2001a) direkt an ihren Handlungen und Beschlüssen teil (CONAIE 2001a). Die Nationalitäten üben in Koordination mit dem Zentralstaat eine Autonomie in ihren Territorien aus. So können sie über ihre eigenen Autoritäten in den Bereichen der Verwaltung, des Rechts, der Politik, der Wirtschaft, des Sozialen und der Kultur Entscheidungen treffen und Kontrolle ausüben ohne sich dadurch von den anderen Teilen des Staates abzugrenzen (CONAIE 2001: 5; CONAIE 2001a). Im Rahmen der Gleichheit, Einheit und Solidarität der Nationalitäten Ecuadors soll jede Nationalität das Recht haben,

,ihren eigenen Prozess der wirtschaftlichen, sozialen, kulturellen, wissenschaftlichen und technologischen Entwicklung zu bestimmen, um die Entwicklung ihrer kulturellen und politischen Identität und so die gesamte Entwicklung der Nation zu garantieren“ (CONAIE 1994: 12).

Der Gesellschaftsvertrag soll der - ethnischen, geschlechtlichen etc. - Differenz geöffnet werden und so der Rassismus, die Exklusion und die Ungleichheit des modernen Nationalstaates gegen das Andere überwunden werden. Dadurch soll die Teilhabe aller an Staat und Gesellschaft garantiert werden (Chuji 2008).

Der plurinationale Staat soll, im Sinne der „Einheit in der Vielfalt“ (CONAIE 2007: 10), Rassismus und Regionalismus im Land zerstören und mit ihnen „die 
kolonialen und monokulturellen Schatten, die ihn seit fast 200 Jahren begleitet haben“ (CONAIE 2007: 5). Über eine Veränderung der politischen und wirtschaftlichen Strukturen soll er die „Entkolonisierung unserer Nationen und Völker“ (CONAIE 2007a: 5) und des afroecuadorianischen und montubischen Volkes (CONAIE 2010: 2) ermöglichen. Der plurinationale Staat stellt den bisher bestehenden, uninationalen Staat in Frage, indem er ihn der Differenz öffnen und demokratisieren will (Walsh 2009: 176).

„Seine Annahme bedeutet, eine dezentralisierte politisch-administrative Struktur aufzubauen, die kulturell heterogen und offen für die selbstständige und partizipative Repräsentation aller sozialer Sektoren ist.“ (CONAIE 1997a: 10)

In diesem Sinne baut er auf die Interkulturalität als Möglichkeit der Rekonfiguration des Staates und der Gesellschaft (Walsh 2008: 26; Walsh 2009b: 238). So sollen die Prinzipien der Demokratie, ,Gleichheit, Freiheit, Brüderlichkeit, sozialer Frieden“" (CONAIE 2001: 2f.), die der uninationale Staat nicht einhalten konnte, ermöglicht werden. Eurozentrismus und Ethnozentrismus sollen Raum machen für ,den Aufbau einer neuen wirtschaftlichen, sozialen, politischen, kulturellen und rechtlichen Ordnung des neuen plurinationalen Staates“ (CONAIE 2001: 2f.).

Schon früh hat die CONAIE die Veränderung der Verfassung Ecuadors in den Mittelpunkt ihres Prozesses des Aufbaus eines plurinationalen Staates gestellt. Seit 1990 war eine Neufassung des ersten Artikels der Verfassung eine wiederkehrende Forderung. Von der Erklärung Ecuadors zu einem plurinationalen und plurikulturellen Land (CONAIE 1992a) ausgehend sollte eine neue Verfassung mit der Tradition der früheren, an anderen Ländern orientierten Verfassungen, brechen. Über diese neue plurinationale Verfassung sollte der Staat gestärkt und separatistische Tendenzen bekämpft werden (García/Tuasa 2007: 9). Diese Stärkung des Staates speist sich auch aus dem Kampf der CONAIE gegen den Neoliberalismus und die Versuche, den Staat und seinen Einfluss zu reduzieren. Die Plurinationalität soll den Staat demokratisieren und ,die soziale und gemeinschaftliche Kontrolle über seine Institutionen und Politiken wiederherstellen“ García/Tuasa 2007: 9).

Die CONAIE hat zu verschiedenen Gelegenheiten konkrete Pläne für den Aufbau der „Neuen Plurinationalen, Kommunitären, Kollektiven, Egalitären, Vielsprachigen und Interkulturellen, gerechten Gesellschaft mit Vision der nachhaltigen Entwicklung“ (CONAIE 2001: 3f.) vorgestellt. Sie ist durch drei großen Dimensionen charakterisiert: den Aufbau einer Interkulturalität als gegenseitige Wertschätzung und Einheit, die Transformation des Staates und des wirtschaftlichen, politischen und soziokulturellen Systems im Sinne einer Demokratisierung und Öffnung und die Anerkennung und Integration der ,gemeinschaftlichen, territorialen Regierungen“ (CONAIE 2007a: 7f.) der indigenen Nationalitäten als den Aufbau des 
Staates von unten. Dazu gehört die Veränderung der Struktur der unabhängigen Gewalten des Staates. Die Struktur von Judikative, Legislative und Exekutive soll verändert werden. Sie müssen nicht nur die Verschiedenheit der Gesellschaft widerspiegeln und die Rechte der Nationalitäten achten (Macdonald 2002: 184; CONAIE 2007a: 5), sondern gesellschaftlich kontrolliert, widerrufbar und rechenschaftspflichtig sein (García/Tuasa 2007: 10; CONAIE 2007a: 7f.). So soll im Rahmen der „Kommunitären und Partizipativen Plurinationalen Demokratie“ (CONAIE 2001: 4f.) die volle Teilhabe der Nationalitäten und Völker und der anderen Teile der Gesellschaft an den großen Entscheidungen ermöglicht werden (CONAIE 2007a: 7f.). Diese Demokratie soll nicht nur repräsentativ, sondern auch partizipativ und gemeinschaftlich, also offener und breiter sein (Macas 2005: 38). ${ }^{17}$ Dazu soll sie dezentral organisiert sein und von den lokalen Gemeinschaften ausgehen (Santillana 2006: 241), im Fall der indigenen Nationalitäten sind das die jeweiligen autonomen und selbstverwalteten Territorien ${ }^{18}$ (Ramón 1998). „Die gemeinschaftliche territoriale Regierung ist eine soziale Macht, ein Ausdruck der Souveränität der Gesellschaft, die die Verwaltung des Staates kontrollieren kann und muss.“ (CONAIE 2007a: 7f.) Die lokalen Gemeinschaften sollen sich gemäß kommunitärer Formen der Demokratie selbst regieren und ihre Territorien als Lebensräume selbstbestimmt verwalten. Dadurch soll die ecuadorianische Nation und der ecuadorianische Staat verbessert werden ,um die harmonische und ausgeglichene Entwicklung“ (CONAIE 2001: 4f.) der Gesellschaft zu ermöglichen. Erst so ist der Aufbau einer neuen Gesellschaft, eines neuen Staates und einer ,wahren Nation, in der wir alle repräsentiert sind“ (Macas 1991: 12) möglich, oder „ein Staat im dem wir alle Platz haben können“ (Macas 2005: 39).

Im Bereich der Wirtschaft soll sich der plurinationale Staat an einem „Modell der plurinationalen Entwicklung“ (CONAIE 1997: 29; CONAIE 2001a) ${ }^{19}$ orientieren, das von familiären-persönlichen, kommunitären-selbstverwalteten, staatlichen und gemischten Besitzformen ausgehend über die Rationalisierung der vorhandenen Ressourcen funktionieren soll (CONAIE 2001a). Der Staat selbst soll diese Besitzformen harmonisieren, um so ein wirtschaftliche, politische, kulturelle, technologi-

17 Andolina (1998: 67f.) behauptet, dass die plurinationale Demokratie das Konsensprinzip an die Stelle des Mehrheitsprinzips stellt, alle Entscheidungen also einstimmig getroffen werden müssen. In den konsultierten Quellen ist davon nicht die Rede.

18 Es sei daran erinnert, dass sich die CONAIE als Regierung der indigenen Nationalitäten begreift und daher in diesem Modell die Kontrolle über die indigenen Territorien und der auf Indigene gerichteten Politikbereiche für sich beansprucht (Ramón 1998; CONAIE 2007a: 7f.).

19 Im Politischen Projekt 1994 noch „Modell der kommunitären ökologischen Planwirtschaft" (CONAIE 1994: 30). 
sche und wissenschaftliche Gleichheit der Gesellschaft zu erreichen und die Befriedigung der Bedürfnisse aller Mitglieder dieser Gesellschaft zu gewährleisten (CONAIE 2001: 4). Der plurinationale Staat steht für das Ende der Ausbeutung und „die Einbettung anderer Rationalitäten in die zur Zeit herrschende wirtschaftliche Rationalität, die mit der physischen Zerstörung unseres Planeten droht" (Macas 2005: 38f.). Er soll „,ein soziales, solidarisches, ökologisches, gerechtes, souveränes, geplantes und einbeziehendes Wirtschaftsmodell“ (CONAIE 2007a: 5) aufbauen. So soll der Staat im Sinne seiner Souveränität die natürlichen Ressourcen kontrollieren und in den Dienst der Gesellschaft stellen, ohne sie zu verstaatlichen. Durch seinen Bezug auf die lokalen und gemeinschaftlichen Produktionsformen soll dieses Entwicklungsmodell die Diversifikation der Produktion, die Stärkung der Ernährungssicherheit, die Entwicklung eigener Technologien und die angemessene Einbindung traditioneller Produktionsformen gewährleisten (Maldonado 2006: 113f.). Das Wasser soll als staatliches Eigentum von den Nutzern verwaltet werden (Cholango, in Tamayo 2007: 15). Wo sie sich in indigenen Territorien befinden sollen die natürlichen Ressourcen der Kontrolle der Nationalitäten unterstellt werden (Macas 1991: 19). Auch die öffentlichen Dienstleistungen und Unternehmen sollen als „öffentliche Verantwortung“ (CONAIE 2007a: 5) des Staates nicht als Waren behandelt werden, sondern allen Bürgern gleichermaßen offenstehen, um so soziale Ungleichheit und Diskriminierung zu bekämpfen. Der plurinationale Staat soll die Preise auf dem Binnenmarkt festsetzen und regulieren, um so die Spekulation und die Bildung von Monopolen zu bremsen. Der Außenhandel soll ausschließlich vom Staat geführt werden, wobei Überschussproduktion exportiert und vor allem im Land nicht vorhandene Rohstoffe importiert werden sollen, um so die wirtschaftliche Entwicklung des Landes zu gewährleisten. „Die Importe von Luxusgütern werden verboten." (CONAIE 2001: 26)

Der plurinationale Staat zeichnet sich durch die rechtliche Anerkennung seiner Nationalitäten im Sinne eines Rechtspluralismus aus. Diese werden als „,kollektive, rechtlich-politische und historisch konstituierte Einheiten und als kollektive Rechtssubjekte“ (Simbaña 2008: 111) anerkannt, wodurch neben individuellen Rechten und einer individuellen Zugehörigkeit zum Staat auch kollektive Rechte und Zugehörigkeiten geschaffen werden (Simbaña 2008: 112). In diesem Sinne soll der plurinationale Staat eine rechtliche Neuordnung durchführen, die eine Stärkung des Rechtssystems jeder Nationalität im Rahmen ihrer Autonomie ermöglicht (CONAIE 2001: 11). So sollen die autonomen Regierungen der Nationalitäten in ihren Territorien ihre internen Angelegenheiten im Rahmen des Nationalstaates auch rechtlich selbst regeln können (Macas 1991: 11). Um dieses System aufbauen zu können, wird eine territoriale Neuordnung des Landes nach den historischen Besitzansprüchen und Bedürfnissen der Nationalitäten durchgeführt (CONAIE 2001: 12). Am Anfang der plurinationalen Neugründung des Staates steht ein grundlegen- 
des Studium der bestehenden Gesetze, das es möglich machen soll, diskriminierende oder unpassende Gesetze aufzuheben oder zu verändern, damit die Rechte der gesamten Bevölkerung gewahrt werden (Macas 1991: 11). So soll das Problem, dass die Territorialität der indigenen Nationalitäten für die nicht-indigenen Bürger des Landes darstellen kann (Santillana 2006: 250), gelöst werden.

\section{c. Plurinationalität als politischer Diskurs}

Der Begriff der Plurinationalität ist die Verbindung der beiden wichtigsten Strömungen in der CONAIE. Er vereint die klassenanalytische mit der ethnischen Perspektive und erlaubte es der CONAIE so, ,sich an die Spitze des gesellschaftlichen Widerstandes gegen die neoliberale Politik“ (Ramírez Gallegos 2009: 65) zu stellen. Dadurch wird die Plurinationalität zu ,einer Art strategisch-politischem Mittel“ (Dávalos 2003: 43), um die inneren Widersprüche der Indigenenbewegung zu überwinden und ihre Konflikte mit dem Staat zu steuern. Die Tatsache, dass dieser Begriff nicht klar definiert ist, hilft, eine breite Basis zu schaffen, die ihre spezifischen Forderungen und Bedürfnisse in den Begriff der Plurinationalität legen kann, ohne dass dies durch festgelegte Definitionen von vorneherein ausgeschlossen wäre (Dávalos 2003: 43). Die Forderung der Plurinationalität erlaubt es - im Gegensatz $\mathrm{zu}$ den Forderungen der Indigenenbewegung bis in die 1980er Jahre nach einer Agrarreform - breite Bündnisse über die Teilung zwischen Stadt und Land hinaus aufzubauen, indem sie verschiedenste Teilforderungen integriert. Die Gleichberechtigung spezifisch indigener Forderungen, etwa nach Territorialität, indigenem Recht oder indigener Medizin, mit allgemeinen Anliegen macht die Plurinationalität zu einem zentralen Begriff für die Indigenenbewegung. In ihr lassen sich die vielfältigen und teils widersprüchlichen Ideen und Forderungen der Indigenenorganisationen zusammenfassen. Dadurch, dass die Plurinationalität neben den sozialen auch die ethnischen Differenzen betont, geschieht diese Integration unter Hervorhebung eben dieser Aspekte. „In diesem Sinne [...] werden sich die ethnischen Behauptungen nur als ein gesellschaftlicher Vorschlag gegen ein bestimmtes Modell der Gesellschaft, das solche Differenzen ausschließt, realisieren lassen." (Sánchez-Parga 1988: 110f.) Gleichzeitig ist dieser Diskurs etwas Neues, dass der Indigenenbewegung als Alleinstellungsmerkmal dient. „Der Begriff der Plurinationalität erlaubt der Bewegung die Behauptung ihrer eigenen politischen Identität.“ (Santillana 2006: 241)

Innerhalb der Indigenenbewegung dient der Begriff der Plurinationalität den anderen indigenen Organisationen zur Abgrenzung von der CONAIE und ihrem vermeintlich ethnizistischen Diskurs. Gerade die FENOCIN entwickelt ihre Forderungen und Begriffe als Antwort auf die Herausforderung der Plurinationalität. Aus ihrer Sicht war es in einem ersten Schritt notwendig, explizit ethnische Forderungen 
aufzustellen, um zeigen zu können, dass Ecuador kein homogener Staat ist und dass Rassismus und Ausgrenzung strukturelle Probleme sind (FENOCIN 1999: 150). In diesem Sinne versteht sie die Forderung nach Plurinationalität als Trägerin anderer Forderungen, wie die nach kollektiven Rechten, die größeren Erfolg hatten (FENOCIN 1999: 151). Sie trennt also bestimmte Forderungen aus dem Komplex Plurinationalität heraus, um sie selbst vertreten und in ihren eigenen Begriff der Interkulturalität integrieren zu können. Dazu gehört das Recht auf Selbstbestimmung der indigenen Völker in ihren autonomen Territorien genauso wie ihr Recht, ihre Entwicklung selbst bestimmen zu können (FENOCIN 1999: 151). So kann die FENOCIN Teile der CONAIE als ,ethnische Fundamentalisten“ (FENOCIN 1999: 150) angreifen, ohne notwendigerweise grundlegend andere Forderungen aufstellen zu müssen.

Die Zentralität des Begriffs der Plurinationalität in ihrem Diskurs hat die CONAIE angreifbar gemacht. Die diskursive Verwendung des Begriffs steht nicht immer im Einklang mit der politischen Praxis der verschiedenen Organisationen, die diesen Begriff verwenden. So sind die relativen Fortschritte der Indigenenbewegung in der ersten Hälfte der 1990er Jahre - etwa die Übertragung von Landrechten oder die Institutionalisierung der zweisprachigen interkulturellen Erziehung - zwar konkrete Gewinne - allerdings stehen sie nur bedingt mit dem Ziel, den gesamten Staat und die gesamte Gesellschaft zu verändern, in Beziehung. Die autonomen Bereiche, die die CONAIE und ihre Organisationen für sich gewinnen konnte, sind nach wie vor unter der Kontrolle des Staates, der versucht, sie für seine Zwecke zu benutzen (Viteri 1997: 54f.).

„Die Plurinationalität funktioniert nicht über die Tatsache, den klassischen Staat in einen gewaltigen Greyerzer-Käse zu verwandeln, um so in seinen Löcher leben zu können und die Abhängigkeit, den Paternalismus und den Charakter vertikaler Führung des Staates zu verewigen. Es geht nicht darum, (den Staat) um Zugeständnisse zu bitten, diese dürfen nicht existieren, wenn wir von einer partizipativen und pluralen Zivilgesellschaft reden. Genauso wenig geht es darum, die Machtpyramide umzudrehen, sondern darum, das Land auf Grundlage einer konzertierten und gleichberechtigten Verwaltung unserer Verschiedenheiten und Konflikte zu verändern.“(Viteri 1997: 55) ${ }^{20}$

Also dient die Plurinationalität auch dazu, die Tagespolitik der CONAIE von ihrem Diskurs aus zu kritisieren - im vorgestellten Fall durch ein Mitglied der CONAIE selbst.

20 Es sei angemerkt, dass der Greyerzer-Käse sich nicht dadurch auszeichnet, viele Löcher zu haben. 
Plurinationalität ist ein doppelter Begriff: er verbindet den Kampf gegen die wirtschaftliche - Ausbeutung mit dem Kampf gegen die - ethnische - Unterdrückung, wodurch die CONAIE in den 1990ern zum Zentrum der Bewegung gegen die neoliberale Reformpolitik werden konnte. Aus einer nicht-marxistischen Perspektive wird der Charakter der Wirtschaft als etwas Politisches und vom Staat nicht $\mathrm{zu}$ Trennendes hervorgehoben und die fehlende demokratische Teilhabe daran problematisiert (Santillana/Herrera 2009: 335). Die Kultur und die kulturelle Unterdrückung der Indigenen ist aus dieser Perspektive auch wirtschaftlich und sozial, die Verbindung der verschiedenen Arten von Unterdrückung lässt sich nur durch „die Dekonstruktion aller sozialer Beziehungen einschließlich des Staates“ (Vargas 2009: 99) bekämpfen. Das Verständnis des ecuadorianischen Staates als „Projekt der herrschenden Klassen, das die Mehrheit der Bevölkerung ausschloss“ (Santillana 2006: 217) erlaubte den Anschluss der CONAIE an die urbanen linken Strömungen. Mit dem Verweis auf die Verbindung von Ungleichheit und kolonialer Ordnung definiert sich die Indigenenbewegung als ältester Akteur des Widerstandes, der mit der Eroberung Amerikas begonnen hat. Auf diese Tradition beruft sich die CONAIE, wenn sie die Plurinationalität als Lösung dieser Probleme entwickelt. Ungleichheit und Diskriminierung ,hat uns dazu gebracht, den Aufbau eines Staates mit einer neuen politischen, wirtschaftlichen, sozialen und kulturellen Ordnung voranzutreiben, wo man die Einheit in der Vielfalt ausübt" (ECUARUNARI/CONAIE 2007: 5). Der Kampf für die Einheit der - neu zu gründenden - Nation und gegen Rassismus und Regionalismus wird so zur Grundbedingung für Gleichberechtigung und Teilhabe die sich wiederum auf die Rechte der indigenen Nationalitäten und ihre territoriale Autonomie gründen (ECUARUNARI/CONAIE 2007: 5).

\section{INTERKULTURALITÄT}

\section{a. Eine kurze Begriffsgeschichte}

Die Entstehung des Begriffes der Interkulturalität und seine Übernahme in Lateinamerika sind nicht völlig klar. Es scheint, dass die Interkulturalität in den USA der 1960er Jahre im Bereich der Erziehungswissenschaften entstanden ist (Ramón 2009: 133). In den 1970er Jahren wurde er - auch auf Betreiben des französischen Soziologen Edgar Morin - in den Sozialwissenschaften übernommen. Interkulturalität sollte die Möglichkeit der Schaffung einer neuen Kultur mit einer neuen Sprache, die dem Rassismus, der Ausgrenzung und der Unterdrückung entgegengesetzt ist, ausdrücken (García/Tuasa 2007: 18). Dieser Entwicklungsgeschichte widerspricht die Existenz des Interkulturellen Dokumentationszentrums (Centro Intercul- 
tural de Documentación, CIDOC) von 1961 bis 1976 in Cuernavaca, Mexiko, das von Ivan Illich geleitet wurde und sich der Befreiungstheologie und dem undogmatischen Sozialismus verpflichtet fühlte (Kaller-Dietrich 2008). Leider ist die Namensgebung des CIDOC nicht genauer nachvollziehbar.

Die erste nachweisbare Verwendung des Begriffs der Interkulturalität auf die indigenen Völker Amerikas findet sich in der Anthropologie. Die venezolanischen Wissenschaftler Esteban Mosonyi und Omar González stellen auf dem 39. Internationalen Kongress der Amerikanisten in Lima 1970 ein Projekt der interkulturellen Erziehung in einer indigenen Gegend in Venezuela vor und fordern eine ,Interkulturisierung“ (Mosonyi/González 1975: 307f.) der Gesellschaft als Alternative zu einer Integration oder Abgrenzung der Indigenen. Damit ist eine wechselseitige Stärkung der verschiedenen Kulturen in einem Land gemeint, die über eine Festigung ihrer Sprachen die Dekulturierung aufhalten soll. Der mexikanische Anthropologe Guillermo Bonfil Batalla spricht kurz nach dem zweiten Treffen von Barbados 1977 von ,,den Problemen der interkulturellen Situation“ (Bonfil Batalla 1978: 212) in den lateinamerikanischen Ländern mit indigener Bevölkerung.

Die Interkulturalität hat seit dieser Zeit eine beeindruckende Karriere gemacht. Genau definiert wurde sie nie, es überwiegen ,deklarative Erklärungen statt schlüssiger Theoreme und verifizierbarer Ergebnisse“ (Földes 2009: 504). Gerade aufgrund dieser mangelnden Klarheit ihrer Inhalte konnte sie in die verschiedensten wissenschaftlichen und gesellschaftlichen Kontexte auf der ganzen Welt integriert werden (Földes 2009: 514f.) - und kann somit als ,ein überaus erfolgreiches kulturelles Globalisierungsprodukt“ (Földes 2009: 515) gelten.

„In der Mehrheit der Publikationen wird ,Interkulturalität' gar nicht definiert, allenfalls rudimentär interpretiert; der Begriff wird zumeist als voranalytisches Konzept gehandhabt und unspezifisch verwendet, sein Signifikat bleibt vielfach vage oder widersprüchlich." (Földes 2009: 510)

Das liegt auch an einer empirisch wenig interessierten Erforschung im wissenschaftlichen Kontext, der sich nach Földes von den geisteswissenschaftlich geprägten cultural studies dominiert wird. Andere, eher empirische Ansätze sind die Kulturvergleichende Psychologie und die Kulturstandardforschung (Földes 2009: 507), die bislang nur wenig Einfluss erlangen konnten. Die cultural studies prägen auch viele der Studien über den Begriff der Interkulturalität in Ecuador, die zentrale Figur ist die Pädagogin Catherine Walsh. Interkulturalität erhält somit ,einen starken politisch-ideologischen Sinn“ (Földes 2009: 511), der ihrer empirischen Erforschung kaum zuträglich sein kann. Dazu kommt eine Verwischung zweier analytischer Ebenen im Begriff der Interkulturalität: die Beschreibung eines Phänomens (einer Beziehung zwischen Kulturen) und die Entwicklung eines Konzepts, das die 
Beziehung zwischen Kulturen interpretieren kann. Die Interkulturalität ist also auch in dieser Hinsicht „ein flexibles Konstrukt“ (Földes 2009: 512), das verschieden ausgelegt werden kann.

Der Begriff der Interkulturalität wurde in den 1980ern und 1990ern vom Europarat und anderen europäischen Institutionen in Dokumenten über Erziehung und Bildung als Alternative zum bisher verwandten Begriff Multikulturalität etabliert, der zunehmend mit einer einfachen Anerkennung der sozialen Situation assoziiert wurde (Bartolomé 2002: 18f.). Zur gleichen Zeit wurde der Begriff von europäischen und US-amerikanischen Organisationen der Entwicklungszusammenarbeit in der Andenregion verankert, die so ihre Projekte der zweisprachigen Erziehung für Indigene benannten (Walsh 2000: 11-13). Ein Regierungstreffen der lateinamerikanischen und karibischen Länder 1981 schlägt die Interkulturalität als Alternative zur bisherigen Integrationspolitik vor (Tamagno 2006: 25). Auf einem regionalen Treffen von Spezialisten der zweisprachigen Erziehung in Mexiko 1982 wurde die Empfehlung verabschiedet, statt von zweisprachiger bikultureller, von zweisprachiger interkultureller Erziehung zu sprechen, um so die Vielfalt und den historischen Charakter der Kultur hervorzuheben (Walsh 2000: 11). Von besonderer Bedeutung ist im Fall Ecuadors die deutsche Gesellschaft für technische Zusammenarbeit (GTZ), deren Engagement im Aufbau ,zweisprachiger interkultureller Schulmodelle“ (Abram 2004: 127) 1985/86 zu einem Abkommen mit dem ecuadorianischen Bildungsministerium und einigen Indigenenorganisationen führte. Daher blieb der Begriff der Interkulturalität zunächst auf den Bereich der Erziehung und Bildung beschränkt, wo er rasch von den meisten Indigenenorganisationen aufgegriffen wurde. Das Bildungsprogramm der FICSHA, SERBISH, das 1972 als Programm der zweisprachigen, bikulturellen Erziehung aufgebaut wurde, bezeichnet sich seit 1980 als zweisprachig und interkulturell (López 2009: 137f.). ECUARUNARI spricht 1989 von ,interkultureller zweisprachiger Erziehung“ (ECUARUNARI 1989: 89) als ein Projekt und eine Forderung der Indigenen. Die Indigenenbewegung hatte in dieser Erziehung schnell ein Werkzeug zur „Bewahrung der kulturellen Andersheit“ (Martínez Novo 2009: 1) und „Stärkung des Eigenen“ (Walsh 2003: 132) der indigenen Völker erkannt. Der Bereich der Erziehung dient traditionell der Konstruktion und Reproduktion von Werten, die der Staat definiert und die ihn stützen - eine Definitionsmacht der Marginalisierten über diese Institution ist also auch eine Infragestellung der Hegemonie des Staates (Walsh 2000: 14). Die interkulturelle, zweisprachige Erziehung ermöglichte den Widerstand gegen diese Bemühungen einer erzwungenen Integration. Nicht nur die indigenen Sprachen, sondern die indigenen Kulturen in ihrer Gesamtheit sollen im Unterricht verankert werden (Bernal 2011: 4). Die interkulturelle Erziehung will neben den Sprachen auch die unterschiedliche Geschichte, Gesellschaft und Wirtschaft der jeweiligen Kulturen thematisieren und über die Behandlung der verschiedenen Lebenswelten 
auch auf die strukturelle Diskriminierung der Indigenen eingehen (Giebeler 2010: 15). Im Politischen Projekt der CONAIE wird die interkulturelle Erziehung als „,befreiende, kritische, reflexive und plurinationalistische Erziehung“ (CONAIE 1994: 44f.; CONAIE 1997: 44; CONAIE 2001: 35) zum Kern des Aufbaus eines plurinationalen Staates.

Bei der Verbreitung des neuen Begriffes spielten die Aktivitäten zur Erinnerung an 500 Jahre indigenen Widerstand 1992 und die Ausrufung des Jahrzehnts der Indigenen Völker durch die UNO 1993 eine zentrale Rolle (Bernal 2011: 5). Bis Mitte der 1990er Jahre konnte sich die Interkulturalität jedoch nicht vom Kontext der Erziehung lösen. Auch wenn Catherine Walsh davon spricht, dass die Interkulturalität bereits seit den 1980ern ein eigenständiges Konzept im Diskurs der Indigenenorganisationen ist, das sich von den Bedeutungen, die ihm die Entwicklungsorganisationen gaben, emanzipiert hat (Walsh 2008: 8; Walsh 2009b: 223), lässt sich die erste Verwendung der Interkulturalität jenseits der Erziehung relativ genau bestimmen. Im Gegensatz zu den meisten hier besprochenen Begriffen geht die Interkulturalität nicht von der CONAIE aus, sondern von der FENOCIN, die die Interkulturalität als Alternative zum Vorschlag der Plurinationalität aufbaut. Deutlich wird der diskursive Wandel, wenn man die Politischen Projekte der CONAIE vergleicht. So ist in der Version von 1994 von Interkulturalität nur im Sinne von interkultureller zweisprachiger Erziehung die Rede (CONAIE 1994: 44). Als ein Ziel der CONAIE wird der „Aufbau der Neuen Humanistischen Gesellschaft“ (CONAIE 1994: 18) angegeben. 1997 und 2001 ist an derselben Stelle die Rede vom „Aufbau der Neuen Interkulturellen Gesellschaft“ (CONAIE 1997: 16; CONAIE 2001: 8). Die Interkulturalität ist in diesen drei Jahren zu einem der ideologischen Prinzipien der CONAIE für eine andere Gesellschaft geworden (Walsh 2008: 8f.). Mit dem eingeschränkteren Begriff der Plurikulturalität fordert die CONAIE schon seit Beginn der 1990er eine allgemeine Anerkennung der kulturellen Diversität des Staates (Barrera 2001: 172) - ohne jedoch zu den weitgehenden Forderungen zu gelangen, die später mit der Interkulturalität verbunden sein würden. Die erste indigene Organisation, die die Interkulturalität als politisches Ziel jenseits der Erziehung definiert, ist die FENOCIN, die sich auf ihrem siebten Kongress im Oktober 1995 als interkulturelle Organisation definiert (FENOCIN 2004: 20). In den folgenden Jahren konkretisiert sie dieses Selbstverständnis, in dem Sinne, dass sie sich als ,einzige nationale Organisation, die zur gleichen Zeit die Differenz der Identitäten und die Interkulturalität anerkennt“ (FENOCIN 2004: 20), versteht. So entwickelt die FENOCIN die Interkulturalität zu einem ,Projekt für das Land und Schlüsselfaktor für die Bürgerbeteiligung [und] das gesellschaftliche Zusammenleben“ (FENOCIN 2004: 39). Sie soll Vorurteile und Rassismus bekämpfen und Toleranz und eine Anerkennung der Diversität erlauben (FENOCIN 2004: 40). Außerdem umfasst die Interkulturalität der FENOCIN die Forderung nach Autonomie für die indigenen 
Völker (FENOCIN 2004: 54) - ganz im Sinne der Plurinationalität der CONAIE also. Es sollen indigene und schwarze Eingrenzungen aufgebaut werden (FENOCIN 1999: 159), die in gemeinschaftlichem Besitz der jeweiligen Gemeinde sind und so der Gemeinde die Teilhabe an Entscheidungen zum Umgang mit den Bodenschätzen gewähren - allerdings nur im Rahmen einer Befragung (FENOCIN 1999: 146). Im Sinne einer Pluralität soll die Wahlmöglichkeit zwischen den verschiedenen Rechts-, Gesundheits- und Erziehungssystemen bestehen, so dass jemand selbst entscheiden kann, ob sein Fall im indigenen oder mestizischen Rechtssystem verhandelt wird. Gleichzeitig soll eine Brücke zwischen den verschiedenen Systemen aufgebaut werden um eine völlige Trennung zu verhindern (FENOCIN 1999: 156). Die verschiedenen territorialen Eingrenzungen sollen eine politische Autonomie erhalten, die ihnen eine Selbstverwaltung erlaubt und sie zugleich funktional in die staatlichen Strukturen integriert. Dort sollen sie repräsentative Funktionen ausüben - und so interkulturelle Räume entstehen (FENOCIN 1999: 157).

Die FENOCIN versteht ihr Projekt der Interkulturalität als Instrument der Dekolonisierung des Staates und der Gesellschaft, da sie die Verschiedenheit nicht nur anerkennt, sondern als zentralen Baustein braucht (Walsh 2009: 178). Diese Übernahme von Forderungen der CONAIE unter einem erweiterten Begriff der Interkulturalität, der Bereiche der Plurinationalität abdeckt, kann das weitreichende Verständnis einiger Wissenschaftler des Begriffes der Interkulturalität erklären. Sowohl Walsh (2003) als auch García/Tuasa (2007) beziehen sich auf den weiteren Interkulturalitätsbegriff der FENOCIN der zwangsläufig die Kritik am „,monokulturellen Staat“ (Walsh 2003: 115; García/Tuasa 2007: 11) als auch „die Veränderung der öffentlichen Politik“" (Walsh 2003: 115; García/Tuasa 2007: 11) umfassen muss.

Die Interkulturalität ist also - im Gegensatz zu Begriffen wie Plurikulturalität oder Multikulturalität, die sowohl der Staat als auch die indigenen Organisationen verwenden - kein deskriptiver, sondern ein normativer Begriff (Walsh 2008: 6; Walsh 2009b: 223). Die unterschiedlichen Kulturen und Ethnien sollen nicht nur anerkannt und respektiert werden, sondern gemeinsam zu etwas Neuem, Anderem beitragen (Ramón 2009: 134f.). Interkulturalität zielt auf eine grundlegende Veränderung des Staates und der Gesellschaft (Walsh 2009b: 223). Gerade weil der ecuadorianische Staat - im Gegensatz zu anderen Staaten, wie etwa Kolumbien - die Verwendung des Begriffes Interkulturalität lange verweigert hat, blieb seine Definition den Indigenenorganisationen überlassen. Diese wendeten die Interkulturalität explizit gegen den bestehenden Staat und seine als kolonial verstandenen Strukturen. Die Interkulturalität sollte mit der herrschenden Hegemonie brechen und den Aufbau von Alternativen erlauben (Walsh 2003: 117).

CONAIE und FENOCIN kooperierten im Umfeld der Verfassungsgebenden Versammlung 1997/1998 (Vogel 2008: 15), um Interkulturalität und Plurinationalität als „grundlegendes Prinzip aller Reformen“ (Walsh 2008: 7) und Leitlinie für 
alle politischen Handlungen und Institutionen durchzusetzen. Dies war - wie der Inti Raymi 1990 für die Plurinationalität - der Moment des Eintritts der Interkulturalität in den nationalen öffentlichen Diskurs jenseits des Erziehungssystems (Walsh 2003: 115/118). Die Kooperation von CONAIE und FENOCIN endete mit den Auseinandersetzungen zwischen den beiden Organisationen über ihren Einfluss in den staatlichen Organisationen DINEIB und CODENPE in den Jahren 2001 und 2002 (Vogel 2008: 15). Schon vor dieser Spaltung hatte sich die FENOCIN zunehmend von der Ideologie der CONAIE und insbesondere dem Begriff der Plurinationalität distanziert (FENOCIN 1999: 151). In der Verfassungsgebenden Versammlung 2007/2008 präsentierte die FENOCIN und ihre Unterstützer die Interkulturalität als Gegenvorschlag zur Plurinationalität. Hier wurde die Interkulturalität als „These, die symmetrischere und nachhaltigere Interaktionen zwischen unterschiedlichen Gruppen zum Ziel hat“ (Vogel 2008: 16f.) entwickelt. Sie soll über die Schaffung eines Dialogs zwischen den Kulturen und Instrumente der positiven Diskriminierung ermöglicht werden.

In den letzten Jahren hat sich auch die FEINE den Begriffen der anderen Indigenenbewegungen geöffnet (Lucero 2006: 52) und spricht sich nun für die Interkulturalität als gesellschaftliches Projekt aus. Die FEINE versteht die Interkulturalität als ein ,einen Prozess des Dialoges und des harmonischen Zusammenlebens zwischen den Kulturen und Völkern“ (FEINE 2010: 19), der das bestehende System der Ausgrenzung und Diskriminierung in Frage stellt. In diesem Sinne erlaubt die Interkulturalität die Suche nach einer neuen Art, die Gesellschaft zu organisieren und die staatlichen Strukturen zu verändern. Eine wirkliche Interkulturalität ist für die FEINE aber noch nicht umsetzbar, da „Kultzentrismus, Ethnozentrismus und Egozentrismus herrscht" (FEINE 2010: 19), die Ausgrenzung also strukturell in der Gesellschaft verankert ist.

\section{b. Definition der Interkulturalität}

Die CONAIE definiert Ecuador als ein plurikulturelles Land in dem Sinne, dass „verschiedene und gleichzeitig unterschiedliche Kulturen in einem Land zusammenleben“ (CONAIE 1994: 52; CONAIE 2001a). Staat und Gesellschaft sollen diese Pluralität anerkennen und die verschiedenen Gruppen integrieren, ohne ihre Identität zu zerstören. Das soll die Interkulturalität leisten, als „Wechselverhältnis mit den verschiedenen Kulturen der Völker die [dort, P.A.] leben, das sich auf den Respekt vor dem kulturellen Unterschied und die Einheit in der Vielfalt gründet“" (CONAIE 1999: 72). Der Ausgangspunkt der Interkulturalität und die Vorbedingung des „Aufbaus einer interkulturellen Gesellschaft“ (Macas 2005: 39) ist somit 
die gegenseitige Wertschätzung der verschiedenen Gruppen der Gesellschaft (CONAIE 2007: 10). Die Interkulturalität - im Gegensatz zu Pluri- oder Multikulturalität - existiert also noch nicht, sie muss erst aufgebaut werden (Walsh 2008: 6). Daher ist sie eine Aufgabe sowohl der Indigenen als auch der mestizischen Mehrheitsgesellschaft (Ospina 2006a: 462) und bricht mit den bislang vorherrschenden indigenistischen oder liberalen Ideologien. Das Schlagwort der Einheit in der Vielfalt wird meist mit der Interkulturalität assoziiert, da es eine Überwindung der bestehenden Spaltungen verspricht und die gesamte Gesellschaft einbezieht (Walsh 2003: 130). Die Interkulturalität wird als ,ein Mittel zur Transformation des Staates und der Gesellschaft" (Andrade 2009: 21) verstanden, das über einen Wandel der Politik die sozialen Strukturen öffnen soll.

Für die CONAIE ist die Interkulturalität der Weg zum Aufbau eines neuen Staates und einer neuen Gesellschaft. Deshalb ist das Verständnis von Kultur, das die Politik dieses Staates prägt, entscheidend. „Die CONAIE definiert die Kultur als die Lebensweise eines Volkes“ (CONAIE 2001: 31), die als soziale Konstruktion andauernd gelebt und neu geschaffen wird. Daher gibt es keine universelle Kultur oder universelle kulturelle Werte, die allgemeinen Geltungsanspruch haben könnten.

,[D]ie wirtschaftlichen und politischen Herrschaftsbeziehungen sind eng verwandt mit den kulturellen Herrschaftsbeziehungen, daher verwandeln sich die Ideen und Praktiken der wirtschaftlich und politisch herrschenden Klassen in die Ideen und Praktiken einer Gesellschaft oder eines Staates. Die Kulturen der beherrschten Völker werden zum Schweigen gebracht, unterdrückt, unsichtbar gemacht oder einfach ignoriert und verachtet." (CONAIE 2001: 31)

Somit ist die Beziehung zwischen den Kulturen, die im Grunde gleich sind, eine ungleiche. Die Beziehungen zwischen Kulturen sind konflikthaft, Kultur ist „ein ideologisches Schlachtfeld“ (Walsh 2003: 116). Die Gruppen, die wirtschaftliche und politische Macht haben, haben auch die Mittel, ihre Kultur als universelle zu behaupten. Dazu versuchen sie, die Kultur von den Herrschaftsbeziehungen zu trennen und so zu naturalisieren - was eine Integration der Differenz als marginal erlaubt und eine Veränderung der Strukturen überflüssig macht (Walsh 2003: 116).

„[I]m Hintergrund gehen die Umrisse, die von jenen Kulturen gezeichnet werden, die mehr symbolische und wirtschaftliche Vorteile haben, weiter, ihnen unterwerfen sich die anderen Kulturen und nehmen die anscheinend richtigen Praktiken an, um zu überleben. Im Unterschied zur hegemonischen Kultur, die Trägerin der wirtschaftlichen und technologischen Gewinne ist, fehlen den anderen diese Ressourcen, um konkurrieren zu können.“ (García/Tuasa 2007: 13) 
Daher verlangt die CONAIE ein breiteres Verständnis von Kultur als in ,einer westlichen Perspektive“ (CONAIE 2007a: 23) üblich. So sollen die Kulturen unter Einschluss ihrer politischen und wirtschaftlichen Aspekte - in die Institutionen des Staates integriert werden. „Wirtschaft, Gesundheit, Erziehung können nicht verdrängt, negiert und, noch schlimmer, ausgeschlossen werden aus der öffentlichen Politik in einem plurinationalen Staat." (ECUARUNARI/CONAIE 2007: 6) Diesen erweiterten Kulturbegriff nimmt die Interkulturalität auf. Sie ,,ist ein integrales politisches Projekt, das die wirtschaftlichen Probleme aus einer kulturellen Logik sehen will und anders herum“ (Simbaña I-2). Der Staat soll die Unterschiede nicht einebnen, sondern anerkennen, wozu geeignete Institutionen nötig sind (de Sousa Santos 2009: 42).

Die Interkulturalität geht davon aus, dass Kulturen voneinander lernen müssen, nicht nur, um sich selbst zu stärken, sondern vor allem, um den Zusammenhalt der gesamten Gesellschaft zu stärken (Ramón 1998). Die Anerkennung des jeweils Anderen ist immer auch die Anerkennung seiner selbst, sie stärkt die eigenen Position und Identität (Sánchez-Parga 1997: 112). Die Kulturen haben viele gemeinsame Werte, die eine Kommunikation erst ermöglichen. Die gegenseitige Anerkennung der jeweiligen Werte erlaubt den Aufbau von Räumen ,der Selbstreflektion und des Selbstwertes aufgrund der eigenen Wertschätzung, das Eigene aufnehmend und das Andere respektierend“ (Saltos/Vázquez 2007: 383). So können unterschiedliche Kulturen lernen, unter Rückgriff auf bestimmte Gemeinsamkeiten zusammenzuleben und ihre Konflikte friedlich zu regeln. Der institutionalisierte Kulturkontakt schafft Vertrauen, Kommunikation, Austausch und die Möglichkeit, voneinander zu lernen (García/Tuasa 2007: 16). Die Interkulturalität ist der Versuch, die Vorherrschaft einer bestimmten Kultur und die Unterdrückung anderer Kulturen zu brechen und die verschiedenen Kulturen in autonomen Strukturen zu stärken (Walsh 2000: 12). In diesem Kontext ist auch das Schlagwort der Einheit in der Vielfalt zu verstehen, das die wirtschaftliche, soziale, kulturelle und politische Einheit der verschiedenen Völker und Nationalitäten im Sinne einer Transformation der bestehenden Strukturen hinzu einem plurinationalen Staat fordert (CONAIE 1994: 13).

Der Kampf der Indigenen für Interkulturalität ist nicht nur politisch im engeren Sinne und bezieht sich nicht nur auf den Staat. Mehr als eine Verteidigung der Identität ist die Interkulturalität der Versuch, ,zwischen hegemonischen und subalternen Positionen mit verschiedenen Formen, Wissen zu produzieren und anzuwenden“ (Walsh 2003: 135), zu vermitteln. So wird die Interkulturalität zu einer ,gegenhegemonischen Praxis“ (Walsh 2003: 135f.), die auf eine Umdeutung des als legitim betrachteten Wissens abzielt und eine Rehabilitation traditioneller und lokaler Wissensformen anstrebt. Ein neues Wissen und eine neue Vernunft sollen geschaffen werden, die durch ,epistemologisches Interkulturalisieren“ (Walsh 2003: 
138) eine Verdeckung der ausschließenden Strukturen der Kolonialität der Macht nicht länger erlaubt.

Die Interkulturalität soll über einige zentrale Punkte umgesetzt werden. Die Institutionen und Strukturen von Staat und Gesellschaft müssen ,interkulturalisiert" (Walsh 2000: 16) werden, wozu eine enge Kooperation der verschiedenen Gruppen der Gesellschaft nötig ist (Walsh 2000: 16). Die Interkulturalität wird so zu einem „Werkzeug der Transformation des Staates und der Gesellschaft“ (Andrade 2009: 21), das die alten kolonialen Strukturen der Politik und Zivilgesellschaft verändern und die Inklusion der Ausgeschlossenen ermöglichen soll. Walsh spricht in diesem Zusammenhang von ,einem neuen Paradigma und sozialem, politischem und epistemologischem Projekt des Interkulturalisierens, das gerade in voller Entwicklung ist" (Walsh 2003: 134). Die Indigenenbewegung schlägt einige konkrete Schritte vor. Die Förderung einer allgemeinen Zweisprachigkeit in Bezug auf die regional vorherrschende indigene Sprache ist hier zentral, da die Sprache auch aus der Sicht der CONAIE der Kern jeder Kultur ist (CONAIE 2001a). Nur über sie sind die Werte einer Kultur zu verstehen - und nur so ist ein voneinander Lernen möglich (García/Tuasa 2007: 15). Das bedeutet neben einer Anerkennung der indigenen Sprachen als Amtssprachen (CONAIE 2001: 14) eine Ausweitung der interkulturellen zweisprachigen Erziehung auch auf nicht-indigene Schulen (Ramón 1998). Die integrationistische und hegemoniale Bildungspolitik des Staates wird von der interkulturellen Erziehung mit ihren identitären und politischen Inhalten herausgefordert (Walsh 2000: 12).

Zur Interkulturalität wird gelegentlich auch die „Harmonisierung und Koordination zwischen Indio-Recht und nationalem Recht und die rechtliche Pluralität" (Ramón 1998) gezählt, die auf ,einen Wandel der politischen Struktur des Staates“ (Chuji 2008) und eine grundlegende Transformation des Rechtssystems bauen muss.

\section{c. Plurinationaler Staat und interkulturelle Gesellschaft?}

Der Zusammenhang zwischen Plurinationalität und Interkulturalität im Diskurs der CONAIE ist unklar. Auch wenn oft von ,einem Plurinationalen Staat und einer interkulturellen Gesellschaft“ (ECUARUNARI/CONAIE 2007: 5) die Rede ist, sind beide Begriffe miteinander verwoben. So bezieht sich die Plurinationalität eher auf Selbstverwaltung und politische und wirtschaftliche Teilhabe, die Interkulturalität eher auf soziokulturelle Beziehungen zwischen den Völker und Nationalitäten keiner der beiden Begriffe schließt den anderen aus und beide beziehen sich aufeinander (Grijalva 2008: 56). Die Plurinationalität braucht die Interkulturalität als Instrument, um umgesetzt werden zu können. Ein plurinationaler Staat muss Diskriminierung bekämpfen und Verständigung herstellen, was er nur über in- 
terkulturelle Politik erreichen kann (Andrade 2009: 18). Die Plurinationalität bricht mit dem uninationalen und ausschließenden Charakter des Staates und öffnet die Institutionen für alle Bevölkerungsgruppen, die Interkulturalität bezieht sich auf die sozialen Beziehungen, die durch diese Öffnung möglich werden sollen (Walsh 2008: 16) ${ }^{21}$. Es geht um die ,Neugründung der politischen Institutionen unter plurinationalen und interkulturellen Kriterien“ (Walsh 2009: 183). So ist für die CONAIE ,der plurinationale Staat durch den Aufbau einer wahren Interkulturalität charakterisiert“ (CONAIE 2007a: 5; García/Tuasa 2007: 9) die den Austausch von Werten, Weltsichten und Wissen in einem Dialog unter Gleichen durch das Ende von Diskriminierung, Kolonialität und Rassismus erlaubt. Daher ist die Interkulturalität für die CONAIE eine der drei Achsen für den Aufbau der Plurinationalität. „Der Aufbau einer wahren Interkulturalität, die den Aufbau eines Projektes für das Land zwischen allen bedeutet“ (CONAIE 2007a: 7f.) ist die ,grundlegende Voraussetzung für eine plurinationale Demokratie und eine gerechte Wirtschaft" (CONAIE 2007a: 7f.). Die Interkulturalität fördert die Diversität der Völker und Nationalitäten und ihre wirtschaftliche, soziale, kulturelle und politische Einheit im Zuge der Transformation der bestehenden Strukturen und des Aufbaus des plurinationalen Staates (CONAIE 2001: 5; García/Tuasa 2007: 12). Dieser muss die Völker und Nationalitäten nicht nur als Kulturen, sondern auch als wirtschaftliche, politische und soziale Einheiten anerkennen und im Sinne der Vielfalt in der Einheit in seine Politik integrieren (ECUARUNARI/CONAIE 2007: 6; CONAIE 2007: 10). Der plurinationale Staat muss neben einer partizipativen und dezentralen Demokratie und einer gerechten Verteilung der Ressourcen den Aufbau einer interkulturellen Gesellschaft anstreben (CONAIE 2005). Dazu stellt er geeignete Gesetze zur Verfügung, die den indigenen Nationalitäten und den anderen Teilen der Gesellschaft die politische Teilhabe „im gesamten Aufbauprozess der Neuen Interkulturellen Gesellschaft, ausgehend von der Anerkennung der Verschiedenheit und Vielheit der ecuadorianischen Gesellschaft" (CONAIE 2001: 8) garantieren sollen. Der plurinationale Staat fördert die Interkulturalität als wechselseitigen Austausch zwischen den Kulturen, an dem alle Bürger des Staates teilnehmen. Dazu stützt er sich insbesondere auf eine allgemeine, für alle gültige zweisprachige, interkulturelle Erziehung (CONAIE 1997a: 17).

21 Es wurde bereits im vorherigen Kapitel verdeutlicht, dass die Plurinationalität nicht rein deskriptiv ist und nicht bei der Anerkennung der Verschiedenheit stehen bleibt, wie es Walsh (2008: 16) behauptet. Daher ist für Walsh die Plurinationalität ein Rückschritt in Bezug auf die Interkulturalität (Walsh 2008: 22) - eine Interpretation, die hier nicht geteilt wird. 
„Die Interkulturalität ist die Möglichkeitsbedingung der Plurinationalität, auf dieselbe Weise wie der plurinationale Staat die einzige Garantie dafür ist, dass die Gesellschaften die Interkulturalität anerkennen, respektieren und aufbauen können.“(Chuji 2008)

In diesem Sinne hat sich der Begriff der Plurinationalität seit den 1990ern in der Beziehung zur Interkulturalität weiterentwickelt. Die Anerkennung der ,ethnischen und kulturellen Rechte der indigenen Nationalitäten“ (Macas 1991: 6) ist nicht länger das Ziel eines plurinationalen Staates, sondern sein Ausgangspunkt. Interkulturalität meint mehr als Anerkennung und Respekt, die Indigenenbewegung hat auch hier neue Begriffe schaffen können.

Die Gegenüberstellung von Plurinationalität und Interkulturalität, wie sie manche Wissenschaftler unternehmen, gründet oft auf Arbeiten aus anderen Kontexten, wie etwa den Forschungen von Will Kymlicka über Kanada. Nicht selten werden diese Übernahmen nicht deutlich gemacht. Insbesondere Catherine Walsh neigt dazu, Interkulturalität und Plurinationalität als zwei mehr oder weniger deutlich voneinander zu trennende Prozesse zu beschreiben, die sich ergänzen müssen, um als dekoloniales Projekt erfolgreich sein zu können (Walsh 2009b: 238). Interkulturalität ist für sie ein Ansatz, der versucht, nicht nur die Beziehungen zwischen bestimmten Gruppen, sondern auch die Strukturen des ecuadorianischen Staates und der ecuadorianischen Gesellschaft zu verändern. Sie soll eine Gesellschaft, die auf Respekt, Gleichberechtigung und gegenseitige Anerkennung gründet, aufbauen und so Differenz erlauben. Dazu soll die Interkulturalität ein Verständnis der verschiedenen Beziehungen und Strukturen ermöglichen (Walsh 2009a: 79f.). Für Walsh beinhaltet Plurinationalität die Anerkennung der Differenz im Land, während Interkulturalität versucht, Beziehungen zwischen den verschiedenen Gruppen (neu) zu schaffen und so das Land neu und besser aufzubauen (Walsh 2008: 16). Wenn sie auch andere Begriffe benutzt, so ist ihr Denken - und das anderer Wissenschaftler, die die Interkulturalität verteidigen - nicht sehr verschieden von dem Denken Will Kymlickas.

Kymlicka stellt dem multikulturellen Staat den interkulturellen Bürger gegenüber (Kymlicka 2003: 148). Der multikulturelle Staat zeichnet sich durch den Abbau institutioneller Diskriminierung - etwa von rassistischen Gesetzen, der Verankerung bestimmter schulischer Inhalte oder dem öffentlichen Feiern bestimmter Feste - aus, um so die Stigmatisierung oder Benachteiligung bestimmter Gruppen zu beenden. Er versucht also, Integration zu befördern, Verschiedenheit zu erlauben und ein offenes Bild seiner selbst zu präsentieren (Kymlicka 2003: 152). Dazu kann der multikulturelle Staat bestimmten Gruppen gewisse Grade an Autonomie erlauben, insbesondere, was deren Erziehungssysteme, politische Repräsentation, Sprachen und Landbesitz angeht (Kymlicka 2003: 152f.). Er erkennt dabei die Verschiedenheit der unterschiedlichen Gruppen an und ermöglicht ihnen die jeweils 
gewünschte Art der Teilhabe an der Gesellschaft - über den Abbau von Diskriminierung oder die Schaffung von Autonomien. Der multikulturelle Staat ermöglicht also verschiedene Arten multikultureller Mitgliedschaft in diesem Staat (Kymlicka 2003: 153). Der Begriff des multikulturellen Staates von Kymlicka könnte somit ein Oberbegriff der Plurinationalität sein, wie sie die CONAIE verteidigt.

Der multikulturelle Staat braucht, um gut funktionieren zu können, interkulturelle Bürger, die in der Lage und willens sind, diesen Staat und seine Integrationsmodelle zu unterstützen. Also muss eine ausreichende Anzahl der Bürger die Prinzipien eines multikulturellen Staates - dass er allen Mitgliedern gleichermaßen gehört, dass Assimilation durch Anerkennung der Differenz ersetzt wird und dass geschehenes Unrecht anerkannt wird - unterstützen (Kymlicka 2003: 153f.). Im Fall von multikulturellen Staaten mit einem hohen Grad an Autonomie (wie Belgien, Quebec, Schweiz) wurde die bestehende Diskriminierung durch Systeme autonomer Selbstverwaltung behoben. Die Schaffung von Strukturen und Institutionen für die verschiedenen Gruppen hat ihnen den Zugang zu Bildung, wirtschaftlichen und politischen Entscheidungen usw. erlaubt - in Institutionen, die unter der Kontrolle der jeweiligen Gruppen waren. Somit wurden in den multikulturellen Staaten autonome Gebiete geschaffen, die untereinander nur wenig Kontakt haben (Kymlicka 2003: 154f.). Es handelt sich also um multikulturelle Staaten mit einem geringen Grad an interkulturellen Beziehungen oder Wissen (Kymlicka 2003: 155). Ein multikultureller Staat kann bestehen, ohne interkulturelle Bürger zu haben - seine Prinzipien werden unterstützt, an interkulturellen Kontakten oder Wissen besteht aber kein großes Interesse. Der Aufbau von Autonomien kann die Notwendigkeit zu interkulturellen Kontakten sogar reduzieren - da diese durch eigene Institutionen schlichtweg nicht mehr notwendig sind (Kymlicka 2003: 157). In diesem Sinne zeichnen sich interkulturelle Bürger nicht nur dadurch aus, dass sie ihren multikulturellen Staat unterstützen, sondern auch dadurch, dass sie persönlich positive Einstellungen den anderen Gruppen und der Verschiedenheit allgemein gegenüber haben. Der interkulturelle Bürger ist neugierig auf das Andere, offen, Neues zu lernen und bereit, die Position Anderer einzunehmen (Kymlicka 2003: 157). Dabei ist für den multikulturellen Staat wichtig, dass die Bürger ,lokalen Interkulturalismus über globalen Interkulturalismus“ (Kymlicka 2003: 161) stellen, sich also auf den Anderen im eigenen Land einlassen - statt sich Kulturen und Sprachen zu widmen, die weiter weg sind und einen höheren persönlichen und wirtschaftlichen Gewinn versprechen (Kymlicka 2003: 159). Dabei geht es weniger um ein tiefgreifendes Verstehen, als um die Anerkennung der Verschiedenheit zwischen den Gruppen, die das Aushandeln von allgemeinen Kompromissen erst möglich macht (Kymlicka 2003: 164f.).

Dieses Verständnis von Interkulturalität als individuelle Fähigkeit der Bürger eines multikulturellen Staates scheint auch dem Begriff der Interkulturalität vieler 
nicht-indigener Wissenschaftler zugrunde zu liegen. In den vorherigen Kapiteln wurde erläutert, dass diese Trennung zwischen Individuum und Staat in den Begriffen der Indigenenbewegung nicht deutlich gemacht wird. Das zeigt die bislang ausführlichste Definition von Interkulturalität der FENOCIN (1999), genauso wie das aktuelle politische Projekt der CONAIE (2001). Die FENOCIN bezieht ihren Begriff der Interkulturalität weniger auf das individuelle Verhalten der Bürger - das gleichwohl eine Rolle spielt - als auf die institutionelle Bekämpfung von Diskriminierung und Ungleichheit, wie sie in Kymlickas Begriff des multikulturellen Staates erläutert wird. Der Begriff der Interkulturalität der Indigenenbewegung ist ein kollektiver, gesamtgesellschaftlicher, in dem staatliche und organisatorische Prozesse eine hohe Bedeutung haben - und nicht ein individueller, der nur über langfristige kulturelle Entwicklungen durchzusetzen wäre. Die Forderung nach Plurinationalität scheint hingegen weniger mit dem Wunsch nach Selbstbestimmung im Rahmen autonomer, nach Ethnien strukturierter Institutionen auf lokaler und überlokaler Ebene (Kymlicka 2003: 152f.) verbunden zu werden, als mit der Verweigerung der Interkulturalität durch Gruppen, für die interkulturelle Beziehungen eine Gefahr für ihre Kultur darstellen (Kymlicka 2003: 161f.). Für Kymlicka ist ein plurinationaler - oder in seiner Sprache multikultureller - Staat mit Bürgern, die interkulturelle Kontakte explizit ablehnen, möglich - ein derart separatistisches Projekt lässt sich durch die Texte der Indigenenbewegung in Ecuador allerdings nicht bestätigen.

\section{d. Kritik der Interkulturalität}

Der Bezug des Begriffs der Interkulturalität auf Kulturen ist fehlleitend, da diese als etwas festes und geschlossenes verstanden werden, die von klar definierten Positionen aus miteinander in Kontakt treten können. Somit ,handelt der interkulturelle Prozess [...] von ethnischen Prozessen und nicht wirklich von Prozessen, um zwischen den Kulturen in Beziehung zu treten" (Giebeler 2010: 17). Daher wird die Interkulturalität oft nicht als Aufgabe der gesamten Gesellschaft „,sondern als Abbild der kulturellen Verfassung der indigenen Welt“ (Walsh 2000: 11) verstanden als erneuter Versuch, die Indigenen in die Mehrheitsgesellschaft zu integrieren. Die Interkulturalität wird mit den Indigenen verbunden und so als ethnisches Problem oder Problem der Indigenen gesehen, das vom Willen der jeweiligen Gruppe abhängt, sich zu integrieren (Walsh 2003: 134). Zwischen der Ideologie der mestizaje, die als kulturelle Hybridität verstanden wird, und der Interkulturalität als politischem Ideal der Indigenenbewegung kann aus dieser Perspektive kaum unterschieden werden (Portocarrera 2003: 203). Daher ist bei einem solchen Verständnis von Interkulturalität der Aufbau einer universellen, einschließenden und partizipativen Gesellschaft, die ihren kolonialen Hintergrund aufarbeitet, wenig wahrscheinlich (Walsh 2003: 132). Zur gleichen Zeit lädt der Begriff der Interkulturalität dazu ein, 
alles Denkbare zu integrieren - bis hin zum Extrem, alles Soziale als interkulturell zu verstehen (Giebeler 2010: 18). Die Neigung, verschiedenste Prozesse des Kulturkontakts, von Migration bis hin zu Kommunikation in Gruppen, als interkulturell zu beschreiben, führt dazu, dass „sich dieser Begriff wie ein großer Topf öffnete“ (Giebeler 2010: 15), in den all diese Phänomene passen. Dadurch droht die analytische Schärfe der Interkulturalität verloren zu gehen. Auch unter den Führern der Indigenenbewegung herrscht wenig Klarheit im Verständnis der Interkulturalität. Sie wird als allägliche Praxis verstanden oder als ethisches Verhalten, als Bestehen einer kulturellen Diversität oder deren Anerkennung. Sie soll Diskriminierung, Exklusion und Marginalisierung angreifen und zu überwinden helfen und so zum Aufbau einer neuen Gesellschaft beitragen (Walsh 2003: 127f.). In einigen Fällen wird sie sehr breit ausgelegt, so dass die Interkulturalität eine weitgehende Autonomie der indigenen Territorien bedeutet (Montaluisa 2008: 100). Kurz, die Interkulturalität wird in einem fetischistischen Sinne verwenden, ,[a]ls eine Art Zauberformel“ (Portocarrera 2003: 203).

Die verschiedenen Bedeutungen, die der Interkulturalität zugeschrieben werden, entstammen verschiedenen Bereichen und haben daher nicht immer Gemeinsamkeiten. Sie widersprechen sich und kritisieren sich gegenseitig. Selten nur gehen sie über die Schlagworte, sich kennenlernen“ und, Einheit in der Vielfalt" hinaus (Walsh 2003: 127) ${ }^{22}$. Auch deshalb wird der Begriff nur selten mit konkreten Inhalten gefüllt und als „schwebender Begriff“ (Walsh 2009a: 74) von den verschiedensten Gruppen mit den verschiedensten Zielen benutzt. Daher wird die Interkulturalität auch verwendet, um die Indigenenorganisationen anzugreifen und den Indigenen Autonomierechte abzusprechen (Montaluisa 2008: 99).

\section{Zum SONdERFall Sumak Kawsay}

Zusätzlich zu den bisher untersuchte Begriffen wurde ab 2000 ein weiterer Begriff geprägt, der mit der Indigenenbewegung assoziiert und von ihr verwendet wird. Das Gute Leben, auf Kichwa Sumak Kawsay oder Alli Kawsay, auf Spanisch Buen Vivir, wurde in Ecuador zuerst von den Sarayaku-Kichwa des Amazonasgebietes als politisches Konzept definiert. Der Sarayaku Carlos Viteri nahm bis 2002 an einem Forschungsprojekt des Instituts der Friedrich-Ebert-Stiftung ILDIS teil, das Nachhaltigkeit im ecuadorianischen Amazonasgebiet untersuchte ${ }^{23}$. In einem Arti-

22 Es wurde bereits erläutert, dass Einheit in der Vielfalt für die CONAIE (1994: 13) weit mehr als ein Schlagwort ist, zu dem es von einigen der hier kritisierten Denkern gemacht wird.

23 Persönliche Kommunikation von Alberto Acosta. 
kel, der seit 2000 in verschiedenen Versionen diskutiert und 2002 zum ersten Mal publiziert wurde, verwendet er den Begriff Alli Kawsay, um ein indigenes Modell wirtschaftlicher Entwicklung zu erklären. Dort beschreibt Viteri das Gute Leben als „die Entwicklung von kohärent an die Bedingungen der Umgebung angepassten Produktionssystemen“ (Viteri 2002: 2), die sich auf die lokale und autonome Verwaltung der Bedürfnisse und Gegebenheiten und das damit verknüpfte Wissen bezieht. Diese Weltsicht verbindet, vermittelt durch weise Menschen, ,physische Räume mit dem Unberührbaren, das Materielle mit dem Spirituellen“ (Viteri 2002: 3). Die indigene Bewegung ist der Beweis, dass das Gute Leben als Rückgriff auf die Tradition und Einbindung und Anpassung fremder Wirtschaftsformen in die autonomen lokalen Strukturen möglich ist (Viteri 2002: 5). Im selben Jahr 2002 greift der Wirtschaftswissenschaftler Alberto Acosta - der auch an dem Projekt des ILDIS beteiligt war - den Begriff des Guten Lebens auf und verwendet ihn, um das westliche Verständnis von wirtschaftlicher Entwicklung $\mathrm{zu}$ kritisieren (Acosta 2002: 46). Er entwirft - noch wenig konkret - eine alternative Vision von Entwicklung, in der wirtschaftliches Wachstum zweitrangig ist.

Dieser neue Begriff wurde vermutlich in Bolivien entwickelt. Dort fanden im Jahr 2000 eine Reihe von Veranstaltungen im Rahmen des ,Nationalen Dialogs 2000“ (Diálogo Nacional 2000) statt, der von der deutschen Gesellschaft für Technische Zusammenarbeit (GTZ) unterstützt wurde. Diese Veranstaltungsreihe widmete sich den kulturellen Bestandteilen des Kampfes gegen die Armut. Ein Schwerpunkt untersuchte in Zusammenarbeit mit der Föderation kommunaler Assoziationen (Federación de Asociaciones Municipales) unter dem Titel Suma Qamaña die Alternativen zum Begriff der Entwicklung. Diese Kampagne wurde weitergeführt und konnte einige Bedeutung zur Zeit der Verfassungsgebenden Versammlung Boliviens entwickeln, in der die vorgeschlagenen Begriffe viel diskutiert und schließlich in die Verfassung integriert wurden (Medina 2011). Mit einer Reihe von Veröffentlichungen, zu denen Javier Medina entscheidend beitrug, wurden die Begriffe des Guten Lebens (damals noch Buena Vida) entwickelt und verbreitet. Die erste größere Veröffentlichung besorgte Medina 2001 (Medina 2001). In ihr untersuchten eine Reihe von Wissenschaftlern und Aktivisten das indigene Verständnis des Guten Lebens und begann die Entwicklung der heute verwendeten Begriffe. Auf einem Seminar der indigenen Organisationen Amerikas in Panama 2002 - ebenfalls von der GTZ organisiert - wurden die neuen Begriffe auf kontinentaler Ebene vorgestellt und diskutiert (GTZ 2002). Im Material der GTZ wird vor allem der Unterschied zwischen westlichem und indigenem Denken betont und die Unterschiede zwischen westlichen und indigenen Vorstellungen eines Guten Lebens herausgearbeitet (GTZ 2002: 22f.). In diesem Zusammenhang werden die Bezeichnungen der drei größten Völker Boliviens für dieses indigene Gute Leben dargestellt (Aymara: Suma Qamaña, Guaraní: Ñande Reku, Quechua: Kausay) und 
eine provisorische Definition aufgestellt: ,,bescheiden und in Harmonie und Gleichgewicht mit sich selbst, der Gemeinschaft und dem Kosmos leben“(GTZ 2002: 24).

Der Begriff des Suma Qamaña wurde also um das Jahr 2000 von einer Reihe von Aymara-Intellektuellen und Anthropologen um Simón Yampara (Spedding, in: Uzeda o.J.: 1f.) und Javier Medina erfunden (Uzeda o.J.: 2). Es handelt sich um „eine postmoderne Erfindung der Aymara-Intellektuellen des 21. Jahrhunderts (die deswegen nicht aufhören, Indigene zu sein)“ (Uzeda o.J.: 20). Das Gute Leben in diesem Sinn ist in der Tradition und dem Alltag der Aymara nicht zu finden. Dennoch baut der neue Begriff auf das traditionelle Denken der Aymara auf, ohne ihm zu widersprechen ${ }^{24}$. Somit handelt es sich beim Guten Leben um eine ,kulturelle Innovation“ (Uzeda o.J.: 20), die auf einer bestimmten Tradition aufbaut. Der Begriff des Guten Lebens wurde anschließend von den Quechua übernommen, jedoch ohne den philosophischen Hintergrund, den die Aymara verwenden. Obwohl die Bezüge auf die Natur, die Gottheiten und die Menschen dieselben sind, ist die Reflexionsarbeit der Verfechter des Sumak kawsay geringer als die der Erfinder des Suma Qamaña (Uzeda o.J.: 14). Auch wenn das Vorhandensein ähnlicher Gedanken in verschiedenen Sprachen und Kulturen - neben Aymara und Quechua auch weitere amerikanische und europäische - betont wird, heben einige der Verfechter des Guten Lebens hervor, dass es sich dabei nicht um Synonyme, sondern um komplementäre Begriffe handelt, die sich auf der Grundlage einiger Gemeinsamkeiten ergänzen (Gudynas 2011: 12). Jede Formulierung des Guten Lebens ist spezifisch für die Kultur und Sprache, in der sie formuliert wird. Es gibt also kein einheitliches Gutes Leben, dass sich auf alle Kulturen oder Länder ausweiten ließe (Gudynas 2011: 8).

Der Begriff Suma Qamaña ist ein Neologismus, dessen ursprüngliche Bedeutung umstritten ist. Die Anthropologin Alison Spedding übersetzt qamaña als, zuhause bleiben' im Sinne von ,wegen Krankheit oder Faulheit nicht arbeiten', weswegen sie behauptet, suma qamaña sei kein anzustrebendes Ideal. Den Ausdruck selbst hat sie vor seiner Präsentation als politischen Begriff nie gehört. Als passendere Alternative für eine wirtschaftlich-politisches Ideal schlägt sie sum sarnaqaña vor, das sich auf das erfolgreiche wirtschaftliche Leben der Familie in Kooperation mit der Gemeinschaft bezieht (Spedding 2010: 6).

Aníbal Quijano - und mit ihm Walter Mignolo (2005: 19) - hat also Unrecht, wenn er das Gute Leben als „wahrscheinlich älteste Formulierung des ,indigenen“ Widerstandes gegen die Kolonialität der Macht“ (Quijano 2010: 120) bezeichnet. Er bezieht sich auf einen Text von Carolina Ortiz Fernández, die über Guamán

24 Die essentialistische Diskussion, ob das Gute Leben, indigen“ ist, wie Uzeda (o.J.) nachzuweisen versucht, wird hier übergangen. Für die vorliegende Arbeit ist nur relevant, dass es sich um einen neuen Begriff handelt, der in einem bestimmten Kontext entstanden ist. 
Poma de Ayala forscht, der ungefähr 1615 in seiner Nueva Corónica y buen gobierno den Begriff bien vivir verwendet (Quijano 2010: 120). Guamán Poma verwendet zwar diesen Begriff, allerdings hat er nicht viel mit dem heutigen Guten Leben gemein. In der Nueva Corónica verbindet Guamán Poma schriftliche und mündliche Quellen, um aus ihnen die Gute Regierung zu rekonstruieren, die vor der Ankunft der Spanier bestand (Ortiz Fernández 2009: 270). Das Gute Leben bedeutet hier die Abwesenheit von Unterdrückung und Ausbeutung, also nichts anderes als die Gute Regierung selbst (Ortiz Fernández 2009: 271).

Der neue Begriff des Guten Lebens baut auf den in den 1990ern häufig zu findenden Begriff der Biodiversität auf, mit dem verschiedene indigene Organisationen bereits versuchten, eine Verbindung von Diversität der Natur und Diversität der Kultur im Sinne von „,Biodiversität‘ als ,Territorium plus Kultur““ (Escobar 1998: 70) herzustellen. Die Biodiversität war und ist ein Diskurs, der in einem komplexen Netzwerk zwischen Nichtregierungsorganisationen des globalen Nordens, Wissenschaftlern und sozialen Bewegungen entwickelt wurde. Dadurch konnten die sozialen Bewegungen eine besondere Form der Kulturpolitik entwickeln, die kulturelle Differenz, Verteidigung lokaler Territorien und gewisse Grade von Autonomie vereinigte. Mit der Biodiversität wird diese Politik um einen ökologischen Rahmen erweitert. Diese Einbettung macht aus dem Begriff der Biodiversität einen umfassenden Begriff, der über die Forderung nach Umweltschutz weit hinausgeht. Das Lokale und die Politik der Gemeinschaften vor Ort treten so in das Zentrum der Aufmerksamkeit (Escobar 1998: 53f.). Der Bezug auf Biodiversität wurde in viele Kämpfe der sozialen Bewegungen um territoriale Selbstverwaltung verankert auch und gerade in Lateinamerika (Escobar 1998: 61). Allerdings täuscht sich Escobar, wenn er Ecuador zu einem Beispiel dieser Integration macht. Die kulturelle Diversität wird in den Texten der CONAIE oft angerufen - jedoch nur selten die biologische Diversität ${ }^{25}$, auf die sich eher staatliche Organisationen beziehen.

Im Jahr 2003 wurde der Begriff des Guten Lebens durch zwei wichtige Veröffentlichungen im gesellschaftlichen Diskurs Ecuadors verankert. Im Oktober 2003 wurde der Begriff zum ersten Mal von einer Organisation der Indigenenbewegung verwandt. Die Basisorganisation der OPIP „Autonomes Territorium der Ureinwohnernation des Kichwa-Volkes von Sarayaku“ (Territorio Autónomo de la Nación Originaria del Pueblo Kichwa de Sarayaku) veröffentlichte unter Führung des

25 Ein Beispiel ist der Vorschlag von ECUARUNARI/CONAIE (2007: 5) an die Verfassungsgebende Versammlung von 2007, der von Biodiversität, aber nicht vom Guten Leben spricht. Es wäre näher zu untersuchen, inwieweit der Begriff der Biodiversität den aufkommenden Begriff des Guten Lebens abwehren sollte bzw. warum ECUARUNARI in den Jahren 2007/2008 von Biodiversität, die CONAIE aber bereits vom Guten Leben spricht. 
späteren CONAIE-Präsidenten Marlón Santi das „Buch des Lebens von Sarayaku, um unsere Zukunft zu verteidigen“ (Sarayaku Sumak Kawsayta Ñawpakma Katina Killka/El libro de la vida de Sarayaku para defender nuestro futuro). Dieser Text begründet die Ablehnung der Sarayaku, Erdölförderung auf ihrem Territorium zuzulassen, und ihre Forderungen nach autonomer Selbstverwaltung, einschließlich indigener Justiz und Wirtschaft, im Rahmen eines plurinationalen Staates (Sarayaku 2003: 1). Das Sumak Kawsay, „das Leben in Fülle“ (Sarayaku 2003: 10), „das Leben in Harmonie“ (Sarayaku 2003: 26f.), wird hier in enger Verbindung mit dem Territorium als von den Vorfahren geerbter Lebensraum und Ort autonomer Selbstverwaltung verstanden. Die Anpassung an einen Lebensraum über Generationen hinweg, ist ein Kernbestandteil des Guten Lebens (Sarayaku 2003: 3f.). Seine genauere Definition erfolgt mit Rückgriff auf die Mythologie:

„Unsere Hauptgottheiten, Amazanga y Nunguli, erinnern uns daran, dass wir nur das Notwendige aus dem Urwald nutzen sollen, wenn wir eine Zukunft haben wollen. Sie haben nie akzeptiert, dass wir mehr als das Erlaubte jagen oder dass wir sähen, ohne die Regeln des Ukupacha und des Kaypacha zu respektieren. Ihr Zorn, Wohlgefallen und Weisheit wurden uns durch unsere Weisen und Frauen enthüllt, die uns die Geheimnisse beigebracht haben, um die Harmonie mit sich selbst und der Natur zu erlangen, unser Grundsatz des Sumak Kawsay. So musste man der Natur eine Regenerationszeit geben, um unser eigenes Leben erneuern zu können. Wir sind in ständiger Bewegung gewesen und erlauben uns und anderen Lebensformen so, ihren Kreislauf weiterzugehen. Mushuk Allpa, die Erde in ständiger Erneuerung, war ein Grundsatz des Sumak Kawsay.“ (Sarayaku 2003: 3)

Von dieser Grundlage ausgehend fordern die Sarayaku die Erklärung ihres Territoriums zur ,Zone von biologischen, kulturellem und historischem Interesse für das Land und die Menschheit“ (Sarayaku 2003: 26), in der ein von den Sarayaku selbst aufgestellter und kontrollierter „Lebensplan für die nächsten 25 Jahre“ (Sarayaku 2003: 26) umgesetzt wird. Im Territorium sollen mehr oder weniger geschützte Bereiche definiert werden und mit Entwicklungs- und Bildungsprojekten und gemeinschaftlichem Tourismus eine nachhaltige Wirtschaft garantiert werden. In diesem Sinne fordern die Sarayaku eine Übertragung der staatlichen Kompetenzen im Umweltschutz und Tourismus an sie, sowie die Zuteilung der entsprechenden Mittel (Sarayaku 2003: 26f.).

Im selben Jahr greift die erste staatliche Institution den neuen Begriff auf. 2003 wird auch der Strategische Plan der CODENPE vorgestellt, der eine Entwicklung vorschlägt, die alle sozialen, wirtschaftlichen und Umweltaspekte umfasst:

„Sie schließt die Nachhaltigkeit in Bezug auf eine produktive Beziehung in Übereinstimmung mit der Bewahrung eines andauernden Prozesses der Suche nach dem Guten Leben oder 
persönlichen, familiären, gemeinschaftlichen und kollektiven Wohlergehen ein." (CODENPE 2003, in: Maldonado 2006: 114)

Im folgenden Jahr 2004 wird der Begriff des Guten Lebens in einer Publikation zur Gründung der der CONAIE nahestehenden Interkulturellen Universität Amawtay Wasi (Universidad Intercultural Amawtay Wasi) als bien vivir in einem weiteren Kontext verwendet. Sumak kawsay wird hier zu einer Lehrphilosophie, die der Interkulturalität verpflichtet ist. Die Erziehung wird

,als eine von der Gesamtheit der sozialen Akteure der verschiedenen Kulturen, die in den Prozess der Veränderung und Verwaltung der lokalen, nationalen und internationalen menschlichen Entwicklung eingreifen, geteilte Verantwortung angenommen, um ein angemessenes Gutes Leben für die aktuellen und zukünftigen Generationen zu erreichen“ (UIAW 2004: 168).

Sie soll also ,als eine Art und Weise zwischen anderen, zur Weisheit zu gehen, sich dem Verstehen des ,Guten Lebens“ anzunähern“ (UIAW 2004: 172f.) dienen.

In den Diskussionen um die Verfassungsgebende Versammlung 2007/2008 wurde der Begriff des Guten Lebens oder Sumak kawsay wieder aufgegriffen. In der Präambel der Verfassung von 2008 wird das Gute Leben als gesellschaftliches Ziel und in weiteren Artikeln (unter anderem Artikel 14, 275, 387) als Verantwortung und Aufgabe des Staates definiert. Diese Verankerung des Guten Lebens dient den Indigenen als Bezugspunkt, als etwas, auf das man in den täglichen Kämpfen verweisen kann und somit als Mittel zur Veränderung des Staates (Tenesaca 2010: 108f.). Damit ist die Verfassung von 2008 die zweite nach der von 1998, die explizit indigene Werte enthält und weit über das ,nicht faul sein, nicht lügen, nicht stehlen“ (ama quilla, ama llulla y ama shua), das $1998 \mathrm{zu}$ den verfassungsmäßigen Pflichten jeden Bürgers gemacht wurde (Art. 97, Nr. 20) (Galán 2009), hinausgeht. Die Verfassung von 2008 verwendet das Gute Leben als Begriff für eine Gesellschaft, die aufgebaut werden soll (Acosta 2010: 6), was sie, verbunden mit den mangelnden Umsetzungsmöglichkeiten, zu einer „nominalen Verfassung“ (Galán 2009) macht, die erst mit der Zeit zu einer normativen Verfassung werden kann sofern sie das Ideal des Guten Lebens überhaupt anwenden kann und nicht zu einer „Pseudoverfassung oder semantischen Verfassung“ (Galán 2009) wird.

Der Begriff des Guten Lebens wurde erst in diesen Diskussionen von der gesamten Indigenenbewegung aufgegriffen, obwohl die Inhalte des Begriffes für sie nichts neues sind. So spricht die CONAIE schon in ihrem Politischen Projekt von 1994 von einem „Integralen Humanismus, in dem der Mensch und die Natur in enger und harmonischer Beziehung stehen“ (CONAIE 1994: 11) und von der „Beziehung Mensch-Natur-Gesellschaft“ (CONAIE 1994: 11) als Prinzip und Forde- 
rung der Indigenen. Damit baut sie auf Überlegungen auf, die Nina Pacari 1984 veröffentlichte (Pacari 1984: 115). Die Vorstellung eines Staates, der auf eine Mischung der traditionellen Lebensweisen der Indigenen mit denen der anderen ethnischen Gruppen baut, war schon seit der Gründung der CONAIE vorhanden. Zu diesem Ideal gehört eine angemessene Verwaltung und eine nachhaltige Verwendung der natürlichen Ressourcen und der Schutz der indigenen Territorien (Montaluisa 2008: 101).

In den Diskussionen ab 2007 baut die CONAIE das Gute Leben zu einem zentralen Begriff ihres Diskurses auf. Sie wird zu ,einer Theologie der Erde, der Mutter Erde, der Pachamama“ (Tenesaca 2010: 108f.), die zuerst in den indigenen Territorien umgesetzt werden kann und von dort aus ein Modell für die gesamte Gesellschaft sein soll. So kämpfen sie für

„den Aufbau einer postkapitalistischen und postkolonialen Gesellschaft, eine Gesellschaft, die das ,Gute Leben“ fördert, das von unseren alten Vätern und Müttern von Generation zu Generation weitergegeben wurde, eine Gesellschaft, die die Lehren seiner alten Völker wiedergewinnt und in Harmonie mit unserer Pacha Mama leben kann“ (CONAIE 2007: 1).

Besonders die Wirtschaft soll sich nicht nach Gewinn, sondern nach menschlichem Wohlergehen richten. „Die Wirtschaft ist nur ein Werkzeug im Dienst der Gemeinschaft.“ (CONAIE 2007: 7) Daher soll sie sich an den Prinzipien des Guten Lebens ausrichten, die auf gemeinschaftliche Solidarität und Reziprozität bauen (CONAIE 2007: 21). Die Harmonie zwischen Menschen und Natur kann so etwas Neues schaffen. „[W]enn diese Mutter [...] nicht vergiftet ist, nicht ausgeplündert wird, nicht verseucht wird, ist es möglich, dass es wirklich einen Sozialismus gäbe.“ (Tenesaca 2010: 109) In diesem Sinne kritisieren einige Mitglieder der CONAIE das staatliche Verständnis des Guten Lebens.

„Sumak Kawsay ist das Leben in Fülle. Das Leben in materieller und spiritueller Exzellenz. Die Herrlichkeit und das Erhabene drücken sich in der Harmonie, im inneren und äußeren Gleichgewicht einer Gemeinschaft aus. Hier ist die strategische Perspektive der Gemeinschaft in Harmonie, das Höchste zu erreichen.“ (Macas 2010: 14)

Das mit der Verfassungsgebenden Versammlung von 2007/2008 gestiegene Interesse am Begriff des Guten Lebens wurde auch von nicht-indigenen Linken aufgegriffen, die sich nicht auf die Diskussion der indigenen Organisationen beziehen und eigene Bedeutungen ins Spiel bringen. Auch sie sehen das Gute Leben als ein „,neues Entwicklungsparadigma für Lateinamerika“ (Ramírez 2010: 5), das eine Alternative zum Kapitalismus darstellen kann. Die intellektuelle Linke geht aber weiter und versteht das Gute Leben als Kern einer „,biozentrische Wende“ (Hernández 
2009: 62). Die Verfassung von 2008 stellt so einen „Sozialismus des sumak kawsay oder republikanischen Bio-Sozialismus“ (Ramírez 2010a: 73) dar, der „eine biozentrische Ethik des Zusammenlebens wiedergewinnt“ (Ramírez 2010a: 73). Diese geringe Konkretisierung durch nicht-indigene Denker zeigt sich auch in der fragwürdigen Verortung des Ursprungs des neuen Begriffes:

„Das Sumak Kawsay, diese Weise des Seins in der Welt, die uns die ursprünglichen Völker und die Völker afrikanischen Ursprungs zeigen und von uns verlangen, bildet ein Streben nach Wohlergehen, dass der Staat unter Bedingung der Gerechtigkeit und des Zugangs für alle Bürger gewährleisten muss.“ (Fernández-Juárez 2010: 17f.)

Auch die inhaltliche Analyse von Catherine Walsh wird nur wenig konkret:

„In seinem allgemeinsten Sinne bezeichnet, organisiert und konstruiert buen vivir ein Wissens- und Lebenssystem, das auf die Gemeinschaft von Menschen und Natur und auf die räumliche-zeitliche-harmonische Totalität der Existenz baut. Also auf die notwendige Wechselbeziehung von Wesen, Wissen, Logiken und Rationalitäten von Gedanken, Taten, Existenz und Leben. Diese Auffassung ist Teil und Bestandteil der Weltsicht, Kosmologie oder Philosophie der indigenen Völker von Abya Yala.“ (Walsh 2010: 18)

Der gestiegene Einfluss des Begriffes des Guten Lebens zwingt auch die anderen Indigenenorganisationen dazu, ihn in ihre Forderungen zu integrieren. So erklärt die FEINE in einem programmatischen Text von 2010 zu einem ihrer Ziele: „Die organisatorische kirchliche Identität durch den Aufbau einer indigenen Theologie stärken, um zum Sumak Kawsay beizutragen“ (FEINE 2010: 7).

Es bleibt festzuhalten, dass Sumak Kawsay ein neu entwickelter Begriff ist, der den Aufbau einer neuen Gesellschaft, die über die Anerkennung der Verschiedenheit in Harmonie mit der Natur existieren kann, fordert. Dazu versucht dieser neue Begriff, durch die Kolonialität der Macht verlorene Wissensformen wiederzuentdecken (Acosta 2010: 9f.) und dadurch eine Alternative zu westlichen Vorstellungen von Wohlstand und Wachstum zu entwickeln. Als neuer Begriff integriert das Gute Leben neben der indigenen Weltsicht auch andere ,universelle philosophische Prinzipien“ (Acosta 2010: 13), darunter marxistische, ökologische, feministische, die alle „substanzielle Veränderungen unter Berufung auf andere Beziehungen zwischen den Menschen und der Umwelt" (Gudynas 2011: 11) erreichen wollen. Die Tatsache, dass dieser neue Begriff teilweise auf Vorstellungen indigener Völker aufbaut und manchmal in indigenen Sprachen formuliert wird, wird von einigen nicht-indigenen Intellektuellen als Kritik an der Kolonialität der Macht (Acosta 2010: 13) verstanden, die ein „dekolonialisierendes Potential“ (Gudynas 2011: 3) hat. „Anders gesagt: heute kann das Gute Leben nur als eine alternative soziale 
Existenz Sinn haben, als eine Des/Kolonialität oder Umverteilung der Macht.“ (Quijano 2010: 113) Der Begriff des Guten Lebens verleiht aus dieser Perspektive den indigenen Wissensformen als seinem Auslöser eine besondere Rolle (Gudynas 2011: 10). So wird das Gute Leben zum Widerstand gegen die geographische und kulturelle Beanspruchung von Lebensräumen (Gudynas 2011: 11). Die darin enthaltene Mischung aus Dekolonisierung des eurozentrisch geprägten Wissens und des Respekts der kulturellen Diversität macht das Gute Leben anschlussfähig für eher kulturell ausgerichtete Begriffe wie Interkulturalität oder Plurinationalität (Gudynas 2011: 12). Das Gute Leben ist ein normativer Begriff, der von der Ablehnung der Ungleichheit und der ökologischen Zerstörung ausgehend Vorstellungen einer idealen Gesellschaft entwickelt, die er wiederum für die Kritik der heutigen Gesellschaft nutzen will (Spedding 2010: 19f.).

\section{ZUSAMMENFASSUNG}

Mitte der 1970er Jahre beginnt in ganz Lateinamerika ein Prozess der Entwicklung spezifisch indigener Begriffe, die Alternativen zu den bisherigen eurozentrischen Begriffen darstellen. Einer der ersten dieser Begriffe ist Abya Yala als Bezeichnung für Amerika. Abya Yala bedeutet in der Sprache der Kuna aus Panama in etwa ,fruchtbares Land‘. $1975^{26}$ nahm der Aymara-Intellektuelle Takir Mamani (Constantino Lima) als Ersatzrepräsentant der Indigenen Boliviens am Ersten Weltkongress der Indigenen Völker in Kanada teil, auf dem der Weltrat der Indigenen Völker gegründet wurde. Auf dem Rückweg besuchte er eine Reihe von Ländern und indigenen Völkern, darunter die Kuna in Panama. Die dortigen Autoritäten stellten ihm den Begriff Abya Yala als Alternative zum Begriff Amerika vor und trugen ihm auf, diesen Gedanken zu verbreiten (Portugal 2008), was er, etwa mit einer Publikation von 1986 (Kummels 2008: 174) ${ }^{27}$, auch tat. Von Bolivien breitete sich die neue Bezeichnung rasch in den indigenen Organisationen des Kontinents aus und konnte sich im Zuge des Jahrestages der Entdeckung Amerikas 1992 auch außerhalb der Indigenenbewegung etablieren. Abya Yala ist also der Versuch, eine eigene Bezeichnung für den amerikanischen Kontinent zu finden, die auf Bezüge auf europäische Entdecker oder - wie bei der Bezeichnung ,Lateinamerika' - europäische Sprachen verzichtet (Estermann 2011: 1).

26 Nach Estermann (2011: 1) und Kummels (2008: 184) fand die Reise 1977 statt, der Kongress 1975 ist allerdings belegt.

27 Laut Kummels tauchte der Begriff Abya Yala 1986 zum ersten Mal auf, der Verlag Abya-Yala trägt diesen Namen allerdings schon seit 1983 (Dietz 1996: 1). 
Einige Jahre zuvor hat Constantino Lima die Verbreitung der Wiphala als Flagge der Indigenenbewegung in den Ländern des Andenraums angestoßen. Bis Ende der 1960er Jahre war die Wiphala nur wenig bekannt. Lima bekam Zugang zu einem Buch über die Indigenen Perus, in dem diese Flagge abgebildet war und verwendete sie zum ersten Mal im April 1970 bei einer Vereidigung von Sekundarschülern in Pacajes. Die damaligen Wiphalas hatten noch nicht die Farben, die heute verwendet werden. Die heutige Farbgebung in den Farben des Regenbogens tauchte aufgrund weiterer Forschung und der Kreativität der damaligen Aktivisten erst in den folgenden Jahren auf - begleitet von einem Prozess der relativen Entpolitisierung dieses Symbols (Portugal 2008). Die Wiphala wird in Anlehnung an den Nationalstaat europäischer Prägung als Flagge verwendet und von den Indigenen als „Symbol von Verschiedenheit und Einheit der ,originären Völker“ der Amerikas" (Kummels 2008: 187) verstanden.

Die 1970er und 1980er Jahre sind vom Entstehen eines neuen Diskurses der Indigenenbewegung geprägt, manchmal als ,Indianismus‘ umschrieben (Landa 2006: 13). In dieser Zeit werden einige Begriffe neu entdeckt - wie der der indigenen Nationalitäten - und andere eingeführt. In Ecuador entsteht eine neue Indigenenbewegung, die weder rein gewerkschaftlich - wie FEI und FENOC bis in die 1990er , noch als reine Verteidigungsorganisation der Indigenen vor Ort - wie die FICSHA in den ersten Jahren - funktioniert. Diese neue Indigenenbewegung ist dadurch charakterisiert, dass sie auf gesamtstaatlichem Niveau agiert und Forderungen formuliert, die die gesamte Gesellschaft betreffen.

Auf ideengeschichtlicher Ebene geht dem Entstehen des von Indigenen selbst entwickelten Indianismus die Krise des weitgehend von mestizischen und weißen Intellektuellen betriebenen Indigenismus voran, der vor allem von den 1940ern bis in die 1960er bestimmend war (Landa 2006: 16). Der Indigenismus wurde mit der Gründung des Interamerikanischen Indigenistischen Instituts 1940 in institutionelle Bahnen gelenkt. 1942 wurde das Ecuadorianische Indigenistische Institut (Instituto Indigenista Ecuatoriano, IIE) unter Pío Jaramillo Alvarado und Víctor Gabriel Garcés gegründet, das den ecuadorianischen Indigenismus institutionalisierte (Landa 2006: 31/37). Der Versuch, die FEI als kommunistischen Indigenismus aufzufassen (Landa 2006: 31), also als ein Bemühen um die Indigenen von Seiten der nichtIndigenen, wurde von Marc Becker (2007) überzeugend widerlegt. Im vorliegenden Text wurde darauf aufmerksam gemacht, dass die FEI keinesfalls von der Kommunistischen Partei oder der CTE abhängig war, sondern auf die Bemühungen Indigener zurückgeht und von diesen gesteuert wurde und wird. Tatsächlich ist kein gröBerer Einfluss der ecuadorianischen Indigenisten auf die Indigenenbewegung dieses Landes nachweisbar - was auch an der sozialistischen Ausprägung dieser Bewegung zur fraglichen Zeit (ca. 1940-Mitte der 1960er) liegen kann. Zur Zeit der relativen Stärke des Indigenismus war der Diskurs der wirklichen Indigenen ein 
völlig anderer, Fragen der Integration als kulturell Andere, wie sie der Indigenismus aufwarf, waren für FEI und FENOC eher sekundär.

Die 1970er Jahre sind gekennzeichnet durch die Krise des Indigenismus und das Aufkommen explizit ethnisch ausgerichteter Indigenenbewegungen in Amerika. Der Wandel der Indigenenbewegungen führt $\mathrm{zu}$ einem ,unklaren ideologischen Netzwerk, dass Indianismus, Pan-Indianismus oder einfach indianisches politisches Denken genannt wurde“ (Landa 2006: 39f.). Dabei ist die inhaltliche Ausrichtung dieses neuen Denkens zuerst wenig klar - abgesehen von einer Kritik am Indigenismus und seiner Versuche, für die Indigenen zu sprechen (Landa 2006: 39f.). Es war ,ein Diskurs der Indigenen selbst für ihre eigene Befreiung“ (Landa 2006: 40). Als solcher versuchte der Indianismus, sich von jedem Einfluss der damals dominanten Denkrichtungen - vor allem Sozialismus und indigenistischer Integrationismus - abzugrenzen (Landa 2006: 40). Landa bezeichnet vier Gruppen, die diesen neuen Indianismus betreiben: die Indigenen selbst, indigene Theoretiker, radikale Indigenisten und indianistische Anthropologen (Landa 2006: 41). Vor allem für die letzten beiden Gruppen sind die Treffen von Barbados 1971 und 1977 wichtig, in denen sich die - vor allem lateinamerikanische - Anthropologie einer scharfen Selbstkritik unterzieht und der klassische Indigenismus zu seinem Ende kommt. Auch wenn oft von dem Indianismus gesprochen wird, handelt es sich keinesfalls um eine einheitliche Strömung. Vielmehr geht es um einige grundlegende Punkte, die in den konkreten Forderungen der Indigenen in ihren Ländern andere Ausprägungen erfahren können (Landa 2006: 41).

Das Ende des Indigenismus beginnt auf dem 39. Internationalen Kongress der Amerikanisten (International Congress of Americanists, ICA) in Lima 1970, auf dem eine Gruppe von Anthropologen zusammenfindet, die ein Jahr später ein Treffen auf Barbados durchführen sollte. Die auf diesem Treffen verabschiedete Erklärung von Barbados bezeichnet die Indigenenpolitik der lateinamerikanischen Staaten als Ethnozid und Genozid, ein Verständnis, das sich im Indianismus verankern sollte (Landa 2006: 56). Die so entstandene Gruppe von Barbados hat in drei Treffen 1971, 1977 und 1993 eine andere Indigenenpolitik von den lateinamerikanischen Staaten gefordert und den Indigenismus kritisiert. Sie forderten, den indigenen Völkern die Möglichkeit zur Selbstorganisation zu geben und ihre Abhängigkeit von Missionaren, Anthropologen und Indigenisten zu beenden (Landa 2006: 57f.). Diese Abhängigkeit wurde auf dem Treffen 1971 als Ethnozid beschrieben, also als Zerstörung der Kultur der Indigenen, ohne sie physisch zerstören zu wollen (Landa 2006: 59). Barbados I und die dort gefassten Beschlüsse blieben allerdings auf die Wissenschaftswelt und die Anthropologie beschränkt und wurden von den Indigenenbewegungen dieser Zeit nicht zur Kenntnis genommen (Landa 2006: 59f.). 
Das Treffen Barbados II 1977 hatte weitreichendere Folgen. In ihrer abschließenden Erklärung forderten die Anthropologen und Indigenen, die an dem Treffen teilnahmen, die Selbstorganisation der Indigenen als Befreiungsbewegung unter einer kohärenten und für die Gesamtbevölkerung verständlichen Ideologie. So soll die Bewegung selbst zusammengehalten und zur gleichen Zeit die Mobilisierung eines Großteils der Bevölkerung ermöglicht werden. Weiterhin wird neben der Bewahrung und Förderung der indigenen Sprachen der Aufbau von Kommunikationswegen zwischen den Völkern verschiedener Sprachen und der Aufbau eines Erziehungssystems, dass die kulturellen Werte des jeweiligen Volkes erhält, gefordert (Landa 2006: 57f.). Dieses Programm wird oft als die Grundlage für den Indianismus als genuin eigene Ideologie der Indigenenbewegungen Lateinamerikas gesehen (Landa 2006: 59). Obwohl den Indigenenbewegungen eine besondere Rolle zugeschrieben wurde und die Indigenen erstmals nicht nur als Objekte des Indigenismus im Zentrum der Aufmerksamkeit standen, sondern als Akteure in eigener Sache, stand der beschriebene Wandel vor allem bis zum II. Treffen von Barbados unter dem entscheidenden Einfluss der Anthropologen, die für die Indigenen Genozid und Ethnozid anklagten (Landa 2006: 58).

Auch wenn die Veränderungen in der Anthropologie mit Sicherheit Einfluss auf die Indigenenbewegungen ausgeübt hat und sie mit Theorien und Begriffen unterstützen konnte (Landa 2006: 58), so sind Indigenismus und Indigenismuskritik im bisher referierten Sinne und Indianismus als Ideologie der Indigenen selbst streng voneinander getrennt - nicht nur personell und strukturell, sondern auch in Bezug auf die Weltsicht und die konkreten Forderungen. Daher muss Landa in diesem Punkt widersprochen werden. Ein Beispiel für den Gegensatz zwischen Wissenschaftlern - so kritisch sie auch sein mögen - und sozialen Bewegungen ist das dritte Treffen von Barbados 1993, auf dem wieder nur Anthropologen teilnahmen, die die Führer der Indigenenorganisationen kritisierten, weil diese - nach Beurteilung der Anthropologen - ihre Basis nicht länger repräsentieren und sich stattdessen einer persönlichen politischen Karriere widmen, also klientelistisch und korrupt agieren und so das politische Projekt der Indigenenbewegung selbst in Gefahr bringen. Die Akademiker empfehlen daher den Indigenenbewegungen, über diese Probleme nachzudenken und sie zu korrigieren (Landa 2006: 59).

Tatsächlich gab es ab Anfang der 1970er Jahre einen Anstieg an politischen Aktivitäten der Indigenenbewegungen Amerikas. Die Anzahl von Organisationen, die sich als ethnisch oder indigen beschreiben, nahm stark zu, ebenso wie ihre Mobilisierungsfähigkeit und ihre konkreten Mobilisierungen, die schon früh zum Aufbau von strukturellen Verbindungen zwischen den verschiedenen nationalen Organisationen führten (Bonfil Batalla 1978: 209). Es wäre zu untersuchen, ob dieser Wandel mit einem allgemeinen Anstieg an Aktivitäten verschiedener, auch nichtindigener, Gruppen in Verbindung steht, den etwa Jenson (1995: 109) im Kanada 
der 1970er Jahre diagnostiziert und deren Ergebnis neue Gesetze zu Zweisprachigkeit und Bikulturalität waren. Zu den ersten der transnationalen strukturellen Verbindungen gehört das I. Amerikanische Indio-Parlament des Cono Sur (I Parlamento Indio Americano del Cono Sur), das 1974 in Asunción, Paraguay, tagte. An ihm nahmen Vertreter der Völker Maquiritare, Quechua, Aymara, Guaraní, Chulupi, Toba, Kolla, Mapuche, Pai-Tavitera, Parixi und Mataca aus Argentinien, Bolivien, Brasilien, Paraguay und Venezuela teil. Weißen war die Teilnahme untersagt, um so eine Einflussnahme von außen zu vermeiden (Barre 1982: 58). Das Parlament beschäftigte sich in erster Linie mit der Lebenssituation der verschiedenen indigenen Völker (Barre 1982: 62) und war vom Entstehen eines ethnischen Bewusstseins auf kontinentalem Niveau geprägt. Es wurde eine stärkere Organisierung der Indigenen angeregt. Die Basisorganisationen sollten sich in regionalen Föderationen zusammenschließen, die nationale und internationale Konföderationen bilden sollten. Jede Indigenenorganisation sollte dabei ihre Unabhängigkeit vom Staat und den politischen Parteien bewahren, um so eine Vereinnahmung zu vermeiden (Barre 1982: 59).

Ein Jahr später wurde auf einem Kongress in Port Alberni, Kanada, der Weltrat der Indio-Völker (Consejo Mundial de Pueblos Indios, CMPI) gegründet (AAVV 1985: 162). An diesem Kongress nahmen Vertreter aus Argentinien, Australien, Bolivien, Kanada, Kolumbien, Ecuador, Finnland, Grönland, Guatemala, Mexiko, Neuseeland, Nicaragua, Norwegen, Panama, Paraguay, Peru, Schweden, den USA und Hawaii, und Venezuela teil. Es wurden einige Beschlüsse zu wirtschaftlichen, kulturellen und politischen Rechten der Indigenen verabschiedet und der Schutz der Umwelt und der Naturschätze angemahnt. Im CMPI waren die später gegründeten Regionalrat der indigenen Völker Zentralamerikas (Consejo Regional de los Pueblos Indígenas de América Central, CORPI) und Indio-Rat Südamerikas (Consejo Indio de América del Sur, CISA) organisiert (Barre 1982: 59).

Der I. Kongress der Indio-Bewegungen Südamerikas (I Congreso de Movimientos Indios de Sudamérica) wurde vom CMPI und peruanischen und argentinischen Indigenenorganisationen organisiert und fand vom 27.2. bis 3.3.1980 in Ollantaytambo in Cusco, Peru, statt (Barre 1982: 61). Es beteiligten sich Vertreter der Indigenenbewegungen Perus, Ecuadors, Kolumbiens, Venezuelas, Brasiliens, Boliviens, Paraguays, Argentiniens und Chiles und weitere Vertreter aus Panama, Nicaragua, Guatemala, El Salvador, Mexiko, den USA, Kanada, Skandinavien und anderen Ländern. Auf dem Kongress wurde über die Geschichte der indigenen Völker, ihre Philosophie und ihre politischen und wirtschaftlichen Organisationsformen diskutiert. Aus den Ergebnissen sollten die Grundlagen der Indianität und ihre Bedeutung für den Rest der Welt als spezifisch politische Theorie herausgearbeitet werden. Die indigenen Völker und ihre Auffassung von Politik wurden in diesem Sinne als Indianität definiert, die dem westlichen Politikverständnis gegenübersteht (Ibarra 
Illanez 1987: 25). So wurde auf dem Kongress die Dekolonisierung und Befreiung der Indigenen als Ziel der Indigenenbewegungen festgehalten (Barre 1982: 62). Die vor allem von den Kataristas aus Bolivien vertretene ethnische Strömung, die von den Repräsentanten der peruanischen, ecuadorianischen, argentinischen und kolumbianischen Organisationen unterstützt wurde, konnte sich auf dem Kongress gegen die marxistische Strömung durchsetzen, die von den Repräsentanten Venezuelas, Brasiliens und Chiles vertreten wurde (Barre 1982: 61).

Auf diesem Kongress wurde die indianistische Ideologie weiterentwickelt und der Indio-Rat Südamerikas (Consejo Indio de Sudamérica, CISA) mit Sitz in La Paz, Bolivien, gegründet (Barre 1982: 61; AAVV 1985: 162), der in den folgenden Jahren zum wichtigsten und einflussreichsten Vertreter des Indianismus in Südamerika werden sollte (Ibarra Illanez 1987: 27). Er erklärt in seinen Statuten das als indigen beschriebene Verständnis der Welt als in ständiger Bewegung begriffene harmonische Beziehungen sich ergänzender Gegensätze zur Grundlage des Indianismus. Der Mensch ist als Faktor dieses Gleichgewichts integraler Teil der Welt, seine Entwicklung hängt aber gleichzeitig von dieser Funktion ab - er ist also keinesfalls Herrscher über die Umwelt. Im Sinne des Indianismus will der CISA das Schicksal der indigenen Völker mit Rückgriff auf ihre Geschichte und Tradition lenken. Besondere Bedeutung haben für den Indianismus des CISA die kollektivistischen und kommunitären Aspekte der indigenen Völker im Sinne eines Gemeinwohls für alle (Statuten CISA 1980, in: Landa 2006: 42; AAVV 1985: 163ff.). Der indianistische Kommunitarismus gründet auf die verschiedenen kommunitären Systeme der indigenen Völker, wie etwa ayni, mink'a, camayali oder yanapacu und das moralische Prinzip des ama swa, ama llulla y ama qhe $1 \mathrm{a}^{28}$, wobei betont wird, dass diese Art des Kommunitarismus ,dem Kapitalismus und dem westlichen Sozialismus völlig fremd ist“" (AAVV 1985: 164).

So kommt der CISA auf seinem Gründungskongress zum Schluss, dass der Indianismus das Zeichen der Befreiung des Kontinents ist (AAVV 1985: 163) und empfiehlt für die Länder, in denen die Indigenen in der Mehrheit sind, die direkte Machtergreifung, für die Länder, in denen sie in der Minderheit sind, aber den Kampf für eine Autonomie. Diese Empfehlung sollte zur Entwicklung von Forderungen nach einem plurinationalen Staat, der seine verschiedenen Nationalitäten respektiert, beitragen (Barre 1982: 74). Der Kampf kann nach den Empfehlungen des CISA auch in Kooperation mit anderen, nicht-indigenen Bewegungen stattfinden, ohne dass dadurch jedoch die kulturelle Identität und Unabhängigkeit der Indigenen gefährdet werden soll (AAVV 1985: 164). Aus denselben Gründen lehnt er den Indigenismus ab, ,denn er entspricht der dominanten Ideologie, die die Flag-

28 In der Schreibweise dieses Prinzips wird den jeweiligen Quellen gefolgt. 
ge des Indios nur erhebt, um Ruhm und Geld in seinem Namen zu verdienen und den Interessen der Unterdrücker zu dienen“ (AAVV 1985: 164).

Aus einer marxistischen Position beschreibt Alicia Ibarra Illanez den Indianismus als „Ethnopopulismus“ (Ibarra Illanez 1987: 25). Dabei bezieht sie sich auf Lenin, der im Russland um das Jahr 1900 eine politische Strömung kritisiert, die den Kapitalismus als Dekadenz versteht und die russischen und insbesondere bäuerlichen Wirtschaftsformen als davon verschiedene und historisch legitimierte Lebensformen beschreibt (Ibarra Illanez 1987: 45). In diesem Sinne liegt dem Ethnopopulismus eine ahistorische und übermäßig positive Vorstellung der ursprünglichen ethnischen Gruppen zugrunde. Die Indigenen werden so den nicht-Indigenen gegenüber gestellt und müssen sich gegen sie verteidigen. Das kann mittelfristig nur über ethnisch und kulturell legitimierte Autonomien geschehen, die die indigene Identität bewahren und als fest definierte Einheiten in einen multinationalen Kontext integrieren. Langfristig ist eine Rückkehr in die Vergangenheit, etwa zum InkaReich oder Tahuantinsuyo angestrebt (Ibarra Illanez 1987: 25-26). Die Situation der Indigenen wird somit eher als politische Unterdrückung eigenständiger Völker denn als wirtschaftliche Ausbeutung einer Klasse verstanden. Es handelt sich um den Kampf zwischen zwei Lebenswelten, der nur durch Autonomie oder Machtergreifung beendet werden kann. Daher arbeiten die Ethnopopulisten mit Regierungen zusammen, die sie als Ethnien anerkennen und ihnen bestimmte Grade an Autonomie zugestehen. Sie streben das Ende der Unterdrückung der indigenen Völker durch den Aufbau von autonomen Gebieten mit unterschiedlichen Entwicklungsformen innerhalb eines multinationalen Staates an (Ibarra Illanez 1987: 26). Es muss angemerkt werden, dass Ibarra Illanez eine Übertragung des kontinentalen Programms des Indianismus auf Ecuador unternimmt, die nicht durch Material der dortigen Indigenenorganisationen gestützt ist. Sie beschreibt somit Gruppen als indianistisch oder ethnopopulistisch, die sich nicht des indianistischen Diskurses bedienen.

Für Bonfil Batalla ist eines der wichtigsten Charakteristika des Indianismus die dichotome Einteilung der Welt in westliche, europäisch geprägte und indigene Zivilisationen, die entgegengesetzt sind und sich widersprechen. Dabei kommt der europäischen Zivilisation die Rolle des Aggressors zu, während die indigene Welt trotz Unterdrückung überleben konnte. Dieser Aggressor und seine Weltsicht sind für die Indianisten gescheitert (Landa 2006: 44). Aus den sehr verschiedenen und geographisch weit verstreuten Kulturen konstruieren die Indianisten so ein einheitliches Denken, welches im Sinne der Pan-Indianität - ein weiteres Charakteristikum des Indianismus für Bonfil Batalla - davon ausgeht, dass die verschiedenen indigenen Völker Teil einer Zivilisation sind, die als solche erst organisatorisch und ideologisch geschaffen werden muss (Bonfil Batalla 1981: 20). Diese indigene Zivilisation zeichnet sich durch eine harmonische Beziehung zwischen Natur und Mensch 
aus und durch die kommunitäre Organisation der Gesellschaft (Landa 2006: 45) beides zentrale Unterschiede zur europäischen Zivilisation. Der so beschriebene Indianismus wurde in den 1960er und - vor allem - 1970er von einer der ersten universitär gebildeten Generationen indigener Führer geprägt, die ihre wissenschaftliche Ausbildung und die Kontakte zu mestizischen Intellektuellen für politische Zwecke einsetzten (Landa 2006: 45). In den 1980er Jahren begann die indianistische Bewegung, sich zunehmend von nicht-Indigenen und Anthropologen zu emanzipieren. Das führte zu einer scharfen Kritik der Indigenenbewegungen an der Anthropologie und dem Verhalten der Anthropologen - auf die diese von der Wissenschaftswelt aus nicht angemessen reagieren konnten. Die zunehmende Autonomie der so ausgerichteten Indigenenbewegungen brachte in diesen Jahren auch einen zunehmenden Einfluss auf die internationale Politik mit sich, vor allem im Rahmen der Vereinten Nationen und deren Organe (Landa 2006: 58f.).

Die relative Annäherung an eine einheitliche Ideologie und ein allgemeines politisches Programm des Indianismus in den 1970er und 1980er ist spätestens seit Beginn der 1990er nicht mehr vorhanden. Abgesehen von einem Weiterbestehen der beschriebenen Überzeugungen gibt es nur noch wenige Bemühungen, diese in pan-indianistischen Strukturen auf kontinentalem Niveau zu verfestigen (Landa 2006: 62). Stattdessen werden die Elemente des Indianismus nach Belieben in die nationalen Kontexte und politische Gelegenheiten eingebaut, ohne sich an einem festen Diskurs zu orientieren (Landa 2006: 63). Zu diesen Elementen gehören der Bezug auf die Geschichte vor der Eroberung und die Tatsache, dass zu dieser Zeit die Indigenen das Land besaßen. Dazu kommt je nach Fall eine Identifikation mit einem indigenen Sozialismus oder Kommunitarismus, die Ablehnung der Ideologie der mestizaje, die Befreiung der Indigenen, die Betonung der ökologischen Aspekte der indigenen Weltsicht und die Darstellung der indigenen Lebensweise als Alternative zu den bestehenden politischen und wirtschaftlichen Systemen (Landa 2006: 63-66). Seit der Jahrtausendwende ist insbesondere der Ökologismus der Indigenen ein Motiv, dass aus den Diskursen der meisten Indigenenbewegungen nicht wegzudenken ist (Landa 2006: 67).

Eine Schwierigkeit bei der Definition des Indianismus ist die, dass sie von der Kritik am Indigenismus, insbesondere von der Gruppe von Barbados aus gemacht wird. Eine Gegenströmung gegen ein kritisiertes Phänomen wird von den Kritikern dieses Phänomens beschrieben - ihre Objektivität ist also keinesfalls selbstverständlich. Die ersten Texte, die bestimmte Denkströmungen über Staatengrenzen hinweg einem pan-indigenen Indianismus zuschreiben, stammen von Guillermo Bonfil Batalla. Mit Utopía y Revolución 1981 hat er eine Reihe von Texten von 1970 bis 1980 unter der Kategorie Indianismus versammelt und interpretierend eingeleitet (Landa 2006: 42) - ohne dass diese Texte selbst eindeutige Anknüpfungspunkte haben. Bonfil Batalla ist somit der erste, der einen diskursiven Wandel 
in den 1970er feststellt - und damit diesen Wandel selbst mit erzeugt. Spätere Autoren (etwa Barre 1983 und Ibarra Illanez 1987) übernehmen seine Kategorienbildung und verstärken sie weiter. In diesem Sinne werden völlig verschiedene Gruppen, die oft nur wenige Kontakte und Gemeinsamkeiten hatten, dem Indianismus zugeordnet. Landa beschreibt vier Gruppen. Dazu gehören die

„Theoretiker der Indianität“ (Landa 2006: 60f.) wie Guillermo Carnero Hoke, Fausto Reinaga oder Virgilio Roel, deren Texte die zentralen Ideen des Indianismus entwickeln. Barre beschreibt diese „Indio-Ideologen“ (Barre 1982: 57f.) als Intellektuelle, die als Mitglieder der ethnischen Organisationen die Realität der Indigenen untersuchen und in einen Dialog mit nicht-indigenen Intellektuellen, vor allem den engagierten Anthropologen, treten, wodurch sie den Indianismus entwickeln. Die zweite Gruppe sind die kritischen Anthropologen um die Gruppe von Barbados und andere nicht-indigene Verbündete, die ihre Verbindungen zu sowohl Indigenenbewegungen als auch Wissenschaft dazu nutzen, um die indigenen Forderungen und Ideologien bekannt $\mathrm{zu}$ machen. So konnten sie eine wissenschaftlich fundierte Verteidigung der Indigenen formulieren. Die dritte Gruppe sind die radikalen Indigenisten, die noch innerhalb der indigenistischen Ideologie die Position der Indigenen zu stärken versuchten. An vierter Stelle folgen für Landa die indigenen Aktivisten selbst, deren Beitrag zum Indianismus weniger in der Formulierung klarer Programme liegt, sondern vielmehr im Ausüben von Druck, um die bereits bestehenden Forderungen umzusetzen (Landa 2006: 60f.). Schon diese imaginierte Arbeitsteilung ist hochgradig problematisch und lässt sich am ecuadorianischen Fall - den Landa (2006: 68f.) allerdings explizit ausklammert - nicht überprüfen.

Ein weiteres Problem - für die vorliegende Arbeit - dieser Erzeugung des Indianismus ist die Tatsache, dass die kategorienbildenden Texte und Zusammenstellungen sich nur sehr marginal auf den ecuadorianischen Fall beziehen. Sowohl Bonfil Batalla (1981), als auch Barre (1982) beziehen sich auf einige wenige Texte der Shuar-Föderation - die wohl auch dadurch zu ihrem Ruf gelangt ist, besonders stark ethnisch ausgerichtet zu sein. Andere Indigenenorganisationen Ecuadors werden beim Aufbau der Kategorie des Indianismus nicht berücksichtigt - was später ein Problem für diejenigen Forscher sein sollte, die von dieser Kategorie ausgehen (etwa Ibarra Illanez 1987). Tatsächlich konnte nachgewiesen werden, dass nicht die Shuar-Föderation selbst, sondern vielmehr FENOC und CONAIE - also ihre ,Mutterorganisationen“ - bis in die späten 1980er Jahre im CISA - der Zentralinstanz des Indianismus - aktiv waren. Trotz dieses Engagements zu den Entstehungszeiten der Indigenenbewegung werden die ecuadorianischen Indigenenorganisationen von den aktuellen Verfechtern der Kategorie des Indianismus als Sonderfall ausgeklammert. So sprechen die ecuadorianischen Indigenen nicht in den Begriffen des Indianismus. 
„Die erfolgreichen indigenen Führer Ecuadors schreiben ihre Dokumente nur sehr selten nach diesen Parametern. Ihre Texte scheinen diese ideologische Form, sich zu identifizieren, zu vermeiden und ziehen es vor, schlicht von ,Indigenenbewegung ' zu sprechen: in Ecuador gibt es keine Indianisten, sondern einfach Indigenenbewegung.“ (Landa 2006: 68f.)

Ein direkter Einfluss der Entwicklung der internationalen Anthropologie auf die Indigenenbewegungen allgemein und die ecuadorianische Indigenenbewegung im Besonderen ist schwer nachzuweisen. Die Einwirkung einzelner Anthropologen (Yuri Zubritzki, Ileana Almeida) auf die Entwicklung des Diskurses bestimmter Organisationen wurde bereits untersucht, eine systematische Einflussnahme ist allerdings nicht festzustellen. Dennoch ist es interessant zu beobachten, wie der mexikanische Anthropologe Guillermo Bonfil Batalla - der keinerlei Kontakt zu den Indigenen Ecuadors hatte - im Anschluss an das zweite Treffen von Barbados 1977, auf dem sich eine Reihe vor allem lateinamerikanischer Anthropologen mit 16 Indigenen ausgetauscht haben (Bonfil Batalla 1977: 100), einige der zentralen Begriffe der ecuadorianischen Indigenenbewegung diskutiert - Jahre, bevor diese die Begriffe zum ersten Mal verwendet. So kommentiert er eine offenbar bereits bestehende Diskussion über den Begriff der Nationalitäten oder Nationen als Bezeichnung ethnischer Gruppen, die eine „,politische Sichtbarkeit“ (Bonfil Batalla 1977: 97) erreicht haben. Die Definition, die Bonfil Batalla findet, ist der Definition die die CONAIE dem Begriff der Nationalität geben sollte, sehr ähnlich. In beiden Fällen bezeichnet Nationalität eine ethnische Gruppe mit historisch konstituierter Identität und der Fähigkeit, autonome soziale Einheiten zu bilden (Bonfil Batalla 1977: 98). Darauf aufbauend fordert er von den jeweiligen Staaten die Anerkennung dieser Nationalitäten und damit auch die Anerkennung ihres eigenen Charakters als „multiethnisch oder multinational“ (Bonfil Batalla 1977: 98) oder auch „plurinational“ (Bonfil Batalla 1977: 99). Er verweist auf die Erfahrungen aus Finnland, der Schweiz und Spanien als Beispiele für solche Staaten (Bonfil Batalla 1977: 100). In einem späteren Text beschreibt er die Lage der indigenen Völker als „die Probleme der interkulturellen Situation“ (Bonfil Batalla 1978: 212), womit er einen weiteren Begriff der ecuadorianischen Indigenenbewegung vorwegnimmt.

Zur gleichen Zeit - in den 1970ern und 1980ern - entsteht aus dem Zusammenfließen zum einen der Organisationen des Hochlands und des Amazonasgebietes und zum anderen der klassistischen und ethnischen Strömungen eine neue Indigenenbewegung in Ecuador. Die Verbindung verschiedener Traditionen und Weltsichten führt zum Aufkommen neuer Forderungen und Bedeutungen, die es erlauben, die Ideen der verschiedenen Gruppen in einer „globalen Strategie“ (Iturralde 1997: 83) auszudrücken. Die Indigenenbewegung begnügt sich nicht länger mit einer Anerkennung der Indigenen ,als einfache Bürger, ohne Sprache, ohne Gebräuche, ohne Territorium“ (ECUARUNARI 1984: 46), sondern fordert „eine eth- 
nische Bürgerschaft in Ecuador, eine Bürgerschaft, die individuelle und kollektive Rechte beinhaltet" (Viteri 1997: 54). Dadurch erzeugt die Indigenenbewegung „eine neue nationale und indigene Seinsform“ (Iturralde 1997: 83), die mit einem hohen Niveau ethnischen Bewusstseins, großer Anerkennung in der Gesellschaft und einem unterstützenden internationalen Umfeld einhergeht (Iturralde 1997: 84f.). Die Indigenenbewegung wird zur Trägerin von Forderungen nach einem neuen Verständnis von Staatsbürgerschaft, das kulturelle Unterschiede und politische Autonomie erlaubt - und daher im Widerspruch zum klassischen Modell der liberalen Demokratie und zum Projekt der mestizaje ist (Zamosc 1994: 38). Diese Entwicklung wird vom Übergang zur Demokratie und der so nötig gewordenen Neudefinition der nationalen Identität in der Gesellschaft begünstigt (Sánchez-Parga 1988: 106f.; Sánchez-Parga 2010a: 86f.). In diesem Moment verstärkt sich die Entwicklung eines ethnischen Bewusstseins und eines indigenen Diskurses, der auf vorherige Diskurse und ,ethnische Ansammlungen“ (Sánchez-Parga 1988: 107), also die Lektüre und Neuinterpretation der Geschichte der indigenen Völker durch diese selbst, aufbaut (Sánchez-Parga 1988: 107f.). Dadurch hat die ethnische Bewusstwerdung ein revolutionäres Potential und kann die Grundlage für die Ausbildung eines politischen Bewusstseins sein (Barre 1982: 73f.). Das schließt auch die Neubewertung der Räume ein, die die jeweiligen indigenen Völker besitzen - und die ein Resultat des Kolonialismus sind. Diese ,symbolische Territorialität“ (Bonfil Batalla 1981: 25) wird die Grundlage für Forderungen nach eigenen, selbstbestimmten Räumen - territorial, wirtschaftlich, politisch oder kulturell -, die die freie Entwicklung jedes Volkes ermöglichen sollen (Bonfil Batalla 1981: 31). Der Kampf für diese Räume und ,die Verteidigung bestimmter raumbasierter geschichtlicher Weltsichten und Praktiken der Schaffung der Welt“ (Escobar 2004: 222f.) sollte innerhalb einer neuen, transnationalen „Raum-Politik“ (Escobar 2004: 222f.) ab den 1990ern auch international an Bedeutung gewinnen. Diese Formen der „Identitätspolitik“ (Ibarra 1999: 72), verbunden mit den komplexen organisatorischen Prozessen der Indigenenbewegung, führten $\mathrm{zu}$ einer neuen Definition des Indios oder Indigenen, in der kulturelle Bestandteile, wie Sprache und Traditionen, ebenso wie die organisierten Forderungen betont werden (Ibarra 1999: 76). Die ethnische Identität der Indigenen wurde so von einem Stigma durch Staat und Gesellschaft zu einem positiven und politisch wirksamen Selbstverständnis (Ibarra 1999: 72).

Das Jahrzehnt von Mitte der 1970er bis Mitte der 1980er ist gewissermaßen die Sattelzeit der modernen ecuadorianischen Indigenenbewegung - nicht zufällig überschneidet sie sich mit dem ,gewonnenen Jahrzehnt“ (Ramón 1992) dieser Bewegung in den 1980ern. In ihr findet eine wichtige organisatorische und diskursive Entwicklung statt, verbunden mit einer Steigerung des ethnischen Bewusstseins der Indigenen (Sánchez-Parga 1988: 108). Sie ist daher von schärfer werdenden Kon- 
flikten zwischen den sozialistischen und ethnischen Strömungen geprägt - 1977 wird die FENOC sozialistisch (FENOCIN 1999: 39), 1980 für kurze Zeit auch ECUARUNARI (Santana 1983: 166). Diese konfliktreiche Phase beginnt mit der ersten öffentlichen Forderung nach einer pluralistischen Neugestaltung des ecuadorianischen Staates 1976 (Federación de Centros Shuar 1976: 129) und endet mit der Gründung der CONAIE 1986 bei gleichzeitig anhaltender Schwäche der anderen Indigenenorganisationen und sozialen Bewegungen. In der CONAIE kommen die beiden Strömungen zu einem - zerbrechlichen - Gleichgewicht, das auch vom mangelnden Druck anderer Organisationen stabilisiert wird. Vor der Sattelzeit konzentrierten sich die Forderungen der indigenen Organisationen (etwa Federación de Centros Shuar (1981) [1973]) auf Gleichberechtigung, den Kampf gegen Rassismus und für Entwicklungsprojekte, jedoch noch nicht auf gesamtgesellschaftliche Veränderungen, wie sie in den Forderungen nach Pluralismus (Federación de Centros Shuar 1976) und Autonomie deutlich werden. In dieser Sattelzeit wird Ende der 1970er - der Begriff der indigenen Nationalitäten entwickelt, der als erster Begriff der Indigenenbewegung kulturelle mit wirtschaftlichen Forderungen verbindet. Er ist auch die Grundlage für den weitergehenden Begriff der Plurinationalität, der ab 1983 entsteht. Der Begriff der Interkulturalität, der deutlich später 1995- geprägt wird, ist ein Gegenbegriff gegen die Plurinationalität (FENOCIN 1999: 151) und lehnt auch den Begriff der indigenen Nationalitäten explizit ab (FENOCIN 1999: 153). Der 2002 entstehende Begriff des Guten Lebens ist ebenfalls ein Gegenbegriff mit dem eine Basisorganisation der CONAIE die öffentliche Aufmerksamkeit auf für sie akute Probleme lenken will. Die Sattelzeit ist von einer Stärkung der indigenen Organisationen geprägt, die neue, ethnisch geprägte Forderungen auf nationalem Niveau präsentieren (Viteri 1997: 55f.). Im Zentrum des neu entstehenden Diskurses sind drei Begriffe bzw. Inhalte: die Territorialität, die Anerkennung der indigenen Kulturen (also die Plurikulturalität) und das Selbstverständnis als indigene Nationalitäten mit Recht auf Selbstbestimmung. Alle drei Begriffe zeichnen sich dadurch aus, allgemein formuliert zu sein, so dass sie nur schwer in konkrete Forderungen übersetzbar sind (Sánchez-Parga 1988: 201). Sie sind umstrittene politische Grundbegriffe im Sinne der Begriffsgeschichte, die eine Geschichte besitzen, politische Konsequenzen erzeugen und auch außerhalb der Vertreter dieser Begriffe verwendet werden (Richter 2005: 220).

Es wird also davon ausgegangen, dass sich bis Mitte der 1980er ein relativ stabiler Diskurs um die eindeutig definierten Begriffe indigene Nationalität, Territorialität und Plurinationalität herausgebildet hat. Dieser stabile Diskurs prägt bereits die Veröffentlichungen von CONACNIE (Conterón/de Viteri 1984) und ECUARUNARI (1984) - noch ohne Verwendung des Begriffs Plurinationalität - und ist zum ersten Mal deutlich in Pacari (1984) zu erkennen, die aus den Begriffen Nationalität und Territorialität die Forderung nach einem multinationalen Staat ableitet. In der 
ab 1987 tagenden Sonderkommission für indigene Angelegenheiten wird aus diesem neuen Diskurs und seinen zentralen Begriffen ein genau definiertes politisches Programm entwickelt, das 1988 dem Parlament - und damit Staat und Gesellschaft - vorgelegt wird (Comisión Especial de Asuntos Indígenas 1988). Im selben Jahr erscheint die erste Auflage des programmatischen Buches Las nacionalidades indígenas en el Ecuador. Nuestro Proceso organizativo der CONAIE (hier: 1989), das ebenfalls um die Begriffe indigene Nationalität, Territorialität und Plurinationalität die Vision eines anderen Landes entwickelt. Spätestens mit diesen beiden Texten liegt eine genaue, von den Organisationen - und nicht wie bei Pacari (1984) Einzelpersonen - getragene Definition der verwendeten Begriffe in einem in diesen Organisationen allgemein geteilten Diskurs vor.

Die erste Auseinandersetzung einer breiten Öffentlichkeit mit dem Diskurs und den Forderungen der Indigenenbewegung findet erst im Rahmen der Erhebung 1990 statt. Jetzt werden Kritiken laut, die vor allem die Begriffe indigene Nationalität und Plurinationalität als Angriff auf den ecuadorianischen Staat und den Diskurs der mestizaje verstehen (etwa in León 1994: 34-37). Auf diese sich entwickelnden Kritiken reagiert die CONAIE Ende 1993 mit der Zusammenstellung eines Politischen Projektes, das um die Begriffe Nationalität und Plurinationalität die Vision eines neuen Staates und einer neuen Gesellschaft in Ecuador entwickelt. Dieser Text vervollständigt die 1988 vorgelegten Programme, ohne mit ihnen zu brechen. Wie die gesamte diskursive Entwicklung der Sattelzeit ist auch das Politische Projekt, das als Ergebnis der Wechselwirkungen zwischen Indigenenbewegung und Gesellschaft gesehen werden kann, von klassistischen und ethnischen Ideen geprägt, die miteinander in einen Dialog gebracht werden. Somit ist das Politische Projekt ,ein allgemeiner Vorschlag von konkreten Veränderungen und Forderungen“ (Ibarra 1999: 89f.), die im Zentrum eines neuen Diskurses stehen - und der erste programmatische Text der CONAIE, der die Ansichten der nicht-indigenen Mehrheitsgesellschaft explizit aufnimmt.

Nur wenig später, im Jahr 1995, wird dieser in sich geschlossene Diskurs von innerhalb der Indigenenbewegung angegriffen. Die FENOC-I reorganisiert sich nach einer langen Krise um den Begriff der Interkulturalität, der explizit als Gegenbegriff zur Plurinationalität entwickelt wird (FENOCIN 1999: 151). Gleichzeitig kritisiert sie den Begriff der indigenen Nationalitäten - ohne ihn aber gänzlich abzulegen (FENOCIN 1999: 153f.). Sie versucht, einen alternativen Diskurs in der Indigenenbewegung zu etablieren, der mit anderen Begriffen teilweise andere Inhalte verbindet - so wird der Begriff der Territorialität kritisiert und durch ,territoriale Umgrenzungen“ (FENOCIN 1999: 146) ersetzt, wobei die Bewohner dieser Umgrenzungen nur noch ein Recht auf Mitbestimmung bei der Ausbeutung von Bodenschätzen haben sollen (FENOCIN 1999: 146) und nicht wie für die CONAIE, 
eine weitgehende Kontrolle über diese (CONAIE 1994: 20) ${ }^{29}$. Der Begriff der Interkulturalität wird auch wegen dieser abgeschwächten Inhalte nicht angegriffen, sondern relativ schnell von Indigenenbewegung und Gesellschaft übernommen von der CONAIE etwa in der zweiten Version ihres Politischen Projektes (CONAIE 1997: 9). Der Preis dieser Übernahme ist allerdings die inhaltliche Entleerung des Begriffs Interkulturalität - die deutlichen Forderungen, die auch in Auseinandersetzung mit dem Diskurs der CONAIE noch 1999 (FENOCIN 1999) aufgestellt wurden, werden von der FENOCIN selbst wenige Jahre später zugunsten einer wenig konkreten Definition von Interkulturalität (etwa FENOCIN 2004: 39) nicht durchgehalten. So sind auch die nicht durch Texte von CONAIE und FENOCIN gedeckten Interpretationen von Interkulturalität zu erklären, die einige nichtindigene Intellektuelle aufstellen.

Ein zweiter diskursiver Angriff auf das jetzt um den Begriff Interkulturalität erweiterte Programm der CONAIE erfolgt von einer ihrer Mitgliedsorganisationen. 2003 veröffentlicht die Basisorganisation der OPIP „Autonomes Territorium der Ureinwohnernation des Kichwa-Volkes von Sarayaku“ (Territorio Autónomo de la Nación Originaria del Pueblo Kichwa de Sarayaku) das „Buch des Lebens von Sarayaku, um unsere Zukunft zu verteidigen“ (Sarayaku Sumak Kawsayta Nawpakma Katina Killka/El libro de la vida de Sarayaku para defender nuestro futuro), mit dem unter Rückgriff auf den Begriff des Guten Lebens die Erdölförderung in der Region angegriffen und eine alternative Lebensweise in Harmonie mit der Umwelt gefordert wird (Sarayaku 2003). Der Begriff des Guten Lebens baut stark auf die bereits etablierten Begriffe Nationalität und Territorialität (Sarayaku 2003: 26f.), erweitert sie aber um eine begriffliche Fassung der schon früh in der Indigenenbewegung präsenten Vorstellung einer Harmonie zwischen Universum, Welt und Mensch (etwa in Pacari 1984: 115). Im Rahmen eines Projektes der Plurinationalität - wie es das Politische Projekt der CONAIE ja ist - bedeutet das Gute Leben eine Betonung der ethnischen Aspekte bei relativer Missachtung der klassistischen Elemente - auch wenn nicht-indigene Intellektuelle wie Acosta (2010) oder Gudynas (2011) versuchen, den sozialistischen Gehalt des Guten Lebens hervorzuheben. Dieser neue Begriff ist somit auch ein Gegenbegriff gegen das zu dieser Zeit vorherrschende Verständnis von Plurinationalität. Verbunden mit dem Kampf der Sarayacu gegen eine Erdölförderung auf ihrem Territorium - der noch immer andauert und von den Indigenenorganisationen des Kontinents aufmerksam verfolgt wird - führte der neue Begriff zu einer diskursiven und politischen Verschiebung innerhalb der CONAIE, die von der Wahl des damaligen Vorsitzenden der Sarayacu Marlón Santi zum Präsidenten der CONAIE 2008 gekrönt wurde. Daher ist es

29 Ab der zweiten Version ihres Politischen Projektes wird dieser Teil noch stärker betont (CONAIE 1997: 50). 
naheliegend, dass die sich zur selben Zeit verstärkende Opposition der CONAIE gegen die Regierung Correa vor allem von umweltpolitischen Aspekten geprägt ist, die sich dem Guten Leben verschreiben (CONAIE 2011: 7) und die Regierung Correa nach diesen Prinzipien beurteilen (CONAIE 2010). Soziopolitische oder sozioökonomische Aspekte treten in dieser Zeit in den Hintergrund.

Der Diskurs ist für Foucault die Summe alles Sagbaren (Steinmetz 2008: 184). In diesem Sinne sind Begriffe Knotenpunkte des Diskurses, zu denen man sich zwangsweise verhalten muss, wenn man über das jeweilige Thema spricht. In Ecuador ist es nach dem Inti Raymi 1990 und der ersten öffentlichen Präsentation der Forderung nach einem plurinationalen Staat nicht mehr möglich, über die Beziehung zwischen Mehrheitsgesellschaft oder Staat und indigenen Völkern in Ecuador zu sprechen, ohne sich auf die Plurinationalität zu beziehen. Die Entwicklung von Gutem Leben und Interkulturalität als Gegenbegriffe zur Plurinationalität ist auch in diesem Zusammenhang zu verstehen. Sie sind der Versuch, die diskursive Hegemonie der CONAIE und bestimmter Strömungen in ihr zu brechen oder zu verschieben, können aber nur in Abgrenzung vom Begriff der Plurinationalität funktionieren - dabei kann diese Abgrenzung als Ablehnung - bei FEINE und FENOCIN - oder als Integration im Sinne einer Erweiterung - bei den Sarayacu - stattfinden. Diese zwangsläufige Auseinandersetzung mit einem bereits bestehenden Diskurs, der nicht vollkommen abgelehnt werden kann, erleichtert die marginale Integration der Gegenbegriffe in diesen Diskurs - wobei sie einen Teil ihrer Bedeutung einbüßen. So lässt sich die relative Stabilität des Diskurses der CONAIE seit Mitte der 1980er und die geringere Bedeutung - und die oft kritisierte Willkürlichkeit - der späteren Begriffe erklären. Der Diskurs der CONAIE wurde erweitert und verschoben, dreht sich aber weiterhin um die Begriffe Nationalität und Plurinationalität.

Eine besondere Rolle bei der Entwicklung der zentralen Begriffe und der Gegenbegriffe des Diskurses der Indigenenbewegung spielen Bewegungsintellektuel$\mathrm{le}^{30}$. Solche akademisch gebildeten Personen haben - wie Claudia Zapata (2007: 170f.) in einem anderen Kontext beschreibt - trotz der mit ihrer Bildung gewonnenen Aufstiegschancen und ihrer Verankerung in der Stadt nicht den Kontakt zu ihren Herkunftsdörfern verloren und identifizieren sich darüber hinaus mit den Zielen der dort aktiven sozialen Bewegung. Gerade in den Organisationen der Indigenenbewegung können diese Intellektuellen Einfluss erlangen (Fernández 2010: 6) und ihre Entwicklung zu zentralen Akteuren unterstützen. Ihre Ausbildung in Institutionen, die Indigenen nur selten offen stehen, erlaubt ihnen, sich Instrumenten und Techniken zu bedienen, die zuvor ihrer Beherrschung dienten, ,ihre Projekte erscheinen im Schoß der westlichen Moderne und des Nationalstaates, den sie zu

$30 \mathrm{Zu}$ unterscheiden von anderen Arten von Intellektuellen, wie sie etwa Fernández (2010: 5) - unter Bezug auf Gramsci - definiert. 
hinterfragen und zu verändern beabsichtigen“ (Fernández 2010: 5). Diese Art von Intellektuellem entsteht gegen Ende der 1970er Jahre und zeichnet sich durch eine Affinität zu den Sozial- und Geisteswissenschaften aus. Im Gegensatz zum anwendungsorientierten técnico, dem technisch gebildeten Spezialisten vor Ort, z.B. dem Agraringenieur, ist der Bewegungsintellektuelle sehr an gesellschaftlich diskutierter Forschung und der Verbreitung seiner Ideen interessiert (Zapata 2007: 170). Dadurch bewegen sich die indigenen Bewegungsintellektuellen zwischen verschiedenen Ebenen: ,der der ethnischen Gruppe (Mapuche, Aymara, Quechua, usw.), der der Nation in der sie eingebettet sind und der der ,Indios', die einen Kontinent und eine Herrschaftsgeschichte teilen“ (Zapata 2007: 171). Diese verschiedenen Einflussräume führen dazu, dass sich die mit der Indigenenbewegung verbundenen indigenen Intellektuellen mit deren dekolonisierendem Projekt identifizieren. Sie reflektieren die Wirklichkeit, die sie als Indigene erleben und versuchen sie - oft in Zusammenarbeit mit nicht-indigenen Intellektuellen, etwa sozial engagierten Anthropologen - theoretisch zu verstehen. Daraus entstehen neue Ansätze und Diskurse, wie etwa der bereits diskutierte Indianismus (Barre 1982: 57f.). Indigene Bewegungsintellektuelle fungieren auch als Herausgeber und Übersetzer von Texten über ihre Völker, wodurch diese sich neu verstehen können (Fernández 2010: 7). So widmen sie sich der Untersuchung der „kolonialen Verfassung“ (Zapata 2007: 171) der Indigenen und versuchen, ihre wissenschaftlichen Disziplinen ,,von einem identitären Gebrauch der Schrift aus“"(Zapata 2007: 171) zu erweitern.

In diesem Sinne interpretieren indigene, bewegungsafine Historiker die Geschichte ihrer Länder neu, ohne den gleichen Wert auf die Unterscheidung zwischen Kolonie und Republik zu legen wie ihre nicht-indigenen Kollegen. Die Geschichte entwickelt sich stattdessen als Abfolge verschiedener „kolonialer Etappen“ (Zapata 2007: 172), die unter wechselnder Herrschaft der Spanier, der Criollos und der Mestizen standen - die allesamt die Indigenen unterdrückten und als Kinder oder Wilde betrachteten (Zapata 2007: 172) ${ }^{31}$. Diese Position haben - so die indigene Auslegung von Fanon und anderen - die Indigenen verinnerlicht und sind so zu unterdrückten Subjekten geworden, zum exotischen und unfähigen Anderen (Zapata 2007: 172). Durch die Erforschung dieses Phänomens wollen die indigenen Bewegungsintellektuellen die koloniale Situation beenden und das kolonisierte Subjekt auflösen (Zapata 2007: 174). Eine Neuinterpretation der Vergangenheit erlaubt es, neue Schlüsse aus der Gegenwart zu ziehen. So können sie zur Entwicklung eines Projekts einer anderen Gesellschaft durch die Indigenenbewegungen beitragen (Zapata 2007: 176). Zu diesen neuen Ansätzen gehört auch eine Kritik der Methoden der jeweiligen Disziplin, von einer Erzählung aus der Position eines ,Ich` oder

31 Diese Sicht auf Geschichte bestätigt sich auch in Ecuador. Es sei auf die explizit indigene Geschichtsschreibung in CONAIE 1989 verwiesen. 
- häufiger - ,Wir', über die Neubewertung indigener Quellen und die Kritik bestimmter Kategorien - wie der der Ethnie - bis zur Beschäftigung mit alternativen Theorieansätzen, wie dem internen Kolonialismus oder der Kolonialität der Macht (Zapata 2007: 177).

Die erste Generation von Bewegungsintellektuellen in der ecuadorianischen Indigenenbewegung wurde gegen Ende der 1970er Jahre aktiv. Zu ihr gehören neben anderen Luis Macas, Nina Pacari und Auki Tituaña, meist Juristen, Sozial- oder Wirtschaftswissenschaftler. Sie waren die ersten, die sich innerhalb der Bewegung kritisch und abstrakt mit deren Strukturen und Zielen und der Entwicklung anderer Bewegungen an anderen Orten beschäftigt haben. Von besonderer Bedeutung ist die ,intellektuelle, ethnisch-kulturelle Strömung“ (Barrera 2001: 143) in und um Otavalo, die vom „Otavalo-Faktor“ (Selverston 1992: 13), also dem wirtschaftlichen Erfolg der Region, getragen wurde. Sie manifestiert sich in einer Reihe kultureller Organisationen wie Conjunto Rumiñahui, Indo América, Ñanda Mañachi, Peguche und Obraje - und im Taller Cultural Causanacunchic, der 1976 von Ariruma Kowii gegründet wurde (UASB o.J.). In ihm beschäftigte sich eine Gruppe von Schülern und Studenten der Region unter anderem mit der Lektüre sozialistischer und postkolonialer Literatur (Abad o.J.) - darunter auch Texte der bolivianischen Kataristas und von Anthropologen und Sozialwissenschaftlern wie Flores Galindo und Bonfil Batalla (Flores 2005: 37)-, eine Aktivität, die 1978 zum Aufbau der sozialwissenschaftlichen Bibliothek Amauta mit Schwerpunkt auf indigene Themen führte (Kowii o.J.: 1f.; Huarcaya 2010: 311). Weiterhin analysierte die Kulturwerkstatt Entwicklung und Aktionen anderer indigener Organisationen, wobei vor allem die kolumbianischen Organisationen Indigener Regionalrat des Cauca (Consejo Regional Indígena del Cauca, CRIC) und Nationale Indigenenorganisation Kolumbiens (Organización Nacional Indígena de Colombia, ONIC) eine wichtige Rolle einnahmen - begünstigt durch den direkten Kontakt, den einige der Mitglieder über ihre Familien, die als Händler in der Region tätig waren, hatten (Flores 2005: 37). Vielleicht ist so die Übernahme vorher rein akademischer und auch in der Wissenschaft marginaler Begriffe zu erklären, wie sie in den Schriften um das zweite Treffen von Barbados zu finden sind - einen personellen Zusammenhang gibt es erwiesenermaßen nicht. Dem Taller Cultural Causanacunchic gehörten etwa 50 indigene Schüler und Studenten aus Otavalo und Cotacachi an (Huarcaya 2010: 310), darunter neben Kowii Luis Maldonado (1976-1982) (Cultura Andina o.J.), Nina Pacari (1980-1985), Auki Tituaña, Blanca Chancoso und Mario Conejo (Hoy 2001); alles Kichwa, die zu den wichtigsten Vordenkern der Indigenenbewegung werden sollten (Huarcaya 2010: 312f.). Er widmete sich neben der Lektüre der Erforschung und Wiederbelebung der Traditionen der Kichwa und versuchte, die Beziehungen zwischen Indigenen und nicht-Indigenen demokratischer zu gestalten (Huarcaya 2010: 310). Dazu kritisierten die Mitglieder marxisti- 
sche und sozialistische Strömungen, die die ethnischen Gegensätze zu einem Nebenwiderspruch reduzieren oder als falsches Bewusstsein diskreditieren wollten. Diese autonome Position hatten sie auch aufgrund ihres Hintergrundes in der urbanen Mittelschicht inne (Ortiz Crespo 2009: 16) - ein Umfeld, in dem die sozialistischen und kommunistischen Organisationen weit weniger Einfluss hatten als auf dem Land. So rückten die Intellektuellen im Umfeld des Taller Cultural Causanacunchic kulturelle und traditionelle Symbole und Feste in den Vordergrund - auch als Mittel der Verteidigung der eigenen Identität gegen Diskriminierung (Huarcaya 2010: 311f.). Dazu gehörte eine Präsentation der indigenen Identität über eine bestimmte Kleidung, wie Hüte oder Ponchos (Flores 2005: 39). Es wurde von den Indigenen erwartet, dass sie ihre Hüte abnehmen, wenn sie mit nicht-Indigenen sprechen, sowie bei offiziellen Anlässen; dieser Brauch wurde als der indigenen Kultur fremd bekämpft. Die Mitglieder des Taller Cultural Causanacunchic koordinierten in diesem Sinne die kollektive Weigerung indigener Schüler und Studenten, ihre Hüte abzunehmen, wenn die Nationalhymne gespielt wurde (Huarcaya 2010: 311f.). Ein wichtiges Beispiel ist auch die Umgestaltung des christlich konnotierten Festes San Juan in das indigene Inti Raymi ab 1976 (Kowii o.J.: 1f.) auf dem indigene Musik und Tänze präsentiert wurden (Ortiz Crespo 2009: 16). Tituaña, Pacari und Kowii änderten in diesem Zusammenhang ihre spanischen Namen in Kichwa-Namen. So ersetzte Tituaña den Namen Segundo Antonio durch Auki, der Name eines Sohnes des Inka und eines Berges in Peru, María Esther Vega nahm den Namen Nina Pacari an, was Licht des Morgens bedeutet und Jacinto Conejo den Namen Ariruma Kowii, was nachdenklicher Mann bedeutet (Abad o.J.). Nina Pacari war die erste Indigene, die den Begriff der indigenen Nationalitäten systematisch auf den ecuadorianischen Kontext anwendete und daraus die Forderung nach einem multinationalen Staat ableitete (Pacari 1984).

Der Taller Cultural Causanacunchic hat schon in den 1970ern den Dialog mit nicht-indigenen Intellektuellen gesucht und so seinen Diskurs weiterentwickelt und zunehmend in der Öffentlichkeit verankert (Ortiz Crespo 2009: 16). Die dort engagierten Kichwa waren auch in den Organisationen der Indigenenbewegung aktiv, insbesondere der OZG der Region, FICI, die sie beim Aufbau von Programmen in den Bereichen Land, Bildung, Gesundheit und Kommunikation unterstützten (Ortiz Crespo 2009: 17). So konnten sich die indigenen Bewegungsintellektuellen professionalisieren und ihre Ideen in die Bewegung selbst einbringen - ein bedeutender Faktor, der zur Distanzierung der FICI vom damals vorherrschenden Klassismus beigetragen hat (Flores 2005: 39; Ortiz Crespo 2009: 18). Eine besondere Rolle spielen die Programme der zweisprachigen Bildung, die seit den 1980ern eine wichtige institutionelle Einbindungsmöglichkeit für indigene Intellektuelle sind (Ortiz Crespo 2009: 18). 
Auch nicht-indigene Intellektuelle haben seit Anfang der 1980er mit den verschiedenen Indigenenorganisationen kooperiert und dabei auch deren Diskurs beeinflusst. Diese Kooperation kann individuell sein, wie im Falle von Ileana Almeida und Pablo Dávalos, die vor allem die CONAIE unterstützen (Flores 2005: 65), oder institutionell verankert, wie das Andine Zentrum der Volksaktion (Centro Andino de Acción Popular, CAAP) um Manuel Chiriboga und das Zentrum für Forschung und Entwicklung der sozialen Bewegungen Ecuadors (Centro para la Investigación y el Desarrollo de los Movimientos Sociales del Ecuador, CEDIME) um Jorge León, die in den 1980ern und 1990ern vor allem mit den Organisationen des Hochlandes kooperierten ${ }^{32}$. Ileana Almeida war eine der Wissenschaftler, die den Begriff der indigenen Nationalität in Ecuador einführten - ein Begriff, der 1983 auch von Chiriboga und León in der Sonderausgabe der Zeitschrift Cuadernos de Nueva ausgearbeitet und um den Begriff Multinationalität bzw. Plurinationalität erweitert wurde. Es ist zu vermuten, dass diese Begriffe auch in den jeweiligen Beratungen, Expertisen und Weiterbildungen verwendet wurden.

Die Auseinandersetzung über die Begriffe der Indigenenbewegung ist nicht nur von endogenen Faktoren geprägt, wie Konflikten über die genaue Bedeutung bestimmter Begriffe oder dem Aufkommen von Gegenbegriffen, die teilweise die Bedeutung bereits bestehender Begriffe übernehmen (wie im Fall von Interkulturalität und Sumak Kawsay gezeigt), sondern auch von exogenen Faktoren. So ist die frühe Übernahme der Begriffe der Indigenenbewegung schon durch Jaime Roldós auch der Versuch, sich diese Begriffe anzueignen und sie der Deutungshoheit der Indigenenbewegung zu entziehen. Niemandem ist das so gut gelungen wie Rafael Correa und Alianza PAÍS, die die hier diskutierten Begriffe der Indigenenbewegung längst in ihren eigenen Diskurs integriert haben - ohne jedoch die spezifisch indigenen Forderungen zu beachten. So wird aus einer Plurinationalität, die die weitgehende Autonomie indigener Territorien bedeutet, eine Plurinationalität, die sich in wenig weitreichenden Mitbestimmungsrechten erschöpft. Diese Strategie führte für die Indigenenbewegung zu einem weitgehenden Verlust ihrer nicht-indigenen Verbündeten - viele der Politiker von Alianza PAÍS waren noch vor einigen Jahren Mitglieder der MUPP-NP. Gleichzeitig verliert die Indigenenbewegung aber auch Legitimität nach innen. Wie gezeigt, sind die politischen Begriffe der Indigenenbewegung auch Mobilisierungsmechanismen. Ihre Übernahme durch andere Akteure führt so für die Indigenenbewegung zwingend zu einem Verlust an Mobilisierungsfähigkeit. Wenn die CONAIE unter Berufung auf Plurinationalität und Sumak Kawsay gegen eine Regierung protestiert, die sich ebenfalls auf Plurinationalität und Sumak Kawsay beruft, wird es schwierig, die eigenen Position zu vermitteln. Neben der zunehmenden Stärke der kleineren Indigenenorganisationen ist das der

32 Persönliche Kommunikation Chiriboga und León. 
Haupterklärungsansatz für die Krise der CONAIE, die mit ihrer Regierungsbeteiligung 2003 begann und seither nicht endet. 


\section{Plurinationalität/Interkulturalität und kulturelle Ungleichheit}

Der diskursive Wandel der ecuadorianischen Indigenenbewegung ist mit einer veränderten Wahrnehmung der erlebten Unterdrückung einhergegangen. Die Situation der Indigenen wird seit den 1970ern zunehmend aus einer ethnischen und identitären Perspektive als Kolonialismus und Kolonialität beschrieben, was auch auf die konkreten Kritiken und die daran angelehnten Forderungen wirkt. Die in dieser Perspektive implizite Betonung der Differenz unterscheidet sich grundlegend von der Zentralität der Gleichheit der gewerkschaftlich geprägten Bewegungen bis in die 1970er. Im Folgenden soll die Diagnose der Situation der Indigenen durch die Indigenenbewegung in Bezug auf den Ansatz der Kolonialität der Macht analysiert werden $^{1}$. Im Anschluss werden die bereits diskutierten Begriffe der Indigenenbewegung auf ihr dekoloniales Potential untersucht. So soll die Frage geklärt werden, ob die Alternativen, die die Indigenenorganisationen entwickeln, die beschriebene Situation als eine koloniale beheben wollen, ob sie also dekolonial im Sinne der Kolonialität der Macht sind.

\section{KOLONIALITÄT UND KULTURELLE UNGLEICHHEIT}

Für die Theoretiker der Kolonialität manifestiert sich diese in verschiedenen Bereichen des Lebens, die auf bestimmte Art und Weise von der Kolonialität geprägt sind und werden. Bei Quijano sind diese Bereiche Arbeit, Sex bzw. Geschlecht, Subjektivität, kollektive Autorität und die Beziehungen zwischen Mensch und Umwelt. Jeder dieser Bereiche ist in das Machtsystem der Kolonialität integriert

1 Es geht also nur um den Diskurs der Indigenenbewegung. Eine Untersuchung, ob Ecuador als solches kolonial strukturiert ist, kann hier nicht vorgenommen werden. Daher ist die folgende Beschäftigung mit den Begriffen der Kolonialität nur im Kontext des Diskurses der verschiedenen Organisationen zu sehen. 
und wird durch diese kontrolliert (Quintero 2010: 4). Mignolo reduziert die Bereiche auf vier: Subjektivität, kollektive Autorität, Wirtschaft und Wissen, die alle miteinander verbunden und nach den Regeln der Kolonisierer strukturiert sind (Mignolo 2009: 48f.). Der folgende Versuch, Diagnose und Alternativen der Kolonialität durch die Indigenenbewegung nachzuzeichnen, geht von drei Bereichen aus, in denen die Indigenenbewegung Kolonialität feststellt. Diese Bereiche sind ,Machtsystem und Staat', ,Identität und Gesellschaft' und als dritter Bereich der Versuch, soziale, politische, kulturelle und wirtschaftliche Ungleichheit als einen zusammenhängenden Komplex zu begreifen. Somit werden die von der Theorie getrennten analytischen Bereiche von der Indigenenbewegung als Problemfelder neu angeordnet. Der von Quijano definierte Bereich Geschlecht wird in den Texten der verschiedenen Organisationen meist vernachlässigt - abgesehen von wenigen Bemühungen (etwa: FENOCIN 2004: 20; CONAIE 2007a: 6), die trotz der zentralen Rolle von Frauen in der Indigenenbewegung bislang nur geringe Wirkung hatten.

\section{a. Machtsystem und Staat}

Für die ecuadorianische Indigenenbewegung ist der Staat das Zentrum eines bis heute bestehenden kolonialen Machtsystems und damit einer der wichtigsten Faktoren der Unterdrückung und Ausbeutung der Indigenen. Der Staat und seine Organe

,sind seit dem Aufkommen des republikanischen Regimes 1830 Instrumente des herrschenden Sektors, die die Funktion des Repräsentanten und Verteidiger seiner wirtschaftlichen und politischen Interessen gegenüber den Interessen und Forderungen der indigenen Völker und Nationalitäten und sonstiger ausgebeuteter und unterdrückter Sektoren Ecuadors erfüllen“ (CONAIE 1994: 18).

Der koloniale Charakter des Staates hat sich also aus der Sicht der Indigenenbewegung seit der Unabhängigkeit nicht grundlegend verändert (CONAIE 2007: 9; CONAIE 2007a: 6f.). Er wurde vielmehr um bestimmte Inhalte und Strukturen aus den neuen Hegemonialmächten Großbritannien und Frankreich - und später den USA - ergänzt und erweitert (Del Valle 2006: 126). Die CONAIE spricht daher von „mehr als fünf Jahrhunderten kolonialistischer und republikanischer Invasion, Unterdrückung und Ausbeutung“ (CONAIE 2001: 2f.). Der Prozess der (staatlichen) Dekolonialisierung wurde nie um einen Prozess der (gesellschaftlichen) Dekolonialität ergänzt, die Kolonialität als Machtsystem wirkt auch nach der Unabhängigkeit weiter, ,sie ist das zentrale Element der Strukturierung der Gesellschaft in Lateinamerika“" (Quintero 2010: 11). Es handelt sich in Lateinamerika noch immer um „unabhängige Staaten verbunden mit kolonialen Gesellschaften“ (Quijano 2006a: 
18). Tatsächlich verschlechterte die Unabhängigkeit Amerikas in manchem die Situation der Indigenen, die nun mit aller Kraft in Nationalstaaten nach europäischen Vorbild integriert werden sollten (Barre 1983: 29). Die politische Elite Ecuadors hat seit der Unabhängigkeit versucht ,eine nationale Identität zu schaffen und eine Reihe von Institutionen, um sie zu stützen“ (Selverston 1994: 148). Diese Nationalstaaten sind allerdings, wie Quijano feststellt, weder national, da sie einen Großteil der Bevölkerung ausschließen, noch demokratisch, da die sich nicht auf eine Mehrheit der Bürger stützen können (Quijano 2006a: 19). Stattdessen sind sie „Instrumente der herrschenden Klassen, um die Nationalitäten und Völker und alle sozialen Sektoren unterjocht zu halten“ (CONAIE 2001: 2f.), weswegen sie politische Teilhabe verhindern und die ,individuellen und kollektiven historischen Rechte" (CONAIE 2001: 2f.) ablehnen müssen. Daher sind sie kaum am Anspruch des Ideals europäischer Nationalstaaten zu messen. „[D]ie grundlegenden Prinzipien der Demokratie: Gleichheit, Freiheit, Brüderlichkeit und sozialer Frieden wurden nicht erfüllt“ (CONAIE 2001: 2f.). Die Indigenenbewegung akzeptiert also die vom Staat selbst postulierte Überzeugung, dieser sei eine Institutionalisierung der Gesellschaft im Sinne eines Gesellschaftsvertrages und kritisiert ihn danach. Dadurch wird eine Art konkreter Kritik möglich, die in ihren Grundsätzen liberaldemokratisch anmutet und dadurch breite Koalitionen möglich macht.

„Die aktuelle öffentliche Verwaltung ist ineffizient, korrupt, unmoralisch, segregationistisch und antidemokratisch; die gesamte Gesellschaft und die indigenen Völker und Nationalitäten sind Opfer einer mangelhaften Organisation und Funktion des Staatsapparates." (CONAIE 1994: 18)

Die klassistische Sichtweise der Indigenenbewegung wird in den 1980ern auch von den sozialistischen Organisationen erweitert, die dadurch eine andere Position zum Staat einnehmen. ECUARUNARI spricht 1984 noch im traditionellen Duktus von einem ,imperialistischen bürgerlichen Staat“ (ECUARUNARI 1984: 46), der im Widerspruch zum Volk und den indigenen Nationalitäten steht, die in ihn integriert werden sollen (ECUARUNARI 1984: 48). So beginnt die Indigenenbewegung, die „Natur eines Nationalstaates, der unsere verschiedenen kulturellen Identitäten nicht anerkennt“ (CONAIE 1989: 268f.), zu hinterfragen und nähert sich einer Diagnose der „strukturellen und Macht-Monokulturalität“ (Walsh 2008: 3) an. Die Tatsache, dass der bestehende Staat die kulturelle Diversität des Landes ignoriert und die Anderen systematisch ausschließt, wird betont.

„Der aktuelle Staat fördert die bürgerliche Gleichheit mit der Bedingung der Verzichts auf kulturelle Besonderheit. Er nimmt die Existenz einer einzigen Nation an und zwingt die indigenen und afroecuadorianischen Völker und Nationalitäten dazu, den Sektoren, die das 
Land wirtschaftlich beherrschen und kontrollieren, untergeordnet $\mathrm{zu}$ leben." (CONAIE 1997a: 9)

Er bleibt also der Vorstellung einer einzigen, einheitlichen Nationalkultur treu, in die alle anderen Kulturen integriert werden müssen. Das Ideal des liberalen, europäisch geprägten Nationalstaates trifft auf eine Wirklichkeit, die ihm grundlegend widerspricht. Gerade die postulierte Einheit von Volk und Staat im Nationalstaat der Neuzeit ist in Lateinamerika offensichtlich nicht vorhanden. Die ,mestizaje wurde die Ideologie der nationalen Homogenität“" (Mignolo 2006: 133), die starke und andauernde Präsenz der Indigenen zu einem Zeugnis ihres Scheiterns. Die Nationalidentität, die den Nationalstaat ausmacht, ist eine Konstruktion, die auf den kolonialen Unterdrückungsmechanismen aufbaut - der Kolonialität der Macht also (Quintero 2010: 11). Die Ausgrenzung und Unterdrückung der in diesem Machtsystem marginalen Gruppen macht ihre Teilhabe an Entscheidungsprozessen unmöglich und verhindert somit eine wirkliche Demokratisierung. Gleichzeitig bleibt die koloniale Nationalidentität immer prekär, da die ,,sozialen Vorstellungen und historischen Erinnerungen, die die Nationalidentität bilden“ (Quintero 2010: 12) die realen Ungleichheiten verdecken.

Aus dieser Perspektive ist der Versuch der Integration in den Nationalstaat ein Angriff auf alle Gruppen, die nicht die Nationalidentität teilen. Die CONAIE sieht daher die staatlichen Entwicklungs- und Bildungsprogramme für Indigene als „Methoden der Vernichtung, Beherrschung, Verminderung und Unterwerfung“ (CONAIE 1994: 22). Die Politik des Staates den Indigenen gegenüber wird als ,kolonialistische Kulturpolitik“ (CONAIE 1994: 41) charakterisiert, die ,als Hauptziel hat, die kulturelle Identität und damit die politische historische Identität unserer indigenen Völker und Nationalitäten zu töten“ (CONAIE 1994: 41). Die Bestandteile dieser Politik sind

,a) die Verfolgung und der Mord der Amautas (indigenen Weisen); b) das Verbot, unsere kulturellen Werte zu praktizieren; c) die Folklorisierung; und d) die Auferlegung einer elitären und ausschließenden ,westlichen Kultur““ (CONAIE 1994: 41).

Gerade die Erziehungsprojekte für die Indigenen und die Gesamtbevölkerung werden als „,weiteres Instrument der Unterwerfung, Beherrschung, Akulturisierung, Desideologisierung und Ausbeutung“ (CONAIE 1994: 43) verstanden, das von den Eliten benutzt wird, um ,die Abhängigkeit, die Unterentwicklung und ihr wirtschaftliches, politisch-ideologisches und kulturelles System aufrechtzuerhalten“ (CONAIE 1994: 43). Die Verankerung europäischer Denktraditionen bei gleichzeitigem Übergehen der indigenen Alternativen wird als Versuch gesehen, den Eurozentrismus und die entsprechenden Wissensformen als natürlich und unhinterfrag- 
bar in der Bevölkerung zu festigen (Quijano 2000b: 343). Diese Wissensformen sorgen dafür, dass sich die Beherrschten am vorgestellten Ideal - Europa bzw. der Westen - messen und sich ,mit den Augen des Beherrschenden sehen“ (Quintero 2010: 10). Institutionen wie der Staat, die Kirche, die Massenmedien entwickeln und verbreiten Wissen, eine „eurozentrische Epistemologie [...] [, die] Völker, Territorien und Räume gemäß der politischen, ideologischen und wirtschaftlichen Absichten der Beherrschenden" (Del Valle 2006: 125) definiert. Somit umschreibt die CONAIE schon 1994, was Quijano später als Kolonialität des Wissens (Walsh 2005: 19) definieren sollte, also die Vernachlässigung und Herabsetzung aller Wissensformen, die nicht der europäischen Art zu wissen oder Wissenschaft zu betreiben entsprechen.

Die Struktur eines ausschließenden und unterdrückenden Staates unter der Kontrolle der wirtschaftlichen und politischen Elite, die die Teilhabe der Indigenen am politischen Leben verhindern will, bezeichnet die CONAIE als „uninationalen Staat“ (CONAIE 2001a). Zusammenfassend gibt sie in ihrem Politischen Projekt ihre Sicht auf die formellen Machtstrukturen wider:

,der weiß-mestizische hegemonische bürgerliche uninationale Staat ist in seiner rechtlichpolitischen und wirtschaftlichen Natur ausschließend, antidemokratisch, repressiv und proimperialistisch“ (CONAIE 2001: 2f.).

Diesem Staat und seinen Versuchen, die Indigenen zu integrieren, stellt die Indigenenbewegung verschiedene Alternativen entgegen, wie die Multinationalität (Pacari 1984: 119), den Plurinationalen Staat (CONAIE 1999: 51) oder die Interkulturalität (FENOCIN o.J.: 11) ${ }^{2}$.

\section{b. Identität und Gesellschaft}

Die Theoretiker der Kolonialität der Macht gehen davon aus, dass „Amerika die erste moderne und weltweite geokulturelle Identität war" (Quijano 2000a: 221), eine Identität, die auf der „,Rassifizierung ‘ der Beziehungen zwischen Kolonisierern und Kolonisierten“ (Quijano 2006a: 14) gründet. Der Aufbau eines „hierarchischen Systems sozialer Klassifizierung“ (Walsh 2009a: 67) in den Kolonien war mit der Erzeugung von rassistisch fundierten Identitäten verbunden, die der Position der jeweiligen Gruppe in der Gesellschaft entsprachen und den Kolonisierern und ihren Nachkommen die Kontrolle über die gesamte Bevölkerung sichern sollten. Die Kategorie des Indigenen ist damit zutiefst kolonial und ohne den Rahmen der Kolonialität der Macht mit einer sozialen, wirtschaftlichen und kulturellen Struktu-

2 Die im folgenden Kapitel auf ihren dekolonialen Gehalt untersucht werden. 
rierung der Gesellschaft nach ethnischen Kriterien nicht denkbar (Quijano 2006a: 14). Gleichzeitig eröffnet sie Möglichkeiten, sich aus der Unterdrückung zu befreien und die Kolonialität zu bekämpfen (Mignolo 2005: 5). Die Anerkennung der gemeinsamen Erfahrungen innerhalb der Kategorie der Indigenen erlaubt es den Kolonisierten, eine geteilte Identität zu entwickelt, die über die verschiedenen Ethnien hinausgeht (Bonfil Batalla 1981: 20) - als Indigene, im Sinne einer Positionierung im kolonialen Machtsystem. Somit treten zwei Identitäten in Verbindung:

„Auch wenn man ideologisch eine Indio-Identität bekräftigt, steht diese nicht im Widerspruch mit der ethnischen Identität [...], denn es handelt sich um zwei verschiedene aber ineinander verschränkte Identifikationsebenen.“ (Bonfil Batalla 1981: 20f.)

Die „historische Dichte der ethnischen Gruppen“ (Bonfil Batalla 1981: 27) erlaubt es ihnen, sich auf eine Vergangenheit vor der Unterwerfung zu beziehen und so deren vorübergehenden Charakter zu betonen. Gleichzeitig häufen sie Erinnerungen und Erfahrungen an, die ihre Identität stärken können (Bonfil Batalla 1981: 27). Daher sind die unter der Kategorie ,Indigene' subsumierten Ethnien als politische Phänomene zu verstehen (Bonfil Batalla 1978: 217f.). Auch die Verbindung von Weißheit und Fortschritt im Sinne der mestizaje (Walsh 2009a: 67) und der damit einhergehende „Mechanismus der subjektiven Des-Indianisierung“ (Quijano 2006a: 24f.) der Gesellschaft und der Indigenen, deren Vergangenheit zur Vergangenheit des mestizisch geprägten Nationalstaates gemacht wird, während sie zur Integration in die vermeintliche Mehrheitsgesellschaft angehalten werden, konnte weder indigene Ethnien noch die Indigenen als koloniale Kategorie beenden.

Gerade im Andenraum ist die Kolonialität der Macht im Alltag präsent. Im Zuge der Kolonisierung wurden die gesellschaftlichen Strukturen und das Wissen der Eroberten weitgehend zerstört und ihre Reste in eine eurozentrische Weltsicht integriert (Quijano 2000b: 378f.). Indigene Wissensformen werden somit als wertlos oder unterlegen wahrgenommen, ihre eigene Perspektive von dieser Weltsicht überdeckt (Quintero 2010: 10) und somit den Weißen ein Status der Universalität zugesprochen. „Das Weiße ist nicht markiert, sondern es wird zum Bezugspunkt, um die Andersheit der Anderen zu messen." (Kaltmeier 2010: 3) Daher war es ein wichtiger Bestandteil des Kampfes der Indigenenbewegung in Ecuador, die Existenz der indigenen Völker und Nationalitäten und ihre spezifischen Erfahrungen deutlich zu machen. Im ersten programmatischen Text der neuen Indigenenbewegung, dem Vorschlag für ein Nationalitätengesetz von 1988, heißt es: „Es gibt in Ecuador eine Nation, die aus der mestizaje entstanden ist, aber es gibt auch verschiedene indigene Nationalitäten, die aus dem Widerstand entstanden sind.“ (Comisión Especial de Asuntos Indígenas 1988: 7) Im dritten Artikel wird die Position der Indigenen näher erläutert: 
„Die indigenen Völker Ecuadors, so wie andere aus Amerika, haben sich mit ihrem Widerstand und ihrem Kampf für die Erhaltung ihrer Identität, territorialen, wirtschaftlichen, politischen und kulturellen Rechten als andere Nationalitäten innerhalb des ecuadorianischen Staates geschmiedet.“ (Comisión Especial de Asuntos Indígenas 1988: 17f.)

Die Indigenenbewegung selbst entwickelt ohne den institutionellen Rahmen der Sonderkommission, die unter Führung der Sozialistischen Partei und Mitwirkung ranghoher Theologen den Vorschlag für das Nationalitätengesetz ausgearbeitet hat, ein radikaleres Verständnis. Einer der ersten Texte der noch jungen CONAIE präzisiert die Position der indigenen Ethnien und der Kategorie des Indigenen in der Gesellschaft und begründet so eine weitgehende Kritik am Staat.

„[W]ir, die Indio-Völker, haben uns vereint, ausgehend, an erster Stelle, von der Anerkennung unserer Verschiedenheit sowie der ähnlichen kulturellen Züge; der Überzeugung, Teilhaber einer selben Geschichte der Unterdrückung und Ausbeutung zu sein; und, dass wir auch Teil einer größeren Gesellschaft sind, in der wir die Natur eines Nationalstaates hinterfragen, der unsere verschiedenen kulturellen Identitäten nicht anerkennt, weshalb er ein Problem ist, das gelöst werden muss.“ (CONAIE 1989: 268f.)

Die kulturelle Ungleichheit und die Unterdrückung der Indigenen und ihrer Kulturen werden also schon früh von den Akteuren der Indigenenbewegung angesprochen: „Unsere kulturellen Werte wurden verschmäht und uns wurde eine entfremdende Kultur auferlegt, die unsere eigenen kulturellen Ausdrücke unterdrückt.“ (Macas 1991: 6) Daher versteht sich die Indigenenbewegung bereits seit den 1980er als ,eine Bewegung, die die Flagge des Kampfes gegen die Diskriminierung und für die Verteidigung der indigenen Nationalitäten erhebt“" (ECUARUNARI 1984: 46). Dieser Kampf beinhaltet konkrete Forderungen, die die einzelnen Völker betreffen, ebenso wie allgemeine Inhalte, die sich auf das gesamte ecuadorianische Volk beziehen. Die Indigenen sollen sich, nach der Vorstellung der Indigenenbewegung, ,als Volk, als Nationalität innerhalb eines größeren Kontextes des Vaterlandes oder Staates verstehen“ (UNAE o.J.: 22f.).

„Die indigenen Kämpfe [...] sind Verteidigungskämpfe um das Territorium, um das Überleben der Gruppe; Kämpfe für die sozio-kulturelle und organisatorische Unabhängigkeit; Kämpfe für die Freiheit von einem kolonialen Unterdrückungssystem, für ein freies Volk.“ (UNAE o.J.: 22)

Die Indigenenbewegung sieht die Indigenen als ,politisch und kulturell unterdrückt“ (Pacari 1984: 118f.), was schon bei der mangelnden Anerkennung ihrer Existenz durch Staat und Gesellschaft anfängt (Pacari 1984: 118f.). Nina Pacari 
selbst beschreibt diese Situation als „Herrschaft eines Volkes über ein anderes Volk“ (Pacari 1984: 121). Und da die Indigenen eine ,unterworfene, besiegte Nationalität“" (UNAE o.J.: 46) sind, befinden sie sich in einer „Rebellion, um ihre ethnisch-kulturelle Befreiung zu gewinnen“ (UNAE o.J.: 46). Das ICCI spricht wenig später von

„einem tiefen Rassismus, der verschiedene Ausschließungs- und Ausbeutungsmechanismen mit Bezug auf die indigenen Völker und Nationalitäten unterhält" (ICCI o.J.).

Dieser Rassismus umfasst auch „eine Position der Unwissenheit und der Unsichtbarkeit“ (ICCI o.J.) den Indigenen und ihrer Kultur gegenüber. Noch in aktuellen Texten spricht die Indigenenbewegung von ,fünf Jahrhunderten wirtschaftlicher Ungleichheiten, sozialer Ausgrenzung und kultureller Diskriminierung" (ECUARUNARI/CONAIE 2007: 5).

\section{c. Kolonialismus, Imperialismus, Kolonialität und Unterdrückung}

Die Verbindung kultureller oder ethnischer mit sozialen und wirtschaftlichen Inhalten seit Ende der 1970er in der neuen Indigenenbewegung - die bereits erläuterte Wahrnehmung der "doppelten Dimension des indigenen Problems“ (CONAIE 1989: 223) - hat zu einer Sichtweise geführt, die die Probleme der Indigenen kombiniert verstehen will. Aus dieser Perspektive sind die Indigenen „Teil der Ausgebeuteten dieses Landes, aber [...] auch politisch und kulturell unterdrückt“ (Pacari 1984: 118f.), wodurch sie sich nicht nur in einem „Klassenkampf, sondern auch [...] in einem Volkskampf, als Volk“ (Pacari 1984: 118f.) befinden. Beide Formen der Unterdrückung und Ausbeutung werden gleichermaßen abgelehnt (Pacari 1984: 121). Daher will sich die neue Indigenenbewegung schon in den ersten Jahren ihrer Existenz an den Kämpfen des gesamten Volkes beteiligen (Pacari 1984: 118f.). Zu diesem Zeitpunkt war das Verständnis der Ursachen der kritisierten Situation allerdings noch ein anderes. ECUARUNARI beschreibt sich 1984 als „eine antiimperialistische Organisation, die für die endgültige Befreiung unseres Vaterlandes, für eine Gesellschaft ohne Unterdrückung kämpft" (ECUARUNARI 1984: 45). Erst langsam bricht die Indigenenbewegung mit dieser Sichtweise zugunsten einer Beschreibung der von ihr erlebten Ausgrenzung als komplexe Form des Rassismus (ICCI o.J.) und schließlich als „Kolonialismus und Neokolonialismen“ (CONAIE 2005), „Kolonisierung“ (Ramírez Gallegos 2009: 71) oder als ,interner und externer Kolonialismus [und] Rassismus und Paternalismus“ (FENOCIN o.J.: 11) - ohne sich allerdings völlig von den antiimperialistischen Wurzeln zu verabschieden (etwa: CONAIE 2001: 7). Die „kolonialen Ketten, die uns über Jahrhunderte unterdrückt haben“ (CONAIE 2008: 17) umfassen gleichermaßen Armut und Dis- 
kriminierung (CONAIE 2008: 17). Die Indigenen haben fünf Jahrhunderte gegen „kolonialistische und republikanische Invasion, Unterdrückung und Ausbeutung“ (CONAIE 2001: 2f.) Widerstand geleistet. So kommt die Indigenenbewegung zur Beschreibung einer dreifachen Ungleichheit: ,wirtschaftliche Ungleichheiten, soziale Ausgrenzung und kulturelle Diskriminierung“ (ECUARUNARI/CONAIE 2007: 5) bzw. „Ausgrenzungen, Rassismus und Beherrschung“ (FENOCIN o.J.: 11). Für die CONAIE steht fest:

„Dass die wirtschaftlichen, politischen, sozialen, psychologischen und kulturellen Rückstände der Kolonie, sowie der von den herrschenden Sektoren geförderte Eurozentrismus und Ethnozentrismus die Entwicklung der Nationalitäten und Völker der gesamten Gesellschaft verhindern.“ (CONAIE 2001: 2f.)

Daher sieht die Indigenenbewegung im ,entschiedenen Kampf für die politische und wirtschaftliche Befreiung" (CONAIE 2001: 2f.) die einzige mögliche Lösung der Probleme der Indigenen und der restlichen Bevölkerung.

Für die zentralen Organisationen der Indigenenbewegung existiert in Ecuador also ein Komplex aus wirtschaftlicher Ausbeutung und ethnischer Unterdrückung, der mit entsprechenden, eurozentrischen Wissensformen einhergeht und die gesamte Gesellschaft betrifft. Diese Beschreibung der erlebten Wirklichkeit entspricht dem ,weltweiten hegemonischen Machtmodell, das [...] Begriffe von Rasse (und Differenz) mit der Ausbeutung von Arbeit verbindet“" (Jáuregui 2008: 16), also der Kolonialität als Grundstein des modernen Kapitalismus (Quijano 2000b: 342). Damit versteht die Indigenenbewegung alle von Quijano beschriebenen Bestandteile des bestehenden Machtsystems - neben der Kolonialität der Macht die kapitalistische Wirtschaft, ein Nationalstaat als Träger kollektiver Autorität und der Eurozentrismus als vorherrschende Wissensform (Quijano 2000: 1) - als problematisch und will sie verändern. Insbesondere die Hervorhebung des doppelten Charakters der Probleme der Indigenen als kulturell unterdrückt und wirtschaftlich ausgebeutet spiegelt die enge Verbindung von Rassismus bzw. Kolonialität und Kapitalismus wider, die aufeinander aufbauen und ohne einander nicht denkbar sind (Quintero 2010: 8). In den Worten der CONAIE:

„Unser Kampf ist eine direkte Alternative gegen das hegemonische, unterdrückerische und versklavende kapitalistische Wirtschafts-, Politik- und Ideologiesystem, sowohl national als auch international, das die Selbstbestimmung und die wirtschaftliche und politische Unabhängigkeit der indigenen Völker und Nationalitäten und der anderer sozialen Sektoren Ecuadors verhindert.“(CONAIE 2001: 3) 


\section{PluRinationalität Und InterkUlturalität ALS DEKOLONIALISIERENDE BEGRIFFE}

Nachdem im ersten Kapitel dieses Teils die Wahrnehmung der Situation durch die Indigenenbewegung als eine koloniale analysiert wurde, sollen im zweiten Kapitel die Perspektiven zur Überwindung dieser Situation erläutert werden. Dazu wird zuerst die Indigenenbewegung als solche als eine Kritik der Kolonialität der Macht interpretiert, um anschließend ihre konkreten Vorschläge, allen voran Plurinationalität und Interkulturalität, auf ihren dekolonisierenden Gehalt hin untersuchen zu können.

\section{a. Indigene und Indigenenbewegung als dekolonialisierende Akteure}

Da die Konstitution der Indigenen in der Kolonialität der Macht die Grundlage für den Aufbau der Moderne, des Kapitalismus und der europäischen Wissensformen ist, spielen diese auch eine besondere Rolle in der ,epistemischen/theoretischen/historischen/ästhetischen/ethischen und politischen Subversion dieses Machtmodells in der Krise“ (Quijano 2010: 120) und können Alternativen dazu entwickeln. Die Indigenenbewegungen ,könnten zu einer anderen sozialen Existenz führen, die von Herrschaft, Ausbeutung und Gewalt befreit ist: zur Globalen Dekolonialität der Macht“ (Quijano 2010: 120). Für Quijano sind die aktuellen Indigenenbewegungen „eindeutigstes Signal, dass die Kolonialität der Macht in der schwersten ihrer Krisen seit ihrem Entstehen vor 500 Jahren ist“ (Quijano 2006a: 25). Sie, ebenso wie die lateinamerikanischen Schwarzenbewegungen, konnten in den letzten Jahren „die europäische Version der Modernität/Rationalität“ (Quijano 2005: 17f.) und den Nationalstaat, der diese Ideen verkörpert, in Frage stellen und ihre eigenen Gegenentwürfe anbieten. Ihre Ablehnung einer ethnischen oder rassischen Klassifizierung der Gesellschaft enthält eine neue Idee sozialer Gleichheit, die auch auf den indigenen Idealen und Traditionen von Solidarität und Gegenseitigkeit basiert. Quijano geht daher soweit, die Erzeugung einer heterogenen, nicht unbedingt von allen geteilten, lateinamerikanischen Identität - also das Produkt der Kolonialität selbst - als „Laufbahn der unvermeidlichen Zerstörung der Kolonialität der Macht, eine sehr besondere Art der Dekolonisierung und der Befreiung: die De/Kolonialität der Macht“ (Quijano 2005: 17f.) zu beschreiben. Diese Überlegung teilt auch Walter Mignolo, der „Identität in der Politik“ (Mignolo 2006: 120), also die politische Reflexion und Dekonstruktion der Identität als Produkt der Kolonialität der Macht, als Grundlage jeder „dekolonialen Option“ (Mignolo 2006: 120) sieht. Nur der Bezug auf Identität innerhalb der Kolonialität erlaubt es, „dekolonial zu denken 
(was bedeutet, politisch in dekolonialen Begriffen und Projekten zu denken)“ (Mignolo 2006: 120), also mit der Logik des bestehenden Machtsystems zu brechen.

Tatsächlich ist in Ecuador der europäisch geprägt Nationalstaat nicht in der Lage gewesen, die Indigenen als mehr als nur individuelle Bürger anzuerkennen. „Seine starre und vertikale Struktur erlaubt es ihm nicht, andere Identitäten als aktive soziale Subjekte anzuerkennen.“ (Viteri 1997: 52) Daher wurden alle Forderungen der Indigenen an den Staat, die der staatlichen Logik der Indigenen als Bürger nicht entsprachen, als subversiv oder illegal verstanden - ohne dass die Indigenenbewegung den Staat explizit in Frage stellen musste (Viteri 1997: 52). Eine ,,politische Anerkennung der Ethnien“ (Bonfil Batalla 1977: 98) - in der Regel mit Fragen der Autonomie verbunden - beinhaltet damit eine weitgehende Kritik am liberalen Nationalstaat und ist Teil „eines Kampfes der Dekolonialisierung“ (Bonfil Batalla 1977: 98) der indigenen Völker. Die Verteidigung der ethnischen und kulturellen Diversität gegenüber einem homogenisierenden und integrierenden Nationalstaat im Sinne von „Identitätspolitiken“ (Maldonado 2006: 99f.) entstammt auch einer Ablehnung der Kolonisierung und der damit einhergehenden „,kolonialen Identifikationsformen“ (Maldonado 2006: 99f.). Die Indigenenbewegung definiert sich über ihre koloniale Erfahrung, wodurch die ,nationale Identität der Ureinwohner“ (Jenson 1995: 112) zu einer antikolonialen Identität wird, die sie den kolonialen Identitätskategorien gegenüberstellen. Die mangelnde Bereitschaft des Staates, die ethnischen Identitäten jenseits der Teilung der Bevölkerung in Mestizen und Indigene anzuerkennen - sein koloniales Wissen also -, war einer der Faktoren, die eine tiefgreifende Kritik dieses Staates förderten. Wenn der Nationalstaat eine Anerkennung der verschiedenen Identitäten nicht erlaubt, muss er verändert werden (CONAIE 1989: 268f.). Von der Differenz aus entwickeln die Indigenen ein neues Verständnis von Nation, Demokratie und Wissen, das das gesamte Land miteinschließt (Walsh 2003: 127). So kommt die Indigenenbewegung zu ihrem Projekt, zusammen mit der restlichen Bevölkerung ,die strukturelle Umformung der Gesellschaft und die Einrichtung einer egalitären, gerechten und plurikulturellen Gesellschaft, die in einem plurinationalen Staat repräsentiert ist“ (CONAIE 1989: 268f.) zu betreiben.

Die Indigenenbewegung in Ecuador versteht die Geschichte der indigenen Völker und Nationalitäten als ,die Geschichte von 500 Jahre Widerstand der IndioNationalitäten und der Volksbewegung gegen den Kolonialismus und den Neokolonialismus“ (Coordinadora Popular, in: León 1994: 90)- Daher kämpfen sie nicht nur ,für eine endgültige Befreiung unserer Nationalitäten“ (Conterón/de Viteri 1984: 21), sondern wollen dadurch ,eine neue Gesellschaft ohne Ausbeutung, ohne Unterdrückung, wo man die Nationalitäten, unsere Kultur, unsere Formen der Selbstorganisation anerkennt“ (Conterón/de Viteri 1984: 21) schaffen. Die Organi- 
sation der Indigenen ist somit eine „historische Notwendigkeit“ (ECUARUNARI/ CONAIE 1989: 39), um

„eine andere Gesellschaft aufzubauen, eine andere Welt, in der wir Respekt vor uns selbst, vor unserer Kultur haben [und] es keine Leute gibt, die sich mit der Arbeit anderer bereichern und keine Beherrschenden oder Beherrschten“ (ECUARUNARI/CONAIE 1989: 39).

Diese Organisation entsteht nicht ohne weiteres. Tatsächlich kann sie auf die jahrhundertelange Erfahrung der Unterdrückung und des Kampfes der indigenen Völker gegen diese bauen. In diesem Sinne war der

„historische Widerstand der Völker ein entscheidender Faktor für die Stärkung und politische Entwicklung der Nationalitäten und bildet die Grundlage für die Veränderung der Strukturen des aktuellen Systems, das von den herrschenden Klassen aufgezwungen wurde, und den Aufbau der neuen Plurinationalen Gesellschaft“"(CONAIE 2001: 2f.).

\section{b. Diskurs und Dekolonisierung}

Schon früh entwickelte die Indigenenbewegung im Kampf gegen ,die hegemonische Kraft der momentanen rechtlich-politischen und wirtschaftlichen Ordnung, aufgezwungen vom herrschenden Sektor“ (CONAIE 1994: 13) einen Diskurs mit bestimmten Begriffen, die „orientierende Prinzipien der fordernden Kämpfe“ (García/Tuasa 2007: 18) wurden und sich um die Veränderung der Gesellschaft und den Bruch mit „,dem monokulturellen und ausschließenden Staat“ (García/Tuasa 2007: 18) drehen. Die Diagnose der Indigenenbewegung, Staat und Gesellschaft in ihrer aktuellen Verfassung würden die politische Teilhabe und die kollektiven und individuellen Rechte der indigenen Völker und Nationalitäten verhindern, führt so zu einer weitgehenden Kritik am ,bürgerlichen uninationalen Staat“" (CONAIE 2001: 2f.).

Seit den 1970ern präsentiert die Indigenenbewegung eine Ordnung der Selbstbestimmung der verschiedenen Völker ,,in einer neuen Konzeption eines pluralistischen ecuadorianischen Staates“ (Federación de Centros Shuar 1976: 129) oder „eine Pluralistische und Demokratische Nation“ (CONAIE 1994: 1) als Alternative zu diesem Staat, die die Lösung allgemeiner gesellschaftlicher Probleme erlaubt (CONAIE 1994: 1). Der plurinationale Staat enthält

„den Aufbau einer neuen dezentralen politischen und Verwaltungsstruktur, die kulturell heterogen ist und offen für die selbständige und partizipative Repräsentation aller indigenen Nationalitäten und sozialen Sektoren, insbesondere derer, die von der herrschenden staatlichen Struktur und dem sozio-ökonomischen Entwicklungsmodell ausgeschlossen wurden“ (CONAIE 1999: 51f.). 
Das soll über eine Demokratisierung des Staates (CONAIE 2001: 2f.) erfolgen, die dem ,Modell der liberalen und kaum repräsentativen Demokratie [...] eine Vielzahl demokratischer Mechanismen“ (CONAIE 2007a: 6) gegenüberstellt, die über Bürgerversammlungen partizipativ, über paritätische Wahlmechanismen repräsentativ und über die Wahl der lokalen Autoritäten vor Ort kommunitär sein sollen (CONAIE 2007a: 6). Somit bedeutet der plurinationale Staat eine institutionelle Erweiterung, die es erlaubt, die ,soziokulturelle Diversität Ecuadors in einem neuen Begriff von Staat, Entwicklung und Bürgerschaft [...] sowohl wirtschaftlich als auch politisch“ (CONAIE 1999: 51f.) aufzugreifen und zu fördern. Dazu richtet sich dieser neue Staat nach dem Prinzip der ,Einheit in der Vielfalt, mit Respekt vor der Weltanschauung und dem eigenen Denken der Nationalitäten und Völker“ (ECUARUNARI/CONAIE 2007: 5) und will auf „,den tiefen Respekt und das Bemühen, die individuellen und kollektiven Rechte zu konkretisieren“ (CONAIE 2008: 51), bauen. Über die Stärkung einer „Kultur des Friedens“ (CONAIE 2001: 33f.) soll der plurinationale Staat den „Dialog der Wissen und Lebensvorstellungen zwischen den verschiedenen Kulturen“ (CONAIE 2001: 33f.) fördern und Rassismus, Ausgrenzung und Ausbeutung in der ecuadorianischen Gesellschaft beenden. Die so umgesetzte Einheit in der Vielfalt soll den neuen Staat dadurch unterstützen, dass sie Rassismus und Regionalismus - mithin die größten Bedrohungen für diesen Staat - zerstört (CONAIE 2007: 10; ECUARUNARI/CONAIE 2007: 5).

Der plurinationale Staat soll ,,antikolonialistisch, antikapitalistisch, antiimperialistisch und antisegregationistisch“ (CONAIE 1994: 12) sein und „die kolonialen und monokulturellen Schatten, die ihn seit fast 200 Jahren begleitet haben“ (CONAIE 2007: 5; CONAIE 2007a: 5), verwerfen. Die wirtschaftlichen, politischen, sozialen und kulturellen Reste der Kolonie sollen genauso verschwinden wie der von den Eliten genährte Eurozentrismus und Ethnozentrismus (CONAIE 2001: 2f.). Die CONAIE präsentiert den plurinationalen Staat also explizit als ,ein Modell der politischen Organisation für die Dekolonialisierung unserer Nationalitäten und Völker“ (CONAIE 2007: 9; CONAIE 2007a: 6f.), das „,das Vergessen, die Verarmung und die Diskriminierung der ursprünglichen Zivilisationen“ (CONAIE 2010: 2) überwinden soll. Aus der Perspektive der Indigenenbewegung ist ein zentraler Bestandteil der Plurinationalität die Anerkennung eines hohen Grades von territorialer Selbstverwaltung in den indigenen Gebieten, die auch die Kontrolle über die jeweiligen Bodenschätze, Bildung, Gesundheit und das Rechtssystem beinhalten (CONAIE 2007a: 5). So kommt etwa Luis Macas zum Schluss: „Um diesen kolonialen Staat abzubauen, müssen wir das von der Selbstbestimmung der Völker aus tun.“ (Macas 2010: 16)

Der plurinationale Staat soll die „strukturellen Formen der Exklusion“ (Walsh 2008: 20) bekämpfen, wodurch schon sein Aufbau „,von dekolonialer Natur“ (Walsh 2008: 20) ist. Er soll ,,tatsächlich mehr als eine Nation repräsentieren kön- 
nen“ (Quijano 2006a: 37), also über eine multiple Staatsbürgerschaft die volle Teilhabe der Indigenen unter Bewahrung ihrer Eigenheiten erlauben - und damit den Nationalstaat nach europäischem Ideal beenden (Quijano 2006a: 37). Die explizite Öffnung der ehemals homogenisierenden Idee des Nationalstaates für die ausgeschlossenen Anderen bedeutet auch „,den Weg zur Pluri-versalität als universellem Projekt“ (Mignolo 2006: 134), in dem Sinne, dass der Kampf für die Anerkennung von Verschiedenheit den universalistischen Status des Kampfes für Gleichheit in Frage stellen kann. Die Plurinationalität ist dekolonial, da sie versucht, den Nationalstaat als pluri-identitär, partizipativ und grundlegend demokratisch neu zu denken. Der Versuch, über eine Veränderung der Struktur des Staates auch Wirtschaft und Kultur zu verändern, mithin die Staatszentriertheit der Forderungen der Indigenenbewegung, vor allem der CONAIE, ist dabei operativ sinnvoll. Der Staat ist in Ecuador tatsächlich an vielen Bereichen, die im Sinne der Plurinationalität verändert werden sollen - Erziehung, Gesundheitswesen, kommunale Selbstverwaltung - entscheidend beteiligt. Da er - nach Sichtweise der CONAIE seit seiner Gründung unter der Kontrolle derselben Eliten stand, die Rassismus und Eurozentrismus im Denken der Bevölkerung verankert haben, ist die Verdrängung dieser Eliten der erste Schritt, die Kolonialität der Macht als Ganzes, also als politisches, wirtschaftliches, gesellschaftliches und kulturelles Phänomen in Frage zu stellen.

Die Interkulturalität setzt bei der Gesellschaft und der Kultur an, ohne sich sehr mit dem Staat zu beschäftigen - daher bleibt sie auch wenig konkret. Im Sinne der Interkulturalität ist die plurinationale Umformung des Staates und der Gesellschaft „ein dekoloniales Projekt, an dem wir alle notwendigerweise beteiligt und miteingeschlossen sind“" (Walsh 2008: 26; Walsh 2009b: 238). Die Grundlage für die Interkulturalität ist die Neustrukturierung der Gesellschaft in einer Weise, die eine unterschiedliche Wertigkeit von Kulturen ausschließt und Verschiedenheit erlaubt. Darauf aufbauend sollen „Räume des Dialogs und der Erzeugung von neuen Wissen, die von diesen Dialogen zwischen den Kulturen ausgehen“ (CONAIE 2007a: 21f.) geschaffen werden. Dieser ,interkulturelle Dialog“ (FENOCIN o.J.: 11) muss

„kritisch mit dem internen und externen Kolonialismus sein, proaktiv in der Konfrontation mit allen Formen des Rassismus und Paternalismus und aktiv, in dem Sinne, dass er die Veränderungen im Prozess aufbaut“ (FENOCIN o.J.: 11).

Dazu muss die Interkulturalität die Forderungen der verschiedenen Bevölkerungsgruppen aufgreifen, was ,persönliche und Verhaltensänderungen mit sich bringt, und auch strukturelle Veränderungen der gesamten Gesellschaft" (FENOCIN o.J.: 11). Somit ist die „geopolitische und dekolonisierende“ (Andrade 2009: 21) Interkulturalität „ein Werkzeug der Veränderung von Staat und Gesellschaft“ (Andrade 
2009: 21), das die alten kolonialen Strukturen überwinden und die volle Teilhabe der Ausgeschlossenen erlauben soll. Dabei reicht eine reine Anerkennung der Verschiedenheit nicht aus, die unterschiedlichen Kulturen müssen akzeptiert und auf Augenhöhe integriert werden, um ,die Strukturen in den Geopolitiken des Wissens“ (Kaltmeier 2010: 7) zu verändern.

Interkulturalität ist daher für die Indigenenbewegung ein zentraler Begriff, um Kolonialität und koloniale Differenz zu hinterfragen und mit den ,,monokulturellen und ausschließenden Vorstellungen“ (Walsh 2003: 119f.) zu brechen. Sie ist

„das Implodieren in den kolonialen Strukturen der Macht als Herausforderung, Vorschlag, Prozess und Projekt von der Differenz aus; sie ist das Schaffen, Neukonzeptualisieren und Neu-Gründen von Strukturen, die unterschiedliche kulturelle Logiken, Praktiken und Arten zu denken, zu handeln und zu leben, inszenieren und in gleichberechtigte Beziehung bringen“ (Walsh 2008: 8).

So verstanden ist die Interkulturalität ein konzeptuelles Instrument, das im Sinne eines „epistemologischen Interkulturalisierens“ (Walsh 2003: 138) auf eine langfristige Veränderung der Gesellschaft und ihrer Wissensformen ausgerichtet ist. Als „kontrahegemonische Praxis“ (Walsh 2003: 135f.) soll die Interkulturalität der Verständnis bestimmten Wissens als universell und legitim und anderen, marginalen Wissens als lokal, traditionell und folkloristisch - also die Grundstrukturen der Kolonialität - hinterfragen (Walsh 2003: 135f.) und neue Kriterien für das Wahre und Vernünftige und dadurch neue Bedingungen für Wissen erzeugen (Walsh 2003: 138). Im Gegensatz zum staatlich durchgesetzten Multikulturalismus, der Kultur erlaubt, ohne dadurch das Wissen zu verändern, zielt Interkulturalität gerade auf die Grundlagen dieses Wissens, auf die ,epistemische Rechte“ (Mignolo 2006: 143f.) der Marginalisierten. Der Dialog der verschiedenen Wissensarten ist daher für Mignolo eine „Inter-Epistemologie“ (Mignolo 2006: 143f.) zwischen den westlichen und den nicht-westlichen Weltsichten. Möglich wird dieser Dialog erst durch ein „epistemisches Entkoppeln“ (Mignolo 2006: 121) von den Grundlagen westlicher Begriffe und Wissensformen, also eine Verschiebung der Geopolitik des Wissens von ihrem Ursprung im imperialen Westen zu den multiplen Politiken der kolonisierten Völker (Mignolo 2006: 121). Dieser Prozess bedeutet ein Öffnen des Denkens und ein Loslösen von der etablierten Rhetorik (Mignolo 2005: 8). So erlaubt er den Einschluss bislang verdeckter Möglichkeiten, insbesondere des im Sinne der Kolonialität als primitiv oder traditionell qualifizierten und abgewerteten Wissens (Mignolo 2005: 6). Von jeder dieser Denktraditionen ausgehend wird das dekoloniale Denken pluri-versell statt uni-versell, in dem Sinne, dass jedes Denken eigene Hintergründe und Bedeutungen einführt und gleichzeitig eine Kritik der Kolonialität von diesen Bedeutungen aus (Mignolo 2005: 26). 
Die Wiedereinführung von Identität in die Politik im Rahmen der Begriffe der ecuadorianischen Indigenenbewegung führt zu einer Krise der bestehenden, von der Kolonialität der Macht geprägten, Weltsichten (Mignolo 2006: 130) und macht eine Neudefinition von Nation und Bürgerschaft notwendig. Die Begriffe Nationalität, Plurinationalität und Interkulturalität und die in ihnen enthaltene Neudeutung von Nationalstaat und Gesellschaft müssen daher ,als Teil eines antikolonialen Projektes interpretiert werden“ (Andolina 1998: 56). Das Besondere ist dabei weniger der Wunsch, einen vorkolonialen Staat aufzubauen - wie er in einigen Spielarten des Indianismus präsent ist -, sondern vielmehr der Versuch, über die Veränderung der Machtbeziehungen und denen ihnen zugrunde liegenden Strukturen ein Land für alle Ecuadorianer zu denken, ein Land also, das interkulturell sein muss (Quijano 2006: 15). Dabei haben die verschiedenen Begriffe unterschiedliche Ansatzpunkte. Die Plurinationalität dekolonisiert den Staat auf doppelte Weise, die sich auch im Sinne des Mottos der Einheit in der Vielfalt verstehen lässt. Zum einen ist sie universell, da sie die gesamte Gesellschaft über eine Demokratisierung und Erweiterung der Repräsentation einbinden will. Zum anderen ist sie partikular, da sie die Indigenen über ein Regime der Autonomie und Selbstbestimmung aus der Entmündigung eines paternalistischen und monokulturellen Nationalstaates befreien will. Die Interkulturalität hingegen richtet sich auf die kulturellen Strukturen der Gesellschaft, konkret das Wissen, das sie über das Schaffen eines respektvollen Umgangs der verschiedenen Kulturen auf Augenhöhe dekolonisieren will. Zusammen mit dem Guten Leben, das eine Dekolonisierung der Beziehungen zwischen Menschen und Natur anstrebt, steht dieser Diskurs für ,eine alternative soziale Existenz, [...] eine De/Kolonialität oder Umverteilung der Macht“"(Quijano 2010: 113). 


\section{Schluss}

Die Geschichte Ecuadors seit der ersten Hälfte des 20. Jahrhunderts wurde von der Indigenenbewegung in ihren verschiedenen Ausprägungen und mit unterschiedlichen Organisationen geprägt. An den meisten der wichtigen gesellschaftlichen Veränderungen - den Agrarreformen, dem Widerstand gegen die Militärdiktaturen, der anschließenden Demokratisierung und Alphabetisierung und schließlich dem langsamen Prozess der Öffnung von Staat und Gesellschaft für andere Identitäten war die Bewegung und ihre Organisationen beteiligt. Auch wenn die staatliche Übernahme der zentralen Begriffe der Indigenenbewegung in der Verfassung von 2008 ein klares Anzeichen der Bedeutung dieser Bewegung ist, ist spätestens bei den Diskussionen über diese Verfassung klar geworden, dass nicht der Diskurs der Organisationen der Indigenenbewegung, sondern sein Verständnis in der Gesellschaft oft verkürzt ist. Die vorliegende Arbeit ist ein Versuch, den Diskurs der ecuadorianischen Indigenenbewegung in Gänze und im jeweiligen Kontext zu verstehen.

Im ersten Teil dieser Arbeit konnte deutlich gemacht werden, dass es sich bei der ecuadorianischen Indigenenbewegung keinesfalls um einen homogenen Akteur handelt, sondern um eine Vielzahl von Organisationen, die miteinander konkurrieren und kooperieren und sich in Aktionsformen und Diskurse unterscheiden. Dabei konnte zumindest zu einem Teil und unter der hier verwendeten Perspektive die Rolle der frühen Indigenenorganisationen im Aufbau der Indigenenbewegung und als Vorläufer der neuen Indigenenbewegung untersucht werden - und so die von Sánchez-Parga (2010: 63) diagnostizierte Forschungslücke, was FEI und FENOCIN angeht, etwas geschlossen werden. Insbesondere die Beziehung zwischen FENOCIN und CONAIE seit der zweiten Hälfte der 1990er Jahre haben die Indigenenbewegung entscheidend geprägt und die Durchführung von Aktionen und die Entwicklung eines eigenständigen Diskurses befördert.

Im zweiten Teil dieser Arbeit konnte das Aufkommen oder die Neudefinition der zentralen politischen Begriffe der heutigen Indigenenbewegung in einer Sattelzeit zwischen Mitte der 1970er und Mitte der 1980er beleuchtet werden. In diesem 
Zeitraum formt sich der Diskurs der Pluralität um die Begriffe Nationalität, Territorium und Plurinationalität heraus, der ab 1995 um den Begriff der Interkulturalität und ab 2002 um den Begriff des Guten Lebens erweitert wird. Die Begriffe und ihre Interpretation sind auch von den Gruppen abhängig, die sie verwenden, wodurch die Interkulturalität für FENOCIN und FEINE ein Gegenbegriff gegen die Plurinationalität der CONAIE ist - die beide ablehnen -, für die CONAIE aber eine Ergänzung des bisherigen Diskurses, wie das Politische Projekt von 1997 deutlich macht. Insbesondere bei der Interpretation der Plurinationalität wurde sich hier bemüht, eine neue Sichtweise zu eröffnen, die von der bisherigen Forschung abweicht. Robert Andolina interpretiert die Forderung der CONAIE nach einem plurinationalen Staat mit Rückgriff auf die Subaltern Studies als Plurinationalismus. Die CONAIE erscheint so als nationalistisch in dem Sinne, dass sie die einzelnen indigenen Nationalitäten wiederaufbauen bzw. stärken will (autonomistisch) und in dem Sinne transformistisch, dass sie die gesamte ecuadorianische Nation neu gründen will. Dieser Plurinationalismus ist also ein alternativer Nationalismus, da er zwar essentialistisch an Ecuador als Nation festhält, den traditionellen Nationalismus aber dekonstruiert und für die bislang Ausgeschlossenen öffnet und so zu einem antikolonialen Plurinationalismus wird (Andolina 1998: 63ff.). Auch Sánchez-Parga spricht von nationalistischen Kämpfen - ,ethnischer Nationalismus oder eine nationalistische Ethnizität“ (Sánchez-Parga 2010a: 87f.) -, die die Indigenenbewegung mit dem Klassenkampf kombiniert und so eine Alternative zum bestehenden Staat entwickeln kann. Tatsächlich ist nur im Politischen Projekt der CONAIE von 1994 (CONAIE 1994: 12) von Plurinationalismus die Rede, weder zuvor noch später sind Referenzen an einen Nationalismus der Ethnien zu finden. Daher wurden obige Beschreibungen als Interpretationen aufgefasst, die hier nicht geteilt werden.

Der dritte Teil der vorliegenden Arbeit hat klar gemacht, dass die Indigenenbewegung eine dekoloniale Bewegung ist. Sie stellt den kolonialen oder eurozentrischen Nationalstaat nicht nur durch ihre Existenz in Frage, da es nur eine Nationalität in einem Staat geben kann, weswegen sich können die Indigenen nicht als solche organisieren, sondern entwickelt eigenständige Alternativen, die die gesamte Bevölkerung umfassen und sich auf Politik, Wirtschaft, Kultur und Gesellschaft auswirken.

Der Diskurs der Indigenenbewegung in Ecuador geht also weit über die eingangs problematisierten Begriffe Interkulturalität und Plurinationalität hinaus. Diese befinden sich zwar im Zentrum des Diskurses, bauen aber auf eigenständige Begriffe, die wiederum einem beständigen Wandel unterworfen sind. Der Diskurs der Indigenenbewegung ist nicht nur eine Ansammlung von Forderungen an Staat und Gesellschaft, sondern auch Ausdruck eines Selbstverständnisses, das mehr ist als die Grundlage für Forderungen. Vielleicht ist das eigentlich Neue der ecuadorianischen Indigenenbewegung nicht so sehr der Bezug auf ethnische Identitäten oder 
die Zivilgesellschaft - den es ja durchaus auch bei anderen sozialen Bewegungen oder den Vorläufern der heutigen Indigenenbewegung in den 1930er und 1940ern gab -, sondern vielmehr die Entwicklung abstrakter Forderungen, die die Vision eines neuen Landes enthalten. Diese Forderungen sind nicht mehr die externer Akteure, etwa von kommunistischen oder konservativen Kadern, wie noch vor einigen Jahrzehnten, stattdessen wurden sie von der Indigenenbewegung selbst durchaus mit externer Unterstützung - entwickelt. So tritt die Vision eines plurinationalen Staates oder einer interkulturellen Gesellschaft, eine Vision, die von den Indigenen ausgeht und den Rest der Gesellschaft umfasst, an die Stelle der Vision einer nachholenden Entwicklung oder des Aufbaus des Sozialismus, eine Vision, in der die Indigenen nur als aus der Zeit gefallene Besonderheiten vorkommen, die aufhören werden, zu existieren. Plurinationalität und Interkulturalität vermögen also, der gesamten Gesellschaft das anzubieten, was (kapitalistische) Entwicklung und (sozialistische) Revolution den Indigenen nie anbieten konnten: eine Teilhabe als Andere, unter Bewahrung der eigenen Andersheit.

Abschließend sei auf die offenen Fragen und Problematiken dieser Arbeit hingewiesen. Viele Themen konnten hier nur angeschnitten werden, hätten aber eine Position im Zentrum einer weiteren Forschung verdient. Andere Probleme erwachsen aus dem theoretischen Zuschnitt der vorliegenden Forschung. So muss sich Wissenschaft, die sich mit dekolonialen Fragestellungen beschäftigt, bewusst sein, dass sie zwangsläufig selbst innerhalb der Regeln der Kolonialität agiert. Wissenschaft und Forschung sind somit notwendigerweise kolonial. Schon Anfang der 1980er gab es Beschwerden der Organisationen, dass sie ohne ihre Zustimmung erforscht werden und nicht die Ergebnisse dieser Forschung erhalten, weswegen sie fordern, dass die Indigenenorganisationen alle Forschung kontrollieren müssen und ihre Ergebnisse zur Lösung der Probleme der Indigenen beitragen sollen (Conterón/de Viteri 1984: 22). Das ICCI spricht ein Problem an, dem sich jede Forschung über die Indigenenbewegung stellen muss:

„Die offiziell anerkannten Sozialwissenschaften haben die indigenen Völker und Nationalitäten in Studienobjekte verwandelt und haben das emanzipatorische Projekt der Indigenenbewegung, das ist der Vorschlag des Plurinationalen Staates, in eine ethnische Agenda eines sozialen Akteurs, der für seine eigenen Forderungen innerhalb einer bestimmten kollektiven Aktion Druck ausüben.“ (ICCI o.J.)

Auch in der Wissenschaft gibt es ein Bewusstsein für das Problem, dass die Indigenen immer noch als Forschungsobjekte dienen, ,während der Westen als Ort dient, der die Begriffe anbietet, um dieses Forschungsobjekt zu kategorisieren“ (Del Valle 2006: 126). Schon deswegen ist es schwer, den ,dekolonialen epistemischen 
Bruch“ (Mignolo 2006: 141) aus der Perspektive der europäisch geprägten Wissenschaft nachzuvollziehen.

Auch auf die Gefahr hin, des Eurozentrismus beschuldigt zu werden, muss ein weiteres Problem angesprochen werden. Viele der nicht-indigenen Wissenschaftler, die über die Indigenenbewegung Ecuadors forschen - vor allem, wenn sie dort ansässig sind - können nicht von sich behaupten, einen neutralen Standpunkt zu haben. Sie sind in die Organisationen und Institutionen direkt oder indirekt eingebunden. Diese Einbindung kann von einer Position als Berater, wie sie etwa Catherine Walsh oder Enrique Ayala Mora in der FENOCIN oder Susana Andrade (2005) in der FEINE haben, über institutionell verfestigtere Positionen, wie etwa Pablo Dávalos, der an der Interkulturellen Universität Amawtay Wasi der CONAIE lehrt, bis hin zu eindeutigen Integrationen, wie Alicia Ibarra Illanez, die in den 1980ern in der staatlichen indigenistischen Behörde Oficina de Asuntos Indígenas (Oficina Nacional de Asuntos Indígenas 1984) arbeitete, gehen. In vielen Fällen ist diese Nähe in den jeweiligen Studien erkennbar. Problematischer ist allerdings die Tatsache, dass manche dieser Wissenschaftler als Ghostwriter für verschiedenen Organisationen dienen - nachweisbar im Falle Dávalos -, was zu einer Vermischung wissenschaftlicher Theorien und Interpretationen und der Forderungen der Organisationen führt. Auch der Einfluss Ileana Almeidas auf die organisatorischen und diskursiven Prozesse der Organisationen, die 1986 die CONAIE gründen sollten (Flores 2005: 65) ist unklar. Daher wäre zu fragen - und näher zu untersuchen - inwieweit diese Wissenschaftler als ,Teil der Indigenenrechtsbewegung oder ihrer Organisationen angesehen werden können“ (Bebbington 2007: 806f.). Der Historiker Hernán Ibarra beschreibt die ideologische Erneuerung der Indigenenbewegung in den 1980ern als Produkt eines Neoindigenismus, der vor allem von Anthropologen und Sozialwissenschaftlern betrieben wurde, die über eine Neubewertung der Indigenen und ihrer Identität Einfluss auf die öffentlichen Debatten nahmen (Ibarra 1999: 76f.). Die Schlussfolgerung, ,die Indigenen assimilierten Diskurse, die extern produziert wurden“ (Ibarra 1999: 84), die Indigenenbewegung sei also von der Wissenschaft abhängig, wird im vorliegenden Text nicht geteilt. Eine genauere Untersuchung der Beziehungen zwischen dem Diskurs der Indigenenbewegung und dem akademischen Diskurs in Ecuador könnte zu einer Klärung dieses Problems beitragen.

Diese Arbeit war nicht im Stande, sich ausreichend den internationalen Zusammenhängen der Indigenenbewegung zu widmen. Die Erforschung der in Ecuador verwendeten Begriffe in anderen Ländern der Region, gerade in Bolivien, könnte sehr aufschlussreich sein. Ebenso würde die Erforschung der internationalen Verbindungen der Indigenenbewegungen, etwa durch kontinentale Treffen, die CLOC oder regionale Verbände eine genauere Geschichte der Begriffe der Indigenenbewegung erlauben. Auch die Transnationalisierung der lokalen Widerstandsbewe- 
gungen gegen die kapitalistische Globalisierung - die ,gegen-hegemonische Globalisierung“ (Escobar 2004: 223f.) - und die verschiedenen Alternativen, die in diesem Kontext entwickelt werden, konnte hier nicht untersucht werden. Wenn es tatsächlich eine Tendenz zu Bewegungen gibt, die gleichzeitig für Gleichheit und Differenz kämpfen (Escobar 2004: 223f.), ist die ecuadorianische Indigenenbewegung sicher ein Teil davon. Sie betreibt „Raumpolitik“ (Escobar 2004: 225) als Verteidigung lokaler Gemeinschaften gegen internationale Logiken, leitet davon bestimmte Ideen und Forderungen ab und sucht für deren Umsetzung Verbündete auch auf internationaler Ebene (Escobar 2004: 222f.). Ob diese Politik allerdings Teil ,eines globalen Flusses von Akteuren und Diskursen innerhalb von etwas, das man als Weltgesellschaft bezeichnen kann“ (Büschges 2009: 43) ist, muss in einer anderen Forschung geklärt werden. Dazu kommen auch konkrete Fragen. Es ist etwa schwer zu erklären, dass die Indigenenbewegung Ecuadors in bestimmten Teilen Inhalte der Antiglobalisierungsbewegung vorwegnimmt (so Escobar 2004: 222f.), aber nie eine internationale Solidarität empfangen hat wie andere Gruppen (etwa die Zapatisten).

Eine streng materialistische Untersuchung der ecuadorianischen Indigenenbewegung und ihrer Organisationen könnte die hier vorliegende Studie um einige wichtige Details ergänzen. Neben der genauen Mitgliedszahl ist unklar, wie sich die verschiedenen Organisationen finanzieren und ob und wie ihre Funktionäre entlohnt werden. Zum letzten Punkt liegen nur wenige und widersprüchliche Daten vor. Die - eher kleine - FICSHA verfügte in den 1970ern über 52 hauptamtliche Mitarbeiter (Zallez/Gortaire 1978: 49), Ende der 1990er trat ein Vertreter aus dem politischen Rat der - wesentlich größeren - ECUARUNARI zurück, weil diese keine Entlohnung enthalten (ECUARUNARI 1998: 391). Eine Untersuchung, wie viel welcher Funktionär zu welchem Zeitpunkt erhält und woher dieses Geld kommt, kann also aufschlussreich sein. Auch die internen Entscheidungsmechanismen der verschiedenen Organisationen wurden bislang nur wenig untersucht. Daher ist die Kritik Carlos Viteris, seit den 1970ern hätte sich in der Indigenenbewegung ,eine vertikale Machtstruktur westlicher Art“ (Viteri 1997: 55f.) mit repräsentativen Wahlprozessen etabliert, die den traditionellen gemeinschaftlichen Konsens verdrängen würde, nicht überprüfbar.

Es wäre noch zu untersuchen, wie genau die Indigenenbewegung die Identität der Indigenen beeinflusst hat - inwieweit sie Teil und Agent der Identitätskonstruktion auch der Indigenen ist, die nicht direkt an den Aktionen der Bewegung beteiligt sind (vgl. Escobar 1992a: 414). Es gehörte nicht zu den Zielsetzungen dieser Arbeit, die Identitätsbildung der Indigenen in Bezug zu den Machtstrukturen vor Ort zu untersuchen. Somit kann die Hypothese Leon Zamoscs, die Auflösung der traditionellen Herrschaftsformen auf dem Land, vor allem der haciendas, führte zu einem Machtvakuum, das wiederum das Entstehen der Indigenenbewegung beförder- 
te (Zamosc 1994: 53f.) genau sowenig überprüft werden, wie seine Behauptung, dass Entstehen und Wachstum der Indigenenbewegung - zusammen mit anderen Faktoren - zu einer Homogenisierung und Stärkung der indigenen Identität führte (Zamosc 1994: 57f.).

Ein wenig beleuchteter Aspekt der Entwicklung der Begriffe der Indigenenbewegung ist die Sprache selbst. So sind diejenigen Begriffe, die sich abstrakten und gesamtgesellschaftlichen Fragestellungen widmen, auf spanisch formuliert, und werden nur in wenigen Fällen durch Begriffe in Kichwa - und nur in sehr seltenen Fällen mit konkretem Bezug auf das jeweilige Volk in anderen Sprachen - ergänzt. Die Struktur Ayllu-Llacta Ayllu-Mama Ayllu oder das moralische Grundprinzip Ama quilla-Ama llulla-Ama shua (Pacari 1984: 115) verlieren seit den 1980ern an Präsenz in den Texten der Indigenenbewegung - was nicht bedeuten muss, dass sie an Bedeutung verlieren. $\mathrm{Zu}$ fragen ist, ob die Tatsache, dass die Begriffsentwicklung auf Spanisch - also für die meisten Beteiligten der zweiten Sprache - stattfindet, einem Übereinkommen geschuldet ist, Spanisch als interindigene Kommunikationssprache zu benutzen - es sich also um einen Kompromiss aus pragmatischen Gründen handelt - oder ob Spanisch gewählt wurde, weil sich die Begriffe an die spanischsprachige Mehrheitsbevölkerung richten. Die Kongresse - und damit auch die programmatischen Entwicklungen - der sprachlich einheitlichen Gruppen fanden zumindest zu Beginn in der jeweiligen Sprache statt (nachweisbar ist dies beim Kongress der ECUARUNARI von 1977 (Zubritzki 1987: 78)). Diese Frage gewinnt an Brisanz, wenn man das Theorem der imperialen Sprachen von Walter Mignolo (etwa 2005: 6) bedenkt, demzufolge die Sprache, in der Gedanken ausgedrückt werden, die Struktur dieser Gedanken beeinflusst. 


\section{Abkürzungen}

\begin{tabular}{|c|c|}
\hline CAAP & $\begin{array}{l}\text { Centro Andino de Acción Popular (Andines Zentrum der } \\
\text { Volksaktion) }\end{array}$ \\
\hline CAOI & $\begin{array}{l}\text { Coordinadora Andina de Organizaciones Indígenas (Andine } \\
\text { Koordination der indigenen Organisationen) }\end{array}$ \\
\hline CAN & Coordinadora Agraria Nacional (Nationale Landkoordination) \\
\hline CEDIME & $\begin{array}{l}\text { Centro para la Investigación y el Desarrol lo de los Movimientos } \\
\text { Sociales del Ecuador (Zentrum für Erforschung und Entwicklung der } \\
\text { sozialen Bewegungen Ecuadors) }\end{array}$ \\
\hline CEDOC & $\begin{array}{l}\text { Confederación Ecuatoriana de Obreros Católicos (Ecuadorianische } \\
\text { Konföderation katholischer Arbeiter), ab 1975: Confederación } \\
\text { Ecuatoriana de Organizaciones Clasistas (Ecuadorianische } \\
\text { Konföderation klassistischer Organisationen) }\end{array}$ \\
\hline
\end{tabular}

CEDOCUT Confederación Ecuatoriana de Organizaciones Clasistas Unitarias de Trabajadores (Ecuadorianische Konföderation klassistischer Arbeiterorganisationen)

CEOSL Confederación Ecuatoriana de Organizaciones Sindicales Libres (Ecuadorianische Konföderation der freien gewerkschaftlichen Organisationen)

CESA Central Ecuatoriana de Servicios Agrícolas (Ecuadorianische Zentrale der landwirtschaftliche Dienstleistungen)

CISA Consejo Indio de Sudamérica (Indio-Rat Südamerikas)

CLOC Coordinadora Latinoamericana de Organizaciones del Campo (Lateinamerikanische Koordination der Landorganisationen)

CMPI Consejo Mundial de Pueblos Indios (Weltrat der Indio-Völker)

CODENPE Consejo de Desarrollo de las Nacionalidades y Pueblos del Ecuador (Entwicklungsrat der Völker und Nationalitäten Ecuadors)

COICA Coordinadora de las Organizaciones Indígenas de la Cuenca Amazónica (Koordination der indigenen Organisationen des Amazonasbeckens) 
CONAIE

CONFENIAE

CONPLADEIN

CTE

DINEIB

ECUARUNARI

FADI

FEI

FEINE

FENACLE

FENOC

FENOC-I
Confederación de Nacionalidades Indígenas del Ecuador (Konföderation der indigenen Nationalitäten Ecuadors)

Confederación de Nacionalidades Indígenas de la Amazonía Ecuatoriana (Konföderation der indigenen

Nationalitäten des ecuadorianischen Amazonasgebietes), seit September 2013: Regierung der Ureinwohnernationen des ecuadorianischen Amazonasgebietes (Gobierno de las Naciones Originarias de la Amazonía Ecuatoriana, GONOAE)

CONFEUNASSC-CNC- Confederación Única Nacional de Afiliados al Seguro Social Campesino-Consejo Nacional Campesino (Nationale Einheitskonföderation der Mitglieder der Bauernsozialversicherung-Nationaler Bauernrat) Consejo Nacional de Planificación y Desarrollo de los Pueblos Indígenas y Negros (Nationaler Planungs- und Entwicklungsrat der indigenen und schwarzen Völker) Confederación de Trabajadores del Ecuador (Arbeiterkonföderation Ecuadors)

Dirección Nacional de Educación Intercultural Bilingüe (Nationale Direktion der Zweisprachigen Interkulturellen Erziehung)

Ecuador Runacunapac Riccharimui (Das Erwachen der ecuadorianischen Indigenen)

Frente Amplio de Izquierda (Breite Linksfront)

Federación Ecuatoriana de Indios (Ecuadorianische Indioföderation)

Consejo de Pueblos y Organizaciones Indígenas Evangélicas del Ecuador (Rat der Indigenen Evangelischen Völker und Organisationen Ecuadors)

Federación Nacional de Trabajadores Agroindustriales, Campesinos e Indígenas Libres del Ecuador (Nationale Föderation der freien Agroindustriellen Arbeiter, Bauern und Indigenen Ecuadors)

Federación Nacional de Organizaciones Campesinas (Nationale Föderation der Bauernorganisationen) Federación Nacional de Organizaciones Campesinas e Indígenas (Nationale Föderation der Bauern-und Indigenenorganisationen) 
FENOCIN

FICSHA

FOIN

FUT

ICCI

IERAC

MUPP-NP

OPIP

PCE

PRODEPINE

PSE

SERBISH

UNORCAC
Confederación Nacional de Organizaciones Campesinas, Indígenas y Negras (Nationale Föderation der Bauern-, Indigenen- und Schwarzenorganisationen)

Federación Interprovincial de Centros Shuar y Achuar (Interprovinzielle Föderation der Shuar- und AchuarZentren)

Federación de Organizaciones Indígenas del Napo (Föderation der indigenen Organisationen Napos) Frente Unitario de Trabajadores (Einheitsfront der Arbeiter)

Instituto Científico de Culturas Indígenas (Wissenschaftliches Institut der Indigenen Kulturen)

Instituto Ecuatoriano de Reforma Agraria y Colonización (Ecuadorianisches Institut für Agrarreform und Kolonisierung)

Movimiento de Unidad Plurinacional Pachakutik Nuevo País (Bewegung der Plurinationalen Einheit

Pachakutik - Neues Land)

Organización de Pueblos Indígenas de Pastaza

(Organisation indigener Völker Pastazas)

Partido Comunista del Ecuador (Kommunistische Partei Ecuadors)

Proyecto de Desarrollo de los Pueblos Indígenas y Negros del Ecuador (Entwicklungsprojekt der indigenen und schwarzen Völker)

Partido Socialista Ecuatoriano (Ecuadorianische Sozialistische Partei)

Sistema de Educación Radiofónica Bicultural Shuar (Shuar-System der bikulturellen radiophonen Erziehung) Unión de Organizaciones Indígenas y Campesinas de Cotacachi (Union der Indigenen- und Bauernorganisationen von Cotacachi) 



\section{Abbildungen und Tabellen}

Abbildung 1: Struktur der Indigenenbewegung nach Bretón 2003: 219f., eigene Abbildung

Abbildung 2: Struktur der FICSH/CONAIE, eigene Abbildung

Abbildung 3: Struktur und Verbindungen der sozialen Bewegungen in Ecuador, eigene Abbildung

Tabelle 1: $\quad$ Kongresse der FEI, eigene Abbildung

Tabelle 2: Kongresse der FENOCIN, eigene Abbildung

Tabelle 3: Kongresse ECUARUNARI, eigene Abbildung

Tabelle 4: Kongresse von CONACNIE und CONAIE, eigene Abbildung

Tabelle 5: $\quad$ Wahlergebnisse der MUPP-NP, nach Meléndez (2007), eigene Abbildung

Tabelle 6: Kongresse der MUPP-NP, eigene Abbildung

Tabelle 7: Organisatorische Hegemonie und symbolische Dominanz in der Indigenenbewegung, eigene Abbildung 



\section{Literatur}

AAVV (1985) [1980]: Anexo II: Primer Congreso de Movimientos Indios de Sudamérica, in: AA.VV. (Hrsg.): Del indigenismo a las organizaciones indígenas, Quito: Colección Ethnos, S. 161-177.

Abad, Gustavo (o.J.): Auki Tituaña, bajo el símbolo de la energía, in: Revista Simposium 2, http://www.portalces.org/index.php?option=com_content $\&$ view= article\&id=26\&Itemid=100000054 (8.8.2013).

Abram, Matthias (2004): Indigene Völker, Bildung und Kultur: Interkulturelle zweisprachige Erziehung, in: GTZ (Hrsg.): Indigene Völker in Lateinamerika und Entwicklungszusammenarbeit, Eschborn: GTZ.

Abya-Yala (o.J.): ¿Quiénes somos?, http://www.abyayala.org/presentacion.php (8.8.2013).

Acosta, Alberto (2001): Breve Historia Económica del Ecuador, Quito: Corporación Editorial Nacional.

- (2002): En la encrucijada de la globalización: algunas reflexiones desde el ámbito local, nacional y global, in: Ecuador Debate 55, S. 37-55.

- (2009): El Estado Plurinacional, puerta para una sociedad democrática. A manera de prólogo, in: Acosta, Alberto/Martínez, Esperanza (Hrsg.): Plurinacionalidad. Democracia en la Diversidad, Quito: Abya-Yala, S. 15-20.

- (2010): El Buen Vivir en el camino del post-desarrollo. Una lectura desde la Constitución de Montecristi, Policy Paper 9, Quito: FES-ILDIS.

Agualsaca, José (o.J.): El Proceso de Lucha del Movimiento Indígena del Ecuador, http://www.cte-ecuador.org/pdf/historia\%20de\%201a\%20fei.pdf (23.4.2011).

Albó, Xavier (2007): Bolivia: avances y tropezones hacia un nuevo país plurinacional e intercultural, in: Martí i Puig, Salvador (Hrsg.): Pueblos Indígenas y Política en América Latina. El reconocimiento de sus derechos y el impacto de sus demandas a inicios del siglo XXI, Barcelona: CIDOB, S. 335-359.

- (2009): Movimientos y poder indígena en Bolivia, Ecuador y Perú, in: Calderón, Fernando (Hrsg.): Movimientos socioculturales en América Latina: ambientalismo, feminismo, pueblos originarios y poder empresarial, Buenos Aires: Siglo XXI, S. 131-332. 
Albornoz, Oswaldo (1976): Las luchas indígenas en el Ecuador, Guayaquil: Editorial Claridad.

— (1983): Breve sintesis: Historia del movimiento obrero ecuatoriano, Quito: Letra Nueva.

Alcántara, Manuel/Marenghi, Patricia (o.J.): Los partidos étnicos de América del Sur: Algunos factores que explican su rendimiento electoral, http://americo. usal.es/oir/opal/pdfs/Manuel_alcantara/Ponencia-Alcantara-Marenghi.pdf (8.8. 2013).

Almeida, Ileana (1979): Consideraciones sobre la nacionalidad kechua, in: Instituto Otavaleño de Antropología (Hrsg.): Lengua y Cultura en el Ecuador, Otavalo: Instituto Otavaleño de Antropología, S. 11-48.

- (2008): Nacionalidad ayuda a los indígenas, Interview in der Tageszeitung Hoy, 23.3.2008, http://www.hoy.com.ec/noticias-ecuador/nacionalidad-ayuda-a-losindigenas-291695-291695.html (8.8.2013).

Almeida, Ileana/Arrobo, Nidia (o.J.): Autonomía Indígena frente al Estado Nación, http://www.latautonomy.org/ECUADORLIBRO_LATAUTONOMY.pdf (8.8.2013).

Almeida Vinueza, José (1997): Identidades múltiples y estado unitario en el Ecuador, in: ILDIS (Hrsg.): Identidad nacional y globalización, ILDIS: Quito, S. 173-181.

Almeida, José/Prieto, Mercedes (1983): Modalidades organizativas, in: Cuadernos de Nueva 7, S. 35-39.

Alvarez, Sonia/Dagnino, Evelina/Escobar, Arturo (1998): Introduction: The Cultural and the Political in Latin American Social Movements, in: dies. (Hrsg.): Cultures of Politics/Politics of Cultures. Re-visionings Latin American Social Movements, Boulder: Westview Press, S. 1-29.

Andolina, Robert (1998): El Proyecto Político de la CONAIE como Lucha Anticolonial. Una (otra) reconsideración de Nación y Ciudadanía en el Ecuador, in: Almeida, Ileana/Arrobo, Nidia (Hrsg.): En Defensa del Pluralismo y la Igualdad. Los Derechos de los Pueblos Indios y el Estado, Quito: Abya-Yala, S. 5574.

- (1999): Colonial Legacies and Plurinational Imaginaries: Indigenous Movement Politics in Ecuador and Bolivia, Dissertation, University of Minnesota.

Andrade, Susana (2005): El despertar político de los indígenas evangélicos en Ecuador, in: Íconos. Revista de Ciencias Sociales 22, S. 49-60.

- (2009): El Reto de la Interculturalidad: Interculturalidad, plurinacionalidad y ciencias sociales en el Ecuador, in: Antropología. Cuadernos de Investigación 8, S. 13-23.

Andrango, Alberto (1983): Contra la discriminación y la pobreza, in: Cuadernos de Nueva 7, S. 50-52.

Ayala Mora, Enrique (2011): La interculturalidad: camino para el Ecuador, Quito: FENOCIN. 
Ayala Mora, Enrique (o.J.): Interculturalidad en el Ecuador, http://www.uasb. edu.ec/UserFiles/380/File/Interculturalidad\%20en\%20el\%20Ecuador.pdf $\quad$ (8.8. 2013).

Barabas, Alicia/Bartolomé, Miguel (1979): Presentación (las dinámicas étnicas), in: Revista Mexicana de ciencias políticas y sociales 97, S. 5-10.

Barre, Marie-Chantal (1982): Políticas indigenista y reivindicaciones indias en América Latina 1940-1980, in: Rojas, Francisco (Hrsg.): América Latina. Etnodesarrollo y etnocidio, San José: FLACSO, S. 39-82.

- (1983): Ideologías indigenistas y movimientos indios, Mexiko: Siglo XXI.

Barrera, Augusto (2001): Acción Colectiva y crisis política. El movimiento indígena ecuatoriano en la década de los noventa, Quito: CIUDAD/Abya-Yala.

- (2002): El movimiento indígena ecuatoriano: entre los actores sociales y el sistema político, in: Nueva Sociedad 182, S. 90-105.

— (o.J.): Ecuador: el movimiento indígena entre lo social y lo político, in: Duterme, Bernard (Hrsg.): Movimientos y Poderes de Izquierda en América Latina, Madrid: Editorial Popular, S. 149-161.

Barrientos, Ignacio (o.J.): ¿Nacionalismo Indígena? El Tránsito de una Identidad Étnica a una Identidad Nacional, http://halshs.archives-ouvertes.fr/docs/00/ 10/47/31/PDF/Ignacio_Barrientos.pdf (8.8.2013).

Bartolomé, Margarita (2002): Introducción: un reto a la educación intercultural, in: dies. (Hrsg.): Identidad y ciudadanía. Un reto a la educación intercultural, Madrid: Narcea, S. 13-25.

Bartolomé, Miguel (1979): Las nacionalidades indígenas emergentes en México, in: Revista Mexicana de ciencias políticas y sociales 97, S. 11-26.

Bauer, Otto (1907): Die Nationalitätenfrage und die Sozialdemokratie, Wien: Volksbuchhandlung Ignaz Brand.

Bebbington, Anthony (2007): Social Movements and the Politicization of Chronic Poverty, in: Development and Change 38 (5), S. 793-818.

Becker, Marc (1997): Class and Ethnicity in the Canton of Cayambe: The Roots of Ecuador's Modern Indian Movement, http://www.yachana.org/research/diss.pdf (8.8.2013).

- (2001): Nina Pacari, in: Tompkins, Cynthia/Foster, David (Hrsg.): Notable Twentieth-Century Latin American Women: A Biographical Dictionary, Westport, CT: Greenwood Publishing, S. 218-222.

- (2002): Mariátegui y el problema de las razas en América Latina, in: Revista Andina 35, S. 191-220.

- (2006): La Historia del Movimiento Indígena Escrita a través de las páginas de Ñucanchic Allpa, in: Sosa-Buchholz, Ximena/Waters, William (Hrsg.): Estudios Ecuatorianos. Un aporte a la discusión. Ponencias escogidas del II Encuentro de la Sección de Estudios Ecuatorianos de LASA Quito 2004, Quito: FLACSO, Abya-Yala, S. 133-153. 
Becker, Marc (2007): Comunistas, indigenistas e indígenas en la formación de la Federación Ecuatoriana de Indios y el Instituto Indigenista Ecuatoriano, in: Íconos. Revista de Ciencias Sociales 27, S. 135-144.

- (2008): Indians and Leftists in the Making of Ecuador's Modern Indian Movements, Durham/London: Duke University Press.

- (2008a): Pachakutik and Indigenous Political Party Politics in Ecuador, in: Stahler-Sholk, Richard/Vanden, Harry/Kuecker, Glen David (Hrsg.): Latin American Social Movements in the Twenty-first Century: Resistance, Power, and Democracy, o.O.: Rowman \& Littlefield Publishers, S. 165-180.

- (2008b): Indigenous Nationalities in Ecuadorian Marxist Thought, in: A contracorriente 5 (2), S. 1-46.

- (2009): Ecuador, indigenous and popular struggles, in: Ness, Immanuel (Hrsg.): International Encyclopedia of Revolution and Protest, London: Blackwell Publishing, S. 1056-1057.

- (2009a): Ecuador, left and popular movements, 1940s to present, in: Ness, Immanuel (Hrsg.): International Encyclopedia of Revolution and Protest, London: Blackwell Publishing, S. 1057-1058.

- (2011): Correa, Indigenous Movements, and the Writing of a New Constitution in Ecuador, in: Latin American Perspectives 176 (38, 1), S. 47-62.

- (2011a): Indigenous Movements from Oppressed Nationalities to an EthnoNationalist Discourse, in: Hunefeldt, Christine/Zamosc, Leon (Hrsg.): Ethnicity from Various angles and Through Varied Lenses. Yesterday's today in Latin America, Brighton: Sussex, S. 192-208.

Becker, Marc/Tutillo, Silvia (2009): Historia agraria y social de Cayambe, Quito: FLACSO, Abya-Yala.

Benford, Robert (1997): An Insider's Critique of the Social Movement Framing Perspective, in: Sociological Inquiry 67 (4), S. 409-430.

Benford, Robert/Snow, David (2000): Framing Processes and Social Movements: An Overview and Assessment, in: Annu. Rev. Sociol. 26, S. 611-639.

Bernal, Angélica (2000): De la exclusión étnica a los derechos colectivos. Un análisis político del Ecuador, in: dies. (Hrsg.): De la Exclusión a la Participación. Pueblos indígenas y sus derechos colectivos en el Ecuador, Quito: Abya-Yala, S. 35-55.

Bernal, Gabriela (2011): ¿Cuál interculturalidad? Reflexiones desde las experiencias de Sudamérica. Thematic Paper for the Commons-Conference in Rome, 28/29 April 2011, http://rosalux-europa.info/userfiles/file/Bernal_Cual_inter culturalidad_2011.pdf (8.8.2013).

Black, Chad (1999): The Making of an Indigenous Movement: Culture Ethnicity, and Post-Marxist Social Praxis in Ecuador, Research Paper Series 32, Department of History, University of New Mexico.

Bonfil Batalla, Guillermo (1977): Sobre la liberación del Indio, in: Nueva Antropología. Revista de Ciencias Sociales 8, S. 95-101 
Bonfil Batalla, Guillermo (1978): Las nuevas organizaciones indígenas (hipotesis para la formulación de un modelo analitico), in: Journal de la Société des Américanistes 65 , S. 209-219.

- (1981): Utopía y revolución: El pensamiento político de los indios en América Latina, in: ders. (Hrsg.): Utopía y revolución: El pensamiento político contemporáneo de los indios en América Latina, Mexiko: Nueva Imagen, S. 11-53.

Boris, Dieter (2009): Soziale Bewegungen in Lateinamerika. Bilanz und Perspektiven, in: Mittag, Jürgen/Ismar, Georg (Hrsg.): ¿,El pueblo unido“? Soziale Bewegungen und politischer Protest in der Geschichte Lateinamerikas, Münster: Westfälisches Dampfboot, S. 555-570.

Borochov, Ber (1905): The National Question and the Class Struggle, http:// www.marxists.org/archive/borochov/1905/national-class.htm (8.8.2013).

Botero, Luis Fernando (1998): Estado, cuestión agraria y movilización india en Ecuador. Los desafíos de la democracia, in: Nueva Sociedad 153, S. 56-72.

- (2000): Movilización indígena, etnicidad y procesos de simbolización en Ecuador. El caso del líder indígena Lázaro Condo, Quito: Abya-Yala.

Bottasso, Juan (2003): Los Salesianos y la lengua de los Shuar, in: Academia Nacional de Historia del Ecuador (Hrsg.): Los Salesianos y la lengua de los Shuar. Discurso de incorporación de Juan Bottasso Boetti sdb a la Academia Nacional de Historia del Ecuador, Jueves 27 de marzo del 2003, Quito: Abya-Yala, S. 1733.

Bretón, Víctor (2003): Desarrollo rural y etnicidad en las tierras altas de Ecuador, in: Bretón, Víctor/García, Francisco (Hrsg.): Estado, etnicidad y movimientos sociales en América Latina. Ecuador en crisis, Barcelona: Icaria, S. 217-253.

- (2008): De la Ventriloquia a la Etnofagia o la Etnitización del Desarrollo Rural en los Andes Ecuatorianos, in: Martínes, Mónica/Rodríguez, Eugenia (Hrsg..): Intelectuales, Mediadores y Antropólogos. La Traducción y la Reinterpretación de lo Global en lo Local, Donostia-San Sebastián: ANKULEGI antropologia elkartea, S. 113-136.

Bruners, Jan (o.J.): Historische Semantik, http://janeden.net/pdf/2286.pdf (8.8. 2013).

Buendía, Fernando (2000): Multiculturalidad e interculturalidad en la experiencia de los movimientos sociales, in: Fernández Salvador, Consuelo (Hrsg.): Diálogo Intercultural. Memorias del Primer Congreso Latinoamericano de Antropología Aplicada, Quito-Ecuador 25 al 29 de enero de 1999, Quito: Abya-Yala, S. 4966.

Büschges, Christian (2009): Políticas de identidad entre integración y autonomía: movimiento indígena, sociedad y Estado en Ecuador y Nepal desde una perspectiva comparada y transnacional, in: Ospina, Pablo/Kaltmeier, Olaf/Büschges, Christian (Hrsg.): Los Andes en movimiento. Identidad y poder en el nuevo paisaje político, Quito: Corporación Editora Nacional/Universidad Andina Simón Bolívar, S. 41-63. 
Bustamante, Teodoro (1983): Cómo se ha entendido lo indígena, in: Cuadernos de Nueva 7, S. 115-117.

Burbano de Lara, Felipe (1998): Cultura política y democracia en el Ecuador: Una Aproximación a nuestros vacíos, Quito: CORDES.

Burguete, Araceli (2010): Autonomía: la emergencia de un paradigma en las luchas por la descolonización en América Latina, in: González, Miguel/Burguete, Araceli/Ortiz, Pablo (Hrsg.): La autonomía a debate. Autogobierno indígena y Estado plurinacional en América Latina, Quito: FLACSO/GTZ/IWGIA/CIESAS/ UNICH, S. 63-94.

Busse, Dietrich (1987): Historische Semantik. Analyse eines Programms, Stuttgart: Klett-Cotta.

- (2003): Begriffsgeschichte oder Diskursgeschichte? Zu theoretischen Grundlagen und Methodenfragen einer historisch-semantischen Epistemologie, in: Dutt, Carsten (Hrsg.): Herausforderungen der Begriffsgeschichte, Heidelberg: Winter Verlag, S. 17-38.

Cabrera, Flor (2002): Hacia una nueva concepción de la ciudadanía en una sociedad multicultural, in: Bartolomé, Margarita (Hrsg.): Identidad y ciudadanía. Un reto a la educación intercultural, Madrid: Narcea, S. 79-104.

Canessa, Andrew (2007): Who Is Indigenous? Self-Identification, Indigeneity, And Claims to Justice In Contemporary Bolivia, in: Urban Anthropology 36 (3), S. 195-237.

Caniglia, Beth/Carmin, JoAnn (2005): Scholarship on Social Movement Organizations: Classic views and emerging trends, in: Mobilization. An International Journal 10 (2), S. 201-212.

CEDOC (1976): CEDOC. Desde la dirección de los conservadores hasta la dirección de los trabajadores. 1938-1976, Quito: CEDOC.

Cerulo, Karen (1997): Identity Construction: New Issues, New Directions, in: Annual Review Sociol. 23, S. 385-409

Chancoso, Blanca (1983): ,El despertar indígena se da en la lucha por la tierra', in: Cuadernos de Nueva 7, S. 40-43.

Chiriboga, Manuel (1983): La cuestión indígena en el Ecuador, in: Cuadernos de Nueva 7, S. 122-124.

- (1986): Crisis económica y movimiento campesino e indígena, in: CLACSO/ILDIS (Hrsg.): Movimientos sociales en el Ecuador, Buenos Aires/Quito: CLACSO, ILDIS, CAAP, CEDIME, IEE, CEPLAES, CIUDAD, S. 63-91.

- (1987): Movimiento campesino e indígena y participación política en Ecuador. La construcción de identidades en una sociedad heterogenea, in: Ecuador Debate 13, S. $87-121$.

Chisaguano, Silverio (2008): Visibilización de la población indígena del Ecuador, in: Revista Ecuatoriana de Estadística, S. 97-107.

Cholango, Humberto (2011): Nuevos retos del movimiento indígena, in: La Tendencia 12 , S. 60-62. 
Chuji Gualinga, Mónica (2008): Diez conceptos básicos sobre plurinacionalidad e interculturalidad, http://alainet.org/active/23366 (8.8.2013).

Collins, Jennifer (2006): Democratizing formal politics: indigenous and social movement political parties in Ecuador and Bolivia, 1978-2000, Diss., University of California, San Diego, http://escholarship.org/uc/item/8hz1j6gx (8.8.2013).

Comisión Especial de Asuntos Indígenas (1988): Proyecto de Ley de Nacionalidades Indígenas, Quito: Congreso Nacional de la República del Ecuador.

CONAIE (1989): Las nacionalidades indígenas en el Ecuador. Nuestro Proceso organizativo, Quito: Tincui/CONAIE, Abya-Yala.

- (1992): Achievements of the Indigenous Movement, http://conaie.nativeweb.org/ conaie3.html (8.8.2013).

- (1992a): The present Situation, http://conaie.nativeweb.org/conaie2.html (8.8. 2013).

- (1993): Political Declaration of Ecuador's Indigenous Peoples, http://conaie. nativeweb.org/conaie4.html (8.8.2013).

- (1994): Proyecto Político de la CONAIE, Quito: CONAIE.

- (1997): Proyecto Político de la CONAIE, Quito: CONAIE.

- (1997a): Las nacionalidades indígenas y el Estado Plurinacional, Quito: CONAIE.

- (1999): Las Nacionalidades Indígenas y sus derechos colectivos en la Constitución, Quito: CONAIE.

- (2001): Proyecto Político de las Nacionalidades y Pueblos del Ecuador, in: http://www.conaie.org/congresos-de-la-conaie/ii-congreso-ed-la-conaie/99proyecto-politico-de-las-nacionalidades-y-pueblos-del-ecuador?format=pdf (12.9.2010).

- (2001a): Proyecto Político de la Confederación de Nacionalidades Indígenas del Ecuador (CONAIE). Aprobado en el I Congreso Nacional de las Nacionalidades y Pueblos Indígenas del Ecuador (12 de octubre de 2001), http://www.llacta. org/organiz/coms/com862.htm (8.8.2013).

- (2004): Propuesta de Ley de Biodiversidad, Quito: CONAIE.

- (2005): Constitución de la Confederación de Nacionalidades Indígenas del EcuaEcua-

dor,http://www.nacionmulticultural.unam.mx/declaraciones/docs/decl_031.pdf (8.8.2013).

- (2007): Propuesta de la CONAIE frente a la Asamblea Constituyente. Principios y lineamientos para la nueva constitución del Ecuador. Por un Estado Plurinacional, Unitario, Soberano, Incluyente, Equitativo y Laico, Quito: CONAIE.

- (2007a): La CONAIE frente a la Asamblea Constituyente. Propuesta de nueva constitución- desde la CONAIE- para la construcción de un Estado Plurinacional, Unitario, Soberano, Incluyente, Equitativo y Laíco, http://www. cebem.org/cmsfiles/archivos/principios-lineamientos-conaie.pdf (8.8.2013). - 
(2010): Declaración al pie de taita Imbabura y mama Cotacachi. Los Pueblos y Nacionalidades Indígenas del Ecuador frente a la Cumbre de los Presidentes del ALBA-TCP con , autoridades indigenas y afrodecendientes', http://www.bio diversidadla.org/content/download/76173/436968/version/1/file/CONAIE+ ante+Cumbre+del+ALBA.pdf (8.8.2013).

CONAIE (2011): Informe del Gobierno de los pueblos y nacionalidades al IV. Congreso de la CONAIE. Enero 2008-Marzo 2011, http://www.movimientos.org/ ima-

gen/INFORME\%20DEL $\% 20$ GOBIERNO $\% 20$ DE $\% 20$ LA $\% 20$ CONAIE $\% 2020$ 11\%20final.pdf (15.8.2011).

- (o.J.): ¿Qué es la CONAIE? El 22 de Octubre del 2007, Quito: CONAIE.

Conferencia de Cabecillas Indígenas (1935): Hoy se clausura la Conferencia de Cabecillas Indígenas, Quito: El Correo, in: http://www.yachana.org/earchivo/ 1935nov7.pdf (8.8.2013).

Conterón, Lourdes/de Viteri, Rosa (1984): Causaimanta Allpamanta Quishpirincacaman Tantanacushunchic. Organizaciones Indígenas del Ecuador, Quito: Ministerio de Educación y Cultura.

Costa, Sérgio/Gurza, Adrián (2006): Cohesión social y coexistencia intercultural en América Latina, in: Cotler, Julio (Hrsg.): La cohésion social en la agenda de América Latina y de la Unión Européa., Lima: Instituto de Estudios Peruanos, S. 247-279.

Crnic, Zelijko (2009): Die Indigenen Lateinamerikas. Zwischen Ausgrenzung und sozialer Aktion, in: Gerheim, Udo/Storozenko, Victoria/Teubl,Thorsten/Trogisch, Corinna (Hg.): Widersprüche, Bewegungen, Konflikte. Zwölftes und dreizehntes DoktorandInnen-Seminar der Rosa-Luxemburg-Stiftung, Karl Dietz Verlag Berlin, S. 45-74.

CTE (o.J.): Reseña historica de la Confederación de Trabajadores del Ecuador, http://www.cte-ecuador.org/pdf/HISTORIA\%20DE\%20LA\%20CTE.pdf (22.4. 2011).

Cultura Andina (o.J.): Dr. Luis Eduardo Maldonado Ruíz, http://cultura andina.khipu.net/index.php?option=com_content\&view=article\&id=46:dr-luiseduardo-maldonado-ruiz\&catid=37: expositores\&Itemid=123 (15.4.2012).

Cuvi, María/Poats, Susan (2011): La interculturalidad en la gestión social de los ecosistemas andinos, Quito: ECOBONA.

Dávalos, Pablo (2003): Plurinacionalidad y poder político en el movimiento indígena ecuatoriano, in: Observatorio Social de América Latina 9, S. 43-50.

Dávila, Jorge (1995): El FUT. Trayectoria y perspectivas, Quito: Cooperación Editora Nacional.

Davis, Diane (1999): The Power of Distance: Re-Theorizing Social Movements in Latin America, in: Theory and Society 28 (5), S. 585-638.

de la Cruz, Rodrigo (1992): Plurinacionalidad y movimiento indígena, in: Corporación Editora Nacional (Hrsg.): Pueblos Indios, estado y derecho, Quito: Corpo- 
ración Editora Nacional, Abya-Yala, ILDIS, CORPEA, Taller Cultural Causanacunchic, S. 135-147.

de la Torre, Luz María (2005): Construcción de ciudadanía desde la diferencia. El caso del movimiento indígena en el Ecuador, Tesis, Quito: FLACSO.

Della Porta, Donatella/Tarrow, Sidney (2005): Transnational Processes and Social Activism: An Introduction, in: dies. (Hrsg.): Transnational Protest and Global Activism, Lanham: Rowman \& Littlefield, S. 1-17.

del Valle Escalante, Emilio (2006): Latinoamericanismo, Barroco de Indias y Colonialidad del Poder: Reflexiones sobre políicas de exclusión, in: Procesos. Revista Ecuatoriana de Historia 23 (1), S. 115-133.

de Sousa Santos, Boaventura (2009): Las paradojas de nuestro tiempo y la Plurinacionalidad, in: Acosta, Alberto/Martínez, Esperanza (Hrsg.): Plurinacionalidad. Democracia en la Diversidad, Quito: Abya-Yala, S. 21-62.

Diani, Mario (1992): The concept of social movements, in: The sociological Review 40 (1), S. 1-25.

Diario Hoy (2000): La Historia de Abya Yala, 23.2.2000, S. 9-A.

Diaz-Bone, Rainer et al. (2007): The Field of Foucaultian Discourse Analysis: Structures, Developments and Perspectives, Forum Qualitative Sozialforschung 8 (2), Art. 30, http://nbn-resolving.de/urn:nbn:de:0114-fqs0702305 (18.4.2012).

Dietz, Christoph (1996): ¿Qué factores contribuyen al éxito de una editorial especializada? Lecciones de la editorial antropológica salesiana ,Abya Yala‘ (Ecuador), in: Catholic Media Council Information Bulletin 2.

ECUARUNARI (1984): El movimiento campesino indígena ,ECUARUNARI“, in: Oficina Nacional de Asuntos Indígenas (Hrsg.): Población Indígena y desarrollo amazónico, Quito: Abya-Yala, S. 43-50.

- (1989): Experiencias educativas y de comunicación del Movimiento Indígena y campesino Ecuarunari, in: ECUARUNARI, FENOC, ALAI: Nuestra voz, nuestra cultura. Memoria del Taller Andino de Intercambio de Experiencias en Educación y Comunicación de Organizaciones Campesino Indígenas, Quito, 711.10.1987, Quito: ECUARUNARI/FENOC/ALAI, S. 87-90.

- (1998): Historia de la nacionalidad y los pueblos quichuas del Ecuador, Quito: FUDEC, CODENPE, ILDIS.

ECUARUNARI (o.J.): Proceso Organizativo de ECUARUNARI, http://ecuarunari. org/portal/info/historia, (8.8.2013).

ECUARUNARI/CONAIE (2007): Propuesta para la Asamblea Constituyente, http://www.ecuarunari.org/es/info_especiales/anc/propuesta_asamblea.pdf (8.8.2013).

ECUARUNARI/CONAIE (1989): Lineamientos políticos generales del movimiento indígnea en el Ecuador, in: ECUARUNARI, FENOC, ALAI: Nuestra voz, nuestra cultura. Memoria del Taller Andino de Intercambio de Experiencias en Educación y Comunicación de Organizaciones Campesino Indígenas, Quito, 7-11.10.1987, Quito: ECUARUNARI/FENOC/ALAI, S. 38-42. 
ECUARUNARI/FENOC (1989): La política agraria en Ecuador. Respuestas del movimiento campesino e indígena, in: ECUARUNARI, FENOC, ALAI: Nuestra voz, nuestra cultura. Memoria del Taller Andino de Intercambio de Experiencias en Educación y Comunicación de Organizaciones Campesino Indígenas, Quito, 7-11.10.1987, Quito: ECUARUNARI/FENOC/ALAI, S. 25-34.

Ennis, James (1987): Fields of Action: Structure in Movements' Tactical Repertoires, in: Sociological Forum 2 (3), S. 520-533.

Escobar, Arturo (1992): Culture, Economics, and Politics in Latin American Social Movements Theory and Research, in: Escobar, Arturo/Alvarez, Sonia (Hrsg.): The Making of Social Movements in Latin America. Identity, Strategy and Democracy, Bolder/Oxford: Westview Press, S. 62-85.

- (1992a): Culture, Practice and Politics: Anthropology and the study of social movements, in: Critique of Anthropology 12, S. 395-432.

- (1998): Whose Knowledge, Whose nature? Biodiversity, Conservation, and the Political Ecology of Social Movements, in: Journal of Political Ecology, Vol.5, S. 53-82.

- (2004): Beyond the Third World: imperial globality, global coloniality and antiglobalisation social movements, in: Third World Quarterly 25 (1), S. 207-230.

Escobar, Manuel (1983): ,La FEI... el indio de poncho colorado‘, in: Cuadernos de Nueva 7, S. 48-49.

Espinosa, Simón (1992): El papel de la iglesia católica en el movimiento indígena, in: ILDIS (Hrsg.): Indios. Una reflexión sobre el levantamiento indígena de 1990, Quito: ILDIS, S. 179-219.

Estermann, Josef (2011): ,Vivir bien“ como utopía política. La concepción andina del , vivir bien“ (suma qamaña/allin kawsay) y su aplicación en el socialismo democrático en Bolivia, http://de.scribd.com/doc/95943707/Vivir-Bien-JosefEstermann-2 (8.8.2013).

Fantasia, Rick/Hirsch, Eric (1995): Culture in Rebellion: The Appropriation and Transformation of the Veil in the Algerian Revolution, in: Johnston, Hank/ Klandermans, Bert (Hrsg.): Social Movements and Culture, Minneapolis: University of Minnesota Press, S. 144-159.

Federación de Centros Shuar (1981) [1973]: Qué es la Federación Shuar, in: Bonfil Batalla, Guillermo (Hrsg.): Utopía y revolución: El pensamiento político contemporáneo de los indios en América Latina, Mexiko: Nueva Imagen, S. 319320.

— (1976): Solución original a un problema actual, Sucúa: Federación Shuar.

FEI (o.J.): Federación Ecuatoriana de Indígenas, http://www.cte-ecuador.org/ (10.10.2010).

- (o.J.a): Historia, http://www.confederacionfei.com/index.php?option=com_ content $\&$ view=article\&id=2\&Itemid=6 (24.4.2011).

FEINE (2010): Plan Estratégico 2010-2014, http://www.feine.org.ec/pacha/wpcontent/uploads/2011/12/Plan-Estrategico-de-FEINE-2010-2014.pdf (8.8.2013). 
- (o.J.): FEINE: Treinta años de lucha en favor de los pueblos indígenas, http://www.feine.org.ec/esp/?p=258 (8.5.2011).

Feldt, Heidi (2004): Indigene Völker und Staat, in: GTZ (Hrsg.): Indigene Völker in Lateinamerika und Entwicklungszusammenarbeit, Eschborn: GTZ.

FENOC (o.J.): Las luchas campesinas 1950-1983. Movilización campesina e historia de la FENOC, o.O.: CEDOC, CEDEP.

FENOCIN (1999): Hacia el nuevo Milenio. Porque en el campo está la fuerza del Desarrollo de la Identidad y la Vida, o.O.: FENOCIN.

- (2004): Noveno Congreso Ambato, 20-22 de mayo de 2004, Quito: FENOCIN.

- (2008): X Congreso Nacional Ordinario de la Confederación Nacional de Organizaciones Campesinas, Indígenas y Negras, FENOCIN, PORTOVIEJO 5, 6 y 7 de Septiembre de 2008, http://www.fenocin.org/RESOLUCIONESxcongres odefinitivo2008.doc (8.8.2013).

- (2011): Construcción de la línea base de las organizaciones de FENOCIN, in: Del Campo 1, S. 7.

- (o.J.): Consolidación organizacional, Revolución agraria, Interculturalidad, Soberanía alimentaria, Construcción del Socialismo, http://www.uasb.edu.ec/ UserFiles/380/File/Presentacion\%20FENOCIN\%202009_Patricio\%20

Sandoval.pdf (25.9.2010).

Fernández, Blanca (2010): ¿Quiénes son los intelectuales indígenas ecuatorianos? Aportes para una construcción intercultural de saber en América Latina, in: A Parte Rei 71, http://serbal.pntic.mec.es/ cmunoz11/blanca71.pdf (8.8.2013).

Fernández-Juárez, Gerardo (2010): Sumak Kawsay: retos y paradojas de la Salud Intercultural, in: Ministerio de Salud Pública (Hrsg.): Salud, Interculturalidad y Derechos. Claves para la reconstrucción del Sumak Kawsay-Buen Vivir, Quito: Ministerio de Salud Pública, S. 17-51.

Fernández Sebastián, Javier (2007): Iberconceptos. Hacia una historia transnacional de los conceptos políticos en el mundo iberoamericano, in: Isegoría. Revista de Filosofía Moral y Política 37, S. 165-176.

Flores, Alejandra (2005): Intelectuales indígenas del Ecuador y su paso por la escuela y universidad, Masterarbeit, FLACSO Ecuador, http://hdl.handle.net/ 10469/768 (8.8.2013).

Földes, Csaba (2009): Black Box ,Interkulturalität‘. Die unbekannte Bekannte (nicht nur) für Deutsch als Fremd-/Zweitsprache. Rückblick, Kontexte und Ausblick, in: Wirkendes Wort 59 (3), S. 503-525.

Fontaine, Guillaume (2001): Discurso ético y praxis política. La institucionalización de la etnicidad en Colombia y Ecuador, in: Indiana 17/18, S. 57-67.

- (2007): El precio del petróleo. Conflictos socio-ambientales y gobernabilidad en la región amazónica, Quito: Abya-Yala.

Foucault, Michel (1983): Der Wille zum Wissen. Sexualität und Wahrheit 1, Frankfurt a. M.: Suhrkamp. 
Frank, Erwin/Patiño, Ninfa/Rodriguez, Marta (Hrsg.) (1992): Los políticos y los indígenas. Diez entrevistas a candidatos presidenciales y máximos representantes de partidos políticos del Ecuador sobre la cuestión indígena, Quito: Abya-Yala/ILDIS.

Freston, Paul (2004): Protestant Political Parties. A Global Survey, Hampshire/Burlington: Ashgate.

Galán, Gabriel (2009): El Buen Vivir. Breves reflexiones constitucionales, http://www.derechoecuador.com/articulos/detalle/archive/doctrinas/derechocons titucional/2009/12/29/el-buen-vivir-breves-reflexiones-constitucionales (9.8.2013).

Gallie, W. B. (1956): Essentially Contested Concepts, in: Proceedings of the Aristotelian Society 56, S. 167-198.

Gamson, William/Meyer, David (1996): Framing political opportunity, in: McAdam, Doug/ McCarthy, John/Zald, Mayer (Hrsg.): Comparative Perspectives on Social Movements: Political Opportunities, Mobilizing Structures, and Cultural Framings, Cambridge: Cambridge University Press, S. 275-290.

García, Fernando (2001): Política, Estado y movimiento indígena: nuevas estrategias de negociación en tiempos de la dolarización, in: Indiana 17/18, S. 69-81.

- (2005): De movimiento social a partido político: el caso del movimiento de unidad plurinacional Pachakutik-Ecuador, http://www.rls.org.br/publique/ media/PartAL_Garcia.pdf (21.3.2010).

- (o.J.): La igualación de los derechos ciudadanos y la vigencia de las diferencias étnicas y culturales en el Ecuador post-constitucional, http://lanic.utexas.edu/ project/etext/liilas/vrp/f_garcia.pdf (9.8.2013).

García, Fernando/Tuasa, Luis Alberto (2007): Estudio sobre legislación intercultural en el Ecuador, www.cebem.org/cmsfiles/archivos/derechos-ecuador.pdf (9.8. 2013).

Giebeler, Cornelia (2010): Conceptos de Inter-,Trans-, e Intraculturalidad en la Educación, in: Ströbele-Gregor, Juliana/Kaltmeier, Olaf/Giebeler, Cornelia (Hrsg.): Fortalecimiento de Organizaciones Indígenas en América Latina: Construyendo Interculturalidad: Pueblos Indígenas, Educación y Políticas de Identidad en América Latina, Eschborn: GTZ, S. 15-20.

González Casanova, Pablo (1969): Sociología de la explotación, Mexiko: Siglo XXI.

- (2006): Colonialismo interno [una redefinición], in: Boron, Atilio/ Amadeo, Javier/González, Sabrina (Hrsg.): La teoría marxista hoy. Problemas y perspectivas, Buenos Aires: CLACSO, S. 409-434.

Grijalva, Agustín (2008): El Estado Plurinacional e Intercultural en la Constitución Ecuatoriana del 2008, in: Ecuador Debate 78, S.49-62.

Grupo Internacionalista (2003): El marxismo y la cuestión indígena en el Ecuador, in: El Internacionalista, Juni 2003, http://www.internationalist.org/ecuador indigena.html (9.8.2013). 
Gnerre, Mauricio/Bottasso, Juan (1985): Del indigenismo a las organizaciones indígenas, in: AA.VV. (Hrsg.): Del indigenismo a las organizaciones indígenas, Quito: Colección Ethnos, S. 7-27.

GTZ (2002): Cooperación con pueblo indígenas en América Latina. Taller, 28 al 30 de abril del 2002, Boquete, Panamá, o.O.: GTZ.

- (2004): Erfahrungsauswertung der Zusammenarbeit mit indigenen Bevölkerungsgruppen und Organisationen in Bolivien, Ecuador und Guatemala, Band 1: Schlussbericht, o.O.: GTZ.

- (2004a): Estudio sobre la Cooperación Alemana con Nacionalidades y Organizaciones Indígenas en Bolivia, Ecuador y Guatemala, Tomo 4: Anexos del Estudio de Ecuador, o.O.: GTZ.

Guamán, Julián (2003): Indígenas evangélicos ecuatorianos. Evangelización, organización e ideología, Quito: FEINE.

- (2006): FEINE, la organización de los indígenas evangélicos en Ecuador, Quito: Abya-Yala, Corporación Editora Nacional.

Guatemal, Miguel (2006): La situación del movimiento indígena en Ecuador, in: Escárzaga, Fabiola/Gutiérrez, Raquel (Hrsg.): Ganamos pero perdimos. Elementos para un balance del movimiento indígena en el Ecuador, Mexiko: Centro de Estudios Andinos y Mesoamericanos, S. 8-24.

Gudynas, Eduardo (2011): Buen vivir: Germinando alternativas al desarrollo, in: América Latina en movimiento 462, S. 1-20.

Guerrero, Andrés (1983): Haciendas, capital y lucha de clases andina, Quito: El Conejo.

Guerrero, Fernando/Ospina, Pablo (2003): El poder de la comunidad. Ajuste estructural y movimiento indígena en los Andes ecuatorianos, CLACSO, http://biblioteca.clacso.edu.ar/subida/uploads/FTPtest/clacso/becas/2011012012 4937/guerrero.pdf (9.8.2013).

Guerrero, Rafael (2010): Discurso y Sujeto en los Movimientos Campesinos en la Costa del Ecuador, 1964-2009, Quito: IEE/FLACSO.

Hall, Stuart (1999): Kulturelle Identität und Globalisierung, in: Hörning, Karl; Winter, Rainer (Hrsg.): Widerspenstige Kulturen. Cultural Studies als Herausforderung, Frankfurt a. M.: Suhrkamp, S. 393-441.

Hanratty, Dennis (Hrsg.) (1989): Ecuador: A Country Study, Washington: GPO for the Library of Congress, http://countrystudies.us/ecuador/ (9.8.2013).

Hernández, Maribel (2009): Sumak kawsay y sumak qamaña, el reto de aprender del sur. Reflexiones en torno al Buen Vivir, in: Obets 4, S. 55-65.

Hidalgo Flor, Francisco (2005): Los movimientos indígenas y la lucha por la hegemonía: el caso de Ecuador, in: Dávalos, Pablo (Hrsg.): Pueblos Indígenas, Estado y democracia, Buenos Aires: CLACSO, S. 341-347.

Houtart, François (2011): El Concepto de Sumak Kawsai (Buen Vivir) y su correspondencia con el Bien Común de la Humanidad, in: Revista Espacio Crítico 15 , S. 2-23. 
Hoy (2001): Los orígenes del poder indígena, 17.2.2001, http://www.hoy.com.ec/ suplemen/blan123/byn.htm (9.8.2013).

Huanacuni, Fernando (2010): Buen Vivir / Vivir Bien. Filosofía, políticas, estrategias y experiencias regionales andinas, Lima: CAOI.

Huarcaya, Sergio (2010): Othering the Mestizo: Alterity and Indigenous Politics in Otavalo, Ecuador, in: Latin American and Caribbean Ethnic Studies 5 (3), S. 301-315.

- (2011): Othering National Identity Alterity and Indigenous Activism in Otavalo, Ecuador, PhD-Arbeit, http://deepblue.lib.umich.edu/bitstream/2027.42/ 84490/1/shuarcay_1.pdf (9.8.2013).

Ibarra Illanez, Alicia (1987): Los indígenas y el Estado en el Ecuador, Quito: AbyaYala.

- (1996): Los indios del Ecuador y su demanda frente al Estado, in: González Casanova, Pablo/Roitman, Marcos (Hrsg.): Democracia y Estado multiétnico en América Latina, Mexiko: Demos, S. 293-320.

Ibarra, Hernán (1999): Intelectuales indígenas, neoindigenismo e indianismo en el Ecuador, in: Ecuador Debate 48, S. 71-94.

- (2010): Refundar al Estado. Procesos Constituyentes en América Latina, Quito: Centro Andino de Acción Popular.

Instituto de Estudios Ecuatorianos (IEE) (o.J.): Mapeo, Actores y Poder. Anexos, http://www.iee.org.ec/investigaciones/MAPEO\%20actores\%20y\%20poder/acto respoder_anexo.pdf (9.8.2013).

Instituto Nacional de Estadística y Censos (INEC) (2010): Censo de Población y Vivienda 2010, http://redatam.inec.gob.ec/cgibin/RpWebEngine.exe/Portal Action?\&MODE=MAIN\&BASE=CPV2010\&MAIN=WebServerMain.inl $\quad(10$. 9.2011).

Iturralde, Diego (1983): Las relaciones Estado-Sectores Indígenas en la historia, in: Cuadernos de Nueva 7, S. 80-82.

- (1997): Demandas indígenas y reforma legal: retos y paradojas, in: Alteridades 7 (14), S. 81-98.

- (2000) [1998]: La CONAIE va más rápido que el Estado, in: Aguirre, Milagros (Hrsg.): Controversia Ecuador hoy: cien miradas, Quito: El Comercio, S. 168170.

Iza, Leonidas (2005): Ascenso y retos del movimiento indígena en Ecuador, in: Escárzaga, Fabiola/Gutiérrez, Raquel (Hrsg.): Movimiento Indígena en América Latina: Resistencia y Proyecto alternativo, Puebla: Benemérita Universidad Autónoma de Puebla, S. 108-118.

Jasper, James (2007): Cultural Approaches in the Sociology of Social Movements, in: Klandermans, Bert/Roggeband, Conny (Hrsg.): Handbook of Social Movements across Disciplines, New York: Springer, S. 59-109.

Jasper, James (2010): Social Movement Theory Today: Toward a Theory of Action? In: Sociology Compass 4 (11), S. 965-976. 
Jáuregui, Carlos (2008): Canibalia. Canibalismo, calibanismo, antropofagia cultural y consumo en América Latina, Madrid: Iberoamericana.

Jenkins, Craig/Perrow, Charles (1977): Insurgency of the powerless. Farm worker movements (1946-1972), in: American Sociological Review 42, S. 249-268.

Jenson, Jane (1995): What's in a Name? Nationalist Movements and Public Discourse, in: Johnston, Hank/Klandermans, Bert (Hrsg.): Social Movements and Culture, Minneapolis: University of Minnesota Press, S. 107-126.

Jijón, Víctor Hugo (2006): Pachakutik en la encrucijada. Movimientos sociales y proyecto político en el Ecuador, in: Entre voces/Renovación, Edición Especial Mai, S. 46-49.

Junge, Kay (2011): Self-Concepts, Counter-Concepts, Asymmetrical Counter-Concepts. Some Aspects of a Multi-Faceted Agenda, in: Junge, Kay/Postoutenko, Kirill (Hrsg.): Asymmetrical Concepts after Reinhart Koselleck. Historical Semantics and Beyond, Bielefeld: transcript, S. 9-49.

Kaller-Dietrich, Martina (2008): Ivan Illich (1926-2002). Sein Leben, sein Denken, Weitra: Bibliothek der Provinz.

Kaltmeier, Olaf (2009): Das Land neu gründen. Gesellschaftliche Kontexte, politische Kulturen und indigene Bewegungen in Südamerika, in: Mittag, Jürgen/Ismar, Georg (Hrsg.): ¿,El pueblo unido“? Soziale Bewegungen und politischer Protest in der Geschichte Lateinamerikas, Münster: Westfälisches Dampfboot, S. 339-363.

-(2010): Educación Intercultural y Políticas de identidad, in: Ströbele-Gregor, Juliana/Kaltmeier, Olaf/Giebeler, Cornelia (Hrsg.): Fortalecimiento de Organizaciones Indígenas en América Latina: Construyendo Interculturalidad: Pueblos Indígenas, Educación y Políticas de Identidad en América Latina, Eschborn: GTZ, S. 3-8.

Kollmeier, Kathrin (2012): Begriffsgeschichte und Historische Semantik, Version: 1.0, in: Docupedia-Zeitgeschichte, 2.12.2011, http://docupedia.de/docupedia/ images/f/f1/Begriffsgeschichte_und_Historische_Semantik_Version_2.0 _Kathrin_Kollmeier.pdf (9.8.2013).

Korovkin, Tanya (1999): Weak Weapons, Strong Weapons? Hidden Resisteance and Political Protest in Highland Ecuador, CERLAC Working Paper Series, Centre for Research on Latin America and the Caribbean.

- (2008): The indigenous movement and left-wing politics in Ecuador, Draft Paper, University of Waterloo.

Koselleck, Reinhart (1996): A Response to Comments on the Geschichtliche Grundbegriffe, in: Lehmann, Hartmut/Richter, Melvin (Hrsg.): The Meaning of Historical Terms and Concepts. New Studies on Begriffsgeschichte, Washington: German Historical Institute, S. 59-70.

Kowii, Ariruma (o.J.): Pawkar Raymi, la Fiesta del Florecimiento, http://www.ipanc.org/images/muestra_docu.php?archivoDonde=vAsp2YZaVF2 hD2lwu2e\%2FZUT9\%2BURDPinPU7\%2F5m9k97d7M6gbErp4JMPTBktZ4Er 
AJwVHT7se8Z5vm\%2BC23Fb5SRjQaeS4g4hKi\&archivoCodificado=0 (18.4. 2012).

Krainer, Anita (1996): Educación Bilingüe Intercultural en el Ecuador, Quito: Abya-Yala.

- (2010): La educación intercultural en Ecuador: logros, desafíos y situación actual, in: Ströbele-Gregor, Juliana/Kaltmeier, Olaf/Giebeler, Cornelia (Hrsg.): Fortalecimiento de Organizaciones Indígenas en América Latina: Construyendo Interculturalidad: Pueblos Indígenas, Educación y Políticas de Identidad en América Latina, Eschborn: GTZ, S. 38-44.

Kummels, Ingrid (2008): Von Zuania bis Abya Yala: Indigene Amerika-Bilder und -projekte, in: Lehmkuhl, Ursula/Rinke, Stefan (Hrsg.): Amerika? Amerikas! Zur Geschichte eines Namens und eines Konzepts, Stuttgart: Akademischer Verlag Hans-Dieter Heinz, S. 174-192.

Kymlicka, Will (2003): Multicultural states and intercultural citizens, in: Theory and Research in Education 1 (2), S. 147-169.

Lalander, Rickard/Gustafsson, Maria-Therese (o.J.): ¿Proceso social o actores políticos? Reflexiones sobre Pachakutik y CONAIE desde Imbabura, http://www. uasb.edu.ec/UserFiles/369/File/PDF/CentrodeReferencia/Temasdeanalisis2/poli ticaspublicasyderechoshumanos/articulos/actualidad/lalander-gustafsson(1).pdf (9.8.2013).

Landa, Ladislao (2006): Pensamientos indígenas en nuestra América, in: CLACSO (Hrsg.): Crítica y teoría en el pensamiento social latinoamericano, Buenos Aires: CLACSO, S. 11-75.

Larrea, Ana María (2008): La Plurinacionalidad: Iguales y Diversos en busca del ,Sumak Kawsay', in: Peñaranda, Raúl (Hrsg.): Democracia, Interculturalidad, Plurinacionalidad y Desafíos para la Integración Andina, La Paz: CEBEM, S. $35-44$.

Lazos, Elena/Lenz, Evelyne (2004): La Educación Indígena en el Páramo Zumbahueño del Ecuador: Demandas, Exitos y Fracasos de una Realidad, http://www.latautonomy.org/SEIC_18feb.pdf (9.8.2013).

Lechón, Miguel (1976): Federación Ecuatoriana de Indios, Filial de la C.T.E., in: Nueva Antropología. Revista de Ciencias Sociales 3, S. 85-93.

León Trujillo, Jorge (1983): Estado, nacionalidades y etnias, in: Cuadernos de Nueva 7 , S. 6-10.

- (1991): Las organizaciones indígenas: Igualdad y diferencia. La afirmación de los conquistados, in: Cornejo, Diego (Hrsg.): Indios. Una reflexión sobre el levantamiento indígena de 1990, Quito: ILDIS, El Duende, Abya-Yala, S. 373417.

- (1994): De campesinos a ciudadanos diferentes. El levantamiento indígena, Quito: CEDIME/Abya-Yala.

León Trujillo, Jorge (2010): Las organizaciones indígenas y el gobierno de Rafael Correa, in: Íconos. Revista de Ciencias Sociales 37, S. 13-23. 
León Trujillo, Jorge/Ycaza, Patricio (1989): La Historiografía Sindical en el Ecuador: Doctrinas y Acción Política, in: Zubillaga, Carlos (Hrsg.): Trabajadores y sindicatos en América Latina. Reflexiones sobre su historia, Montevideo: CLACSO, S. 175-197.

León, Juan (1994): La Sociedad Ecuatoriana, in: Grijalva, Agustín (Hrsg.): Datos Básicos de la Realidad Nacional, Quito: Cooperación Editora Nacional, S. 6179.

Leonhard, Jörn (2004): Grundbegriffe und Sattelzeiten - Languages and Discourses. Europäische und anglo-amerikanische Deutungen des Verhältnisses von Sprache und Geschichte, in: Habermas, Rebekka (Hrsg.): Interkultureller Transfer und nationaler Eigensinn: Europäische und anglo-amerikanische Positionen der Kulturwissenschaften, Göttingen: Wallstein, S. 71-86.

Le Quang, Matthieu (2010): La reestructuración de la izquierda en Ecuador. Del movimiento indígena hasta Rafael Correa, in: Gaudichaud, Franck (Hrsg.): El volcán latino-americano. Izquierdas, movimientos sociales y neoliberalismo al sur del Río Bravo. Balance de una década de luchas: 1999-2009, http:// alainet.org/images/El_volcan_latino_FG.pdf (9.8.2013), S. 111-120.

López, Luis Enrique (2009): Interculturalidad, educación y política en América Latina: perspectivas desde el Sur. Pistas para una investigación comprometida y dialogal, in: ders. (Hrsg.): Interculturalidad, educación y ciudadanía. Perspectivas latinoamericanas, La Paz: FUNPROEIB, Plural, S. 129-218.

Lucas, Kintto (2000): La Rebelión de los Indios, Quito: Abya-Yala.

Lucero, José Antonio (2003): Locating the ,Indian Problem‘: Community, Nationality, and Contradiction in Ecuadorian Indigenous Politics, Latin American Perspectives 30 (1), S. 23-48.

Lucero, José Antonio (2006): Representing , Real Indians‘. The Challenges of Indigenous Authenticity and Strategic Constructivism in Ecuador and Bolivia, in: Latin American Research Review 41 (2), S. 31-56.

- (2008): Indigenous Political Voice and the Struggle for Recognition in Ecuador and Bolivia, in: Bebbington, Anthony et al. (Hg.): Institutional pathways to equity: addressing inequality traps, Washington D.C.: World Bank, S. 139-167.

Macas, Luis (1983): ,Unidad con los grupos marginados', in: Cuadernos de Nueva 7, S. 53-56.

- (1991): El Levantamiento Indígena visto por sus protagonistas, Quito: Instituto Científico de Culturas Indígenas.

- (2005): La necesidad política de una reconstrucción epistémica de los saberes ancestrales, in: Dávalos, Pablo (Hrsg.): Pueblos Indígenas, Estado y democracia, Buenos Aires: CLACSO, S. 35-42.

- (2010): Sumak Kawsay: La vida en plenitud, in: ALAI 452, S. 14-16.

Macdonald, Theodore (1998): Indigenous Politics and ,Local Heritage“ in the 1990s: Shifting Concepts of Land Use, Land Tenure, and Self, Yale F\&ES Bulletin, S. 86-103. 
- (2002): Ecuador's Indian Movement: Pawn in a Short Game or Agent in State Reconfiguration, in: Maybury-Lewis, David (Hrsg.): The Politics of Ethnicity: Indigenous Peoples in Latin American States, Cambridge: Harvard University Press, S. 169-198.

Maldonado, Luis (2006): Pueblos y Nacionalidades Indígenas del Ecuador: De la reivindicación al protagonismo político, Ibarra: ESGOPP.

Marmon, Tangmar/Kramer, Andrea (2006): Ethnische Parteien der Andenländer: Von sozialen Bewegungen zu politischen Gestaltern, GIGA Focus 11.

Martínez Novo, Carmen (2009): Is the Cultural Project of the Indigenous Movement in Crisis? Some Ethnographic Remarks on the Ambiguities of Intercultural Bilingual Education in Ecuador, http://lasa.international.pitt.edu/members/ congress-papers/lasa2009/files/MartinezCarmen.pdf (9.8.2013).

- (2009a): La crisis del proyecto cultural del movimiento indígena, in: dies. (Hrsg.): Repensando los Movimientos Indígenas, Quito: FLACSO, Ministerio de Cultura del Ecuador, S. 173-196.

- (2010): The ,Citizen's Revolution' and the Indigenous Movement in Ecuador: Re-centering the Ecuadorian State at the Expense of Social Movements, Vortragstext SARR Conference 27.-29. Mai 2010, Quito, Ecuador, http://sarr. emory.edu/documents/Andes/MartinezNovo.pdf (9.8.2013).

Martínez-Torres, María Elena/Rosset, Peter (2010): La Vía Campesina: the birth and evolution of a transnational social movement, in: Journal of Peasant Studies, 37 (1), S. 149-175.

Martínez Valle, Luciano (2006): Las organizaciones de segundo grado como nuevas formas de organización de la población rural, in: Grammont, Hubert (Hrsg.): La construcción de la democracia en el campo latinoamericano, Buenos Aires: CLACSO, S. 107-132.

Maugé, René (2008): La experiencia del Frente Amplio de Izquierda (FADI) en Ecuador, in: Ramírez Gallegos, Franklin (Hrsg.): La innovación partidista de las izquierdas en América Latina, Quito: ILDIS-FES, S. 114-120.

Massal, Julie (2010): El proyecto político indígena ecuatoriano. Convergencia y divergencias con su entorno político, in: Colombia Internacional 71, S. 9-33.

Mato, Daniel (2010): Las iniciativas de los movimientos indígenas en educación superior: un aporte para la profundización de la democracia, in: Nueva Sociedad 227, S. 102-119.

Mayer, Stefan (2008): Participación política del movimiento indígena ecuatoriano: El Movimiento Pachakutik en el Gobierno de Lucio Gutiérrez, Diplomarbeit, Universität Wien, http://othes.univie.ac.at/1109/1/2008-09-01_0106264.pdf (9.8.2013).

Mayring, Philipp (2000): Qualitative Inhaltsanalyse, in: Forum Qualitative Sozialforschung 1 (2), Art. 20, http://nbn-resolving.de/urn:nbn:de:0114-fqs0002204 (9.8.2013). 
McCarthy, John/Zald, Mayer (1977): Resource Mobilization and Social Movements: A Partial Theory, in: American Journal of Sociology 82 (6), S. 12121241.

Medina, Javier (Hrsg.) (2001): Suma qamaña. La comprensión indígena de la Buena Vida, La Paz: GTZ.

- (2011): Suma qamaña, vivir bien y de vita beata. Una cartografía boliviana, http://lareciprocidad.blogspot.de/2011/01/suma-qamana-vivir-bien-y-de-vitabeata.html (8.8.2013).

Meléndez, Carlos (2007): Los nuevos escenarios políticos en los países andinos, in: Roncagliolo, Rafael/Meléndez, Carlos (Hrsg.): La política por dentro. Cambios y continuidades en las organizaciones políticas de los países andinos, Stockholm: IDEA, S. 303-326.

Melucci, Alberto/Avritzer, Leonardo (2000): Complexity, cultural pluralism and democracy: collective action in the public space, in: Social Science Information 39 (4), S. 507-527.

Meyer, David/Whittier, Nancy (1994): Social Movement Spillover, in: Social Problems 41 (2), S. 277-298.

Meyer, David/Staggenborg, Suzanne (1996): Movements, Countermovements, and the Structure of Political Opportunity, in: The American Journal of Sociology 101 (6), S. 1628-1660.

Mignolo, Walter (2000): La colonialidad a lo largo y a lo ancho: el hemisferio occidental en el horizonte colonial de la modernidad, in: Lander, Edgardo (Hrsg.): La colonialidad del saber: eurocentrismo y ciencias sociales. Perspectivas latinoamericanas, Buenos Aires: CLACSO, S. 55-85.

- (2005): El Pensamiento Des-Colonial, Desprendimiento y Apertura: Un Manifiesto, in: Tristestópicos, http://www.tristestopicos.org/walter\%20mignolo_ descolonial_tristestopicos.pdf (2.6.2012).

- (2006): The de-colonial option and the meaning of identity in politics, in: Magalhães Neto, Hamilton (Hrsg.): Desarrollo e Interculturalidad, Imaginario y Diferencia: la Nación en el Mundo Andino, Rio de Janeiro: Academia de la Latinidad, S. 119-156.

- (2009): La colonialidad: la cara oculta de la modernidad, in: Breitwieser, Sabine/Klinger, Cornelia/Mignolo, Walter (Hrsg.): Modernologías. Artistas contemporáneos investigan la modernidad y el modernismo, Barcelona: MACBA, S. 39-48.

Minkner-Brünjer, Mechthild (2009): Zwischen Erfolgen und Ausbootung. Soziale Bewegungen in Ecuador, in: Mittag, Jürgen/Ismar, Georg (Hrsg.): ¿,El pueblo unido'? Soziale Bewegungen und politischer Protest in der Geschichte Lateinamerikas, Münster: Westfälisches Dampfboot, S. 133-165.

Moreno Yánez, Segundo (1997): Lectura para Asambleistas, Diario HOY 24.9.1997, S. 9, http://www.explored.com.ec/noticias-ecuador/lectura-paraasambleistas-109446-109446.html (9.8.2013). 
- (2010): Casas-territorios, Diario Hoy, 29.9.2010, http://www.hoy.com.ec/ noticias-ecuador/casas-territorios-432581.html (9.8.2013).

Moreno Yánez, Segundo/Figueroa, José (1992): El Levantamieno Indígena del Inti Raymi de 1990, Quito: FESO, Abya-Yala.

Montaluisa, Luis (2003): Participación Comunitaria en la Educación Intercultural Bilingüe del Ecuador, in: World Learning (Hrsg.): La Educación Indígena en las Américas, SIT Occasional Papers 1, http://digitalcollections.sit.edu/sop/1 (9.8.2013), S. 85-92.

- (2008): Interculturalidad y empoderamiento, in: UNICEF (Hrsg.): Interculturalidad: reflexiones desde la práctica, Quito: UNICEF, S. 99-104.

Mosonyi, Esteban/González, Omar (1975): Ensayo de educación intercultural en la zona arahuaca del Río Negro (Venezuela), in: XXXIX Congreso Internacional de Americanistas (Hrsg.): Lingüística e indigenismo moderno de Amércia. Trabajos presentados al XXXIX Congreso Internacional de Americanistas, Vol. 5, Lima: Instituto de Estudios Peruanos, S. 307-314.

Moya, Ruth (1987): Educación bilingüe en el Ecuador: retos y alternativas, in: Indiana 11, S. 387-406.

MUPP-NP (2006): Plan de Gobierno Democrático del Estado Plurinacional, http://www.llacta.org/organiz/pachakutik/plan_de_gobierno_2006.pdf (10.9.2013).

Negreiros, Janaina (2009): La FENACLE y la organización de los asalariados rurales en la provincia del Guayas, in: Ecuador Debate 78, S. 125-140.

Noboa, Particio (2005): La matriz colonial, los movimientos sociales y los silencios de la modernidad, in: Walsh, Catherine (Hrsg.): Pensamiento crítico y matriz (de)colonial. Reflexiones latinoamericanas, Quito: Abya-Yala, S. 71-109.

Oficina Nacional de Asuntos Indígenas (Hrsg.) (1984): Población Indígena y desarrollo amazónico, Quito: Abya-Yala.

Ojeda Segovia, Lautaro (2004): Análisis politológico del proceso de construcción de la autonomía multicultural en el Ecuador, in: LATAUTONOMY Politological Studies, http://www.latautonomy.org/EstudioPolitico_Ecuador.pdf (9.8.2013).

Ortiz, Pablo (2010): Entre la cooptación y la ruptura: la lucha por el derecho a la autodeterminación de las nacionalidades indígenas del centro sur amazónico del Ecuador, in: González, Miguel/Burguete, Araceli/Ortiz, Pablo (Hrsg.): La autonomía a debate. Autogobierno indígena y Estado plurinacional en América Latina, Quito: FLACSO/GTZ/IWGIA/CIESAS/UNICH, S. 455-508.

Ortiz Crespo, Santiago (2009): Ciudadanía étnica y Desigualdad en Otavalo y Cotacachi, Ecuador, Observatory on Structures and Institutions of Inequality in Latin America, University of Miami, Working Paper Series \#20.

Ortiz Fernández, Carolina (2009): Felipe Guaman Poma de Ayala, Clorinda Matto de Turner, Trinidad Henríquez y la teoría crítica - Sus legados a la teoría social 
contemporánea, in: Yuyaykusun. Revista del Departamento Académico de Humanidades de la Universidad Ricardo Palma 2, S. 263-284.

Ospina, Pablo (2006): Movimiento indígena ecuatoriano, gobierno territorial local y desarrollo económico: los casos del Gobierno Municipal de Cotacachi y el Gobierno Provincial de Cotopaxi, in: ders. (Hrsg.): En las fisuras del poder. Movimiento indígena, cambio social y gobiernos locales, Quito: Instituto de Estudios Ecuatorianos, S. 15-118.

- (2006a): Der Plurinationale Staat und territoriale Selbstverwaltung. Indigene Forderungen in Ecuador, in: Peripherie 108, S. 456-468.

- (2008): La deriva de una promesa. Movimientos sociales, democracia y neoliberalismo, in: Andrade, María/Herrera, Gonzalo/Ospina, Pablo: Mapa de movimientos sociales en el Ecuador. Informe final, Instituto de Estudios Ecuatorianos,

http://www.iee.org.ec/investigaciones/MOVIMIENTOS\%20SOCIALES/movi mientosSOCIALES.pdf (9.8.2013), S. 66-86.

- (2009): ,Nos vino un huracán político“: la crisis de la CONAIE, in: Ospina, Pablo/Kaltmeier, Olaf/Büschges, Christian (Hrsg.): Los Andes en movimiento. Identidad y poder en el nuevo paisaje político, Quito: Corporación Editora Nacional/Universidad Andina Simón Bolívar, S. 123-146.

- (2010): Estado plurinacional y autogobierno territorial. Demandas indígenas en Ecuador, in: González, Miguel/Burguete, Araceli/Ortiz, Pablo (Hrsg.): La autonomía a debate. Autogobierno indígena y Estado plurinacional en América Latina, Quito: FLACSO/GTZ/IWGIA/CIESAS/UNICH, S. 201-218.

Pacari, Nina (1984): Las culturas nacionales en el estado multinacional ecuatoriano, in: Cultura. Revista del Banco Central del Ecuador VI (18a), S. 113-123.

- (2007): Todo puede ocurrir, Mexiko: UNAM.

Pajuelo, Ramón (2003): Fronteras, representaciones y movimientos étnicos en los países centroandinos en tiempos de globalización, in: Daniel Mato (Hrsg.): Políticas de identidades y diferencias sociales en tiempos de globalización, Caracas: FaCES, Universidad Central de Venezuela, S. 283-302.

- (2004): Identidades en Movimiento. Tiempos de globalización, procesos sociopolíticos y movimiento indígena en los países centro andinos, Caracas: Programa Globalización, Cultura y Transformaciones Sociales, CIPOST, FaCES, Universidad Central de Venezuela.

Palacios, Paulina/Chuma, Vicenta (2001): El Sistema de Formación de Mujeres Líderes Indígenas ,Dolores Cacuango“. La construcción de una utopia, Boletín ICCI RIMAY 3 (28), http://www.icci.nativeweb.org/boletin/28/palacios.html (9.8.2013).

Paz y Miño, Juan (2009): Ecuador en la Globalización: 1975-2005, in: Historia Actual Online 18, S. 25-39.

Perreault, Thomas/Bebbington, Anthony/Carroll, Thomas (1998): Indigenous Irrigation Organizations and the Formation of Social Capital in Northern Highland 
Ecuador, in: Keeling, David/Wiley, James (Hrsg.): Conference of Latin Americanist Geographers Yearbook, S. 1-15.

Petras, James/Veltmeyer, Henry (2005): Movimientos sociales y poder estatal. Argentina, Brasil, Bolivia, Ecuador, Mexiko: Lumen.

Polletta, Francesca (1997): Culture and Its Discontents: Recent Theorizing on the Cultural Dimensions of Protest, in: Sociological Inquiry 67 (4), S. 431-450.

- (1999): Snarls, Quacks, and Quarrels: Culture and Structure in Political Process Theory, in: Sociological Forum 14 (1), S. 63-70.

- (1999a): ,Free spaces" in collective action, in: Theory and Society 28, S. 1-38.

- (2008): Culture and Movements, in: The ANNALS of the American Academy of Political and Social Science 619, S. 78-96.

Polletta, Francesca/Jasper, James (2001): Collective Identity and Social Movements, in: Annual Reviews Sociol. 27, S. 283-305.

Porras, Angélica (2005): Tiempo de Indios. La construcción de la identidad política colectiva del movimiento indio ecuatoriano (Las movilizaciones de 1990, 1993 y 1997), Quito: Abya-Yala.

Portocarrera, Gonzalo (2003): Comentarios, in: Fuller, Norma (Hrsg.): Interculturalidad y política: desafíos y posibilidades, Lima: Red para el Desarrollo de las Ciencias Sociales en el Perú, S. 201-205.

Portugal, Pedro (2008): Constantino Lima: Memorias de un luchador indianista, in: Pukara 32.

Quijano, Aníbal (2000): Colonialidad del Poder, Globalización y Democracia, Lima: o. V., http://www.rrojasdatabank.info/pfpc/quijan02.pdf (9.8.2013).

- (2000a): Colonialidad del poder, eurocentrismo y América Latina, in: Lander, Edgardo (Hrsg.): La colonialidad del saber: eurocentrismo y ciencias sociales. Perspectivas latinoamericanas, Buenos Aires: CLACSO, S. 201-246.

- (2000b): Colonialidad del Poder y Clasificacion Social, in: Journal of worldsystems research, VI-2, Special Issue: Festschrift for Immanuel Wallerstein Part I, S. 342-386.

— (2004): El laberinto de América Latina: ¿hay otras salidas?, in: OSAL 13, S. 1530.

- (2005): Don Quijote y los molinos de viento en América Latina, http://www.archivochile.com/Ideas_Autores/quijanoa/quijanoa00003.pdf (9.8.2013).

- (2006): Estado-nación y ,movimientos indígenas‘ en la región Andina: cuestiones abiertas, OSAL 19, S. 15-24.

- (2006a): El ,Movimiento Indígena‘ y las cuestiones pendientes en América Latina, in: San Marcos 24, S. 13-42.

- (2010): ,Bien Vivir“ para redistribuir el poder. Los pueblos indígenas y su propuesta alternativa en tiempos de dominación global, in: Oxfam (Hrsg.): Pobreza, Desigualdad y Desarrollo en el Perú. Informe Anual 2009-2010, Lima: Oxfam, S. 112-121. 
Quintero, Pablo (2010): Notas sobre la teoría de la Colonialidad del Poder y la Estructuración de la sociedad en América Latina, Papeles de Trabajo 19, o.O.: Centro de Estudios Interdisciplinarios en Etnolingüística y Antropología SocioCultural.

Radcliffe, Sarah (2002): Indigenous Women, Rights and the Nation-State in the Andes, in: Molyneux, Maxine/Craske, Nikki (Hrsg.): Gender and the Politics of Rights and Democracy in Latin America, New York: Palgrave, S. 149-172.

Ramírez, René (2010): Socialismo y sumak kawsay, in: SENPLADES (Hrsg.): Los nuevos retos de América Latina: Socialismo y Sumak Kawsay, Quito: SENPLADES, S. 5-6.

- (2010a): Socialismo del sumak kawsay o biosocialismo republicano, in: SENPLADES (Hrsg.): Los nuevos retos de América Latina: Socialismo y Sumak Kawsay, Quito: SENPLADES, S. 55-76.

Ramírez Gallegos, Franklin (2009): El movimiento indígena y la reconstrucción de la izquierda en Ecuador: el caso del Movimiento de Unidad Plurinacional Pachakutik-Nuevo País, in: Ospina, Pablo/Kaltmeier, Olaf/Büschges, Christian (Hrsg.): Los Andes en movimiento. Identidad y poder en el nuevo paisaje político, Quito: Corporación Editora Nacional/Universidad Andina Simón Bolívar, S. 65-94.

- (2010): Desencuentros, convergencias, polarización (y viceversa). El gobierno ecuatoriano y los movimientos sociales, in: Nueva Sociedad 227, S. 83-101.

Ramón, Galo (1983): Supervivencia y ritualidad en la reivindicación indígena de la tierra, in: Cuadernos de Nueva 7, S. 100-101.

- (Hrsg.) (1992): Actores de una década ganada: Tribus, Comunidades y Campesinos en la Modernidad, Quito: COMUNIDEC.

- (1992a): Estado Plurinacional: una propuesta innovadora atrapada en viejos conceptos, in: Corporación Editora Nacional (Hrsg.): Pueblos Indios, estado y derecho, Quito: Corporación Editora Nacional, Abya-Yala, ILDIS, CORPEA, Taller Cultural Causanacunchic, S. 9-24.

- (1998): Nuevos avances en la propuesta del país plurinacional, in: Agencia Latinoamericana de Información: Autonomías Indígenas - Diversidad de Culturas, Igualdad de Derechos, Aportes para el Debate 6, http://www.alainet.org/ active/974 (9.8.2013).

- (2001): El índice de capacidad Institucional de las OSGs en el Ecuador, in: Bebbington, Anthony/Torres, Víctor (Hrsg.): Capital Social en los Andes, Quito: Abya-Yala, S. 39-68.

- (2009): ¿Plurinacionalidad o interculturalidad en la Constitución?, in: Acosta, Alberto/Martínez, Esperanza (Hrsg.): Plurinacionalidad. Democracia en la Diversidad, Quito: Abya-Yala, S. 125-160.

Raschke, Joachim (1985): Soziale Bewegungen: ein historisch-systematischer Grundriss, Frankfurt a.M./New York: Campus. 
Resina de la Fuente, Jorge (2011): Participación y percepción de la CONAIE en el Ecuador plurinacional, in: Ciências Sociais Unisinos 47 (2), S. 108-115.

Rhon Dávila, Francisco (2003): Estado y movimientos étnicos en Ecuador, in: Seoane, José (Hrsg.): Movimientos sociales y conflicto en América Latina, Buenos Aires: CLACSO, S. 127-140.

Richter, Melvin (1997): Appreciating a Contemporary Classic. The Geschichtliche Grundbegriffe and Future Scholarship, in: Finnish Yearbook of Political Thought 1, S. 25-38.

- (2005): More than a two-way traffic: Analyzing, Translating, and Comparing Political Concepts from other cultures, in: Redescriptions. Yearbook of Political Thought and Conceptual History 9, S. 217-228.

Rodríguez, Nemesio (1982): La fragmentación lingüística. Prolongación de la fragmentación colonial, in: Rojas, Francisco (Hrsg.): América Latina. Etnodesarrollo y etnocidio, San José: FLACSO, S. 185-205.

Rodríguez, Orlando/Martínez, Maria José (2000): Neoindigenismo, interculturalidad y desarrollo local, in: Fernández Salvador, Consuelo (Hrsg.): Diálogo Intercultural. Memorias del Primer Congreso Latinoamericano de Antropología Aplicada, Quito-Ecuador 25 al 29 de enero de 1999, Quito: Abya-Yala, S. 7188.

Rodríguez Chávez, Héctor (2008): Gobernanza local en Ecuador, un proceso visto desde la comunidad, Masterarbeit, Mexiko: FLACSO.

Rohr, Elisabeth (2006): Politisierungstendenzen des religiösen Fundamentalismus in Lateinamerika. Das Beispiel Guatemala, Brasilien und Ecuador, Vortrag in Arnoldshain am 19. November 2006, http://www.evangelische-akademie. de/admin/projects/akademie/pdf/material/061131_80.pdf (16.11.2011).

Ruiz, Lucy (1991): Pueblos indígenas y etnicidad en la Amazonía, in: Cornejo, Diego (Hrsg.): Indios. Una reflexión sobre el levantamiento indígena de 1990, Quito: ILDIS, El Duende, Abya-Yala, S. 449-497.

Ruiz, Jorge (2009): Sociological Discourse Analysis: Methods and Logic, in: Forum Qualitative Sozialforschung 10 (2), Art. 26.

Rusterholz, Peter (1998): Poststrukturalistische Semiotik, in: Posner, Roland/Robering, Klaus/Sebeok, Thomas (Hrsg.): Semiotik. Ein Handbuch zu den zeichentheoretischen Grundlagen von Natur und Kultur, Berlin/New York: de Gruyter, S. 2329-2337.

Salamea, Marco (2006): Movimientos sociales e izquierda en el Ecuador, in: Entre voces/Renovación, Edición Especial, S. 52-56.

Salazar, Ernesto (1981): La Federación Shuar y la frontera de la colonización, in: Whitten, Norman (Hrsg.): Amazonía Ecuatoriana. La otra cara del progreso, o.O.: Mundo Shuar, S. 59-82.

Saltos, Napoleón (2000): La rebelión del arcoiris: Testimonios y análisis, Quito: Fundación José Peralta. 
Saltos, Napoleón/Vázquez, Lola (2005): Ecuador: su realidad 2005-2006, Quito: Fundación José Peralta.

Saltos, Napoleón/Vázquez, Lola (2007): Ecuador: su realidad 2007-2008, Quito: Fundación José Peralta.

Sánchez López, Francisco/Freidenberg, Flavia (1998): El proceso de incorporación política de los sectores indígenas en el Ecuador. Pachakutik, un caso de estudio, in: América Latina Hoy 19, S. 65-79.

Sánchez-Parga, José (1988): Actores y discursos culturales. Ecuador: 1972-88, Quito: CAAP.

- (1992): Comunidad indígena y estado nacional, in: Corporación Editora Nacional (Hrsg.): Pueblos Indios, estado y derecho, Quito: Corporación Editora Nacional, Abya-Yala, ILDIS, CORPEA, Taller Cultural Causanacunchic, S. 6178.

- (1993): Estado Nacional y Nacionalidades Indígenas, in: Vintimilla, María (Hrsg.): 500 años: Historia, Actualidad y Perspectiva, Cuenca: Universidad de Cuenca, S. 173-183.

- (1997): Identidad nacional, cultura y globalización, in: ILDIS: Identidad nacional y globalización, ILDIS: Quito, S. 95-123.

- (2007): El movimiento indígena ecuatoriano, Quito: Centro Andino de Acción Popular.

- (2009): Qué significa ser indígena para el indígena, Quito: Abya-Yala.

- (2010): El movimiento indígena ecuatoriano, Quito: Abya-Yala.

- (2010a): El movimiento indígena ecuatoriano. La larga ruta de la comunidad al partido, Quito: Abya-Yala.

Santana, Roberto (1983): Campesinado indígena y el desafío de la modernidad, Quito: CAAP.

- (1992): Les Indiens d'Equateur, citoyens dans l'ethnicité?, Paris: CNRS.

Santillana, Alejandra (2006): Proceso organizativo y límites del proyecto político de Pachakutik, in: Ospina, Pablo (Hrsg.): En las fisuras del poder. Movimiento indígena, cambio social y gobiernos locales, Quito: Instituto de Estudios Ecuadorianos, S. 215-265.

Santillana, Alejandra/Herrera, Stalin (2009): Génesis, experiencia, transformación y crisis del Movimiento Indígena Ecuatoriano, in: Coscione, Marco (Hrsg.): América Latina desde abajo. Experiencias de luchas cotidianas, Quito: Abya-Yala, S. 330-352.

Sarango, Luis Fernando (2009): Universidad Intercultural de las Nacionalidades y Pueblos Indígenas ,Amawtay Wasi‘, Ecuador/Chinchaysuyu, in: Mato, Daniel (Hrsg.): Instituciones Interculturales de Educación Superior en América Latina. Procesos de construcción, logros, innovaciones y desafíos, Caracas: UNESCOIESALC, S. 191-214.

Sarayaku (Territorio Autónomo de la Nación Originaria del Pueblo Kichwa de Sarayaku) (2003): Boletín de Prensa. Sarayacu propone un acuerdo integral 
sobre autodeterminación y manejo de sus territorios/“Sarayaku Sumak Kawsayta Nawpakma Katina Killka"/ "El libro de la vida de Sarayaku para defender nuestro futuro", http://148.206.53.231/especiales/231026//html/Documents/ sarayaku.pdf (8.8.2013).

Schilling-Vacaflor, Almut (2010): Die indigenen Völker Lateinamerikas: Zwischen zunehmender Selbstbestimmung und anhaltender Marginalisierung, GIGAFocus Lateinamerika 8.

Selverston, Melina (1992): Politicized Ethnicity and the Nation-State in Ecuador, Draft Presented at LASA Congress 1992.

- (1994): The Politics of Culture: Indigenous Peoples and the State in Ecuador, in: van Cott, Donna Lee (Hrsg.): Indigenous Peoples and democracy in Latin America, New York: St. Martin's Press, S. 131-152.

Simbaña, Floresmilo (2005): Plurinacionalidad y derechos colectivos. El caso ecuatoriano, in: Dávalos, Pablo (Hrsg.): Pueblos Indígenas, Estado y democracia, Buenos Aires: CLACSO, S. 197-215.

- (2006): Derechos territoriales y pluriculturalidad, Quito: Fundación Kawsay/ ECUARUNARI.

- (2007): El movimiento indígena y el actual proceso de transición, in: América Latina en movimiento 423, S. 21-24.

- (2008): La Plurinacionalidad en la nueva Constitución, in: ILDIS (Hrsg.): Análisis Nueva Constitución, Quito: ILDIS, S. 102-117.

- (2008a): Los derechos culturales y colectivos en la nueva constitución de Ecuador, in: Peñaranda, Raúl (Hrsg.): Seminario Regional Andino: Democracia, Interculturalidad, Plurinacionalidad y Desafíos para la Integración Andina, La Paz: CEBEM, S. 237-242.

- (2009): El movimiento indígena ecuatoriano y la construcción de la plurinacionalidad, in: Hoetmer, Raphael (Hrsg.): Repensar la política desde América Latina. Cultura, Estado y movimientos sociales, Lima: Programa Democracia y Transformación Global/Fondo Editorial de la Facultad de Ciencias Sociales, S. 153-167.

- (2010): Las relaciones entre el movimiento indígena y el gobierno, in: La Tendencia 10, S. 43-47.

Spedding, Alison (2010): 'Suma qamaña' ¿kamsañ muni? (¿Qué quiere decir 'vivir bien'?), http://de.scribd.com/doc/114500901/SPEDDING-Alison-Sumaqamana-Kamsan-muni-Que-quiere-decir-vivir-bien (8.8.2013).

Stavenhagen, Rodolfo (1997): Indigene Völker: Neue Akteure in Lateinamerika, in: von Gleich, Utta (Hrsg.): Indigene Völker in Lateinamerika: Konfliktfaktor oder Entwicklungspotential?, Frankfurt a.M.: Vervuert, S. 15-33.

Steinmetz, Willibald (2008): Vierzig Jahre Begriffsgeschichte - The State of the Art, in: Kämper, Heidrun/Eichinger, Ludwig (Hrsg.): Sprache - Kognition Kultur. Sprache zwischen mentaler Struktur und kultureller Prägung, Berlin/New York: Walter de Gruyter, S. 174-197. 
Swindler, Ann (1995): Cultural Power and Social Movements, in: Johnston, Hank/Klandermans, Bert (Hrsg.): Social Movements and Culture, Minneapolis: University of Minneapolis, S. 25-40.

Tamagno, Liliana (2006): Interculturalidad. Una revisión desde y con los pueblos indígenas, in: Diario del Campo (Hrsg.): Diversidad y Reconocimiento. Aproximaciones al Multiculturalismo y la Interculturalidad en América Latina, Suplemento 39, S. 20-31.

Tamayo, Eduardo (1996): Movimientos sociales. La Riqueza de la Diversidad, Quito: ALAI.

- (2007): Movimiento indígena: actor social y político determinante. Entrevista con el dirigente indígena Humberto Cholango, in: América Latina en Movimiento 418, S. 7-15.

Tenesaca, Delfín (2010): Pueblos indígenas: exclusión histórica, aportes civilizatorios y nuevo contexto constitucional, in: Lang, Miriam/Santillana, Alejandra (Hrsg.): Democracia, Participación y Socialismo. Bolivia, Ecuador, Venezuela, Quito: Rosa-Luxemburg-Stiftung, S. 107-110.

Tibán, Lourdes/García, Fernando (2006): Entre la oposición y el enfrentamiento al diálogo y las alianzas: la experiencia de la CONAIE y el MICC en Ecuador, http://www.flacsoandes.org/web/imagesFTP/1242842398.Entre_la_Oposicion.p df (9.8.2013).

Tilly, Charles (2001): Mechanisms in Political Processes, in: Annual Review of Political Sciences 4, S. 21-41.

Torres, Edelberto (1972): Reflexiones en torno a una interpretación histórico-social de Guatemala, in: Revista Mexicana de Sociología 34 (1), S. 115-131.

Trujillo, Julio (1992): El Estado Plurinacional en Ecuador: las reformas constitucionales, in: Corporación Editora Nacional (Hrsg.): Pueblos Indios, estado y derecho, Quito: Corporación Editora Nacional, Abya-Yala, ILDIS, CORPEA, Taller Cultural Causanacunchic, S. 167-179.

- (2009): El Ecuador como Estado Plurinacional, in: Acosta, Alberto/Martínez, Esperanza (Hrsg.): Plurinacionalidad. Democracia en la Diversidad, Quito: Abya-Yala, S. 63-79.

UASB (о.J.): Diccionario de nombres kichwas, http://www.uasb.edu.ec/index_ publicacion.php?cd=345 (9.8.2013).

UIAW (Universidad Intercultural Amawtay Wasi) (2004): Aprender en la Sabiduría y el Buen Vivir, Quito: UNESCO.

UNAE (o.J.) [ca. 1986]: Problemática indígena y colonización en el oriente ecuatoriano. Reflexiones nacidas al interior de la U.N.A.E. (Unión de Nativos de la Amazonía Ecuatoriana), o.O.: o.V.

Uzeda, Andrés (o.J.): Suma Qamaña, visiones indígenas y desarrollo, http://www.bibliocomunidad.com/web/libros/Suma\%20Qama\%F1a,\%20visione s\%20ind\%EDgenas\%20y\%20desarrollo.pdf (8.8.2013). 
Van Cott, Donna Lee (2005): From movements to parties. The evolution of ethnic parties, New York: Cambridge University Press.

Vargas, Edwar (2009): La Plurinacionalidad. Un paradigma de transformación social, in: Acosta, Alberto/Martínez, Esperanza (Hrsg.): Plurinacionalidad. Democracia en la Diversidad, Quito: Abya-Yala, S. 99-105.

Verdesoto, Luis (1986): Los movimientos sociales, la crisis y la democracia en el Ecuador, in: CLACSO/ILDIS (Hrsg.): Movimientos sociales en el Ecuador, Buenos Aires/Quito: CLACSO, ILDIS, CAAP, CEDIME, IEE, CEPLAES, CIUDAD, S. 13-61.

Villavicencio, Gladys (1973): Relaciones interétnicas en Otavalo ¿Una nacionalidad india en formación?, Mexiko: Instituto Indigenista Interamericano.

Viteri, Alfredo (1983): Los pueblos de la Amazonía se unen, in: Cuadernos de Nueva 7, S. 44-47.

Viteri, Carlos (1997): Ecuador: un país ficticio. El nuestro es un país al que le cuesta asumir y manejar sus diversidades, in: Íconos 2, S. 51-58.

- (1998): Nuevos retos del movimiento indígena amazónico, in: Restrepo, Marco (Hrsg.): III Jornadas Internacionales Amazónicos. Construyendo el futuro desde la experiencia, o.O.: CEDIME, UNICEF, S. 92-98.

- (1999): La Paz: Una rectificación de equívocos, Íconos 6, S. 36-44.

- (2002): Visión indígena del desarrollo en la Amazonía, in: Polis, Revista de la Universidad Bolivariana 1 (3), S. 1-6, y http://polis.revues.org/7678 (9.8.2013).

Vogel, Sara (2008): Los Alcanzes y Límites de Participación Ciudadana: la Conaie y la Plurinacionalidad en la Asamblea Constituyente de 2008, ISP Collection. Paper 33, http://digitalcollections.sit.edu/isp_collection/33 (9.8.2013).

Walsh, Catherine (2000): Políticas y Significados Conflictivos, in: Estudios Interculturales 2, S. 9-24.

- (2003): (De)Construir la interculturalidad. Consideraciones críticas desde la política, la colonialidad y los movimientos indígenas y negros en el Ecuador, in: Fuller, Norma (Hrsg.): Interculturalidad y política: desafíos y posibilidades, Lima: Red para el Desarrollo de las Ciencias Sociales en el Perú, S. 115-142.

- (2005): (Re)pensamiento crítico y (de)colonialidad, in: dies. (Hrsg.): Pensamiento crítico y matriz (de)colonial. Reflexiones latinoamericanas, Quito: AbyaYala, S. 13-35.

- (2008): Interculturalidad y Plurinacionalidad: Elementos para el debate constituyente, Quito: Universidad Andina Simón Bolívar.

- (2009): Estado Plurinacional e intercultural. Complementaridad y complicidad hacia el ,Buen Vivir‘, in: Acosta, Alberto/Martínez, Esperanza (Hrsg.): Plurinacionalidad. Democracia en la Diversidad, Quito: Abya-Yala, S. 161-184.

- (2009a): The Plurinational and Intercultural State: Decolonization and State Refounding in Ecuador, in: Kult 6 - Special Issue: Epistemologies of Transformation: The Latin American Decolonial Option and its Ramifications, S. 65-84. 
Walsh, Catherine (2009b): Estado e interculturalidad: reflexiones críticas desde la coyuntura andina, in: Ospina, Pablo/Kaltmeier, Olaf/Büschges, Christian (Hrsg.): Los Andes en movimiento. Identidad y poder en el nuevo paisaje político, Quito: Corporación Editora Nacional/Universidad Andina Simón Bolívar, S. 217-248.

- (2010): Development as Buen Vivir: Institutional arrangements and (de)colonial entanglements, in: Development 53, 1, S. 15-21.

Wolff, Jonas (2008): Buscando respuestas a la doble transformación: El movimiento indígena ecuatoriano, in: de la Fontaine, Dana/Aparicio, Pablo (Hrsg.): Diversidad cultural y Desigualdad social en América Latina y el Caribe: Desafíos de la integración global, o.O.: Heinrich Böll Stiftung, S. 113-129.

- (2010): Elitenwandel in Ecuador Soziopolitische Akteure und politische Perspektiven, http://library.fes.de/pdf-files/iez/07116.pdf (9.8.2013).

Ycaza, Patricio (1991): Historia del movimiento obrero ecuatoriano. De la influencia de la tactica del frente popular a las luchas del FUT, Quito: CEDIME.

Yépez, Julián (2010): Antecedentes: las Luchas de Nuestro Partido, http://jcepichincha.blogspot.com/2010/04/antecedentes-de-las-luchas-denuestro.html (10.9.2011).

Yumbay, Mariana (2008): Educación intercultural bilingüe en el Ecuador: Logros y limitaciones, in: Fondo Indígena (Hrsg.): Hacia el buen vivir: experiencias de gestión indígena en Centro América, Colombia, Costa Rica, Ecuador y Guatemala, La Paz: Fondo Indígena, S. 229-285.

Zald, Mayer (1979): Macro Issues in the Theory of social movements. SMO Interaction, the Role of Counter-Movements and Cross-National Determinants of the Social Movement Sector, CRSO Working Paper 204, Center for Research on Social Organization, University of Michigan.

Zald, Mayer/Ash, Roberta (1966): Social Movement Organizations: Growth, Decay and Change, in: Social Forces 44 (3), S. 327-341.

Zald, Mayer/McCarthy, John (1979): Social Movement Industries: Competition and Cooperation among Movement Organizations, CRSO Working Paper 201, Center for Research on Social Organization, University of Michigan.

Zallez, Jaime/Gortaire, Alfonso (1978): Organizarse o sucumbir... La Federación Shuar, Sucúa: Mundo Shuar.

Zamosc, Leon (1994): Agrarian Protest and the Indian Movement in the Ecuadorian Highlands, Latin American Research Review 29 (3), S. 37-68.

- (2005): El Movimiento Indígena Ecuatoriano: de la Política de la Influencia a la Política del Poder, in: Postero, Nancy/Zamosc, Leon (Hrsg.): La lucha por los derechos indígenas en América Latina, Quito: Abya-Yala, S. 193-228.

Zapata, Claudia (2007): Desplazamientos teóricos y proyectos políticos en la emergente historiografía mapuche y aymara, in: Estudios de Filosofía Práctica e Historia de las Ideas. Revista anual de la Unidad de Historiografía e Historia de las Ideas 8 (9), S. 169-180. 
Zubritski, Yuri (1979) [1975]: Los Incas-Quechuas, Übersetzung von Vidal Vidal Villanueva, Moskau: Editorial Progreso.

- (1987): Las funciones sociales de la lengua quechua en el area OtavaloCotacachi, in: Instituto Otavaleño de Antropología (Hrsg.): Ecuador indígena. Simbolismo y Cotidianidad, Quito: Abya-Yala, S. 73-95. 


\section{Sonstige Quellen}

Alberto Acosta, Wissenschaftler an der FLACSO Ecuador: Persönliche Kommunikation (Gruppendiskussion im Umfeld des ICA 2012), 19.7.2012.

Marc Becker, Wissenschaftler an der Truman State University: Persönliche Kommunikation (E-Mail), 3.-12.5.2011, 28.7.2011, 14.8.2011.

Manuel Chiriboga, Wissenschaftler des Andinen Zentrums der Volksaktion (Centro Andino de Acción Popular, CAAP): Persönliche Kommunikation (E-Mail), 12.2.2012.

Tomás Condori, permanenter Repräsentant des unterstützenden Außenkomitées des Indiorates Südamerikas (Comité de Exterior de apoyo al Consejo Indio de Sud América, CEA-CISA) vor der UNO: Persönliche Kommunikation (E-Mail), 25.7.2011.

Jorge León Trujillo, Wissenschaftler des Zentrums für Erforschung und Entwicklung der sozialen Bewegungen Ecuadors (Centro para la Investigación y el Desarrollo de los Movimientos Sociales del Ecuador, CEDIME): Persönliche Kommunikation (E-Mail), 6.5.2011, 4.-13.2.2012.

Juan Paz y Miño, Professor an der Pontificia Universidad Católica del Ecuador (PUCE): Interview, 16.9.2008.

Floresmilo Simbaña, Wissenschaftler und Mitglied von ECUARUNARI, Interviews (Simbaña I-1: 5.2.2009 und Simbaña I-2: 19.6.2009).

Teilnehmende Beobachtung: IV Cumbre Continental de los Pueblos Indígenas de la Abya Yala, Universidad Nacional del Altiplano, Puno, Peru, 27.-31.5.2009. 



\section{Internetseiten}

Centro Andino de Acción Popular (CAAP) (http://www.caapecuador.com/)

Coordinadora Andina de Organizaciones Indígenas (CAOI) (http://minkan dina.org/)

Coordinadora de las Organizaciones Indígenas de la Cuenca Amazónica (COICA) (http://www.coica.org.ec/)

Coordinadora Latinoamericana de Organizaciones del Campo (CLOC) (http://movi mientos.org/cloc/ und http://www.cloc-viacampesina.net/)

Confederación Ecuatoriana de Organizaciones Clasistas Unitarias de Trabajadores (CEDOCUT) (http://www.cedocut.org/cms/)

Confederación de las Nacionalidades Indígenas del Ecuador (CONAIE) (http://www.conaie.org/)

Confederación de las Nacionalidades Indígenas de la Amazonia Ecuatoriana (CONFENIAE) (http://www.ecuanex.net.ec/confeniae/)

Confederación Kichwa del Ecuador ECUARUNARI (http://ecuarunari.org/portal/)

Confederación Nacional de Organizaciones Campesinas, Indígenas y Negras (FENOCIN) (http://www.fenocin.org/)

Confederación de Pueblos y Organizaciones Indígenas Campesinas del Ecuador (FEI) (http://www.confederacionfei.com/)

Confederación de Trabajadores del Ecuador (CTE) (http://www.cte-ecuador.org/)

Confederación Sindical de Trabajadoras y Trabajadores del Ecuador (CSE) (http://www.cse-ec.org/index.php)

Confederación Única Nacional de Afiliados al Seguro Social Campesino-Consejo Nacional Campesino (CONFEUNASSC-CNC) (http://movimientos.org/cloc/ confeunassc-ec/)

Consejo de Pueblos y Organizaciones Indígenas Evangélicas del Ecuador (FEINE) (http://www.feine.org.ec/)

Federación de Comunas Unión de Nativos de la Amazonía Ecuatoriana (FCUNAE) (http://fcunae.nativeweb.org/)

Federación Interprovincial de Centros Shuar (FICSH) (http://ficsh.org.ec/)

Federación Nacional de Trabajadores Agroindustriales, Campesinos e Indígenas Libres del Ecuador (FENACLE) (http://www.fenacle.org.ec/) 
Instituto Científico de Culturas Indígenas (ICCI) (http://icci.nativeweb.org/)

Unión de Organizaciones Campesinas Indígenas de Cotacachi (UNORCAC) (http://unorcac.nativeweb.org/index.html)

Universidad Intercultural de las Nacionalidades y Pueblos Indígenas ,Amawtay Wasi` (http://www.amawtaywasi.edu.ec/) 


\section{Global Studies}

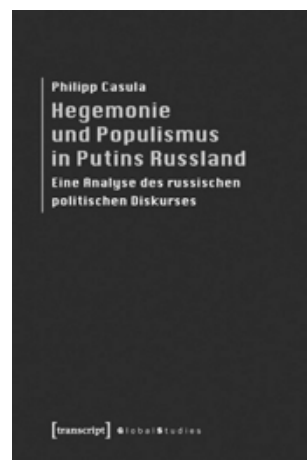

Philipp Casula

Hegemonie und Populismus in Putins Russland Eine Analyse des russischen politischen

Diskurses

20I2, 350 Seiten, kart., 33,80€,

ISBN 978-3-8376-2105-I

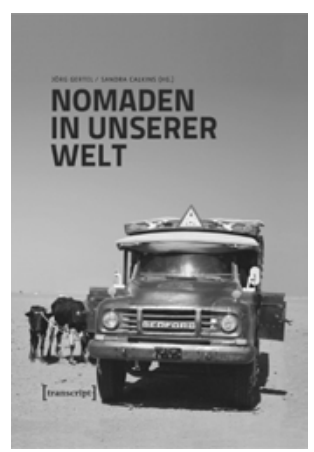

Jörg Gertel, Sandra Calkins (Hg.)

Nomaden in unserer Welt

Die Vorreiter der Globalisierung: Von Mobilität und Handel, Herrschaft und Widerstand

201I, 304 Seiten, Hardcover,

durchgehend vierfarbig bebildert, I9,80 €,

ISBN 978-3-8376-1697-2

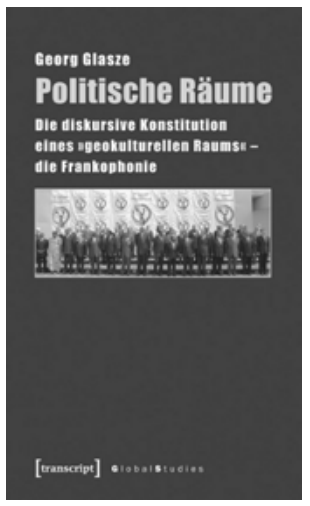

Georg GLasze

Politische Räume

Die diskursive Konstitution eines

»geokulturellen Raums« - die Frankophonie

März 2013, 272 Seiten, kart., 29,80€,

ISBN 978-3-8376-I232-5 


\section{Global Studies}
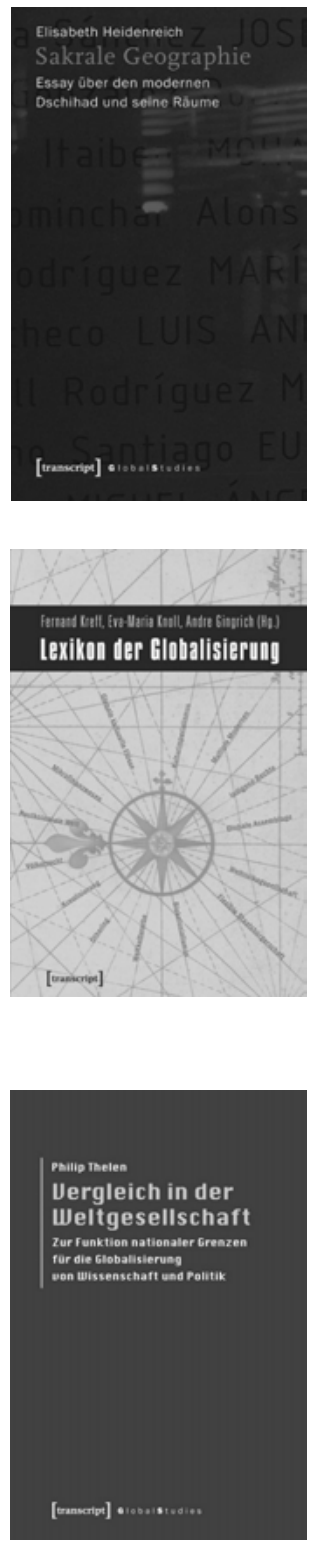

Elisabeth Heidenreich

Sakrale Geographie

Essay über den modernen Dschihad und seine Räume

20I0, 328 Seiten, kart., 27,80€, ISBN 978-3-8376-I604-0

Fernand Kreff, Eva-Maria Knoll, ANDRE Gingrich (Hg.) Lexikon der Globalisierung

20II, 536 Seiten, kart., 29,80 €, ISBN 978-3-8376-I822-8
Philip Thelen

Vergleich in der Weltgesellschaft Zur Funktion nationaler Grenzen für die Globalisierung von Wissenschaft und Politik 20II, 378 Seiten, kart., $32,80 €$, ISBN 978-3-8376-19I3-3 


\section{Global Studies}

SEYHAN BAyrakTAR

Politik und Erinnerung

Der Diskurs über den Armeniermord

in der Türkei zwischen Nationalismus und Europäisierung

20I0, 3I4 Seiten, kart., 29,80€,

ISBN 978-3-8376-I3I2-4

Tobias Boos

Ethnische Sphären

Über die emotionale Konstruktion von Gemeinschaft bei syrisch- und libanesischstämmigen Argentiniern

Februar 2013, 276 Seiten, kart., zahlr. Abb., 34,80€, ISBN 978-3-8376-2336-9

UlRike CAPDEPón

Vom Fall Pinochet zu den Verschwundenen des Spanischen Bürgerkrieges

Die Auseinandersetzung mit Diktatur und Menschenrechtsverletzungen

in Spanien und Chile

Februar 20I4, ca. 380 Seiten, kart., ca. $34,80 €$, ISBN 978-3-8376-2347-5

\section{PATRICK EsER}

Fragmentierte Nation globalisierte Region?

Der baskische und katalanische Nationalismus im Kontext von Globalisierung und europäischer Integration

März 20I3, 538 Seiten, kart., 39,80 €, ISBN 978-3-8376-2344-4

Schirin Fathi (Hg.)

Komplotte, Ketzer und Konspirationen

Zur Logik des Verschwörungsdenkens

- Beispiele aus dem Nahen Osten

20Iо, 326 Seiten, kart., 29,80 €,

ISBN 978-3-8376-I34I-4
BARBARA GRIMPE

Ökonomie sichtbar machen

Die Welt nationaler Schulden in

Bildschirmgröße. Eine Ethnographie 20I0, 290 Seiten, kart.,

zahlr. z.T. farb. Abb., $32,80 €$,

ISBN 978-3-8376-I608-8

HeIKo GRÜNWEDEL

Schamanismus zwischen Sibirien und Deutschland

Kulturelle Austauschprozesse in globalen religiösen Diskursfeldern

Juli 2013, 364 Seiten,

kart., zahlr. Abb., $34,80 €$,

ISBN 978-3-8376-2046-7

Ernst Halbmayer, Sylvia Karl (Hg.)

Die erinnerte Gewalt

Postkonfliktdynamiken

in Lateinamerika

20I2, 340 Seiten, kart., 32,80€,

ISBN 978-3-8376-1858-7

KATHARINA INHETVEEN

Die politische Ordnung

des Flüchtlingslagers

Akteure - Macht - Organisation.

Eine Ethnographie im Südlichen Afrika

2о1о, 444 Seiten, kart., zahlr. Abb., 35,80€,

ISBN 978-3-8376-I378-o

Stefan Luft, Peter Schimany (Hg.)

Integration von Zuwanderern

Erfahrungen, Konzepte, Perspektiven

2010, 360 Seiten, kart., 29,80€,

ISBN 978-3-8376-I438-I

MARgarete Misselwitz,

Kiaus Schlichte (Hg.)

Politik der Unentschiedenheit

Die internationale Politik und

ihr Umgang mit Kriegsflüchtlingen

20I0, 300 Seiten, kart., 29,80€,

ISBN 978-3-8376--зіо-о 
